\title{
INCLUSION CHEMISTRY OF \\ TRIS(5-ACETYL-3-THIENYL)METHANE (TATM) \\ MATERIALS
}

by

PAUL S. SIDHU

BSc., Dalhousie University, Halifax, Nova Scotia, 1992

MSc., University of Guelph, Guelph, Ontario, 1994

A THESIS SUBMITTED in PARTIAL FULFILMENT of the REQUIREMENTS for the DEGREE of DOCTOR of PHILOSOPHY

to

THE DEPARTMENT OF CHEMISTRY

and

THE FACULTY of GRADUATE STUDIES and RESEARCH

\section{CARLETON UNIVERSITY \\ OTTAWA, ONTARIO}

CANADA

April 2005

(C) Copyright

Paul S. Sidhu, 2005 
Library and

Archives Canada

Published Heritage

Branch

395 Wellington Street Ottawa ONK1A ON4

Canada
Bibliothèque et

Archives Canada

Direction du

Patrimoine de l'édition

$0-494-06746-2$

395, rue Wellington

Ottawa ON K1A ON4

Canada

NOTICE:

The author has granted a nonexclusive license allowing Library and Archives Canada to reproduce, publish, archive, preserve, conserve, communicate to the public by telecommunication or on the Internet, loan, distribute and sell theses worldwide, for commercial or noncommercial purposes, in microform, paper, electronic and/or any other formats.

The author retains copyright ownership and moral rights in this thesis. Neither the thesis nor substantial extracts from it may be printed or otherwise reproduced without the author's permission.
AVIS:

L'auteur a accordé une licence non exclusive permettant à la Bibliothèque et Archives Canada de reproduire, publier, archiver, sauvegarder, conserver, transmettre au public par télécommunication ou par l'Internet, prêter, distribuer et vendre des thèses partout dans le monde, à des fins commerciales ou autres, sur support microforme, papier, électronique et/ou autres formats.

L'auteur conserve la propriété du droit d'auteur et des droits moraux qui protège cette thèse. $\mathrm{Ni}$ la thèse ni des extraits substantiels de celle-ci ne doivent être imprimés ou autrement reproduits sans son autorisation.
In compliance with the Canadian

Privacy Act some supporting forms may have been removed from this thesis.

While these forms may be included in the document page count, their removal does not represent any loss of content from the thesis.
Conformément à la loi canadienne sur la protection de la vie privée, quelques formulaires secondaires ont été enlevés de cette thèse.

Bien que ces formulaires aient inclus dans la pagination, il n'y aura aucun contenu manquant.

\section{Canada}




\begin{abstract}
A wide variety of materials characterization techniques are used to investigate physicochemical properties of tris(5-acetyl-3-thienyl)methane (TATM) inclusion compounds with, in part, alkyl halide guests and alkyl halide guest families. The results are presented as nine different projects. In the first, we use NMR and X-ray diffraction to characterize the TATM inclusion compound with 1,2-dichloroethane that crystallizes in at least two different forms. We came up with a simple NMR model to describe the motion of the guest, however since it was not consistent with the XRD data, we came up with a second model that was consistent with both experiments. In the second, we characterized TATM inclusion compounds with alkyl halide guest families. We found, based on DSC results, that TATM forms more thermally stable inclusions with guests that have smaller, more electronegative substituents, perhaps due to stronger dipole interactions between host and guest. The third and fourth projects dealt with competitive inclusion experiments and the nature of the inclusion mixtures respectively, for the same guests as in the second project above. Alkyl bromides were the most preferred guests, followed by alkyl chlorides and alkyl iodides. We reasoned this on the basis of a balance of forces of cage stretching for larger guests and lack of extensive dispersion forces between guest and host for smaller guests. The alkyl bromides bring about a balance of the forces to qualify as the most preferred guest. In the fifth project, we characterize TATM inclusion compounds with $\mathrm{Cl}\left(\mathrm{CH}_{2}\right)_{11} \mathrm{Cl}$ guests $\left(n=1\right.$ to 10 ), using techniques such as PXRD, ${ }^{13} \mathrm{C} \mathrm{CP} / \mathrm{MAS}$ NMR and DSC. Following this, we report a TATM inclusion compound with 1,3-dichloropropane guest that crystallizes in five 2:1 H:G forms. This is an unprecedented discovery, and suggests that minor changes in synthetic procedures can have a profound effect on the structure of the resulting complex. In the next chapter, we report a study similar to that described above for $\mathrm{Cl}\left(\mathrm{CH}_{2}\right)_{n} \mathrm{Cl}$ guests, but now we study $\mathrm{Br}\left(\mathrm{CH}_{2}\right)_{n} \mathrm{CH}_{3}(n=1$ to 15) guests, and compare results among the two groups. Next, we report the structure of the hydrophobic TATM host with water as a guest. Finally, we describe the synthesis and characterization of a novel crystalline guest-free form of TATM.
\end{abstract}




\section{ACKNOWLEDGEMENTS}

This work would not have been possible were it not for the kind assistance of several people for whose efforts I wish to acknowledge here:

First and foremost, I would like to show appreciation to my graduate supervisor, Professor John Ripmeester, who helped to guide me through to the completion of this project. $\mathrm{He}$ was always willing to listen, sometimes providing interesting ideas, and keeping me on a straight and narrow course. Thank you Dr Ripmeester.

Secondly, I wish to thank Dr. Gary Enright, who obtained all of the crystal structures in this thesis. Without his kind help, my thesis would have been very incomplete and onedimensional. He never once complained when I had "yet another" batch of crystals to examine. Thank you Dr Enright. I would also like to thank Konstantin Udachin, for preparing most all of the single crystal packing diagrams contained in this thesis, taking time out of his busy schedule to help me. Thank you Kostiya.

I would like to thank Don Leek, who measured all of the ${ }^{1} \mathrm{H}$ and ${ }^{13} \mathrm{C}$ liquid NMR spectra contained in my thesis (and a lot more not in the thesis). Thank you for your kind assistance Don. Thanks to our glass-blowers, Mike Vandenhoff and Darcy Doering, for giving your time to help me. Also thanks to the computer support group Randy Holmes, Lyne Seguin and Claude Cantin. Thank you to our librarian Bonnie Bullock for your help.

I wish to thank the Natural Sciences and Engineering Research Council (NSERC) committee for me awarding a post-graduate scholarship that I held for two years. I am also grateful to the Ontario Graduate Scholarship (OGS) committee for awarding me a postgraduate scholarship that I held for one year. I also wish to acknowledge Carleton University for awarding me an entrance scholarship and the H. J. Bernstein memorial award.

Finally, I wish to thank my thesis committee, for taking time out their busy schedules to read my thesis and ask me interesting questions. I hope we all can learn something new. 


\section{MOTIVATION}

My $\mathrm{PhD}$ project involves physicochemical characterization of TATM inclusion compounds, at least in part, with alkyl halide guests and alkyl halide guest families. One type of study (Chapters VII and XII) involves characterization of a single guest (1,2dichloroethane and 1,3-dichloropropane, respectively), while other projects (Chapters VIII/IX/X, XI and XIII) encompass guest families (alkyl and aryl halides, $\alpha,(\omega)$ dichloroalkanes and 1-bromoalkanes, respectively). We wish to examine how the physical properties of the TATM inclusion compound change as the guest's structure is systematically altered (eg. changing halogen substituents or alkyl chain lengthening). The other two projects involve preparation and characterization of an expressly hydrophobic TATM host with water as a guest, and the synthesis and description of a novel polycrystalline guest-free TATM structure. This fact that TATM will incorporate practically anything in which it can be dissolved itself justifies a detailed study of this host system, namely the driving forces and intermolecular interactions in TATM inclusion compounds that lead to its remarkable inclusion versatility.

The results presented in this thesis are primarily focused on using several physical characterization techniques to describe properties of these TATM inclusion compounds. For example, single crystal X-ray diffraction allows one to visualize the intermolecular interactions that lead to the remarkable versatility of the TATM host. However, this technique measures only a single selected crystal, so we also used powder X-ray diffraction and ${ }^{13} \mathrm{C}$ CP/MAS NMR to determine whether the selected crystal was representative of the bulk sample. This is important, since a number of TATM inclusions are known to crystallize in more than one form. Deuterium solid-state NMR allows a description of the dynamics of an enclathrated guest molecule. Thermal techniques (TGA and DSC) allow a measurement of relative thermal stabilities for a series of related guests. Competitive inclusion experiments may provide complementary information, in that more thermally stable samples may be preferentially incorporated by TATM from a guest mixture. In summary, by employing a comprehensive range of techniques, we hope to characterize our TATM compounds in as complete a manner as possible. 


\section{PUblications}

(1) Sidhu, P.S., Enright, G.D., Udachin, K.A. and Ripmeester, J.A. "The first example of a crystalline guest-free form of the tris(5-acetyl-3-thienyl)methane host material" Chemical Communications 2092-2094 (2005).

(2) Sidhu, P.S., Enright, G.D., Udachin, K.A. and Ripmeester, J.A. "Polymorphism and structure in a pentamorphic guest-host material: The tris(5-acetyl-3thienyl)methane inclusion compound with 1,3-dichloropropane" Crystal Growth and Design 4(6), 1249-1257 (2004).

(3) Sidhu. P.S., Udachin, K.A. and Ripmeester, J.A. "Water and tris(5-acetyl-3thienyl)methane assemble into a one-dimensional channel compound" Chemical Communications 1358-1359 (2004).

(4) Sidhu, P.S. and Ripmeester, J.A. "Physicochemical characterization of tris(5acetyl-3-thienyl)methane inclusion compounds with $\alpha,(1)$-dichloroalkane guests ( $n=$ 1 to 10). A combined TGA, ${ }^{13} \mathrm{C}$ CP/MAS NMR, powder X-ray diffraction and DSC study" Supramolecular Chemistry 15(6), 433-446 (2003).

(5) Sidhu. P.S.. Enright, G.D.. Ripmeester, J.A. and Penner, G.H. (2002) -Polymorphism. structure, guest conformation and dynamics in the inclusion compound of 1.2-dichloroethane with tris(5-acetyl-3-thienyl)methane. A combined single erystal and powder X-ray diffraction, ${ }^{13} \mathrm{C} C \mathrm{CP}$ /MAS and ${ }^{2} \mathrm{H}$ NMR study" The Journal of Phisical Chemistry B 106, 8569-8581 (2002).

(6) Sidhu, P.S. and Ripmeester, J.A. "Structural type and thermal stability of tris (5acetyl-3-thienyl) methane inclusion compounds. A combined ${ }^{13} \mathrm{C}$ CP/MAS NMR, powder $X$-ray diffraction and differential scanning calorimetric study" The Journal of Supramolecular Chemistry 1, 63-76 (2001).

(7) Falk, M., Sidhu. P.S. and Walter, J.A. "Conformation of kainic acid in solution from molecular modelling and NMR spectra" Natural Toxins 6, 159-171 (1998).

(8) Sidhu, P.S.. Penner. G.H., Jeffrey, K.R., Zhao. B., Wang. Z.L. and Goh, I. "A solid-state NMR study of guest molecule dynamics in 4-alkyl-tert-butylbenzene / thiourea inclusion compounds" The Journal of Physical Chemistry B 101.90879097 (1997).

(9) Sidhu, P.S., Bell, J., Penner, G.H. and Jeffrey, K.R. (1996) "A deuterium NMR study of guest molecular dynamics in tris (5-acetyl-3-thienyl) methane inclusion compounds" The Canadian Journal of Chemistry 74, 1784-1794 (1996).

(10) Sidhu, P.S., Bell, J., Penner, G.H. and Jeffrey, K.R. “A deuterium NMR study of guest molecular dynamics of acetone in two organic inclusion compounds" The Canadian Journal of Chemistry 73, 2196-2207 (1995). 
(1) Ontario graduate scholarship Carleton University January 2002 - December 2002

$\$ 15000$

(2) NSERC PGS B post-graduate scholarship

Carleton University

May 1999 - December 2001

$\$ 38200$

(3) Harold J. Bernstein memorial award

Carleton University

$1998-1999$

$\$ 2000$

(4) Entrance scholarship

Carleton University

$1998-1999$

$\$ 2000$

(5) In-course scholarship University of Guelph $1993-1994$

$\$ 1600$

(6) Entrance scholarship University of Guelph $1992-1993$

$\$ 1600$

(7) Entrance scholarship Dalhousie University $1985-1986$

$\$ 1000$ 


\section{TABLE OF CONTENTS}

Title Page

Acceptance Form

i

Abstract

iii

Acknowledgements iv

Motivation

Publications

v

Awards

Table of Contents

List of Tables

List of Figures

List of Abbreviations and Symbols

vi

vii

viii-xii

xiii-xvi

$x v i-x x i i i$

xxiii-xxxiii

Chapter I

Introduction to inclusion compounds

References

2

5

Chapter II

Halogenated hydrocarbons

7

References

10

Chapter III TATM literature review

(A) J. Org. Chem. (USSR) 9, 1549 (1973) 11

(B) J. Chem. Soc. Chem. Commun. 21, 741 (1977) 12

(C) Acta Cryst. C47. 2468 (1991) 14

(D) Acta Cryst. C48, 2229 (1992) 14

(E) Acta Cryst. C48, 1882 (1992) 14

(F) Acta Cryst. C47.2718 (1991) 14

(G) Acta Cryst. B50, 112 (1994) 19

(H) Acta Cryst. C50, 615 (1994) 25

(I) Acta Cryst. C50. 1947 (1994) 27

(J) Can. J. Chem. 72, 2318 (1994) 29

(K) Acta Cryst. B53, 168 (1997) 38

(L) Can. J. Chem. 73, $2196(1995)$

(M) Can. J. Chem. 74, 1784 (1996) 55

Concluding remarks 72

References $\quad 73$

$\begin{array}{lll}\text { Chapter IV } & \text { Other host systems } & 74\end{array}$

(A) Urea $\quad 74$

(B) Tri-ortho-thymotide (TOT)

(C) Hydroquinone ( $\beta$-quinol) $\quad 77$

(D) Dianin's compound 78

(E) Gossypol $\quad 79$

(F) Perhydrotriphenylene (PHTP) 80

References 81

viii 
$\begin{array}{lll}\text { Chapter V Techniques employed } & 84\end{array}$

References $\quad 89$

Chapter VI Experimental details 91

References $\quad 95$

Chapter VII 1,2-Dichloroethane / TATM 96

Abstract $\quad 97$

Introduction $\quad 98$

Experimental $\quad 98$

Results

(A) Single crystal X-ray Diffraction 99

(B) Powder X-Ray Diffraction 103

(C) ${ }^{13} \mathrm{C} \mathrm{CP/MAS} \mathrm{NMR} \quad 104$

(D) ${ }^{2} \mathrm{H}$ Solid-State NMR 106

(1) Model 1: Trans-Gauche Equilibrium $\quad 106$

(2) Model 2: Restricted Cl Motion 112

(3) Model 3: Large Scale Cl Motion 113

Concluding remarks 116

References $\quad 117$

$\begin{array}{lll}\text { Chapter VIII } & \text { Structural Type and Thermal Stability } & 118\end{array}$

Abstract $\quad 119$

Introduction 119

Experimental $\quad 121$

Results

(A) ${ }^{13} \mathrm{C}$ CP/MAS NMR $\quad 122$

(B) Powder X-Ray Diffraction 127

(C) Differential Scanning Calorimetry 133

Discussion

(D) Solid solution model 137

(E) Cyclodextrins $\quad 139$

(F) Cholic acid 146

(G) Gossypol 147

(H) Other host systems

(1) 1,2,3,4,5-penta-O-berzoyl-6-O-tosyl myo-inositol 148

(2) 2,4,6-tris(4-halophenoxy)-1,3,5-triazine $\quad 149$

(3) $\mathrm{Cd}(\mathrm{pm}) \mathrm{Ni}(\mathrm{CN})_{4} \quad 150$

Concluding remarks $\quad 152$

References 153 
Introduction

Experimental

Results

Discussion

(A) $\beta$-quinol

(B) Dianin's compound

(C) 1,1-bis(4-hydroxyphenyl)cyclohexane

172

(D) Cyclophane

(E) 2,5-bis(9-hydroxyfluoren-9-yl)thieno[2.3-b]thiophene

Concluding remarks

References

177

Supplemental Material

Chapter X

The Nature of Inclusion Mixtures

Abstract

Introduction

Experimental

Results

(A) 1,3-Dihalopropane / TATM pure phases

(1) Powder X-Ray Diffraction 192

(2) ${ }^{13} \mathrm{C}$ CP/MAS NMR 195

(3) Single Crystal Structures 198

(a) 1.3-difluoropropane TATM 199

(b) 1.3-dichloropropane TATM 201

(c) 1,3-dibromopropane TATM 201

(B) Nature of Inclusion Mixtures 204

(4) Methylene Halide Guests 205

(5) 1,3-Dihalopropane Guests 207

(6) 2-Halobutane Guests 209

(7) Cyclohexyl Halide Guests 211

Discussion 213

(C) $\left[\mathrm{N}\left(\mathrm{CH}_{3}\right)_{4} \cdot \mathrm{xG}\right]\left[\mathrm{Cd}_{3}(\mathrm{CN})_{7}\right] \quad 216$

(D) 1,1,2,2-tetraphenylethane-1,2-diol 217

(E) Cholic acid 218

Concluding remarks 221

References 223 
Results
(A) Thermogravimetric Analysis
(B) ${ }^{13} \mathrm{C} \mathrm{CP/MAS} \mathrm{NMR}$
(C) Powder X-Ray Diffraction
(D) Differential Scanning Calorimetry
Discussion
(E) Urea
(F) Cyclodextrins
256
(G) 2,2,6,6-tetramethyl-4-oxopiperidine-l-oxyl 256
(H) Graphite 258
Concluding remarks $\quad 260$
References

The Pentamorphic TATM/1.3-Dichloropropane Complex 263

Abstract

Introduction

Experimental

Results

(A) Powder Diffraction and ${ }^{13} \mathrm{C} \mathrm{CP} / \mathrm{MAS} N M R \quad 268$

(1) Forms 1 and $3 \quad 269$

(2) Form 2

(3) Forms 4 and $5 \quad 273$

(B) Thermogravimetric Analysis $\quad 275$

(C) Differential Scanning Calorimetry 276

(D) Polycrystalline Densities 281

(E) Single Crystal X-Ray Diffraction 281

$\begin{array}{ll}\text { (4) Form 1 } & 283 \\ \text { (5) Form 2 } & 284 \\ \text { (6) Form 3 } & 286 \\ \text { (7) Form 4 } & 288 \\ \text { (8) Form 5 } & 289\end{array}$

Discussion

(F) Conformational preferences 292

(G) Polymorphism in other systems 299

(9) Dianilinegossypol 299

(10) 5-methyl-2-[(2-nitrophenyl)amino]-3-thiophenecarbonitrile 301

Concluding remarks $\quad 304$

References $\quad 305$ 
Chapter XIII

Chapter XIV

Chapter XV

Chapter XVI
1-Bromoalkane Guest Family $\mathrm{Br}\left(\mathrm{CH}_{2}\right)_{n} \mathrm{CH}_{3}(n=1$ to 15$) 308$

Abstract

Introduction

Experimental

312

Results
(A) TGA and ' $\mathrm{H}$ Solution NMR
312
(B) PXRD and ${ }^{13} \mathrm{C}$ CP/MAS Solid NMR
318
(C) ${ }^{13} \mathrm{C}$ CP/MAS NMR with Dipolar Dephasing
327
(D) Differential Scanning Calorimetry
331
(E) Polycrystalline Densities
339
Discussion
342
Concluding remarks
347
References
348

Water / TATM Inclusion Compound

350

Abstract

350

Introduction

351

Experimental

352

Results

352

References

358

359

360

361

362

Experimental

(A) Single Crystal X-Ray Diffraction 363

(B) Powder X-Ray Diffraction

373

378

379

(D) Thermogravimetric Analysis

$\begin{array}{ll}\text { (E) Differential Scanning Calorimetry } & 381 \\ \text { (F) Polycrystalline Densities } & 388\end{array}$

(G) Vapor Diffusion $\quad 390$

Discussion

(H) Effect of Guest Size upon Host Lattice 393

(I) Gossypol 396

(J) Hydroquinone 398

(K) Perhydrotriphenylene 401

(L) Dianin's compound 403

Summary $\quad 405$

Concluding remarks 406

References 407

Summary

Thesis Summary

411

Suggestions for Further Work $\quad 412$

Final Remarks 


\section{LIST OF TABLES}

\section{Chapter II: Halogenated hydrocarbons}

1. Radiative forcing, relative to $\mathrm{CO}_{2}$, for some common greenhouse gases

\section{Chapter III: TATM literature review}

1. Crystal data for literature TATM inclusion compounds with four guests 18

2. Bond lengths and angles for literature TATM acetyl thienyl rings 23

3. Bond lengths and angles for the ethanol and ethyl acetate 25

4. Crystal data for literature TATM inclusion compounds with six guests 31

5. Crystallographic data for literature inclusion complexes of TATM 41

6. Torsion angles for literature TATM inclusion compound structures 43

7. Values of non-bonded $\mathrm{H} \cdots \mathrm{H}$ distances in the 3 TATM conformational types 45

\section{Chapter VII: 1,2-Dichlorocthane / TATM}

1. Crystal data for the two forms of 1,2-dichloroethane / TATM

2. Guest conformational/dynamic parameters of 1,2-dichloroethane- $\mathrm{d}_{4} /$ TATM

\section{Chapter VIII: Structural Type and Thermal Stability}

1. Guest families with the asymmetric unit multiplicity and isostructuralism $\quad 128$

2. Guest families / TATM hot-stage microscopy and DSC melting points

3. A table of TATM guest family DSC enthalpy and entropy of decomposition 136

4. Association constants for $\alpha$ - and $\beta-C D$ with monohalobenzene guests

5. Association constants for $\alpha$ - and $\beta-C D$ with 1,4-disubstituted benzene guests 143

6. Association constants for $\alpha-, \beta$ - and $\gamma$-CD with monohalobenzene guests 144

7. Association constants for $\alpha-\beta$ - and $\gamma-C D$ with para-dihalobenzene guests 145

8. Crystal data for gossypol/halogenomethane derivatives 147

9. Crystal data for a myo-inositol derivative host with $\mathrm{CH}_{2} \mathrm{Cl}_{2}$ and $\mathrm{CH}_{2} \mathrm{Br}_{2} \quad 149$

10. Lattice type for $\mathrm{Cd}(p n) \mathrm{Ni}(\mathrm{CN})_{4}$ host with alkyl halide guest families 
Chapter IX: Competitive Inclusion Experiments

1. DSC $T_{\mathrm{m}}$ and $\Delta_{\text {fus }} H$ data for four TATM guest alkyl halide families 159

2. Competitive inclusion results for alkyl halide guest families 161

3. Competitive inclusion results for monohalobenzene guest families 165

4. Summary of competitive inclusion results for monohalobenzenes 165

5. Thermal and structural properties of $\beta$-quinol adducts vs. guest size 167

6. Relative accommodation factors for Dianin's compound inclusions 170

Chapter X: The Nature of Inclusion Mixtures

1. Unit cell parameters for 1,3-dihalopropane / TATM complexes 194

2. Single crystal data for 1,3-dihalopropane / TATM complexes 197

3. Occupancies of $m$-xylene and $p$-xylene in cholic acid single crystal mixtures 220

4. Occupancies of $m$-xylene and $p$-xylene in cholic acid bulk mixtures $\quad 220$

Chapter XI: $\quad \alpha, \omega$-Dichloroalkane Guest Family $\mathrm{Cl}\left(\mathrm{CH}_{2}\right)_{n} \mathrm{Cl}(n=1$ 10 10)

1. TGA data for the nine $\alpha, \omega$-dichloroalkane/TATM complexes 233

2. ${ }^{13} \mathrm{C}$ CP/MAS NMR data for the nine $\alpha,(1)$-dichloroalkane/TATM complexes 236

3. Percent increase in size on going from an $n$ to an $n+1$ carbon chain 240

4. Alkyl halide guests with asymmetric unit multiplicity and isostructuralism 241

5. $T_{\mathrm{m}}, \Delta_{\text {fus }} H$ and $\Delta_{\text {fus }} S$ for nine $\alpha,(1)$-dichloroalkane/TATM complexes 242

6. $\mathrm{H}: \mathrm{G}$ stoichiometric ratios, $T_{\mathrm{m}}$ and $\Delta_{\text {fus }} H$ data for urea/fatty acid complexes 245

7. Stoichiometric ratios and $\Delta H_{\text {dec }}$ for urea complexes with five guest families 247

8. Decomposition temperatures of urea / $n$-paraffin inclusion compounds 248

9. Enthalpy of decomposition for urea / n-paraffin inclusion compounds $\quad 249$

10. Decomposition temperatures of urea / n-substituted paraffin complexes 254

11. Enthalpy of decomposition for urea / n-substituted paraffin complexes $\quad 254$

12. $T_{\mathrm{m}}$ and $\Delta_{\text {fus }} H$ data for TATM complexes with $\alpha,(\omega$-dihaloalkane guests $\quad 255$

13. $\Delta G^{0}$ and $K_{\mathrm{a}}$ for the inclusion of $\beta-\mathrm{CD}$ with sodium alkyl sulfates 256

14. $T_{\mathrm{m}}$ and $\Delta_{\text {fus }} H$ data for tano / n-alkane inclusion compounds 258 
Chapter XII: The Pentamorphic TATM/1.3-Dichloropropane Complex

1. Synthetic techniques for the six samples of 1,3-dichloropropane/TATM 267

2. Synthesis, PXRD and SCXRD data for 1,3-dichloropropane/TATM 268

3. Unit cell data for 1,3-dichloropropane/TATM inclusions (form 2) 272

4. Unit cell data for 1,3-dichloropropane/TATM inclusions (form 5) 274

5. DSC data and densities for the six samples of 1,3-dichloropropane/TATM 277

6. Crystal data for the five forms of 1,3-dichloropropane/TATM 282

7. Torsion angles for TATM host molecules of this study 293

8. Torsion angles for TATM host molecules as reported in the literature 294

9. Torsion angles for 1.3-dichloropropane guest molecules of this study 298

10. Crystal data for 5-methyl-2-[(2-nitrophenyl)amino]-3-thiophenecarbonitrile 303

11. Structure data for 5-methyl-2-[(2-nitrophenyl)amino]-3-thiophenecarbonitrile 304

Chapter XIII: I-Bromoalkane Guest Family Br( $\left.\mathrm{CH}_{2}\right)_{n} \mathrm{CH}_{3}(n=1$ to 15$)$

1. H:G stoichiometric ratios for 1-bromoalkane/TATM inclusion compounds 315

2. Asymmetric unit contents / isostructuralism for l-bromoalkanes in TATM 324

3. Percent increase in size on going from an $n$ to an $n+1$ carbon chain 325

4. ${ }^{13} \mathrm{C}$ NMR solution chemical shifts for l-bromoalkanes 328

5. ${ }^{13} \mathrm{C}$ NMR solution chemical shifts for $\alpha,()$-dichloroalkanes 330

6. DSC $T_{\mathrm{m}}$ and $\Delta_{\text {fus }} H$ for the fifteen 1-bromoalkane/TATM inclusions 334

7. Crystal data for commensurate urea / 1,6-dihaloalkane inclusion compounds 344

Chapter XIV: Water / TATM Inclusion Compound

1. Crystal data for the single-crystal structure of $2 \mathrm{H}_{2} \mathrm{O} \cdot$ TATM 357

Chapter XV: Crystalline Guest-Free TATM

1. Crystal data for guest-free TATM and the three starting materials 364

2. Torsional angles for the three starting materials and guest-free TATM 369

3. Torsion angles for TATM host molecules as reported in the literature $\quad 370$

4. Dimensions of the host channel for TATM inclusion compounds 372

5. SCXRD and PXRD unit cell parameters for the three starting materials 374 
6. SCXRD and PXRD unit cell parameters for the three guest-free samples

7. DSC data for the three starting materials and the three guest-free samples

8. Residual guest and residual water content of guest-free TATM samples

9. Polycrystalline densities measured in bulk using a pycnometer apparatus

10. Crystal data for $(\alpha+1),(\omega-1)$-alkanedione/urea complexes

11. Crystal data for clathrates of the Dianin's compound thia-analogue

12. Crystal data for gossypol guest-free forms $P_{1}, P_{2}$ and $P_{3}$

13. Crystal data for selected $\beta$-quinol inclusion compounds

14. Crystal data for $\alpha$-quinol and $\gamma$-quinol

15. Crystal data for the two guest-free forms of perhydrotriphenylene

\section{LIST OF FIGURES}

\section{Chapter III: TATM literature review}

1. Perspective view of ethyl acetate / TATM with atomic numbering scheme 15

2. Perspective view of benzene / TATM with atomic numbering scheme $\quad 16$

3. Perspective view of $n$-hexane / TATM with atomic numbering scheme 16

4. Stereopair of $n$-hexane / TATM showing the crystal packing 17

5. Perspective view of ethanol / TATM with atomic numbering scheme 17

6. Electron density map of the ethyl acetate/TATM inclusion compound 21

7. Electron density map of the ethanol/TATM inclusion compound 21

8. An acetyl thienyl ring fragment of TATM, showing the numbering scheme 23

9. A view of the unit cell of TATM / $\mathrm{CCl}_{4}$ down the $c$ axis 26

10. A comparison of the molecular orientations of $\mathrm{CCl}_{4}$ and benzene 27

11. Structure of cyclononanone/TATM with the atomic numbering scheme 28

12. A stereoscopic view of the unit cell contents of cyclononanone/TATM 29

13. The unit cell contents of the TATM / cyclohexane $\mathbf{A}$ inclusion compound 33

14. The unit cell contents of the TATM cyclohexane B inclusion compound 35

15. Molecular orientation of cyclohexane, -heptane, and -octane inclusions 36

16. Molecular dynamics simulations for: cycloheptane and -octane inclusions 37

17. TATM structure, indicating the six bonds about which torsion can occur 41 
18. Molecular fragment used in search for syn and anti conformations

19. ${ }^{13} \mathrm{C} \mathrm{CP/MAS} \mathrm{NMR} \mathrm{spectrum} \mathrm{of} \mathrm{the} \mathrm{acetone} \mathrm{/} \mathrm{TATM} \mathrm{inclusion} \mathrm{compound}$ 48

20. Deuterium NMR spectra for acetone- $d_{6} /$ TATM at various temperatures 49

21. Graph of $\ln k$ vs $1000 / T$ for the two-fold flip of the acetone- $\mathrm{d}_{6} /$ TATM 50

22. Variable temperature ${ }^{2} \mathrm{H} T_{1}$, for the acetone- $\mathrm{d}_{6} /$ TATM 51

23. ${ }^{2} \mathrm{H}$ NMR spectrum of acetone- $\mathrm{d}_{6} /$ TATM at $84 \mathrm{~K}$, along with simulation 54

24. ${ }^{2} \mathrm{H}$ NMR spectra for acetonitrile- $\mathrm{d}_{3} /$ TATM at various temperatures 56

25. ${ }^{2} \mathrm{H}$ NMR spectra for nitromethane- $\mathrm{d}_{3} /$ TATM at various temperatures 57

26. $\eta_{\mathrm{efr}}$ and $\chi_{\mathrm{efr}}$ for acetonitrile and nitromethane / TATM inclusions 59

27. Experimental and simulated ${ }^{2} \mathrm{H}$ NMR spectra for DMSO- $\mathrm{d}_{6} /$ TATM 61

28. Rate of internal rotation for DMSO- $\mathrm{d}_{6} / \mathrm{TATM}$ from ${ }^{2} \mathrm{H} T_{1}$ and ${ }^{2} \mathrm{H}$ spectra $\quad 62$

29. ${ }^{2} \mathrm{H} T_{1}$, as a function of reciprocal temperature, for DMSO-d $\mathrm{d}_{6} /$ TATM 63

30. ${ }^{2} \mathrm{H}$ NMR spectrum of benzene- $\mathrm{d}_{6} /$ TATM at $294 \mathrm{~K}$

31. ${ }^{2} \mathrm{H} T_{1}$ and $\chi_{\text {erf }}$ for benzene- $\mathrm{d}_{6} / \mathrm{TATM}$ as a function of $1000 / \mathrm{T}$

32. ${ }^{2} \mathrm{H}$ variable temperature NMR spectra of mesitylene- $\mathrm{d}_{9} /$ TATM 68

33. ${ }^{2} \mathrm{H}$ echo intensity, as a function of jump rate, for in-plane $\mathrm{C}_{3}$ rotation $\quad 69$

34. Simulated ${ }^{2} \mathrm{H}$ NMR spectra for $C_{3}$ rotation of a mesitylene molecule $\quad 70$

35. ${ }^{2} \mathrm{H}$ NMR spectra for: $o$-xylene- $d_{10} /$ TATM and $p$-xylene- $\mathrm{d}_{10} /$ TATM 71

Chapter IV: Other host systems

1. Urea host architecture 75

2. Stereoview of $\beta$-quinol host with MeNC guest 77

Chapter V: Techniques employed

1. Idealized TGA-DSC plot of an inclusion compound with a volatile guest 88

Chapter VII: 1,2-Dichloroethane / TATM

1. The unit cell of triclinic 1,2-dichloroethane/TATM 100

2A. Triclinic 1,2-dichloroethane/TATM down the $a$ axis 100

2B. Monoclinic 1,2-dichloroethane/TATM approximately down the $c$ axis 101

3. PXRD spectrum of 1,2-dichloroethane/TATM (pure triclinic form) 103

xvii 
4. PXRD spectrum of 1,2-dichloroethane/TATM (mixture) 104

5. ${ }^{13} \mathrm{C} \mathrm{CP/MAS} \mathrm{NMR} \mathrm{spectra} \mathrm{for} \mathrm{1,2-dichloroethane/TATM} 105$

6. Deuterium NMR spectra for 1,2-dichloroethane-d 4 /TATM (Model 1) 107

7. Energy diagram of the conformers of 1,2-dichloroethane 107

8. A plot of $\ln K_{\text {eq }}$ vs $1 / T$ for 1,2-dichloroethane-d di $_{4}$ TATM (Model 1) 108

9. P.E. diagram depicting the rates and barriers for 1,2-dichloroethane 110

10. ${ }^{2} \mathrm{H}$ NMR spectra for 1,2-dichloroethane- $\mathrm{d}_{4} / \mathrm{TATM}$ at $226 \mathrm{~K}$ and $246 \mathrm{~K} \quad 110$

11. A plot of $\ln k$ vs $1 / T$, for the 1,2-dichloroethane-d guest (Model 1) $\quad 112$

12. The reorientation of 1,2-dichloroethane in TATM (Model 3) 113

13. ${ }^{2} \mathrm{H}$ NMR spectra of 1,2-dichloroethane-d $+\mathrm{d}_{4} / \mathrm{TATM}$ (Model 3) 114

14. A plot of $\ln k$ vs $1 / T$ for the 1,2-dichloroethane-d 4 guest (Model 3) 114

15. The angle of libration vs $T$, for the 1,2-dichloroethane- $\mathrm{d}_{4}$ guest (Model 3) $\quad 114$

16. A plot of ${ }^{2} \mathrm{H} T_{1}$ vs $1 / T$ for 1,2-dichloroethane- $\mathrm{d}_{4} /$ TATM 115

Chapter VIII: Structural Type and Thermal Stability

1. Structure of alkyl halide / TATM guest molecule families 121

2. ${ }^{13} \mathrm{C}$ NMR spectrum and dipolar dephasing for guest-free TATM 123

3. ${ }^{13} \mathrm{C} \mathrm{CP/MAS} \mathrm{NMR} \mathrm{spectrum} \mathrm{of} \mathrm{the} \mathrm{ethanol} \mathrm{/} \mathrm{TATM} \mathrm{complex} 124$

4. ${ }^{13} \mathrm{C}$ CP/MAS NMR spectrum of the cyclohexane B / TATM complex 125

5. ${ }^{13} \mathrm{C} \mathrm{CP/MAS} \mathrm{NMR} \mathrm{spectrum} \mathrm{of} \mathrm{the} n$-hexane / TATM complex 126

6. PXRD and ${ }^{13} \mathrm{C} \mathrm{CP} / \mathrm{MAS}$ NMR of the 2-halobutanes in TATM 127

7. PXRD and ${ }^{13} \mathrm{C} C P / M A S ~ N M R$ of the monohalobenzenes in TATM 130

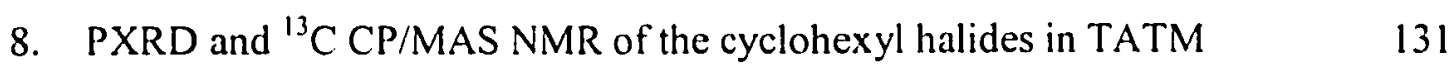

9. PXRD and ${ }^{13} \mathrm{C} C P / M A S N M R$ of the methylene halides in TATM 131

10. PXRD and ${ }^{13} \mathrm{C} C \mathrm{CP} / \mathrm{MAS} N \mathrm{NR}$ of the 1,3-dihalopropanes in TATM 132

11. DSC traces of the cyclohexyl halide / TATM inclusion compounds 133

12. Equilibrium constant vs refractive index for $\alpha$ - and $\beta-C D$ with halobenzenes 141

13. Depiction of orientation and location of monohalobenzenes in $\alpha$ - and $\beta-C D \quad 141$

14. Morphological transitions of gossypol host with halogenomethane guests 148

15. Sinusoidal chains of $\mathrm{C}-\mathrm{X} \cdots \pi$ and $\mathrm{C}-\mathrm{H} \cdots \pi$ interactions 150 
Chapter IX: Competitive Inclusion Experiments

1. DSC traces for monohalobenzene / TATM inclusion compounds

Chapter X: The Nature of Inclusion Mixtures

1. Mechanisms of selective and unselective competitive inclusion 189

2. The 1,3-dihalopropane guests focused on as pure phases with TATM 192

3. PXRD patterns for the pure phase 1,3-dihalopropane/TATM complexes 193

4. ${ }^{13} \mathrm{C}$ CP/MAS NMR spectra for pure 1,3-dihalopropane/TATM complexes 196

5. A schematic depiction of the asymmetric unit of 1,3-difluoropropane/TATM 198

6. A schematic depiction of the asymmetric unit of 1.3-dichloropropane/TATM 198

7. A schematic depiction of the asymmetric unit of 1,3-dibromopropane/TATM 199

8. Packing diagram for 1,3-difluoropropane/TATM in the $b c$ plane 200

9. Packing diagram for 1,3-difluoropropane/TATM in the ac plane 200

10. Packing diagram for 1,3-dichloropropane/TATM in the $a b$ plane 202

11. Packing diagram for 1,3-dichloropropane/TATM in the ac plane 203

12. Packing diagram for 1,3-dichloropropane/TATM in the $b c$ plane 203

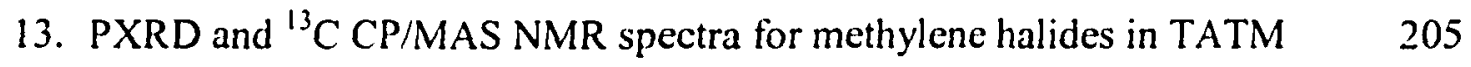

14. PXRD patterns for the methylene halide/TATM guest family mixtures 206

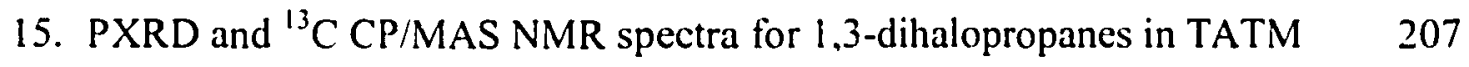

16. PXRD patterns for the 1,3-dihalopropane/TATM guest family mixtures 208

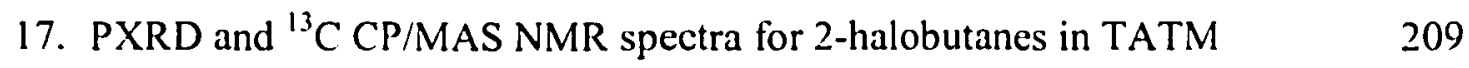

18. PXRD patterns for the 2-halobutane/TATM guest family mixtures 210

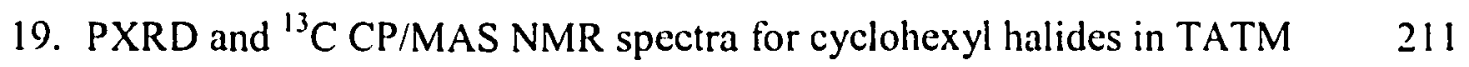

20. PXRD and ${ }^{13} \mathrm{C} \mathrm{CP} / \mathrm{MAS}$ NMR spectra for monohalobenzenes in TATM 212

21. PXRD patterns for the cyclohexyl halide/TATM guest family mixtures 214

22. PXRD data for single and mixed guest $\left[\mathrm{N}\left(\mathrm{CH}_{3}\right)_{4} \times \mathrm{G}\right]\left[\mathrm{Cd}_{3}(\mathrm{CN})_{7}\right]$ complexes 217 
Chapter XI: $\quad \alpha,(1)$-Dichloroalkane Guest Family $\mathrm{Cl}\left(\mathrm{CH}_{2}\right)_{11} \mathrm{Cl}(\mathrm{n}=1$ to 10$)$

1. The structure of the nine $\alpha, \omega$-dichloroalkane TATM guests of this study 230

2. TGA curves of the four smallest size $\alpha,(1)$-dichloroalkane/TATM guests 231

3. ${ }^{13} \mathrm{C}$ CP/MAS NMR spectra of three $\alpha, \omega$-dichloroalkane/TATM complexes 235

4. PXRD patterns of inclusions with the 4 smallest size $\alpha, \omega$-dichloroalkanes 238

5. PXRD patterns of inclusions with the 2 middle size $\alpha,(\omega)$-dichloroalkanes 238

6. PXRD patterns of inclusions with the 3 largest size $\alpha, \omega$-dichloroalkanes 239

7. DSC traces for the 4 smallest size guest $\alpha,(\omega$-dichloroalkanes in TATM $\quad 242$

8. $T_{\mathrm{m}}$ vs. guest chain length for $\alpha$,(1)-dichloroalkane/TATM inclusions 243

9. $\Delta_{\text {fus }} H$ vs. guest chain length for $\alpha,(1)$-dichloroalkane/TATM inclusions $\quad 243$

10. $\Delta_{\text {fus }} H$ vs. $T_{\mathrm{m}}$ for $\alpha, \omega$-dichloroalkane/TATM inclusions $\quad 244$

11. Schematic of urea host assembly 245

12. $\Delta_{\text {fus }} H$ vs. $T_{\mathrm{m}}$ for urea/long chain fatty acid inclusions 246

13. DTA traces for urea and urea / n-paraffin complexes $\quad 249$

14. $\Delta_{\text {lus }} H$ vs carbon chain length for urea / n-paraffin complexes 249

15. Melting temperatures of $n$-paraffins and urea / $n$-paraffin complexes $\quad 250$

16. TGA traces for a series of urea / n-paraffin inclusion compounds 251

17. TGA curves for a series of urea / $n$-alcohol complexes 253

18. Melting temperatures for even- and odd-numbered pure $n$-paraffins 258

19. DSC signal of $n$-hexadecane/graphite in the melting region 258

20. Solid-solid and solid-liquid transitions for $n$-paraffin/graphite complexes $\quad 259$

Chapter XII: The Pentamorphic TATM/1.3-Dichloropropane Complex

1A. Expt ${ }^{`} 1$ and calc'd PXRD of 1,3-dichloropropane/TATM (forms 1 and 3) 270

1B. ${ }^{13} \mathrm{C} \mathrm{CP/MAS} \mathrm{NMR} \mathrm{of} \mathrm{1,3-dichloropropane/TATM} \mathrm{(forms} 1$ and 3) 270

2. PXRD and ${ }^{13} \mathrm{C}$ NMR of 1,3-dichloropropane/TATM (form 2) 272

3. PXRD and ${ }^{13} \mathrm{C}$ NMR of 1,3-dichloropropane/TATM (form 5) 274

4. TGA of the six 1,3-dichloropropane/TATM products 276

5. DSC of 1,3-dichloropropane/TATM complexes (forms 1, 2 and 3) 278

6. DSC of 1,3-dichloropropane/TATM complexes (forms 4 and 5) 279

7. TGA and DSC of 1,1 -binaphthyl-2,2'-carboxylic acid with xylene guests 280 
8. Structure (of form 1) of 1,3-dichloropropane/TATM (both down $c^{*}$ ) 283

9. Structure (of form 2) of 1,3-dichloropropane/TATM ( $b c$ and $a b$ planes) 285

10. Structure (of form 3) of 1,3-dichloropropane/TATM (both down [001]) 287

11. Structure (of form 4) of 1,3-dichloropropane/TATM (both in $a b$ plane) 288

12. Structure (of form 5) of 1,3-dichloropropane/TATM ( $a b$ and $b c$ planes) 290

13. Schematic of the channels of forms $\mathbf{2}$ and $\mathbf{5}$ of 1,3-dichloropropane/TATM 291

14. ORTEP diagram of the $\beta$-dianilinegossypol / ethyl acetate unit cell 300

15. ORTEP diagram of the $\alpha$ - and $\beta$-dianilinegossypol / acetone unit cells 301

16. 5-methyl-2-[(2-nitrophenyl)amino]-3-thiophenecarbonitrile crystals 302

Chapter XIII: I-Bromoalkane Guest Family Br$\left(\mathrm{CH}_{2}\right)_{n} \mathrm{CH}_{3}(n=1$ to 15

1. A list of the fifteen 1-bromoalkane guests $\left\{\mathrm{Br}\left(\mathrm{CH}_{2}\right)_{n} \mathrm{CH}_{3}(n=1\right.$ to 15$\left.)\right\} \quad 311$

2. TGA curves of two 1-bromoalkane/TATM inclusion compounds 313

3. 'H NMR spectra of two I-bromoalkane/TATM inclusion compounds 314

4. Plot of stoichiometric ratio of 1 -bromoalkane inclusions vs. guest size $\quad 316$

5. Stoichiometric ratio vs guest chain length for urea/n-paraffin complexes 317

6. Stoichiometric ratio vs guest chain length for urea/carboxylic acid complexes 317

7. Stoichiometric ratio for thiourea / branched-paraffin complexes 318

8. Stoichiometric ratio for thiourea / $\alpha, \omega$-dicyclohexyl-paraffin complexes 318

9. PXRD and ${ }^{13} \mathrm{C}$ NMR for inclusions with the five smallest 1 -bromoalkanes 320

10. PXRD and ${ }^{13} \mathrm{C}$ NMR for inclusions with the five middle 1-bromoalkanes 321

11. PXRD and ${ }^{13} \mathrm{C}$ NMR for inclusions with the five largest 1-bromoalkanes 322

12. ${ }^{13} \mathrm{C}$ dipolar dephasing NMR spectra of the 1-bromoalkane inclusions 329

13. ${ }^{13} \mathrm{C}$ dipolar dephasing NMR spectra of the $\alpha,(1)$-dichloroalkane inclusions 330

14. DSC traces for six 1-bromoalkane / TATM inclusions 333

15. Plot of $T_{\mathrm{m}}$ and $\Delta_{\text {tus }} H$ vs guest size for the l-bromoalkane / TATM inclusions 334

16. Plot of $\Delta_{\text {fus }} H$ vs $T_{\mathrm{m}}$ for 1-bromoalkane / TATM inclusions $(\mathrm{Br} 2-\mathrm{Br} 9)$

17. Plot of secondary endotherm $T_{\mathrm{m}}$ and $\Delta_{\text {fus }} H$ vs. size of 1-bromoalkane guest 337

18. Plot of DSC and hot-stage melting points for 1-bromoalkane inclusions 338

19. Plot of polycrystalline densities vs size of I-bromoalkane guest 340

20. $2 \mathrm{D}$ representation of a urea inclusion compound, illustrating $c_{\mathrm{g}}, c_{\mathrm{h}}$ and $\Delta_{\mathrm{g}} \quad 342$ 
21. Schematic of hexagonal and orthorhombic urea inclusion compounds

22. Stereoview of the urea $/ \mathrm{Br}\left(\mathrm{CH}_{2}\right)_{6} \mathrm{Br}$ complex down the channel axis

23. Guest repeat distances for urea/ $\alpha, \omega$-dibromoalkane $(n=6$ to 10$)$ complexes 345

24. $\mathrm{X} \cdots \mathrm{X}, \mathrm{X} \cdots \mathrm{Y}$ and $\mathrm{Y} \cdots \mathrm{Y}$ end group interactions for $\mathrm{X}\left(\mathrm{CH}_{2}\right)_{\mathrm{n}} \mathrm{Y}$ guest molecules 346

\section{Chapter XIV: Water / TATM Inclusion Compound}

1. ${ }^{13} \mathrm{C}$ CP/MAS NMR spectra of TATM hydrate and sublimed TATM 353

2. View of $2 \mathrm{H}_{2} \mathrm{O} / \mathrm{TATM}$ approximately down the channel $(a)$ axis 353

3. View of $2 \mathrm{H}_{2} \mathrm{O} /$ TATM down the $c$ axis (perpendicular to the channel) 353

4. TGA trace of $2 \mathrm{H}_{2} \mathrm{O} /$ TATM as a function of temperature 355

5. Isothermal $\left(100^{\circ} \mathrm{C}\right) \mathrm{TGA}$ trace of $2 \mathrm{H}_{2} \mathrm{O} / \mathrm{TATM}$ as a function of time 355

6. Deuterium NMR spectrum of $2 \mathrm{D}_{2} \mathrm{O} / \mathrm{TATM}$ at room temperature 356

Chapter XV: Crystalline Guest-Free TATM

1. General scheme of formation and decomposition of an inclusion compound 362

2. TGA, DSC and PXRD techniques for following phase changes of a complex 362

3. Structure of acetone/TATM (in $b c$ and $a b$ planes) 366

4. Structure of ethanol/TATM (in $b c$ and $a c$ planes) 367

5. Structure of methanol/TATM (unit cell and $b c$ plane) 368

6. Structure of crystalline guest-free TATM (asymmetric unit and $b c$ plane) 369

7. PXRD and ${ }^{13} \mathrm{C} \mathrm{CP} / \mathrm{MAS} N M R$ spectra for the three starting materials 374

8. PXRD and ${ }^{13} \mathrm{C} C P / M A S N M R$ spectra for the three guest-free materials 376

9. ${ }^{13} \mathrm{C}$ CP/MAS NMR and PXRD spectra of vacuum sublimed TATM 377

10. TGA traces of the 3 guest-free materials with their starting materials 380

11. TGA and DSC traces for vacuum sublimed guest-free TATM 381

12. DSC traces for 3 guest-free TATM samples with their starting materials 382

13. 'H solution NMR spectra of acetone/TATM and its guest-free product 385

14. Low temperature DSC trace for amorphous guest-free TATM 388

15. PXRD and ${ }^{13} \mathrm{C}$ NMR for acetone/TATM (from vapor and solution) 391

16. PXRD and ${ }^{13} \mathrm{C}$ NMR for $\mathrm{CH}_{2} \mathrm{Cl}_{2} / \mathrm{TATM}$ (from vapor and solution) 392

17. PXRD and ${ }^{13} \mathrm{C}$ NMR for $\mathrm{BrCH}_{2} \mathrm{CH}_{3} / \mathrm{TATM}$ (from vapor and solution) 392 
18. Unit cell- vs guest-molecular volume for TOT cage-type inclusions

19. Unit cell- vs guest-molecular volume for TOT channel-type inclusions

20. Stereoview of a lattice cage in unsolvated $\beta$-hydroquinone

21. Stereoview of the structure of $\alpha$-hydroquinone

22. Stereoview of the crystal structure of $\gamma$-hydroquinone

23. Molecular packing (down $c$ ) of modification I of crystalline PHTP

24. Molecular packing (down $c$ ) of modification II of crystalline PHTP

\section{LIST OF ABBREVIATIONS AND SYMBOLS}

\begin{tabular}{|c|c|}
\hline$a b$ & lattice plane perpendicular to the $c$ direction \\
\hline$a c$ & lattice plane perpendicular to the $b$ direction \\
\hline $\mathrm{amu}$ & atomic mass unit $\left(1 \mathrm{amu}=1 \mathrm{~g} \mathrm{~mol}^{-1}\right)$ \\
\hline anneal $50 \mathrm{C}$ & anneal sample at $50^{\circ} \mathrm{C}$ \\
\hline anti $(A)$ & opposite side stereochemistry $\left(\tau \sim 180^{\circ}\right)$ \\
\hline $\mathrm{atm}$ & atmospheres (unit of pressure) \\
\hline$a, b, c$ & unit cell dimensions (in $\AA$ ) \\
\hline$b$ & van der Waals covolume \\
\hline$b c$ & lattice plane perpendicular to the $a$ direction \\
\hline bp (or BP) & boiling point \\
\hline ca. & approximately \\
\hline cal & calorie $(1$ calorie $=4.184$ Joules $)$ \\
\hline $\mathrm{cc}$ & cubic centimeter $\left(\mathrm{cm}^{3}\right)$ \\
\hline$c_{\mathrm{g}}$ & periodicity of guest substructure in the host lattice channel \\
\hline & periodicity of host lattice channel \\
\hline ct & centimeter $\left(10^{-2} \mathrm{~m}\right)$ \\
\hline c.l & "compare with" \\
\hline alc & calculated crystal density $\left(\mathrm{g} \mathrm{cm}^{-3}\right)$ \\
\hline exis & measured crystal density $\left(\mathrm{g} \mathrm{cm}^{-3}\right)$ \\
\hline elta $\mathrm{H}_{\mathrm{m}}$ & enthalpy (or heat) of fusion (or melting) \\
\hline $\mathrm{dM} / \mathrm{dT}$ & rate of mass loss as a function of increasing temperature (TGA) \\
\hline$d(\mathrm{C}-\mathrm{H})$ & bond distance \\
\hline & ethyl substituent \\
\hline lipsed & $0^{\circ}$ torsion angle \\
\hline do & endothermic, and shared C--C bonds (for PHTP) \\
\hline$e q_{z}$ & $\begin{array}{l}\text { principal component of diagonalized electric field gradient tensor } \\
\text { estimated standard deviation }\end{array}$ \\
\hline & especially \\
\hline & exothermic, and unshared C-C bonds (for PHTP) \\
\hline & nuclear quadrupole moment \\
\hline
\end{tabular}




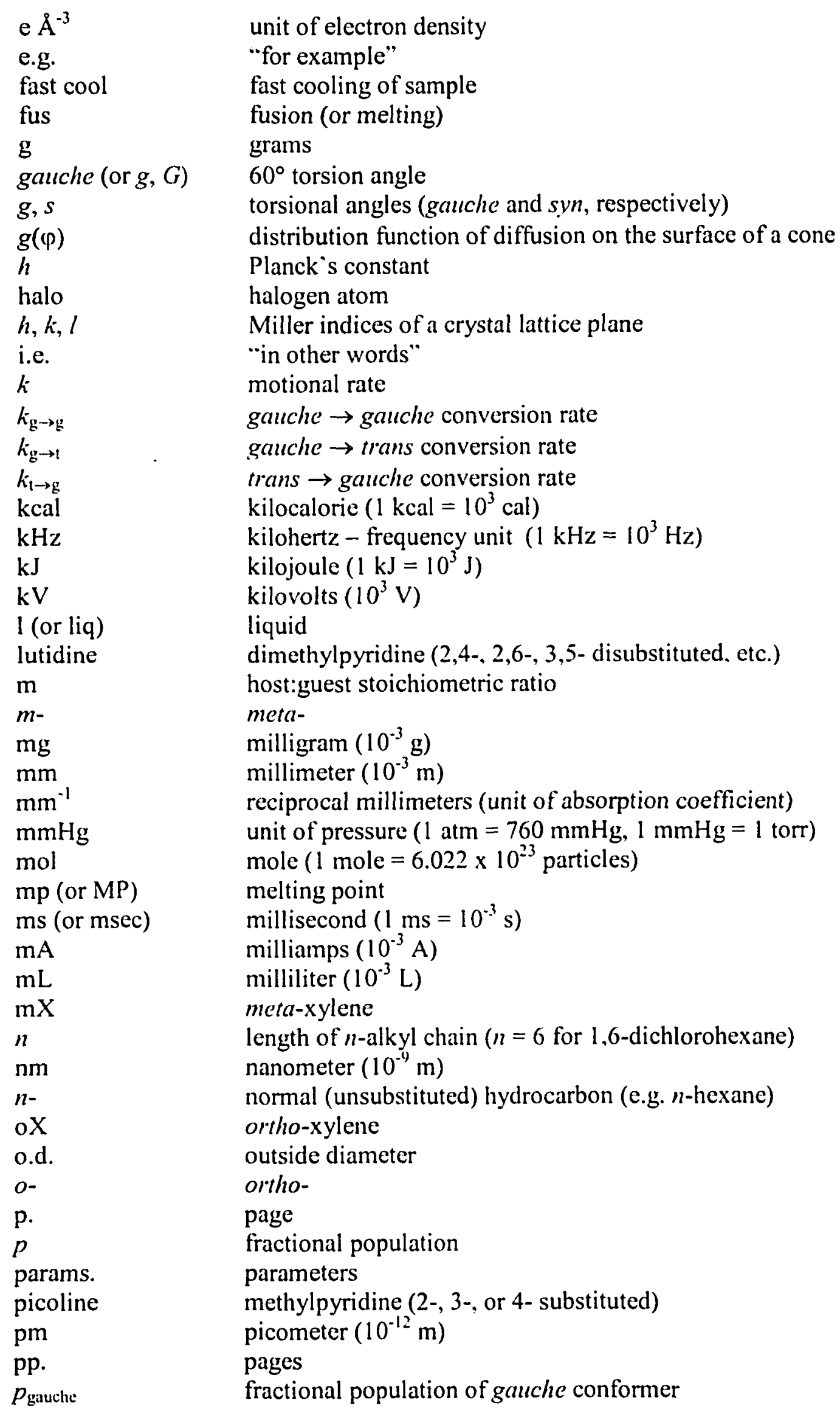




\begin{tabular}{|c|c|c|}
\hline$p_{\text {trans }}$ & \multicolumn{2}{|c|}{ fractional population of trans conformer } \\
\hline$p n$ & \multicolumn{2}{|c|}{ 1,2-diaminopropane ligand } \\
\hline ppm & \multicolumn{2}{|c|}{ parts per million (NMR chemical shift) } \\
\hline $\mathrm{pX}$ & \multicolumn{2}{|l|}{ para-xylene } \\
\hline$p, q$ & \multicolumn{2}{|c|}{ integers such that $p c_{\mathrm{h}} \sim q c_{\mathrm{g}}$ (for commensurate inclusions) } \\
\hline$p-$ & \multicolumn{2}{|l|}{ para- } \\
\hline$p-\mathrm{X}\left(\mathrm{C}_{6} \mathrm{H}_{4}\right) \mathrm{Y}$ & \multicolumn{2}{|c|}{ para-disubstituted benzene ring } \\
\hline $\mathrm{rms}$ & \multicolumn{2}{|l|}{ root-mean-square } \\
\hline rpm & \multicolumn{2}{|c|}{ revolutions per minute (stirring rate) } \\
\hline $\mathrm{s}($ or sec) & \multicolumn{2}{|l|}{ second } \\
\hline $\mathbf{S}$ & \multicolumn{2}{|c|}{ solid } \\
\hline slow cool & \multicolumn{2}{|c|}{ slow cooling of sample } \\
\hline slow evap & \multicolumn{2}{|c|}{ allow excess solvent of sample to evaporate at room temperature } \\
\hline stir 4D RT & \multicolumn{2}{|c|}{ stir sample for four days at room temperature } \\
\hline sub & \multicolumn{2}{|c|}{ sublimation } \\
\hline sym & \multicolumn{2}{|c|}{ identical functional groups (e.g. 1,4-sym-dihalobenzene) } \\
\hline $\operatorname{syn}(S)$ & \multicolumn{2}{|c|}{ same side stereochemistry $\left(\tau-0^{\circ}\right)$} \\
\hline$s^{-1}$ & \multicolumn{2}{|c|}{ unit of motional rate $\left(1 \mathrm{~s}^{-1}=1 \mathrm{~Hz}\right)$} \\
\hline $\operatorname{tano}$ & \multicolumn{2}{|c|}{ 2,2,6,6-tetramethyl-4-oxopiperidine-1-oxyl host system } \\
\hline thia- & \multicolumn{2}{|c|}{ sulfur substituted } \\
\hline torr & \multicolumn{2}{|c|}{ unit of pressure $(1 \mathrm{~atm}=760 \mathrm{torr}, 1$ torr $=1 \mathrm{mmHg})$} \\
\hline trans (or $t, T)$ & \multicolumn{2}{|c|}{$180^{\circ}$ torsion angle } \\
\hline $\mathrm{v}$ & \multicolumn{2}{|l|}{ vapor (or gas) } \\
\hline vac-sub & \multicolumn{2}{|l|}{ vacuum sublimed } \\
\hline vap & vaporization & \\
\hline vol & volume & \\
\hline wrto & "with respect to" & \\
\hline xylene & dimethylbenzene ( & ho-, meta-or para-disubstituted) \\
\hline A & anti & \\
\hline $\mathbf{A}$ and $\mathbf{B}$ & polymorphs of cyc & nexane and 1,2-dichloroethane with TATM \\
\hline AccuPyc & gas pycnometer (fo & density measurement) \\
\hline $\mathrm{AlCl}_{3}$ & aluminum chloride & \\
\hline $\operatorname{AMX} 300$ & solid-state Bruker & AR spectrometer \\
\hline A.U. & crystallographic as & ametric unit \\
\hline$A, B, C$ & different TATM m & ecules in the asymmetric unit \\
\hline $\mathrm{A} 2 / \mathrm{a}$ & monoclinic space & \\
\hline $\mathrm{Br}$ & bromine & \\
\hline $\mathrm{Br} 2$ & 1-bromoethane & $\mathrm{Br}\left(\mathrm{CH}_{2}\right)_{1} \mathrm{CH}_{3}$ \\
\hline $\mathrm{Br} 3$ & 1-bromopropane & $\mathrm{Br}\left(\mathrm{CH}_{2}\right)_{2} \mathrm{CH}_{3}$ \\
\hline $\mathrm{Br} 4$ & 1-bromobutane & $\mathrm{Br}\left(\mathrm{CH}_{2}\right)_{3} \mathrm{CH}_{3}$ \\
\hline $\mathrm{Br} 5$ & 1-bromopentane & $\mathrm{Br}\left(\mathrm{CH}_{2}\right)_{4} \mathrm{CH}_{3}$ \\
\hline Br6 & 1-bromohexane & $\mathrm{Br}\left(\mathrm{CH}_{2}\right)_{5} \mathrm{CH}_{3}$ \\
\hline $\mathrm{Br} 7$ & 1-bromopentane & $\mathrm{Br}\left(\mathrm{CH}_{2}\right)_{6} \mathrm{CH}_{3}$ \\
\hline $\mathrm{Br} 8$ & 1-bromooctane & $\mathrm{Br}\left(\mathrm{CH}_{2}\right)_{7} \mathrm{CH}_{3}$ \\
\hline $\mathrm{Br} 9$ & 1-bromononane & $\mathrm{Br}\left(\mathrm{CH}_{2}\right)_{8} \mathrm{CH}_{3}$ \\
\hline $\mathrm{Br} 10$ & 1-bromodecane & $\mathrm{Br}\left(\mathrm{CH}_{2}\right)_{9} \mathrm{CH}_{3}$ \\
\hline $\mathrm{Brll}$ & l-bromoundecane & $\mathrm{Br}\left(\mathrm{CH}_{2}\right)_{10} \mathrm{CH}_{3}$ \\
\hline
\end{tabular}




\begin{tabular}{|c|c|}
\hline $\mathrm{Br} 12$ & 1-bromododecane $\quad \mathrm{Br}\left(\mathrm{CH}_{2}\right)_{11} \mathrm{CH}_{3}$ \\
\hline $\mathrm{Br} 13$ & 1-bromotridecane $\quad \mathrm{Br}\left(\mathrm{CH}_{2}\right)_{12} \mathrm{CH}_{3}$ \\
\hline $\mathrm{Br} 14$ & 1-bromotetradecane $\mathrm{Br}\left(\mathrm{CH}_{2}\right)_{13} \mathrm{CH}_{3}$ \\
\hline $\operatorname{Br} 15$ & 1-bromopentadecane $\mathrm{Br}\left(\mathrm{CH}_{2}\right)_{14} \mathrm{CH}_{3}$ \\
\hline $\mathrm{Br} 16$ & 1-bromohexadecane $\mathrm{Br}\left(\mathrm{CH}_{2}\right)_{15} \mathrm{CH}_{3}$ \\
\hline $\mathrm{Br}\left(\mathrm{CH}_{2}\right)_{\mathrm{n}} \mathrm{Br}$ & $\alpha, \omega$-dibromoalkanes ( $n=$ carbon chain length) \\
\hline $\mathrm{Br}\left(\mathrm{CH}_{2}\right)_{11} \mathrm{CH}_{3}$ & 1-bromoalkane guest family ( $n=1$ to 15 ) \\
\hline $\mathrm{Br}\left(\mathrm{CH}_{2}\right)_{3} \mathrm{Br}$ & 1,3-dibromopropane \\
\hline $\mathrm{Bu}^{\mathrm{n}} \mathrm{OH}$ & $n$-butanol \\
\hline $\mathrm{Bz}$ & benzyl functional group $\left(-\mathrm{CH}_{2}-\mathrm{C}_{6} \mathrm{H}_{5}\right)$ \\
\hline BP & boiling point \\
\hline BPP & Bloembergen-Purcell-Pound spectral density function \\
\hline $\mathrm{C}$ & carbon \\
\hline $\mathrm{Cc}$ & monoclinic space group \\
\hline $\mathrm{Cl}$ & chlorine \\
\hline $\mathrm{CllCl}$ & 1,1-dichloromethane $\mathrm{Cl}\left(\mathrm{CH}_{2}\right)_{1} \mathrm{Cl}$ \\
\hline $\mathrm{Cl} 2 \mathrm{Cl}$ & 1,2-dichloroethane $\mathrm{Cl}\left(\mathrm{CH}_{2}\right)_{2} \mathrm{Cl}$ \\
\hline $\mathrm{Cl} 3 \mathrm{Cl}$ & 1,3-dichloropropane $\mathrm{Cl}\left(\mathrm{CH}_{2}\right)_{3} \mathrm{Cl}$ \\
\hline $\mathrm{Cl} 4 \mathrm{Cl}$ & 1,4-dichlorobutane $\mathrm{Cl}\left(\mathrm{CH}_{2}\right)_{4} \mathrm{Cl}$ \\
\hline $\mathrm{Cl} 5 \mathrm{Cl}$ & 1,5-dichloropentane $\mathrm{Cl}\left(\mathrm{CH}_{2}\right)_{5} \mathrm{Cl}$ \\
\hline $\mathrm{Cl} 6 \mathrm{Cl}$ & 1,6-dichlorohexane $\mathrm{Cl}\left(\mathrm{CH}_{2}\right)_{6} \mathrm{Cl}$ \\
\hline $\mathrm{Cl} 8 \mathrm{Cl}$ & 1,8-dichlorooctane $\mathrm{Cl}\left(\mathrm{CH}_{2}\right)_{8} \mathrm{Cl}$ \\
\hline $\mathrm{Cl} 9 \mathrm{Cl}$ & 1,9-dichlorononane $\mathrm{Cl}\left(\mathrm{CH}_{2}\right)_{9} \mathrm{Cl}$ \\
\hline $\mathrm{Cl} 10 \mathrm{Cl}$ & 1,10-dichlorodecane $\mathrm{Cl}\left(\mathrm{CH}_{2}\right)_{10} \mathrm{Cl}$ \\
\hline $\mathrm{Cl}\left(\mathrm{CH}_{2}\right)_{10} \mathrm{Cl}$ & $\alpha,(\omega$-dichloroalkanes ( $n=$ carbon chain length) \\
\hline $\mathrm{Cl}\left(\mathrm{CH}_{2}\right)_{3} \mathrm{Cl}$ & 1,3-dichloropropane \\
\hline $\mathrm{C}_{n} \mathrm{H}_{2 \mathrm{n}+1} \mathrm{OSO}_{3} \cdot \mathrm{Na}$ & sodium alkyl sulfates (anionic surfactant) \\
\hline Co $K_{u}$ & cobalt source $\mathrm{K}$-shell X-ray radiation $(\lambda-1.79 \AA)$ \\
\hline $\mathrm{Cu} K_{u}$ & copper source $\mathrm{K}$-shell X-ray radiation $(\lambda \sim 1.54 \AA)$ \\
\hline $\mathrm{CA}$ & cholic acid host system \\
\hline $\mathrm{CCl}_{4}$ & carbon tetrachloride \\
\hline $\mathrm{CCD}$ & charged coupled device (X-ray diffraction detector) \\
\hline $\mathrm{CD}$ & cyclodextrin $\left(\alpha-, \beta-\right.$, or $\left.\gamma_{-}\right)$ \\
\hline $\mathrm{CDCl}_{3}$ & deuteriochloroform (NMR solvent) \\
\hline CFC's & chlorofluorocarbons \\
\hline CFC-11 & $\mathrm{CFCl}_{3}$ \\
\hline CFC- 12 & $\mathrm{CF}_{2} \mathrm{Cl}_{2}$ \\
\hline $\mathrm{CHBr}_{3}$ & bromoform \\
\hline $\mathrm{CHCl}_{3}$ & chloroform \\
\hline $\mathrm{CH}_{2} \mathrm{Br}_{2}$ & methylene bromide \\
\hline $\mathrm{CH}_{2} \mathrm{Cl}_{2}$ & methylene chloride \\
\hline $\mathrm{CH}_{2} \mathrm{I}_{2}$ & methylene iodide \\
\hline $\mathrm{CH}_{3} \mathrm{CN}$ & acetonitrile \\
\hline $\mathrm{CH}_{3} \mathrm{NC}$ & methyl isocyanide \\
\hline $\mathrm{CH}_{3} \mathrm{OH}$ & methanol \\
\hline CP/MAS & cross polarization / magic angle spinning \\
\hline $\mathrm{Cx}$ & carbon chain length (e.g. $\mathrm{C} 7=n$-heptane, $\mathrm{x}=7$ ) \\
\hline
\end{tabular}




\begin{tabular}{|c|c|}
\hline$C-n$ & position in a carbon chain (eg. C-2 of pentane, $n=2$ ) \\
\hline $\mathrm{C} 2 / \mathrm{c}$ & monoclinic space group \\
\hline$C_{2}$ & two-fold axis of symmetry (or axis of rotation) \\
\hline $\mathrm{C} 222_{1}$ & orthorhombic space group \\
\hline$C_{3}$ & three-fold axis of symmetry (or axis of rotation) \\
\hline $\mathrm{C}_{4} \mathrm{H}_{9} \mathrm{Br}$ & 2-bromobutane \\
\hline $\mathrm{C}_{4} \mathrm{H}_{9} \mathrm{Cl}$ & 2-chlorobutane \\
\hline $\mathrm{C}_{4} \mathrm{H}_{9} \mathrm{I}$ & 2-iodobutane \\
\hline $\mathrm{C}_{6}$ & six-fold axis of symmetry (or axis of rotation) \\
\hline $\mathrm{C}_{6} \mathrm{H}_{5} \mathrm{Br}$ & bromobenzene \\
\hline $\mathrm{C}_{6} \mathrm{H}_{5} \mathrm{Cl}$ & chlorobenzene \\
\hline $\mathrm{C}_{6} \mathrm{H}_{5} \mathrm{~F}$ & fluorobenzene \\
\hline $\mathrm{C}_{6} \mathrm{H}_{5} \mathrm{I}$ & iodobenzene \\
\hline $\mathrm{C}_{6} \mathrm{H}_{6}$ & benzene \\
\hline $\mathrm{C}_{6} \mathrm{H}_{11} \mathrm{Br}$ & bromocyclohexane \\
\hline $\mathrm{C}_{6} \mathrm{H}_{11} \mathrm{Cl}$ & chlorocyclohexane \\
\hline $\mathrm{C}_{6} \mathrm{H}_{11} \mathrm{Cl}$ & iodocyclohexane \\
\hline $\mathrm{C}_{19} \mathrm{H}_{16} \mathrm{O}_{3} \mathrm{~S}_{3}$ & molecular formula of TATM host material \\
\hline $\mathrm{C}-\mathrm{H} \cdots \mathrm{O}$ & schematic of weak hydrogen bond \\
\hline $\mathrm{C}-\mathrm{H} \cdots \pi$ & schematic of weak hydrogen bond \\
\hline $\mathrm{C}-\mathrm{X} \cdots \mathrm{O}$ & schematic of weak halogen bond \\
\hline$C-X \cdots \pi$ & schematic of weak halogen bond \\
\hline $\mathrm{C}=\mathrm{O}$ & carbonyl functional group \\
\hline D & deuterium nucleus $\left({ }^{2} \mathrm{H}\right)$ \\
\hline DaGp & dianilinegossypol host system \\
\hline DD & doubly disordered \\
\hline DMSO & dimethylsulfoxide \\
\hline DRX Win & powder X-ray diffraction software program \\
\hline DRX400 & high resolution solution Bruker NMR spectrometer \\
\hline DSC & differential scanning calorimetry \\
\hline DTA & differential thermal analysis \\
\hline $\mathrm{D}_{2} \mathrm{O}$ & deuterio-water $\left({ }^{2} \mathrm{H}_{2} \mathrm{O}\right)$ \\
\hline$D_{3}$ & point group \\
\hline$E_{\mathrm{a}}$ & activation energy \\
\hline$E \mathrm{a}_{\mathrm{g} \rightarrow \mathrm{g}}$ & energy barrier to gauche $\rightarrow$ gauche conversion \\
\hline$E \mathrm{ag}_{\mathrm{g} \rightarrow \mathrm{l}}$ & energy barrier to gauche $\rightarrow$ trans conversion \\
\hline$E \mathrm{a}_{\mathrm{l} \rightarrow \mathrm{g}}$ & energy barrier to trans $\rightarrow$ gauche conversion \\
\hline $\mathrm{EtOH}$ & ethanol \\
\hline $\mathrm{F}$ & fluorine \\
\hline$F$ & structure factor \\
\hline$F_{\text {calc }}$ & calculated structure factor \\
\hline$F_{\text {obs }}$ & observed structure factor \\
\hline $\mathrm{F}\left(\mathrm{CH}_{2}\right)_{3} \mathrm{~F}$ & 1,3-difluoropropane \\
\hline$F(000)$ & effective number of electrons in the crystal unit cell \\
\hline G & guest \\
\hline$G$ & gauche \\
\hline $\mathrm{Gp}$ & gossypol host system \\
\hline
\end{tabular}




\begin{tabular}{|c|c|}
\hline $\mathrm{G}_{\mathrm{g}}$ & gaseous guest \\
\hline $\mathrm{G}_{1}$ & $\begin{array}{l}\text { liquid guest } \\
\text { solid guest }\end{array}$ \\
\hline $\mathrm{G}_{\mathrm{s}}$ & solid guest \\
\hline GoF (or GOF) & goodness-of-fit of a crystal structure \\
\hline $\mathrm{H}$ & hydrogen \\
\hline $\mathrm{H}$ & host \\
\hline $\mathrm{Hz}$ & unit of frequency or motional rate $\left(1 \mathrm{~Hz}=1 \mathrm{~s}^{-1}\right)$ \\
\hline $\mathrm{HBr}$ & hydrogen bromide \\
\hline $\mathrm{HCl}$ & hydrogen chloride \\
\hline HCFC- 123 & $\mathrm{C}_{2} \mathrm{~F}_{3} \mathrm{HCl}_{2}$ \\
\hline HCFC- 124 & $\mathrm{C}_{2} \mathrm{~F}_{4} \mathrm{HCl}$ \\
\hline HCFC-22 & $\mathrm{CF}_{2} \mathrm{HCl}$ \\
\hline HFC-134a & $\mathrm{C}_{2} \mathrm{~F}_{4} \mathrm{H}_{2}$ \\
\hline $\mathrm{HH}$ & head-to-head arrangement of guests $(X \cdots X)$ \\
\hline $\mathrm{HO}-\mathrm{C}_{6} \mathrm{H}_{4}-\mathrm{OH}$ & hydroquinone \\
\hline HT & head-to-tail arrangement of guests $(X \cdots Y)$ \\
\hline $\mathrm{H}_{2} \mathrm{~N}-\mathrm{C}_{6} \mathrm{H}_{4}-\mathrm{NH}_{2}$ & phenylenediamine \\
\hline $\mathrm{H}_{2} \mathrm{O}$ & water \\
\hline $\mathrm{H}_{2} \mathrm{~S}$ & hydrogen sulfide \\
\hline H-bonding & hydrogen bonding \\
\hline H:G SR & host:guest stoichiometric ratio \\
\hline $\mathrm{H} \cdots \mathrm{H}, \mathrm{C} \cdots \mathrm{C}$ & through space (non-bonded) interactions / contacts \\
\hline $\mathrm{H} \cdots \mathrm{O}-\mathrm{H}$ & schematic of a strong hydrogen bond \\
\hline $\mathrm{H} \cdots \mathrm{N}-\mathrm{H}$ & schematic of a strong hydrogen bond \\
\hline I & one \\
\hline I & iodine \\
\hline I-, II- & two forms of guest-free perhydrotriphenylene \\
\hline I-, II-, III- & three forms of $\beta$-quinol inclusion compounds \\
\hline IQNS & incoherent quasielastic neutron scattering \\
\hline IR & infrared \\
\hline $\mathrm{I}\left(\mathrm{CH}_{2}\right)_{3} \mathrm{I}$ & 1,3-diiodopropane \\
\hline $\mathrm{J}$ & Joule (unit of energy) \\
\hline$J_{1}\left(\omega_{0}\right), J_{2}\left(2 \omega_{0}\right)$ & spectral density functions \\
\hline & one thousand \\
\hline $\mathrm{K}$ & degree Kelvin \\
\hline$K$ & $T_{1}$ pre-exponential factor $\left(\mathrm{s}^{-2}\right)$ \\
\hline $\mathrm{K}_{\mathrm{i}}$ & association constant (or binding constant) \\
\hline$K_{\mathrm{eq}}$ & equilibrium constant \\
\hline $\mathrm{KI}$ & potassium iodide \\
\hline $\mathrm{L}$ & liquid (or molten) host material \\
\hline $\mathrm{L}$ & liter (unit of volume) \\
\hline $\mathrm{M}$ & molarity (unit of concentration in moles $\mathrm{L}^{-1}$ ) \\
\hline $\mathrm{MeOH}$ & methanol \\
\hline Mo $K_{a}$ & molybdenum source $K$-shell $X$-ray radiation $(\lambda \sim 0.71 \AA)$ \\
\hline Mol. Wt. & molecular weight (in amu or $\mathrm{g} \mathrm{mol}^{-1}$ ) \\
\hline $\mathrm{MHz}$ & Megahertz - frequency unit $\left(1 \mathrm{MHz}=10^{6} \mathrm{~Hz}\right)$ \\
\hline MM & molecular mechanics (calculations) \\
\hline
\end{tabular}




\begin{tabular}{|c|c|}
\hline MP & melting point \\
\hline MPA & hot-stage (visual) melting point apparatus \\
\hline MT & empty (guest-free) TATM material \\
\hline MW & molecular weight \\
\hline MXQET & deuterium NMR spectral simulation program \\
\hline $\mathrm{M} 1, \mathrm{M} 2, \mathrm{M} 3$ & crystallographically independent molecules in the asymmetric unit \\
\hline $\mathrm{N}$ & nitrogen \\
\hline $\mathrm{N}$ & number of carbon atoms of guest hydrocarbon \\
\hline $\mathrm{NaOH}$ & sodium hydroxide \\
\hline NE-SW & upper right to lower left direction \\
\hline NMR & nuclear magnetic resonance \\
\hline NOE & nuclear Overhauser enhancement \\
\hline NW-SE & upper left to lower right direction \\
\hline $\mathrm{O}$ & oxygen \\
\hline ON & orange needles \\
\hline OP & orange plates \\
\hline ORP & orange-red plates \\
\hline ORTEP & thermal ellipsoid plot \\
\hline $\mathrm{O} \cdots \mathrm{O}$ & hydrogen bond \\
\hline$P_{b c a}$ & orthorhombic space group \\
\hline $\mathrm{Ph}$ & phenyl ring \\
\hline $\mathrm{PhBr}$ & bromobenzene \\
\hline $\mathrm{PhCl}$ & chlorobenzene \\
\hline $\mathrm{PhF}$ & fluorobenzene \\
\hline PhI & iodobenzene \\
\hline$P_{n}$ & different forms of guest-free gossypol ( $n=1$ to 7$)$ \\
\hline$P_{\text {nain }}$ & orthorhombic space group \\
\hline$P_{\text {numa }}$ & orthorhombic space group \\
\hline PriEndo & primary melting endotherm \\
\hline PriOH & isopropanol \\
\hline $\mathrm{PC}_{\text {cavity }}$ & ratio of volume of guest to that of host cavity \\
\hline PHTP & perhydrotriphenylene host system \\
\hline$P_{N}$ & repeat distance along channel axis \\
\hline POW & powder \\
\hline PXRD & powder X-ray diffraction \\
\hline P1 & triclinic space group \\
\hline $\mathrm{P} \bar{l}$ & triclinic space group \\
\hline $\mathrm{P} 2,1 / \mathfrak{a}$ & monoclinic space group \\
\hline $\mathrm{P}_{1} / \mathrm{c}$ & monoclinic space group \\
\hline $\mathrm{P} 2, / \mathrm{n}$ & monoclinic space group \\
\hline P3 & hexagonal space group \\
\hline $\mathrm{P} 3_{1}$ & trigonal space group \\
\hline $\mathrm{P} 3{ }_{1} 12$ & trigonal space group \\
\hline$P 3,21$ & trigonal space group \\
\hline $\mathrm{P} 3{ }_{1}^{*}$ & trigonal space group \\
\hline $\mathrm{P} 6_{1}$ & hexagonal space group \\
\hline $\mathrm{P} 6,22$ & hexagonal space group \\
\hline
\end{tabular}




\begin{tabular}{|c|c|}
\hline $\mathrm{P}_{2}$ & hexagonal space group \\
\hline $\mathrm{P}_{3}$ & hexagonal space group \\
\hline $\mathrm{P} 6_{3} / \mathrm{m}$ & hexagonal space group \\
\hline $\mathrm{P}_{3} / \mathrm{mmc}$ & hexagonal space group \\
\hline $\mathrm{P} 65_{5} 22$ & hexagonal space group \\
\hline QCC & quadrupole coupling constant (or $\chi$ ) \\
\hline$R$ & gas constant \\
\hline $\mathrm{R}$ & generic alkyl functional group \\
\hline $\mathrm{R}$ & chiral molecule with clockwise arrangement of substituents \\
\hline $\mathbf{R}$ & red prisms \\
\hline$R_{\text {int }}$ & average agreement between equivalent reflections \\
\hline $\mathrm{R}_{\mathrm{m}}$ & molar refraction \\
\hline RT & room temperature \\
\hline$R\left(\right.$ or $\left.R_{1}\right)$ & residual error in a crystal structure \\
\hline$R_{w}\left(\right.$ or $\left.w R_{2}\right)$ & weighted residual error in a crystal structure \\
\hline R 3 & hexagonal space group \\
\hline $\mathrm{R} \overrightarrow{3}$ & hexagonal space group \\
\hline $\mathrm{S}$ & syn \\
\hline $\mathrm{S}$ & sulfur \\
\hline $\mathrm{S}$ & chiral molecule with counterclockwise arrangement of substituents \\
\hline SecEndo & secondary melting endotherm \\
\hline $\mathrm{SC}$ & single crystal \\
\hline SCXRD & single crystal $\mathrm{X}$-ray diffraction \\
\hline SD & symmetry disordered \\
\hline $\mathrm{SO}_{2}$ & sulfur dioxide \\
\hline S.R. & stoichiometric ratio \\
\hline S.G. & space group \\
\hline $\mathrm{S}_{u \alpha}$ & dense guest-free host lattice (stable) \\
\hline$S_{\beta}$ & host-guest inclusion compound \\
\hline $\mathrm{S}_{\beta}^{\circ}$ & hypothetical guest-free empty host lattice (metastable) \\
\hline$S_{\beta n}^{o}$ & different structural polytypes of the empty $S_{\beta}^{\circ}$ host lattice \\
\hline$T$ & temperature (in $\mathrm{K}$ or ${ }^{\circ} \mathrm{C}$ ) \\
\hline$T$ & trans \\
\hline$T_{\mathrm{d}}$ & decomposition temperature \\
\hline$T_{\mathrm{g}}$ & glass transition temperature (glass phase $\rightarrow$ rubber phase) \\
\hline$T_{\mathrm{m}}$ & melting temperature (in $\mathrm{K}$ or ${ }^{\circ} \mathrm{C}$ ) \\
\hline$T_{\text {meit }}$ & melting temperature (in $\mathrm{K}$ or ${ }^{\circ} \mathrm{C}$ ) \\
\hline$T_{\text {ond }}\left(\right.$ or $\left.T_{\text {onset }}\right)$ & onset transition temperature \\
\hline Ts & tosyl functional group \\
\hline TA & thermal analysis \\
\hline TATM & tris(5-acetyl-3-thienyl)methane host material \\
\hline TBB & 1,3,5-tribromobenzene \\
\hline TG & thermogravimetry \\
\hline TGA & thermogravimetric analysis \\
\hline TIB & 1,3,5-triiodobenzene \\
\hline TMS & tetramethylsilane $\mathrm{Si}\left(\mathrm{CH}_{3}\right)_{4}$ \\
\hline
\end{tabular}




\begin{tabular}{|c|c|}
\hline TOT & tri-ortho-thymotide host material \\
\hline TT & tail-to-tail arrangement of guests $(\mathrm{Y} \cdots \mathrm{Y})$ \\
\hline$T_{1}$ & spin-lattice relaxation time \\
\hline$T_{1 \min }$ & spin-lattice relaxation time minimum \\
\hline$T_{2}$ & transverse relaxation time \\
\hline$U_{\mathrm{eq}}$ & equivalent isotropic displacement parameter \\
\hline$U_{\mathrm{ij}}$ & anisotropic displacement parameter \\
\hline UIC & urea inclusion compound \\
\hline UV & ultraviolet electromagnetic radiation \\
\hline V & five \\
\hline$V$ & unit cell volume (in $\AA^{3}$ ) \\
\hline VIS & visible electromagnetic radiation \\
\hline W & Watt \\
\hline $\mathrm{W} \mathrm{g}^{-1}$ & heat flow (DSC) \\
\hline $\mathrm{X}$ & ten \\
\hline $\mathrm{X}$ & halogen atom (generic) \\
\hline$X$ & site occupancy factor of guest $(0 \leq X \leq 1)$ \\
\hline$X_{\mathrm{A}}$ & mole fraction of species $\mathrm{A}\left(0<X_{\mathrm{A}}<1\right)$ \\
\hline XRD & $\mathrm{X}$-ray diffraction \\
\hline Y & yellow prisms \\
\hline$Y$ & interaction between thienyl and methine hydrogens of TATM \\
\hline YN & yellow needles \\
\hline$Z$ & number of formula units in the unit cell \\
\hline${ }_{\mathrm{H}}^{1,2,3,4,5}$ & $\begin{array}{l}\text { polymorphs of 1,3-dichloropropane / TATM inclusions } \\
\text { hydrogen (mass }=1 \mathrm{amu} \text { ) nucleus }\end{array}$ \\
\hline${ }^{1} \mathrm{H} \rightarrow{ }^{13} \mathrm{C}$ & cross polarization of magnetization from ${ }^{1} \mathrm{H}$ to ${ }^{13} \mathrm{C}$ \\
\hline${ }^{13} \mathrm{C}$ & carbon $($ mass $=13 \mathrm{amu})$ nucleus \\
\hline${ }^{2} \mathrm{H}$ & deuterium (mass $=2 \mathrm{amu}$ ) nucleus \\
\hline 2THETA & $2 \theta$ (angle of reflection of X-rays) \\
\hline$-d_{n}$ & guest molecule substituted with $n$ deuterium atoms \\
\hline$-\mathrm{CH}$ & methine functional group \\
\hline$-\mathrm{CH}_{2}$ & methylene functional group \\
\hline$-\mathrm{CH}_{3}$ & methyl functional group \\
\hline$-\mathrm{C}_{4} \mathrm{H}_{9}$ & 2-substituted butyl functional group \\
\hline$-\mathrm{C}_{6} \mathrm{H}_{5}$ & monosubstituted phenyl functional group \\
\hline$-\mathrm{C}_{6} \mathrm{H}_{11}$ & monosubstituted cyclohexyl functional group \\
\hline$-\left(\mathrm{CH}_{2}\right)_{3^{-}}$ & 1,3-disubstituted propyl functional group \\
\hline$-[\mathrm{OH}-]_{6}$ & $\begin{array}{l}\text { hydrogen-bonded hexamer (Dianin's compound or } \beta \text {-quinol) } \\
\text { number of }\end{array}$ \\
\hline$\%$ & percent \\
\hline$<$ & less than \\
\hline$=$ & equal to \\
\hline$>$ & greater than \\
\hline 〜 & approximately equal to \\
\hline \pm & absolute error \\
\hline$\alpha$ & molar polarizability \\
\hline
\end{tabular}




\begin{tabular}{|c|c|}
\hline$\alpha-$ & dense, empty (guest-free) host phase \\
\hline$\alpha-, \beta-$ & polymorphs of dianilinegossypol with acetone and ethyl acetate \\
\hline$\alpha, \beta, \gamma$ & unit cell angles \\
\hline$\alpha_{1}, \beta_{2}, \gamma_{3}$ & conformation $\alpha$, orientation 1 (molecular dynamics simulations) \\
\hline$\alpha-, \beta-, \gamma-$ & three forms of quinol $\left\{\mathrm{C}_{6} \mathrm{H}_{4}(\mathrm{OH})_{2}\right\}$ \\
\hline$\alpha, \omega-$ & diterminally substituted alkyl chain (e.g. 1,6-dichlorohexane) \\
\hline$\beta-$ & host:guest inclusion compound phase \\
\hline$\beta_{0^{-}}$ & "empty" open (low density) guest-free host phase \\
\hline$\chi_{\mathrm{ent}}$ & effective (motionally averaged) quadrupole coupling constant \\
\hline$\chi_{\text {static }}$ & static (low temperature limit) quadrupole coupling constant \\
\hline$\delta$ & distance between $\pi-\pi$ stacked thienyl rings of TATM \\
\hline$\delta_{\mathrm{C}}$ & ${ }^{13} \mathrm{C}$ NMR chemical shift (ppm) \\
\hline$\delta_{H}$ & ${ }^{1} \mathrm{H}$ NMR chemical shift (ppm) \\
\hline$\phi$ & half angle of two-site libration \\
\hline$\gamma-$ & partially occupied $(<100 \%)$ host:guest inclusion compound phase \\
\hline$\eta$ & ${ }^{2} \mathrm{H}$ NMR spectral asymmetry parameter \\
\hline$\lambda$ & wavelength of electromagnetic radiation \\
\hline$\mu$ & absorption coefficient of a crystal $\left(\mathrm{mm}^{-1}\right)$ \\
\hline$\mu \mathrm{m}$ & micrometer $\left(10^{-6} \mathrm{~m}\right)$ \\
\hline$\mu \mathrm{s}$ & microsecond $\left(10^{-6} \mathrm{~s}\right)$ \\
\hline$\mu \mathrm{L}$ & microliter $\left(10^{-6} \mathrm{~L}\right)$ \\
\hline$\pi$ & $180^{\circ}$ \\
\hline$\pi$ & pi electrons of an aromatic ring (e.g. thienyl rings of TATM) \\
\hline$\pi-\pi$ & pi stacking of thienyl rings of TATM \\
\hline$\pi / 2$ & $90^{\circ}$ \\
\hline$\theta$ & angle between $C-D$ bond and axis of rotation \\
\hline$\theta$ & half angle of precession of symmetry axis on the surface of a cone \\
\hline$\theta-\theta$ & X-ray diffraction scan mode \\
\hline$\theta($ or $2 \theta)$ & angle of reflection of X-rays \\
\hline$\theta(\mathrm{C}-\mathrm{C}-\mathrm{H})$ & bond angle \\
\hline$\theta_{\text {nitro }}$ & nitro group torsion angle \\
\hline$\theta_{\text {phen }}$ & phenyl ring torsion angle \\
\hline$\theta_{\text {thio }}$ & thiophene ring torsion angle \\
\hline$\tau$ & deuterium $T_{1}$ relaxation delay \\
\hline$\tau_{\mathrm{c}}$ & correlation time, $\tau \propto \mathrm{k}^{-1}$ (in seconds) \\
\hline$\tau_{\mathrm{Q}}$ & interpulse spacing in quadrupole echo pulse sequence \\
\hline$\tau_{m}$ & correlation time at infinite temperature \\
\hline$\tau_{1}, \tau_{2}, \tau_{3}$ & torsional angles of thienyl rings wrto methine $\mathrm{C}-\mathrm{H}$ of TATM \\
\hline$\tau_{4}, \tau_{5}, \tau_{6}$ & steroechemistry of carbonyl wrto ring sulfur of TATM \\
\hline$\omega$ & X-ray diffraction scan mode \\
\hline$\omega_{0}$ & Larmor precession frequency \\
\hline$\omega_{0} \tau_{c}<<1$ & extreme narrowing limit (at high temperatures) \\
\hline$\omega_{\mathrm{Q}}$ & $3 / 4 \chi$ \\
\hline$\omega / 2 \theta$ & X-ray diffraction scan mode \\
\hline
\end{tabular}


$\Delta$

$\Delta_{\mathrm{ds}} G$

$\Delta_{\text {fus }} G$

$\Delta_{\text {lus }} H$

$\Delta_{\text {lus }} S$

$\Delta \mathrm{g}$

$\Delta_{\text {inp }}\left(\right.$ or $\left.T_{\text {inpai }}-T_{\text {dsc }}\right)$

$\Delta w$

$\Delta E_{\mathrm{gt}}$

$\Delta G^{0}$

$\Delta H$

$\Delta H_{\mathrm{d}}$

$\Delta H_{f}\left(\right.$ or $\left.\Delta H_{\text {finmation }}\right)$

$\Delta H_{\mathrm{gt}}$

$\Delta a$

$(\Delta / \sigma)_{\max }$

$\circ$

${ }^{\circ} \mathrm{C}$

$\angle$

$\cdots$

$\AA$ pairwise interactions between thienyl hydrogens of TATM

Gibbs free energy of association

Gibbs free energy of fusion (or melting)

enthalpy (or heat) of fusion (or melting)

entropy of fusion (or melting)

offset of guests in adjacent channels of the host lattice

difference between MPA and DSC melting points

mass loss in a TGA experiment

energy difference between trans and gauche conformers

standard Gibbs free energy change

enthalpy change or difference between two states

heat (or enthalpy) of decomposition

heat (or enthalpy) of formation

enthalpy difference between trans and gauche conformers

$\mathrm{X}$-ray diffraction scan width

maximum shift / estimated standard deviation

degrees (temperature and angles)

degree Celsius

bond angle $\left({ }^{\circ}\right)$

hydrogen bond or intermolecular contact

Angstrom - distance unit $\left(1 \AA=10^{-11} \mathrm{~m}\right)$ 


\section{CHAPTERS I TO VI}

\section{Introduction, Literature Review and Experimental Techniques}

\begin{tabular}{|c|c|}
\hline Chapter I & $\begin{array}{l}\text { Introduction to inclusion compounds } \\
\text { References }\end{array}$ \\
\hline Chapter II & $\begin{array}{l}\text { Halogenated hydrocarbons } \\
\text { References }\end{array}$ \\
\hline Chapter III & $\begin{array}{l}\text { TATM literature review } \\
\text { (A) J. Org. Chem. (USSR) 9, 1549 (1973) } \\
\text { (B) J. Chem. Soc. Chem. Commun. 21, 741 (1977) } \\
\text { (C) Acta Cryst. C47, 2468 (1991) } \\
\text { (D) Acta Cryst. C48, } 2229(1992) \\
\text { (E) Acta Cryst. C48, } 1882(1992) \\
\text { (F) Acta Cryst. C47, 2718 (1991) } \\
\text { (G) Acta Cryst. B50, 112 (1994) } \\
\text { (H) Acta Cryst. C50, 615 (1994) } \\
\text { (I) Acta Cryst. C50, 1947(1994) } \\
\text { (J) Can. J. Chem. 72, 2318(1994) } \\
\text { (K) Acta Cryst. B53, 168 (1997) } \\
\text { (L) Can. J. Chem. 73, } 2196(1995) \\
\text { (M) Can. J. Chem. 74, 1784(1996) } \\
\text { Concluding remarks } \\
\text { References }\end{array}$ \\
\hline Chapter IV & $\begin{array}{l}\text { Other host systems } \\
\text { (A) Urea } \\
\text { (B) Tri-ortho-thymotide (TOT) } \\
\text { (C) Hydroquinone ( } \beta \text {-quinol) } \\
\text { (D) Dianin's compound } \\
\text { (E) Gossypol } \\
\text { (F) Perhydrotriphenylene (PHTP) } \\
\text { References }\end{array}$ \\
\hline Chapter V & $\begin{array}{l}\text { Techniques employed } \\
\text { References }\end{array}$ \\
\hline Chapter VI & $\begin{array}{l}\text { Experimental details } \\
\text { References }\end{array}$ \\
\hline
\end{tabular}




\section{CHAPTER I}

Since the middle of the $19^{\text {th }}$ century, molecular chemistry, particularly synthetic chemistry, has resulted in an increasing mastery in formation of the covalent bond [1]. A parallel evolution is now being encountered for noncovalent intermolecular forces. Beyond molecular chemistry, based on the covalent bond, lies the field of supramolecular chemistry, [2] the aim of which is to gain control over the intermolecular bond,

Thus, supramolecular chemistry has been defined as chemistry beyond the molecule, referring to the organized entities of higher complexity that result from the association of two or more chemical species held together by intermolecular forces.

The field grew out of studies in the early 1960's of molecular recognition of alkali metal ions using natural antibiotics and synthetic macro(poly)cyclic polyethers $[3,4]$. Although it was not conceived as such at that time, its roots can be traced back to Paul Ehrlich's receptor idea [5,6], Alfred Werner's coordination theory $[\mathbf{7 , 8}]$, and Emil Fischer's lock and key image $[9, \mathbf{1 0} \mid$. In addition, there had been early investigations, already in the mid-1930s, of associations in solution, which were even then termed "Ubermolekeln" (supermolecules) [11].

The general concept was recognized and formulated in the $1960^{\circ} \mathrm{s} /$ early $1970^{\circ} \mathrm{s}$ [12-14|. Its breadth and unifying power became progressively more and more apparent, so that recent years have seen an explosive growth in the number of laboratories that are working in this field, and whose work has been reported in a vast range of publications, books, journals, meetings and symposia $[15,16]$.

Supramolecular chemistry has developed into a coherent and extremely lively body of concepts and objects, progressively generating and incorporating novel areas of investigation. A whole vocabulary, however still incomplete, has been produced and is becoming more and more widely accepted and used [17-19].

In summary, supramolecular chemistry is a highly interdisciplinary field that has rapidly expanded at the frontiers of chemical science with physical and biological phenomena $[20,21]$. Its roots extend over organic chemistry and the synthetic procedures for molecular construction [22,23]; coordination chemistry and metal ion / ligand complexes [24,25]; physical chemistry and the experimental and theoretical studies of interactions [26,27]; biochemistry and the biological processes that all start with substrate 
binding and recognition [28,29]; and materials science and the mechanical properties of solids [30,31]. A major feature is the range of perspectives offered by the crossfertilization of supramolecular chemical research due to its location at the intersection of chemistry, biology and physics. Drawing on the physics of organized condensed matter and expanding over the biology of large molecular assemblies, supramolecular chemistry expands into a supramolecular science $[32\}$. Such wide horizons are a challenge and a stimulus to the creative imagination of chemists.

Most chemical compounds consist of more or less orderly arrays of atoms reasonably strongly bonded together. One exception to this generalization is the group of substances known as inclusion compounds. Distinction from other types of chemical complexes comes from inclusion compounds existing as a combination of two distinct molecular species, associated not through chemical bonding, but rather through the ability of one species to fit into a crystal lattice made up of the second species. The guest molecule, in many cases, cannot readily leave its position since it is surrounded by the host lattice (although not actually bonded to it), and both contributing molecular species are usually recoverable in their normal thermodynamically stable forms upon destruction of the lattice by solvent dissolution, thermal decomposition etc. Between each pair of molecular species forming an inclusion compound, there is usually some definite (although often non-integral) proportionality, which generally distinguish inclusion compounds from non-stoichiometric compositions such as eutectics or solid solutions. The unique opportunity for studying the properties of individual molecules of one species, isolated from their fellows by the host lattice structure in a number of types of inclusion compounds has long been recognized, and deserves further exploitation.

The essential property of a host molecule is that it is able to form a solid structure containing a regular system of voids into which guest molecules of particular size and shape can fit. In many instances, this structure is not the normal crystal lattice of the pure host material, but an entirely new lattice that will exist only so long as it accommodates at least a fraction of the potential maximum content of the guest species.

The basic feature of a guest atom or molecule is that its size and shape alone, relative to that of the space available within the host lattice, determines whether or not it will participate in an inclusion compound. Thus, it becomes a question of fitting the 
correct size and shape of peg into the available hole, with the usual considerations of chemical reactivity becoming of secondary importance. There are also certain interactions between the guest species and the surrounding host lattice, ranging from weak van der Waals types of attraction to fairly highly oriented dipole contacts. The resultant forces of these attractions contribute in a positive manner to the overall stability of the inclusion compound, but are generally not as strong as the forces encountered in normal chemical bonding.

A particular confusion continues to exist in the literature as to the interchangeable use of the terms clathrate, inclusion compound and molecular complex. The original definition of the term "clathrate" [12] clearly specifies a three-dimensional closed structure in which the guest species is "locked up" within the lattice of the host species, and may only be released when this lattice is destroyed (e.g. $\beta$-quinol $[33,34]$, tri-orthothymotide $[35,36]$, gas hydrates $[37,38]$ etc.).

There are other substances which are definitely inclusion compounds, satisfying all the requirements of host and guest species, but in which the host lattice contains regular openings at some point. These openings are of sufticient size to permit loss of the guest species, provided that any internal interaction with the host lattice can be overcome. Once the host cavity has been vacated, the lattice may collapse (e.g. urea $[39,40]$ ), or may remain structurally stable (e.g. zeolites $|\mathbf{4 1 , 4 2}|$ ). Thus, the term clathrate should be used to denote a particular class of inclusion compounds, and should not be misused in a generic sense.

While inclusion compounds are frequently classified on the basis of the nature of the cavity shape or dimensions within the host lattice, it is also convenient to distinguish between those types in which the empty host lattice may exist in spite of the absence of guest, and those in which the presence of the guest species is required to keep the host lattice from transforming into a dense guest-free phase. Examples of compounds in which the empty host lattice remains despite the absence of a guest species include zeolites $[\mathbf{4 1}, \mathbf{4 2}]$, fibrous clays $[43,44]$, layer type lattices (e.g. montmorillonite) $[45]$ and various organic compounds (e.g. cyclodextrins $[46.47]$ and Dianin`s compound $[33,48]$ ). On the other hand, those hosts in which the original lattice exists only in the presence of a 
suitable guest species include urea $[39,40]$ and thiourea $[39,40 \mid$ channel compounds, hydroquinone $[33,34]$ and phenol $[33,34]$ clathrates, and gas hydrates $[37,38]$.

\section{CHAPTER I}

REFERENCES

(1) Organic Chemistry in Action: The Design of Organic Synthesis. Felix Serratosa and Josep Xicart. $2^{\text {nd }}$ edition, Elsevier: Amsterdam, 1996.

(2) Solid-state Supramolecular Chemistry: Crystal Engineering. In Comprehensive Supramolecular Chemistry, J.L. Atwood, J.E.D. Davies, D.D. MacNicol and F. Vogtle, Eds. Volume 6, Pergamon / Elsevier: Oxford, 1996.

(3) Antibiotics and Their Complexes: Metal Ions in Biological Systems. Volume 19, M. Dekker: New York, 1985.

(4) Macrocyclic Chemistry: Aspects of Organic and Inorganic Sturamolecular Chemistry. B. Dietrich, P. Viout and J.M. Lehn. VCH: Weinheim, Germany, 1993.

(5) C.R. Prull. Medical History 47(3), 332 (2003).

(6) A.M. Silverstein. Paul Ehrlich's Receptor Immunology: The Magnificent Obsession. Academic Press: London, 2002.

(7) G.B. Kauffman. ACS Symposium Series 565, 3 (1994).

(8) L.M. Venanzi. Chimia 48(1), 16 (1994).

(9) F. Cramer. Pharm. Acta Helvetiae 69(4), 193 (1995).

(10) R.U. Lemieux and U. Spohr. Adv. Carb. Chem. Biochem. 50, 1 (1994).

(11) E.J.M. Honigmann. Natumwissenschaften 20,635 (1932).

(12) Clathrate Inclusion Compounds. M.M. Hagen. Reinhold Publication Corp: New York, 1962.

(13) Non-Stoichiometric Compounds. L. Mandelcorn. Academic Press: New York, 1964.

(14) Clathrate Compounds. V.M. Bhatnagar. Chemical Publishing Company: Delhi, 1970.

(15) Supramolecular Chemistry. Gordon and Breach Science Publishers: New York, 1992.

(16) Journal of Supramolecular Chemistry. Elsevier Science Ltd: London, 2001 -.

(17) Supramolecular Chemistry: Concepts and Perspectives - A Personal Account. J.M. Lehn. VCH: Weinheim, Germany, 1995.

(18) Supramolecular Chemistry. J.W. Steed and J.L. Atwood. John Wiley and Sons: New York, 2002.

(19) Principles and Methods in Supramolecular Chemistry. H.J. Schneider and A.K. Yatsimirski. John Wiley and Sons: New York, 2000.

(20) Supramolecular Design for Biological Applications. N. Yui, Ed. CRC Press: Boca Raton, Florida, 2002.

(21) Molecules as Components of Electronic Devices. M. Lieberman, Ed. Oxford University Press: Washington, D.C., 2003.

(22) Design of Organic Solids. E. Weber, Ed. Springer: Berlin, 1998.

(23) Molecular Self-Assembly: Organic vs. Inorganic Approaches. M. Fujita, Ed. Springer: Berlin 2000. 
(24) Transition Metals in Supramolecular Chemistry. L. Fabbrizzi and A. Poggi Eds. Kluwer Academic Publishers: Dordrecht, 1994.

(25) Perspectives in Coordination Chemistry. A.F. Williams, C. Floriani and A.E. Merbach, Eds. VCH: Weinheim, 1992.

(26) Physical Supramolecular Chemistry. L. Echegoyen and A.E. Kaifer, Eds. Kluwer Academic Publishers: Dordrecht, 1996.

(27) Computational Approaches in Supramolecular Chemistry. G. Wipff, Ed. Kluwer Academic Publishers: Dordrecht, 1994.

(28) Journal of Supramolecular Structure and Cellular Biochemistry. A.R. Liss Inc.: New York, 1981 -.

(29) Biological Complexity and the Dynamics of Life Processes. J. Richard. Elsevier: Amsterdam, 1999.

(30) Advanced Macromolecular and Supramolecular Matcrials and Processes. K.E. Geckeler, Ed. Kluwer Academic / Plenum Publishers: New York, 2003.

(31) Supramolecular Structurc and Function. G. Pifat-Mrzljak. Springer-Verlag: Berlin, 1986.

(32) Supramolecular Science. Elsevier Science: Oxford 1994-1998 (Vols 1-5).

(33) D.D. MacNicol. Structure and Design of Inclusion Compounds: The Clathrates of Hydroquinone, Phenol, Dianin's Compound and Related Systems. In Inclusion Compounds. J.L. Atwood, J.E.D. Davies and D.D. MacNicol, Eds. Volume 2, Chapter 1. Academic Press: London, 1984.

(34) T.C.W. Mak and B.R.F. Bracke. Hydroquinone Clathrates and Diamondoid Host Lattices. In Comprehensive Supramolecular Chemistry, J.L. Atwood, J.E.D. Davies, D.D. MacNicol and F. Vogtle, Eds. Volume 6, Chapter 2. Pergamon / Elsevier: Oxford, 1996.

(35) R. Gerdil. Tri-o-Thymotide Clathrates. In Topics in Current Chemistry. E. Weber, Ed. Volume 140, Chapter 4. Springer-Verlag: Berlin, 1987.

(36) R. Gerdil. Tri-o-Thymotide and Related Hosts. In Comprehensive Supramolecular Chemistry, J.L. Atwood, J.E.D. Davies, D.D. MacNicol and F. Vogtle, Eds. Volume 6, Chapter 8. Pergamon / Elsevier: Oxford, 1996.

(37) Gas Hydrates: Challenges for the Future. G.D. Holder and P.R. Bishnoi, Eds. New York Academy of Sciences: New York, 2000.

(38) Clathrate Hydrates of Natural Gases. E. Dendy Sloan Jr. Marcel Dekker: New York, 1998.

(39) K. Takemoto and N. Sonoda. Inclusion Compounds of Urea. Thiourea and Selenourea. In Inclusion Compounds. J.L. Atwood, J.E.D. Davies and D.D. MacNicol, Eds. Volume 2, Chapter 2. Academic Press: London, 1984.

(40) M.D. Hollingsworth and K.D.M. Harris. Urea, Thiourea and Selenourea. In Comprehensive Supramolecular Chemistry, J.L. Atwood, J.E.D. Davies, D.D. MacNicol and F. Vogtle, Eds. Volume 6, Chapter 7. Pergamon / Elsevier: Oxford, 1996.

(41) Inclusion Chemistry with Zeolites: Nanoscale Materials by Design. N. Herron and D.R. Corbin, Eds. Kluwer Academic Publishers: Dordrecht, 1995.

(42) Introduction to Zeolite Science and Practice. H.V. Bekkum, Ed. Elsevier: Amsterdam, 2001. 
(43) Clay Microstructure. R.H. Bennett and M.H. Hulbert. Reidel Publishing Corporation: Boston, 1986.

(44) Clay Mineralogy. R.E. Grim. McGraw-Hill: New York, 1968.

(45) Fuller's Earth: A History of Calcium Montmorillonite. R.H.S. Robertson. Volturna Press: Hythe, Kent, 1986.

(46) Cyclodextrin Technology. J. Szejtli. Kluwer Academic Publishers: Dordrecht, 1988.

(47) Cyclodextrins and Their Inclusion Complexes. J. Szejtli. Akademiai Kiado: Budapest, 1982.

(48) P. Finocchiaro and S. Failla. Other Potentially Important Hosts. In Comprehensive Supramolecular Chemistry, J.L. Atwood, J.E.D. Davies, D.D. MacNicol and F. Vogtle, Eds. Volume 6, Chapter 18. Pergamon / Elsevier: Oxford, 1996.

\section{CHAPTER II}

\section{HALOGENATED HYDROCARBONS}

Halocarbons are halogen-substituted hydrocarbons that contain carbon, hydrogen and halogen atoms. These compounds are formed when hydrogen atoms of hydrocarbons are partially or fully replaced by halogen atoms. Some of the widely used halocarbons include chloroform $\left(\mathrm{CHCl}_{3}\right)$, methylene chloride $\left(\mathrm{CH}_{2} \mathrm{Cl}_{2}\right)$, methyl iodide $\left(\mathrm{CH}_{3} \mathrm{I}\right), 1,1,1$ trichloroethane $\left(\mathrm{CH}_{3} \mathrm{CCl}_{3}\right)$ and vinyl chloride $\left(\mathrm{CH}_{2}=\mathrm{CHCl}\right)$. On the other hand, carbon tetrachloride $\left(\mathrm{CCl}_{4}\right)$, carbon tetrabromide $\left(\mathrm{CBr}_{4}\right)$, tetrachloroethylene $\left(\mathrm{Cl}_{2} \mathrm{C}=\mathrm{CCl}_{2}\right), 1,1,2-$ trichloro-1,2,2-trifluoroethane $\left(\mathrm{Cl}_{2} \mathrm{FC}-\mathrm{CF}_{2} \mathrm{Cl}\right)$ and hexachlorobenzene $\left(\mathrm{CCl}_{6}\right)$ are examples of fully substituted halocarbons that do not contain hydrogen atoms.

Although most of the halogenated hydrocarbons that are commonly in use are liquids at ambient temperature and pressure, lower molecular weight compounds, such as 1,2-difluoroethane, methyl bromide or vinyl chloride, are gases. Conversely, high molecular weight halocarbons, such as iodoform $\left(\mathrm{CHI}_{3}\right)$ or hexachloronaphthalene, are solids at room temperature. Halogenated hydrocarbons are used as organic solvents, in extractions, as intermediates, and in the manufacture of numerous chemicals of wide commercial use. Many fluorocarbons are used as refrigerants and in fire extinguishers. Because of health hazards, many anesthetic halocarbons used earlier on in clinical anesthesia have been replaced by less toxic compounds.

Halogenated hydrocarbons, in general, exhibit low acute toxicity. Inhalation toxicity is greater for gaseous or volatile liquid compounds. The health hazards from exposure to these compounds may be due to their anesthetic actions, damaging effects on 
the liver and kidneys, and in the case of some halocarbons, carcinogenicity. The toxic symptoms include drowsiness, incoordination, anesthesia, hepatitis and necrosis of the liver. Vapors may cause irritation of the eyes and respiratory tract. Anesthetic effects in animals or humans occur at high levels of exposure, which may be several percentages of concentration in air. Death may result from cardiac arrest due to prolonged exposure to high concentrations. Oral ingestion may produce nausea, vomiting and liver injury. Fatal doses in humans vary with the compound. Fluorocarbons are less toxic than the chlorobromo- and iodo- compounds. The toxicity increases with an increase in the mass number of the halogen atom(s). Some of the halogenated hydrocarbons cause cancer in humans. These include vinyl chloride, vinyl bromide, methyl iodide, 1,2-dibromoethane, chloroform, carbon tetrachloride and hexachlorobutadiene. In addition, many other compounds of commercial interest have been shown to cause cancer in animals, such as methylene chloride, 1,1,2-trichloroethane, trichloroethylene, tetrachloroethylene, 1,1dichloroethylene, 4,4'-dichloro-diphenyl-dichloroethylene, chlorobutadiene, 1,2dichloroethane, hexachloroethane, $n$-propyliodide and chlorofluoromethane.

Halocarbons play an important role in the photochemistry of the stratosphere [1], since they are the source of halogen radicals that catalytically destroy ozone. In addition, most halocarbons are efficient greenhouse gases, as they absorb thermal radiation in the spectral region of the atmospheric window, between about 8 and $13 \mu \mathrm{m}$. Thus, depending on the strength of $I R$ absorption bands and atmospheric lifetimes, their greenhouse "warming potential", relative to that of $\mathrm{CO}_{2}$ as a molecular base, can reach high values.

In the second half of the last century, some 23 million metric tonnes of chlorofluorocarbons (CFC's), about 11 million tonnes of methyl chloroform, 2.5 million tonnes of carbon tetrachloride and 4 million tonnes of a hydrofluorocarbon (HCFC-22) were released into the atmosphere. All of these halocarbons have sufficiently long atmospheric lifetimes for them to be transported into the stratosphere. The amount of chlorine in the stratosphere today is between six and seven times what it was in 1950. There is an ozone hole over Antarctica from September to December each year, less systematic but nevertheless large ozone losses in the Arctic, and moderate ozone losses in the mid to high latitudes of both hemispheres, greatest during winter and spring. The 
weight of the evidence indicates that decay products from human-activity-related emissions of various halocarbons, particularly $\mathrm{CFC}$ 's such as $\mathrm{CFCl}_{3}$ and $\mathrm{CF}_{2} \mathrm{Cl}_{2}$, and halons, such as $\mathrm{CF}_{3} \mathrm{Br}$, are responsible for this ozone decrease $[2,3 \mid$. Chlorine and bromine atoms contained in these molecules react catalytically in the stratosphere to destroy ozone. Indeed, atmospheric measurements show that natural amounts of ozone in the atmosphere have decreased globally by more than $10 \%$ since 1970 [2-4|. As a result of this damage, there will be serious impacts on human health, including a significant increase in the incidence of skin cancer.

Infrared (IR) active gases, principally water vapor, carbon dioxide and ozone, naturally present in the Earth's atmosphere, absorb thermal IR radiation emitted by the Earth's surface and atmosphere. The atmosphere is warmed by this mechanism and, in turn, emits $I R$ radiation, with a significant portion of this energy acting to warm the surface and lower atmosphere. As a consequence, the average surface air temperature of the Earth is about $30^{\circ} \mathrm{C}$ higher than it would be without this atmospheric absorption and reradiation of IR energy [5-7].

Atmospheric measurements also show that concentrations of several of these radiatively important gases, termed greenhouse gases, are changing, and in many cases, growing. The rapid increase in concentrations of greenhouse gases since the industrial period began has given rise to concern over potential resultant climate changes. Budget analyses of the sources and sinks of these gases indicate that these changes result primarily from human activities. Concern about the effects of these changes on climate has centered on carbon dioxide $\left(\mathrm{CO}_{2}\right)$, because it is an important greenhouse gas, and because its atmospheric concentration is rapidly increasing. However, other greenhouse gases have contributed about half the overall increase in the radiative forcing effect on climate [8,9]. The most important of these greenhouse gases are methane $\left(\mathrm{CH}_{4}\right)$, nitrous oxide $\left(\mathrm{N}_{2} \mathrm{O}\right)$ and a variety of halocarbons, including chlorofluorocarbons and halons. Relative to carbon dioxide, the various halocarbon chemicals in the atmosphere, although in a significantly lower abundance, have a very high radiative forcing potential, 10000 or more times more effective (per molecule) than $\mathrm{CO}_{2}$, as depicted in Table II-1. 
Table II-1: Radiative forcing relative to $\mathrm{CO}_{2}$ on a per-molecule basis for some common greenhouse gases [10].

\begin{tabular}{lll}
\hline Gas & molecular formula & relative radiative forcing \\
carbon dioxide & $\mathrm{CO}_{2}$ & 1 \\
methane & $\mathrm{CH}_{4}$ & 21 \\
nitrous oxide & $\mathrm{N}_{2} \mathrm{O}$ & 206 \\
CFC-11 & $\mathrm{CFCl}_{3}$ & 12400 \\
CFC-12 & $\mathrm{CF}_{2} \mathrm{Cl}_{2}$ & 15800 \\
$\mathrm{HCFC}-22$ & $\mathrm{CF}_{2} \mathrm{HCl}$ & 10700 \\
HCFC-123 & $\mathrm{C}_{2} \mathrm{~F}_{3} \mathrm{HCl}$ & 9920 \\
HCFC-124 & $\mathrm{C}_{2} \mathrm{~F}_{4} \mathrm{HCl}$ & 10790 \\
HFC-134a & $\mathrm{C}_{2} \mathrm{~F}_{4} \mathrm{H}_{2}$ & 9550 \\
perfluoromethane & $\mathrm{CF}_{4}$ & 5460 \\
sulfur hexafluoride & $\mathrm{SF}_{6}$ & 38400 \\
\hline
\end{tabular}

\section{CHAPTER II}

REFERENCES

(1) P. Warneck. Chemistry of the Natural Atmosphere Academic Press: San Diego, 2000.

(2) World Meteorological Organization. Scientific Assessment of Ozone Depletion Global Ozone Research and Monitoring Project - Report No. 37, 1994, Geneva

(3) World Meteorological Organization. Scientific Assessment of Ozone Depletion. Global Ozone Research and Monitoring Project - Report No. 25, 1991, Geneva

(4) G.C. Reinsel, G.C. Tiao, D.J. Wuebbles, J.B. Kerr and A.J. Miller. J. Geophys. Res. 99, 5449 (1991).

(5) A. Henderson-Sellers and P.J. Robinson. Contemporary Climatologv John Wiley and Sons Inc.: New York, 1986.

(6) W.K. Kellogg. Greenhouse Effect In Encyclopedia of Climate and Weather, S.H. Schneider, Ed. Oxford University Press: New York, 1996.

(7) J.P. Peixoto and A.H. Oort. Physics of Climate American Institute of Physics: New York, 1992.

(8) Intergovernmental Panel on Climate Change Radiative Forcing of Climate Change Cambridge University Press: Cambridge, UK, 1994.

(9) Intergovernmental Panel on Climate Change The IPCC Scientific Assessment Cambridge University Press: Cambridge, UK, 1990.

(10) D.J. Wuebbles. Annu. Rev. Energy Environ. 20, 45 (1995). 
CHAPTER III

TATM LITERATURE REVIEW

The tripodal host tris(5-acetyl-3-thienyl) methane (TATM), 1 , is a tripodal host molecule that can exist in a wide variety of conformationally isomeric states [1]. It has been found to form inclusion compounds with a large and wide variety of organic compounds [2]. It is a host that is similar in structure to the triphenylmethane group of host materials [3].

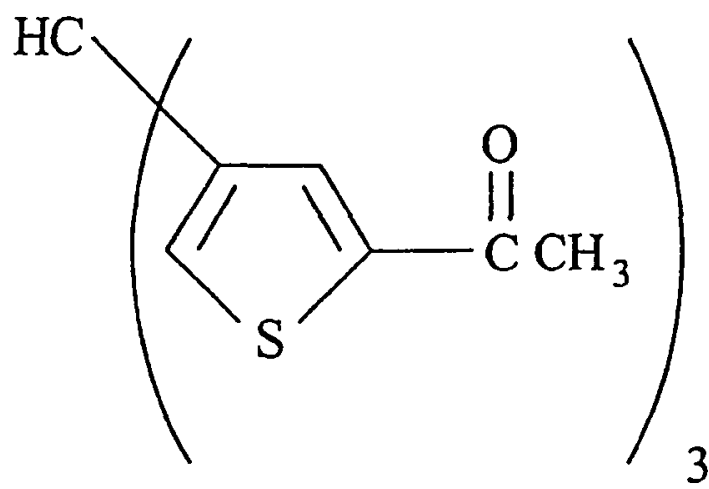

(A) A.P. Yakubov, Y.K. Sudarushkin, L.I. Belen'kii and Y.L. Gol'dfarb. J. Org. Chem. (USSR) 9, 1549 (1973)

TATM was first prepared by Y. L. Gol'dfarb et al, in 1973, from 2-acetylthiophene and chloroform, in the presence of an excess of the Lewis acid aluminum chloride [4]. Like other electrophilic substitution reactions, the alkylation of 2-acetythiophene, in the presence of aluminum chloride and a stoichiometric quantity of chloroform, is directed primarily to position 4 (position 3 of TATM) of the thiophene ring. The preferential formation of this product is explained [5] by strengthening of the electron-withdrawing power of the carbonyl group as a result of complex formation with aluminum chloride, leading to greater deactivation of positions 3 and 5 of 2-acetylthiophene compared to position 4, which then becomes the most reactive. It was also established [6] that it is not essential to use a large excess of aluminum chloride, which acts as a unique kind of solvent, leading to a reduction in the melting point of the complex of 2-acetylthiophene with aluminum chloride. Similar reactions can also be carried out in an excess of (rather than a stoichiometric amount of) conventional solvents (c.g. $\mathrm{CH}_{2} \mathrm{Cl}_{2}, \mathrm{CHCl}_{3}$ or $\mathrm{CCl}_{4}$ ) that 
are capable of dissolving these complexes without appreciable dissociation, and then it is sufficient to use only 1.5 moles of aluminum chloride for each mole of 2-acetylthiophene. In this case, the alkyl chloride serves both as a reactant and as a solvent medium.

It was found that the alkylation of 2-acetylthiophene by chloroform takes place only at elevated temperatures. The reaction carried out at room temperature after extended periods of time (e.g. 7 hours) gave only negligible amounts of TATM product. On the other hand, the alkylation, in the presence of an excess of aluminum chloride, at the boiling point of the solvent $\left(61^{\circ} \mathrm{C}\right)$, leads to tris (5-acetyl-3-thienyl) methane with a yield of $\sim 70 \%$, calculated based on the amount of 2-acetylthiophene used. The greater degree of conversion of 2-acetylthiophene when carbon tetrachloride $(79 \%)$ and chloroform (75\%) are used, compared to methylene chloride (13\%), is due not only to the higher temperature at which the reaction is carried out, but also clearly to the higher reactivity of $\mathrm{CCl}_{4}$ and $\mathrm{CHCl}_{3}$ compared to $\mathrm{CH}_{2} \mathrm{Cl}_{2}$.

The author's general synthetic procedure [4] was as follows: A mixture of 0.25 moles aluminum chloride and 0.1 moles of 2-acetylthiophene in $50 \mathrm{~mL}$ of chloroform was heated (with vigorous stirring) at the boiling point of chloroform $\left(61^{\circ} \mathrm{C}\right)$ for one hour. The mixture was then mixed with dilute hydrochloric acid and extracted with chloroform. The organic extract was washed with water, the solvent was distilled off under vacuum, and the remaining residue was then sublimed under vacuum $\left(220^{\circ}-240^{\circ} \mathrm{C}\right.$, 0.3 torr) to give an amorphous guest-free pale orange solid. Found: $\mathrm{C} 58.66 \%, \mathrm{H} 4.15 \%$, S $24.76 \%$. Calculated: C $58.70 \%, \mathrm{H} 4.12 \%$, S $24.70 \%$. The visually measured melting point of the vacuum sublimed product was $50^{\circ}-58^{\circ} \mathrm{C}$.

The authors also discovered that during crystallization of TATM from various solvents (e.g. benzene, ethanol and pyridine), TATM incorporates them in a stoichiometric ratio of $2 \mathrm{H}: 1 \mathrm{G}$ into its lattice. The solvent-free product was obtained by vacuum sublimation. The melting points were as follows: benzene/TATM $\left(94^{\circ}-97^{\circ} \mathrm{C}\right)$, ethanol/TATM $\left(103.5^{\circ}-105.5^{\circ} \mathrm{C}\right)$ and pyridine/TATM $\left(104^{\circ}-107^{\circ} \mathrm{C}\right)$.

(B) L. Bin Din and O. Meth-Cohn J. Chem. Soc. Chem. Commun. 21, 741 (1977) In 1977, Bin Din and Meth-Cohn found that TATM forms 2:1 host:guest clathrates with a wide variety of organic compounds [2]. They characterized TATM inclusion compounds 
with 39 different guests. For each one, they measured stoichiometric ratios and melting points. Almost all of the inclusion compounds melted between $80^{\circ}-140^{\circ} \mathrm{C}$ (vacuum sublimed TATM melts between $50^{\circ}-58^{\circ} \mathrm{C}$ ), and almost all were comprised of a $2 \mathrm{H}: 1 \mathrm{G}$ stoichiometric ratio. In addition, their systematic study, to that point in time, failed to reveal a solvent that was not incorporated. Furthermore, studies in our laboratory, over the past several years, involving a numerous (up to 100) and extremely wide variety of guests, have also found no evidence of an unsuitable solvent. However, the problem with incorporating compounds that exist as solids (at room temperature) into TATM is that it is almost impossible to find a common solvent that itself is not incorporated into the host lattice.

In the 1977 study, Bin Din and Meth-Cohn concurred with the proposed structure [4] from NMR data that prove the 3,5-substitution: (1) thiophene $\mathrm{H}^{`} \mathrm{~s}: 7.51 \mathrm{ppm}$ and 7.23 ppm; (2) methine $\mathrm{CH}: 5.59 \mathrm{ppm}$; and (3) acetyl $\mathrm{CH}_{3}: 2.51 \mathrm{ppm}$. They found that the TATM compound is readily prepared in 50-100 $\mathrm{g}$ amounts by minor modifications to the initial procedure reported by Gol'dfarb and co-workers in 1973 [4|. Their systematic study $[2]$ yielded the following results $(2 \mathrm{H}: 1 \mathrm{G}$ stoichiometric ratio unless otherwise noted): benzene $106^{\circ} \mathrm{C}-110^{\circ} \mathrm{C}$, toluene $109^{\circ} \mathrm{C}-110^{\circ} \mathrm{C}$, ortho-xylene $120^{\circ} \mathrm{C}-121^{\circ} \mathrm{C}$, metaxylene $97^{\circ} \mathrm{C}-98^{\circ} \mathrm{C}$, para-xylene $112^{\circ} \mathrm{C}-113^{\circ} \mathrm{C}$, mesitylene $77^{\circ} \mathrm{C}-78^{\circ} \mathrm{C}$, ethylbenzene $106^{\circ} \mathrm{C}-110^{\circ} \mathrm{C}$, cumene $85^{\circ} \mathrm{C}-89^{\circ} \mathrm{C}$, tert-butylbenzene $(3 \mathrm{H}: 1 \mathrm{G}) 136^{\circ} \mathrm{C}-139^{\circ} \mathrm{C}$, fluorobenzene $106^{\circ} \mathrm{C}-110^{\circ} \mathrm{C}$, chlorobenzene $116^{\circ} \mathrm{C}-119^{\circ} \mathrm{C}$, bromobenzene $128^{\circ} \mathrm{C}-130^{\circ} \mathrm{C}$, iodobenzene $131^{\circ} \mathrm{C}-132^{\circ} \mathrm{C}$, naphthalene $140^{\circ} \mathrm{C}-142^{\circ} \mathrm{C}$, cyclohexane $98^{\circ} \mathrm{C}-100^{\circ} \mathrm{C}$, decalin $74^{\circ} \mathrm{C}-75^{\circ} \mathrm{C}$, cyclooctene $98^{\circ} \mathrm{C}-99^{\circ} \mathrm{C}$, methanol $108^{\circ} \mathrm{C}-114^{\circ} \mathrm{C}$, ethanol $108^{\circ} \mathrm{C}-114^{\circ} \mathrm{C}$, isopropanol $106^{\circ} \mathrm{C}-111^{\circ} \mathrm{C}, 2$-butanol $97^{\circ} \mathrm{C}-100^{\circ} \mathrm{C}$, tert-butanol $103^{\circ} \mathrm{C}-107^{\circ} \mathrm{C}, n$-decanol $121^{\circ} \mathrm{C}-123^{\circ} \mathrm{C}$, methoxybenzene $102^{\circ} \mathrm{C}-106^{\circ} \mathrm{C}$, acetone $111^{\circ} \mathrm{C}-113^{\circ} \mathrm{C}$, methylethylketone $111^{\circ} \mathrm{C}-114^{\circ} \mathrm{C}$, methylphenylketone $101{ }^{\circ} \mathrm{C}-104^{\circ} \mathrm{C}$, ethyl acetate $100^{\circ} \mathrm{C}-101^{\circ} \mathrm{C}$, acetonitrile $108^{\circ} \mathrm{C}-111^{\circ} \mathrm{C}$, acetic acid $102^{\circ} \mathrm{C}-104^{\circ} \mathrm{C}$, pyridine $104^{\circ} \mathrm{C}-107^{\circ} \mathrm{C}$, piperidine $102^{\circ} \mathrm{C}-105^{\circ} \mathrm{C}$, triethyl amine $114^{\circ} \mathrm{C}-115^{\circ} \mathrm{C}$, nitromethane $109^{\circ} \mathrm{C}-112^{\circ} \mathrm{C}$, dimethylsulfoxide $113^{\circ} \mathrm{C}-116^{\circ} \mathrm{C}$, methylene chloride $109^{\circ} \mathrm{C}-115^{\circ} \mathrm{C}$, chloroform $100^{\circ} \mathrm{C}$ $107^{\circ} \mathrm{C}$, carbon tetrachloride $(6 \mathrm{H}: 1 \mathrm{G}) 98^{\circ} \mathrm{C}-99^{\circ} \mathrm{C}$, dimethylformamide $110^{\circ} \mathrm{C}-113^{\circ} \mathrm{C}$. 
The authors noted that solids can sometimes be incorporated from solutions in appropriate solvents (e.g. naphthalene is incorporated from a mesitylene solution), while solvents which readily dissolve the host compound (i.e. do not crystallize easily) may yield the clathrate by addition of light petroleum (e.g. for chloroform) or water (e.g. for dimethyl sulfoxide). When TATM inclusion compounds were dissolved in various pure solvents, no evidence of host-guest interactions was observed, since ' $H$ solution NMR signals generally showed unchanged chemical shifts (compared to the neat compounds). The melting points of the TATM clathrates are often considerably higher than the boiling points of the pure guest solvents, while the higher melting ortho-xylene/TATM $\left(120^{\circ}\right.$ $\left.121^{\circ} \mathrm{C}\right)$ is preferentially, but not exclusively $(\sim 5: 1)$ incorporated from a $50: 50$ mixture of ortho-xylene and meta-xylene (meta-xylene/TATM melts at $97^{\circ}-98^{\circ} \mathrm{C}$ )

In the 1977 study, the authors prepared a variety of TATM analogues, by replacement of the acetyl group of the 2-acetylthiophene starting material with other functionalities, such as $\mathrm{OCCH}_{2} \mathrm{CH}_{3}$ (yield $86 \%$, mp $147^{\circ}-150^{\circ} \mathrm{C}$ ), $\mathrm{OCCH}_{2} \mathrm{CH}_{2} \mathrm{CH}_{3}$ (yield $53 \%, \mathrm{mp} 76^{\circ} \mathrm{C}$ ), $\mathrm{OCCH}\left(\mathrm{CH}_{3}\right)_{2}$ (yield $11 \%, \mathrm{mp} 153^{\circ}-155^{\circ} \mathrm{C}$ ), $\mathrm{OCOCH}_{3}$ (yield $52 \%$ ) and $\mathrm{OCOCH}_{2} \mathrm{CH}_{3}$ (yield $20 \%$, bp $264^{\circ} \mathrm{C}$ at $0.7 \mathrm{mmHg}$ ) among others. Oddly enough, only the methyl ester showed any promise of clathration ability. It was obtained as a viscous oil, but crystallized slowly from ethanol, cyclohexane or carbon tetrachloride (melting points $74^{\circ}-77^{\circ} \mathrm{C}, 83^{\circ}-86^{\circ} \mathrm{C}$ and $84^{\circ}-86^{\circ} \mathrm{C}$ respectively), incorporating the solvent in each case in a $2 \mathrm{H}: \mathrm{IG}$ stoichiometric ratio. Finally, attempts at resolving a racemic mixture of 2-butanol, to utilize the potentially chiral crystalline clathrates with TATM, were found to be ineffective.

(C) P.H. van Rooyen and H.M. Roos Acta Cryst. C47, 2468 (1991)

(D) J.L.M. Dillen and H.M. Roos Acta Cryst. C48, 2229 (1992)

(E) H.M. Roos and J.L.M. Dillen Acta Cryst. C48, 1882 (1992)

(F) P.H. van Rooyen and H.M. Roos Acta Cryst. C47, 2718 (1991)

Between 1991 and 1992, four crystal structures of TATM inclusion compounds were published by H. M. Roos and co-workers [7-10]. All four crystallized in the triclinic $\mathrm{P} \overline{\mathrm{l}}$ space group. Ethyl acetate [7] and ethanol [8] each form 2:1 host:guest complexes in 
which the guest is enclathrated by two of the three acetyl thienyl substituents of each of the two host molecules in the unit cell. For ethyl acetate/TATM, the shortest nonhydrogen non-bonded distance between host and guest is $3.416 \AA[\mathrm{O}(7)-\mathrm{C}(26)]$

On the other hand, the $n$-hexane/TATM structure [9] consists of three TATM host molecules and one $n$-hexane guest molecule. The $n$-hexane guest molecules fill the channels formed by two acetyl thienyl substituents from each TATM host molecule. The remaining acetyl thienyl ring links the host molecules into parallel stacks with an alternating orientation. Benzene/TATM appears to be unique [10] in that the guest interacts with only one thienyl ring of each host molecule. In this structure, the shortest non-hydrogen non-bonded distance between host and guest is $3.437 \AA[\mathrm{O}(6)-\mathrm{C}(43)]$.

In all four structures, the isotropic thermal displacement factors of the guest's atoms are at least two to three times as large as those measured for the host's. This suggests a lack of strong bonding between host and guest, perhaps resulting in disorder on the part of the guest. All four structures were solved by direct methods using the MULTAN80 suite of programs [11] and refined by blocked-full-matrix least squares on $F$ using SHELX76 software [12] with $\sigma^{-2}(F)$ weights. All TATM host non-hydrogen atoms were refined anisotropically, and all guest non-hydrogen atoms were refined with a common isotropic thermal parameter. All TATM thiophene hydrogen atoms were refined in experimental positions and all other hydrogens (host and guest) were placed in calculated positions $\left(\mathrm{C}-\mathrm{H}=1.08 \AA, \angle \mathrm{H}-\mathrm{C}-\mathrm{H}=109.4^{\circ}\right)$ with a common thermal parameter that was also refined. Scattering factors were taken from International Tables for X-ray Crystallography (1974, Vol. IV).

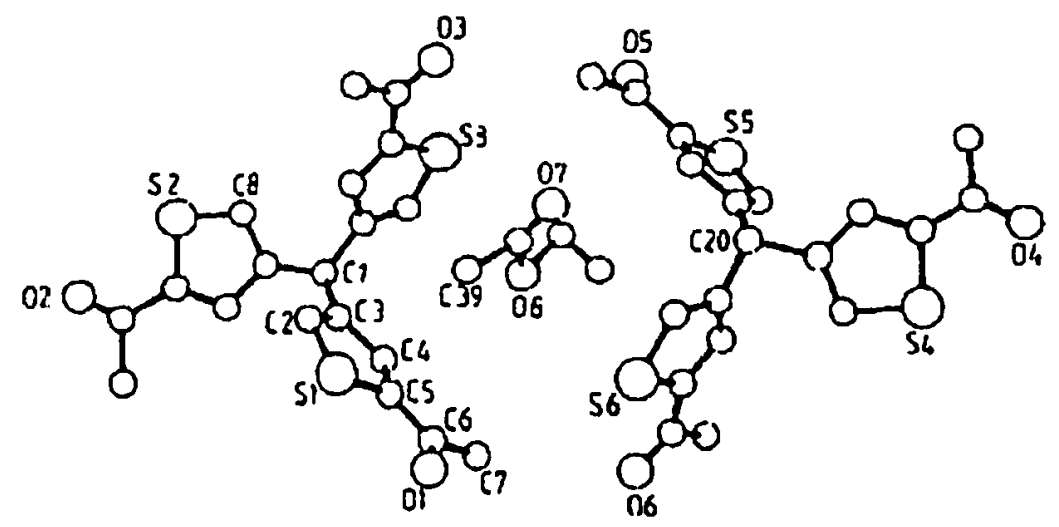

Figure III-1: Perspective view of ethyl acetate/TATM with atomic numbering scheme [7] 


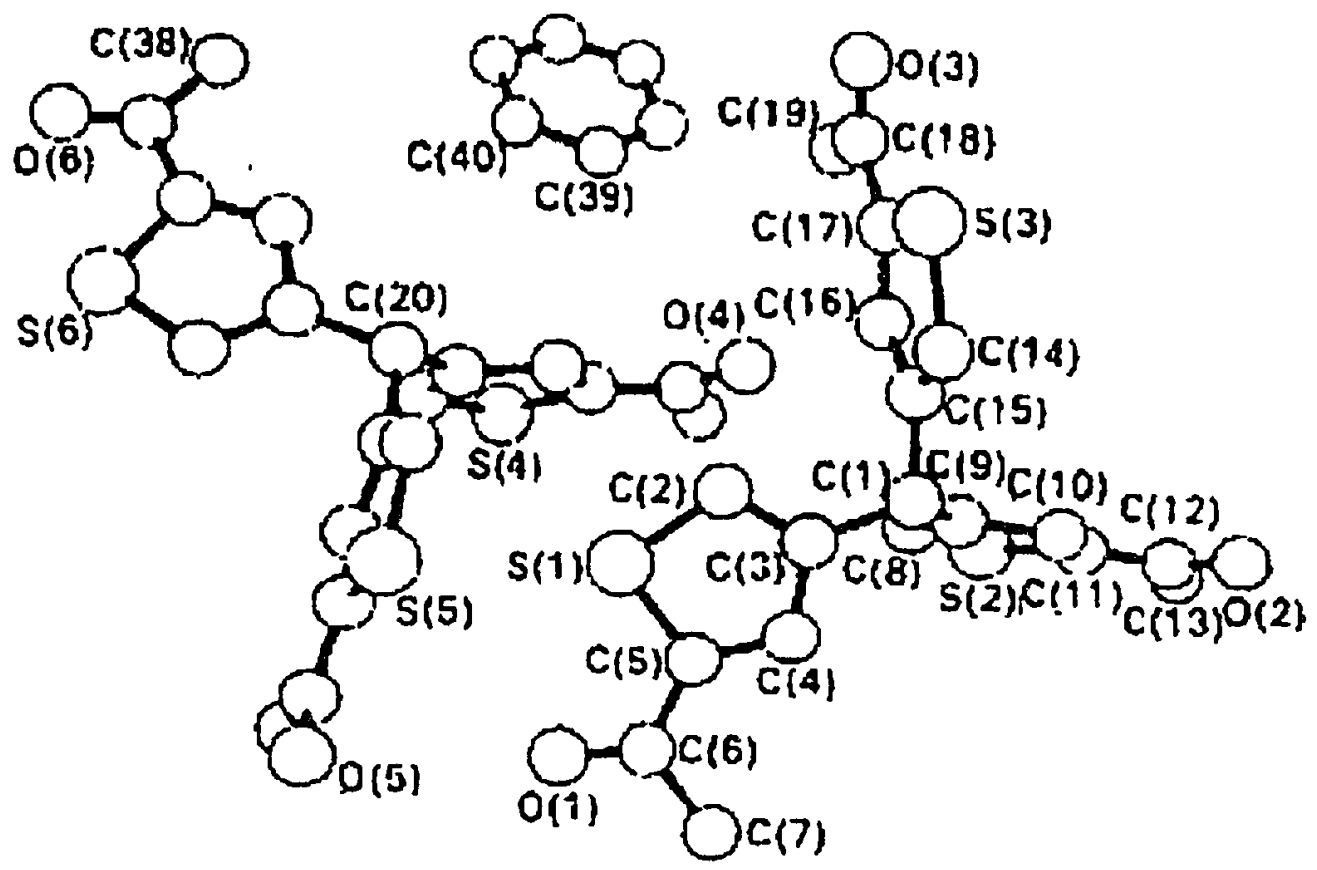

Figure III-2: Perspective view of benzene / TATM with atomic numbering scheme [10]

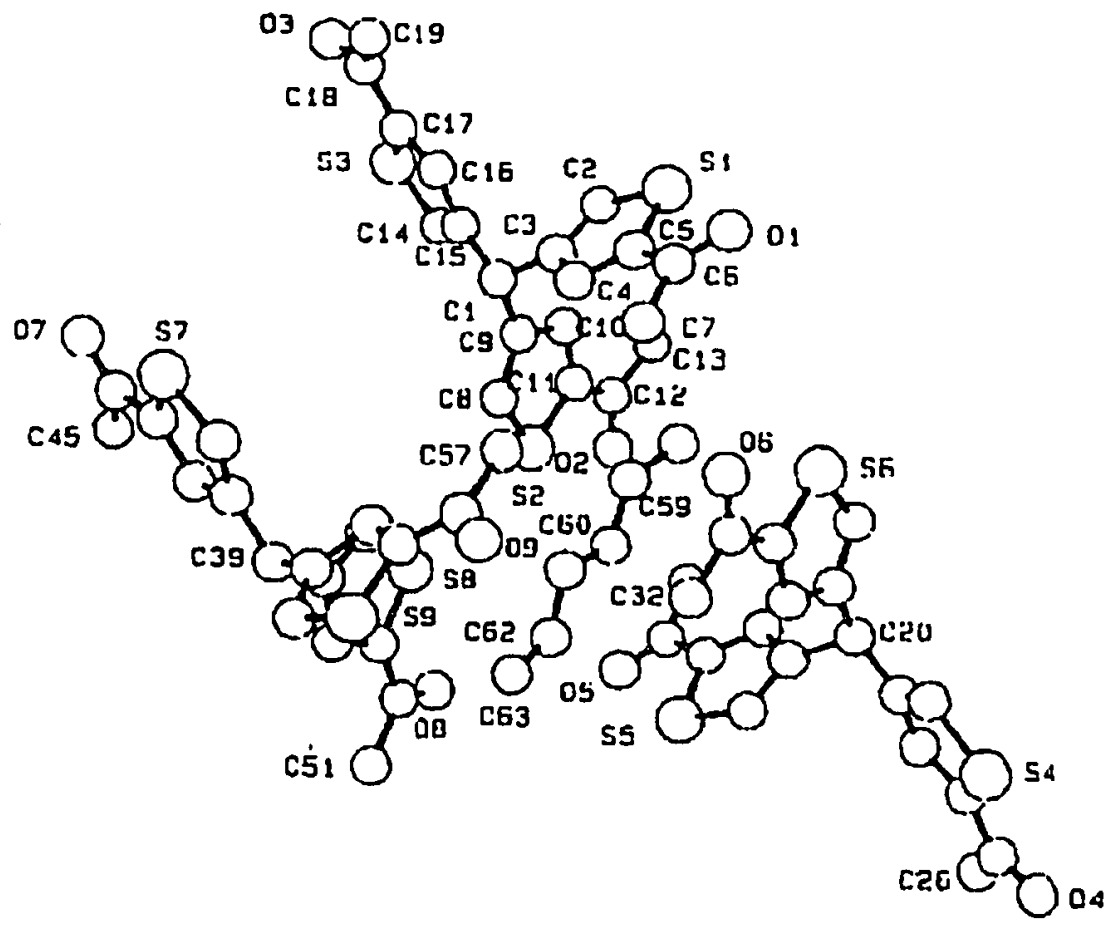

Figure III-3: Perspective view of $n$-hexane / TATM with atomic numbering scheme [9] 

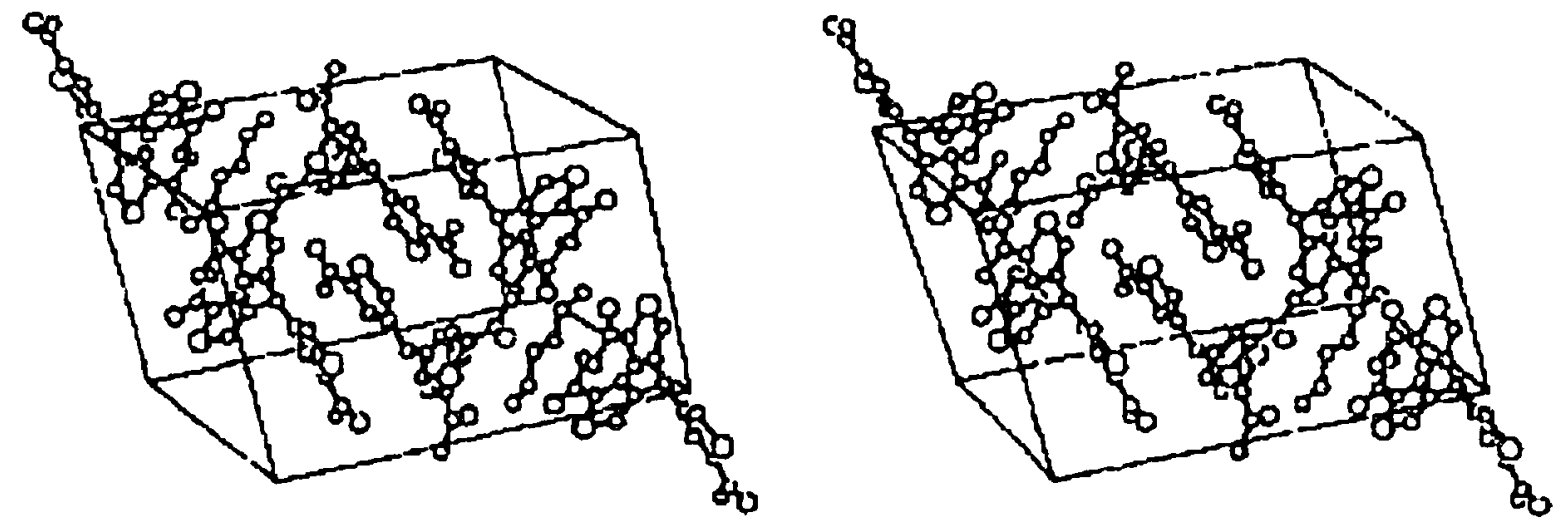

Figure III-4: Stereopair of $n$-hexane / TATM showing the crystal packing [9]

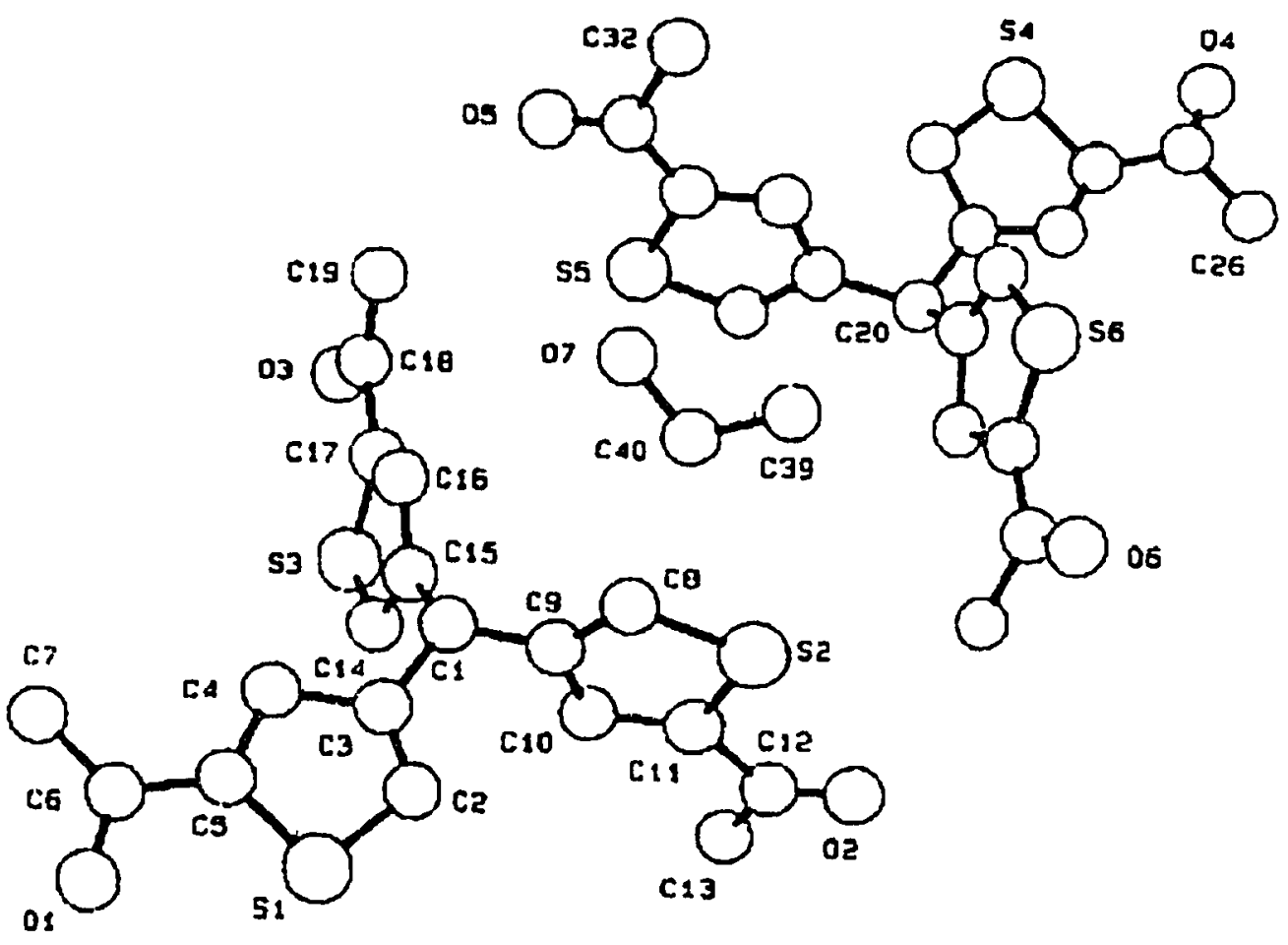

Figure III-5: Perspective view of ethanol / TATM with atomic numbering scheme [8] 
Table III-1: Crystal data for TATM inclusions with four different guests [7-10]

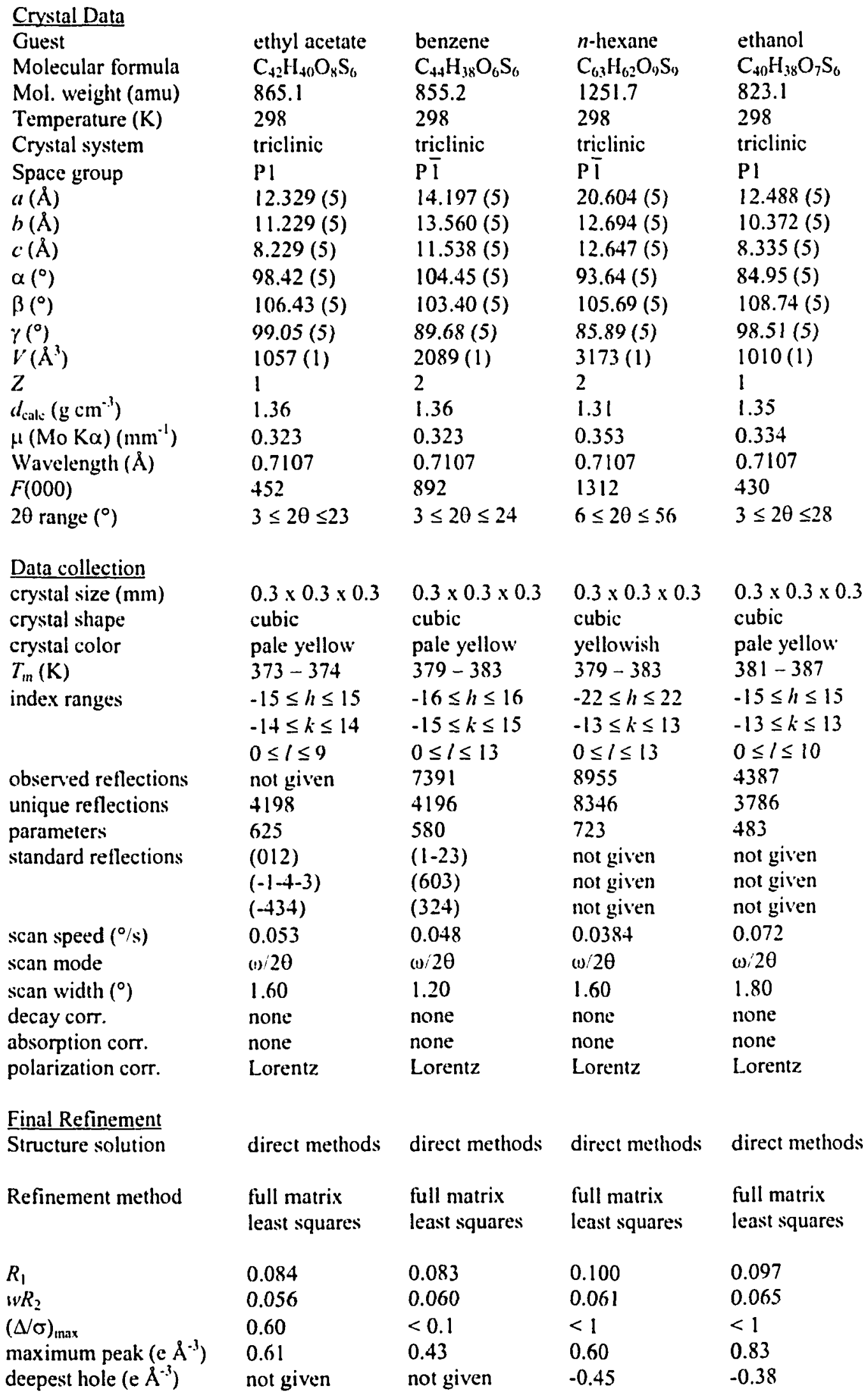


(G) R.E. Marsh Acta Cryst B50, 112 (1994)

In 1994, R. E. Marsh suggested [13] that the previously reported crystal structures of ethyl acetate / TATM [7] and ethanol / TATM [8] are better described in the triclinic space group $\mathrm{P} \overline{\mathrm{l}}$, rather than $P 1$. He stated that refinements in $\mathrm{P} \overline{\mathrm{l}}$ led to lower e.s.d.'s and lower $R$ values and to much more reasonable bond lengths, bond angles and $U_{\mathrm{ij}}{ }^{\circ}$ s for the TATM host molecules, however the guest molecules are disordered and can be described no better in $\mathrm{P} \bar{l}$ than they were in $P 1$.

In the two original structures, it was reported that there were two molecules of TATM and one guest molecule in the unit cell. However, in both cases, the two TATM molecules are closely related by a center of symmetry, and Marsh noted that refinement in $P I$ led to improbable values for many bond lengths and bond angles, presumably due to the large correlations inherent in the refinement of a nearly centrosymmetric model in a non-centrosymmetric space group. Refinement in $P \overline{1}$, which requires the guest molecules to be disordered, seems preferable, leading to improved $R$ values and more reasonable distances and angles within the TATM molecules, however, because of disorder, the guest molecules cannot be described with confidence.

For the original ethyl acetate / TATM structure, the $P 1$ structure was refined to an $R$ value of 0.084 for 4198 reflections [7]. However, Marsh stated that many details of the resulting structure were surprising. For example, the six structurally independent but chemically equivalent $C-C$ bonds involving the two central methine $C(1)$ and $C(20)$ carbons showed bond lengths ranging from 1.453(8) $\AA$ to 1.638(6) $\AA$, there were also large irregularities in the bond angles and the $U_{\text {eq }}$ values as well. After recasting the structure in $\mathrm{P} \overline{\mathrm{l}}$ (by moving the origin to the approximate inversion center between the two TATM molecules, and averaging the coordinates of equivalent atoms), full-matrix least-squares refinement led to an $R$ value of 0.082 for 4182 reflections. The total number of parameters was 281 , compared to 625 in the original structure.

The new refinement has led to much more reasonable bond lengths and bond angles within the host TATM molecule, equivalent values within the now three crystallographically independent acetyl thienyl rings agreeing nearly as well as the e.s.d.'s would predict. The same cannot be said for the guest ethyl acetate molecule, which is now forcibly disordered about the center of symmetry. Because of this, and the 
overlap that results (see Figure III-6), refinement of the individual guest atoms, especially with anisotropic $U_{\mathrm{ij}}$ 's, is probably inappropriate, however convergence to a somewhat reasonable model resulted, and an alternative manner of representing the guest region could not be found. The final coordinates of the guest's atoms are similar to those reported in the original structure, while the same problems of overlap in the guest region must have also affected the original $P 1$ refinement, since the structure is very nearly centrosymmetric no matter how it is described, and accordingly, extensive correlations could not have been avoided.

For the original ethanol / TATM structure, $[8]$ the structure was refined in the triclinic $P 1$ space group to an $R$ value of 0.097 for 3786 reflections. Once again, there were anomalies in some bond lengths and bond angles, with chemically equivalent distances differing by as much as $0.26(3) \AA$. Furthermore, the $\mathrm{C}-\mathrm{C}$ and $\mathrm{C}-\mathrm{O}$ bond lengths in the enclathrated ethanol molecule needed to be constrained. Refinement in $\mathrm{P} \bar{l}$ was based on the same 3786 reflections. At convergence, $R$ was 0.094 for 240 parameters, compared with 483 parameters for the original $P$ I model. In both cases, the $\mathrm{H}$-atoms were placed in calculated positions. Once again, the $\mathrm{P} \overline{\mathrm{I}}$ refinement led to a completely satisfactory TATM molecule, but to confusion for the guest ethanol molecule.

Attempts by Marsh to model the guest ethanol molecule were frustrating and unsatisfying (as apparently had also been the case with the original $P 1$ model, $[8]$ since constraints were required). A tentative electron density map through the major features in this region of the structure is shown in Figure III-7, together with the atom positions that were finally settled upon. Note that the two electron density maxima in Figure III-7 are not coincident with any of the atom centers, they are accounted for by overlap arising from large $B^{\prime}$ s of the three atoms, and in particular, by the very large $U_{\mathrm{ij}}$ anisotropic coefficients of the central guest atom, which leads to a r.m.s. displacement of nearly 0.9 $\AA$ in that direction. Marsh noted that perhaps the only thing that can be said in favor of these guest positions is that they survived least-squares refinement to convergence. Other arrangements of the ethanol molecule may well be imagined, but attempts to model them were never satisfactory. There is clearly a guest present, and studies of the very loworder reflections clearly indicate that in this region of the structure, about as many electrons that are contained in an ethanol molecule correspond to that required in order to 
match the $F_{\text {calc }}$ with the $F_{\text {obs. }}$. There is no reason to doubt the presence of an enclathrated ethanol molecule, but its orientation within the cavity is unclear.

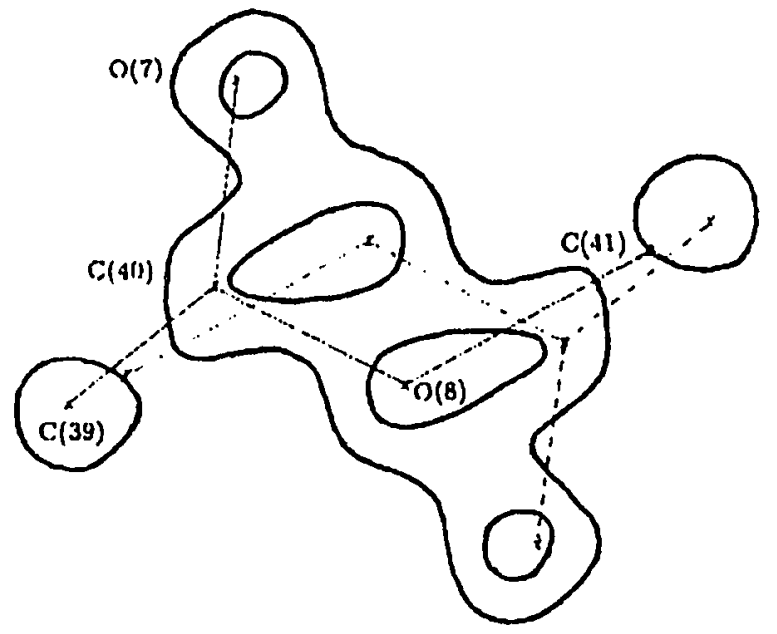

Figure III-6: Electron density map of the ethyl acetate/TATM inclusion compound through most of the major features of the guest area. Contours are at 1.0 and $2.0 \mathrm{e} \AA^{-3}$. the center of the map is at $(0,1 / 2,1 / 2)$. The terminal methyl group $(C(42))$ lies about $0.8 \AA$ out of the plane of the figure. The two centrosymmetrically related guest molecules are indicated by solid and dashed lines [13].

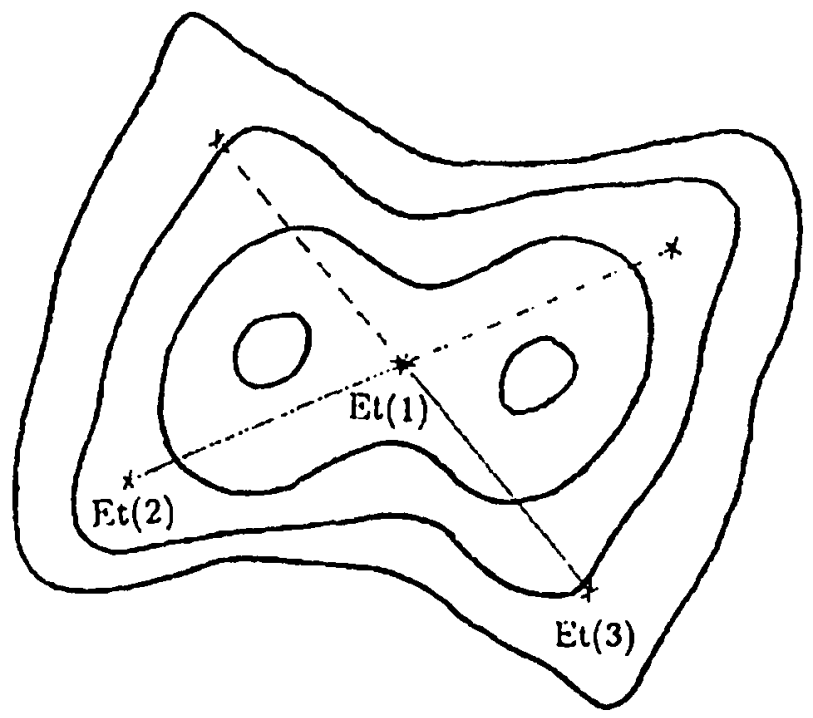

Figure III-7: Electron density map of the ethanol/TATM inclusion compound through most of the major features of the guest area. Contours are at $0.5,1.0,1.5$ and $2.0 \mathrm{e} \AA^{-3}$. the center of the map is at $(1 / 2,0,1 / 2)$. The two centrosymmetrically related guest molecules are indicated by solid and dashed lines [13]. 
Quite conclusive evidence for the centrosymmetric description of the ethyl acetate inclusion compound can be found in an examination of the very weak reflections. From the observed and calculated structure factors (included in the supplementary material of the original structure [7]) for 274 reflections with $F_{\text {obs }}=1$ e, the earlier $P 1$ refinement led to an average value for $F_{\text {calc }}$ of $1.99 \mathrm{e}$ and an $R$ value of 1.09 . On the other hand, the present $\mathrm{P} \overline{\mathrm{l}}$ model leads to an average $F_{\text {calc }}$ of 1.03 e and an $R$ value of 0.59 . As has been noted many times (e.g. [14]), it is these very weak reflections that are most sensitive to the centrosymmetric - noncentrosymmetric ambiguity, and the convincing evidence found in this case leaves little doubt that the centrosymmetric description is most appropriate. The weakest reflections were not available for the ethanol compound, $|8|$ so a comparable test could not be done.

The four crystallographic studies that Roos and co-workers have carried out represent seven independent TATM molecules - two for benzene/TATM, [10] three for $n$-hexane/TATM [9] and one each for ethanol/TATM [8] and ethyl acetate/TATM [7]. Since each TATM molecule has three crystallographically independent acetyl thienyl rings (see Figure III-8), there are 21 independent determinations of the bond lengths and bond angles within these four structures. Marsh calculated the weighted averages of these bond lengths and bond angles, the estimated standard deviations (e.s.d. 's) of the averages, and the goodness-of-fit $(\mathrm{GoF})$ values based on the assumption that all 21 measurements are equivalent. The results are summarized in Table III-2. For the bond lengths and the internal ring angles, the average GoF is about 1.6, quite consistent with conventional belief [13] that formal e.s.d.'s emerging from crystallographic studies tend to be low by approximately this factor. For the external angles at $C(3)$ and $C(5)$ and especially at $\mathrm{C}(1)$, the GoF's are markedly larger, suggesting that the variations are genuine, and hence that the host molecules undergo various types of flexing as they pack together, so as to form the cavities of appropriate sizes and shapes for a particular guest molecule. Therefore, the 21 measurements of these exterior angles cannot be considered as equivalent observations, and there may be some danger in trying to establish standard values for them. On the other hand, the bond lengths and bond angles within the acetyl thienyl rings may be accepted with good confidence. As an example, one notes a significant, and real, difference between the two types of C-S bond lengths. 
Table III-2: Bond lengths and angles for the TATM acetyl thienyl rings. The values were obtained from weighted averages of 21 sets of values from four TATM crystal structures. The e.s.d's of the average values (in parentheses) have been multiplied by the goodness of fit $(\mathrm{GoF})$ values, which were obtained from the sums of the squares of deviations of the 21 individual values from their weighted average [13].

\begin{tabular}{llllll} 
Bond type & Length $(\AA)$ & GoF & Angle type & Angle (9) & GoF \\
\hline$C(1)-C(3)$ & $1.517(2)$ & 1.3 & & & \\
$C(2)-C(3)$ & $1.359(2)$ & 1.4 & $C(3)-C(1)-C(9)$ & $111.9(4)$ & 3.6 \\
$C(3)-C(4)$ & $1.417(2)$ & 1.4 & $C(1)-C(3)-C(2)$ & $124.9(3)$ & 3.0 \\
$C(4)-C(5)$ & $1.369(2)$ & 1.6 & $C(1)-C(3)-C(4)$ & $123.5(3)$ & 2.7 \\
$S(1)-C(2)$ & $1.707(2)$ & 1.6 & $C(2)-C(3)-C(4)$ & $111.5(2)$ & 1.6 \\
$S(1)-C(5)$ & $1.723(2)$ & 2.2 & $C(3)-C(4)-C(5)$ & $113.2(2)$ & 2.0 \\
$C(5)-C(6)$ & $1.466(3)$ & 1.9 & $C(3)-C(2)-S(1)$ & $112.8(2)$ & 1.6 \\
$C(6)-C(7)$ & $1.496(2)$ & 1.3 & $C(2)-S(1)-C(5)$ & $91.5(1)$ & 1.6 \\
$C(6)-O(1)$ & $1.216(2)$ & 0.9 & $S(1)-C(5)-C(4)$ & $111.0(2)$ & 2.0 \\
& & & $S(1)-C(5)-C(6)$ & $119.3(3)$ & 2.8 \\
& & & $C(4)-C(5)-C(6)$ & $129.7(2)$ & 1.8 \\
& & & $C(5)-C(6)-O(1)$ & $120.5(2)$ & 1.5 \\
& & & $C(7)-C(6)-C(7)$ & $118.3(2)$ & 2.0 \\
& & & & $121.2(2)$ & 1.5 \\
\hline
\end{tabular}

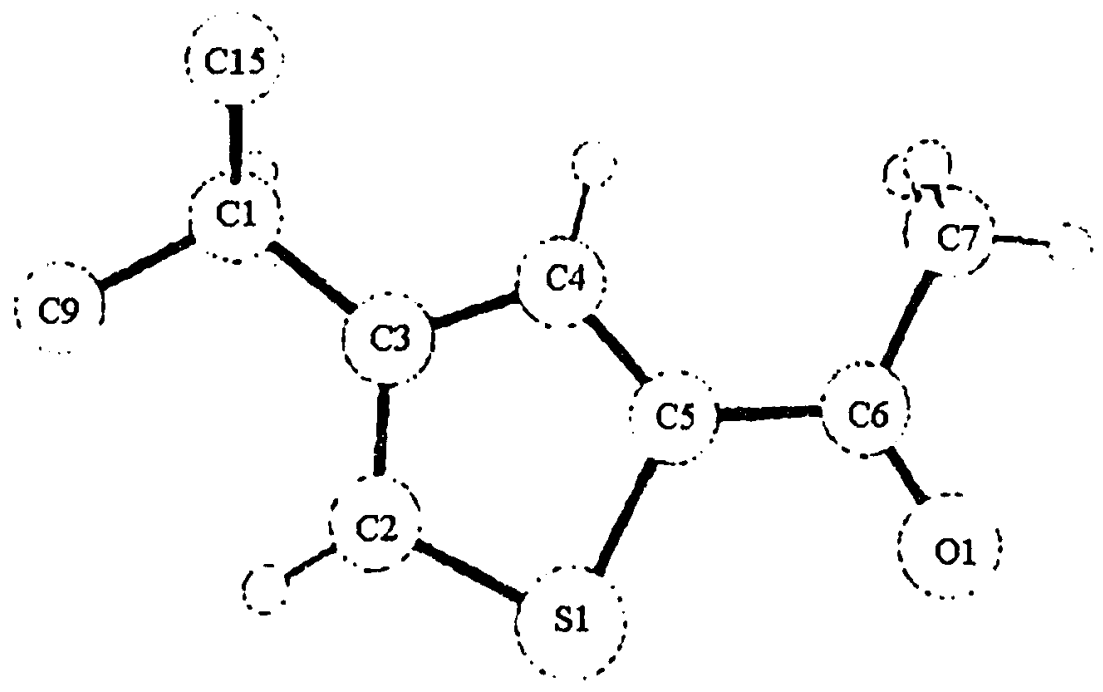

Figure III-8: An acetyl thienyl ring fragment of TATM, showing the numbering scheme used in Table III-2. Atoms $\mathrm{C}(9)$ and $\mathrm{C}(15)$ belong to other thienyl rings and are chemically equivalent to atom $\mathrm{C}(3)\{13\}$ 
An interesting point to which Marsh brought attention is the enclathrating behavior of the TATM molecule. In forming cages with four different guests (ethyl acetate, ethanol, benzene and $n$-hexane) the structure selects each time a different triclinic cell, of space group $\mathrm{P} \overline{\mathrm{l}}$. (The axial lengths of the ethyl acetate and ethanol inclusions are similar, as are the projections of the structures onto (001), but Marsh believes they are far from isostructural). Therefore, the TATM host can form at least four different types of cages, depending on the size and shape of the included guest. However, the guests are confined only loosely: in all four cases the $U_{\text {eq }}$ values of the guest's atoms are large (0.12 $\AA^{2}$ or more), and in the cases of ethanol and ethyl acetate inclusions, the noncentrosymmetric guest molecule is disordered within a centrosymmetric cavity. Given the remarkable inclusion versatility of TATM, it seems surprising that the enclosed guest does not fit in more tightly, particularly in the case of a polar solvent such as ethanol, which might be expected to form a hydrogen bond with an acetyl group of TATM. As Marsh concluded, there is no concentration of electron density toward the outer region of the guest cavity, where a localized $\mathrm{H} \cdots \mathrm{O}-\mathrm{H}$ bond would surely lie. Furthermore, no host acetyl oxygen atom lies within $4 \AA$ of any of the guest's atoms, the nearest neighbors to the guest are a methyl group $C(7)$ of one acetyl thienyl ring, and a ring atom $\mathrm{C}(5)$ of another, both at about $3.5 \AA$.

Marsh noted that it is doubtful, particularly in the case of the ethanol compound, that the coordinates assigned to the guest 's atoms bear much resemblance to reality. It seems quite clear that these ethanol molecules occupy many different sites and are quite likely to be in dynamic flux at room temperature. The situation with respect to the ethyl acetate compound is somewhat clearer, but by no means satisfactory. The bond lengths and bond angles calculated from the final coordinates of the guest ${ }^{\circ}$ atoms are given in Table III-3. There were similar problems in the original $P 1$ refinements.

Finally, Marsh pointed out that the choice between space groups $P 1$ and $P \bar{l}$ is not a clear one, since diffraction intensities are generally incapable of detecting small deviations from centrosymmetry. Marsh could find no indication that either of the ethanol or ethyl acetate structures could be better described in P1. For example, the TATM atoms lying at the edges of the guest cavity do not show the anomalous $U_{\mathrm{ij}}$ 's that would be expected if they actually lay in two sets of positions (as would be necessary to 
create a noncentrosymmetric cavity). And if correct $P 1$ structures do exist, it is clear, from the anomalous bond lengths, that the earlier refinements did not converge to them. The advantages of the $\mathrm{P} \overline{\mathrm{I}}$ descriptions seem overwhelming.

Table III-3: Bond lengths and angles for the ethanol and ethyl acetate guest molecules as obtained from least-squares refinement [13]. The values are subject to significant errors and are only very approximate. See Figures III-6 and III-7 for atomic labeling scheme of the guest molecules

\begin{tabular}{llll}
\hline Ethyl acetate: & & & \\
& Bond length $(\AA)$ & $O(7)-C(40)-C(39)$ & 134 \\
$\mathrm{C}(39)-\mathrm{C}(40)$ & 1.22 & $\mathrm{O}(8)-\mathrm{C}(40)-\mathrm{C}(39)$ & 111 \\
$\mathrm{C}(40)-\mathrm{O}(7)$ & 1.28 & $\mathrm{O}(8)-\mathrm{C}(40)-\mathrm{O}(7)$ & 109 \\
$\mathrm{C}(40)-\mathrm{O}(8)$ & 1.36 & $\mathrm{C}(41)-\mathrm{O}(8)-\mathrm{C}(40)$ & 118 \\
$\mathrm{O}(8)-\mathrm{C}(41)$ & 1.94 & $\mathrm{C}(42)-\mathrm{C}(41)-\mathrm{O}(8)$ & 89 \\
$\mathrm{C}(41)-\mathrm{C}(42)$ & 1.38 &
\end{tabular}

Ethanol:

$\begin{array}{llll}E t(1)-E t(2) & 1.41 & E t(3)-E t(1)-E t(2) & 108\end{array}$

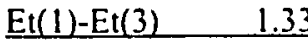

(H) L. Pang, R.C. Hynes and M.A. Whitehead Acta Cryst. C50, 615 (1994)

In 1994, six TATM crystal structures encompassing five different guests were reported by Li Pang and co-workers [15-17]. The first paper dealt with the carbon tetrachloride $\left(\mathrm{CCl}_{4}\right)$ /TATM inclusion compound [15]. The authors noted that the crystal structure obtained for this compound is isostructural with the previously reported structure of benzene/TATM [10]. In $\mathrm{CCl}_{4} /$ TATM, four TATM host molecules and two $\mathrm{CCl}_{4}$ guest molecules reside in the unit cell. All of the TATM host molecules are ordered, while the $\mathrm{CCl}_{4}$ guest molecules have partially disordered structures, in which one $\mathrm{Cl}$ atom is ordered, but the three remaining $\mathrm{Cl}$ atoms are trigonally disordered over two molecular orientations with $65 \%$ and $35 \%$ occupation.

As depicted in Figure $\mathrm{II}-9$, it can be seen that the $\mathrm{CCl}_{4} / \mathrm{TATM}$ inclusion compound is made up of channel-type cavities along the $c$ axis. By comparing the $\mathrm{CCl}_{4}$ clathrate with the previously reported benzene inclusion compound, [10] it is found that the nature of the guest cavity is the same for both. However, the $\mathrm{CCl}_{4}$ molecule experiences a disorder effect so that the ordered axial $\mathrm{C}-\mathrm{Cl}$ bond is nearly perpendicular 
to the plane of the benzene ring in their respective isostructural lattices. Since the benzene guest molecule is in an ordered site (and therefore the six carbon atoms are located in deep potential energy minima), the analogous $\mathrm{CCl}_{4}$ guest molecule would be required to have two possible orientations to produce six disordered equatorial $\mathrm{Cl}$ sites in a plane parallel to the corresponding benzene ring. Figure III-10 shows the respective orientations of the two guests in their isostructural TATM lattices.

The $\mathrm{CCl}_{4}$ / TATM inclusion compound was prepared by recrystallization of the host from the guest solvent. The host-guest ratio of 2:1 was determined by crystal density measurements (flotation in $\mathrm{KI} / \mathrm{H}_{2} \mathrm{O}$ mixtures). A suitable single crystal was selected and removed from the mother liquor, then quickly covered with epoxy to prevent deterioration, and mounted on the tip of a glass fiber. The structure was solved by direct methods with the program SOLVER, [18] and refined by full-matrix least squares using the NRCVAX suite of programs [19]. All non-H atoms were refined with anisotropic displacement parameters. All positions and displacement parameters of the $\mathrm{H}$ atoms were calculated. The $\mathrm{CCl}_{4}$ guest is partially disordered. $\mathrm{Cl}(1)$ and $\mathrm{C}(20)$ are ordered, but there are two orientations of the three remaining $\mathrm{Cl}$ atoms, a major position $(65 \%)$ and a minor position $(35 \%)$.

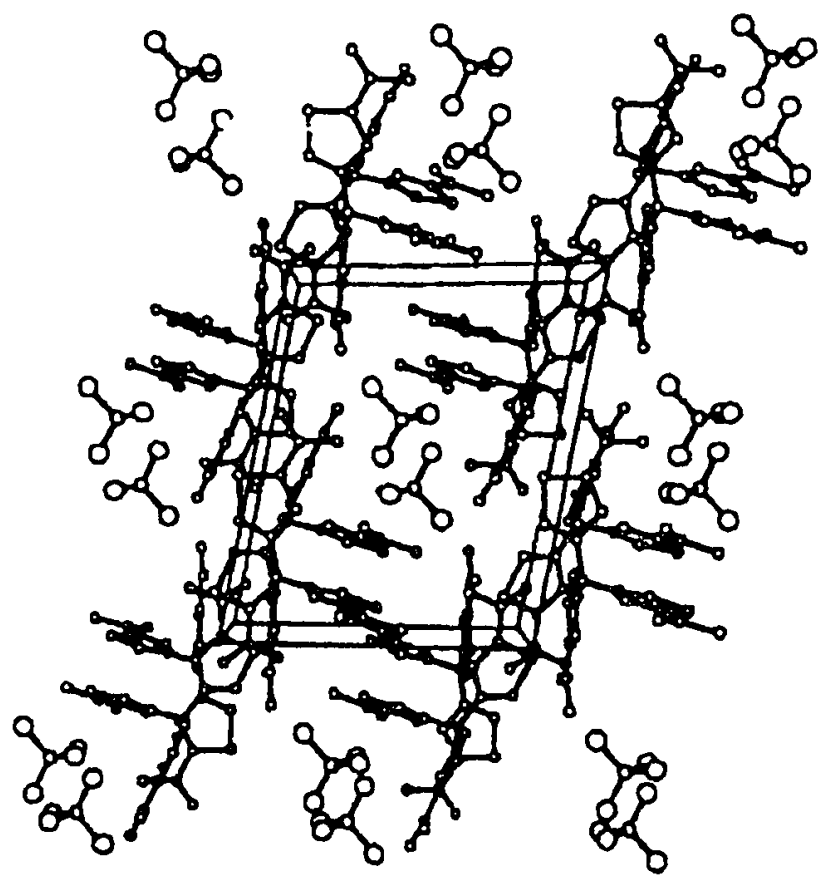

Figure III-9: A view of the unit cell of TATM $/ \mathrm{CCl}_{+}$down the $c$ axis. The minor orientation of $\mathrm{CCl}_{4}$ has been omitted for clarity [15]. 

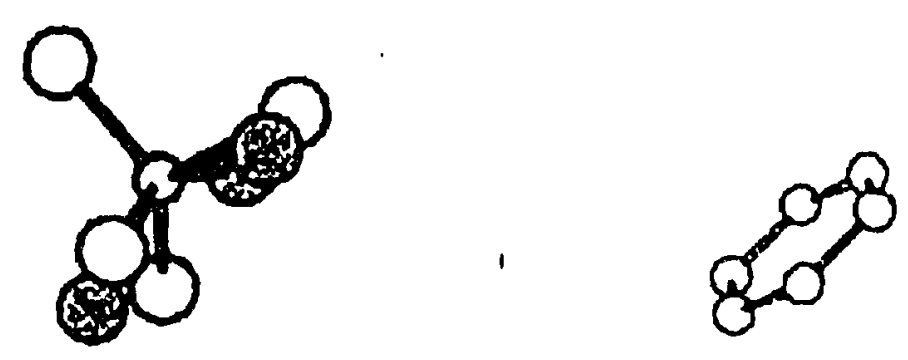

Figure III-10: A comparison of the molecular orientations of disordered $\mathrm{CCl}_{4}$ and ordered benzene in their respective isostructural TATM crystal lattices $[10,15]$.

(I) L. Pang and F. Brisse Acta Cryst. C50, 1947 (1994)

In 1994, the crystal structure of cyclononanone / TATM was reported [16] by Pang and Brisse (see Figure III-1I). They encountered a novel TATM stoichiometric ratio of $1 \mathrm{H}: 1 \mathrm{G}$ for this compound. The measured crystal belongs to the monoclinic $\mathrm{P} 2 / 1 \mathrm{c}$ space group. The TATM host molecules are ordered, but the cyclononanone guest molecules reside in two orientationally disordered positions with occupational factors of $25 \%$ and $75 \%$. Both of the cyclononanone molecules have a ( $g$ gggg gisgs) conformation, in addition to very similar orientations. The cyclononanone guest molecule of the present work is somewhat larger than the guest molecules of previous TATM structures, whose structures were reported with a $2 \mathrm{H}: 1 \mathrm{G}$ stoichiometry. There are four TATM host molecules and four cyclononanone guest molecules in the unit cell.

A stereoscopic view of this inclusion compound (in Figure 111-12) suggests that the TATM molecules form channel-type cavities along the $c$ axis in which the cyclononanone guest molecules are disordered in a zig-zag manner. Since the two cyclononanone molecules have very nearly the same conformation, the authors believe that the disorder effect is of the orientational type. The guest conformation ( $g^{\prime} g g g g^{\prime} s g$ $s$ ) is in agreement with that predicted for neat solid cyclononanone [20]. Perhaps as a result of their disorder, significantly larger thermal factors for the guest's atoms were measured, when compared to those for the host.

The structure was solved by direct methods with MULTAN80, $[11]$ and refined by full-matrix least squares using the NRCVAX suite of programs |19|. The disordered 
structure was refined by constraining the $\mathrm{C}-\mathrm{C}$ distances to $1.53(1) \AA, \mathrm{C}=\mathrm{O}$ distances to $1.21(1) \AA$, and non-bonded $C \cdots C$ distances to $2.58(2) \AA$. All non- $H$ atoms of the TATM host molecule were refined with anisotropic displacement parameters. The structure of the two guest molecules was established from difference Fourier synthesis. Only the non- $\mathrm{H}$ atoms of the major (75\%) guest were refined anisotropically. The occupancy factors of $25 \%$ and $75 \%$ were arrived at after several refinement cycles, keeping the displacement parameters constant. The positions of all the $\mathrm{H}$ atoms were calculated $(\mathrm{C}-\mathrm{H}$ $=0.95 \AA, \angle \mathrm{C}-\mathrm{C}-\mathrm{H}=105^{\circ}-125^{\circ}$ ), except for one $\mathrm{H}$ atom of each $\mathrm{CH}_{3}$ group of the TATM molecule, which was found from the difference Fourier synthesis and refined. The two remaining methyl $\mathrm{H}$-atom positions were calculated. All $\mathrm{H}$ atoms were refined isotropically.

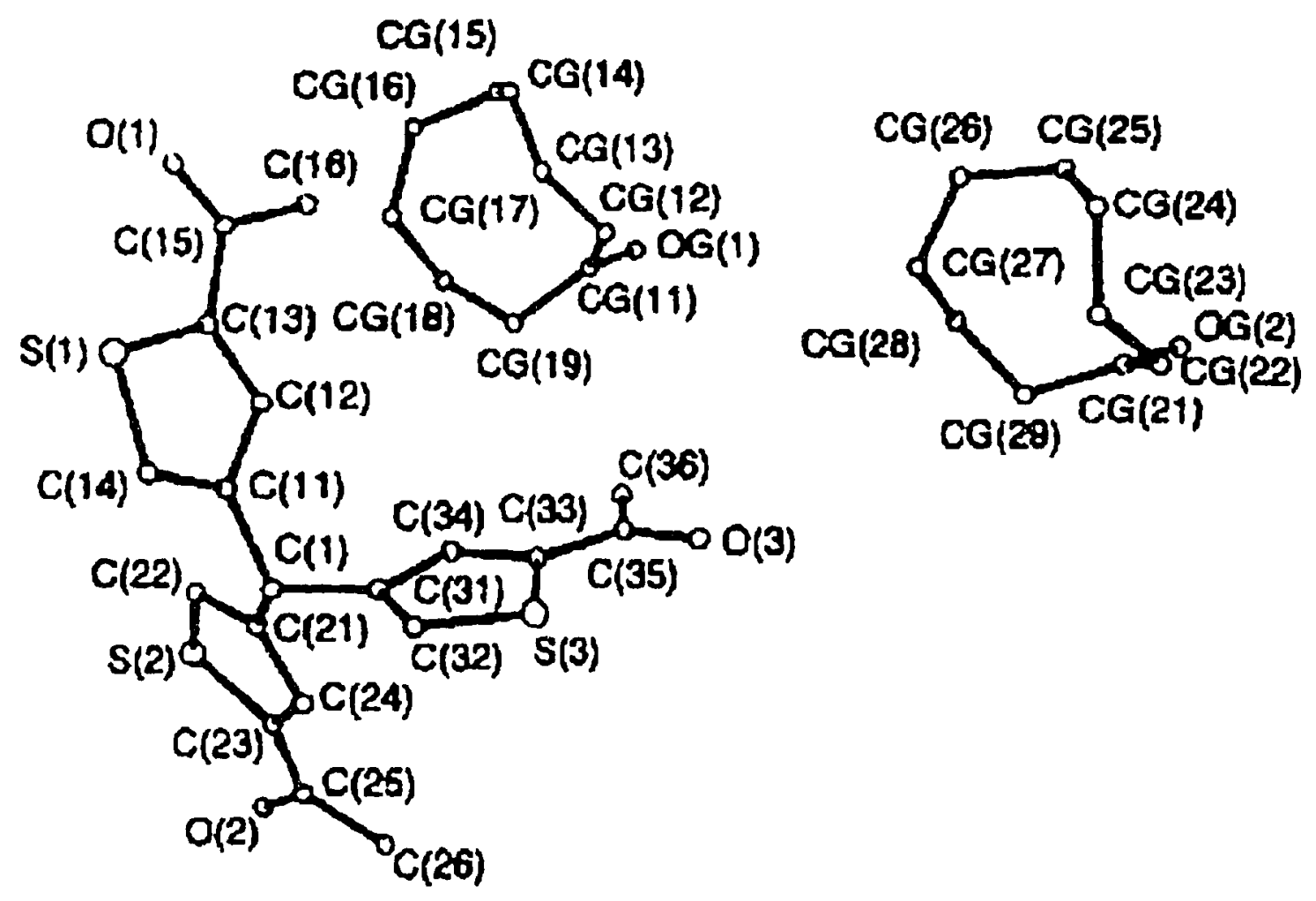

Figure III-11: Molecular structure of cyclononanone/TATM with the atomic numbering scheme. The guest on the right is the major component $(75 \%)[16]$. 

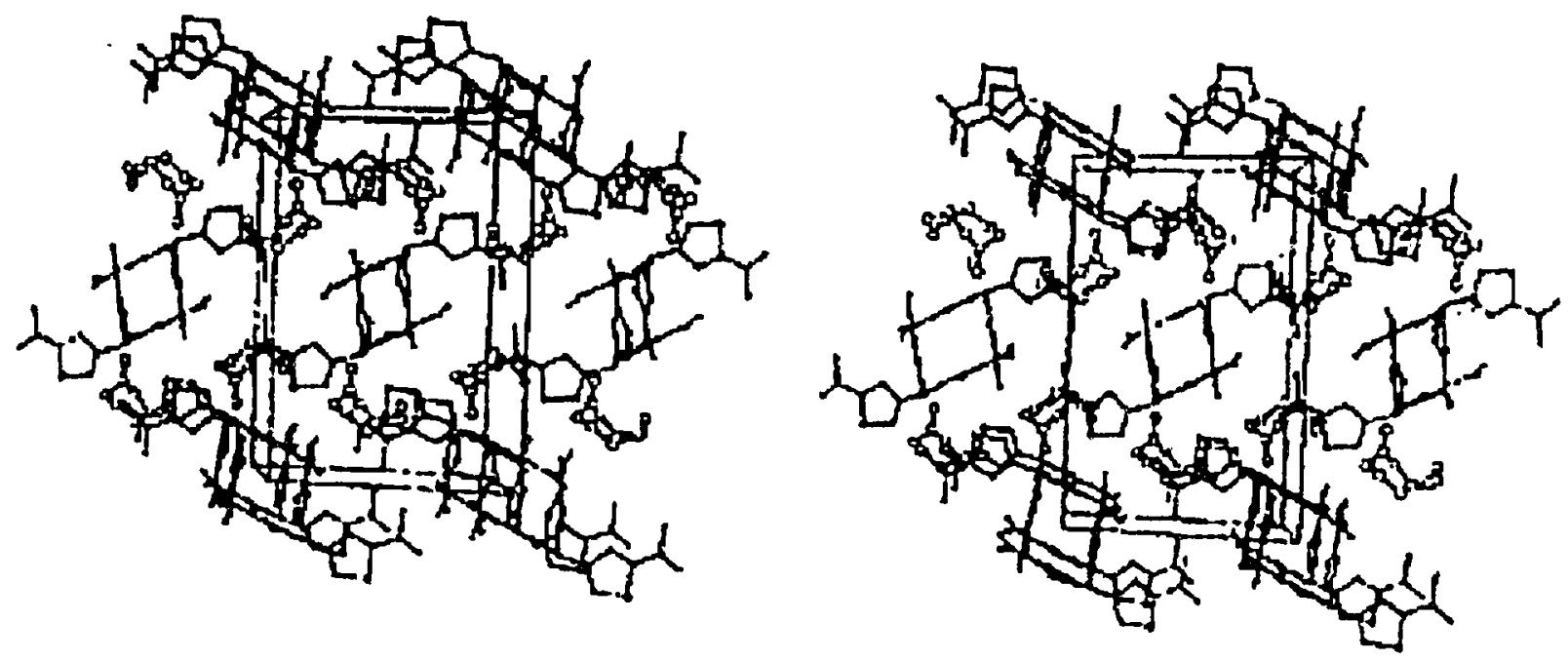

Figure III-12: A stereoscopic view of the unit cell contents of cyclononanone / TATM down the $a$ axis. The minor guest orientation $(25 \%)$ has been omitted for clarity. The $c$ (channel) axis is horizontal in direction [16].

(J) L. Pang and F. Brisse Can. J. Chem. 72, 2318 (1994)

In 1994, Pang and Brisse reported crystal structures of TATM inclusion compounds with cyclohexane, cycloheptane and cyclooctane guests [17]. Cyclohexane was observed to crystallize with TATM in two different forms (A and B), depending on the crystallization conditions. All four structures crystallized in the triclinic $\mathrm{P} \bar{l}$ space group with a stoichiometric ratio of $2 \mathrm{H}: 1 \mathrm{G}$. Cycloheptane / TATM and cyclooctane / TATM are isostructural to cyclohexane B / TATM. In cyclohexane A / TATM, the void space is of a linear channel type, with ordered guest molecules located on centers of inversion. On the other hand, for all three B type structures the void space is of a zig-zag channel type, with disordered guest molecules located in general positions. Conformational analyses of the guest molecules were performed by a combination of molecular dynamics and energy minimization.

The crystals of the inclusion compounds were obtained by slow recrystallization of TATM from cyclohexane, cycloheptane or cyclooctane. The crystals of cyclohexane A / TATM and the three B forms were obtained under different crystallization conditions. The $\mathrm{A}$ form resulted from slow cooling $\left(5^{\circ} \mathrm{C}\right.$ per day) of the TATM-cyclohexane 
solution, whereas the $\mathbf{B}$ forms were obtained by a relatively faster cooling of the three solutions $\left(1^{\circ} \mathrm{C}\right.$ per hour). The 2:1 host:guest ratios were determined by crystal density measurements.

The structures were determined on an Enraf-Nonius CAD4 single crystal diffractometer. Lattice constants were determined by least-squares refinement using 25 well-centered reflections in the range $20^{\circ}<\theta<25^{\circ}$. The crystal of cyclohexane B / TATM was measured at room temperature, the three others at $220 \mathrm{~K}$ (see Table III-4). Intensity data were collected using $\mathrm{Cu} K_{\mu}$ graphite monochromatized radiation in the $\omega / 2 \theta$ scan mode, with a scan width defined by $\Delta \omega=(0.80+0.14 \tan (\theta))^{\circ}$, and a scan rate of $16.48^{\circ}$ per minute. The structures were solved by direct methods using the MULTAN80 program [11] for cyclohexane A / TATM, and the SHELX86 program suite [21] for the remaining three structures. The structures were refined through full-matrix least-squares calculations by using the NRCVAX program [19] for cyclohexane A / TATM and the SHELX76 suite of programs [12] for the remaining three structures. All non-hydrogen atoms of the TATM host were refined with anisotropic thermal parameters. The positions of the hydrogen atoms were calculated, and their isotropic thermal parameters were refined.

As mentioned above, the guest molecules in cyclohexane A / TATM are ordered, while in the three remaining structures the guests are disordered. The modeling of the disordered structures was established from difference Fourier synthesis, and the structures were refined by constraining $\mathrm{C}-\mathrm{C}$ bond distances to $1.54( \pm 0.02) \AA$, and the nonbonded C-C distances to $(I) 2.56( \pm 0.05) \AA$ and (2) $2.95( \pm 0.02) \AA$ for $(I)$ cyclohexane B / TATM and (2) cycloheptane and cyclooctane inclusions, respectively. The non-hydrogen atoms of the guest molecule in cyclohexane A / TATM, and only those of the major structure of the guest in the cycloheptane and cyclooctane inclusions were refined anisotropically. Occupancy factors in cyclohexane B / TATM of 0.40, 0.35 and 0.25 were fixed, while those of $0.709(4)$ and $0.291(4)$ in cycloheptane / TATM, and of $0.763(3)$ and $0.237(3)$ in cyclooctane / TATM were refined. The positions of the hydrogen atoms of the guest molecules were calculated: $\mathrm{d}(\mathrm{C}-\mathrm{H})=0.95 \AA, \theta(\mathrm{C}-\mathrm{C}-\mathrm{H})=$ $109.5^{\circ}$. A summary of the crystal data, data collection and final refinement results is presented in Table III-4. 
Table III-4: Crystal data for TATM inclusion compounds with six different guests [1517]

\begin{tabular}{|c|c|c|c|c|c|c|}
\hline \multicolumn{7}{|l|}{ Crystal Data } \\
\hline Guest & $\mathrm{CCl}_{4}$ & $\mathrm{C}_{1} \mathrm{H}_{1,1} \mathrm{O}$ & $\mathrm{C}_{6} \mathrm{H}_{12} \Lambda$ & $\mathrm{C}_{6} \mathrm{H}_{12} \mathrm{~B}$ & $\mathrm{C}_{7} \mathrm{H}_{14}$ & $\mathrm{C}_{k} \mathrm{H}_{\mathrm{IR}_{1}}$ \\
\hline Molecular formula & $\mathrm{C}_{31} \mathrm{H}_{12} \mathrm{O}_{4} \mathrm{~S}_{1} \mathrm{Cl}_{4}$ & $\mathrm{C}_{1,0}, \mathrm{H}_{10}, \mathrm{O}_{7} \mathrm{~S}_{\mathrm{f}_{1}}$ & $\mathrm{C}_{44} \mathrm{H}_{44} \mathrm{O}_{4} \mathrm{~S}_{4}$ & $\mathrm{C}_{44} \mathrm{H}_{44} \mathrm{O}_{4} \mathrm{~S}_{6}$ & $\mathrm{C}_{4} \mathrm{H}_{48} \mathrm{O}_{6}, \mathrm{~S}_{6}$ & $\mathrm{C}_{40}, \mathrm{H}_{4 x} \mathrm{O}_{6,} \mathrm{~S}_{6}$ \\
\hline Host:Guest & $2 H: 1 \mathrm{G}$ & IH:1G & $2 H: 1 \mathrm{O}$ & $2 \mathrm{H}: 1 \mathrm{G}$ & $2 \mathrm{H}: \mathrm{IG}$ & $2 \mathrm{H}: 1 \mathrm{G}$ \\
\hline Form. Wt. (amu) & 930.8 & 528.7 & 430.59 & 861.19 & 875.22 & 889.24 \\
\hline Temperiture $(K)$ & 295 & 220 & 220 & 295 & 220 & 220 \\
\hline Crystal system & triclinic & monoclinic & triclinic & triclinic & triclinic & triclinic \\
\hline Spacc group & $P \overline{1}$ & $\mathrm{P}_{2} / \mathrm{c}$ & $P \bar{I}$ & $\mathbf{P I}$ & $\overrightarrow{P l}$ & $\mathrm{PI}$ \\
\hline$a(A)$ & $11.638(3)$ & $10.904(2)$ & $8.622(2)$ & $11.668(4)$ & $11.721(5)$ & $11.914(1)$ \\
\hline$b(A)$ & $13.684(3)$ & $19.464(4)$ & $10.194(2)$ & $13.729(3)$ & $13.734(6)$ & $14.013(2)$ \\
\hline$c(A)$ & $14.235(5)$ & $13.417(1)$ & $12.795(2)$ & $14.227(4)$ & $14.177(6)$ & $13.986(2)$ \\
\hline$u\left({ }^{\circ}\right)$ & $88.90(2)$ & 90 & $79.09(1)$ & $89.40(3)$ & $89.22(4)$ & $89.46(1)$ \\
\hline$\beta\left(^{c}\right)$ & $77.46(2)$ & $109.40(1)$ & $72.74(1)$ & $77.15(4)$ & $76.84(4)$ & $77.15(1)$ \\
\hline$\gamma\left({ }^{\circ}\right)$ & $78.77(2)$ & 90 & $84.89(1)$ & $76.24(4)$ & $76.01(3)$ & $75.12(1)$ \\
\hline$V\left(A^{\prime}\right)$ & $2170(1)$ & $2708.1(8)$ & $1053.9(4)$ & $2157(1)$ & $21.54(2)$ & $2198.4(4)$ \\
\hline$Z$ & 2 & 4 & 1 & 2 & 2 & 2 \\
\hline$F(000)$ & not given & not given & 452 & 904 & 920 & 936 \\
\hline$d_{\text {calc }}\left(g \mathrm{~cm}^{2}\right)$ & 1.43 & 1.30 & 1.36 & 1.33 & 1.35 & 1.34 \\
\hline$d_{\text {max }}\left(g \mathrm{~cm}^{-1}\right)$ & 1.42 & 1.24 & 1.32 & 1.30 & 1.30 & 1.32 \\
\hline$\mu\left(m m^{-1}\right)$ & 0.59 & 0.27 & 0.333 & 0.325 & 0.326 & 0.320 \\
\hline Wavelength (A) & $0.7107(\mathrm{Mn})$ & $1.5418(\mathrm{Cu})$ & $1.5418(\mathrm{Cu})$ & $1.5418(\mathrm{Cu})$ & $1.5418(\mathrm{Cu})$ & $1.5418(\mathrm{Cu})$ \\
\hline $200_{\mathrm{tata}}\left({ }^{\circ}\right)$ & 44.9 & 70 & 140 & 140 & 140 & 140 \\
\hline \multicolumn{7}{|l|}{ Data collection } \\
\hline crystal size (tnm) & $0.40 \times 0.33 \times 0.10$ & $0.61 \times 0.23 \times 0.09$ & $0.62 \times 0.26 \times 0.08$ & $0.17 \times 0.12 \times 0.09$ & $0.52 \times 0.14 \times 0.09$ & $0.40 \times 0.20 \times 0.08$ \\
\hline crystal color & pale yellow & $\begin{array}{l}\text { pale yellow } \\
\text { pales }\end{array}$ & pale yellow & pale yellow & palc yellow & palc yellow \\
\hline index ranges & $-12 \leq h \leq 12$ & $-13 \leq h \leq 13$ & $-10 \leq h \leq 10$ & $-14 \leq 11 \leq 14$ & $-14 \leq 11 \leq 14$ & $-14 \leq h \leq 14$ \\
\hline & $0 \leq k \leq 14$ & $0 \leq k \leq 23$ & $0 \leq k \leq 12$ & $-16 \leq k \leq 16$ & $-16 \leq k \leq 16$ & $-17 \leq k \leq 17$ \\
\hline & $-15 \leq / \leq 15$ & $-16 \leq 1 \leq 16$ & $-15 \leq / \leq 15$ & $-17 \leq / \leq 17$ & $-17 \leq 1 \leq 17$ & $-17 \leq 1 \leq 17$ \\
\hline measured retlis & 6016 & 10110 & 78.56 & $|608|$ & 15805 & 16367 \\
\hline unique refl's & 5682 & 5138 & 3996 & 8085 & 8096 & 8316 \\
\hline obsenced refl's & 3227 & 4256 & 3590 & 3960 & 5985 & 6008 \\
\hline$R_{\mathrm{m}}$ & 0.021 & 0.043 & 0.020 & 0.042 & 0.052 & 0.035 \\
\hline paramelers & 523 & 414 & 342 & 609 & 621 & 638 \\
\hline scan speed $\left({ }^{\circ}\right.$ min $)$ & 16 & not given & 16.48 & 16.48 & 16.48 & 16.48 \\
\hline scan mode & (.) & (1) 20 & (.) 20 & $(120)$ & (") 20 & $\because 20$ \\
\hline scan width $\left({ }^{\circ}\right)$ & 1.6 & not given & 2.76 & 1.77 & 2.22 & 1.93 \\
\hline \multicolumn{7}{|l|}{ Final Rofincment } \\
\hline \multirow[t]{2}{*}{ Structure solution } & direct & direct & dircet & direct & direct & direct \\
\hline & methods. & methods & methods & methods & methods. & methods \\
\hline \multirow[t]{2}{*}{ Reline, method } & full matrix & full inatrix & fult matrix & full matrix & lull matrix & full matrix \\
\hline & least squares & least squarts & least squares & least squarcs & least squares & least squares \\
\hline$R_{1}$ & 0.058 & 0.055 & 0.047 & 0.087 & 0.070 & 0.065 \\
\hline$\| \cdot R_{2}$ & 0.058 & 0.059 & 0.054 & 0.071 & 0.076 & 0.066 \\
\hline$(. \Lambda \sigma)_{\text {IIAM }}$ & 0.098 & 0.61 & 0.03 & 0.40 & 0.32 & 0.60 \\
\hline $\max$ peak $\left(e A^{-1}\right)$ & 0.510 & 0.58 & 0.62 & 0.57 & 0.59 & 0.43 \\
\hline decpest hole $\left(c A^{1}\right)$ & -0.370 & -0.38 & -.0 .33 & -0.51 & -0.77 & -0.4 .5 \\
\hline
\end{tabular}


As was previously noted, cyclohexane / TATM crystallizes in two distinct forms. The unit cell of the $\mathbf{A}$ form contains two TATM molecules and one guest molecule, while there are four TATM molecules and two cyclohexane molecules in the unit cell of the B form. Subsequently, the volume of the unit cell of the B form $\left(2157 \AA^{3}\right)$ is about twice that of the $\mathbf{A}$ form (1054 $\AA^{3}$ ). No crystallographic phase transitions were observed between these two forms in the temperature range $220 \mathrm{~K}-295 \mathrm{~K}$, thus it was concluded that the two phases do not interconvert in this temperature region, and that their structures depend upon, in part, the crystallization conditions. The guest molecules in the three B forms are always disordered, perhaps due to a larger channel. The following occupancy factors were measured: $40 / 35 / 25,71 / 29$ and $76 / 24$ for the guest molecules in the cyclohexane B, cycloheptane and cyclooctane inclusions respectively.

The molecular structures of the TATM molecules in the cyclohexane $\mathbf{A}$ and cyclohexane B crystal forms are very similar, and close to those of previously published work $[10,15]$. The acetyl groups are very nearly coplanar with the thienyl rings, since the oxygen atoms and the methyl groups deviate from the plane of their associated thienyl ring by an average of only 0.06(2) $\AA$ and $0.08(2) \AA$ respectively. The hydrogen atoms of the methyl groups display a fair amount of disorder, as is indicated by their high thermal parameters. The dihedral angles formed between the thienyl rings are in the range $68^{\circ}$ $81^{\circ}$.

Since the TATM molecule has a relatively invariant structure, the different crystal structures and cavity types depend primarily upon the size and shape of the guest molecule and the resulting molecular packing. Figure III-13 illustrates two perspectives of the cyclohexane A / TATM inclusion compound. The figure clearly shows that there is a linear-type channel in this structure. Two of the three thienyl rings of two host molecules and two acetyl groups of two neighboring TATM molecules contribute to the formation of any particular channel. The axis of the channel, parallel to $a$. passes through crystallographic inversion centers located at $\left(\begin{array}{lllll}0 & 1 / 2 & 1 / 2\end{array}\right)\left(\begin{array}{llll}1 / 2 & 1 / 2 & 1 / 2\end{array}\right)\left(\begin{array}{lll}1 & 1 / 2 & 1 / 2\end{array}\right)$ etc. The cyclohexane guest molecules are located on these centers of inversion and are ordered. 
(a)
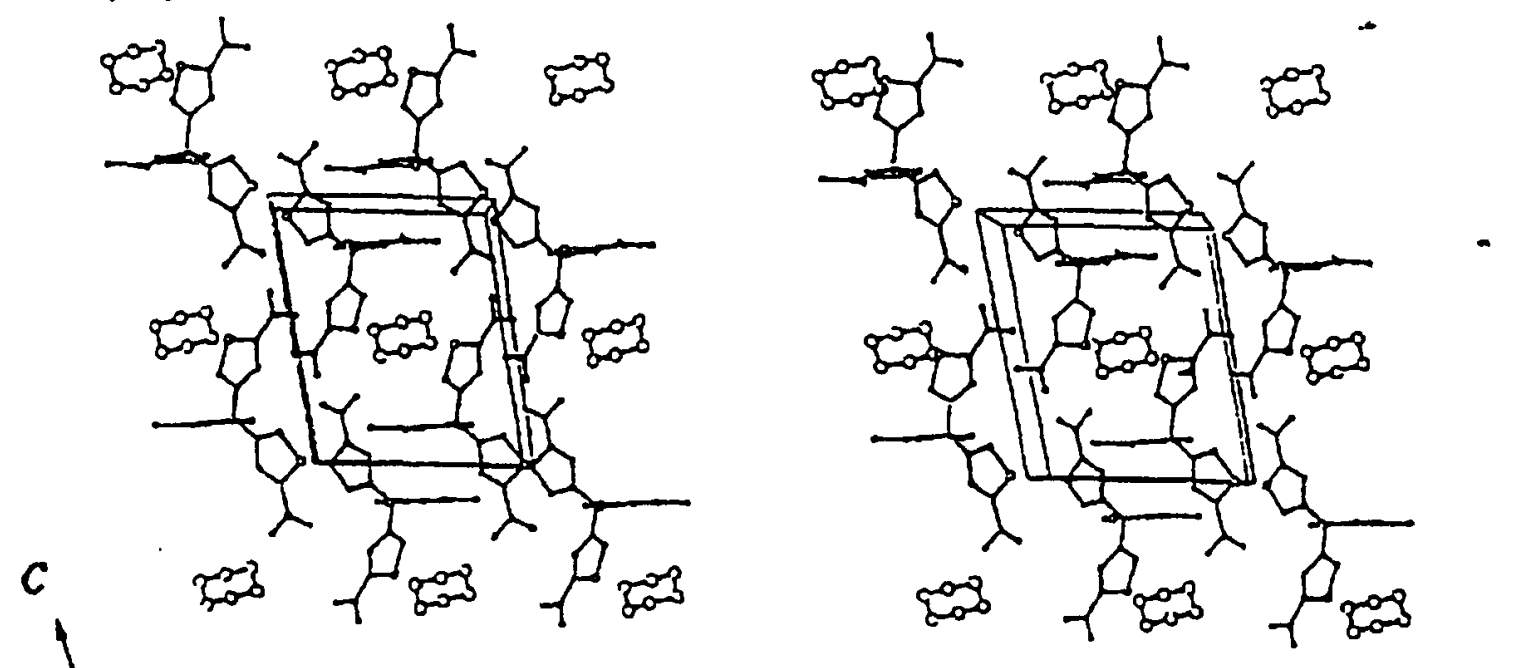

$b$

(b)

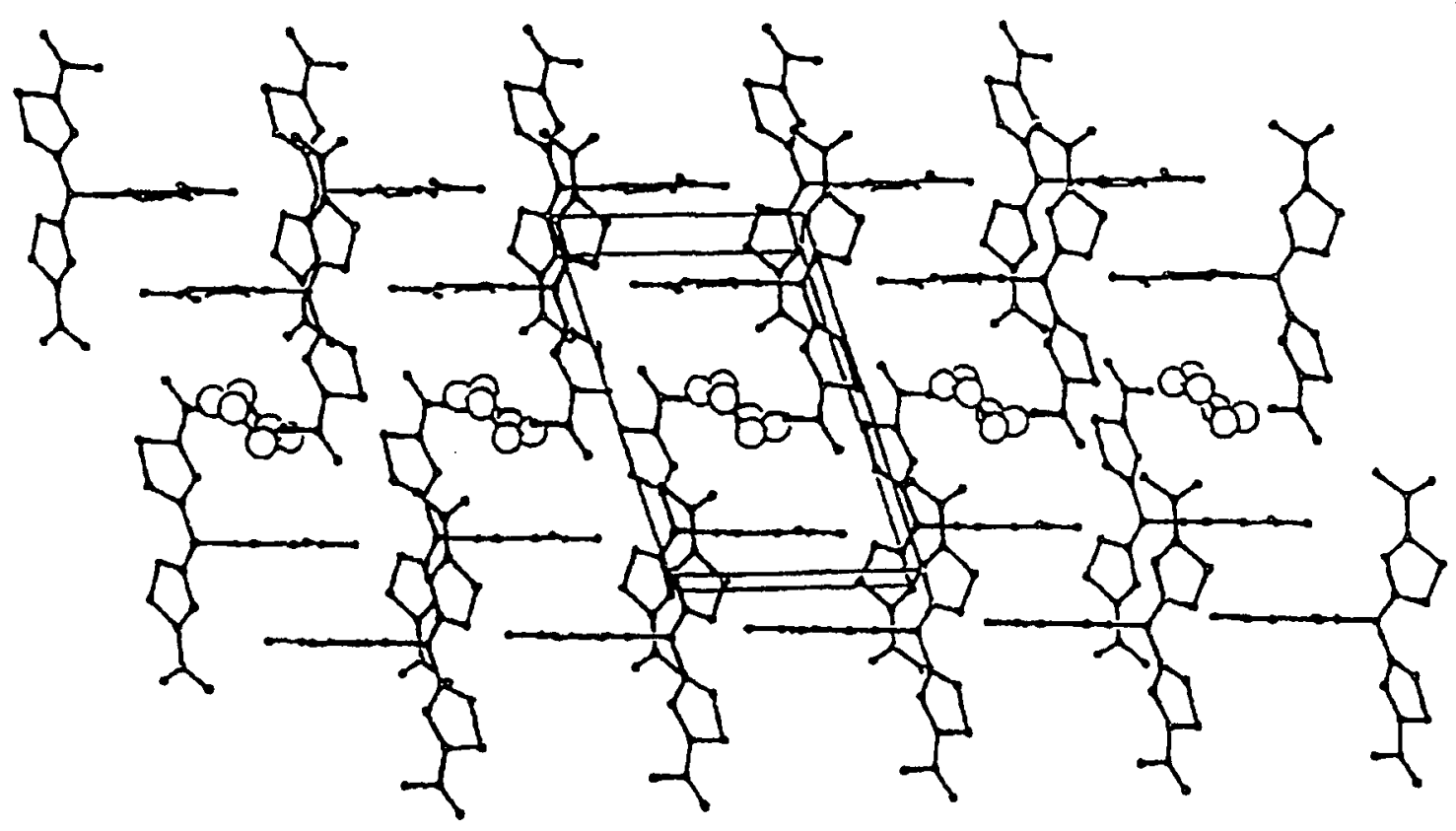

Figure III-13: The unit cell contents of the TATM / cyclohexane inclusion compound in the $\mathbf{A}$ form. (a) Stereo pair showing the channel cross section down the $a$ axis. (b) A side view (the $a$ axis is horizontal) [17] 
The situation for cyclohexane B / TATM is slightly different. Figure III-14 suggests that the void space is also of the channel type. However, the guest molecules in the channel of the B form adopt a zig-zag structure rather than the linear disposition of the $\mathbf{A}$ form. The molecular packing of the TATM molecules in the $\mathbf{B}$ form is slightly different from that in the $\mathbf{A}$ form. In the $\mathbf{B}$ form, the TATM molecules are arranged in such a way that one of the thienyl rings is almost parallel to the $a b$ face of the unit cell, while the remaining two thienyl rings form the sides of the channels. A comparison of the $b$ and $c$ dimensions of the $\mathbf{A}$ form with the $a$ and $b$ dimensions of the $\mathbf{B}$ form, which are measures of the respective channel cross-sections, reveals that the channels in cyclohexane B / TATM are slightly larger than in the A form. This increase in the channel cross-section could explain the disorder of the $\mathbf{B}$ form guest molecules.

The most significant difference between the cyclohexane $\mathbf{A}$ and $\mathbf{B}$ forms is in the nature of the guest molecule(s). In the $\mathbf{A}$ form, the cyclohexane molecule is located on a crystallographic center of symmetry and is ordered. In the cyclohexane B / TATM, the guest is in a general position and has three molecular orientations. These orientations have occupation factors of $40 / 35 / 25$, and thus produce a statistical disorder. The cyclohexane guest in both forms always has the chair conformation, so that the disordered positions in the B form are of the orientational type. Figure III-15(a) shows the disordered structure for the cyclohexane B guest molecule.

The TATM inclusion compounds with cycloheptane and cyclooctane are isostructural to cyclohexane B / TATM. This may be understood if one realizes that these two guest molecules are slightly larger than cyclohexane, so they should not fit comfortably into the smaller channels of the cyclohexane $\mathbf{A}$ form, and if they did, it would result in shorter intermolecular contacts and subsequently a higher potential energy. These two guest molecules are disordered in the TATM lattice. The cycloheptane molecule was found to reside in two orientations with respective occupancy factors of $0.709(4)$ and $0.291(4)$. Both molecules have the same conformation ( $g$-gg-ggg$g$ ), so the authors concluded that the disorder is only of an orientational type. 
(a)
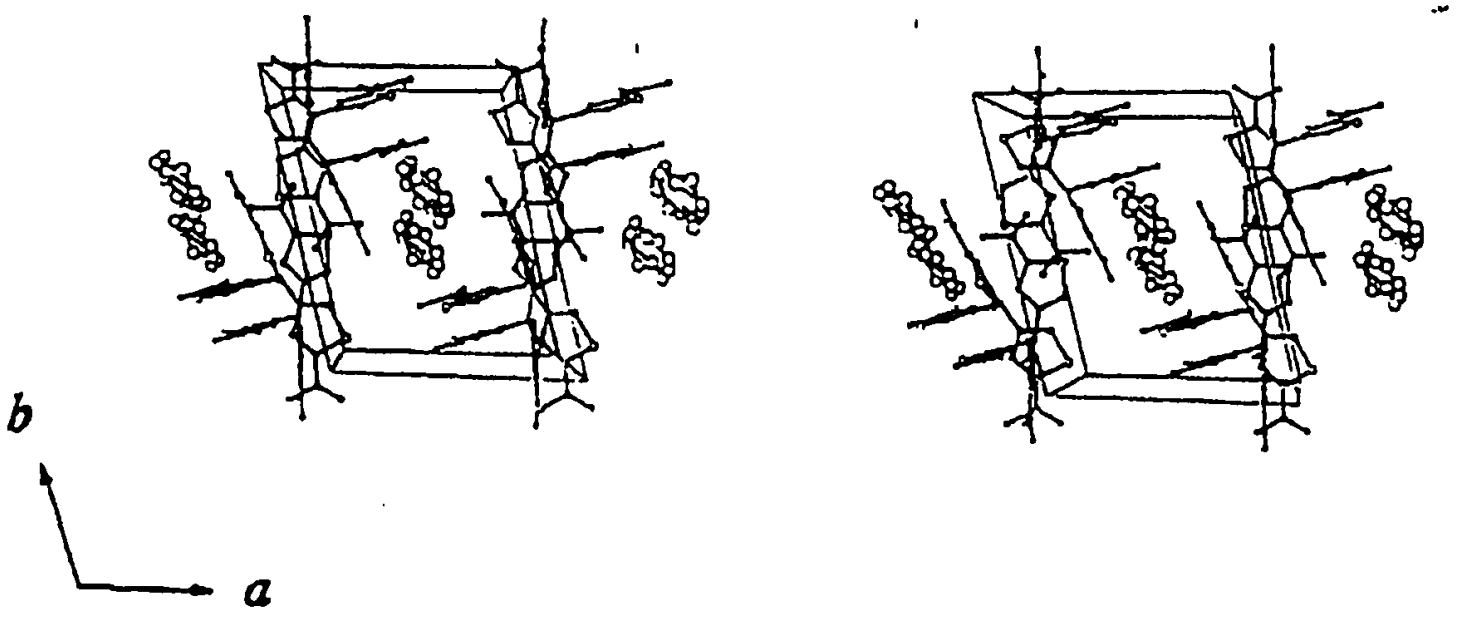

(b)

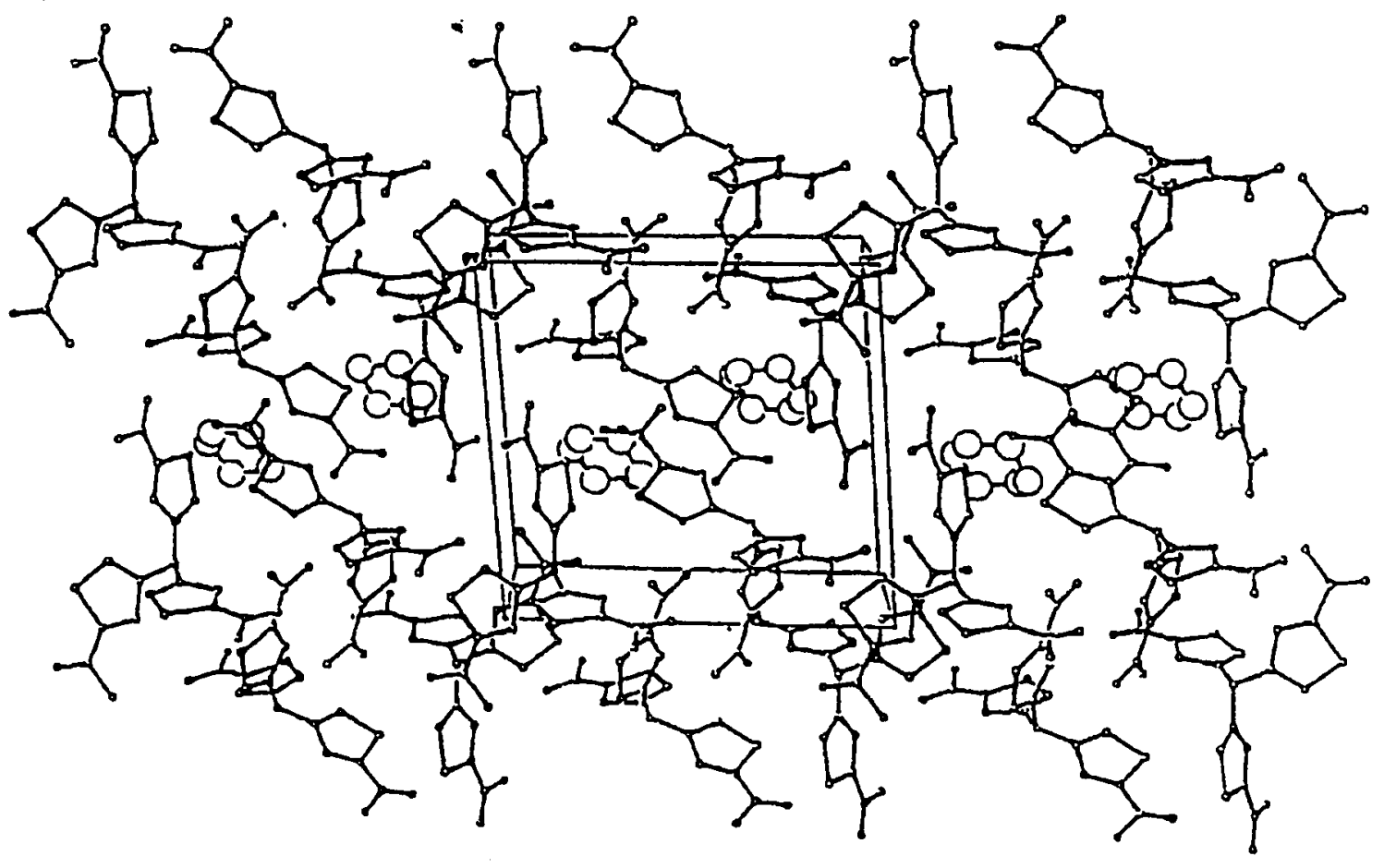

Figure III-14: The unit cell contents of the TATM cyclohexane inclusion compound in the B form. (a) Stereo pair showing the channel cross section down the $c$ axis (b) Side view showing the length of the channel (the $c$ axis is horizontal) [17] 
A similar result was obtained for the cyclooctane / TATM inclusion compound. Two orientations of the guest molecule in the same conformation (gg-gg-ggg-g-) were found, with occupancy factors of $0.763(3)$ and $0.237(3)$ respectively. Figures $1-15$ (b) and (c) show the orientational disorder for cycloheptane and cyclooctane in their respective TATM inclusion compounds.
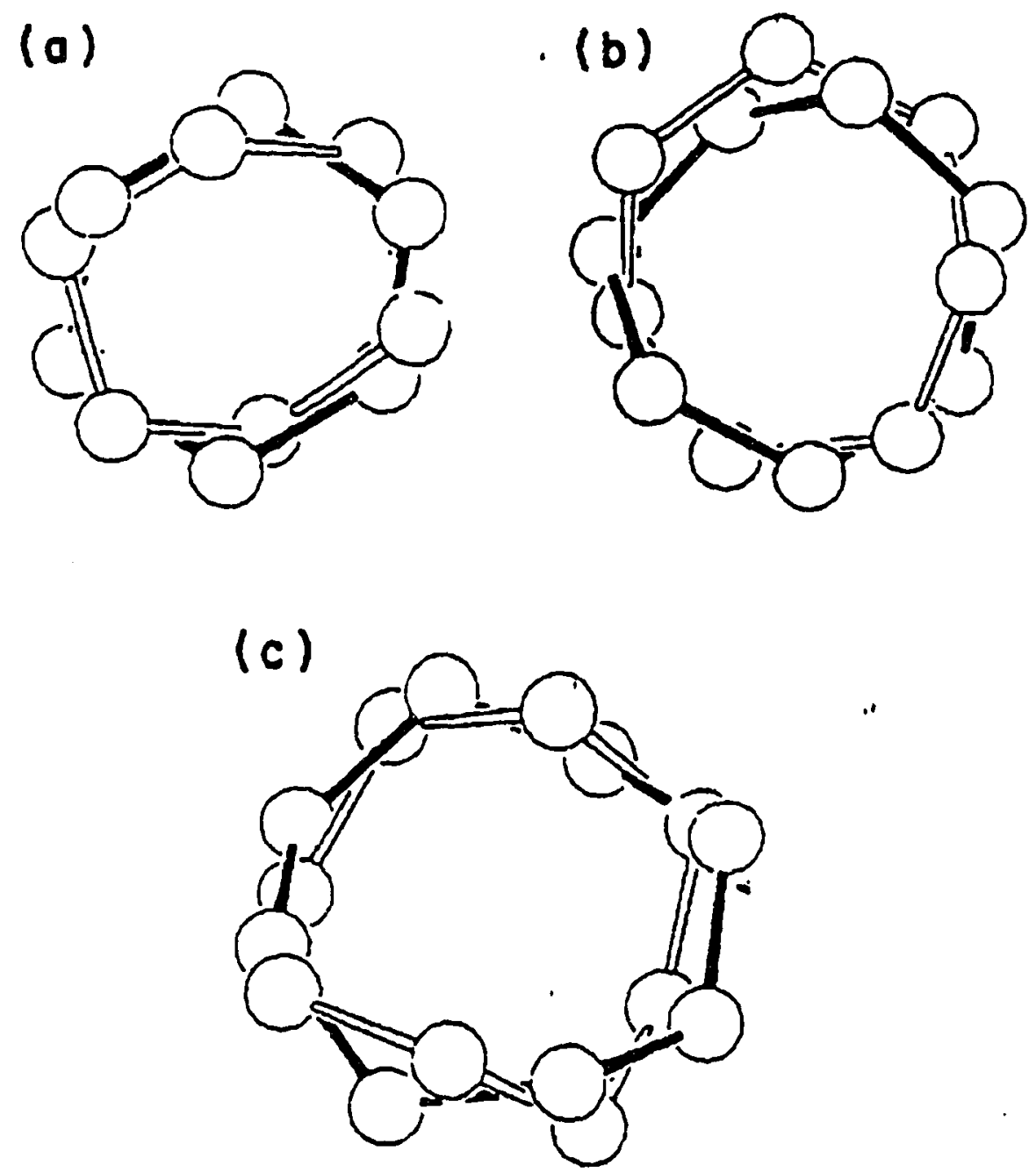

Figure III-15: Molecular orientations of (a) cyclohexane, (b) cycloheptane, and (c) cyclooctane in their respective TATM lattices [17]. 
From a crystallographic point of view, the authors found that the difficulty in refining disordered structures lies in the fact that the electron density map, derived from a difference Fourier synthesis, reflects many possible molecular orientations or conformations within several potential minima. It may therefore be helpful to carry out some molecular dynamics simulations to determine the lower energy orientations or conformations, which can be useful in confirming or predicting the actual structure.

The results of a molecular dynamics simulation for cycloheptane and cyclooctane guests in their TATM lattices are shown in Figure III-16. The calculations were starting using the molecular conformations $\alpha$ ( $g$-gg-ggg-g) and $\beta$ (ggg-ggg-g-) for cycloheptane, and the conformation $\alpha$ (ggg-g-ggg-g-) for cyclooctane.

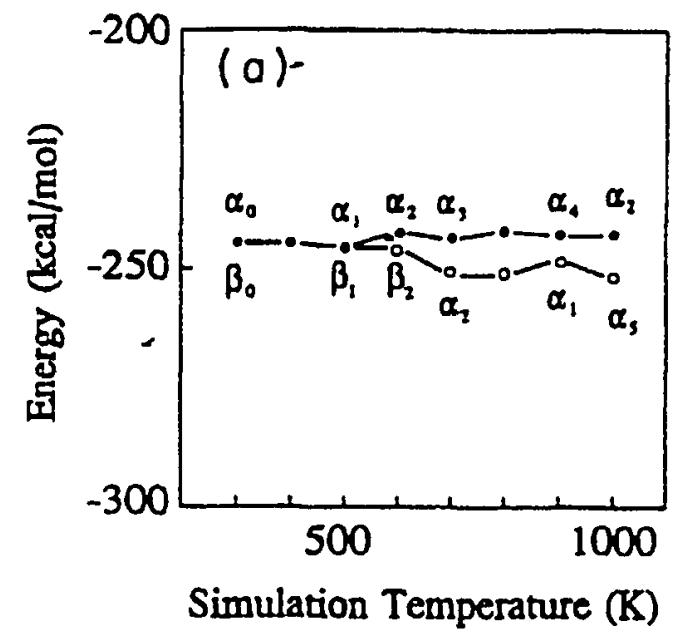

Figure III-16: Molecular dynamics simulation results for: [17]

(a) cycloheptane / TATM

(b) cyclooctane / TATM

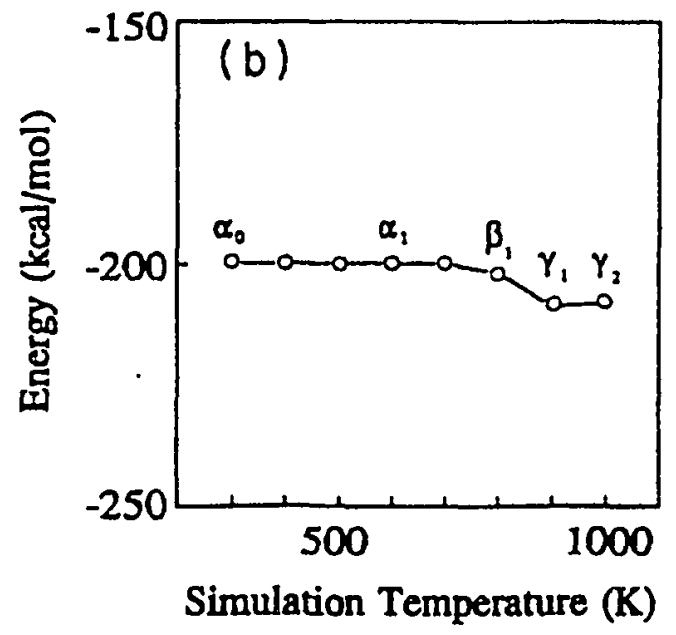


These calculations reveal that the cycloheptane guest can exist in two conformations, $\alpha$ and $\beta$. This guest in its $\alpha$ conformation takes on a number of orientations within the cavity, denoted $\alpha_{1}, \alpha_{2}, \alpha_{3} \ldots$ etc. The energy barrier to reorientation is about $1 \mathrm{kcal} / \mathrm{mol}$. The situation is somewhat different for the cycloheptane guest in the $\beta$ conformation. Besides reorienting itself, the molecule undergoes a conformational transformation at $700 \mathrm{~K}$ (see Figure III-16), which brings it into the more energetically stable $\alpha$ conformation. The calculated energy barrier for this transformation is about $2 \mathrm{kcal} / \mathrm{mol}$.

For the cyclooctane molecule, there are three molecular conformations. When the molecule is in the $\alpha$ conformation (ggg-g-ggg-g-), it goes through two conformational transformations at $800 \mathrm{~K}$ and $900 \mathrm{~K}$, to arrive at the energetically more stable $\beta$ ( $g g$-gg-gggg-) and $\gamma(g g-g g-g g g-g-)$ conformations, respectively. The $\gamma$ conformation was the one encountered in the X-ray diffraction experiment.

(K) F.H. Herbstein Acta Cryst. B53, 168 (1997)

In 1997, Herbstein published a study [22] on conformational isomerism of TATM host molecules, using literature structures of TATM inclusion compounds that had appeared in the literature up to that point $[7-10,15-17]$. At the time, Herbstein noted that TATM is a flexile molecule that can occur in many conformationally isomeric states, and that based on the unit cell parameters of the available structures, five different types of crystal structures (encompassing nine different guests) gave rise to 17 crystallographically independent TATM molecules. Within each of the five isostructural groups, it was noted that each group contains a particular TATM conformer with characteristic torsion angles about the bonds between the central methine carbon and the three thienyl rings $\left(\tau_{1}, \tau_{2}\right.$ and $\tau_{3}$ ), the range of torsion angles in a particular structural group does not exceed $10^{\circ}$. The conformers are also distinguished by the stereochemistry of the acetyl group with respect to its associated thienyl ring. Herbstein noted that there are approximately equal numbers of examples of the carbonyl oxygen being either syn or anti to the thienyl ring sulfur, intermediate conformations were not encountered. Among the five structural groups, three different types of overall conformation have been encountered for the TATM molecule. Herbstein believed that a necessary condition for the existence of a particular 
overall conformation is that the torsion angles $\tau_{1}, \tau_{2}$ and $\tau_{3}$ are such that the thienyl ring hydrogens should not approach more closely than approximately $2.5 \AA$.

As far as the nature of the crystallization process is concerned, the author suggested that crystallization of the inclusion complex from a particular solvent can be imagined to occur as follows: The TATM solution contains a Boltzmann distribution of host conformers. Under suitable temperature and solubility conditions, a guest molecule will crystallize with a TATM molecule that exists in an appropriate conformation to form an inclusion complex nucleus in a molecular recognition stage, and then subsequently grow into larger crystallites of the TATM inclusion complex. The perturbed Boltzmann distribution (depleted in the incorporated conformer), will continuously revert to its equilibrium form by conversion of non-appropriate conformers into the appropriate conformer as crystallization proceeds.

Herbstein noted that in crystalline "host-guest inclusion complexes", the details of the interaction of a larger host molecule with a smaller guest are optimally measured by studying a family of complexes. When a particular host compound interacts with a variety of chemically different guests, it is often possible to discern the structural features (molecular and crystallographic) that are required for the formation of such complexes. This process is often termed "molecular recognition". In a one-component system crystallization requires self-recognition, while in a two-component system both selfrecognition (host-host) and other-recognition (host-guest) interactions are important. In a crystalline host-guest inclusion complex, guest-guest interactions are generally considered to be negligible. When the host molecule is conformationally flexile and the guest molecule is rigid, then the host conformer found in a particular complex will depend both on the relative energies of the possible conformers and the nature of the guest. If the guest is also conformationally tlexile, then the possible guest conformers must also be taken into account. In this context, "rigid" means that conformational isomerism is not possible, and "flexile" means the opposite.

When an organic host molecule is rigid, the possibility of mutual host-guest adaptation is severely limited. However, the preparation of many inclusion compounds of TATM, which has a tripod shape, in addition to the determination of a number of their crystal structures now provides information about the mutual adaptation of a flexile host 
molecule and its guests. This allows correlations to be made between the conformers encountered for TATM and the crystal structures of the five different structural groups encountered so far, with the goal of understanding the relationship between the host conformation in a TATM inclusion complex with the chemical nature of the guest.

Herbstein, employing the literature structural data that was available at the time, wished to address the following questions:

(1) Is there a correlation between the TATM conformer and the crystal structure of the inclusion complex? (Is the same host conformer found within each of the five groupings of isostructural TATM complexes?)

(2) If so, then which TATM conformers occur within each of the five groups of isostructural inclusion compounds, and what is the range of TATM molecular structural variation (i.e. torsion angles) found within each group?

(3) What is the range of structural variation when there is more than one TATM molecule in the asymmetric unit for any one TATM complex?

Herbstein believed that these questions could be addressed, at least in part, by an analysis of the published crystallographic structures [7-10, 15-17]. He also hoped to study how the nature of the guest influences the conformation taken up by the host, however he noted that this would be quite difficult as the guest molecules are generally disordered in most of the available structures. A further possible pitfall is that the overall conclusions obtained from Herbstein's study are dependent on how representative the available structures are of the whole population, and this limitation must be taken into account.

The crystallographic data from which Herbstein drew his conclusions are depicted in Table III-5. The complexes may be divided into a number of groups on the basis of the cell dimensions, and further detail is obtained from the full structure analysis. The terms "clathrate", "cage" and "channel" have been used to describe different varieties of these complexes. Structural analysis of the ethyl acetate complex (Group I in Table III5), and of the A and B forms of cyclohexane / TATM (Groups II and III respectively), show clearly that these are all channel complexes, with the linear channel axis of Groups 
I and II along the shortest cell dimension (a), and the zig-zag channel axis of Group III along the longest $(c)$. The triclinic $n$-hexane complex (Group IV) and the monoclinic cyclononanone complex (Group V) are also channel inclusion complexes. The former is made up of channels along (111), and the latter along (100).

Table III-5: Crystallographic data for literature inclusion complexes of TATM [22]

\begin{tabular}{|c|c|c|c|c|c|c|c|c|}
\hline Guest & $a(\AA)$ & $b(\AA)$ & $c(\AA)$ & $\alpha\left({ }^{\circ}\right)$ & $\beta\left(^{\circ}\right)$ & $\gamma\left(1^{\circ}\right)$ & $V\left(\AA^{3}\right)$ & $Z$ \\
\hline \multicolumn{9}{|c|}{ GROUP I } \\
\hline ethyl acctate & 8.229 & 11.229 & 12.329 & 99.05 & 106.43 & 98.42 & 1057 & 2 \\
\hline \multicolumn{9}{|c|}{ GROUP II } \\
\hline ethimol & 8.335 & 10.372 & 12.488 & 81.49 & 71.26 & 84.95 & 1010 & 2 \\
\hline cyclohexane $A$ & 8.622 & 10.194 & 12.795 & 79.09 & 7274 & 84.89 & 1054 & 2 \\
\hline \multicolumn{9}{|c|}{ GROUP III } \\
\hline bendene & 11.538 & 13.560 & 14.197 & 89.68 & 76.60 & 75.50 & 2089 & 4 \\
\hline $\mathrm{CCl}_{4}$ & 11.638 & 13.684 & 14.235 & 88.90 & 77.46 & 78.77 & 2170 & 4 \\
\hline cyclohexane $\mathbf{B}$ & 11,668 & 13.729 & 14.227 & 89.40 & 77.15 & 76.24 & 2157 & 4 \\
\hline cycloheptanc & 11.721 & 13.734 & 14.177 & 89.22 & 76.84 & 76.01 & 2154 & 4 \\
\hline cyclooctine & 11.914 & 14.013 & 13.986 & 89.46 & 77.15 & 75.22 & 2198 & 4 \\
\hline \multicolumn{8}{|c|}{ GROUP IV } & \\
\hline$n$-hexane & 12.6047 & 12.694 & 20.604 & 85.89 & 74.31 & 86.36 & 3173 & 6 \\
\hline \multirow{2}{*}{\multicolumn{9}{|c|}{ GROUP V }} \\
\hline vclononamons: & & & 13.417 & 90 & 109,4 & 90 & 2708 & 4 \\
\hline
\end{tabular}

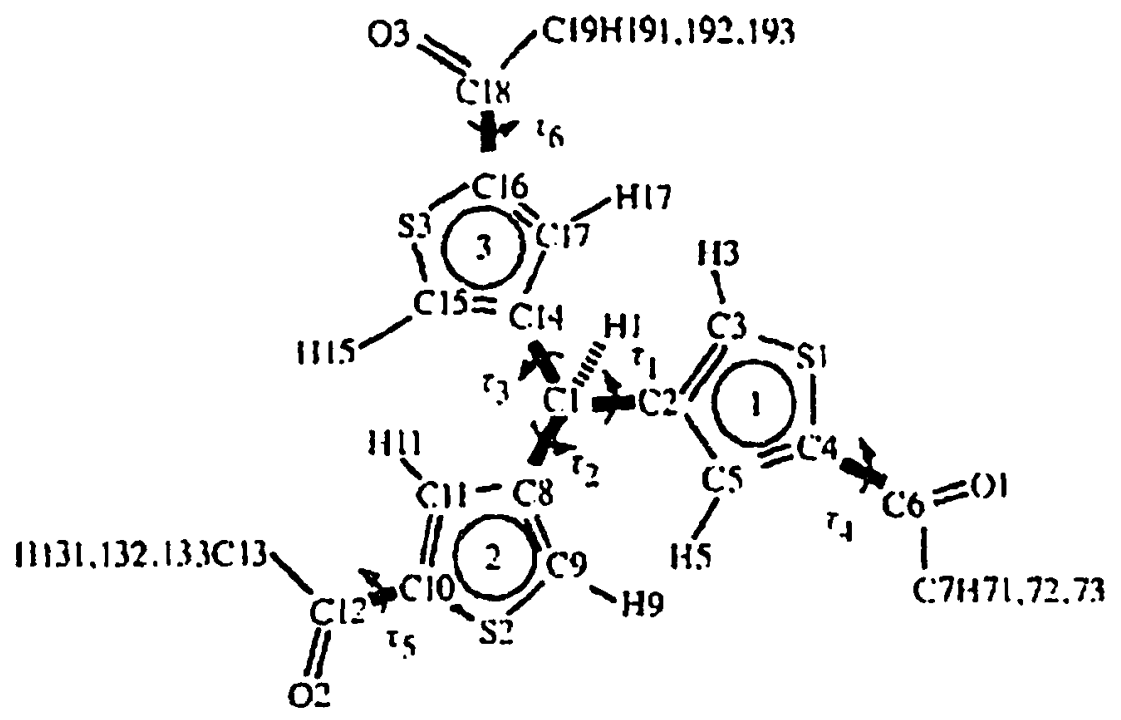

Figure III-17: Schematic representation of a TATM molecule, indicating the six bonds about which torsional flexibility can occur [22]. 
The crystals of the compounds listed in Table III-5 are all racemic, and therefore absolute optical configurations are not required. However, a meaningful comparison of the geometrical structures taken up by the TATM host in its various inclusion complexes requires comparison of conformers of the same optical configuration. As depicted in Figure III-17, Herbstein chose, arbitrarily, that the central methine $\mathrm{Cl}-\mathrm{Hl}$ bond of the host is below the plane of the figure, and the apex $\mathrm{Cl}$ of the central trigonal pyramid points away from the observer. The torsion angles $\tau_{1}, \tau_{2}$ and $\tau_{3}$ are assigned (arbitrarily, but consistently) as $\mathrm{HlClC} 2 \mathrm{C} 3, \mathrm{HIClC} 8 \mathrm{C} 9$ and $\mathrm{HlClCl} 4 \mathrm{Cl} 5$ respectively (see Figure III-17). The TATM molecule will be achiral only for some special values of the torsion angles $\tau_{1}, \tau_{2}$ and $\tau_{3}$, as these values were not encountered, Herbstein concluded that the TATM molecule is chiral. By his convention, the torsion angle with the smallest absolute value is denoted $\tau_{1}$ (in most cases it is close to $0^{\circ}$ ), while rings $2\left(\tau_{2}\right)$ and $3\left(\tau_{3}\right)$ follow in a clockwise sequence with the TATM molecule oriented as described above. Further distinction among conformers depends on whether the carbonyl oxygen atoms are $\operatorname{syn}(S)$ or anti $(A)$ to the thienyl ring sulfur. Herbstein found that these torsion angles (e.g. S1C4C6O1) are either $\sim 0^{\circ}(\mathrm{syn})$ or $\sim 180^{\circ}$ (anti). These torsion angles extracted from the results of the literature structures are summarized in Table III-6. Some of Herbstein"s conclusions are as follows:

(1) Three conformations are encountered for the TATM host molecule: (a) Conformation 1, with $\tau_{1} \sim 0^{\circ}, \tau_{2} \sim 105^{\circ}, \tau_{3} \sim 160^{\circ}$ and SSS for the three acetyl substituents. The guests of this group include ethyl acetate, ethanol, cyclohexane $A$ and $n$-hexane. (b) Conformation 2, with $\tau_{1} \sim 0^{\circ}, \tau_{2} \sim 130^{\circ}, \tau_{3} \sim 150^{\circ}$ and SSA for the three acetyl groups. The guests of this group include benzene, carbon tetrachloride, cyclohexane B, cycloheptane and cyclooctane. (c) Conformation 3, with $\tau_{1} \sim 0^{\circ}, \tau_{2} \sim 100^{\circ}, \tau_{3} \sim-15^{\circ}$ and SSS for the three acetyl groups. The guest in this group is cyclononanone.

(2) When there is more than one molecule in the asymmetric unit (Groups III and IV in Table III-5), analogous torsion angles of the crystallographically independent TATM molecules within each asymmetric unit do not differ by more than $\sim 10^{\circ}$.

(3) Analogous torsion angles within the isostructural groups that have more than one member (Groups II and III - see Table III-5) do not differ by more than $\sim 10^{\circ}$. 
Table III-6: Torsion angles among literature TATM inclusion compound structures [22].

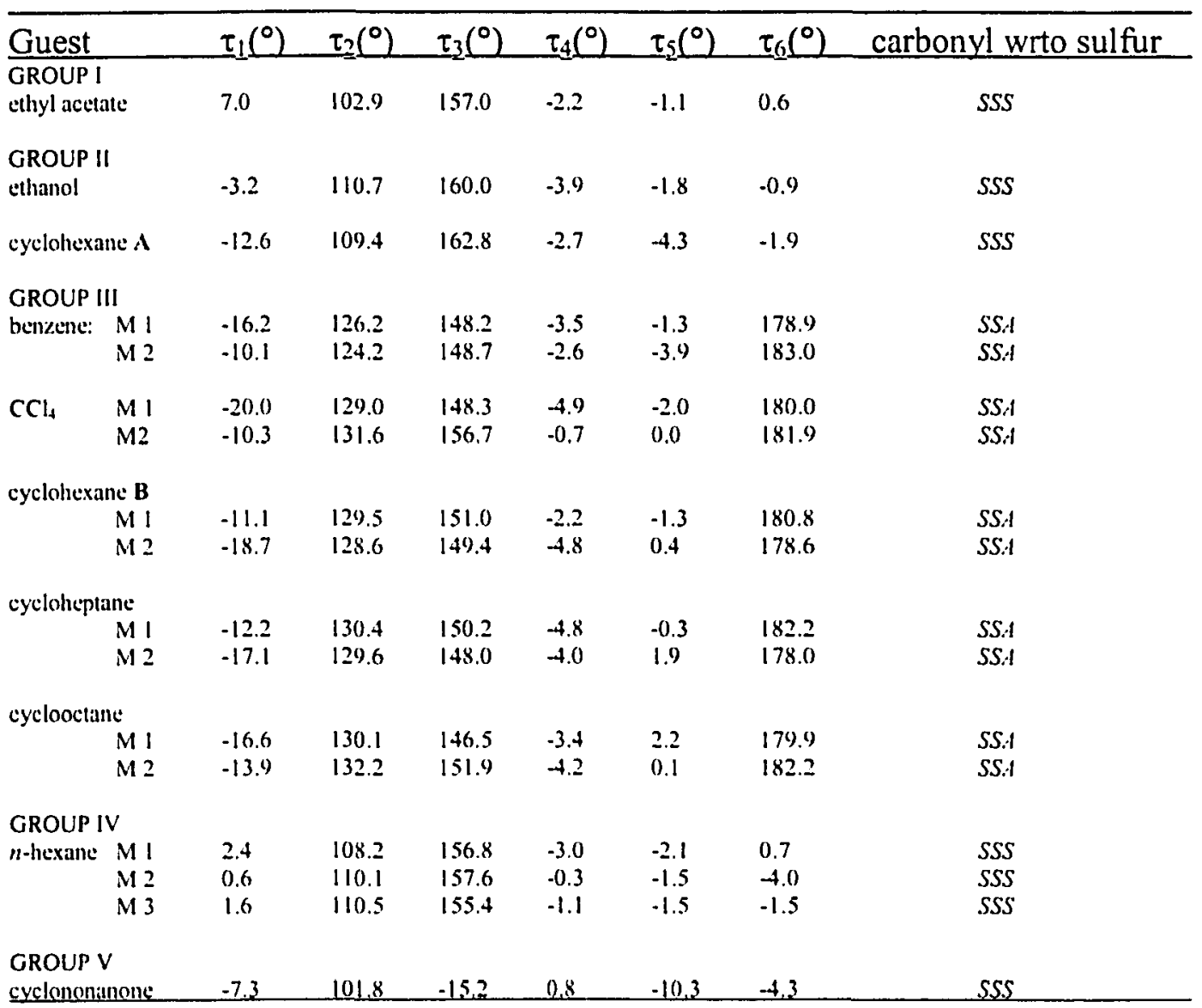

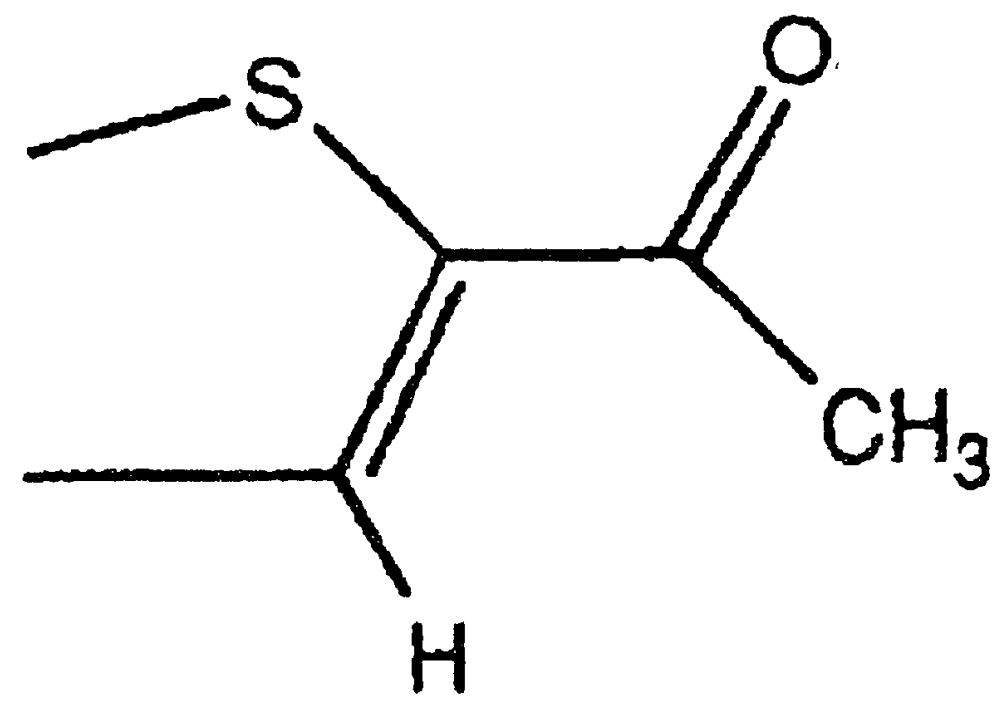

Figure III-18: Schematic of molecular fragment used to search for occurrence of $\operatorname{syn}(S)$ and anti $(A)$ conformations in the literature [22]. 
A search (by Herbstein) of the Cambridge Crystallographic Data Base for the fragment pictured in Figure III-18 produced six hits (not including those for TATM complexes). Three had syn stereochemistry and the three others were anti. On the basis of this result, Herbstein tentatively concluded that the syn and anti conformations for this type of fragment (carbonyl $O$ with respect to $S$ ) appear to be of approximately equal energy, however the factors that favor one conformation over the other are not precisely known.

On the basis of the results noted above, Herbstein suggests that there are two factors that may determine the overall conformation of a TATM molecule:

(1) The values of the ring torsion angles $\tau_{1}, \tau_{2}$ and $\tau_{3}$, which are presumably the major factor affecting the energies of the various conformations. Herbstein suggested that the two sets of conformation $\left(\tau_{1} \sim 0^{\circ}, \tau_{2} \sim 105^{\circ}, \tau_{3} \sim 160^{\circ}\right)$ and $\left(\tau_{1}\right.$ $\sim 0^{\circ}, \tau_{2} \sim 130^{\circ}, \tau_{3} \sim 150^{\circ}$ ) both belong to the same potential energy minimum (as a function of $\tau_{2}$ and $\tau_{3}$ ), and in fact refer to one set of ring conformations, from which conformation $3\left(\tau_{1} \sim 0^{\circ}, \tau_{2} \sim 100^{\circ}, \tau_{3} \sim-15^{\circ}\right)$ is separated by a substantial energy barrier. Therefore, Herbstein merged the first two conformations (1 and 2) into a common conformation denoted $1 / 2$ (with either SSS or SSA appended), instead of the separate designations 1 and 2 .

(2) The values of the acetyl group torsion angles $\left(\tau_{4}, \tau_{5}\right.$ and $\left.\tau_{6}\right)$. Here the literature suggests that syn and anti conformations should have similar energies, and so therefore the combinations SSS, SSA, ASS, SAS, AAS, ASA, SAA and $A A A$ should be equally prevalent. The current evidence shows the occurrence of only SSS and $S S A$, so Herbstein concluded that either the present TATM structural literature is unrepresentative as a whole, or possibly that there are some unknown factors playing a role.

On the basis of these conclusions, Herbstein suggested that there are only three different overall conformations of TATM: (a) conformation $1 / 2-S S S$ (b) conformation $1 / 2-S S A$ and (c) conformation 3 - SSS.

Herbstein noted that there are two different types of non-bonded $\mathrm{H} \cdots \mathrm{H}$ interactions that may be important in influencing the ring conformation of TATM. The first is between the central methine hydrogen $(\mathrm{H} 1)$ and the thienyl ring hydrogens (e.g. 
H3, H11 and H17, see Figure III-17). Herbstein denoted this the $Y$ interaction. The second type is the pairwise interaction between the thienyl ring hydrogens (e.g. $\mathrm{H} 5, \mathrm{H} 9$ and $H 15$, see Figure $111-17$ ), denoted as the $\Delta$ interaction. The values of these nonbonded $\mathrm{H} \cdots \mathrm{H}$ interactions are given in Table III-7, for one example of each of the three TATM conformational types. As mentioned above, it is to be expected that non-bonded $\mathrm{H} \cdots \mathrm{H}$ distances should be no shorter than $2.4 \AA$, the smallest distance in Table III-7 is $2.39 \AA$, for cyclononanone / TATM. There are no other distances smaller than $2.4 \AA$, so Herbstein's hypothesis is experimentally verified.

Table III-7: Values of non-bonded $H \cdots H$ distances (in $\AA$ ) in each of the three overall TATM conformational types (see labeling scheme in Figure III-17) [22]

\begin{tabular}{|c|c|c|c|c|c|c|}
\hline Guest & $\mathrm{HI} \cdots \mathrm{H} 3$ & $\mathrm{H} \mid \cdots \mathrm{H} I \mathrm{I}$ & $\mathrm{H} 1 \cdots \mathrm{H} 17$ & $\mathrm{H} 5 \cdots \mathrm{HO}$ & $\mathrm{H} 9 \cdots \mathrm{H} / \mathrm{s}$ & $\mathrm{H} 5 \mathrm{\cdots H} 15$ \\
\hline $\mathrm{CCl}_{+}$ & 2.44 & 2.78 & 2.61 & 2.96 & 3.55 & 2.92 \\
\hline $\begin{array}{l}\text { thyl } \\
\text { acctate }\end{array}$ & 2.44 & 3.03 & 2.51 & 2.87 & 4.16 & 2.74 \\
\hline & $\mathrm{Hl} \cdots \mathrm{H} 3$ & $\mathrm{H} \mid \cdots \mathrm{H}^{9}$ & $\mathrm{H} 1 \cdots \mathrm{H} 17$ & $\mathrm{H} 5 \cdots \mathrm{HII}$ & $\mathrm{H} \mid \mathrm{W} \cdots \mathrm{H} 15$ & $\mathrm{H} 5 \cdots \mathrm{HI}$ \\
\hline $\begin{array}{l}\text { cyclo. } \\
\text { nonamon }\end{array}$ & 2.46 & 2.43 & 3.05 & 2.39 & 3.25 & 4.16 \\
\hline
\end{tabular}

The three different overall conformations of TATM illustrated above will occur in TATM solutions in relative proportions given by the Boltzmann distribution, these proportions will generally depend on the temperature but not on the nature of the solvent. This assumption holds at high temperature. when TATM is in a dissolved state, but may no longer hold as the solution cools and the inclusion complex begins to crystallize. As an example, if the solvent is benzene, then benzene / TATM will crystallize as the solution cools, and the solution will become depleted in TATM molecules with a conformation of $1 / 2-S S A$. Subsequently, at sufficiently high temperatures, the Boltzmann distribution will be continuously re-established by conversion of dissolved TATM molecules with conformations, such as $1 / 2-S S S$ and $3-S S S$, into conformers $1 / 2-S S A$, and this process will continue until an appropriate amount of the crystalline inclusion compound has been formed.

In terms of this description, Herbstein suggested that the overall process of the formation of a crystalline TATM inclusion complex can be divided into three stages: 
(1) Selection of an appropriate TATM conformer from an ensemble of TATM conformers, requiring recognition between the solvent molecules and the appropriate TATM conformer. In this stage the determining factor is the hostguest interaction.

(2) Formation of nuclei from the TATM-solvent aggregates, requiring predominately host conformer - host conformer recognition.

(3) Growth of the nuclei to form crystals.

This description also provides a possible explanation for the occurrence of an amorphous guest-free product upon vacuum sublimation. In this case, the presence of more than one conformer in the condensing sublimate prevents crystallization.

In summary, Herbstein's analysis of the published crystallographic data shows that within any particular group of isostructural TATM inclusion compounds, the TATM molecules all have very similar conformations. The same holds true for comparisons between different host molecules in any particular inclusion complex when there is more than one TATM molecule in the asymmetric unit. Herbstein suggested that this implies a 1:1 correlation between thienyl ring conformation and crystal structure, but he noted that this may be an oversimplification. This situation is complicated by the fact that while the ring conformations may be a primary factor in determining the conformational energy of a TATM molecule, the conformation of the three carbonyl groups ( $s . \mathrm{n}$ or anti) in relation to the thienyl ring sulfur may also be an essential component in determining the overall molecular shape, and may be just as important as the thienyl ring conformation in determining the crystal structure of the inclusion complex. Thus, the correlation is more correctly noted as between overall molecular conformation (a combination of thienyl rings and acetyl groups) and the resulting crystal structure.

Herbstein noted that the syn and anti conformations of the acetyl groups appear to have similar energies from the statistics of their occurrence in the literature, but only three of the possible combinations of TATM thienyl ring conformation - acetyl group conformation have so far been encountered. It is unclear whether this is because the available structures are unrepresentative of the population as a whole, or because other factors contributing to overall molecular energy have not yet been recognized. 
In 1995, P.S. Sidhu and co-workers reported [23] on the reorientational dynamics of acetone- $\mathrm{d}_{6}$ incorporated into a TATM lattice by employing deuterium $\left({ }^{2} \mathrm{H}\right)$ solid-state NMR spectra and ${ }^{2} \mathrm{H}$ spin-lattice relaxation times. The ${ }^{13} \mathrm{C}$ CP/MAS NMR spectrum of this compound was obtained to verify inclusion. From variable temperature ${ }^{2} \mathrm{H}$ NMR spectra, the authors found that the guest is undergoing two-fold reorientation about the carbonyl bond, exchanging the two $-\mathrm{CD}_{3}$ groups. Identical activation energies of 20.8 $( \pm 1.4) \mathrm{kJ} \mathrm{mol}^{-1}$ and $20.8( \pm 0.5) \mathrm{kJ} \mathrm{mol}^{-1}$ for this motion were determined, independently, from ${ }^{2} \mathrm{H}$ NMR spectra and ${ }^{2} \mathrm{H}$ NMR relaxation times, respectively. A second motion, internal $C_{3}$ methyl reorientation, displays a rate that is in the fast rate limit $\left(k>10^{8} \mathrm{~Hz}\right)$, even at the lowest temperature measured $(84 \mathrm{~K})$. Both abovementioned reorientations are occurring at the same time. Analytical expressions for the ${ }^{2} \mathrm{H}$ spin-lattice relaxation time $\left(T_{1}\right)$, for a two-fold jump, were derived. Calculated values of the effective quadrupolar coupling constant $\left(\chi_{\mathrm{ent}}\right)$ and the spin-lattice relaxation time minimum value $\left(T_{1 \mathrm{~min}}\right)$, agree very well with the experimental data. Finally, the ${ }^{2} \mathrm{H}$ NMR spectrum of this inclusion compound unexpectedly shows some asymmetry, the origin of which is discussed.

The ${ }^{13} \mathrm{C}$ CP/MAS NMR spectrum of acetone/TATM is presented in Figure III-19. The signals were assigned using ${ }^{13} \mathrm{C}$ chemical shift correlation tables [24]. The acetone $\mathrm{CH}_{3}$ signal is located at $29.2 \mathrm{ppm}$, while its carbonyl peak appears at $202.6 \mathrm{ppm}$. The multiplicity of the TATM carbons is two each, one strong and one weak signal for each carbon. This may originate from the fact that in the crystal structures done on TATM inclusion compounds to that point in time, $[7-10,15-17]$ two of the three thienyl rings of each TATM molecule participate in forming the cavity in which the guest resides, while the third thienyl ring does not. Therefore, the two thienyl rings forming the cavity give rise to the strong signals, while the respective weak signals, of approximately $50 \%$ intensity, arise from the non-participating TATM thienyl ring. This is supported by the observation that the central methine carbon of the host gives only a single signal ( $\sim 46$ $\mathrm{ppm}$ ), as there is only one methine carbon in each TATM molecule. 


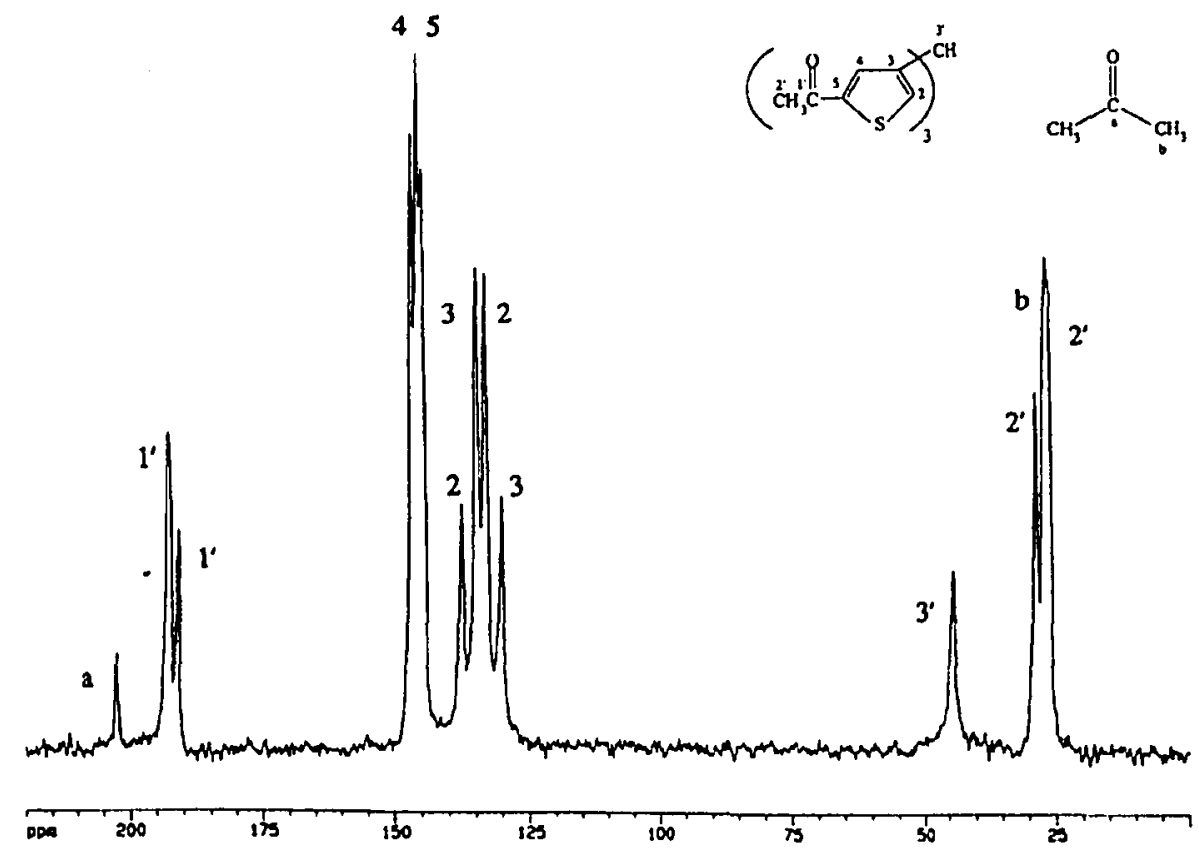

Figure III-19: ${ }^{13} \mathrm{C} \mathrm{CP} / \mathrm{MAS}$ powder NMR spectrum of the acetone / TATM inclusion compound with signal assignments [23].

The ${ }^{2} \mathrm{H}$ NMR powder spectra of acetone- $\mathrm{d}_{6} /$ TATM are presented in Figure III-20, along with simulations. The acetone- $d_{6} /$ TATM complex is characterized by a rapid rate $(k>$ $\left.10^{8} \mathrm{~Hz}\right)$ of methyl rotation at all temperatures studied $(142 \mathrm{~K}-273 \mathrm{~K})$, because of its known very low activation energy $\left(2-5 \mathrm{~kJ} \mathrm{~mol}^{-1}\right),[25-27]$ and the fact that the $142 \mathrm{~K}$ spectrum is narrowed by at least a factor of three over the static spectrum $\left(\chi_{\text {static }} \sim 170\right.$ $\mathrm{kHz}$ ). On top of this motion, there is exchange of the two methyl groups about the carbonyl bond, the molecular $C_{2}$ axis. The rate of this motion is in the intermediate exchange region $\left(10^{3} \mathrm{~Hz}<k<10^{8} \mathrm{~Hz}\right)$ in the temperature range studied. Changes in the rate of this motion with temperature give rise to changes in the lineshape of the experimentally observed ${ }^{2} \mathrm{H}$ spectra. By visually fitting the calculated simulations to their corresponding experimental spectra, it is possible to extract rates for this two-fold reorientation as a function of temperature (see Figure III-20). A plot of $\ln k$ vs 1000/T (Figure III-21) gave an activation energy of $20.8( \pm 1.4) \mathrm{kJ} \mathrm{mol}^{-1}$ for this $C_{2}$ exchange. In the simulations, it was necessary to employ a $\mathrm{CD}_{3^{-}} \mathrm{C}-\mathrm{CD}_{3}$ bond angle of $123^{\circ}\left( \pm 2^{\circ}\right)$ to make a match to the experimental spectra. From microwave data, the angle in the gas phase is $116^{\circ}[28,29]$. 
Experimental
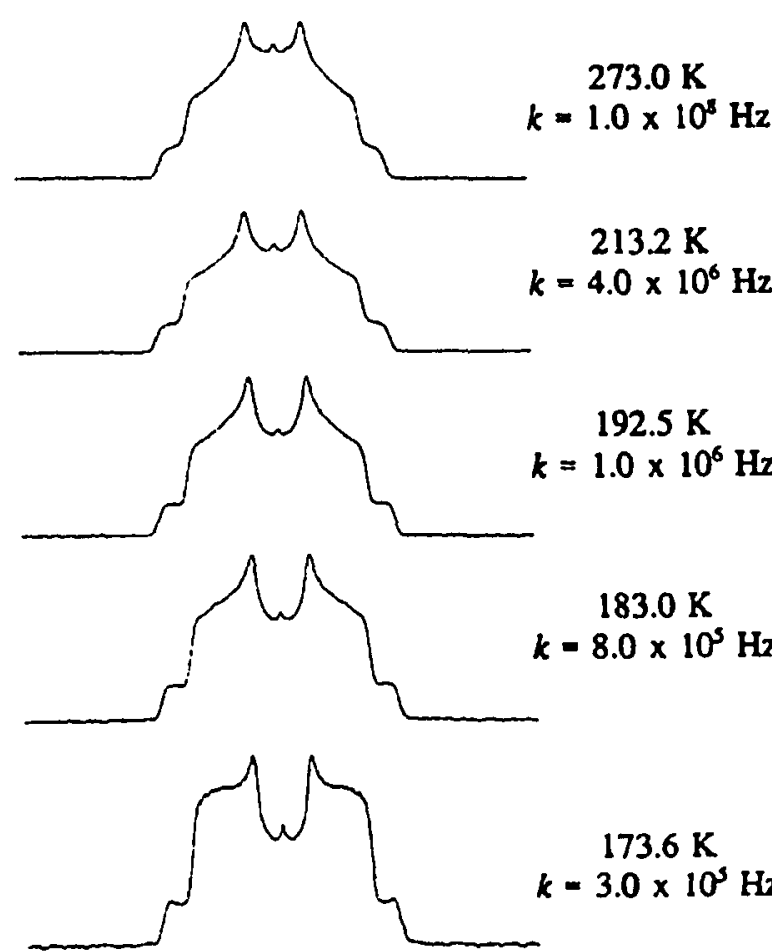

$\sqrt{2}$<smiles>CCCCCCCCCCCCCCCCCCCC</smiles>
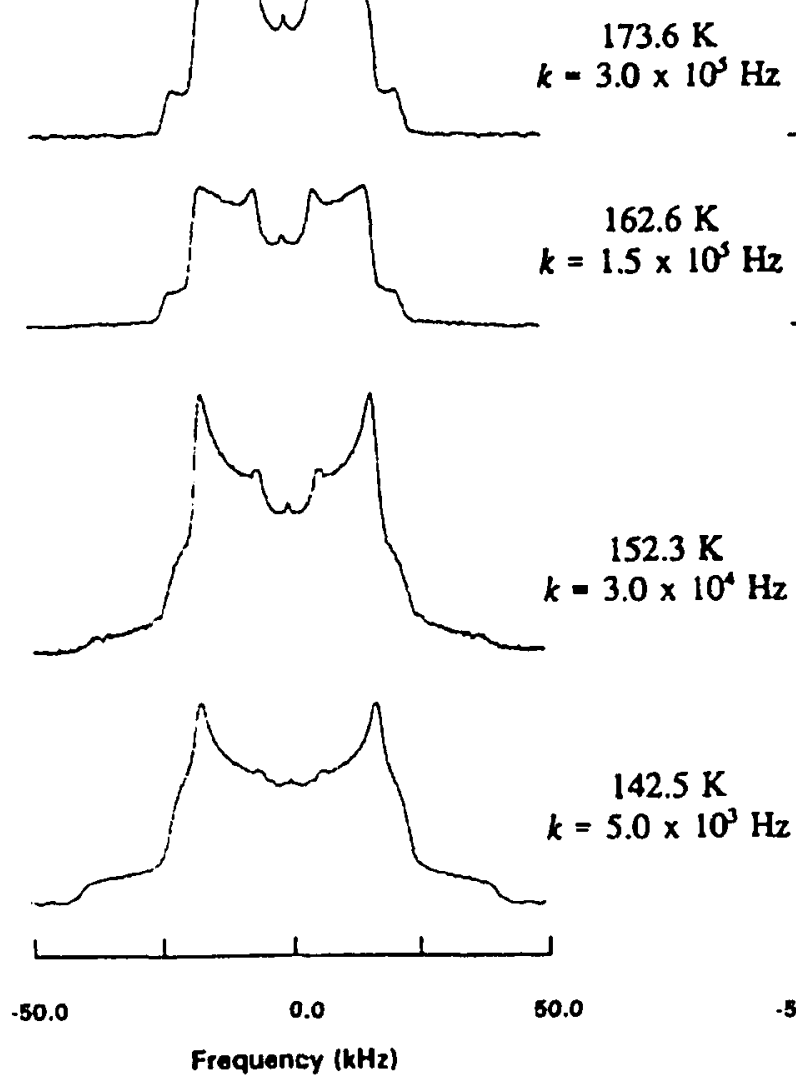

$192.5 \mathrm{~K}$ $k=1.0 \times 10^{6} \mathrm{~Hz}$

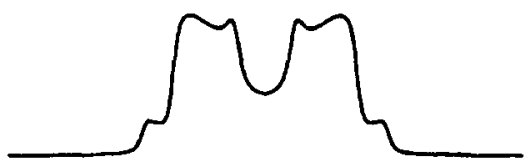

Simulated
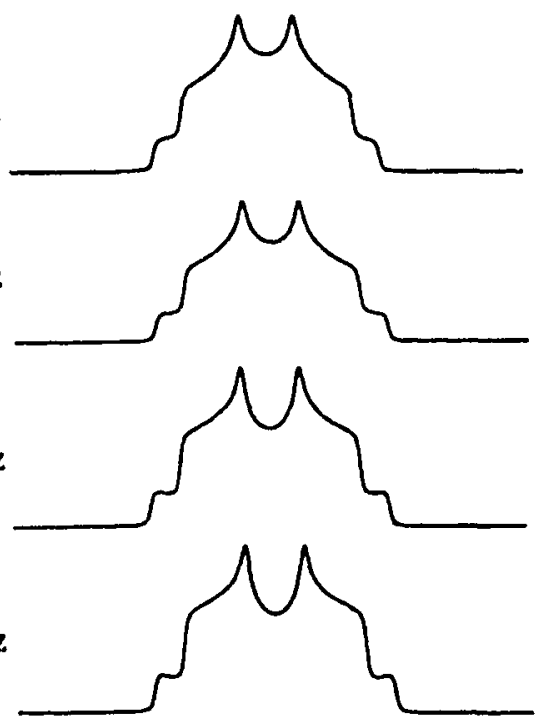

$173.6 \mathrm{~K}$ $k=3.0 \times 10^{3} \mathrm{~Hz}$
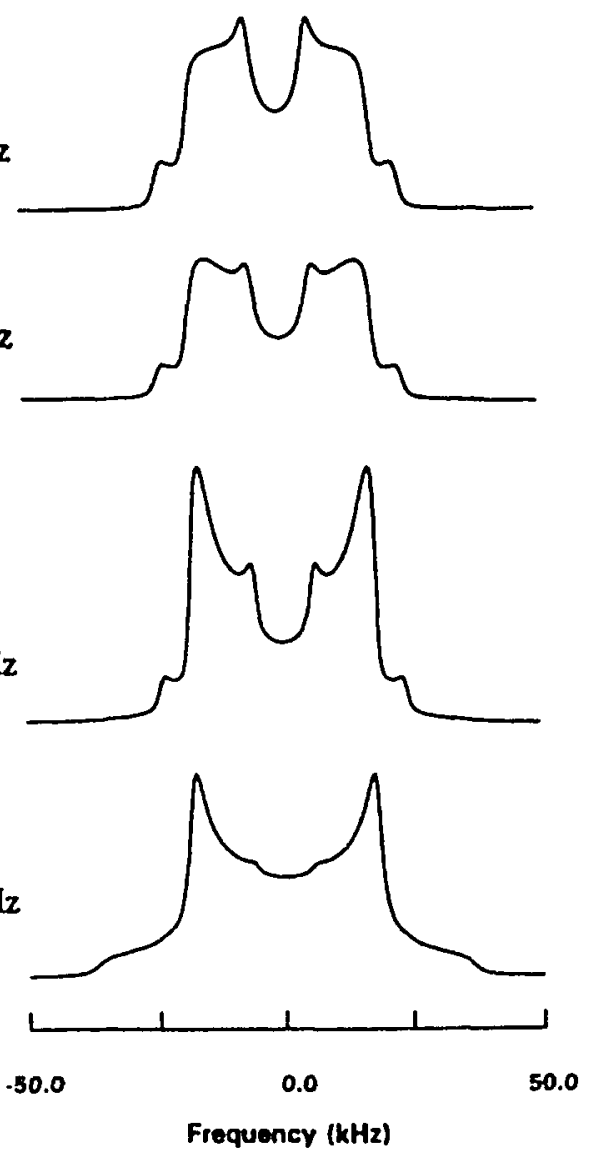

Figure III-20: Deuterium NMR spectra for acetone- $\mathrm{d}_{6}$ in TATM at various temperatures, along with best-fit lineshapes and two-fold flip rate [23]. 


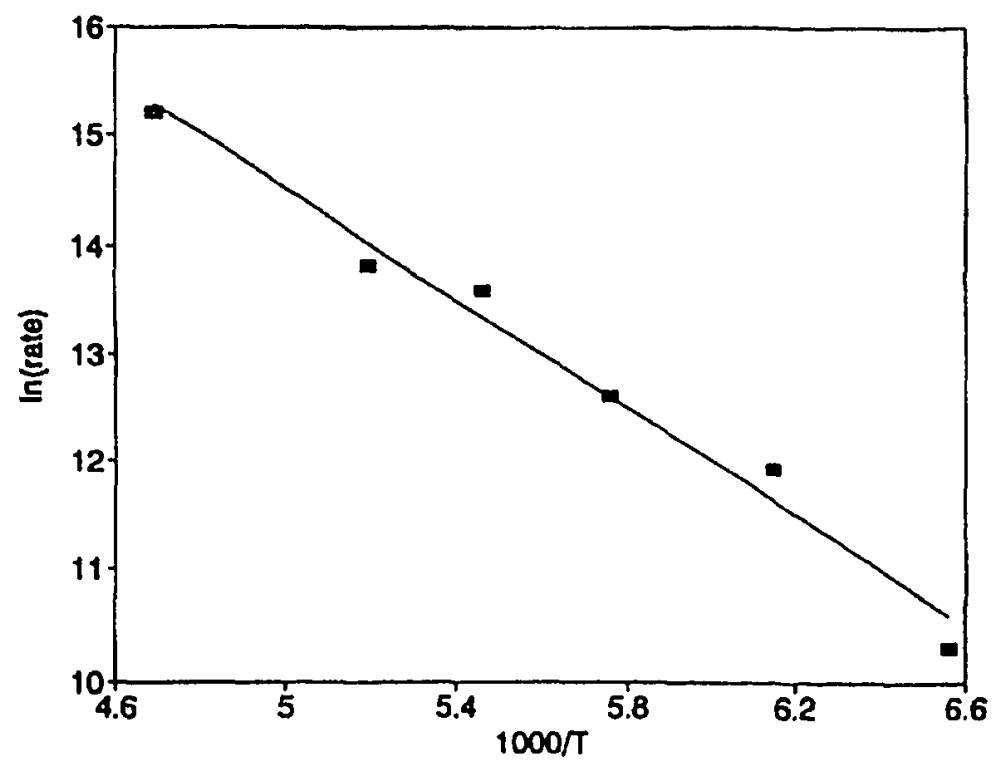

Figure III-21: Graph of $\ln k$ vs 1000/T for the two-fold flip of the acetone- $\mathrm{d}_{6}$ guest in the TATM lattice, along with a least-squares fit to the data. From the slope of the graph, $E_{\mathrm{a}}=20.8 \mathrm{~kJ} \mathrm{~mol}^{-1}[23]$.

The profile of ${ }^{2} \mathrm{H}$ spin-lattice relaxation times for acetone- $\mathrm{d}_{6} /$ TATM, as a function of inverse temperature, is presented in Figure III-22. Changes in ${ }^{2} \mathrm{H} T_{1}$ in this temperature region result from changes in the rate of the abovementioned two-fold flips. Analysis of the ${ }^{2} \mathrm{H} T_{1}$ data was done using the BPP spectral density function [30]. The variable temperature $T_{1}$ data were fit to equations of the following type:

$$
\begin{gathered}
\frac{1}{T_{1}}=K\left[\frac{\tau_{c}}{1+\omega_{0}^{2} \tau_{c}^{2}}+\frac{4 \tau_{c}}{1+4 \omega_{0}^{2} \tau_{c}^{2}}\right] \\
\tau_{c}=\tau_{\infty} \exp \left(\frac{-E_{a}}{R T}\right)
\end{gathered}
$$

In these equations, $\tau_{c}$ is interpreted as the correlation time for the motion, $\tau_{\infty}$ is the correlation time at infinite temperature, and $\omega_{0}$ is the Larmor precession frequency. $K$ is a constant that depends on the static quadrupolar coupling constant $\left(\chi_{\text {static }}\right)$ and the mode of molecular reorientation, while $E_{\mathrm{a}}$ is the activation energy for the motion of interest. 


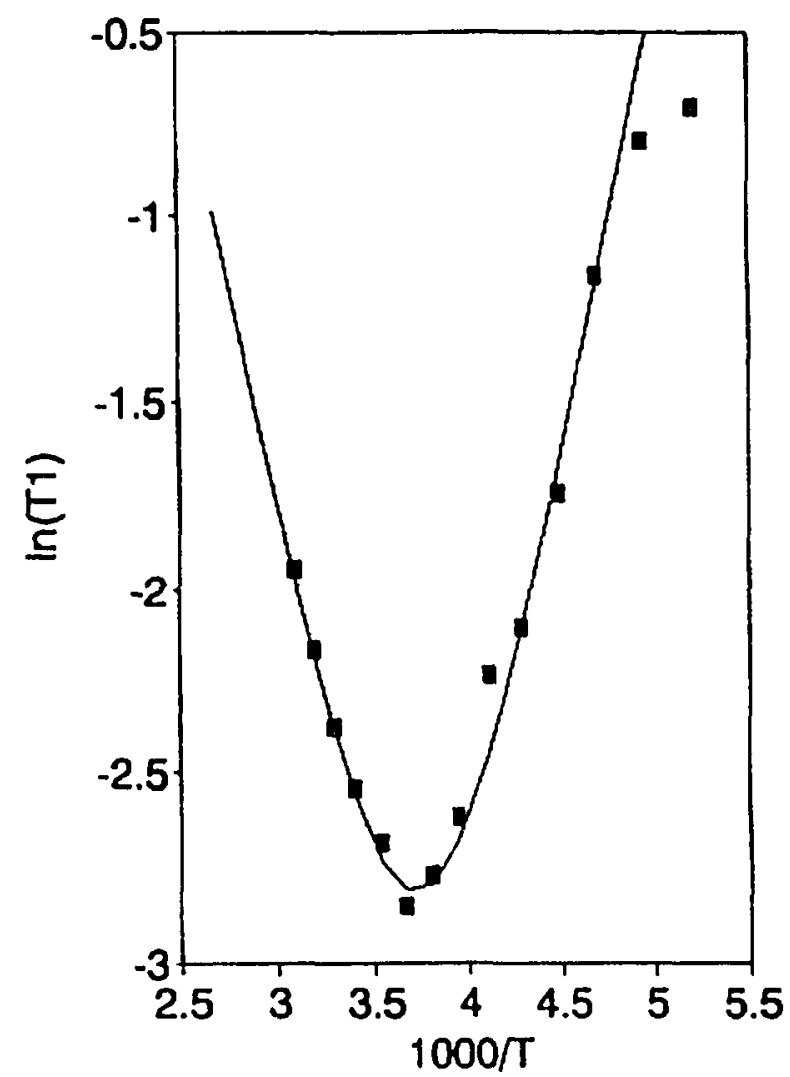

Figure III-22: Deuterium spinlattice relaxation times, as a function of reciprocal temperature, for the acetone- $\mathrm{d}_{6} /$ TATM inclusion compound. [23]

By a least squares fitting of the experimental data (symbols in Figure III-22) to a best-fit curve (also in Figure III-22), incorporating the variables in Equations III-1 and III-2, an activation energy of $20.8( \pm 0.5) \mathrm{kJ} \mathrm{mol}^{-1}$ for the $C_{2}$ exchange was measured. Note that this is exactly the same value determined from the ${ }^{2} \mathrm{H}$ NMR simulations discussed above. The constant $K$ (Equation III-1) was measured as $3.3 \times 10^{9} \mathrm{~s}^{-2}$, and $\tau_{\infty}$ (Equation III-2) was calculated as $2.03 \times 10^{-13} \mathrm{~s}$. Using these values of $\tau_{\infty}$ and $E_{\mathrm{a}}$ in Equation III-2, and the fact that the jump rate $k=\left(2 \tau_{\mathrm{c}}\right)^{-1}$ for the two-fold reorientation, it is possible to compare the rates of reorientation determined from the $T_{1}$ analysis with those found from simulation of the ${ }^{2} H$ NMR spectra, as a function of temperature. The two methods gave the same result within experimental error. In addition, it can be seen that the two lowest temperature points (the two highest $1000 / T$ points) in Figure III-22 fall below the fitted curve. This suggests the presence of a second $T_{1}$ minimum at much lower temperatures that may be associated with internal $C_{3}$ reorientation of the $-\mathrm{CD}_{3}$ groups. 
Analytical expressions for the deuterium spin-lattice relaxation time, for a twosite jump model, were derived from the following general equations: [31]

$$
\begin{gathered}
\frac{1}{T_{1}}=\frac{\chi^{2}}{3}\left[J_{1}\left(\omega_{0}\right)+4 J_{2}\left(2 \omega_{0}\right)\right] \\
\chi=\frac{e^{2} q Q}{h}
\end{gathered}
$$

The quadrupolar coupling constant is defined as $\chi$, with $e q$ and $e Q$ representing the electric field gradient of the surrounding charge distribution and the nuclear quadrupole moment, respectively. The spectral density functions $J_{1}$ and $J_{2}$ are defined with respect to the direction of the applied field. For two-site jump motion about $\mathrm{a} z$ axis fixed in the crystal, with the angle between the two sites being $2 \theta$, the expression for $T_{1}$ is given by:

$$
\frac{1}{T_{1}}=\frac{9}{160}\left(\frac{2 \pi e^{2} q Q}{h}\right)^{2} \sin ^{2} 2 \theta\left[J_{1}^{\prime}\left(\omega_{0}\right)+4 J_{2}^{\prime}\left(2 \omega_{0}\right)\right]
$$

Now $J_{1}$ and $J_{2}^{\prime}$ are defined with respect to the crystal axis. Therefore, the constant $K$ in Equation III-1 is equal to:

$$
K=\frac{9}{160} \chi^{2} 4 \pi^{2}\left(\sin ^{2} 2 \theta\right)
$$

In addition, the spin-lattice relaxation time minimum is determined to be:

$$
\frac{1}{T_{1 \min }}=\frac{9}{160}\left(\frac{2 \pi e^{2} q Q}{h}\right)^{2} \sin ^{2} 2 \theta\left(\frac{1.42}{\omega_{0}}\right)
$$

From the value of $K$, determined from the $T_{1}$ fit above $\left(3.3 \times 10^{9} \mathrm{~s}^{-2}\right)$, and the angle $2 \theta$ $\left(123^{\circ}\right)$, found from the ${ }^{2} \mathrm{H}$ spectral simulations above, the effective quadrupolar coupling constant $\left(\chi_{\text {etf }}\right)$ is approximately $46( \pm 1) \mathrm{kHz}$. This compares with an observed $\chi_{\mathrm{etr}}$ of 45 $( \pm 1) \mathrm{kHz}$ at $273 \mathrm{~K}$ (from the experimental ${ }^{2} \mathrm{H}$ NMR spectrum). This quadrupolar coupling constant is averaged by internal methyl rotation and librational motion (see below), but not by the two-fold flip.

The experimental value of the ${ }^{2} \mathrm{H}$ spin-lattice relaxation time at the $T_{1}$ minimum is about $60 \mathrm{msec}$. In Equation III-7, using $\chi_{\text {elf }}\left(=\mathrm{e}^{2} q Q / h\right)$ of $45 \mathrm{kHz}$ at the $T_{1}$ minimum, and a spectrometer frequency of $44.669 \mathrm{MHz}$, it was calculated that the ${ }^{2} \mathrm{H} T_{1}$ minimum 
should be approximately $62 \mathrm{msec}$. Thus, the agreement between calculated and experimental $T_{\mathrm{l} \text { min }}$ is quite good.

Finally, a third form of motion, modeled as precession of the $C_{2}$ axis on a cone, [32] is present. This was incorporated due to the fact that the ${ }^{2} \mathrm{H}$ NMR spectra were too narrow. In other words, the two types of reorientation described above $\left(C_{2}\right.$ and $\left.C_{3}\right)$ cannot fully account for the observed spectral narrowing. In the ${ }^{2} \mathrm{H}$ simulations, precession of the $C_{2}$ axis on a circular shaped cone was assumed. However, since acetone is not an axially symmetric molecule, one might expect the precession to be much more asymmetric (i.e. elliptical). However, introducing elliptical precession in this case would overcomplicate the motional model. Elliptical precession might be a valid conclusion because the ${ }^{2} \mathrm{H}$ NMR spectrum at $143 \mathrm{~K}$ shows some asymmetry (i.e. $\eta>0$ ). This is because libration of an elliptical nature introduces asymmetry into the spectrum, whereas circular precession does not. For circular precession, the half angle of libration at room temperature is about $16.9^{\circ}$.

In Figure III-23 is depicted the ${ }^{2} \mathrm{H}$ NMR spectrum of acetone- $\mathrm{d}_{6} /$ TATM at $84 \mathrm{~K}$, along with a best-fit simulation. The experimental spectrum unexpectedly shows some asymmetry, as the temperature is low enough to practically "freeze-out" the librational motion. Facey and co-workers [33] argue that the reason their $77 \mathrm{~K}$ spectrum of acetone$\mathrm{d}_{6}$ in tri-ortho-thymotide (TOT) shows some asymmetry is because the three sites visited by the methyl deuterons are inequivalent, as one of the C-D bonds is very close (parallel) to the carbonyl group. Possible consequences for this interaction include a difference in the static quadrupole coupling constant for one of the three deuterons due to a perturbation of the electric field gradient by the adjacent electronegative carbonyl group, [34] and/or a slight deviation in the projected angle of $120^{\circ}$ between two of the deuterons [35]. Facey's argument is valid, because the width of their $77 \mathrm{~K}$ spectrum is characteristic of no precession being present $\left(\chi_{\mathrm{eff}} \sim 57 \mathrm{kHz}\right)$. In the present case, the ${ }^{2} \mathrm{H}$ spectrum of acetone- $d_{6} /$ TATM at $84 \mathrm{~K}$ (Figure III-23) also shows considerable asymmetry. The effective quadrupolar coupling constant is about $55.5 \mathrm{kHz}$ at this temperature. Thus the static quadrupole coupling constant is approximately three times $\chi_{\mathrm{eff}}$, or $166.4 \mathrm{kHz}$. Since static quadrupole coupling constants of saturated aliphatic deuterons are usually in the range $165-175 \mathrm{kHz}$, it was proposed that the magnitude of 
the librational motion (i.e. wobble of the $C_{2}$ axis) is practically zero at this temperature. Yet the experimental spectrum in Figure III-23 shows a considerable amount of asymmetry $(\eta \sim 0.14)$. This implies that the asymmetry does not come from elliptical precession. Indeed, the experimental spectrum was successfully simulated (see Figure III-23) by changing the projected angle between the two methyl deuterons (that are not parallel to the carbonyl group) from $120^{\circ}$ to $124^{\circ}$. These calculations are outlined in more detail in reference [35].

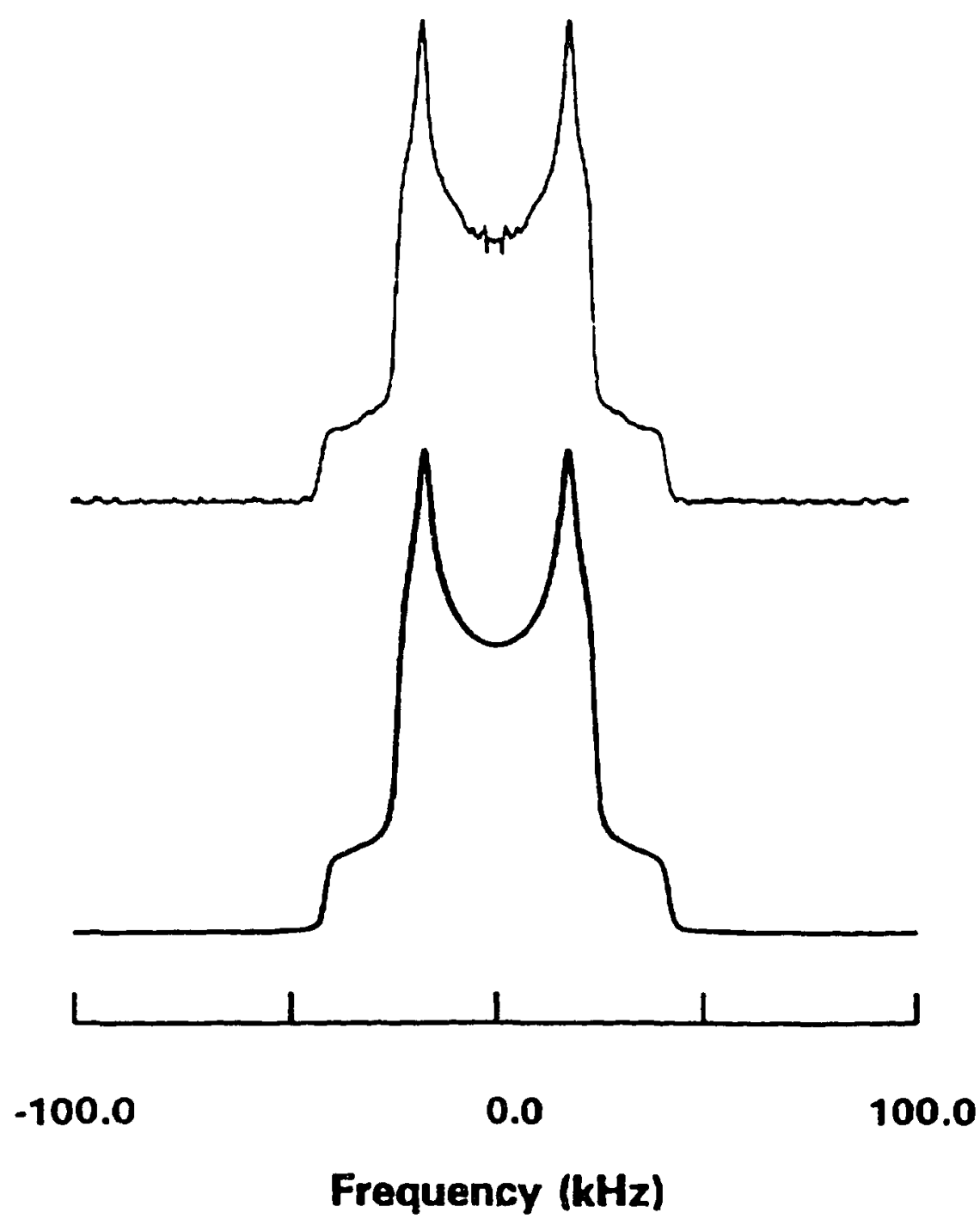

Figure III-23: Deuterium NMR powder spectrum of acetone-d $\mathrm{d}_{6} /$ TATM at $84 \mathrm{~K}$, along with a best fit simulation, as discussed in the text [23]. 
(M) P.S. Sidhu, J. Bell, G.H. Penner and K.R. Jeffrey Can. J. Chem. 74, 1784 (1996)

In 1996, P.S. Sidhu and co-workers again investigated dynamics in TATM guest-host systems, [36] but instead of studying only one guest (i.e. acetone), they measured ${ }^{2} \mathrm{H}$ solid-state variable temperature NMR spectra and ${ }^{2} \mathrm{H}$ NMR spin-lattice relaxation times for a large number of relatively small, deuterated guests. The seven guests include acetonitrile, nitromethane, dimethyl sulfoxide (DMSO), benzene, mesitylene, orthoxylene and para-xylene, and they show a wide variety of motional behavior. The reorientation of acetonitrile- $\mathrm{d}_{3}$ in TATM and nitromethane- $\mathrm{d}_{3}$ in TATM were both modeled as precession of the molecular axis on a cone, the base of which is more elliptical in shape for nitromethane- $d_{3}$, as would be expected considering their respective molecular symmetries. DMSO- $\mathrm{d}_{6}$ in TATM does not undergo any reorientation other than internal methyl rotation at the temperatures studied. At low temperatures, the ${ }^{2} \mathrm{H}$ lineshape and ${ }^{2} \mathrm{H} T_{1}$ both depend on the rate of internal $C_{3}$ methyl rotation. Activation barriers of $11.7( \pm 0.4) \mathrm{kJ} \mathrm{mol}^{-1}$ and $11.2( \pm 0.5) \mathrm{kJ} \mathrm{mol}^{-1}$ were found from the two techniques respectively. Benzene- $d_{6}$ undergoes six-fold reorientation about the principal molecular axis, however the rate of this motion is still greater than $10^{8} \mathrm{~Hz}$ down to 113 $\mathrm{K}$. The variable temperature ${ }^{2} \mathrm{H}$ spin-lattice relaxation time profile does not reach a minimum on decreasing to $112 \mathrm{~K}$, while the slope of this plot provides an activation energy of $4.1( \pm 0.4) \mathrm{kJ} \mathrm{mol}^{-1}$ for the six-fold reorientation. The ${ }^{2} \mathrm{H}$ NMR spectra of mesitylene- $d_{9}\left(1,3,5\right.$-trimethylbenzene- $\left.d_{9}\right)$ in TATM can be simulated by using a model where the guest occupies two different sites in the TATM lattice, with the guest molecules performing in plane $C_{3}$ rotation either very rapidly $\left(k>10^{8} \mathrm{~Hz}\right)$ or very slowly $\left(k<10^{3} \mathrm{~Hz}\right)$, with the relative populations of each changing with temperature. An alternative model suggests that there is a continuous distribution of motional rates, the median of which is increasing with temperature. This second model is more realistic, however there are simply too many parameters to consider a detailed fit. Finally, both ortho-xylene- $\mathrm{d}_{10}$ and para-xylene- $\mathrm{d}_{10}$ are rigidly held in their TATM lattices, while internal rotation of the methyl groups is in the fast rate limit.

Acetonitrile and nitromethane are two guest molecules of TATM somewhat related in that their respective dynamic characteristics are similar. The ${ }^{2} \mathrm{H}$ NMR spectra, along with simulations, for acetonitrile- $d_{3} /$ TATM and nitromethane- $d_{3} /$ TATM are 
depicted in Figures III-24 and III-25 respectively. From the observed spectral averaging (i.e. the spectral width) it is clearly evident that they both exhibit internal methyl $-\mathrm{CH}_{3}$ rotation at rates greater than $10^{8} \mathrm{~Hz}$, down to $173 \mathrm{~K}$.

Experimental
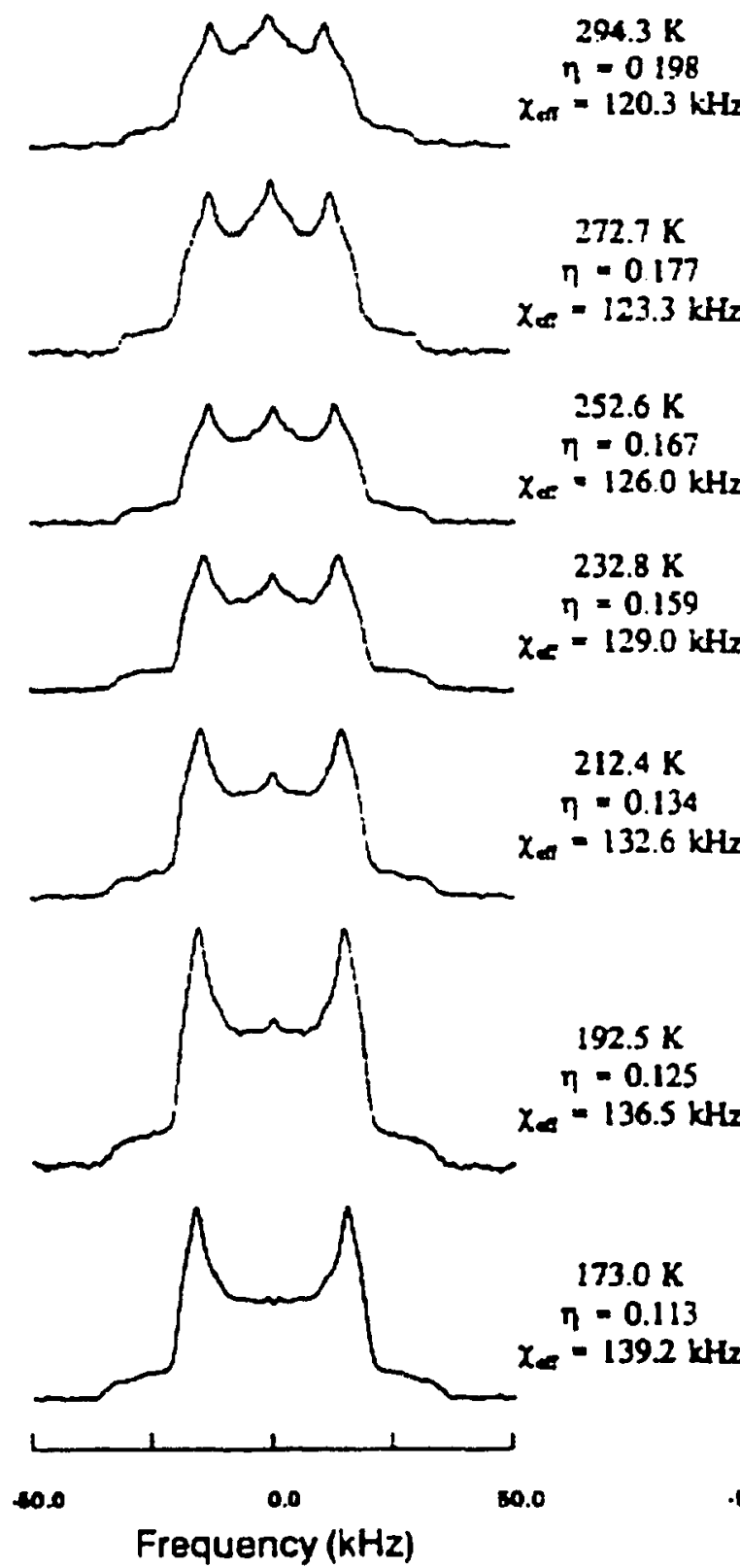

$232.8 \mathrm{~K}$

$\eta=0.159$ $x_{<}=129.0 \mathrm{kHz}$

$\eta=0.134$ $x_{\text {eat }}=132.6 \mathrm{kHz}$

$192.5 \mathrm{~K}$

$\eta=0.125$ $x_{4}=136.5 \mathrm{kHz}$

$173.0 \mathrm{~K}$

$\eta=0.113$ $x=-139.2 \mathrm{kHz}$

0
Simulated
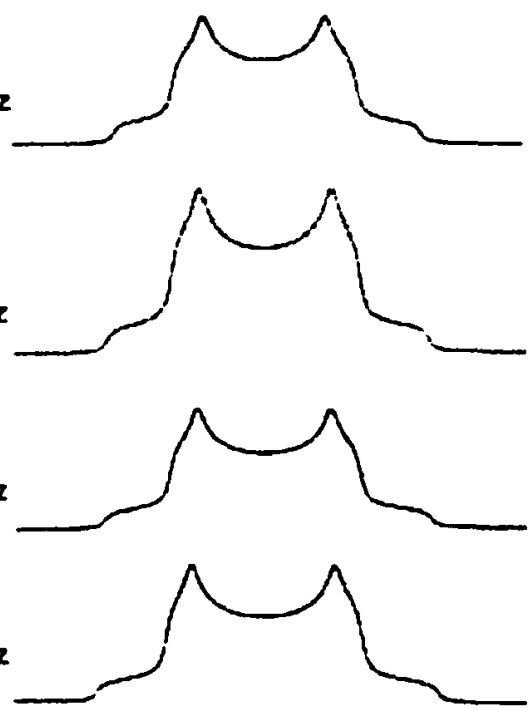

$212.4 \mathrm{~K}$
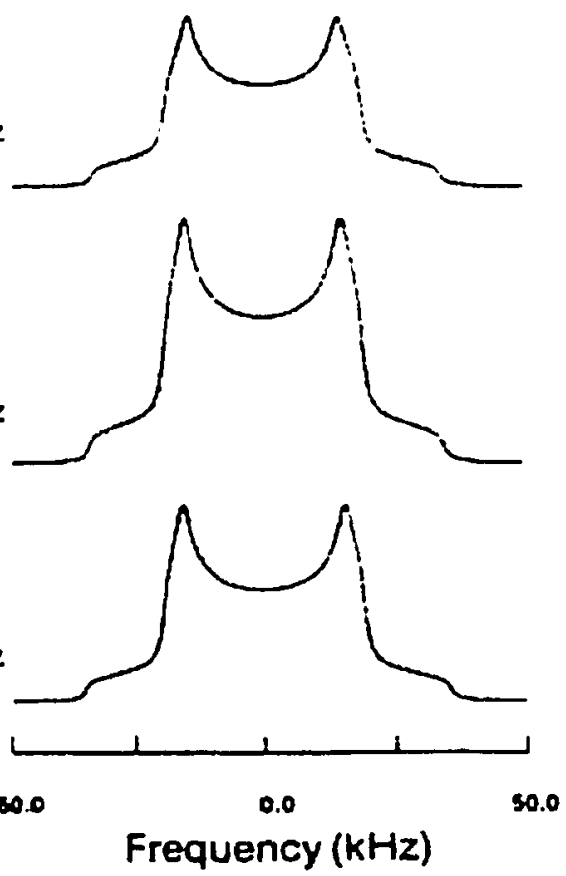

Figure 111-24: Deuterium NMR spectra for the acetonitrile- $\mathrm{d}_{3}$ / TATM inclusion compound at various temperatures, along with simulations [36]. 
Experimental
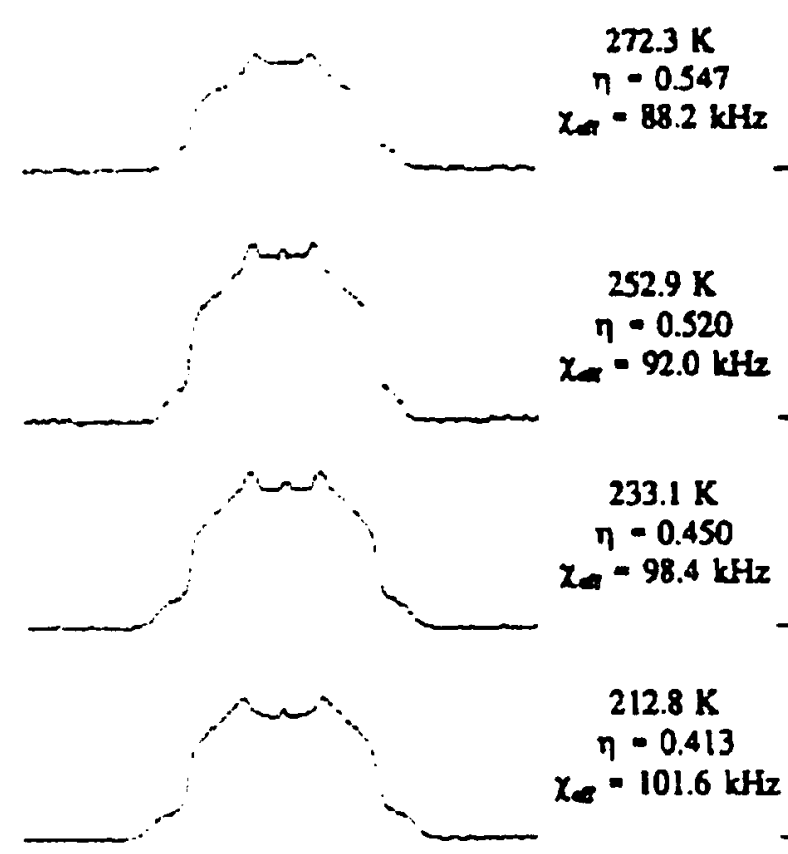

$193.1 \mathrm{~K}$

$\eta=0.330$

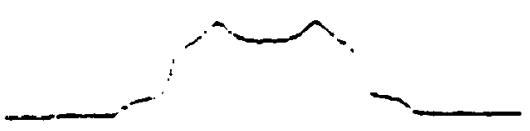

$x_{0 .}-108.8 \mathrm{kHz}$
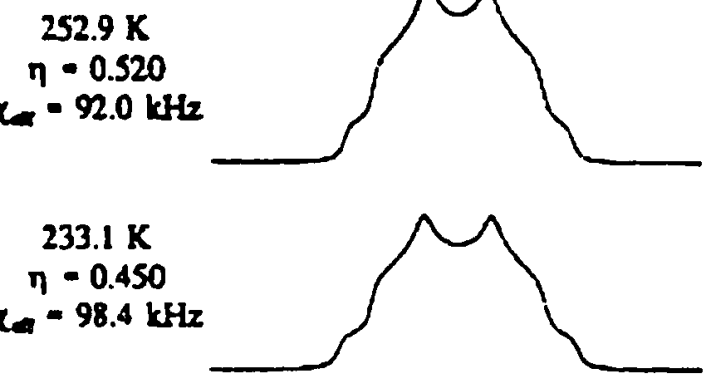

$x=92.0 \mathrm{kHz}$

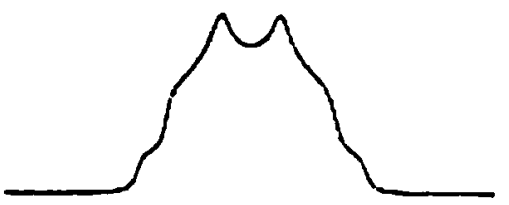

Simulated
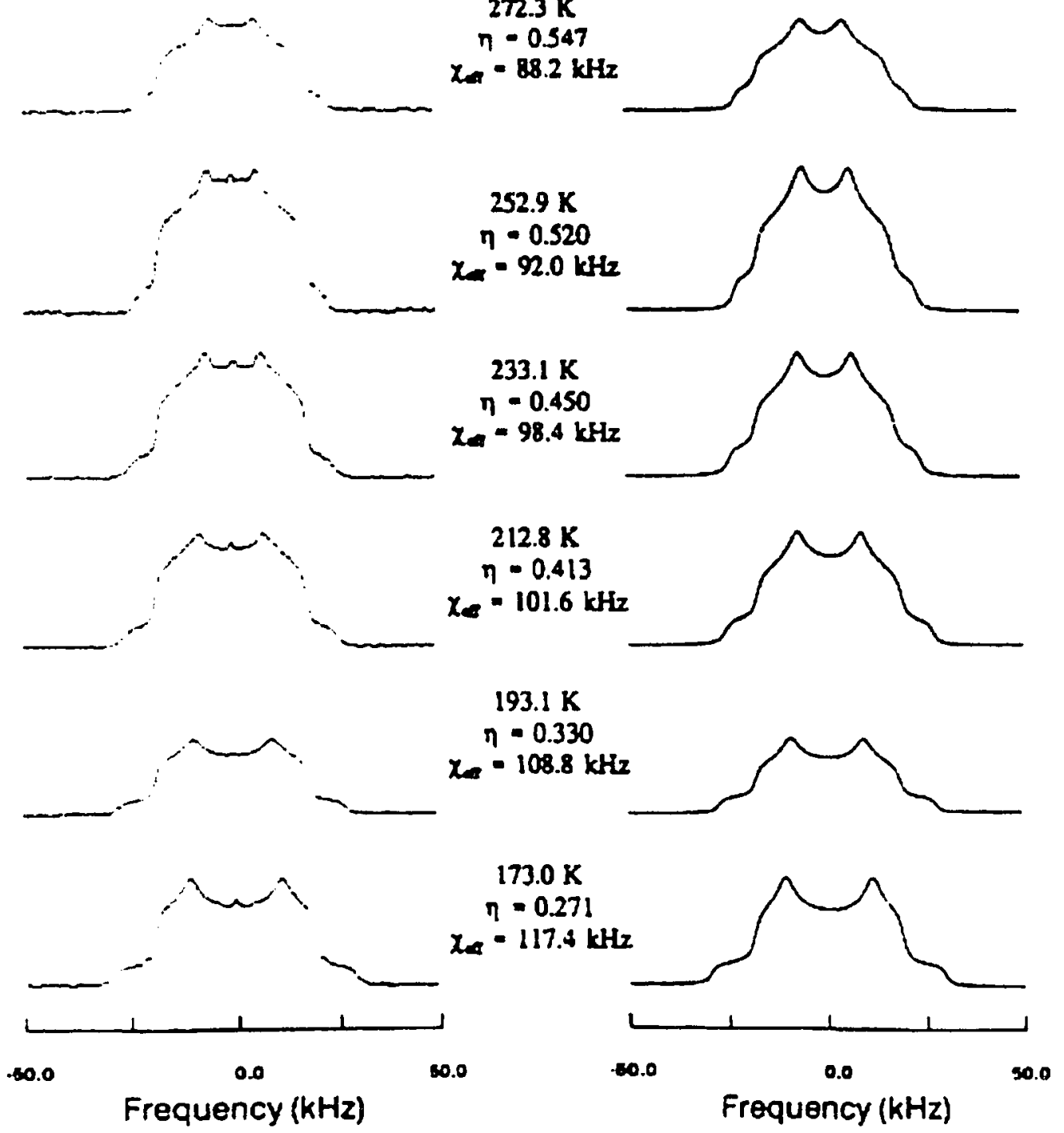

Figure III-25: Deuterium NMR spectra for the nitromethane- $d_{3} /$ TATM inclusion $^{2}$ compound at various temperatures, along with simulations [36].

The other type of common motion is precession of the principal molecular symmetry axis, [32] which affects both $\chi(\mathrm{QCC})$ and $\eta$ (the asymmetry parameter). Although the precessional motion is best described as diffusion of the principal axis within a cone, where the possible angles, $\psi$, are governed by a distribution function $\mathrm{g}(\psi)$, a simpler model was used, in which the molecule is allowed to precess on a cone of half-angle $\theta$. 
The authors noted that many guest molecules in inclusion compounds undergo this type of precessional motion. Actually, a more realistic description of this reorientation would be a small-amplitude whole-body librational motion, but the model of precession employed by the authors gives a simple analytical model. The precession can be thought of as a rotation of the principal symmetry axis on a cone, the base of which is either circular or elliptical. This type of motion normally has a very small activation energy, and the rate of reorientation is usually of the same order as the rate of vibrational motion of a molecule inside a potential energy well, $\sim 10^{12} \mathrm{~Hz}$.

The motion can be broken down into two components, precession on a circular cone (corresponding to an angle $\theta$ ), and a two-site libration (corresponding to an angle $\phi$ ), which transforms the circular base cone into an elliptical cone (see Scheme 1) [32|. For an elliptical cone, the precession leads to a non-zero asymmetry parameter, since it is motion in a preferred direction.

\section{Scheme 1.}

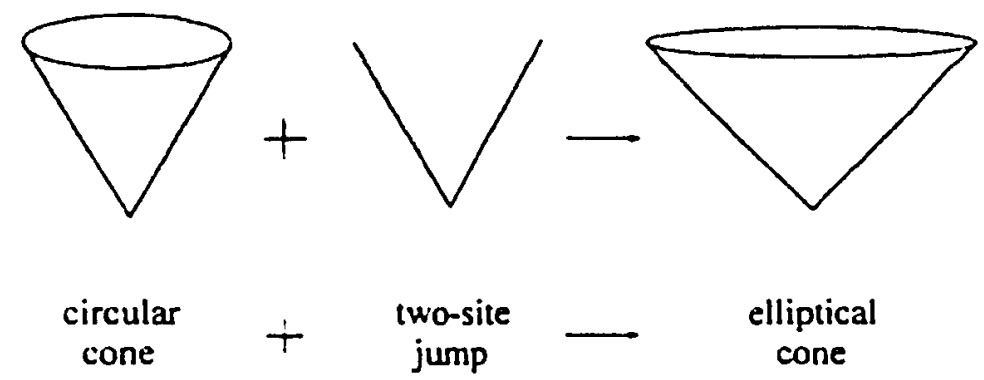

The simulations of Figures III-24 and III-25 were calculated by allowing the guest molecule to reorient between two sites $\phi$ degrees apart. On top of this, the circular cone wobble can be incorporated by simply using a motionally averaged effective quadrupolar coupling constant $\left(\chi_{\mathrm{ertr}}\right)$, and calculating the angle $\theta$ from $\chi_{\mathrm{etr}} / \chi_{\text {static }}=1 / 2\left(3 \cos ^{2} \theta-1\right)$. Rather than reporting the angles $\theta$ and $\phi$ for each spectrum in Figures III-24 and III-25, the authors chose instead to present values of the spectral asymmetry $\eta$ (dependent on $\phi$ ) and the effective quadrupolar coupling constant $\chi_{\text {enr }}$ (inversely dependent on $\theta$ ), as these parameters can be easily measured from each spectrum. Thus, the angles $\theta$ and $\phi$ at each temperature may easily be extracted from each simulation. In Figure III-26 is presented a 
plot of the effective asymmetry parameter and the effective quadrupolar coupling constant for each guest as a function of temperature.

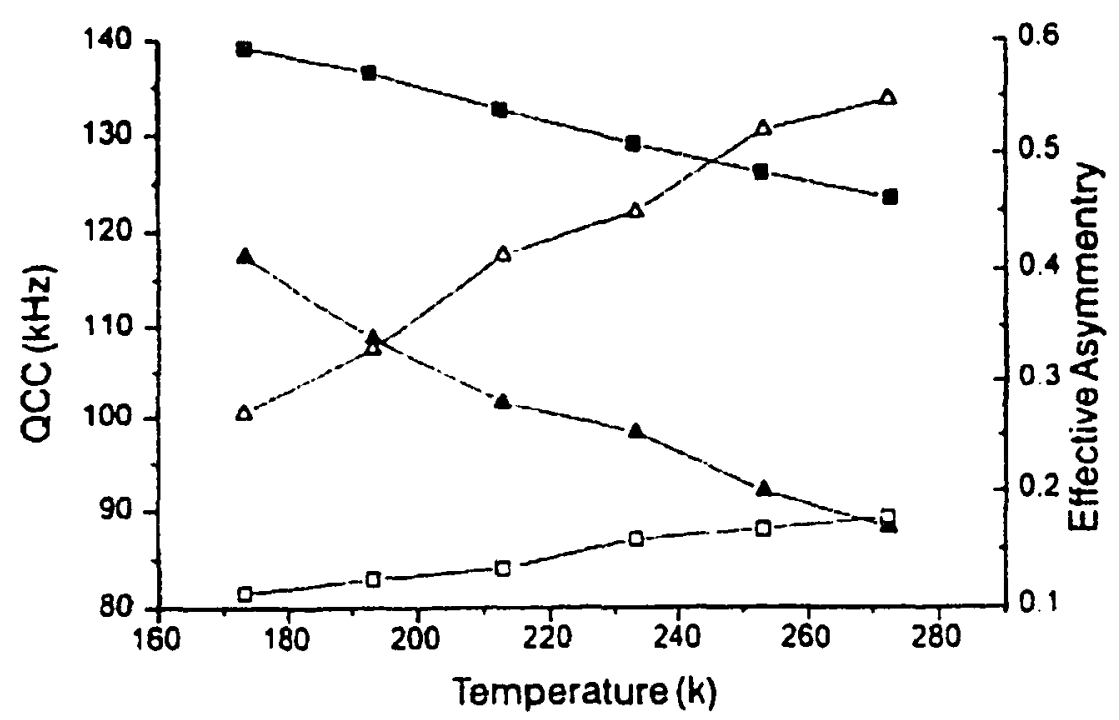

Figure III-26: Graph of the spectral asymmetry ( $\eta_{\mathrm{etf}}$ - right axis) and the effective QCC $\left(\chi_{\mathrm{etr}}-\right.$ left axis), as a function of temperature for the TATM guests acetonitrile and nitromethane. Legend: $\eta$ (acetonitrile) open square; $\chi$ (acetonitrile) filled square; $\eta$ (nitromethane) open triangle; $\chi$ (nitromethane) filled triangle [36].

A number of trends are evident from Figure III-26. Firstly, $\eta_{\text {etr }}$ for nitromethane- $d_{3} /$ TATM is significantly greater than that for acetonitrile- $\mathrm{d}_{3} /$ TATM at all temperatures studied. This suggests that the cone on which nitromethane- $d_{3}$ precesses is much more elliptical than that for acetonitrile- $\mathrm{d}_{3}$ (see Scheme 2). This is to be expected, based on a comparison of their molecular symmetries. Nitromethane would likely prefer to librate parallel to the $\mathrm{NO}_{2}$ plane, since this type of libration minimizes the motion of the oxygen atoms, compared to a libration in the opposite direction (perpendicular to the $\mathrm{NO}_{2}$ plane). Acetonitrile, with its axial symmetry, should show no preferred precessional direction. Perhaps the reason that the ${ }^{2} H$ NMR spectra of acetonitrile- $d_{3} /$ TATM show a non-zero asymmetry may be due to a non-uniform interaction with a TATM cavity of low symmetry. 
Scheme 2:

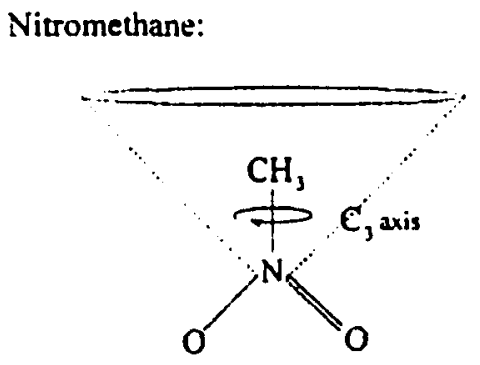

2
Acetonitrile:

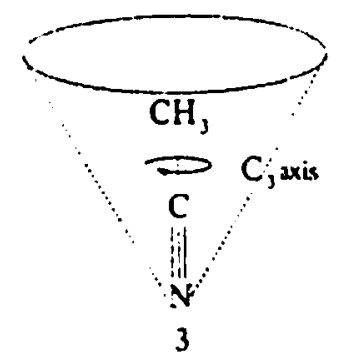

As far as $\chi_{\text {etr }}$ is concerned, Figure III-26 shows that $\chi_{\text {eff }}$ is much greater for acetonitrile- $\mathrm{d}_{3}$ / TATM than for nitromethane- $d_{3}$ / TATM at all temperatures investigated. This is a surprising result, as one would have expected acetonitrile, being a smaller guest molecule, to show more motional averaging (i.e. a smaller $\chi_{\text {err }}$ ) than the nitromethane guest. Therefore, this trend may instead be due to the size, or symmetry, of the respective host cavities. In the TATM crystal structures available at the time, the authors concluded that the shape of the guest cavities show substantial variations in size and shape 17-10, 15-17]. If the TATM cavity that encloses nitromethane is larger than that to enclose acetonitrile, nitromethane would be more easily able to engage in large-amplitude librational motions. This would spectrally manifest itself as a smaller $\chi_{\text {eff }}$ for nitromethane, which is experimentally observed.

The ${ }^{2} \mathrm{H}$ NMR powder spectra of dimethyl sulfoxide- $\mathrm{d}_{6}\left(\right.$ DMSO- $\mathrm{d}_{6}$ ) in its TATM inclusion compound are presented in Figure III-27, along with simulations. The spectrum at room temperature (not shown) is a Pake doublet with an effective QCC of $51.3 \mathrm{kHz}$, signifying fast methyl rotation. The ${ }^{2} \mathrm{H}$ lineshape does not change to any degree from room temperature to $143 \mathrm{~K}$, which implies that the motion giving rise to the lineshape changes below $143 \mathrm{~K}$ is internal $C_{3}$ rotation of the methyl groups of the guest (methyl rotation). Indeed, the ${ }^{2} \mathrm{H}$ NMR spectra in Figure III-27 were simulated on the basis of this model. The rate of methyl rotation at each temperature is indicated along with each spectrum in Figure III-27. It should be noted that the authors were only able to obtain ${ }^{2} \mathrm{H}$ spectra down to $97 \mathrm{~K}\left(k=1.2 \times 10^{6} \mathrm{~Hz}\right)$. Below this temperature, in the intermediate exchange regime, the intensity of the echo becomes very weak, as the effective transverse relaxation time $\left(T_{2 \mathrm{efr}}\right)$ approaches the magnitude of the interpulse spacing $(50 \mu \mathrm{s})$ in the quadrupole echo pulse sequence. This precluded the acquisition of spectra below $97 \mathrm{~K}$. 
Experimental

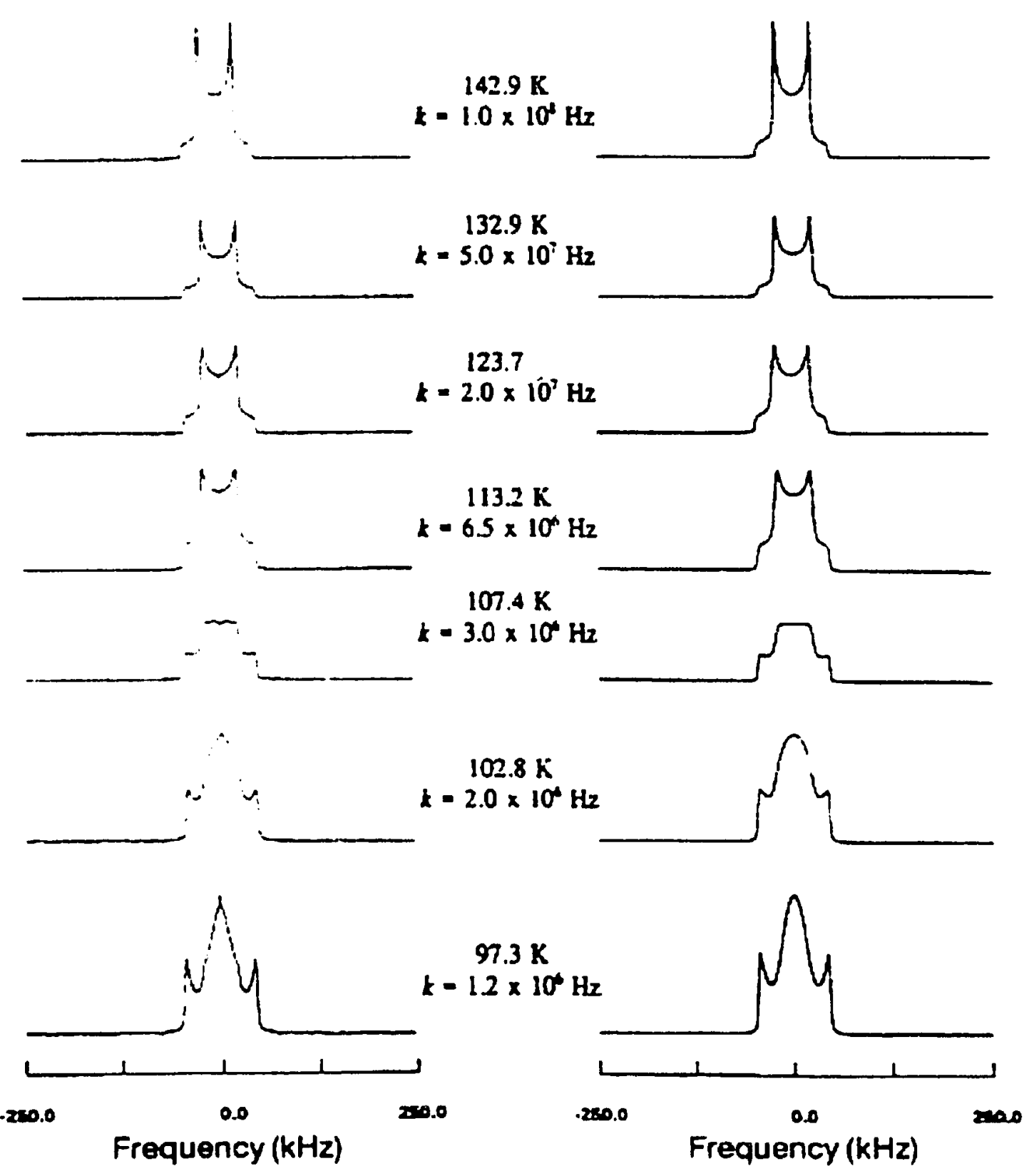

Figure III-27: Experimental and simulated ${ }^{2} \mathrm{H}$ NMR spectra for dimethyl sulfoxide- $\mathrm{d}_{6}$ in its TATM inclusion compound, at the temperatures indicated. The rate of internal methyl rotation at each temperature is also given [36]. 
By plotting ln $k$ vs $1000 / T$ (see Figure III-28), an activation barrier to methyl rotation was calculated to be $11.7( \pm 0.4) \mathrm{kJ} \mathrm{mol}^{-1}$. This compares to an activation energy of $12.0 \mathrm{~kJ} \mathrm{~mol}^{-1}$ determined for DMSO in the gas phase [37]. In the solid-state of pure DMSO, the two methyl groups are crystallographically and dynamically inequivalent [38]. It was determined (from variable temperature proton spin-lattice relaxation times) that the activation energy for methyl rotation of one methyl group was $13.3 \mathrm{~kJ} \mathrm{~mol}^{-1}$ $\left(\tau_{\infty}=5.6 \times 10^{-13} \mathrm{~s}\right)$, while the other methyl group had a significantly higher activation barrier of $21.7 \mathrm{~kJ} \mathrm{~mol}^{-1}\left(\tau_{\infty}=6.8 \times 10^{-14} \mathrm{~s}\right)[39]$. In the TATM inclusion compound and in the gas phase, the activation energy is determined primarily by intramolecular interactions, whereas in the pure solid the reorientation of one of the methyl groups must be significantly hindered by intermolecular interactions.

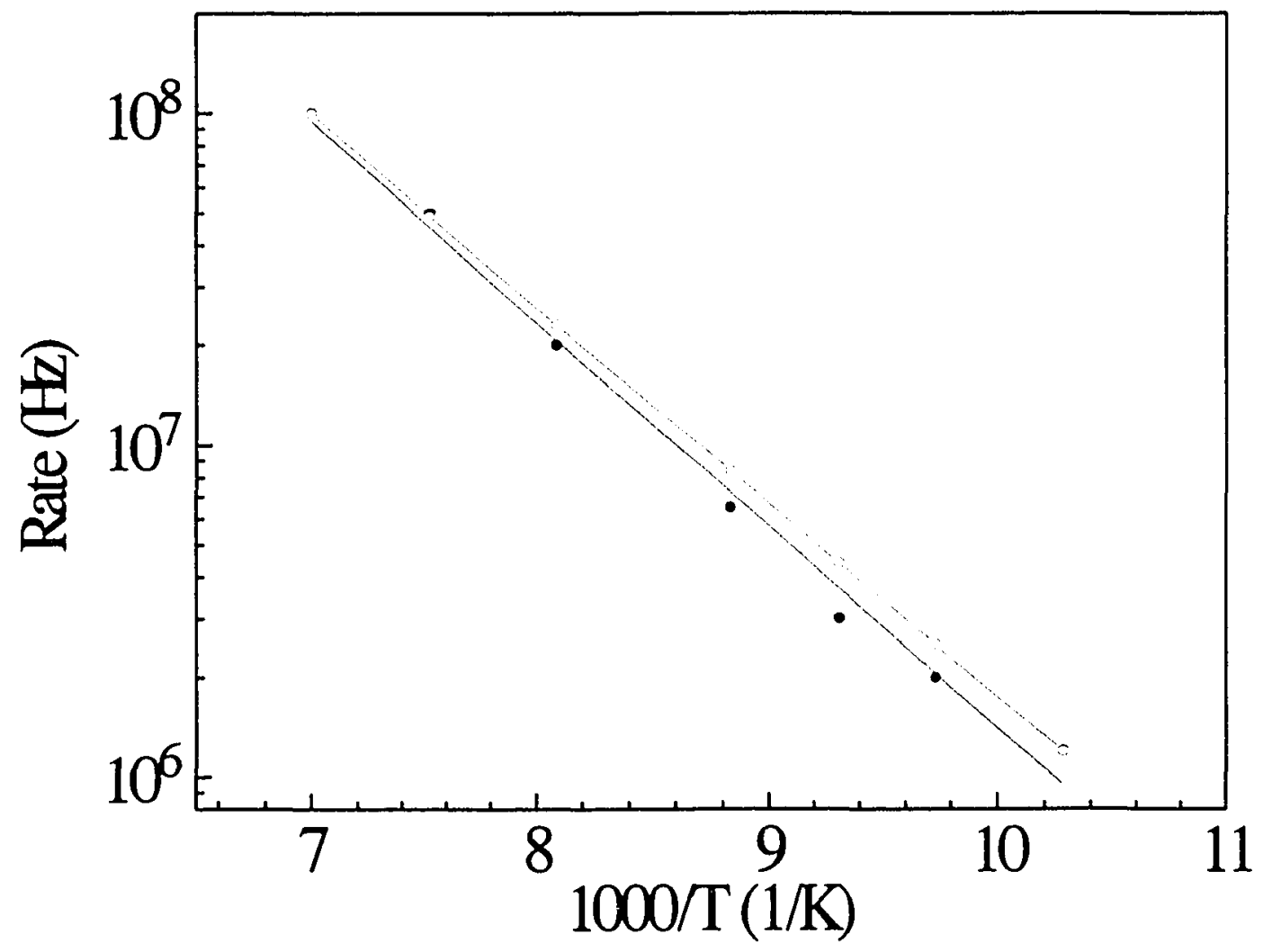

Figure III-28: A plot of the rate of internal methyl rotation for the DMSO- $\mathrm{d}_{6}$ guest in TATM as a function of inverse temperature. Legend: blue line - ${ }^{2} \mathrm{H}$ NMR spectra; red line $-{ }^{2} \mathrm{H}$ spin-lattice relaxation times [36]. 
In addition, the authors noted that the spectrum at $143 \mathrm{~K}$ has an effective asymmetry parameter $\eta \sim 0$ (see Figure III-27). This implies that the methyl groups are not distorted from tetrahedral geometry, in contrast to acetone- $\mathrm{d}_{6} /$ TATM, [23] where the dihedral angle between two of the deuterons goes from $120^{\circ}$ to $124^{\circ}$, which gives rise to a non-zero asymmetry $\eta \sim 0.14$ at $84 \mathrm{~K}$, at which temperature internal methyl rotation is in the fast rate limit $\left(k>10^{8} \mathrm{~Hz}\right)$.

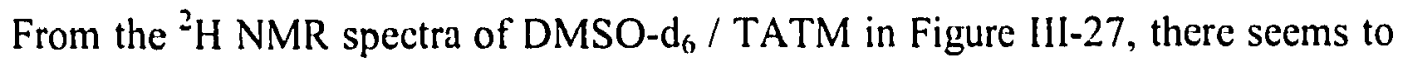
be no motion (other than internal methyl rotation at low temperatures) that is causing modulation of the lineshape. Again, this is in contrast to acetone- $d_{6}$ in the same host, [23] in which the guest was observed to undergo two-fold flips about the carbonyl bond. The lack of a similar motion in DMSO may be due to the fact that DMSO is much larger than acetone. Thus, DMSO would likely interact more strongly with the TATM lattice, and be more restricted in its motion.

The variable temperature ${ }^{2} \mathrm{H} T_{1}$ data for DMSO- $\mathrm{d}_{6} /$ TATM appears in Figure III29. Only one $T_{1}$ minimum is observed, due to internal rotation of the methyl groups. Proof of this statement comes from a consideration of the activation energy determined from the best-fit calculated curve (also in Figure III-29), $E_{\mathrm{a}}=11.2( \pm 0.5) \mathrm{kJ} \mathrm{mol}^{-1}$, which may be compared with $11.7( \pm 0.4) \mathrm{kJ} \mathrm{mol}^{-1}$ determined from the ${ }^{2} \mathrm{H}$ NMR spectral simulations (see above). The correlation time at infinite temperature $\left(\tau_{x}\right)$ is $2.7 \times 10^{-13} \mathrm{~s}$, and the constant $K$ (see Equation III-1 above) is about $8 \times 10^{10} \mathrm{~s}^{-2}$.

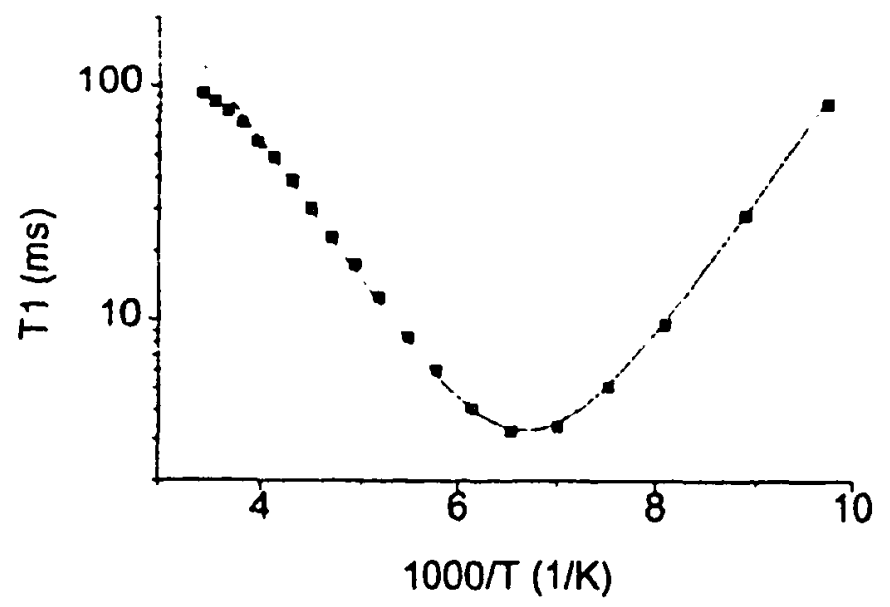

Figure III-29: Deuterium spinlattice relaxation times, as a function of reciprocal temperature, for DMSO-d $\mathrm{d}_{6} /$ TATM [36]. 
It is possible to derive explicit expressions for ${ }^{2} \mathrm{H} T_{1}$ and the $T_{1}$ minimum value by considering a situation in which a C-D bond can jump between three equivalent sites. The expression for $T_{1}$ is: [31]

$$
\frac{1}{T_{1}}=\left(\frac{\omega_{Q}^{2}}{8}\right)\left\{g\left(\tau_{c}, \omega_{0}\right)\left[A_{1} B_{4}+A_{2} B_{5}-8 A_{3} B_{3} \cos 3 \phi\right]+g\left(\tau_{c}, 2 \omega_{0}\right)\left[4 A_{1} B_{5}+A_{2} B_{6}+8 A_{3} B_{3} \cos 3 \phi\right]\right\}
$$

where $\tau_{\mathrm{c}}=(3 k)^{-1}, \omega_{0}$ is the deuterium Larmor precession frequency, and $\omega_{Q}=3 / 4 \chi$. The angles $\theta$ and $\phi$ describe the orientation of the static magnetic field in the crystal-fixed axis system. The $C-D$ bond makes an angle $\Theta$ with respect to the $C_{3}$ rotational axis. The angular functions $A_{\mathrm{i}}$ and $B_{\mathrm{i}}$ depend on $\theta$ and $\Theta$, and are given in reference [31]. It is the $\cos 3 \phi$ term in Equation III-8 that, in principle, leads to non-exponential recovery of the magnetization. However, this effect is small, and it is reasonable to average over the angles $\theta$ and $\phi$. The angle $\Theta$ is set to $109.5^{\circ}$, the tetrahedral angle. This leads to:

$$
\frac{1}{T_{1}}=\frac{\chi^{2}}{15}\left[\frac{\tau_{c}}{1+\omega_{0}^{2} \tau_{c}^{2}}+\frac{4 \tau_{c}}{1+4 \omega_{0}^{2} \tau_{c}^{2}}\right]
$$

Therefore, the constant $K$ in Equation III-l (above) is given by $\chi^{2} / 15$. Using the value of $K$ from the ${ }^{2} \mathrm{H} T_{1}$ fit $\left(8 \times 10^{10} \mathrm{~s}^{-2}\right)$, the quadrupolar coupling constant $\chi$ should be about $174 \mathrm{kHz}$ (for $\Theta=109.5^{\circ}$ ). If the angle between the C-D bond and the three-fold axis is increased to $111.5^{\circ}$, the calculated value for $\chi$ would increase to approximately $178 \mathrm{kHz}$, in good agreement with the experimental value, determined from the splitting of the ${ }^{2} \mathrm{H}$ NMR spectrum, as $178.2 \mathrm{kHz}$. This compares with a static ${ }^{2} \mathrm{H}$ QCC of $177 \mathrm{kHz}$ determined for DMSO- $\mathrm{d}_{6}$ in the DMSO / kaolinite intercalation compound, obtained from ${ }^{2} \mathrm{H}$ solid-state NMR spectra [40].

In addition, the value of the $T_{1}$ minimum is given by: [41]

$$
\frac{1}{T_{1 \min }}=\frac{1.42}{15} \times \frac{\chi^{2}}{\omega_{0}}
$$

As noted by the authors, if one uses the experimental value of $\chi=178.2 \mathrm{kHz}$, and the ${ }^{2} \mathrm{H}$ Larmor frequency of $44.667 \mathrm{kHz}$, the value of $T_{1}$ at the minimum should be about $2.2 \mathrm{~ms}$. This compares with an experimental value for $T_{1 \min }$ of $3.2 \mathrm{~ms}$ at about $148 \mathrm{~K}$. The 
DMSO- $\mathrm{d}_{6}$ guest in the kaolinite intercalate was found to go through a $T_{1}$ minimum of about $7.2 \mathrm{~ms}$, around $205 \mathrm{~K} \mathrm{[40]}$.

The authors noted that it is interesting to compare the motion of DMSO- $\mathrm{d}_{6}$ in TATM with that of pure dimethylsulfone, $\mathrm{SO}_{2}\left(\mathrm{CH}_{3}\right)_{2}$. It is known that dimethylsulfone, with its tetrahedral geometry, undergoes jumps about its $C_{2}$ principal axis to exchange the two oxygens and the two methyl groups [42]. In comparison, DMSO is a molecule of much lower symmetry $\left(C_{\mathrm{s}}\right)$. It has no proper axis of symmetry about which to reorient. This is accompanied by a lack of motion, other than internal methyl rotation, at all temperatures investigated. The activation energy for methyl rotation in pure dimethyl sulfone was measured from variable temperature ${ }^{~} \mathrm{H}$ spin-lattice relaxation times to be $17.0 \mathrm{~kJ} \mathrm{~mol}^{-1}\left(\tau_{s}=1.5 \times 10^{-13} \mathrm{~s}\right)[43]$.

The room temperature ${ }^{2} H$ NMR spectrum of benzene- $d_{6}$ in TATM, as depicted in Figure III-30, indicates that the benzene guest molecules are likely performing in-plane $60^{\circ}$ jumps about the molecular $C_{6}$ axis. This type of reorientation results in a ${ }^{2} \mathrm{H}$ spectrum that is $50 \%$ of the width of the static spectrum (i.c. when $\theta=90^{\circ}, 1 / 2\left(3 \cos ^{2} \theta-1\right)$ $=0.50$ ), with no change in lineshape (axially symmetric motion). The appearance of the spectrum does not change from room temperature down to $113 \mathrm{~K}$, suggesting that the sixfold reorientation is proceeding at a rate greater than $10^{8} \mathrm{~Hz}$ to the lowest temperature measured. The fractional populations of the six sites are equal, as they must be by the symmetry of the guest molecule. However, $\chi_{\text {err }}$ is $86.8 \mathrm{kHz}$ at room temperature and 92.9 $\mathrm{kHz}$ at $113 \mathrm{~K}$. This suggests that the benzene molecules are engaged in small-amplitude librational motions while in the TATM cavity, to cause further spectral averaging on top of the $C_{6}$ reorientation. The experimental value of $\chi_{\text {static }}$ at $113 \mathrm{~K}$ is $185.8 \mathrm{kHz}$, which is typical for a static aromatic deuteron. The value of $\chi_{\text {static }}$ as a function of inverse temperature is depicted in Figure III-31 (circles). At low temperatures (high inverse temperature), the graph is becoming flat, suggesting that the librational motion is being frozen out. 


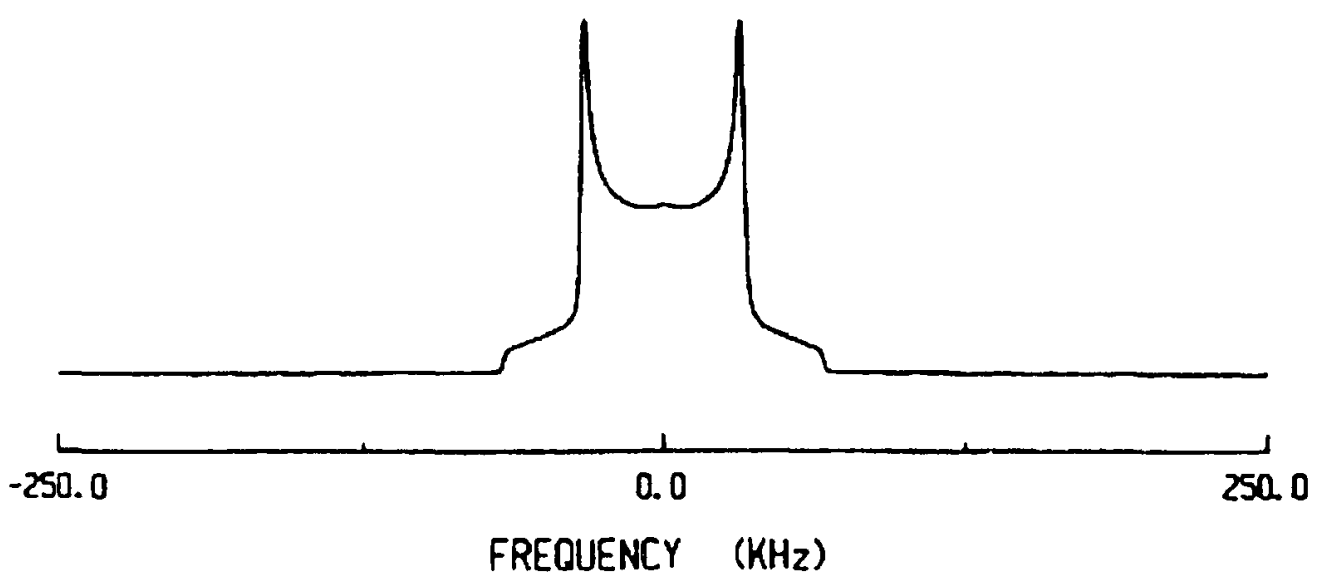

Figure III-30: Deuterium NMR spectrum of benzene- $\mathrm{d}_{6} /$ TATM at $294 \mathrm{~K}$ [36].

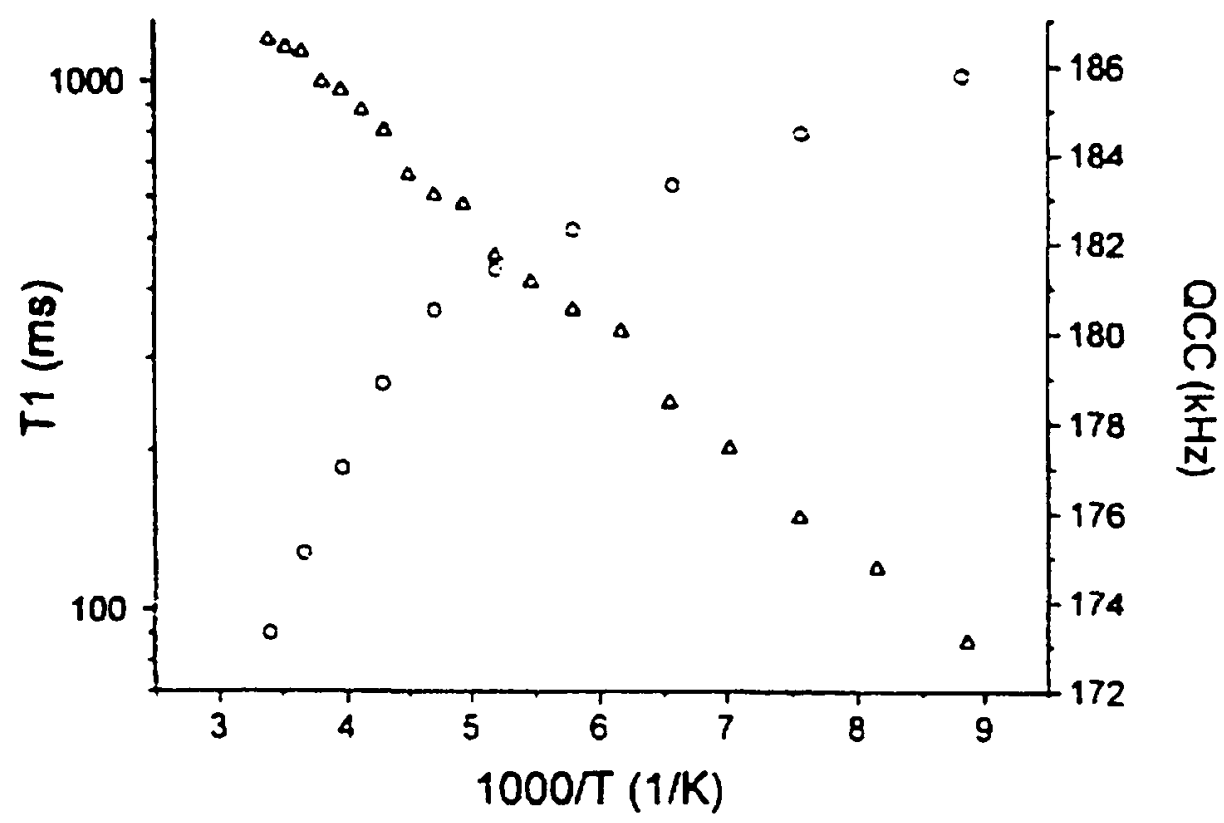

Figure III-31: A graph of ${ }^{2} \mathrm{H}$ spin-lattice relaxation time for benzene- $\mathrm{d}_{6} / \mathrm{TATM}$ (triangles) and effective QCC (circles) as a function of 1000/T [36].

The ${ }^{2} \mathrm{H}$ spin-lattice relaxation times for benzene- $\mathrm{d}_{6} /$ TATM also appear in Figure III-3I (triangles). Notice that the $T_{1}$ curve does not reach a minimum down to the lowest temperature measured. The motion modulating ${ }^{2} \mathrm{H} T_{1}$ is always in the extreme narrowing limit (i.e. $\omega_{0} \tau_{c} \ll<1$ ). This agrees with the ${ }^{2} \mathrm{H}$ NMR spectra, as it indicates that the rate of six-fold rotation is significantly larger than the ${ }^{2} \mathrm{H}$ Larmor precession frequency $\left(\sim 4 \times 10^{7}\right.$ $\mathrm{Hz}$ ) down to $115 \mathrm{~K}$. The lack of a complete $T_{1}$ minimum precluded the authors from 
performing a complete $T_{1}$ fit, however the slope of the curve in Figure III-31 gave an activation energy of $4.1( \pm 0.4) \mathrm{kJ} \mathrm{mol}^{-1}$ for the $C_{6}$ rotation of benzene- $\mathrm{d}_{6}$ in TATM. This compares with an activation energy of $16.5 \mathrm{~kJ} \mathrm{~mol}^{-1}[44]$ and $16.8 \mathrm{~kJ} \mathrm{~mol}^{-1}$ [45] for pure solid benzene, determined from ${ }^{2} \mathrm{H}$ powder NMR and $T_{1}$ relaxation time measurements, respectively. When benzene is in an inclusion compound, $E_{\mathrm{a}}$ for $C_{6}$ rotation varies from $4.0 \mathrm{~kJ} \mathrm{~mol}^{-1}$ in the tri-ortho-thymotide clathrate, [46] to $24.9 \mathrm{~kJ} \mathrm{~mol}^{-1}$ in 1,3cyclohexanedione [44]. As a consequence of the very low activation barrier for $C_{6}$ rotation of benzene- $d_{6}$ in TATM, the authors were unable to slow the reorientation rate below $10^{8} \mathrm{~Hz}$ even down to $113 \mathrm{~K}$.

The ${ }^{2} \mathrm{H}$ NMR spectra of mesitylene-d, in TATM (1,3,5-trimethylbenzene-d, deuterated only on the methyl groups) appear in Figure III-32. One might expect that mesitylene would reorient by $120^{\circ}$ in-plane rotational jumps to exchange the methyl groups about the molecular $C_{3}$ axis. However, this type of motion (or $60^{\circ}$ jumps for that matter) leads to simulations that do not match the experimental spectra at intermediate rates $\left(10^{4}-10^{6} \mathrm{~Hz}\right)$.

An alternative model that may be considered is that there are two dynamically distinct types of mesitylene molecules. One type is performing $C_{3}$ rotation at a rate that is fast on the ${ }^{2} \mathrm{H}$ NMR spectral timescale $\left(k>10^{8} \mathrm{~Hz}\right.$ ), and the other type is rotating slowly $\left(k<10^{3} \mathrm{~Hz}\right)$. In this model, there are no mesitylene guest molecules in the intermediate exchange region. This model would require the existence of two different sites in the TATM lattice. The proportion of these two sites (and hence the ratio of the two dynamically different types of mesitylene guest molecules) is changing with temperature. At high temperatures, most of the mesitylene molecules are reorienting rapidly, while at low temperatures, the majority are in the slow exchange limit. The percentage of guest molecules rotating rapidly $\left(P_{1}\right)$ and the percentage rotating slowly $\left(P_{2}\right)$, at each temperature, are indicated with the simulations in Figure III-32. 
Experimental

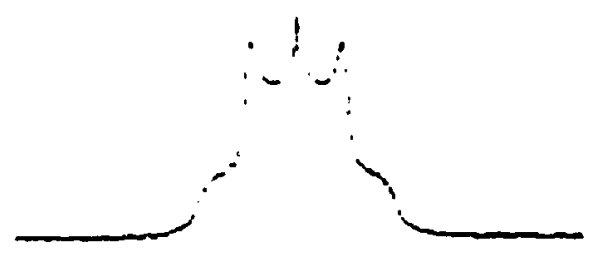

$294.5 \mathrm{~K}$

$P .=100 \%$

$P_{2}=0 \%$

$272.3 \mathrm{~K}$

$P_{1}=70 \%$

$P:=30 \%$

$253.1 \mathrm{~K}$

$P_{1}=57 x$

$P_{2}=43$ \%

$233.1 \mathrm{~K}$

$P_{1}=40 \%$

$P_{:}=60 \%$

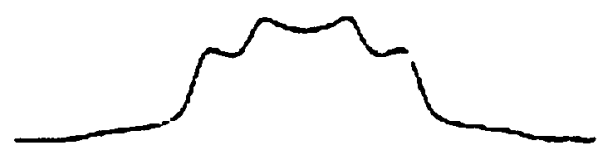

$232.3 \mathrm{~K}$

$P_{1}=21 \%$

$P_{2}=79 \%$

$192.5 \mathrm{~K}$

$P_{1}=10 \%$

$P_{2}=90 \%$

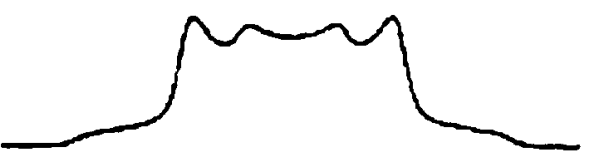

$176.7 \mathrm{~K}$

$P_{1}=5 \%$

$P_{2}=95 \%$

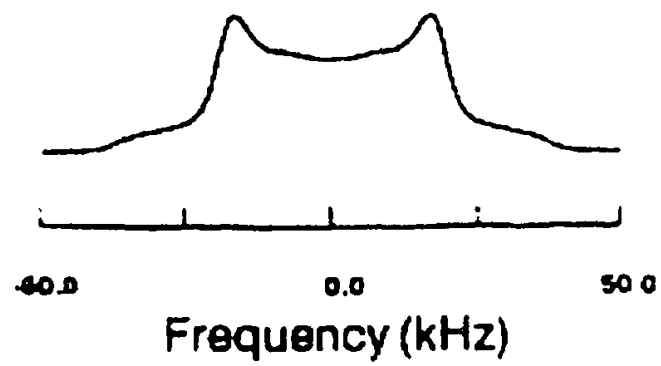

Figure III-32: Simulated and experimental ${ }^{2} \mathrm{H}$ NMR spectra for mesitylene-d, in TATM at the temperatures indicated, along with percentage populations of fast moving $\left(P_{1}\right)$ and slow moving $\left(P_{2}\right)$ mesitylene molecules [36]. 
However, the authors noted that this model is only the simplest one to consider. On the other hand, there may be a continuous distribution of $C_{3}$ jump rates. The mesitylene guest molecules that are reorienting at rates between about $10^{4} \mathrm{~Hz}$ and $10^{6} \mathrm{~Hz}$ would contribute very little to the overall spectral intensity, when compared to guest molecules rotating rapidly or slowly, due to the fact that the echo intensity is attenuated considerably for molecules in the intermediate exchange regime. This model is physically more reasonable, since small differences in the guest-host interaction from one cavity to the next could result in a distribution of motional rates. In Figure III-33, the normalized echo intensity is plotted as a function of jump rate, calculated for in-plane $120^{\circ}$ jumps of the mesitylene guest molecule. It is seen from this figure that the echo (and thus spectral) intensity, at a jump rate of $5 \times 10^{4} \mathrm{~Hz}$, falls to only $15 \%$ of that at very fast $C_{3}$ reorientational rates. Therefore, there would be very little contribution to the overall spectral intensity for molecules rotating at intermediate rates, which is exactly where the agreement between simulated and experimental spectra is at its weakest.

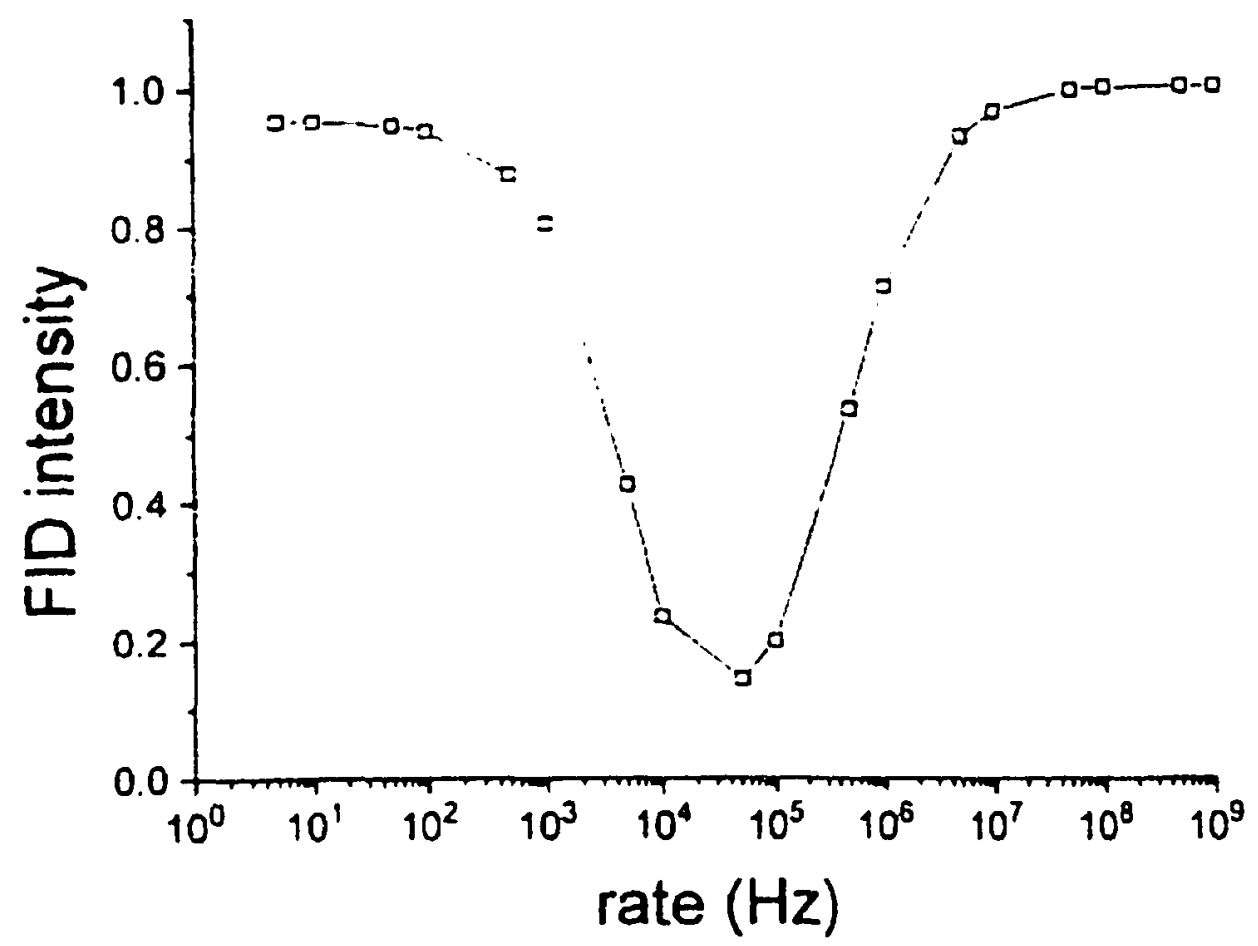

Figure III-33: Plot of normalized echo intensity, as a function of jump rate, calculated for in-plane $C_{3}$ rotation of a mesitylene molecule [36]. 
To investigate this matter further, the authors present in Figure III-34 spectral simulations based on equal populations of mesitylene molecules over five equally spaced rates, with the distribution slowly changing from faster rates to slower rates (corresponding to a decrease in temperature). Although the populations of the five rates in each simulation are equal, the contributing rates have been weighted according to the attenuation of the echo intensity at intermediate rates (see Figure III-33). By comparing the simulations of Figure III-34 with the experimental ${ }^{2} \mathrm{H}$ NMR spectra of mesitylene- $\mathrm{d}_{9}$ / TATM in Figure III-32, it can be seen that the agreement is quite good. This model is somewhat more elaborate than the first one described above, but it is even more realistic to consider the population distribution as Gausssian, rather than rectangular. However, there are simply too many variables to consider a detailed lineshape fit with this scheme.

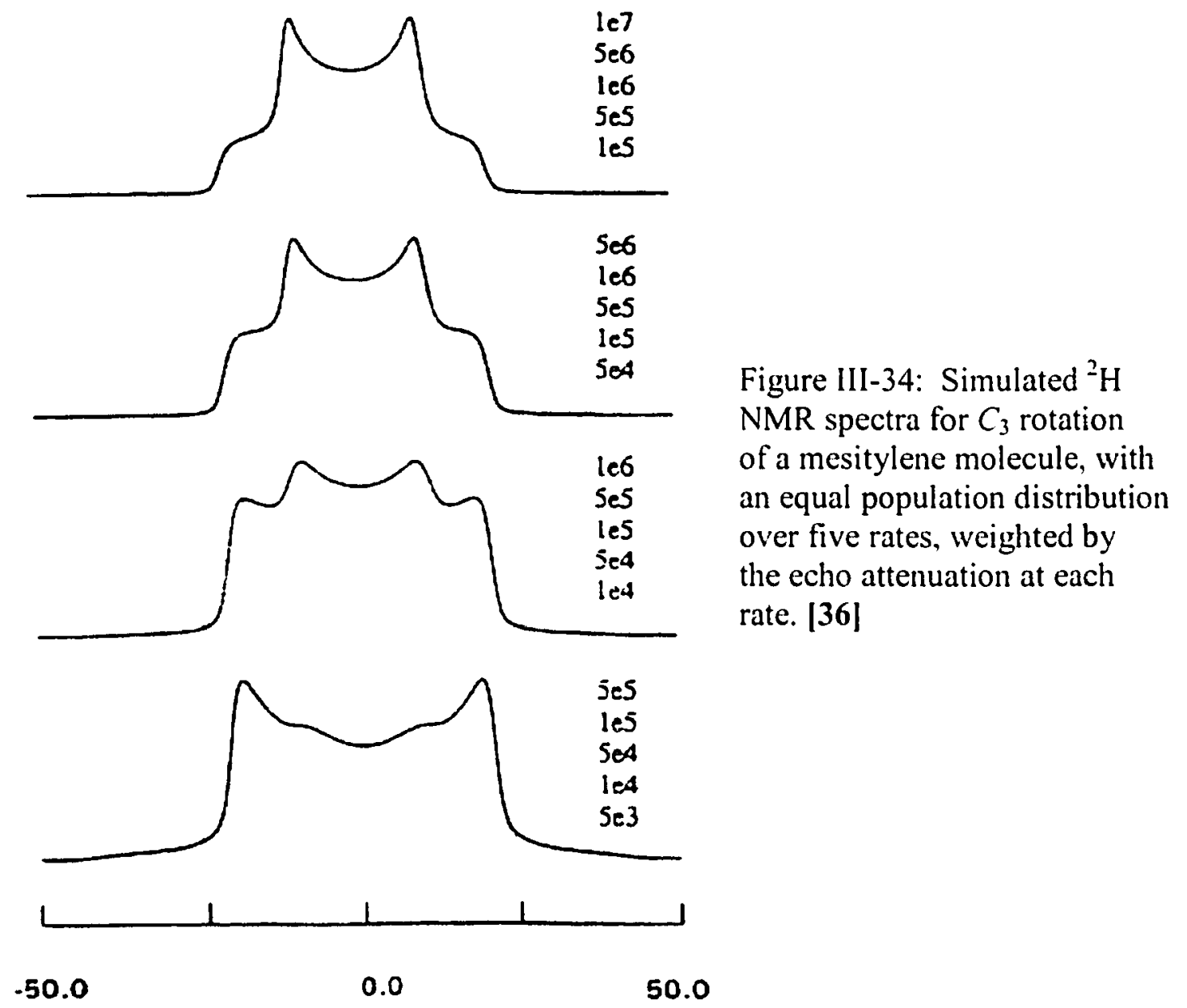

Frequency $(\mathrm{kHz})$ 
The ${ }^{2} \mathrm{H}$ NMR spectrum of ortho-xylene-d $\mathrm{d}_{10}$ (ortho-dimethylbenzene- $\mathrm{d}_{10}$ ) / TATM, at room temperature, is depicted in Figure III-35A. Note that there are two spectral components, aside from the sharp isotropic-type line at zero frequency. The inner component $\left(\chi_{\mathrm{eff}}=47.4 \mathrm{kHz}\right)$ is typical of methyl groups that are undergoing fast methyl rotation. Because $\chi_{\text {err }}$ is less than $56.7 \mathrm{kHz}$, the guest molecule is also performing a precessional or librational type of reorientation, of half-angle $\theta=19.3^{\circ}$ at room temperature. The outer component of the spectrum corresponds to the four static phenyl deuterons, with a non-zero static asymmetry parameter $\eta \sim 0.06$ commonly observed for aromatic deuterons. Their effective QCC is about $165 \mathrm{kHz}$ at room temperature. This is considerably smaller than the typical value of $190( \pm 5) \mathrm{kHz}$ for aromatic deuterons, also suggesting some motional averaging due to libration, of half-angle of $17.2^{\circ}$ at room temperature.

The ${ }^{2} \mathrm{H}$ NMR spectrum of para-xylene- $\mathrm{d}_{10}$ (para-dimethylbenzene- $\mathrm{d}_{10}$ ) / TATM at room temperature is shown in Figure III-35B. Note the general similarity to the ${ }^{2} \mathrm{H}$ NMR spectrum of $o$-xylene- $\mathrm{d}_{10} /$ TATM in Figure III-35A. The inner component, again from the six methyl deuterons, is indicative of methyl rotation as the only motion being present. The quadrupolar coupling constant, $50.5 \mathrm{kHz}$, again suggests that the guest is librating, with a half-angle $\theta=15.7^{\circ}$ at room temperature. The $\chi_{\text {erf }}$ value for the ring deuterons is $175 \mathrm{kHz}$, indicating a partial averaging due to the wobbling motion $(\theta=$ $\left.13.3^{\circ}\right)$.
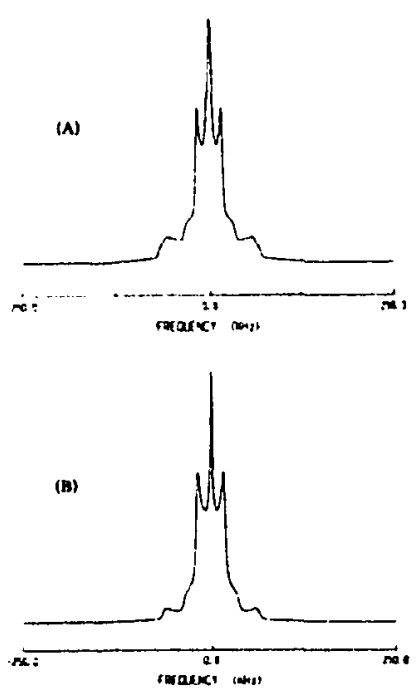

Figure IIl-35: Experimental ${ }^{2} \mathrm{H}$ NMR spectra (at room temperature) for:

(A) ortho-xylene- $d_{10} /$ TATM

(B) para-xylene-d $d_{10} /$ TATM [36] 


\section{CONCLUDING REMARKS}

TATM is a very versatile organic host material that was first prepared in 1973. As is often the case with many experimental discoveries, the original researchers stumbled upon TATM's inclusion abilities by accident, the original motivation behind their work being concerned with organic synthesis, namely the alkylation of 2-acetythiophene by polyhalogenomethanes. As a part of their observations, they noted that one of their products incorporated a few organic solvents upon crystallization. Four years later, in 1977 , the amazing ability of TATM to incorporate practically anything it would dissolve in was reported. These were followed by a number of reported single-crystal X-ray diffraction structures, which permitted a preliminary evaluation of the driving forces behind TATM's remarkable versatility. By employing these literature structures, Herbstein examined the conformational isomerism of TATM as a factor in molecular recognition of these host-guest inclusion complexes, drawing together all of the previous crystallographic work. In 1995 and 1996, the author of the thesis used deuterium NMR spectroscopy to measure reorientational dynamics of small guest molecules within TATM host lattices. The general conclusion was that most of the guests exhibited at least some degree of reorientational freedom within their TATM inclusions, illustrating quite nicely the lack of strong host-guest interactions in most cases. We hope that the studies presented in this thesis can further elaborate upon the previous studies, and may give directions for future work on this very interesting host material. 
(1) F.H. Herbstein. Acta Cryst. B53, 168 (1997).

(2) L. Bin Din and O. Meth-Cohn. J. Chem. Soc. Chem. Commun. 21, 741 (1977).

(3) P. Finocchiaro and S. Failla Solid-State Supramolecular Chemistry: Crystal Engineering. In Comprehensive Supramolecular Chemistry, J.L. Atwood, J. E. D. Davies, D.D. MacNicol and F. Vogtle Eds.; Pergamon / Elsevier: Oxford, 1996; Vol. 6, Chapter 18, pp 593-642.

(4) A.P. Yakubov, Y.K. Sudarushkin, L.I. Belen'kii and Y.L. Gol'dfarb. J. Org. Chem. (USSR) 9, 1549 (1973).

(5) Y.L. Gol'dfarb, B. Yu, B. Vol'kenshtein and L.I. Belen'kii. Angew. Chem. 80, 547 (1968).

(6) L.I. Belen'kii, G.P. Gromova and Y.L. Gol'dfarb. Izv. Akad. Nauk SSSR, Ser. Khim. 951, 1228 (1971).

(7) P.H. van Rooyen and H.M. Roos. Acta Cryst. C47, 2468 (1991).

(8) J.L.M. Dillen and H.M. Roos. Acta Cryst. C48, 2229 (1992).

(9) H.M. Roos and J.L.M. Dillen. Acta Cryst. C48, 1882 (1992).

(10) P.H. van Rooyen and H.M. Roos. Acta Cryst. C47, 2718 (1991).

(11) P. Main, S.J. Fiske, S.E. Hull, L. Lessinger, G. Germain, J.P. Declfreq and M.M. Woolfson. MULTAN80. A system of computer programs for the automatic solution of crystal structures from X-ray diffraction data. University of York, England and University of Louvain, Belgium (1980).

(12) G.M. Sheldrick. SHELX76. Program for crystal structure determination. University of Cambridge, England (1976).

(13) R.E. Marsh. Acta Cryst. B50, 112 (1994).

(14) R.E. Marsh. Acta Cryst. B37, 1985 (1981).

(15) L. Pang, R.C. Hynes and M.A. Whitehead. Acta Cryst. C50, 615 (1994).

(16) L. Pang and F. Brisse. Acta Cryst. C50, 1947 (1994).

(17) L. Pang and F. Brisse. Can. J. Chem. 72, 2318 (1994).

(18) E.J. Gabe, Y. LePage, J.P. Charland, F.L. Lee and P.S. White. J. Appl. Cryst. 22, 384 (1989) (SOLVER program suite).

(19) E.J. Gabe, Y. LePage, J.P. Charland, F.L. Lee and P.S. White. J. Appl. Cryst. 22, 384 (1989) (NRCVAX program suite).

(20) P. Dale. Acta Chem. Scand. Ser. A. 27, 1115 (1973).

(21) G.M. Sheldrick. SHELX86. Program for the solution of crystal structures. University of Gottingen, Germany (1985).

(22) F.H. Herbstein. Acta Cryst. B53, 168 (1997).

(23) P.S. Sidhu, J. Bell, G.H. Penner and K.R. Jeffrey. Can. J. Chem. 73, 2196 (1995).

(24) D.H. Williams and I. Fleming. Spectroscopic methods in organic chemistry. $4^{\text {th }}$ ed., McGraw-Hill Book Co. Ltd. London, England (1987).

(25) M.J. Potrzebowski. Magn. Reson. Chem. 29, 35 (1991).

(26) K. Borner, G. Diezemann, E. Rossler and H.M. Vieth. Chem. Phys. Lett. 181, 563 (1991).

(27) A. Kintanar, T.M. Alam, W.C. Huang, D.C. Schindele, D.E. Wemmer and G. Drobny. J. Am. Chem. Soc. 110, 6367 (1988).

(28) T. Iijima. Bull. Chem. Soc. Japan. 45, 3526 (1972). 
(29) J.D. Swalen and C.C. Costain. J. Chem. Phys. 31, 1562 (1959).

(30) N. Bloembergen, E.M. Purcell and R.V. Pound. Phys. Rev. 73, 679 (1948).

(31) D.A. Torchia and A. Szabo. J. Magn. Reson. 42, 381 (1981).

(32) S.J. Heyes and C.M. Dobson. Magn. Reson. Chem. 28, S37 (1990).

(33) G.A. Facey, C.I. Ratcliffe, R. Hynes and J.A. Ripmeester. J. Phys. Org. Chem. 5, 670 (1992).

(34) R.G. Barnes. Adv. Nucl. Quadrupole Reson. 1, 335 (1974).

(35) M.H. Wann and G.S. Harbison. J. Chem. Phys. 101, 231 (1994).

(36) P.S. Sidhu, J. Bell, G.H. Penner and K.R. Jeffrey. Can. J. Chem. 74, 1784 (1996).

(37) H. Dreizler and G. Dendl. Z. Naturforsch A: Astrophys. Phys. Phys. Chem. 20A, 1431 (1965).

(38) S. Jurga. Ser. Fiz. (Uniw. Adama Mickiewicza Poznaniu) 41 (1980).

(39) T. Hasebe and R. Sato. Fukushima Daigaku Kyoikugakubu Ronshu, Rika Hokoku. 46, 19 (1990).

(40) S. Hayashi. J. Phys. Chem. 99, 7120 (1995).

(41) A. Abragam. The principles of nuclear magnetism. Oxford University Press, London (1961).

(42) C. Schmidt, S. Wefing, B. Blumich and H.W. Spiess. Chem. Phys. Lett. 130, 84 (1986).

(43) F. Koksal and S. Bahceli. Z. Naturforsch A: Phys. Phys. Chem. Kosmophys. 39A, 548 (1984).

(44) J.H. Ok, R.R. Vold, R.L. Vold and M.C. Etter. J. Phys. Chem. 93, 7618 (1989).

(45) E.R. Andrew and R.G. Eades. Proc. Roy. Soc. London, Ser. A. 218, 537 (1953).

(46) A.E. Aliev, K.D.M. Harris and A. Mahdyarfar. J. Chem. Soc. Faraday Trans. 91, 2017 (1995).

\section{CHAPTER IV}

\section{OTHER HOST SYSTEMS}

(A) Urea

Urea $\left(\mathrm{H}_{2} \mathrm{NCONH}_{2}\right)$ inclusion compounds [1] differ from "clathrate" inclusion compounds in that they form channel rather than cage structures. Instead of the normal guest-free close-packed tetragonal lattice $[2,3]$, urea molecules in its inclusion compounds are linked by $\mathrm{N}-\mathrm{H} \cdots \mathrm{O}$ hydrogen bonds into three interpenetrating spirals that form the walls of a hexagonal cell, in the center of which is a channel of $4-5 \AA$ in diameter as depicted in Figure IV-1 [4]. A wide variety of long-chain molecules may be accommodated within these channels, provided that the cross-sectional dimensions of these guests do not radically distort the channel [5]. The organic compounds that may form urea inclusion compounds generally include $n$-paraffins $\left(\mathrm{C}_{7}\right.$ or larger), and some $n$ olefins, $n$-carboxylic acids, ketones and esters [6]. Compounds of propane and $n$-butane with urea have also been prepared, but they are not stable at room temperature. 

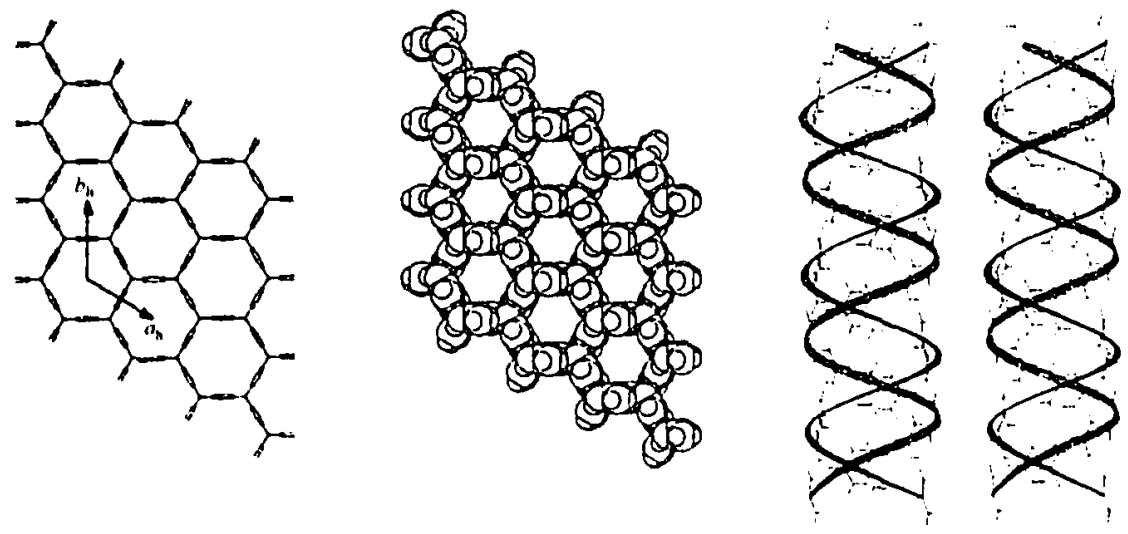

Figure IV-1: Urea host architecture

Generally, branched chain molecules cannot be accommodated into the channels [7]. There is no evidence to indicate that the hexagonal urea structure is stable in the absence of guest species, rather removal of the guest results in immediate reversion to the normal tetragonal urea structure $[8,9]$. Furthermore, it is not possible to prepare adducts comprising partly filled cavities, instead the substance disproportionates into the completely filled adduct and pure tetragonal urea [10]. Therefore, for any particular guest substance, only one adduct composition is possible.

(B) Tri-ortho-thymotide (TOT)

In solid inclusion compounds of tri-ortho-thymotide, 1, appropriately sized guest molecules are located within cavities or tunnels generated from the crystal packing of TOT molecules [11]. The TOT molecule is flexible, and in solution two different chiral conformations predominate [12]. The major conformer is described as a "propeller", with all three carbonyl oxygen atoms pointing in the same direction (i.e. clockwise or counterclockwise) about the central 12-membered ring. The minor conformer is described as "helical", with one carbonyl oxygen atom pointing in the opposite direction (compared to the other two). The pure crystalline phase of TOT is a racemic mixture of the two enantiomers of the propeller conformation [13]. When TOT is crystallized in the presence of appropriate guest molecules, inclusion compounds of TOT may be formed [14]. A wide variety of guest molecules can form such inclusion compounds with TOT, and the TOT host structure can differ significantly, depending on the structural characteristics of the guest $[15,16]$. 


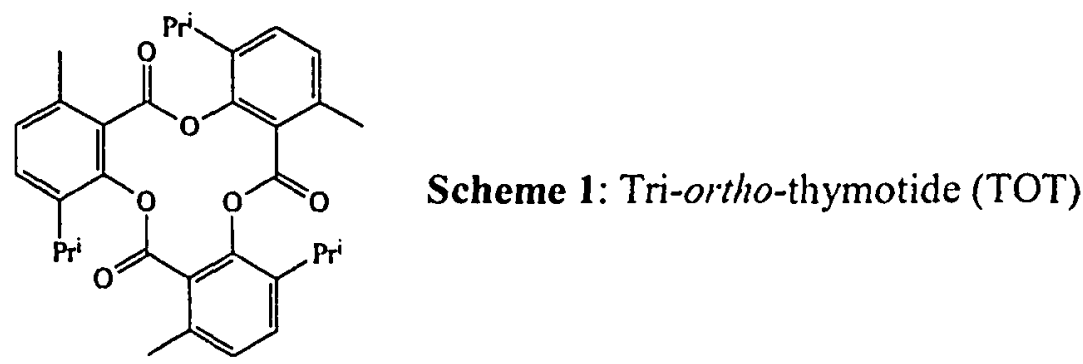

In general, TOT inclusion compounds may be classified as either cage-type [17] or tunnel-type $[18,19]$, depending on the nature of the inclusion cavities within the host structure. In the cage-type inclusion compounds, the guest molecules are enclosed in discrete cavities within the host crystal, and guest molecules in different cages are not in direct contact with each other. Virtually all cage-type TOT inclusion compounds have space group $P 3,21$, and are formed by guest molecules with a maximum dimension less than $\sim 9 \AA$, such as acetone, methanol, bromocyclohexane and pyridine. In general, the cage-type inclusion compounds have a host:guest stoichiometric ratio of 2:1 [20].

The tunnel-type inclusion compounds may be subdivided into two general types, characterized by different tunnel topologies:

(1) Systems of intersecting tunnels. This type of tunnel structure is typically formed with non-linear guests such as benzene, toluene, trans-stilbene and cis-stilbene [21]. The space group is generally $\mathrm{P}$, , and there are three different types of tunnels in the host structure. Two types are parallel to each other and have uniform cross-sectional areas. The third type of tunnel lies along another direction, and its cross-sectional area varies, through a series of constrictions and bulges, on moving along the tunnel.

(2) Parallel, non-intersecting tunnels. This type of tunnel structure is typically formed with long-chain, unbranched guest molecules longer than $\sim 9.5 \AA[22]$. Linear, parallel tunnels run through the crystal, generally along a principal crystallographic axis. The space group is usually $P 6_{1}, P 6_{2}$ or $P 3_{1}$, depending on the guest, with the screw axis lying along the tunnel direction. In common with many other solid organic inclusion compounds, the tunnels are stable only if guest molecules are present. If the guest molecules are removed (e.g. by heating), the empty tunnels collapse to give the pure crystalline phase of TOT [23]. 


\section{(C) Hydroquinone (quinol)}

The hydroquinone (para-dihydroxybenzene) clathrates [24] are formed by threedimensionally closed structures of hydroquinone molecules, hydrogen-bonded in such a way as to produce cavities within which a variety of relatively small guest species may be accommodated [25-27]. The guest species, being totally enclosed by the host lattice, may only enter a cavity during its formation (i.e. during crystallization of the clathrate), and the cavities are capable of relatively limited deformation to accommodate elongated guest species such as acetonitrile [28]. In most cases, it is not necessary to have all of the cavities occupied, as empty cavities are generally stabilized by adjacent occupied cavities [29]. On the other hand, the host-guest interactions do contribute to the overall stability of the clathrate, as is shown by the fact that the heat of inclusion increases linearly with the fraction of available cavities occupied [30].

Hydroquinone is known to exist in three crystal modifications, designated $\alpha$ quinol [31], $\beta$-quinol [32] and $\gamma$-quinol [33]. The $\alpha$ - and $\gamma$-crystal structures essentially consist of hydrogen-bonded helical chains and sheets respectively, while $\beta$-quinol forms a clathrate host structure, which is also held together by hydrogen bonds, but in a different manner. Two six-membered hydrogen bonded $\mathrm{O}-\mathrm{H} \cdots \mathrm{O}$ rings form the top and bottom of the cage. At room temperature, the $\alpha$-form is the most stable of the three forms, but the $\beta$-structure lies only about -0.1 to $-0.2 \mathrm{kcal} \mathrm{mol}^{-1}$ higher in energy [34]. In the presence of guest molecules, even very weakly bound ones, the host-guest $\beta$ compound becomes the stable polymorph. The $\beta$-form [32] is most suitable for clathrate formation because of its maximal number of cages, so that $\beta$-quinol clathrates may contain up to one guest molecule per three quinol molecules. The host-guest crystal structures are generally made up of planar hydroquinone molecules, which form a threedimensional network containing approximately spherical cages, about $4 \AA$ in diameter. One such cage is shown in Figure IV-2.
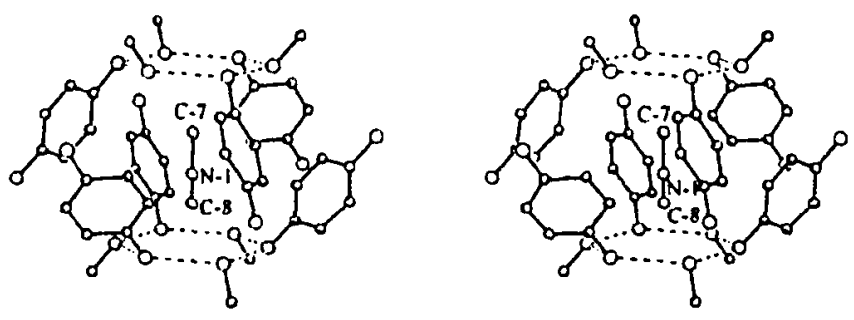

Figure IV-2: Stereoview of $\beta$-hydroquinone host cage with MeNC guest 
(D) Dianin's compound

Dianin's compound, 2, [35] is a well-established organic cage-forming host system that forms clathrate compounds with a wide variety of guest molecules $[36,37]$. Its structural characteristics were first established in the early 1970's [38,39]. These, and other [40] crystallographic investigations indicated a $\beta$-quinol type cage system of the trigonal space group $\mathrm{R} \overline{3}$, with cavities that are bounded by two pairs of three host molecules, that are each linked together by hydrogen bonds between their hydroxyl groups, resulting in puckered six-membered rings with three host molecules pointing up and three pointing down. These hexamer units are stacked directly on top of one another, and the cage is formed when two of these hexamer units stack together, with one hexagon of hydrogen bonded oxygen atoms forming the floor of the cage, and the next hexagon of hydrogen bonded oxygens forming the ceiling of the cage. The three host molecules pointing upward from the floor of the cage, and the three host molecules pointing downward from the ceiling of the cage are physically enmeshed at the point of narrowest diameter $(z=$ 0.5 ), to form the waist of the cage. The cavities are described as hourglass shaped, with the cavity axis along the crystallographic $c$ axis $[41]$. The length of the cavity is $\sim 11.0 \AA$, with a waist diameter of $\sim 4.2 \AA$ at $z=0.5$, and the greatest diameter of $\sim 6.2 \AA$ at both $z=$ 0.3 and $z=0.7[38-40]$. Furthermore, these values are generally unaffected by the type and size of guest molecules $[42,43]$. This cage structure is significantly larger than the familiar $\beta$-quinol structure, which is reflected in the ability of Dianin's compound to include significantly larger guests than the $\beta$-quinol lattice [44]. The guest-free Dianin's compound structure $|45|$ is practically identical, as far as the host framework is concerned, as that of its inclusion compounds (i.e. in the presence of a guest). The space group $R \overline{3}$ requires that the cavity have $\overline{3}$ symmetry, which implies that a single unsymmetrical guest molecule be statistically six-fold disordered [46].

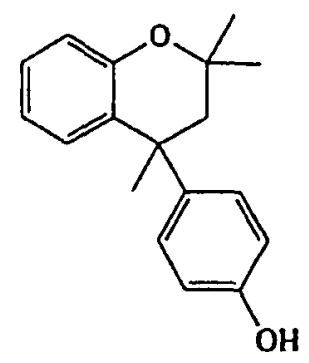

Scheme 2: Dianin's Compound 


\section{(E) Gossypol}

Gossypol, 3, [47] an optically (and biologically) active component of cotton-seed kernels [48-51], is a versatile host material, giving inclusion complexes with an extremely wide variety of organic compounds $[\mathbf{5 2 , 5 3 ]}$. Indeed, one study showed that racemic gossypol formed inclusion complexes with almost every solvent used for recrystallization [54]. Gossypol is soluble in a wide variety of organic solvents, but is practically insoluble in water or petroleum ether. Various crystalline forms can be obtained by changing crystallization solvents, using solvent mixtures, or by recrystallizing gossypol from a solution with an added guest component. Indeed, only a small amount of acetic acid, added to a diethyl ether solution (containing gossypol) is sufficient to obtain a 1:1 complex of gossypol - acetic acid, even though gossypol easily forms inclusion compounds with diethyl ether [55].

Nearly 100 inclusion compounds of gossypol have been prepared, and approximately 80 of them formed single crystals of sufficient quality for crystal data determination [56]. Thus, it is evident that gossypol is an extremely versatile host, which can be easily rearranged to accommodate, in its crystal lattice, guest molecules of various shapes, sizes and chemical nature $[52,53]$. Contrary to the racemic mixture, optically pure gossypol does not crystallize easily, and when forced to crystallize, yields crystals which are of insufficient quality for X-ray diffraction analysis $[57,58]$.<smiles>Cc1cc2c(C(C)C)c(O)c(O)c(C=O)c2c(O)c1-c1c(C)cc2c(C(C)C)c(O)c(O)c(C=O)c2c1O</smiles>

Scheme 3: Gossypol<smiles>[R]NC=C1C(=O)C(O)=C(C(C)C)c2cc(C)c(-c3c(C)cc4c(c3O)C(=CN[R])C(=O)C(O)=C4C(C)C)c(O)c21</smiles>

Scheme 4: Dianilinegossypol 
The dianiline derivative of gossypol (dianilinegossypol), 4 , is also able to form inclusion compounds with a large number of guests $[59,60]$. Interestingly, in some cases it is able to form more than one type of inclusion compound with the same guest (under differing preparatory conditions). For example, dianilinegossypol forms two true polymorphic modifications with both ethyl acetate [61] and acetone [62]. With the exception of TATM, these were the only two reported cases of true polymorphism in the inclusion compound literature. True polymorphic modifications of a certain host-guest pair should have different crystal structures, but the same host-guest stoichiometric ratio [63]. If two complexes of the same host-guest pair have different stoichiometric ratios, then by definition they are not considered "true" polymorphs, rather they are correctly termed "pseudopolymorphs" [64].

\section{(F) Perhydrotriphenylene}

Perhydrotriphenylene (PHTP), 5 [65], is made up of four condensed cyclohexane rings, with three of them being symmetrically placed around the central ring. PHTP $\left(\mathrm{C}_{18} \mathrm{H}_{30}\right)$ was first prepared in 1963 [66] during research into low molecular weight models of optically active polymers, and into the exceptions to the common nomenclature in reference to organic stereochemistry [67-69|. PHTP is prepared by hydrogenation of dodecahydrotriphenylene $\left(\mathrm{C}_{18} \mathrm{H}_{24}\right)$. The hydrogenation processs produces a mixture of up to ten stereoisomers [70,71], from which the trans-anti-trans-anti-trans isomer is most easily isolated ( $60 \%$ yield), and it is this isomer that possesses the inclusion capabilities. The six C-C bonds of the central ring may be divided into two classes: $\{1\}$ three bonds being shared by two cyclohexane rings (endo), while $\{2\}$ the other three bonds are unshared (exo). PHTP is a highly symmetric chiral molecule (point group $D_{3}$ ). As dictated by this point group, it contains one three-fold and three two-fold axes of symmetry. However, it has no elements of mirror symmetry or a center of inversion. As a consequence, it exists in two enantiomeric forms [72]. PHTP is one of the few host materials for which both the enantiomerically pure forms and the racemic form are available $[73,74]$. 
<smiles>C1CCC2C(C1)C1CCCCC1C1CCCCC21</smiles>

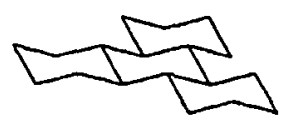

Scheme 5: Perhydrotriphenylene

The strong tendency of PHTP to form inclusion compounds is demonstrated by the great variety of compounds that are able to act as guests, the mild conditions used in their preparation and their thermal properties [75]. The conditions essential for inclusion are the shape and size of the guest. On the other hand, the role of the chemical nature of the guest is more important in determining the thermal properties of the resulting inclusion compound [76]. Due to the generally elongated nature of the host cavities, typical guests are in most cases long and thin molecules [77]. However, because of the absence of strong host-host interactions, and the subsequent flexibility of the host lattice, various bulky guests may also be included [78]. Among the common organic solvents, only methyl ethyl ketone is not included, and is therefore used extensively as a non-interacting medium for PHTP inclusion compound preparation [79].

\section{CHAPTER IV}

REFERENCES

(1) M.D. Hollingsworth and K.D.M. Harris. Urea. Thiourea and Selenourea. In Comprehensive Supramolecular Chemistry, J.L. Atwood, J.E.D. Davies, D.D. MacNicol and F. Vogtle, Eds. Volume 6, Chapter 7. Pergamon / Elsevier: Oxford, 1996.

(2) P. Vaughan and J. Donohue. Acta Cryst. 5, 530 (1952).

(3) A.E. Smith. Acta Cryst. 5, 224 (1952).

(4) K.D.M. Harris and J.M. Thomas. J. Chem. Soc. Faraday Trans. 86, 2985 (1990).

(5) K.D.M. Harris. J. Solid State Chem. 106, 83 (1993).

(6) L.C. Fetterly. In Non-Stoichiometric Compounds. L. Mandelcorn, Ed. Academic Press: New York, 1964.

(7) M.D. Hollingsworth, M.E. Brown, B.D. Santarsiero, J.C. Huffman and C.G. Ross. Chem. Mater. 6, 1227 (1994).

(8) H.G. McAdie and G.B. Forst. Can. J. Chem. 36, 635 (1958).

(9) H.G. McAdie. Can. J. Chem. 40, 2195 (1962).

(10) K.D.M. Harris. J. Phys. Chem. Solids 53, 529 (1992).

(11) R. Gerdil. Tri-o-Thymotide and Related Hosts. In Comprehensive Supramolecular Chemistry, J.L. Atwood, J.E.D. Davies, D.D. MacNicol and F. Vogtle, Eds. Volume 6, Chapter 8. Pergamon / Elsevier: Oxford, 1996.

(12) W.D. Ollis and I.O. Sutherland. Chem. Commun. 402 (1966).

(13) S. Brunie and G. Tsoucaris. Cryst. Struct. Commum. 3, 481 (1974). 
(14) D. Lawton and H.M. Powell. J. Chem. Soc. 2339 (1958).

(15) R. Gerdil. Tri-o-Thymotide Clathrates. In Topics in Current Chemistry. E. Weber, Ed. Volume 140, Chapter 4. Springer-Verlag: Berlin, 1987.

(16) R. Arad-Yellin, B.S. Green, M. Knossow and G. Tsoucaris. J. Am. Chem. Soc. 105, 4561 (1983).

(17) R. Arad-Yellin, B.S. Green, M. Knossow and G. Tsoucaris. In Inclusion Compounds, J.L. Atwood, J.E.D. Davies and D.D. MacNicol, Eds. Volume 2, Chapter 6, Academic Press: New York, 1984.

(18) H. Serrano-Gonzalez and K.D.M. Harris. J. Solid State Chem. 148, 63 (1999).

(19) H. Serrano-Gonzalez and K.D.M. Harris. J. Mol. Struct. 519, 41 (2000).

(20) R. Gerdil. Tri-o-Thymotide and Related Hosts. In Comprehensive Supramolecular Chemistry, J.L. Atwood, J.E.D. Davies, D.D. MacNicol and F. Vogtle, Eds. Volume 6, Chapter 8: (Table 1, p.243). Pergamon / Elsevier: Oxford, 1996.

(21) R. Gerdil. Tri-o-Thymotide and Related Hosts. In Comprehensive Supramolecular Chemistry, J.L. Atwood, J.E.D. Davies, D.D. MacNicol and F. Vogtle, Eds. Volume 6, Chapter 8: (Table 4, p.244). Pergamon / Elsevier: Oxford, 1996.

(22) R. Gerdil. Tri-o-Thymotide and Related Hosts. In Comprehensive Supramolecular Chemistry, J.L. Atwood, J.E.D. Davies, D.D. MacNicol and F. Vogtle, Eds. Volume 6, Chapter 8: (Table 3, p.244). Pergamon / Elsevier: Oxford, 1996.

(23) D.J. Williams and D. Lawton. Tetrahedron Lett. 111 (1975).

(24) T.C.W. Mak and B.R.F. Bracke. Hydroquinone Clathrates and Diamondoid Host Lattices. In Comprehensive Supramolecular Chemistry, J.L. Atwood, J.E.D. Davies, D.D. MacNicol and F. Vogtle, Eds. Volume 6, Chapter 2. Pergamon / Elsevier: Oxford, 1996.

(25) D.E. Palin and H.M. Powell. J. Chem. Soc. 208 (1947).

(26) H.M. Powell. J. Chem. Soc. 61 (1948).

(27) H.M. Powell. In Non-Stoichiometric Compounds. L. Mandelcorn, Ed. Chapter 7. Academic Press: New York, 1964.

(28) T.L. Chan and T.C.W. Mak. J. Chem. Soc. Perkin Trans 2. 777 (1983).

(29) A.I. Kitaigorodsky. Molecular Crystals and Molecules. Academic Press: New York, 1973.

(30) D.F. Evans and R.E. Richards. Proc. Roy. Soc. (London) Series A. 223, 238 (1954).

(31) S.C. Wallwork and H.M. Powell. J. Chem. Soc. Perkin Trans 2. 1435 (1982).

(32) S.V. Lindeman, V.E. Shklover and Y.T. Struchkov. Cryst. Struct. Commun. 10, $1173(1981)$.

(33) K. Maartmann-Moe. Acta Cryst. 21, 979 (1952).

(34) D.F. Evans and R.E. Richards. J. Chem. Soc. 3932 (1952).

(35) P. Finocchiaro and S. Failla. Other Potentially Important Hosts. In Comprehensive Supramolecular Chemistry, J.L. Atwood, J.E.D. Davies, D.D. MacNicol and F. Vogtle, Eds. Volume 6, Chapter 18. Pergamon / Elsevier: Oxford, 1996.

(36) D.D. MacNicol. Structure and Design of Inclusion Compounds: The Clathrates of Hydroquinone, Phenol, Dianin's Compound and Related Systems. In Inclusion Compounds. J.L. Atwood, J.E.D. Davies and D.D. MacNicol, Eds. Volume 2, Chapter 1. Academic Press: London, 1984. 
(37) W. Baker, A.J. Floyd, J.F.W. McOmie, G. Pope, A.S. Weaving and J.H. Wild. J. Chem. Soc. 2010 (1956).

(38) J.L. Flippen, J. Karle and I.L. Karle. J. Am. Chem. Soc. 92, 3749 (1970).

(39) J.L. Flippen and J.Karle. J. Phys. Chem. 75, 3566 (1971).

(40) W. Baker, J.F.W. McOmie and A.S. Weaving. J. Chem. Soc. A. 3516 (1971).

(41) L. Pang, E.A.C. Lucken and G. Bernardinelli. J. Am. Chem. Soc. 112, 8754 (1990).

(42) D.D. MacNicol, J.J. McKendrick and D.R. Wilson. Chem. Soc. Rev. 7, 65 (1978).

(43) F. Lee, E. Gabe, J.S. Tse and J.A. Ripmeester. J. Am. Chem. Soc. 110, 6014 (1988).

(44) H.M. Powell and B.D.P. Wetters. Chem. Ind. (London). 256 (1955).

(45) F. Imashiro, M. Yoshimura and T. Fujiwara. Acta Cryst. C54, 1357 (1998).

(46) R.W.H. Small. Acta Cryst. B59, 141 (2003).

(47) M. Gdaniec, B.T. Ibragimov and S.A. Talipov. Gossypol. In Comprehensive Supramolecular Chemistry, J.L. Atwood, J.E.D. Davies, D.D. MacNicol and F. Vogtle, Eds. Volume 6, Chapter 5. Pergamon / Elsevier: Oxford, 1996.

(48) G.P. Tuszynski and G. Cossu. Cancer Research. 44, 768 (1984).

(49) R.I. Radolff, L.M. Deck, R.E. Royer and J.D.L. Vander. Pharmacol. Res. Commun. 18, 1063 (1986).

(50) B. Polsky, S.J. Segal, P.A. Baron, J.W.H. Gold, H. Ueno and D. Armstrong. Contracep. 39, 579 (1989).

(51) E.M. Continho, J.F. Melo, I. Barbosa and S.J. Segal. Fertil. Steril. 42, 424 (1984).

(52) B.T. Ibragimov, S.A. Talipov and P.M. Zorky. Supramol. Chem. 3, 147 (1994).

(53) B.T. Ibragimov and S.A. Talipov. Mol. Cryst. Liq. Cryst. A276, 305 (1996).

(54) B.T. Ibragimov, S.A. Talipov, B.N. Dadabaev, G.B. Nazarov and T.F. Aripov. Khim. Prir. Soedin. 675 (1988).

(55) K.N. Campbell, R.C. Morris and R. Adams. J. Am. Chem. Soc. 59, 1723 (1937).

(56) Reference 47: Table 1, pp 122-123.

(57) J.M. Dechary and P. Pradel. J. Am. Oil Chem. Soc. 48, 563 (1971).

(58) L. Huang, D.K. Zheng and Y.K. Si. Ethnopharm. 20, 13 (1987).

(59) N.I. Baram, F.G. Kamaev, K.L. Ziyaev, L. Biktimirov, A.I. Ismailov, G.B. Nazarov and B.T. Ibragimov. Khim. Prir. Soedin. 650 (1988).

(60) G.B. Nazarov, B.T. Ibragimov and T.F. Aripov. Khim. Prir. Soedin. 661 (1988).

(61) K.M. Beketov, B.T. Ibragimov, S.A. Talipov, K. Makhkamov and T.F. Aripov. J. Incl. Phenom. Mol. Recognit. Chem. 27, 105 (1997).

(62) K.M. Beketov, B.T. Ibragimov and S.A. Talipov. J. Incl. Phenom. Mol. Recognit. Chem. 28, 141 (1997).

(63) P.S. Sidhu, G.D. Enright, K.A. Udachin and J.A. Ripmeester. Crystal Growth and Design. 4(6), 1249 (2004).

(64) W.C. McCrone. In Physics and Chemistry of the Organic Solid State. D. Fox, M.M. Labes and A. Weissberger Eds. Volume 2, pp 725-767, Interscience: New York, 1965.

(65) M. Farina, G. DiSilvestro and P. Sozzani. Perhydrotriphenylene: $A D_{3}$ Symmetric Host. In Comprehensive Supramolecular Chemistry, J.L. Atwood, J.E.D. Davies, D.D. MacNicol and F. Vogtle, Eds. Volume 6, Chapter 12. Pergamon / Elsevier: Oxford, 1996. 
(66) M. Farina. Tetrahedron Lett. 2097 (1963).

(67) G. Natta and M. Farina. Tetrahedron Lett. 703 (1963).

(68) M. Farina, M. Peraldo and G. Natta. Angew. Chem. Int. Ed. Engl. 4, 107 (1965).

(69) R.S. Cahn, C.K. Ingold and V. Prelog. Angew. Chem. Int. Ed. Engl. 5, 385 (1966).

(70) M. Farina, G. Audisio and P. Bergomi-Bianchi. Chim. Ind. (Milan). 50, 446 (1968).

(71) M. Farina and G. Audisio. Tetrahedron. 26, 1827 (1970).

(72) R. Raban and K. Mislow. Top. Stereochem. 2, 199 (1967).

(73) M. Farina and G. Audisio. Tetrahedron Lett. 1285 (1967).

(74) M. Farina and G. Audisio. Tetrahedron. 26, 1839 (1970).

(75) M. Farina, G. Allegra and G. Natta. J. Am. Chem. Soc. 86, 516 (1964).

(76) G. Allegra, M. Farina, A. Immirzi, A. Colombo, U. Rossi, R, Broggi and G. Natta. J. Chem. Soc. (B). 1020 (1967).

(77) A. Colombo and G. Allegra. Rend. Accad. Naz. Lincei. 43(8), 41 (1967).

(78) A. Immirzi and G. Allegra. Rend. Accad. Naz. Lincei. 43(8), 181 (1967).

(79) M. Farina. In Inclusion Compounds. J.L. Atwood, J.E.D. Davies and D.D. MacNicol, Eds. Volume 2, Chapter 3, Academic Press: London, 1984.

\section{CHAPTER V}

TECHNIQUES EMPLOYED

Single crystal X-ray diffraction has contributed greatly to the understanding of structural properties of inclusion compounds, $[1-4]$ and is a primary source of definitive data on the geometry of host-guest binding in the solid state. For these complexes, $\mathrm{X}$-ray studies provide non-bonded host-guest intermolecular distances, whose ranges reveal the "tightness of fit" of the guest molecule in the cavity or channel provided by the host framework. With increasing levels of coordinative interactions, the diffraction method yields geometrical data for interactions ranging from rather weak binding $[5,6 \mid$ (e.g. C$\mathrm{H} \cdots \pi$ (arene) or $\mathrm{C}-\mathrm{H} \cdots \mathrm{O}$ interactions), to very strong hydrogen bonding $[7]$ (e.g. $\mathrm{N}-\mathrm{H} \cdots \mathrm{O}$ or $\mathrm{O}-\mathrm{H} \cdots \mathrm{O}$ ), that may occur when the host and guest molecules contain suitable complementary acceptor and donor functional groups [8]. Furthermore, by determining the atomic coordinates of a crystalline unit cell, a number of calculations and drawings may be performed [9], and can classify the host as forming cavities, channels or layers etc. into which the guest molecules reside. In addition, calculation of the shape and volume of the host cavity is possible, by volume filling techniques such as generating a large number of points in the unit cell, and determining whether or not each of them lie within the van der Waals radii of the host $\mathrm{s}$ atoms. These and other types of calculations based on single crystal X-ray diffraction data demonstrate the power of this technique to characterize solid inclusion compounds [10]. 
Powders of crystalline materials diffract X-rays [11]. A beam of X-rays passing through a sample of randomly oriented microcrystals produces a pattern of rings. Powder $\mathrm{X}$-ray diffraction (PXRD), as an analytical technique, is useful for confirming the identity of a solid material, by comparison of the positions and intensities of the diffraction lines with spectra in comprehensive data banks, and can be used to determine whether two or more compounds are isostructural (i.e. similar unit cell parameters). In addition, it can measure crystallinity, phase purity, phase diagrams and the relative amount of each phase present in a mixture, as different solid phases give rise to different diffraction patterns. PXRD is also useful as a method for cross calibrating with the solid-state NMR results to define structure type. Powder $\mathrm{X}$-ray diffraction provides less information than singlecrystal X-ray diffraction, however it is generally much simpler and faster, it can characterize the bulk product (rather than a single selected crystal), and can be used on crystalline solids that cannot be prepared as single crystals of sufficient size and quality for conventional single-crystal diffraction studies $[12,13]$.

The ${ }^{13} \mathrm{C}$ NMR spectra of solids are dominated by a number of electronic and magnetic interactions [14-16]. These interactions are present in solutions as well, however the rapid tumbling motion in this phase averages them out partially or completely, usually leaving very sharp lines at frequencies determined by the isotropic chemical shift and scalar coupling. In general, molecules in solids do not possess the same degree of motional freedom and the interactions manifest themselves as very broad resonances that may, in principle, provide much more information than the isotropic lines observed in the NMR spectra of solutions. In solids, these anisotropic interactions can be averaged by a combination of magic angle spinning (MAS) and high power proton decoupling. In addition, cross polarization from protons to carbons greatly increases the ${ }^{13} \mathrm{C}$ NMR signal strength. One important feature of high resolution solid-state cross polarization / magic angle spinning (CP/MAS) NMR spectroscopy is that nuclei which are normally considered to be chemically equivalent in solution may not be magnetically equivalent in the solid state, and hence extra signal splittings can occur. In principle, every crystallographically inequivalent atom can give rise to its own resonance. It is the crystallographic symmetry rather than the molecular symmetry that determines the equivalence or nonequivalence of any group of atoms. Examination of the ${ }^{13} \mathrm{C} C P / \mathrm{MAS}$ 
NMR spectrum can therefore give a quick "snapshot" on the contents of the asymmetric unit and has the potential as a "fingerprinting" technique for polycrystalline solids. It may also be considered as a routine screening method (along with powder X-ray diffraction) before embarking on single crystal X-ray diffraction studies.

In contrast to diffusive Brownian reorientation in the liquid state, molecular motion in solids usually proceeds through discrete jumps. These motions are often masked in solution by overall isotropic molecular tumbling. Inclusion compounds can provide anisotropic environments for small molecules that exist as solids only at very low temperatures. The analysis of molecular motion is fundamental to the understanding of many macroscopic properties of solids. Knowing whether the nature of the guest's motion is determined by the internal symmetry of the guest molecule or the external symmetry of the host cavity provides insight into the nature of the interaction between host and guest.

Solid-state deuterium NMR spectroscopy has become a very powerful technique for the study of dynamic processes in a variety of systems [17-19]. These include crystalline solids [20-22], inclusion compounds [23-25] and zeolites [26-28]. This has been made possible with a combination of technical advances such as the development of the quadrupolar echo pulse sequence [29], the availability of computer programs capable of simulating deuterium NMR powder lineshapes [30] and progress in the chemistry of selective isotope labelling [31|. The deuteron, as compared to the proton, is a much better probe of molecular dynamics. For deuterium, the primary nuclear spin perturbation on the Zeeman interaction is the quadrupolar interaction, which is usually two or more orders of magnitude greater than any other perturbation (such as dipolar coupling or chemical shift anisotropy), generally allowing one to neglect these other contributions to the ${ }^{2} \mathrm{H}$ NMR lineshape. In reality, these other interactions sometimes manifest themselves as excess line broadening in the solid-state ${ }^{2} \mathrm{H}$ NMR spectrum.

Dynamic processes occuring with rates in the range $10^{3}-10^{8} \mathrm{~Hz}$ (when the rate of motion $k$ of the C-D bond is comparable in magnitude to the ${ }^{2} \mathrm{H}$ quadrupolar coupling constant) can be studied by lineshape analysis of the variable temperature ${ }^{2} \mathrm{H}$ NMR spectra of a polycrystalline powder [32]. For motions faster than $10^{8} \mathrm{~Hz}$ (when $k$ approximates the deuterium Larmor frequency), the ${ }^{2} \mathrm{H}$ NMR lineshapes become 
independent of the exchange rate, and an analysis of the ${ }^{2} \mathrm{H}$ spin-lattice relaxation time is used to discriminate among the types and rates of motion [33]. Recent progress in multipulse techniques and two-dimensional exchange experiments allows the study of an extremely broad range of rates $\left(10^{1}-10^{12} \mathrm{~Hz}\right)[34]$.

The most convenient techniques to study the thermal properties of solid inclusion compounds are thermal analysis methods [35-38]. These techniques are among the most fundamental means of studying phases of matter, for example, they can provide information on properties ranging from thermal properties to intermolecular interactions [39]. Although thermal experimental methods primarily relate to bulk matter, their results can be interpreted in microscopic terms. It is the microscopic interpretation that is most important to thermal measurements of supramolecular systems, as this can indicate the role of intermolecular interactions [40].

Thermal techniques exist in two main categories, namely thermal analysis and calorimetry. The term "thermal analysis" is used to denote measurements of properties carried out mostly using commercial equipment developed for analytical purposes. These techniques include differential thermal analysis (DTA) [41], differential scanning calorimetry (DSC) [42] and thermogravimetric analysis (TGA, also known as thermogravimetry - TG) [43]. The term "calorimetry" usually denotes accurate measurements of thermal properties, especially heat capacity; these experiments are usually carried out on equipment that generally is not available commercially. Most of the thermal analysis techniques described above are scanning methods (i.e. measurements as a function of temperature without establishment of thermal equilibrium), whereas calorimetric methods are largely carried with the establishment of thermal equilibrium. Another distinguishing characteristic is that thermal analysis methods are generally differential, that is, measured with respect to standards, while calorimetric measurements are generally absolute measurements.

In differential scanning calorimetry (DSC) [44] thermal events in the sample appear as deviations from the baseline, either endothermic or exothermic, depending on whether more or less energy, respectively, is supplied to the sample relative to the reference material. Thus melting points, phase changes, and the associated enthalpies can 
be determined to give information on the thermodynamic state of the system and relative thermal stabilities [45].

Thermogravimetric analysis (TGA) [46] measures the mass loss of a solid while it is subjected to a selected temperature program under a controlled atmosphere. An idealized TGA-DSC plot for an organic inclusion compound containing a volatile guest is depicted in Figure V-1. The mass loss is generally reproducible to $\pm 1 \%$ in successive experiments, and yields an accurate host-guest ratio for the compound under study. This is particularly important for inclusion compounds that are notoriously non-stoichiometric. The results thus obtained may be usefully employed in crystal structure analysis, where the site occupancy factors of the guest's atoms may be more easily assigned. Furthermore, TGA can provide complementary information to DSC experiments by portraying thermally induced loss of guest from an inclusion compound as it is heated. Perhaps an inclusion compound that loses its guest more easily and rapidly (from TGA) as it is heated will perhaps display a lower DSC melting temperature and a lower heat of decomposition, when compared to an inclusion compound (with the same host) that holds onto its guest more tenaciously when it is heated during the TGA experiment. So by incorporating information from both experiments, we may arrive at a more definite conclusion concerning the relative thermal stabilities of a series of TATM inclusion compounds, rather than by using results from only one of these experiments.

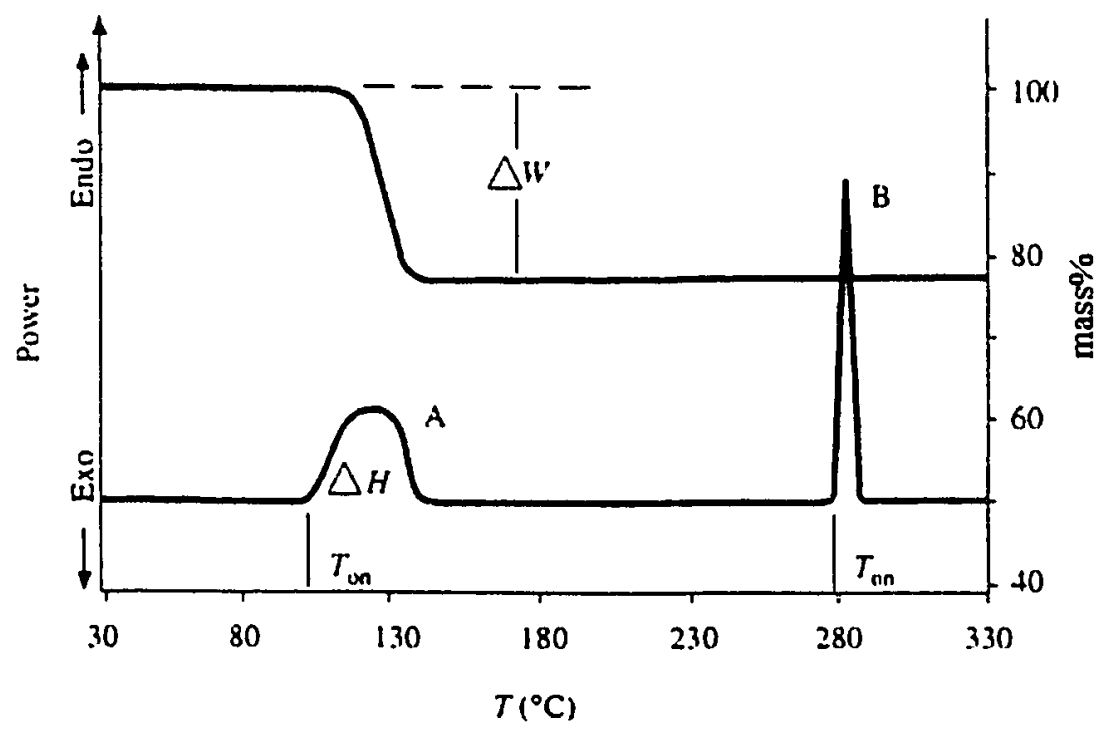

Figure V-1: Idealized TGA-DSC experiment plot of an inclusion compound with a volatile guest 
(1) Z. Dauter and K.S. Wilson. Diffraction techniques. In Comprehensive Supramolecular Chemistry. J.L. Atwood, J.E.D. Davies, D.D. MacNicol and F. Vogtle, Eds. Elsevier Science Ltd, New York, 1996. (Volume 8).

(2) J.L. Atwood. An Introduction to the Crystallography of Supramolecular Compounds, In Crystallography of Supramolecular Compounds, G. Tsoucaris, J.L Atwood and J. Lipkowski, Kluwer Academic Publishers: Dordrecht, 1996.

(3) G.R. Desiraju. The Crystal as a Supramolecular Entity, Wiley: New York, 1996.

(4) G.R. Desiraju. Crystal Engineering: The Design of Organic Solids, Elsevier, Amsterdam, 1989.

(5) G.R. Desiraju and T. Steiner. The Weak Hydrogen Bond: Applications to Structural Chemistry and Biology, Oxford University Press: Oxford, 1999.

(6) M. Nishio, M. Hirota and Y. Umezawa. The CH- $\pi$ Interaction: Evidence, Nature and Consequences, Wiley: New York, 1998.

(7) G.A. Jeffrey. An Introduction to Hydrogen Bonding, Oxford University Press: New York, 1997.

(8) F.H. Allen. Applications of the Cambridge Structural Database in the Study of Noncovalent Interactions, In Crystallography of Supramolecular Compounds, G. Tsoucaris, J.L Atwood and J. Lipkowski, Kluwer Academic Publishers: Dordrecht, 1996.

(9) J. Sauer. Computational Methods for Host-Guest Interactions, In Host-Guest Systems Based on Nanoporous Crystals, F. Laeri, Ed. Wiley: New York, 2003.

(10) G.R. Desiraju. Crystal Design: Structure and Function, Wiley: New York, 1996.

(11) Modern powder diffraction. In Reviews in Mineralogy. D.L. Bish and J.E. Post, Eds. Mineralogical Society of America, Washington, D.C. (1989).

(12) K.D.M. Harris, M. Treymayne and B.M. Kariuki. Angew. Chem. Int. Ed. 40(9), $1626(2001)$.

(13) K.D.M. Harris and M. Treymayne. Chem. Mater. 8(11), 2554 (1996).

(14) J.A. Ripmeester and C.I. Ratcliffe. Solid-State NMR Spectroscopv. In Comprehensive Supramolecular Chemistry J.L. Atwood, J.E.D. Davies, D.D. MacNicol, and F. Vogtle, Eds. Elsevier Science Ltd.: New York, 1996; Vol. 8, pp. $323-380$.

(15) C.A. Fyfe. Solid-State NMR for Chemists, C.F.C. Press: Guelph, 1983.

(16) Solid-State NMR Spectroscopy: Principles and Applications. M.J. Duer, Ed. Blackwell Science: Oxford, 2002.

(17) H.W. Spiess. Adv. Polym. Sci. 66, 23 (1985).

(18) J.H. Davis. In Isotopes in the physical and the biomedical sciences, E. Buncel, J.R. Jones, Eds, Elsevier Science: Amsterdam, 1991.

(19) R.G. Griffin. Methods. Enzymol. 72, 108 (1981).

(20) T. Brauniger, R. Poupko, H. Zimmerman and Z. Luz. Chem. Soc. Perkin Trans. II. 1255 (1997).

(21) C.I. Ratcliffe. J. Phys. Chem. 98(42), 10935 (1994).

(22) G.H. Penner, Y.C.P. Chang and J. Hutzal. Inorg. Chem. 38, 2868 (1999).

(23) P.S. Sidhu, G.H. Penner, K.R. Jeffrey, B. Zhao, Z.L. Wang and I. Goh. J. Phys. Chem. 101B, 9087 (1997). 
(24) P.S. Sidhu, J. Bell, G.H. Penner and K.R. Jeffrey. Can. J. Chem. 74,1784 (1996).

(25) J. Schmider and K. Muller. J. Phys. Chem. 102A, 1181 (1998).

(26) M.J. Duer, H. He, W. Kolodziejski and J. Klinowski. J. Phys. Chem. 98, 1198 (1994).

(27) D.F. Shantz and R.F. Lobo. J. Phys. Chem. 102B, 2339 (1998).

(28) T. Sato, K. Kunimori and S. Hayashi. Phys. Chem. Chem. Phys. 1, 3839 (1999).

(29) J.H. Davis, K.R. Jeffrey, M. Bloom, M.I. Valic and T.P. Higgs. Chem. Phys. Lett. 42, 390 (1976).

(30) M.S. Greenfield, A.D. Ronemus, R.L. Vold, R.R. Vold, P.D. Ellis and T.R. Raidy. J. Magn. Reson. 72, 89 (1987).

(31) A.F. Thomas. Deuterium labelling in organic chemistry. Meredith Corp., New York, 1971.

(32) R.J. Wittebort, E.T. Olejniczak and R.G. Griffin. J. Chem. Phys. 86, 5411 (1978).

(33) D.A. Torchia and A. Szabo. J. Magn. Reson. 49, 107 (1982).

(34) K. Schmidt-Rohr and H.W. Spiess. Multidimensional solid-state NMR and polymers. Academic Press, London, 1994.

(35) L.R. Nassimbeni Cryst. Eng. Commun. 5(35), 200 (2003).

(36) F. Giordano, C. Novak and J.M. Moyano Thermochim. Acta 380(2), 123 (2002).

(37) M.D. Veigamdveiga, H. Merino, D. Fernandez and R. Lozano J. Therm. Anal. Calorim. 68(2), 511 (2002).

(38) M.A. White. Thermal Properties of Materials In Crystal Engineering: The Design and Application of Functional Solids, K.R. Seddon and M. Zaworotko, Eds. Kluwer Academic: Dordrecht, 1999.

(39) M.A. White. Thermal Analysis and Calorimetry Methods. In Comprehensive Supramolecular Chemistry; J.L. Atwood, J.E.D. Davies, D.D. MacNicol and F. Vogtle, Eds. Elsevier Science Ltd., New York, 1996, Volume 8.

(40) P. Pyykko. Theory of Intermolecular Interactions, In Crystal Engineering: From Molecules and Crystals to Materials. D. Braga, F. Grepioni and A.G. Orpen, Eds. Kluwer Academic Publishers: Dordrecht, 1999.

(41) R.C. MacKenzie Differential Thermal Analvsis, Academic Press: London, 1970.

(42) G. Hoehne, W. Hemminger and H.J. Flammersheim Differcntial Scanning Calorimetry - An Introduction for Practitioners, Springer: Berlin, 1996.

(43) C. Duval Thermogravimetric Analysis, Elsvier, Amsterdam, 1953.

(44) R.F. Speyer. Thermal analvsis of materials. Marcel Dekker Inc., New York, 1994.

(45) L.R. Nassimbeni. Thermodynamics and Kinetics of Crystalline Inclusion Compounds In Crystal Engineering: From Molecules and Crystals to Materials. D. Braga, F. Grepioni and A.G. Orpen, Eds. Kluwer Academic Publishers: Dordrecht, 1999.

(46) T. Hatakeyama and F. Quinn. Thermal analysis: Fundamentals and applications to polymer science. John Wiley and Sons Ltd., Chichester, England, 1999. 
TATM was prepared as follows: 1200 grams of chloroform (Caledon, 99.8\%) was added to 400 grams of anhydrous aluminum chloride (Alfa Aesar, $95+\%$ ) in a 2 liter, threenecked round bottom flask, placed on a heating mantle on top of a stir plate. Before the reagents were added, a small magnetic stir bar was placed into the round bottom flask. Excessive exposure of the anhydrous $\mathrm{AlCl}_{3}$ to the atmosphere while weighing and transfer to the round bottom flask was avoided, as it reacts with ambient water vapor. The round bottom flask was connected to a reflux condenser, and the other two necks were sealed with appropriately sized glass stoppers. Then, while the mixture of $\mathrm{CHCl}_{3}$ and $\mathrm{AlCl}_{3}$ was stirring, 2-acetylthiophene (Aldrich, 98\%) was slowly introduced through the top of the reflux condenser, one or two $\mathrm{mL}$ at a time, being very careful that the mixture did not boil over. One or two $\mathrm{mL}$ is approximately one Pasteur pipet full. The reaction mixture was allowed to settle for about $10-30$ seconds between successive additions. A total of 200 grams of 2-acetylthiophene was added over the course of about $30-60$ minutes. Then the mixture was heated at the boiling point of chloroform $\left(61^{\circ} \mathrm{C}\right)$ for $6-8$ hours (the return of solvent from the reflux column to the round bottom flask was about one drop per second). After this time, there were a large number of black lumps in the flask that contained the TATM product. Then the heat was turned off, and the reaction quenching and purification was carried out the next day.

The reaction was quenched by addition of a $\mathrm{NaOH}$ solution, prepared by dissolving about 10 grams of sodium hydroxide pellets in $-500 \mathrm{~mL}$ distilled water. The $\mathrm{NaOH}$ solution was introduced slowly into the top of the reflux condenser, in the same manner as the addition of 2-acetylthiophene described above. The mixture was not allowed to boil over. The $\mathrm{NaOH}$ (a Bronsted base) consumed the unreacted $\mathrm{AlCl}_{3}$ (a Lewis acid), and the mixture became much more liquid in nature. After about $200-400$ $\mathrm{mL} \mathrm{NaOH}$ was added, the mixture was poured into a 4 liter beaker. Then about $100 \mathrm{~mL}$ of fresh chloroform was added to the residual product in the round bottom flask, and was heated to dissolve any TATM that was left behind. Then the washing was poured into the 4 liter beaker with the rest of the product.

There were two layers in the 4 liter beaker, the aqueous layer (that we didn't want) was the upper layer, while the deep red lower chloroform layer (that contained the 
TATM product) is what we wanted to isolate. The upper aqueous layer was decanted off as much as possible, being very careful not to lose any of the lower red chloroform layer. Then about half of the chloroform layer was added into a 2 liter separatory funnel and then washed with 2-3 separate aliquots of distilled water, each time draining the chloroform layer out the bottom of the separatory funnel, leaving the upper water layer behind. This washed chloroform layer was poured into to a clean 4 liter beaker, and the second half of the original chloroform solution was washed with distilled water in a similar manner, and then this second washed chloroform layer was added to the first washed chloroform layer in the 4 liter beaker. Then this 4 liter beaker, containing the washed chloroform layers (deep red in color) was placed into a fume hood, to allow the excess chloroform to evaporate off over the course of $1-2$ days, leaving behind a crude TATM product.

Then, after the beaker contents were completely dry, the crude product was recrystallized with $2-3$ liters of anhydrous ethanol (Commercial Alcohols Inc. 98\%). The ethanol product mixture was heated until it was completely dissolved, then was allowed to cool overnight on a stir plate with continuous stirring. The next day, the purified ethanol / TATM inclusion compound crystallized, and was filtered under suction. Then the remaining ethanol / TATM mixture was concentrated, by heating, down to about $500 \mathrm{~mL}$, and allow to stir overnight again. The additional purified ethanol / TATM product was again filtered under suction. The sample was allowed to dry briefly, then transfered to $2-3$ screw cap bottles. The yield was about 100 grams $(\sim 50$ $\%$ yield). This ethanol / TATM product had a visual melting point of $100.5^{\circ}-104.0^{\circ} \mathrm{C}$.

Single crystal X-ray diffraction data were measured on a Bruker Smart diffractometer equipped with a CCD detector, using graphite monochromatized Mo $K_{\alpha}$ radiation $(\lambda=0.71073 \AA)$ in the $\omega$ scan mode. The acquisition temperatures were either $125 \mathrm{~K}$ or $173 \mathrm{~K}$. The data reduction included correction for Lorentz and polarization effects. The crystal structures were solved by direct methods using the NRCVAX suite of programs, $|1|$ and refined by full-matrix least-squares routines using the SHELXTL program [2]. A multiscan absorption correction was made with SADABS [3] software. Direct methods yielded all non-hydrogen atoms in the unit cell, and all non-hydrogen atoms were refined anisotropically. The host hydrogen atoms were located in the 
difference Fourier map, while the guest hydrogens were placed in calculated positions with a riding temperature factor and bond length constraints.

$\mathrm{X}$-ray powder diffraction patterns (PXRD) were recorded at room temperature on a Rigaku Geigerflex vertical goniometer diffractometer with DRX Win 1.3 software, using graphite monochromatized Co $K_{\alpha}$ radiation $(\lambda=1.79 \AA)$ in the $\theta-\theta$ scan mode. Samples were scanned over a range $10^{\circ}<2 \theta<40^{\circ}$, with a 30 second accumulation time at an increment of $0.02^{\circ}$ in $2 \theta$, giving a total acquisition time for each sample of approximately 12 hours. We found that this acquisition time was necessary on our instrument to obtain good quality spectra. Voltage and current were set at $30 \mathrm{kV}$ and 20 $\mathrm{mA}$, respectively.

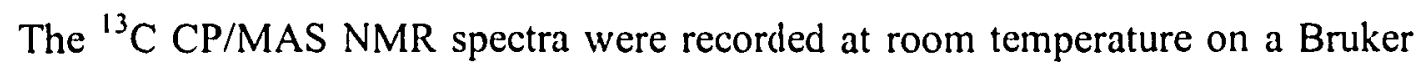
AMX 300 NMR spectrometer at a frequency of $75.483 \mathrm{MHz}$ for ${ }^{13} \mathrm{C}$ and $300.145 \mathrm{MHz}$ for ${ }^{1} \mathrm{H}$. The polycrystalline powder samples were spun in a $5 \mathrm{~mm}$ Doty magic anglespinning (MAS) probe at frequencies between $5 \mathrm{kHz}$ and $6 \mathrm{kHz}$. The numbers of scans collected was approximately ten thousand, with $3.8 \mu \mathrm{s} 90^{\circ}$ pulses, $5 \mathrm{~ms}$ cross polarization contact time, $51.2 \mathrm{~ms}$ acquisition time and a $10 \mathrm{~s}$ recycle delay. Typically 4096 data points were accumulated with zero filling to $16 \mathrm{~K}$, resulting in a digital resolution of about $2.4 \mathrm{~Hz} /$ point. The chemical shifts are given relative to tetramethylsilane at zero $\mathrm{ppm}$, and were referenced externally (by rotor replacement) to the high field signal of hexamethylbenzene $(16.9 \mathrm{ppm})$. Dipolar dephasing experiments $[4 \mid$ were performed by inserting, between the end of cross polarization and the start of ${ }^{\prime} \mathrm{H}$ decoupled signal acquisition, a short delay ( $40 \mu \mathrm{s})$ with no proton decoupling. This pulse sequence eliminates signals from methine $(\mathrm{CH})$ and methylene $\left(\mathrm{CH}_{2}\right)$ carbons, provided they are not reorienting, on the directly bonded ${ }^{13} \mathrm{C}-{ }^{1} \mathrm{H}$ heteronuclear dipolar coupling timescale.

The ${ }^{2} \mathrm{H}$ NMR spectra were recorded on a Bruker AMX 300 NMR spectrometer at a frequency of $46.07 \mathrm{MHz}$. The polycrystalline powder samples were packed in $6 \mathrm{~mm}$ o.d. nmr tubes of length $\sim 2 \mathrm{~cm}$. The deuterium NMR spectra were obtained using the quadrupole echo pulse sequence: $[5](\pi / 2)_{ \pm x}-\tau_{Q}-(\pi / 2)_{y}-\tau_{Q}-$ acquire. The length of the $\pi / 2$ pulses were approximately $3.0 \mu \mathrm{s}$, and a recycle delay of 1 second was used. At each temperature, the echo signals were collected using a $\tau_{\mathrm{Q}}$ value of $20 \mu \mathrm{s}$, and after left shifting to reach the echo maximum, the echo signals were Fourier transformed to obtain 
the NMR spectra. In order to improve the signal / noise ratio, spectra were symmetrized by adding the original spectrum to the same spectrum reflected about zero frequency. The temperature of the sample was lowered by passing cold nitrogen gas through the sample chamber, in conjunction with a heater in the gas stream and the temperature was regulated by coupling the heater to a copper constantan thermocouple located close to the sample itself. The temperature of the sample was stable to within $\pm 0.1^{\circ} \mathrm{C}$ over the course of a measurement.

Quadrupolar echo spectra were simulated using the program MXQET [6]. This program takes into account variables such a $90^{\circ}$ pulse length, pulse spacing $\tau_{\mathrm{Q}}$ and spectral line broadening. Simulated spectra were visually matched to experimental lineshapes.

The deuterium $T_{1}$ data were determined using a home-built, phase-coherent pulsed NMR spectrometer operating a $44.7 \mathrm{MHz}$. The deuterium $T_{1}$ data were acquired using an inversion recovery pulse sequence modified for quadrupolar nuclei: $(\pi)_{x^{-}-\tau-}$ $(\pi / 2)_{ \pm x}-\tau_{\mathrm{Q}}-(\pi / 2)_{y^{-}}-\tau_{\mathrm{Q}}$-acquire. Typically, 12 values of $\tau$ were used to determine $T_{1}$ at each temperature. The pulse spacing, $\tau_{0}$, for the $T_{1}$ determination was $50 \mu \mathrm{s}$. The time between repetitions of the pulse sequence was always greater than $5 T_{1}$. The amplitude of the signal for each $\tau$ value was determined from the integral over the echo peak. The echo integrals were fit using a nonlinear least-squares algorithm employing an exponential fitting function to obtain the relevant relaxation time parameters.

Thermogravimetric analysis (TGA) experiments were performed on a commercial TA Instruments TGA 2050 series system with Thermal Solutions for Windows NT software. Samples of 10 to $20 \mathrm{mg}$ were placed in open aluminum pans and sealed in the furnace. The sample masses were precise to $\pm 0.001 \mathrm{mg}$. Nitrogen was used as a purge gas, flowing at a rate of $40 \mathrm{~mL}$ per minute. The samples were heated at a rate of $5^{\circ} \mathrm{C}$ per minute, from room temperature to about $350^{\circ} \mathrm{C}$.

Differential scanning calorimetry (DSC) experiments were performed on a commercial TA Instruments DSC 2920 series system with Thermal Solutions for Windows NT software. Finely powdered specimens, obtained from continuously stirred solutions, were hermetically sealed in aluminum pans, specified to be able to withstand pressures up to five atmospheres. The sample masses were usually between $1-5 \mathrm{mg}$, and 
were precise to $\pm 0.01 \mathrm{mg}$. The samples were heated from room temperature to about $250^{\circ} \mathrm{C}$, at a rate of $5^{\circ} \mathrm{C}$ per minute. The samples were purged by a stream of helium flowing at a rate of $40 \mathrm{~mL}$ per minute. The calorimeter was calibrated by making a run (at $5^{\circ} \mathrm{C}$ per minute) with an accurately weighed amount of indium metal, whose heat of fusion is known accurately $\left(T_{\mathrm{m}}=156.4^{\circ} \mathrm{C}, \Delta_{\text {fus }} H=28.5 \mathrm{~J} \mathrm{~g}^{-1}\right)$. An empty pan was used for baseline calibration.

Polycrystalline density measurements were performed using a fully automated gas displacement AccuPyc 1330 brand pycnometer. The pycnometer determines sample volume by measuring the pressure drop of an inert gas as it expands from one chamber (containing the sample) into an adjacent empty chamber. The analysis measures sample volume, from which density may be derived automatically if the sample mass is known. All sample and calibration runs were performed ten times in succession, from which the average and standard deviation of the final results were obtained. The final results were obtained twice, on consecutive days. A stainless steel metal ball standard with a very accurately measured volume $(0.718581$ ( \pm 0.000001$)$ cubic centimeters) was used to calibrate the system. The samples were weighed on an analytical balance, precise to \pm $0.01 \mathrm{mg}$, and helium gas was employed for both chamber purge and volume measurements. The chamber purge served to remove ambient air, moisture and other contaminants from the cell.

CHAPTER VI

REFERENCES

(1) E.J. Gabe, Y. LePage, J.P. Charland, F.L. Lee and P.S. White. J. Appl. Cryst. 22, 384 (1989).

(2) Sheldrick, G. M. SHELXTL, Version 6.10; Bruker AXS Inc., Madison, Wisconsin, USA, 2000.

(3) Sheldrick, G. M. SADABS (Siemens $A$ rea Detector $A B$ Sorption), Version 2.03 Bruker-Nonius BV, Madison, Wisconsin, USA, 2002.

(4) S.J. Opella and M.H. Frey. J. Am. Chem. Soc. 101, 5855 (1979).

(5) J.H. Davis, K.R. Jeffrey, M. Bloom, M.I. Valic and T.P. Higgs. Chem. Phys. Lett. 42, 390 (1976).

(6) M.S. Greenfield, A.D. Ronemus, R.L. Vold, R.R. Vold, P.D. Ellis and T.R. Raidy. J. Magn. Reson. 72, 89 (1987). 


\section{CHAPTER VII}

Polymorphism, structure, guest conformation and dynamics in the inclusion compound of 1,2dichloroethane with tris (5-acetyl-3-thienyl) methane:

a combined single crystal and powder X-ray diffraction, ${ }^{13} \mathrm{C} \mathrm{CP} / \mathrm{MAS}$ and ${ }^{2} \mathrm{H}$ NMR study

$\begin{array}{ll}\text { Abstract } & 97 \\ \text { Introduction } & 98 \\ \text { Experimental } & 98 \\ \text { Results } & \\ \text { (A) Single crystal X-ray Diffraction } & 99 \\ \text { (B) Powder X-Ray Diffraction } & 103 \\ \text { (C) }{ }^{13} \text { C CP/MAS NMR } & 104 \\ \text { (D) }{ }^{2} \text { H Solid-State NMR } & 106 \\ & \text { (1) Model 1: Tians-Gauche Equilibrium } \\ & 106 \\ & \text { (2) Model 2: Restricted Cl Motion } \\ \text { (3) Model 3: Large Scale Cl Motion } & 112 \\ \text { Concluding remarks } & 113 \\ \text { References } & 116 \\ \end{array}$




\begin{abstract}
Various physicochemical characterization techniques were used to study the structure and guest dynamics of the 1,2-dichloroethane / tris (5-acetyl-3-thienyl) methane (TATM) inclusion compound. This complex was observed to crystallize in two forms, a triclinic $\mathrm{P} \overline{1}$ structure and a less usual monoclinic $\mathrm{P} 2_{1} / \mathrm{c}$ form. The $X$-ray structures indicate that the TATM molecules form "bottleneck" channels parallel to the crystallographic $a$ and $c$ axes respectively, and that the guests exist exclusively in the trans form. Solid-state deuterium NMR spectroscopy was used to study the dynamics of the 1,2-dichloroethane$\mathrm{d}_{4}$ guest in the triclinic form. The simplest model consistent with the experimental NMR spectra was based on internal rotation about the $\mathrm{C}-\mathrm{C}$ bond (a trans / gallche conformational equilibrium). As this was not consistent with the X-ray structure, another model was developed where the trans conformer performs $180^{\circ}$ flips about an axis through the centre of symmetry of the guest and perpendicular to the $\mathrm{Cl}-\mathrm{C}-\mathrm{C}-\mathrm{Cl}$ plane. The important point to be taken from this work is that complementary methods are often necessary to discriminate between motional models based on a single technique, and that the simplest model is not necessarily correct.
\end{abstract}




\section{INTRODUCTION}

In molecular crystals, structural disorder often arises when molecules are able to take up more than one orientation (not necessarily with equal energy) within the space available to them. Fundamentally, such disorder raises important issues concerning the intermolecular interactions within the crystal. Although the space- and time- averaged crystal structure, as determined by diffraction-based techniques, can generally indicate the existence of disorder, it is often unable to provide information at a sufficiently detailed level. Techniques that can be used to study local structural and/or dynamic properties of solids must then come to the fore, and in this regard solid-state NMR spectroscopy proves to be a versatile and powerful approach, providing important information to be used in close conjunction with the crystallographic techniques.

The void space in inclusion compounds provides a convenient way of confining guests in order to test our ability to describe their dynamic states. In the case of rigid guests, or guests with a limited number of allowed conformers, considerable progress has been made. However, because of the additional degrees of freedom for aliphatic compounds such descriptions are more complicated. In this contribution we use a variety of methods to study the structure and dynamics of 1,2-dichloroethane included in the tris (5-acetyl-3-thienyl) methane (TATM) host compound. In the pure solid, the guest molecule is known to undergo dynamic processes that involve $180^{\circ}$ jumps of the molecule about the $\mathrm{Cl}-\mathrm{Cl}$ axis as a rigid unit [1-4]. In the gas phase, however, the guest motion is isotropic and shows conformational isomerism [5]. In at least one inclusion compound, the clathrate hydrate, it is known that size constraints force the 1,2dichloroethane guest into a gauche conformation [6]. Thus, questions can be asked about the state of the guest in a cavity: is it more like a gas or a solid in the inclusion compound void space? It proved to be a surprisingly challenging task to arrive at a model that was consistent with the data obtained from the various experimental methods employed.

\section{EXPERIMENTAL}

Commercially available reagents were used as received (Aldrich). The 1,2dichloroethane / TATM inclusion compound was crystallized by two different methods. In the first (slow evaporation), a hot saturated solution of about $300 \mathrm{mg}$ TATM in $\sim 2 \mathrm{~mL}$ 
of 1,2-dichloroethane was poured into a glass petri dish and the excess 1,2-dichloroethane was allowed to evaporate at room temperature over the course of approximately 1-2 days. This sample was used for single crystal X-ray diffraction analysis. In the other method (slow cooling), again $300 \mathrm{mg}$ TATM was dissolved in $2 \mathrm{~mL}$ of 1,2-dichloroethane and then sealed in a glass ampoule. The ampoule was then placed in an oven, and cooled from the boiling point of 1,2 -dichloroethane $\left(83^{\circ} \mathrm{C}\right)$ to room temperature, at a rate of $5^{\circ} \mathrm{C}$ per day. The resulting crystals were then filtered and ground into a fine powder (this sample was not used for single crystal X-ray diffraction analysis).

\section{RESULTS}

\section{(A) Single crystal X-ray diffraction}

The crystals used for single crystal $\mathrm{X}$-ray analysis were grown by slow evaporation. That is, a hot saturated solution of about $300 \mathrm{mg}$ TATM in approximately $2 \mathrm{~mL} \mathrm{1,2-}$ dichloroethane was poured into a glass petri dish, and the excess solvent was allowed to evaporate slowly over the course of 1-2 days. As will be shown below, this sample contains a mixture of both crystal forms. The first crystal chosen for analysis crystallized in the triclinic $\mathrm{P} \overline{\mathrm{T}}$ space group. The crystal data, data collection and final refinement data for the structure at $173 \mathrm{~K}$ appear in Table VII-1. There are two host molecules in the unit cell, related by a centre of symmetry, giving one host TATM molecule in the asymmetric unit. There is one-half guest 1.2-dichloroethane molecule in the asymmetric unit, and the guest exists exclusively in the trans form. The centre of inversion of the guest coincides with the centre of inversion of the unit cell. The guest is disordered over three positions. A schematic illustration of the unit cell, showing the atomic numbering scheme, is depicted in Figure VII-1. Only one guest molecule is shown for clarity. The three guest site occupancy factors are $0.58,0.23,0.19$. A molecular packing diagram of the triclinic form, in the $b c$ plane, (at $173 \mathrm{~K}$ ) appears in Figure VII-2A. The TATM molecules form channels parallel to the crystallographic $a$ axis in which the guest molecules reside. The term "channel" should be regarded with some reserve, however, as there is a significant constriction of the channel between adjoining guest molecules that prevents the guests' translation from one guest site to the next. In other words, there is a "bottleneck" in the channel between adjacent guests. Of the three acetylthienyl rings in the asymmetric unit, 
two participate in the formation of the guest channel, while the third interacts via $\pi-\pi$ stacking with the TATM host in the next unit cell to form an endless ribbon. In one layer, many ribbons are arranged parallel to one another to form sheets. Figure VII-2A depicts a short section of a few such ribbons. Immediately on either side of a host cavity / guest molecule is located a pair of $\pi$ stacked thienyl rings of adjacent ribbons, and on either side of the pair of $\pi$ stacked thienyl rings is located a host cavity / guest molecule of adjacent ribbons.

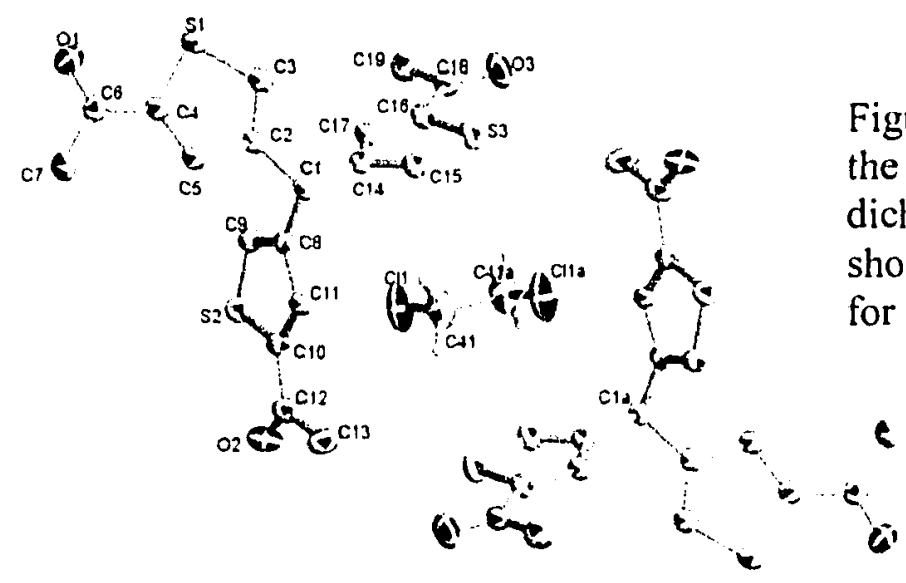

Figure VII-1: A schematic depiction of the unit cell of the triclinic form of 1,2dichloroethane / TATM at $173 \mathrm{~K}$, showing the atomic numbering scheme for both forms

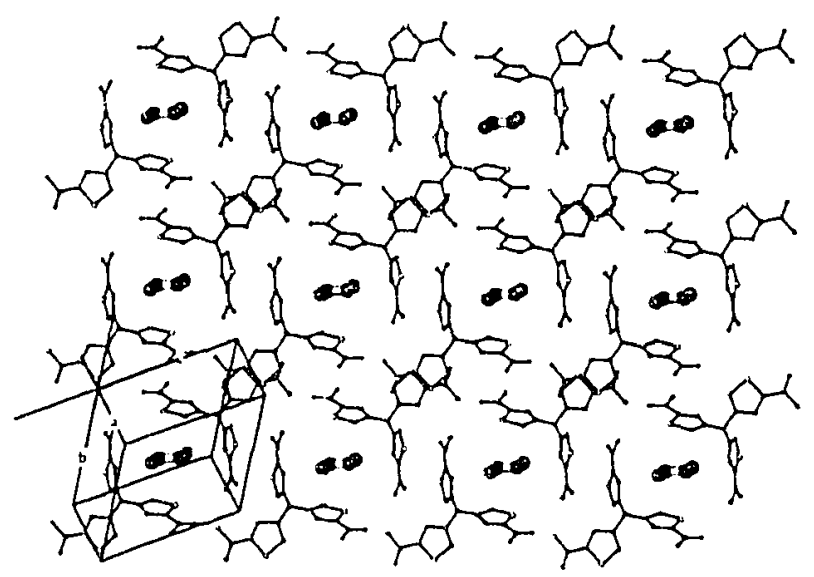

Figure VII-2A: A packing diagram of the triclinic form of 1,2-dichloroethane /TATM approximately down the channel axis ( $a$ direction), showing only one guest position for clarity. 

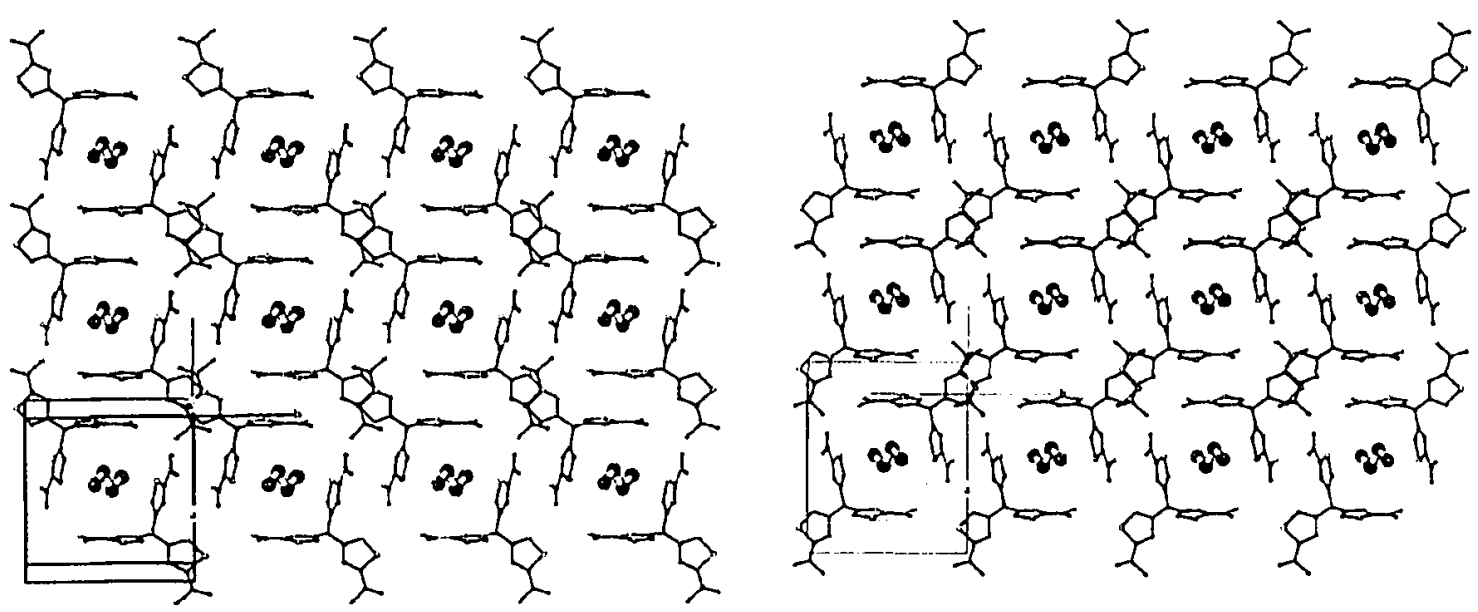

Figure VII-2B:Two packing diagrams (of adjacent layers) of the monoclinic form of 1,2dichloroethane / TATM approximately down the channel axis ( $c$ direction), showing only one guest position for clarity.

We also found that the inclusion compound crystallizes in a monoclinic form, in the $\mathrm{P} 2_{\mathrm{J}} / \mathrm{c}$ space group. A perspective view of two adjacent sheets in the monoclinic form appears in Figure VII-2B. The unit cell axes are shown with each sheet to show that the view is identical for each layer. The difference between the two forms arises from the layering of ribbons in adjacent sheets (within one layer, the two forms are almost isostructural). In the triclinic form, the adjacent sheets are related by a unit cell translation, and the ribbons in adjacent sheets run parallel to one another. In the monoclinic form, the adjacent sheets are related by a $c$ glide, and ribbons in adjacent sheets run in perpendicular directions. This is illustrated in Figure VII-2B, for which in the view to the left, the ribbons run in the NW-SE direction, while the ribbons in the adjacent layer (view to the right) run in the NE-SW direction. This suggests that the two forms should not interconvert on standing (at least during the timeframe between our preparation and characterization). Finally, we found that there is extensive weak aromatic $\mathrm{C}-\mathrm{H} \cdots \mathrm{O}$ hydrogen bonding throughout the structure that apparently stabilizes the framework in both forms. Between layers there are only a few of these interactions, but within a layer there are many more. This suggests that stabilizing interactions within a layer are much stronger than the same interactions between layers. The crystal data for the monoclinic form at $173 \mathrm{~K}$ appears in Table VII-I. 
Table VII-1: Crystal data, data collection and final refinement parameters for the two crystal forms of 1,2-dichloroethane / TATM.

\section{Crystal Data}

Molecular formula

Molecular weight (amu)

Temperature (K)

Crystal system

Space Group

$a(\AA)$

$b(\AA)$

$c(\AA)$

$\alpha\left({ }^{\circ}\right)$

$\beta\left(^{\circ}\right)$

$\gamma\left({ }^{\circ}\right)$

$V\left(\AA^{3}\right)$

$Z$

$d_{\text {calc }}\left(\mathrm{g} \mathrm{cm}^{-3}\right)$

$F(000)$

$\mu(\mathrm{MoK} \alpha)\left(\mathrm{mm}^{-1}\right)$

$\lambda(\AA)$

$2 \theta$ range $\left(^{\circ}\right)$

Data Collection

Crystal dimensions ( $\mathrm{mm}$ )

Index ranges

Reflections measured

Unique reflections

$R$ (int)

Completeness to $2 \theta_{\operatorname{mix}}$

Scan mode

Absorption correction

Final Refinement

Refinement method

Data/restraints/parameters

Goodness of fit

Final $R$ indices $[\mathrm{I}>2 \sigma(\mathrm{I})]$

$R$ indices (all data)

Highest peak (e $\AA^{-3}$ )

Deepest hole $\left(\mathrm{e} \AA^{-3}\right)$
$2 \mathrm{C}_{19} \mathrm{H}_{16} \mathrm{O}_{3} \mathrm{~S}_{3} \quad 1 \mathrm{C}_{2} \mathrm{H}_{4} \mathrm{Cl}_{2}$

875.98

173

Triclinic

$\mathrm{P} \bar{l}$

$8.0332(5)$

$10.9065(7)$

$12.3063(8)$

$97.207(10)$

$106.630(10)$

$96.686(10)$

$1011.70(11)$

1

1.438

454

0.517

0.71073

$1.75^{\circ}<2 \theta<28.72^{\circ}$

$0.30 \times 0.35 \times 0.40$

$-10 \leq h \leq 10$

$-14 \leq k \leq 14$

$-16 \leq l \leq 16$

11818

5170

0.0350

$98.8 \%$

omega

empirical

Full-matrix least squares

$5170 / 3 / 341$

0.788

$R_{1}=0.0443, w R_{2}=0.1190$

$R_{1}=0.0523, w R_{2}=0.1300$

0.583

$-0.461$
$2 \mathrm{C}_{19} \mathrm{H}_{16} \mathrm{O}_{3} \mathrm{~S}_{3} \quad 1 \mathrm{C}_{2} \mathrm{H}_{4} \mathrm{Cl}_{2}$

875.98

173

Monoclinic

$\mathrm{P} 2$ / $/ \mathrm{c}$

$12.6902(7)$

$12.7431(7)$

$13.7376(7)$

90

$109.046(10)$

90

2099.90 (20)

2

1.379

900

0.497

0.71073

$1.70^{\circ}<2 \theta<28.77^{\circ}$

$0.20 \times 0.35 \times 0.40$

$-17 \leq h \leq 17$

$-17 \leq k \leq 17$

$-18 \leq l \leq 18$

24125

5453

0.0266

$95.7 \%$

omega

empirical 
(B) Powder X-ray diffraction (PXRD)

The powder X-ray diffraction pattern of the 1,2-dichloroethane / TATM sample obtained by slow cooling appears in Figure VII-3. The powder X-ray diffraction pattern of the same inclusion compound obtained by slow evaporation appears in Figure VII-4. The second pattern contains many more peaks than the first, so it may be reasonable to assume that the 1,2-dichloroethane / TATM inclusion compound obtained by slow cooling contains only one of the two phases, while the sample obtained by slow evaporation contains a mixture of both phases. In fact, a detailed analysis of the experimental powcier X-ray diffraction peak positions $(2 \theta)$ in comparison with those calculated from the respective single crystal structures show that the sample prepared by slow cooling contains only the triclinic phase, while the sample prepared by slow evaporation contains both the triclinic and monoclinic phases. Figure VII-3 depicts the observed powder X-ray diffraction pattern for the inclusion compound prepared by slow cooling, along with the calculated reflection angles for the triclinic phase and the monoclinic phase. As can be seen in the figure, there is very good correspondence between the observed reflection angles and the calculated reflection angles for the triclinic phase. In contrast, there is no agreement between the observed reflections and the calculated reflection angles for the monoclinic phase. This conclusively shows that the sample of 1,2-dichloroethane / TATM prepared by slow cooling contains only the triclinic phase.

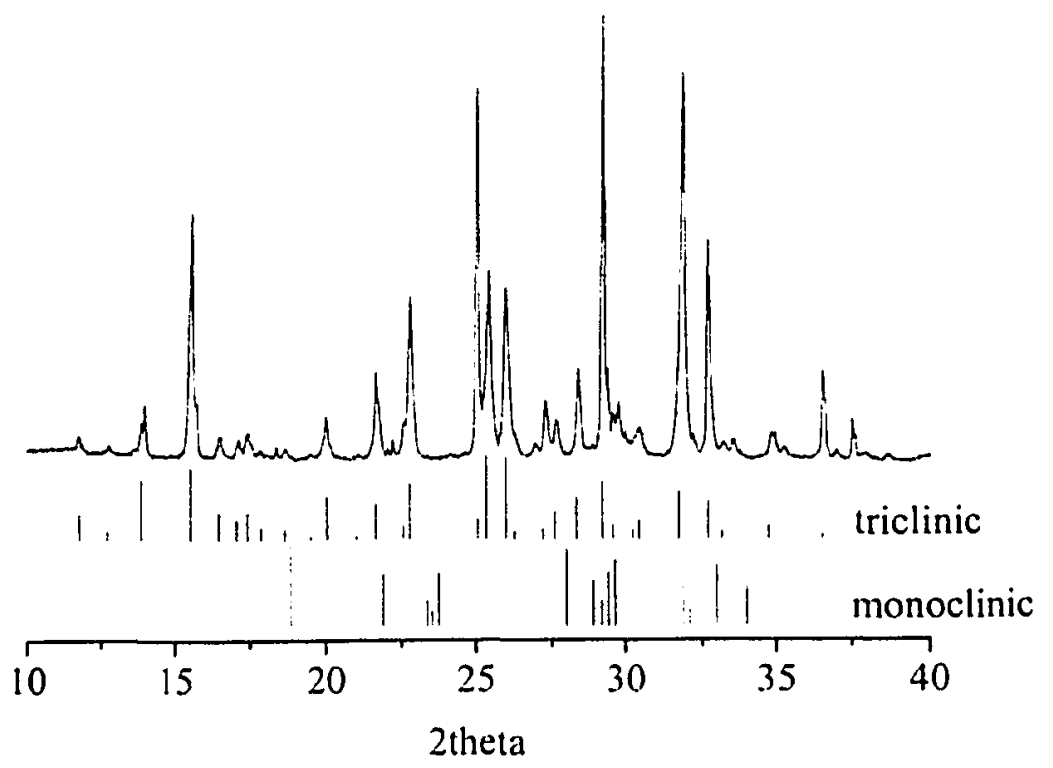

Figure VII-3: Powder $\mathrm{X}$-ray diffractogram for a 1,2-dichloroethane/TATM sample prepared by slow cooling (triclinic form only), along with peak positions calculated from the unit cell parameters of both forms. Note correlation with the triclinic form only. 
The experimental powder X-ray diffraction pattern for the inclusion compound prepared by slow evaporation appears in Figure VII-4, along with the calculated angles for the triclinic phase and the monoclinic phase. In this case, there are a number of reflections that cannot be accounted for by the calculated angles for the triclinic cell. However these reflections can be assigned to calculated reflections for the monoclinic cell. Therefore, this shows that the sample of 1,2-dichloroethane / TATM prepared by slow evaporation contains a mixture of the triclinic phase and the monoclinic phase.

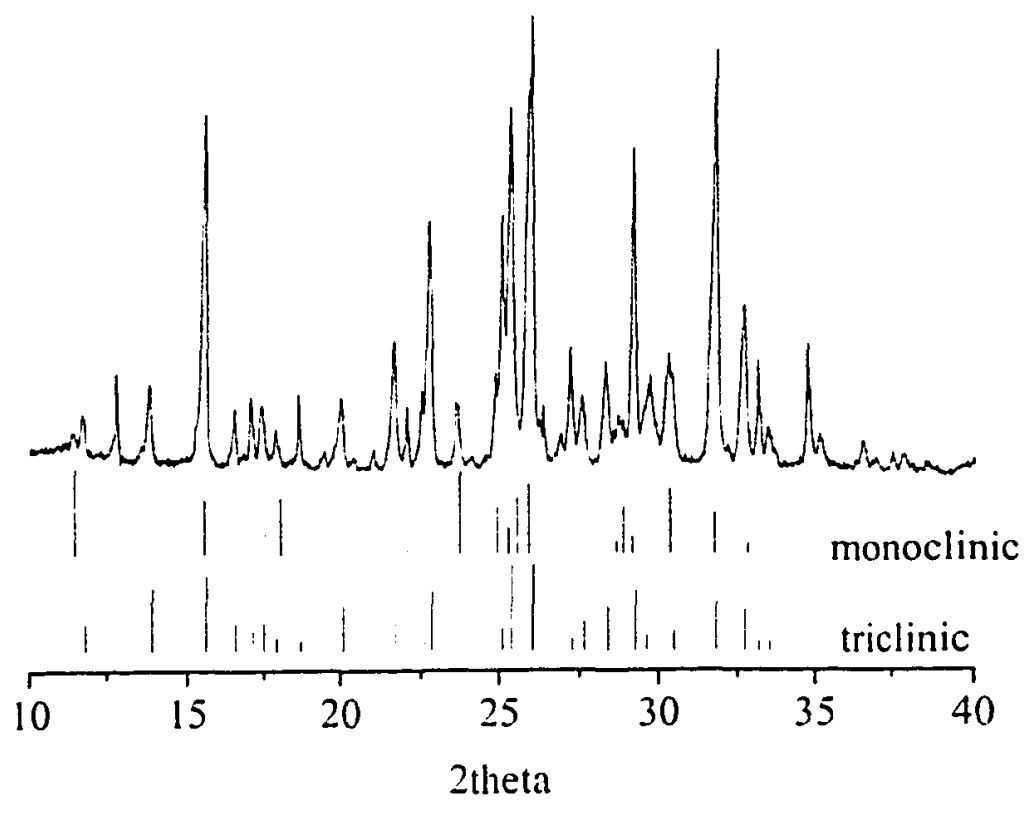

Figure VII-4: Powder $\mathrm{X}$-ray diffractogram for a 1,2-dichloroethane/TATM sample prepared by slow evaporation (triclinic and monoclinic mixture), along with peak positions calculated from the unit cell parameters of both forms. Note correlation with both triclinic and monoclinic forms.

\section{(C) ${ }^{13} \mathrm{C}$ CP/MAS NMR}

The ${ }^{13} \mathrm{C}$ CP/MAS NMR spectrum of 1,2-dichloroethane / TATM prepared by slow cooling appears in Figure VII-5A. As was noted in the previous section, this sample contains only the triclinic phase. The multiplicity of the TATM carbonyl signal at about $192 \mathrm{ppm}$ is three, and the multiplicity of the TATM methyl signal at about $28 \mathrm{ppm}$ is also three. This implies that there is one TATM molecule in the asymmetric unit for this sample, as there are three carbonyl groups and three methyl groups in one TATM molecule. This result agrees with the single crystal X-ray diffraction analysis. In the single crystal structure, there are two TATM molecules in the unit cell, and a centre of symmetry relates these two host molecules. The multiplicity of the TATM thienyl ring quaternary carbons at $146 \mathrm{ppm}$ (they remain on dipolar dephasing) is six, as is the 
multiplicity of the TATM thienyl ring tertiary $\mathrm{C}-\mathrm{H}$ carbons at about $135 \mathrm{ppm}$. As there are six quaternary carbons and six tertiary carbons in one TATM molecule, this provides further support. The 1,2-dichloroethane signal resonates at $44.5 \mathrm{ppm}$ (the signal at 42 $\mathrm{ppm}$ comes from the TATM methine carbon), which is $0.6 \mathrm{ppm}$ downfield of the resonance position of 1,2-dichloroethane in the neat liquid $(43.9 \mathrm{ppm})$. This implies that 1,2-dichloroethane's nuclei are deshielded in the TATM inclusion compound relative to its state in the pure liquid.
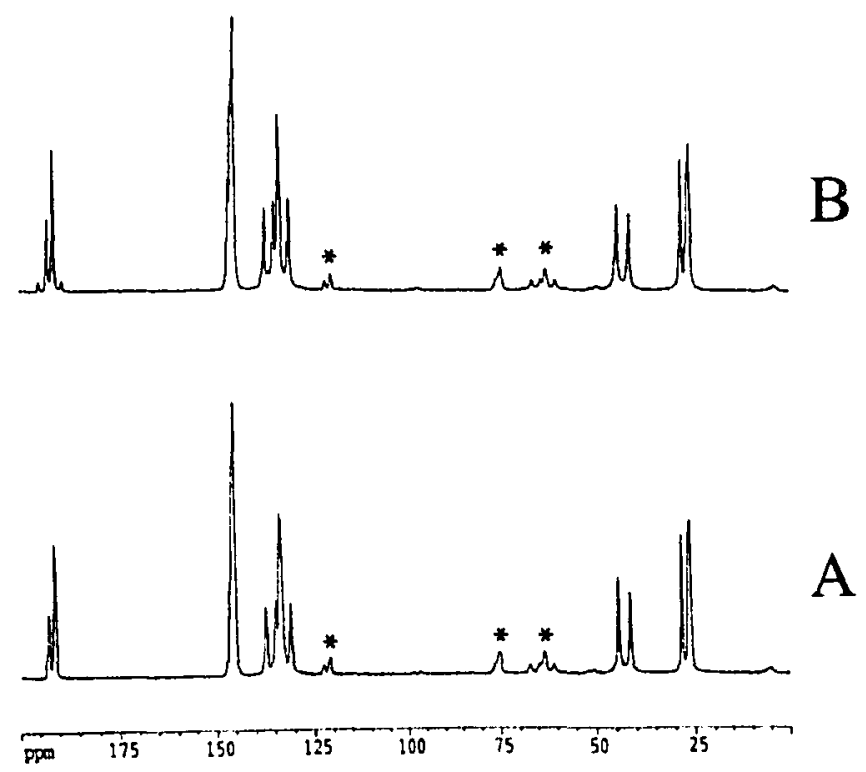

Figure VII-5: ${ }^{13} \mathrm{C}$ CP/MAS NMR spectra for 1,2-dichloroethane/ TATM samples. Spinning sidebands are marked with an asterisk:

(A) Sample prepared by slow cooling (triclinic form only).

(B) Sample prepared by slow evaporation (triclinic and monoclinic mixture).

The ${ }^{13} \mathrm{C}$ CP/MAS NMR spectrum of the 1,2-dichloroethane / TATM inclusion compound prepared by slow evaporation appears in Figure VII-5B. As was noted in the last section, this sample contains a mixture of triclinic and monoclinic phases. The spectrum appears identical to the spectrum of the sample prepared by slow cooling. However now there is one small peak to high field of the TATM carbonyl signals and one small peak to low field of the TATM carbonyl signals. These peaks are not spinning sidebands, as identical spinning rates were used in the acquisition of the two spectra. Therefore these small peaks must be from the small amount of monoclinic 1,2-dichloroethane / TATM in the sample prepared by slow evaporation. There is also a small peak visible at $136 \mathrm{ppm}$ partially hidden under the main signal from the TATM tertiary thienyl signal. There are likely also other weak signals corresponding to the other host TATM carbons and 1,2- 
dichloroethane guest, but they may be hidden underneath the larger signals corresponding to the triclinic 1,2-dichloroethane form. Thus we see agreement between results obtained from powder $\mathrm{X}$-ray diffraction and those obtained from ${ }^{13} \mathrm{C} \mathrm{CP} / \mathrm{MAS} \mathrm{NMR}$, demonstrating the complementary information available from these two experimental techniques.

\section{(D) Deuterium solid-state NMR}

Initially, the NMR dynamic spectra were treated as an independent data set. That means that a likely model was chosen, in this case consistent with rotational isomerism as in the gas phase, and analyzed to be internally consistent. This was then placed alongside the model from single crystal XRD to check for consistency among techniques. The initial NMR model then had to be abandoned, as it was not possible to arrive at a consistent model. A second dynamic model was then chosen to conform to the structural data. Both NMR models are presented, as there is an important lesson to be learned regarding uniqueness of models and the importance of complementary approaches.

(1) Model 1: Trans / gauche conformational equilibrium

The deuterium NMR spectra (from $296 \mathrm{~K}$ to $226 \mathrm{~K}$ ) for the 1,2-dichloroethane- $\mathrm{d}_{4} /$ TATM inclusion compound, along with simulated spectra, appear in Figure VII- 6 . The details of the parameters used in these simulations are outlined below. It should be noted that the sample prepared for analysis by deuterium NMR spectroscopy was made by slow cooling of a hot saturated solution, and therefore contains only the triclinic phase. The ${ }^{2} \mathrm{H}$ NMR spectra were simulated by considering the conformations of the guest that are encountered when one $\mathrm{CD}_{2} \mathrm{Cl}$ group is held fixed, while the other is rotated 360 degrees about the $\mathrm{C}-\mathrm{C}$ bond. The lowest energy trans conformer has a $180^{\circ}$ dihedral angle between the two $\mathrm{C}-\mathrm{Cl}$ bonds. The two higher energy gauche conformers have dihedral angles of $60^{\circ}$ and $-60^{\circ}$ between the two $\mathrm{C}-\mathrm{Cl}$ bonds. This gives three potential energy minima. There are also three potential energy maxima. The highest energy maximum corresponds to a dihedral angle of $0^{\circ}$ between the two $\mathrm{C}-\mathrm{Cl}$ bonds, denoted as the eclipsed $\left(0^{\circ}\right)$ transition state. The two lower energy maxima correspond to a $120^{\circ}$ dihedral angle between the $\mathrm{C}-\mathrm{Cl}$ bonds, denoted the eclipsed $\left(120^{\circ}\right)$ transition state. A 
potential energy diagram based on this scheme is depicted in Figure VII-7, which shows the rotational potential energy of the guest as a function of the dihedral angle between the two $\mathrm{C}-\mathrm{Cl}$ bonds, and Newman projections of the 1,2-dichloroethane- $\mathrm{d}_{4}$ conformers an each potential energy minimum and maximum.

Experimental
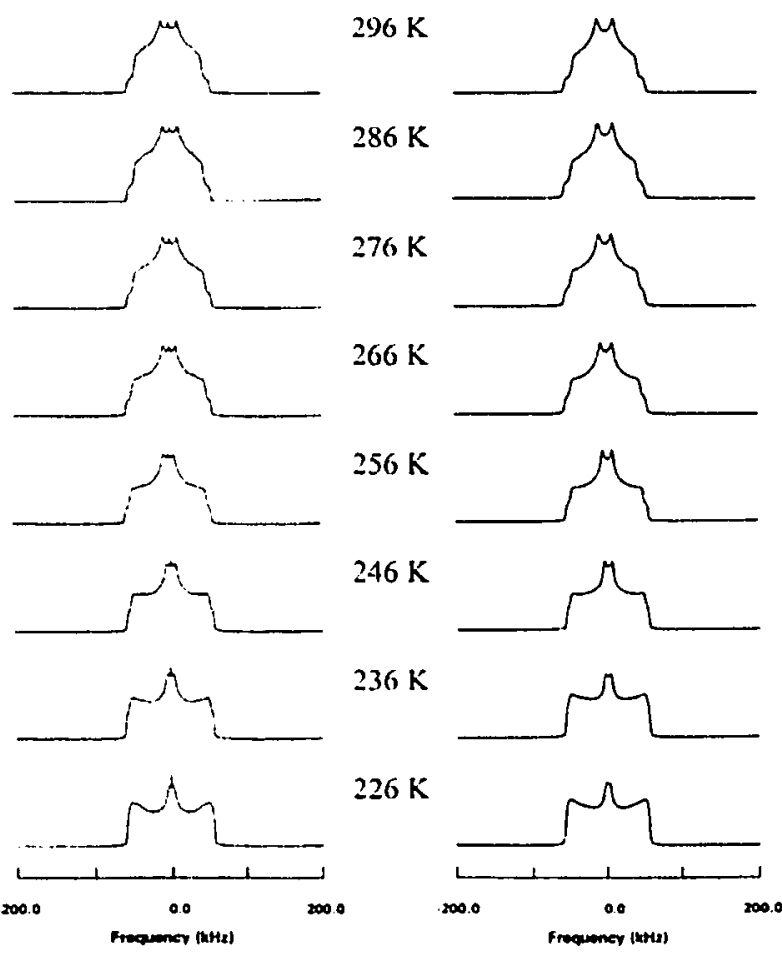

Simulated

$296 \mathrm{~K}$

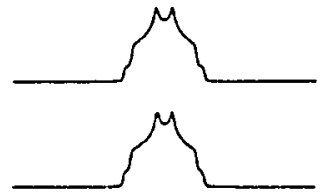

$276 \mathrm{~K}$

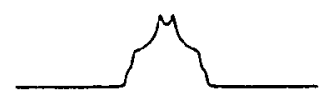

$266 \mathrm{~K}$

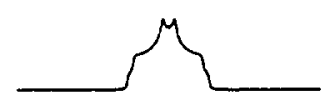

$256 \mathrm{~K}$

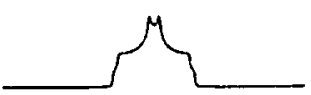

$246 \mathrm{~K}$

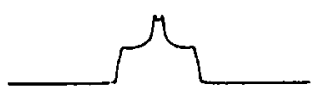

$236 \mathrm{~K}$

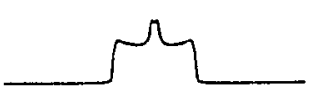

Figure VII-6: Experimental (left) and simulated (right) solid-state deuterium NMR spectra for the 1,2-dichloroethane-d $\mathrm{d}_{4}$ / TATM inclusion compound at the temperatures indicated, based on Model 1 (trans - gauche conformational equilibrium). Dynamic and conformational data extracted by simulating the experimental spectra appear in Table VII-2.

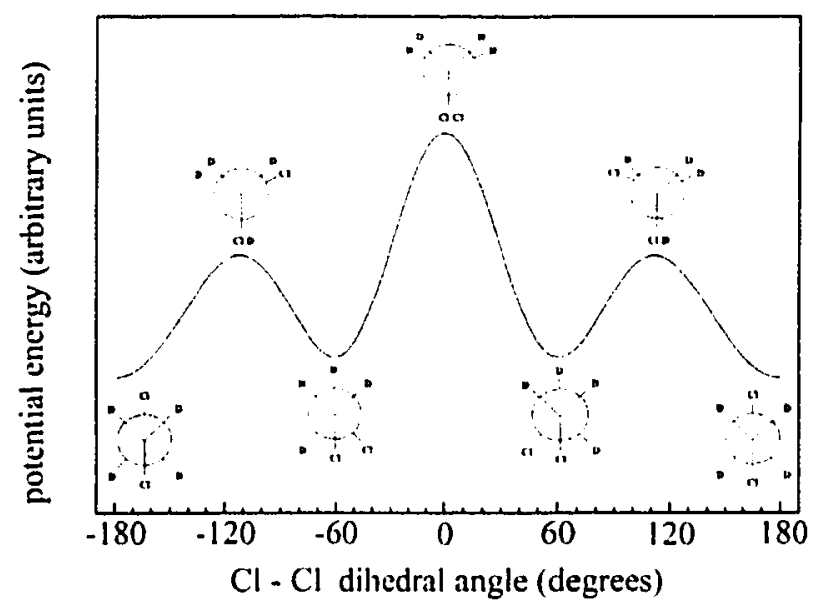

Figure VII-7: A schematic potential energy diagram illustrating the conformers present (as Newman projections) at the three potential energy minima and the three potential energy maxima as the $1,2-$ dichloroethane guest is rotated 360 degrees about the carbon - carbon bond, based on Model 1. 
In this model, the guest 1,2-dichloroethane molecule interconverts between the trans and gauche potential energy minima by performing $120^{\circ}$ jumps about the C-C bond. According to the simulated deuterium NMR spectra, the trans conformer has a higher fractional population (and lower potential energy), while the two gauche conformers have lower, and equal, fractional populations (and higher potential energy). As the temperature is lowered below room temperature, the fractional population of the lower energy trans conformer increases at the expense of the fractional populations of the two higher energy gauche conformers. This temperature - population behaviour is also observed in the pure liquid state and pure gaseous state of 1,2-dichloroethane, for which trans - gauche isomerism is known to exist [5]. According to the simulated ${ }^{2} \mathrm{H}$ NMR spectra for 1,2-dichloroethane- $d_{4} /$ TATM, at room temperature $(296 \mathrm{~K})$ the population of the trans conformer is $48.4 \%$, while the populations of the two gauche conformers are both $25.8 \%$ each. At the lowest temperature measured, $226 \mathrm{~K}$, the population of the trans conformer increases to approximately $60 \%$, while the same for the two gauche conformers decreases to about $20 \%$ each. At cach temperature measured, the fractional populations appear in Table VII-2. If one calculates the equilibrium constant $\left(K_{\text {ey }}\right)$ at each temperature as the ratio of the two populations (see Table VII-2), it is possible to determine the enthalpy difference between the two conformers from the slope of a plot of $\ln K_{\text {ey }}$ against inverse temperature. This graph appears in Figure VII-8, and we calculate the enthalpy difference between the trans and gauche conformers as $3.75 \mathrm{~kJ} \mathrm{~mol}^{-1}$.

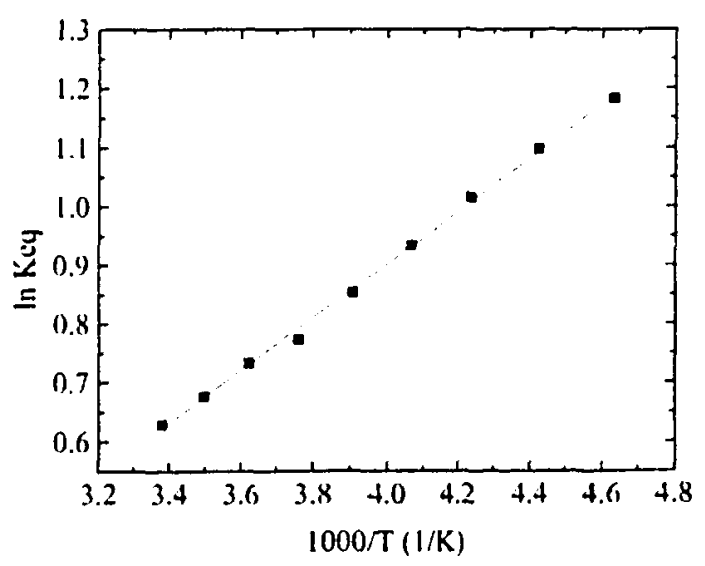

Figure VII-8: A plot of the natural logarithm of the equilibrium constant against inverse temperature for the conformational equilibrium of 1,2-dichloroethane- $d_{4}$ in TATM, based on Model 1. The equilibrium constant was defined at each temperature as the fractional population of the trans conformer divided by the fractional population of the gauche conformer. From the slope of a best fit curve, an energy difference between the trans and gauche conformers of $3.75( \pm 0.06) \mathrm{kJ} \mathrm{mol}^{-1}$ was determined, the trans conformer being lower in energy. 
Table VII-2: A table of guest conformational / dynamic parameters obtained by simulating (Model 1) the solid-state deuterium NMR spectra of 1,2-dichloroethane- $d_{4}$ in TATM at the temperatures indicated.

$\begin{array}{lllllll}T(\mathrm{~K}) & k_{\mathrm{T} \rightarrow \mathrm{G}^{a}} & k_{\mathrm{G} \rightarrow \mathrm{T}}{ }^{b} & k_{\mathrm{G} \rightarrow \mathrm{G}^{c}} & p_{\text {trans }}{ }^{d} & p_{\text {gauchel }{ }^{c}} & p_{\text {gauche }}{ }^{f} \\ 296 & 7.74 \times 10^{7} & 1.45 \times 10^{8} & 0 & 0.484 & 0.258 & 0.258 \\ 286 & 5.04 \times 10^{7} & 9.92 \times 10^{7} & 0 & 0.496 & 0.252 & 0.252 \\ 276 & 2.45 \times 10^{7} & 5.10 \times 10^{7} & 0 & 0.510 & 0.245 & 0.245 \\ 266 & 1.44 \times 10^{7} & 3.12 \times 10^{7} & 0 & 0.520 & 0.240 & 0.240 \\ 256 & 9.20 \times 10^{6} & 2.16 \times 10^{7} & 0 & 0.540 & 0.230 & 0.230 \\ 246 & 4.40 \times 10^{6} & 1.12 \times 10^{7} & 0 & 0.560 & 0.220 & 0.220 \\ 236 & 1.89 \times 10^{6} & 5.22 \times 10^{6} & 0 & 0.580 & 0.210 & 0.210 \\ 226 & 1.20 \times 10^{6} & 3.60 \times 10^{6} & 0 & 0.600 & 0.200 & 0.200\end{array}$

${ }^{a}$ The rate of conversion $\left( \pm 0.01 \times 10^{7}\right)$ from the trans conformer to the gauche conformers, in jumps per second. ${ }^{b}$ The rate of conversion $\left( \pm 0.01 \times 10^{7}\right)$ from the gauche conformers to the trans conformer, in jumps per second. ${ }^{c}$ The rate of conversion of one galuche conformer to the other gauche conformer, in jumps per second. The ${ }^{2} \mathrm{H}$ NMR spectra were simulated at all temperatures by approximating a rate of zero. ${ }^{d}$ The fractional population $( \pm 0.001)$ of the trans conformer at each temperature. "The fractional population $( \pm 0.001)$ of one of the two gauche conformers at each temperature. ${ }^{\prime}$ The fractional population $( \pm 0.001)$ of the other gauche conformer at each temperature.

When considering the rate of interconversion between the trans and gauche conformers, calculated from the deuterium NMR spectra, there are actually three different rates to consider, as depicted in the potential energy diagram of Figure VII-9. We can assign $k_{g \rightarrow l}$ as the rate of conversion of the gauche conformers to the trans conformer. Conversely, $k_{\mathrm{t} \rightarrow \mathrm{g}}$ is the rate of conversion of the trans conformer to the gauche conformers. Finally, $k_{\mathrm{g} \rightarrow \mathrm{g}}$ is the rate of conversion of one gauche conformer to the other gauche conformer. As the trans conformer has the lowest energy in this case, $k_{\mathrm{g} \rightarrow \mathrm{l}}$ has the smallest activation barrier, and hence the fastest rate, of the three at any particular temperature. The rate of conversion $k_{\mathrm{t} \rightarrow \mathrm{g}}$ has a higher activation energy and hence a smaller rate than $k_{\mathrm{g} \rightarrow \mathrm{t}}$. When the 1,2-dichloroethane molecule interconverts from one gauche conformer to the other gauche conformer, it must go through the highest energy maximum, the eclipsed $\left(0^{\circ}\right)$ transition state. Thus $k_{\mathrm{g} \rightarrow \mathrm{g}}$ has the highest activation energy and the smallest rate. The three rates at each measured temperature that we used in the simulations appear in Table VII-2. Note that we approximated a rate of $0 \mathrm{~Hz}$ at all temperatures for $k_{\mathrm{g} \rightarrow \mathrm{g}}$. 


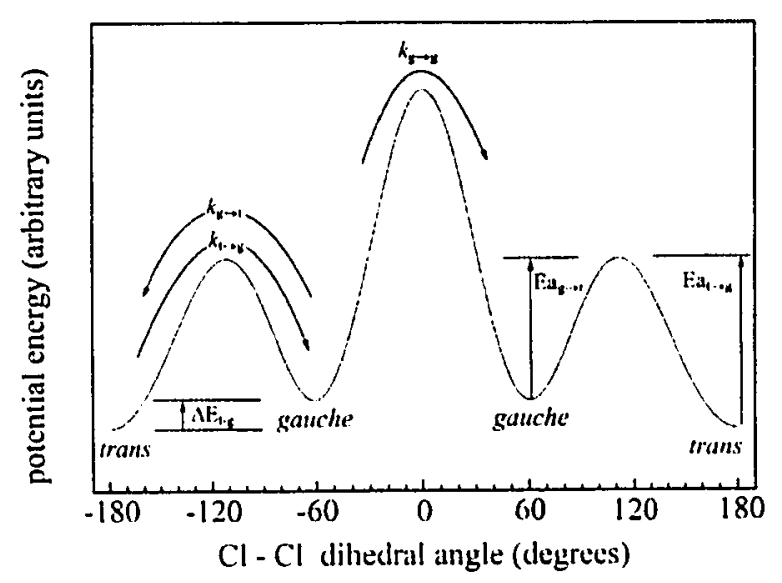

Figure VII-9: A schematic potential energy diagram depicting the three independent rates, two of the three independent activation barriers and the energy difference between the potential energy minima with regards to the conformational equilibrium for 1,2dichloroethane/TATM, based on Model 1 . The conformers present at the three potential energy minima and the three potential energy maxima are the same as in Figure VII-7.

\section{$226 \mathrm{~K}$}
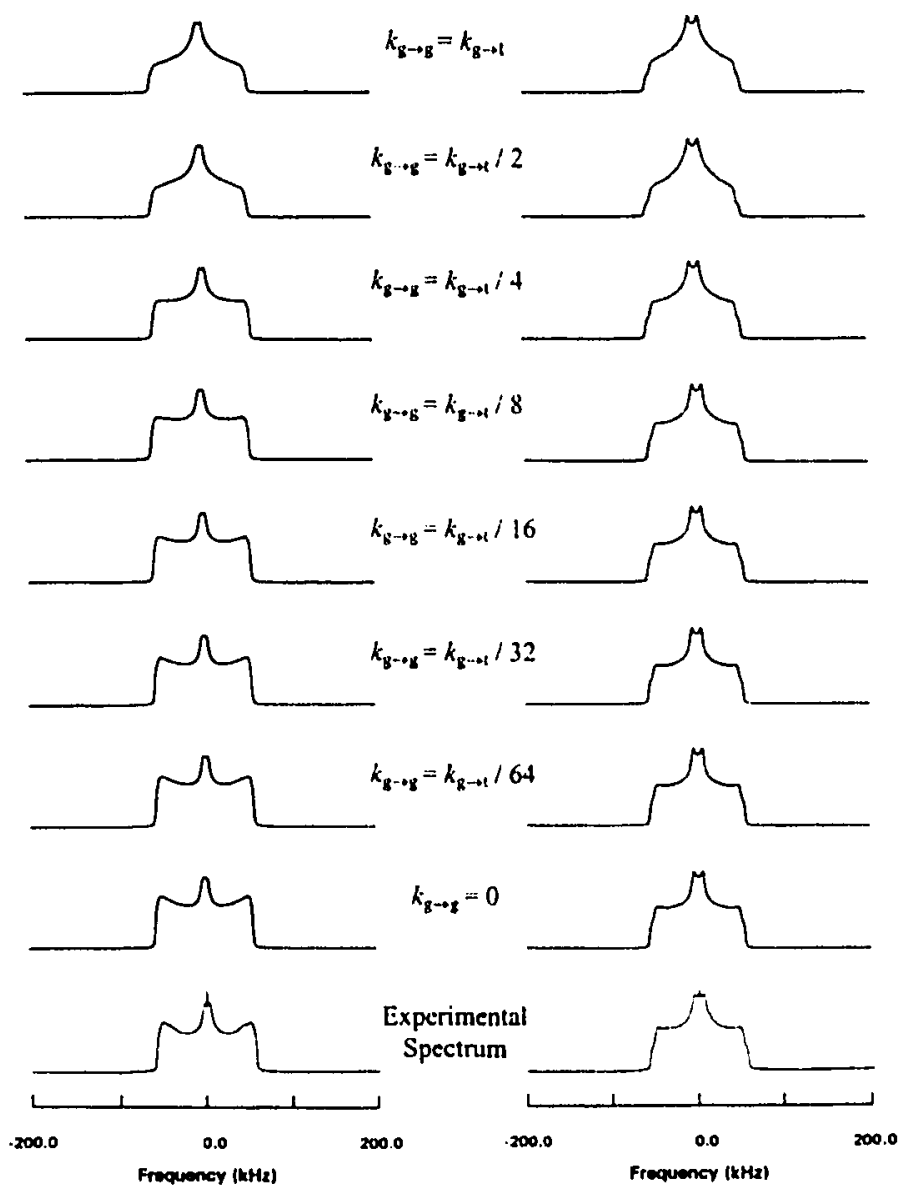

Figure VII-10: Experimental and simulated (Model 1) solid-state ${ }^{2} \mathrm{H}$ NMR spectra for he 1,2-dichloroethane- $\mathrm{d}_{4} /$ TATM inclusion compound at $226 \mathrm{~K}$ and $246 \mathrm{~K}$. The expt'l spectrum at each temperature appears at the bottom. Eight simulated spectra were performed at each temperature, the difference between them being the rate of conversion about the gauche-gauche barrier. The rate $k_{\mathrm{g} \rightarrow \mathrm{g}}$ is varied as a function of the rate $k_{\mathrm{g} \rightarrow 1}$, as depicted in the figure. 
We considered this situation in detail for the deuterium NMR spectrum at two temperatures $\left(226 \mathrm{~K}\right.$ and $246 \mathrm{~K}$ ). We performed simulations using fixed $k_{\mathrm{g} \rightarrow 1}$ and $k_{\mathrm{t} \rightarrow \mathrm{g}}$ rates of interconversion. We varied $k_{\mathrm{g} \rightarrow \mathrm{g}}$ as a function of $k_{\mathrm{g} \rightarrow l}$. The simulations appear in Figure VII-10. By comparing the experimental ${ }^{2} \mathrm{H}$ NMR spectra at $226 \mathrm{~K}$ and $246 \mathrm{~K}$ with the simulated spectra, it can be concluded that the rate $k_{\mathrm{g} \rightarrow \mathrm{g}}$ is at most 1/64 times as large as $k_{\mathrm{g} \rightarrow \mathrm{l}}$. That is, $k_{\mathrm{g} \rightarrow \mathrm{g}}$ is somewhere between this value and zero. However, from the simulated spectra, we are unable to determine the rate $k_{\mathrm{g} \rightarrow \mathrm{g}}$ precisely at each temperature, so we simply used a rate of $0 \mathrm{~Hz}$, this is only an approximation. The spectra are invariant to the rate of $k_{\mathrm{g} \rightarrow \mathrm{g}}$ when it is any value less than $1 / 64$ times as large as $k_{\mathrm{g} \rightarrow \mathrm{l}}$. On the other hand, we were able to determine $k_{\mathrm{g} \rightarrow \mathrm{r}}$ and $k_{\mathrm{t} \rightarrow \mathrm{g}}$ precisely at each temperature, these rates appear in Table VII-2.

By plotting $\ln k_{\mathrm{g} \rightarrow \mathrm{t}}$ and $\ln k_{\mathrm{t} \rightarrow \mathrm{g}}$ against inverse temperature (see Figure VII-11), we calculate that the activation barrier to go from the trans conformer to the gauche conformers $\left(E_{\mathrm{a} \mathrm{t} \rightarrow \mathrm{g}}\right)$ is $33.0( \pm 0.9) \mathrm{kJ} \mathrm{mol}^{-1}$, while the activation barrier to go from the gauche conformers to the trans conformer $\left(E_{\mathrm{a} g \rightarrow \mathrm{c}}\right)$ is $29.2( \pm 0.9) \mathrm{kJ} \mathrm{mol}^{-1}$. In theory, the energy difference $\Delta H_{\mathrm{gt}}$ between the trans and gauche conformers added to $E_{\mathrm{a} g \rightarrow \mathrm{l}}$ should equal $E_{\mathrm{a} t \rightarrow \mathrm{g}}$. The former sum is $3.75 \mathrm{~kJ} \mathrm{~mol}^{-1}+29.2 \mathrm{~kJ} \mathrm{~mol}^{-1}=32.9 \mathrm{~kJ} \mathrm{~mol}^{-1}$, the latter is $33.0 \mathrm{~kJ} \mathrm{~mol}^{-1}$, showing good agreement. From the spectra, we cannot quantitatively determine the magnitude of the gauche $\rightarrow$ gauche barrier, we can only say it is much greater than the other two activation energies. The literature on activation barriers for internal rotation about the $\mathrm{C}-\mathrm{C}$ bond in 1,2-dichloroethane is sparse indeed. From gas phase heat capacity measurements, Gwinn and Pitzer determined an activation barrier of $11.30 \mathrm{~kJ} \mathrm{~mol}^{-1}$ between the trans and gauche conformers of pure gaseous 1,2dichloroethane, $[7]$ they did not specify whether the barrier referred to $E_{\mathrm{a}} \mathrm{g} \rightarrow \mathrm{l}$ or $E_{\mathrm{a}(\mathrm{l} \rightarrow \mathrm{g} \text {. }}$ From a force model calculation, Bernstein found that the trans-gauche barrier of the same system is $11.76 \mathrm{~kJ} \mathrm{~mol}^{-1}$ and the gauche-gauche barrier is a much larger $18.80 \mathrm{~kJ} \mathrm{~mol}^{-1}$ [8]. For our motional model, the barriers $E_{\mathrm{a} g \rightarrow 1}$ and $E_{\mathrm{a} \mathrm{l} \rightarrow \mathrm{g}}$ for 1,2-dichloroethane in TATM are both much larger than not only the two trans-galuche barriers listed, but also the gauche-gauche barrier. 


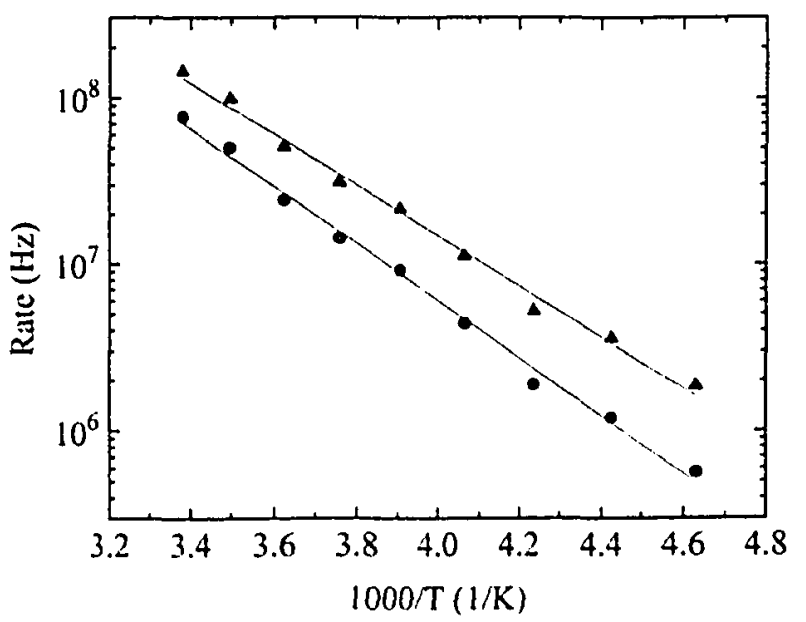

Figure VII-11: A plot of rotation rate against inverse temperature, on a semi-logarithmic scale, for the conformational equilibrium of 1,2-dichloroethane- $\mathrm{d}_{4}$ in TATM, according to Model 1 . The rate $k_{\mathrm{g} \rightarrow l}$ appears as triangles and the rate $k_{t \rightarrow g}$ appears as circles. From the slope of best fit curves, activation barriers $E_{\mathrm{a} g \rightarrow \mathrm{l}}=29.2$ $( \pm 0.9) \mathrm{kJ} \mathrm{mol}^{-1}$ and $E_{\mathrm{a} \rightarrow \mathrm{g}}=33.0$ $( \pm 0.9) \mathrm{kJ} \mathrm{mol}^{-1}$ were determined.

(2) Model 2: $\quad$ NMR model based on motion between disordered positions as shown in the $\mathrm{X}$-ray structure. (restricted $\mathrm{Cl}$ motion)

Unfortunately, the single crystal structure of the triclinic form shows no evidence for the existence of a gauche conformer, or for that matter, rotation about the $\mathrm{C}-\mathrm{C}$ bond of the 1,2-dichloroethane guest. Although the fit between the simulated and experimental ${ }^{2} \mathrm{H}$ NMR spectra using Model 1 are excellent, one must be aware that the dynamic models used in spectral simulations are not unique. More than one set of simulations based on particular motional models may fit the experimental spectra. Still, this model does not agree with the disorder of the guest as elucidated from our crystal structure, so we attempted to simulate the ${ }^{2} \mathrm{H}$ NMR spectra based on the disorder inherent in the crystal structure. The sample used for deuterium NMR analysis was prepared by slow cooling. so it contains only the triclinic form. We performed crystal structure analyses for the triclinic form at $298 \mathrm{~K}$ and $173 \mathrm{~K}$. At both of these temperatures, the guest is disordered over three sites. We considered all possible exchanges between guest sites that do not involve an end-to-end exchange of the terminal $\mathrm{Cl}$ atoms. We determined the Euler angles relating the deuterons exchanging over three sites and entered the angles into the MXQET [9] simulation program. Unfortunately, we were unable to obtain a satisfactory fit between the experimental and simulated ${ }^{2} \mathrm{H}$ powder spectra. Finally, as part of these simulations, we were able to show that the deuterium NMR spectra are relatively insensitive to $180^{\circ}$ flips about the $\mathrm{Cl}-\mathrm{Cl}$ axis, and although this motion may be present it does not explain the observed results. 
(3) Model 3: Two-fold flip (large scale $\mathrm{Cl}$ motion)

The failure of Model 2 led us to consider a model that includes end-to-end exchange of the terminal $\mathrm{Cl}$ atoms. The simplest such motion is a two-fold reorientation about an axis through the centre of mass of the guest and perpendicular to the $\mathrm{Cl}-\mathrm{C}-\mathrm{C}-\mathrm{Cl}$ plane of the trans conformer. The guest performs $180^{\circ}$ jumps about this axis to rotate back into itself (see Figure VII-12). Therefore this motion would be invisible in the single crystal diffraction experiment. We were able to obtain a satisfactory fit with the experimental spectra over the range $296 \mathrm{~K}$ to $186 \mathrm{~K}$, as depicted in Figure VII-13. The rate of the twofold reorientation goes from $5 \times 10^{7} \mathrm{~Hz}$ at $296 \mathrm{~K}$ to $3 \times 10^{4} \mathrm{~Hz}$ at $186 \mathrm{~K}$. A plot of rate vs. inverse temperature appears in Figure VII-14. From the slope of the best-fit line, we calculate an activation barrier of $30.1( \pm 0.6) \mathrm{kJ} \mathrm{mol}^{-1}$.

It should be noted that although the trans - gauche conformational model (Model 1) was able to fit the experimental spectra very well in the region $296 \mathrm{~K}-226 \mathrm{~K}$, it was unable to match the experimental spectra in the region $216 \mathrm{~K}-186 \mathrm{~K}$. This provides further support for the correctness of the twofold flip model (Model 3).

Another feature of this model is that the 1,2-dichloroethane molecule performs small angle librations about the two-fold axis within the two potential wells of the $C_{2}$ flip. That is, this motion $\left(k>>10^{8} \mathrm{~Hz}\right)$ is superimposed on the twofold flip. The amplitude of this motion increases with temperature, going from $\pm 3^{\circ}$ at $226 \mathrm{~K}$ to $\pm 28^{\circ}$ at $296 \mathrm{~K}$. The modeled librational angle at each temperature is plotted in Figure VII-15.
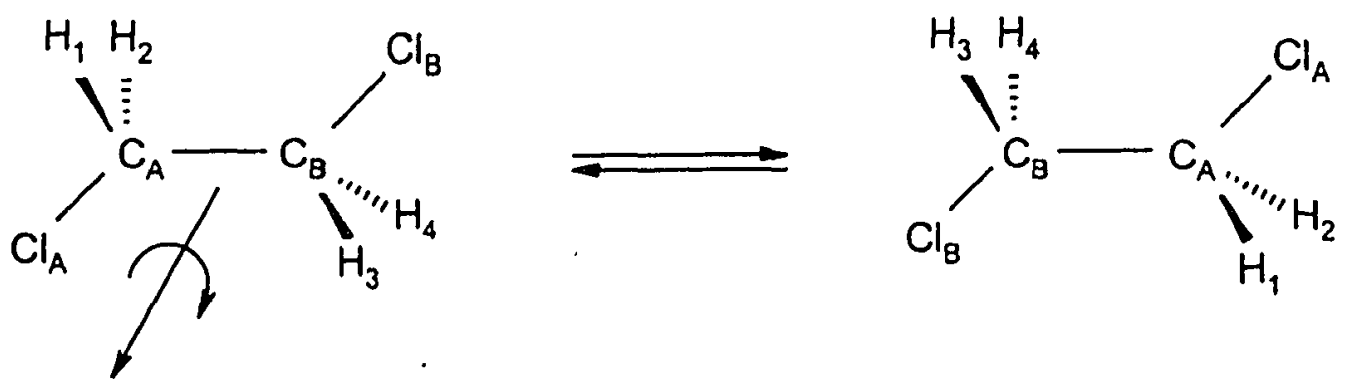

Figure VII-12: A schematic depiction of the reorientation of the 1,2-dichloroethane guest in TATM, according to Model 3 (two-fold flip). 


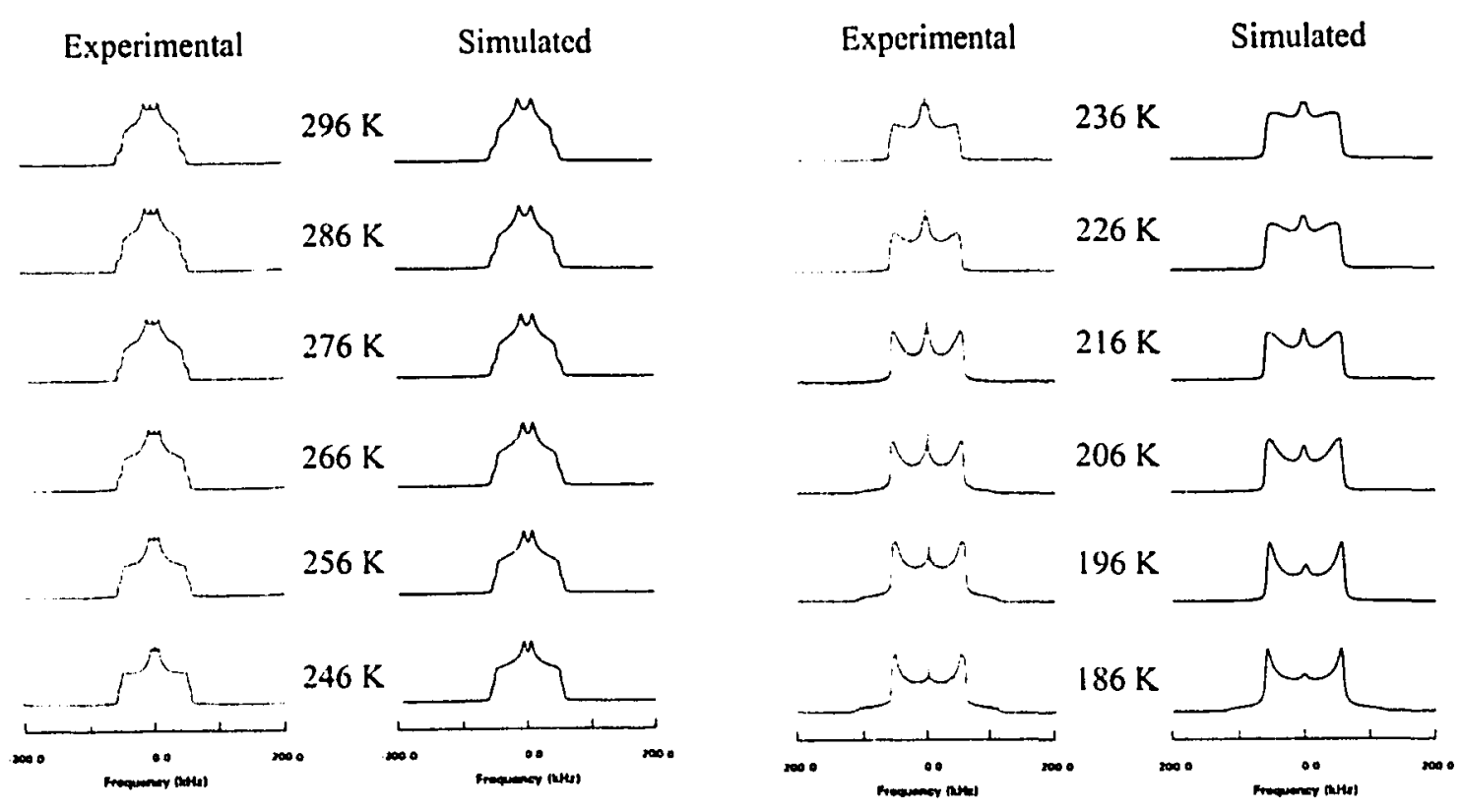

Figure VII-13: Experimental and simulated solid-state deuterium NMR spectra for the 1,2-dichloroethane- $\mathrm{d}_{4} /$ TATM inclusion compound, based on Model 3 (two-fold flip)
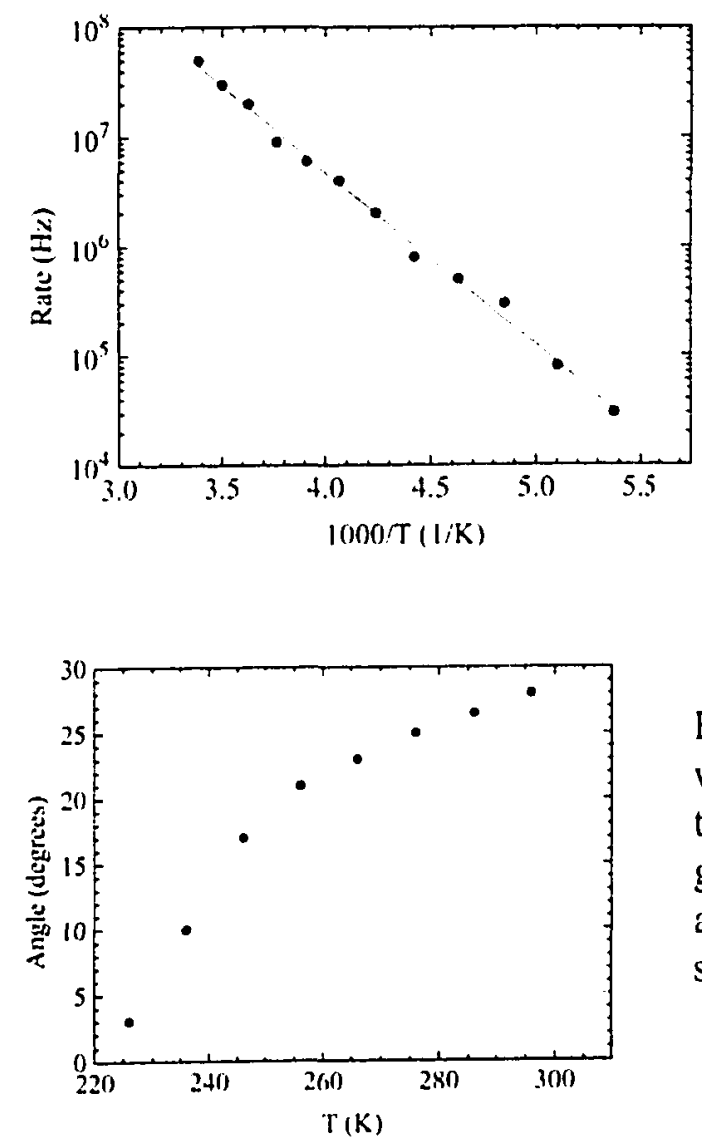

Figure VII-14: A plot of the rate of two-fold reorientation against inverse temperature for the 1,2-dichloroethane- $\mathrm{d}_{4}$ guest (according to Model 3). The rate at each temperature was determined by simulating the experimental ${ }^{2} \mathrm{H}$ NMR spectra. A best fit line indicates an activation barrier of $30.1( \pm 0.6) \mathrm{kJ} \mathrm{mol}^{-1}$.
Figure VII-15: A plot of angle of libration within the two potential energy wells against temperature for the 1,2-dichloroethane- $d_{4}$ guest, based on Model 3. The angle at each temperature was determined by simulating the experimental ${ }^{2} \mathrm{H}$ NMR spectra. 
The profile of ${ }^{2} \mathrm{H}$ spin-lattice relaxation time for 1,2-dichloroethane-d $\mathrm{d}_{4} /$ TATM, as a function of inverse temperature, is presented in Figure VII-16. Changes in $T_{1}$ in this temperature range result from changes in the rate of the above mentioned two-fold flips. It is apparent from the $T_{1}$ profile that the correlation time of the motion is exclusively on the slow motion side of the $T_{1}$ minimum (i.e. the rate of motion $k$ is less than the Larmor precession frequency). The ${ }^{2} \mathrm{H}$ spin-lattice relaxation time at ambient temperature, the highest temperature measured, is $56.1 \mathrm{~ms}$. The relaxation time at $232.8 \mathrm{~K}$, the lowest temperature measured, increases to $202 \mathrm{~ms}$. (We were somewhat hesitant to acquire $T_{1}$ data at temperatures above room temperature, as from our experience, TATM inclusion compounds generally decompose at these temperatures.) From the slope of the curve in the linear region (at intermediate temperatures) we calculate that the activation energy for the two-fold flip is $25.4( \pm 0.3) \mathrm{kJ} \mathrm{mol}^{-1}$. This compares with an activation energy of $30.1( \pm 0.6) \mathrm{kJ} \mathrm{mol}^{-1}$ determined from ${ }^{2} \mathrm{H}$ NMR spectral simulations quoted above. Note that the lack of a complete $T_{1}$ minimum precluded a detailed fit of the $T_{1}$ data, from which one can determine the correlation time $\left(\tau_{c}\right)$ of the motion as a function of temperature $\left(\tau_{\mathrm{i}} \propto k^{-1}\right)$. However the similarity of the activation energies from ${ }^{2} \mathrm{H}$ spectra and ${ }^{2} \mathrm{H} T_{1}$ data lends support to the accuracy of the two-fold flip model.

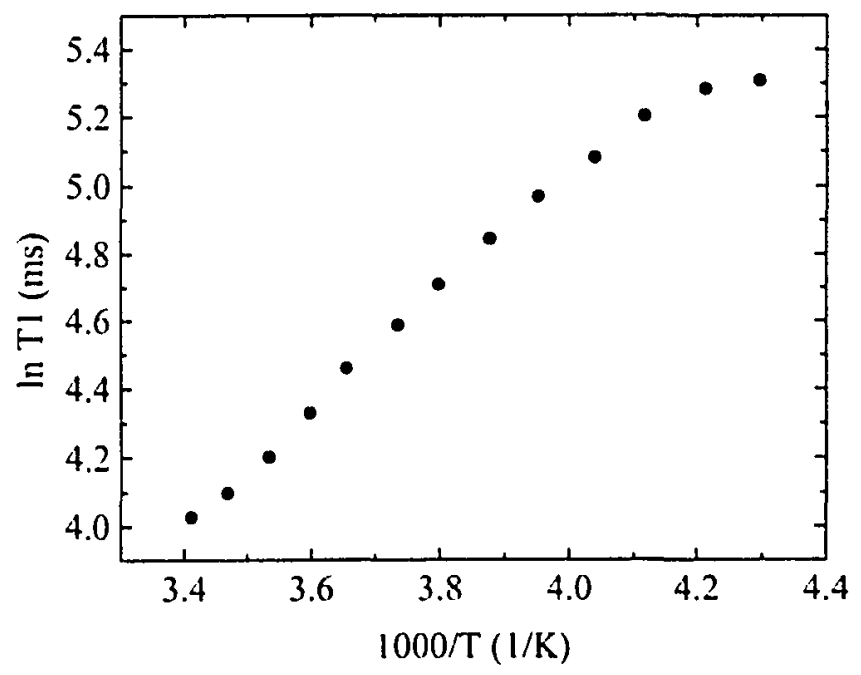

Figure VII-16: A plot of the deuterium spin-lattice relaxation time of the 1,2dichloroethane- $d_{4}$ guest as a function of inverse temperature. From the slope of the curve in the linear region, an activation energy of $25.4( \pm 0.3) \mathrm{kJ} \mathrm{mol}^{-1}$ for the two-fold flip (Model 3) was determined. 


\section{CONCLUDING REMARKS}

It is generally accepted that in many host-guest inclusion compounds, the relatively weak van der Waals interactions between host and guest affords the enclathrated moiety a large degree of motional freedom. As evidenced in this work, deuterium NMR spectroscopy has emerged as a very important technique for the study of anisotropic molecular motion in solids. Determination of the dynamics of a guest molecule provides insight into the nature of the host-guest interaction. For example, the potential for free rotation is undoubtedly hindered in inclusion compounds where the host-guest interaction involves hydrogen bonds. In summary, it seems that solid-state deuterium NMR spectroscopy can provide insight into the type of motion that a molecule, in its solid phase, is performing. However, as mentioned before, one must recognize the limitations of this technique. One-dimensional ${ }^{2} \mathrm{H}$ NMR does not, unequivocally, with $100 \%$ accuracy, tell us what type of motion a molecule is performing. Complementary techniques, such as $2 \mathrm{D}$ exchange NMR, X-ray crystallography, multinuclear powder NMR and differential scanning calorimetry, also provide us with information concerning host-guest interactions in inclusion compounds. Thus, utilization of these other techniques would seem to be generally indicated.

The combined ${ }^{2} \mathrm{H}$ NMR and single crystal structural data suggest that the barrier to internal rotation of the 1,2-dichloroethane molecule is very high in the TATM cavity, as it is in the pure solid. One way that this may happen is if the cavity shape and the trans form of the molecule show some complementarity in terms of guest-host interactions. The $\mathrm{Cl}$ atoms are likely to play a key role in locating the molecule in the cavity, although this must be determined during guest-host assembly of the crystal. We note that the TATM host is one of the most versatile in adopting a variety of structures with different guests.

A crucial point to be taken from this work is that although a simple dynamic model may produce calculated simulations that fit the ${ }^{2} \mathrm{H}$ NMR experimental spectra very accurately, one must realize, as mentioned above, that these models are not unique, and that information gained from other experimental techniques (such as single crystal X-ray diffraction) can often provide information that will support or refute a proposed model, as was the case here for 1,2-dichloroethane / TATM. In coming up with a proposed 
dynamic model, one must make sure that it is consistent with information obtained from all of the other relevant experiments performed.

\section{CHAPTER VII}

\section{REFERENCES}

(1) M.E. Milberg and W.N. Lipscomb. Acta. Cryst. 4, 369 (1951).

(2) H.S. Gutowsky and G.E. Pake J. Chem. Phvs. 18(2), 162 (1950).

(3) S. Mizushima, T. Shimanouchi, I. Harada, Y. Abe and H. Takeuchi. Can. J. Phys. 53, 2085 (1975).

(4) J.L. Ragle. J. Phys. Chem. 63, 1395 (1959).

(5) S. Mizushima. Structure of Molecules Academic Press: New York, 1954.

(6) S.K. Garg, D.W. Davidson, S.R. Gough and J.A. Ripmeester. Can. J. Chem. 57, 635 (1979).

(7) W.G. Gwinn and K.S. Pitzer. J. Chem. Phys. 16, 303 (1948).

(8) H.J. Bernstein. J. Chem. Phvs. 17(3), 258 (1949).

(9) M.S. Greenfield, A.D. Ronemus, R.L. Vold, R.R. Vold, P.D. Ellis and T.R. Raidy. J. Magn. Reson. 72, 89 (1987). 


\section{CHAPTER VIII}

\section{Structural type and thermal properties of tris (5-} acetyl-3-thienyl) methane inclusion compounds: A combined ${ }^{13} \mathrm{C} C \mathrm{CP} / \mathrm{MAS}$ NMR, powder X-ray diffraction and differential scanning calorimetric study

\begin{tabular}{|c|c|c|}
\hline \multicolumn{2}{|c|}{ Abstract } & 11 \\
\hline \multicolumn{2}{|c|}{ Introduction } & 11 \\
\hline \multicolumn{2}{|c|}{ Experimental } & 12 \\
\hline \multicolumn{3}{|c|}{ Results } \\
\hline (A) & ${ }^{13} \mathrm{C}$ CP/MAS NMR & 12 \\
\hline (B) & Powder X-Ray Diffraction & 12 \\
\hline (C) & Differential Scanning Calorimetry & 13 \\
\hline \multicolumn{3}{|c|}{ Discussion and comparison to other host systems } \\
\hline (D) & Solid solution model & $13^{\circ}$ \\
\hline (E) & Cyclodextrins & 13 \\
\hline (F) & Cholic acid & 14 \\
\hline (G) & Gossypol & 14 \\
\hline \multicolumn{2}{|r|}{ Other host systems } & \\
\hline & (1) 1,2,3,4,5-penta-O-benzoyl-6-O-tosyl myo-inositol & 14 \\
\hline & (2) 2,4,6-tris(4-halophenoxy)-1,3,5-triarine & 14 \\
\hline & (3) $C d(p n) N i(C N)_{4}$ & 15 \\
\hline Cor & ading remarks & 15 \\
\hline Refer & nces & 15 \\
\hline
\end{tabular}




\section{ABSTRACT}

Tris (5-acetyl-3-thienyl) methane (TATM) inclusion compounds with a wide variety of guest molecules have been studied by the techniques of solid state ${ }^{13} \mathrm{C}$ cross polarization / magic angle spinning nuclear magnetic resonance spectroscopy (CP/MAS NMR), powder $\mathrm{X}$-ray diffraction and differential scanning calorimetry (DSC). The approach taken was to study guests with the same hydrocarbon backbone but with different halogen substituents $(\mathrm{F}, \mathrm{Cl}, \mathrm{Br}, \mathrm{I})$ at selected sites on that backbone. From the multiplicities of the host signals in the ${ }^{13} \mathrm{C}$ CP/MAS NMR spectra, the number of TATM molecules in the crystallographic asymmetric unit was determined. Powder X-ray diffraction experiments allowed us to investigate structural isomorphism of TATM inclusion compounds with different guests. In some cases, TATM forms isomorphic structures with guests that have the same hydrocarbon backbone, but with different halogen substituents. This was illustrated in the case of cyclohexyl halide and methylene halide guests. On the other hand, TATM will sometimes form one type of structure with a guest that has smaller halogen substituents (e.g. $\mathrm{F}, \mathrm{Cl}, \mathrm{Br}$ ), but a completely different structure with a guest that has larger halogen substituents (e.g. I). This was illustrated in the case of monohalobenzene, 2-halobutane and 1,3-dihalopropane guests. Differential scanning calorimetry experiments allow one to investigate thermal stabilities of inclusion compounds with different guests. The enthalpy of decomposition data show that TATM forms inclusion compounds with a greater heat of fusion for guests that have smaller, more electronegative halogen substituents (for a given hydrocarbon backbone), and the heat of fusion decreases as the size of the halogen substituent increases.

\section{INTRODUCTION:}

The behaviour of organic molecules incarcerated within solid-state inclusion environments is an important fundamental issue, not the least because the included molecules often possess properties that contrast with those of the same molecule in other phases [1]. The conformational behaviour of monohalocyclohexanes included within the thiourea channel structure represents a striking illustrative cxample [2-4]. Underlying all structural aspects of organic molecular crystals is the fundamental question of what 
factors control the molecular packing arrangement, and an important related issue is how to apply an understanding of these factors in the design of crystals with desired structures [5]. The problem of rationalizing a three-dimensional packing arrangement is considerable, since the observed structure often arises from the interplay of several factors of approximately equal importance. However, if the molecules are constrained conformationally or spatially in some way (e.g. by virtue of being included within a host lattice) the number of "variables" that must be considered can be reduced considerably.

Analysis of heats of fusion or melting points in terms of crystal structures can provide a wealth of information about intermolecular forces [6]. However, such measurements do not ordinarily isolate the interaction between functional groups, unless an isostructural series of compounds are found in which the interactions of interest are particularly strong compared to other crystal packing forces. Some success has been achieved in developing a scale of isotropic functional group interaction energies from heat of vaporization data [7], but the magnitudes and directional properties of many functional group interactions are still unknown, and the chemist is generally left with only a vague notion of the strengths of many weak interactions. Since, in the present study, we limit ourselves to only alkyl halide and aryl halide guest families, and furthermore generally compare DSC data within each guest family (as opposed to between guest families), we hope that this limitation may be overcome.

Tris (5-acetyl-3-thienyl) methane (TATM) is a conformationally flexile tripodal host molecule structurally analogous to the triphenylmethane group of hosts [8]. It has been found to form inclusion compounds with a large and wide variety of organic compounds [9]. We note, however, that there have been few attempts to gain a systematic understanding of the inclusion properties, or, to test the material for possible applications, for instance, as a selective sorbent of organics.

The purpose of this study is to examine a suite of TATM inclusions systematically in order to understand structural relationships and the stability for a series of related guests. This kind of information is required in order to build a thermodynamic model for TATM materials and to provide background information for a study of selectivity toward guest mixtures, planned as future work. 
We believe that the most straightforward way to investigate guest-property relationships for TATM inclusion compounds is to choose guests that have the same hydrocarbon backbone, but different substituents at selected positions on the molecule. Accordingly, we have chosen five groupings of guest molecules, depicted in Figure VIII1, with halogen substituents $(\mathrm{F}, \mathrm{Cl}, \mathrm{Br}, \mathrm{I})$. The guest families are the monohalobenzenes, the cyclohexyl halides, methylene halides, 2-halobutanes and 1,3-dihalopropanes. By choosing guests that are very similar in structure, we hope to eliminate extraneous complications in the interpretation of our data, the only two significant differences within any one group of guests being the size and electronegativity / polarity of the halogen substituents.

1)<smiles>[X]C([X])([1H])[2H]</smiles>

2)<smiles>[X]CCC[Y]</smiles>

3)<smiles>[Y]C(C)CC</smiles>

4)<smiles>[Y]C1CCCCC1</smiles>

5)

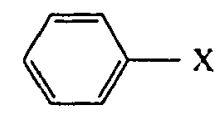

Methylene Halides

\section{1,3-Dihalopropanes}

2-Halobutanes

Cyclohexyl halides

Monohalobenzenes
Figure VIIl-1: Structure of guest molecule families studied as guests in TATM inclusion compounds. $\mathrm{X}$ denotes a halogen atom $(\mathrm{F}, \mathrm{Cl}, \mathrm{Br}, \mathrm{I})$. 
appreciably dissolve in the guest solvent ( $n$-hexane and cyclohexane), a mixture of 300 mg TATM and $50 \mathrm{~mL}$ guest solvent was refluxed for about one week to allow the reaction to go to completion, followed by cooling and filtration. All samples were stirred for extensive periods of time at room temperature (2-4 days) to give fine polycrystalline samples, thus grinding was not necessary.

\section{RESULTS}

\section{(A) ${ }^{13} \mathrm{C}$ CP/MAS NMR}

The ${ }^{13} \mathrm{C}$ CP/MAS NMR spectrum of guest-free TATM (prepared by vacuum sublimation) is presented in Figure VIII-2A. The signals are broad singlets, reflecting the amorphous character of desolvated TATM. For noncrystalline or amorphous solids, the spectral peaks may be broadened as a result of the nuclei having a distribution of electronic environments. In contrast, the ${ }^{13} \mathrm{C}$ CP/MAS NMR signals of TATM in TATM inclusion compounds are usually sharp multiplets, giving information on the crystal symmetry in each case. The NMR signal at lowest field ( $190.7 \mathrm{ppm}$ ) comes from the carbonyl carbon of the acetyl groups of TATM. The signal at highest field $(26.4 \mathrm{ppm})$ originates from the methyl carbon on the same functional group. Both retain their intensity upon dipolar dephasing (Figure VIII-2B), the latter because of rapid internal methyl rotation. In contrast, the central methine $\mathrm{CH}$ host signal at $43.7 \mathrm{ppm}$ is eliminated by this pulse sequence. Finally, the two signals at $132.8 \mathrm{ppm}$ and $145.2 \mathrm{ppm}$ belong to the thienyl ring carbons. Their assignment is assisted by the dipolar dephasing experiment $[10]$. The quaternary carbons ( 3 and 5 ) together give rise to the signal at $145.2 \mathrm{ppm}$, as they retain their full intensity in Figure VIII-2B. On the other hand, the signal at $132.8 \mathrm{ppm}$ belongs to the two tertiary $\mathrm{CH}$ thienyl carbons (2 and 4). They are eliminated by dipolar dephasing, because of strong heteronuclear one bond ${ }^{13} \mathrm{C}-{ }^{1} \mathrm{H}$ dipolar coupling. This experiment also shows that the TATM molecule in guest-free TATM (and also in the inclusion compounds) is not reorienting at room temperature, other than internal methyl rotation of the acetyl groups. 

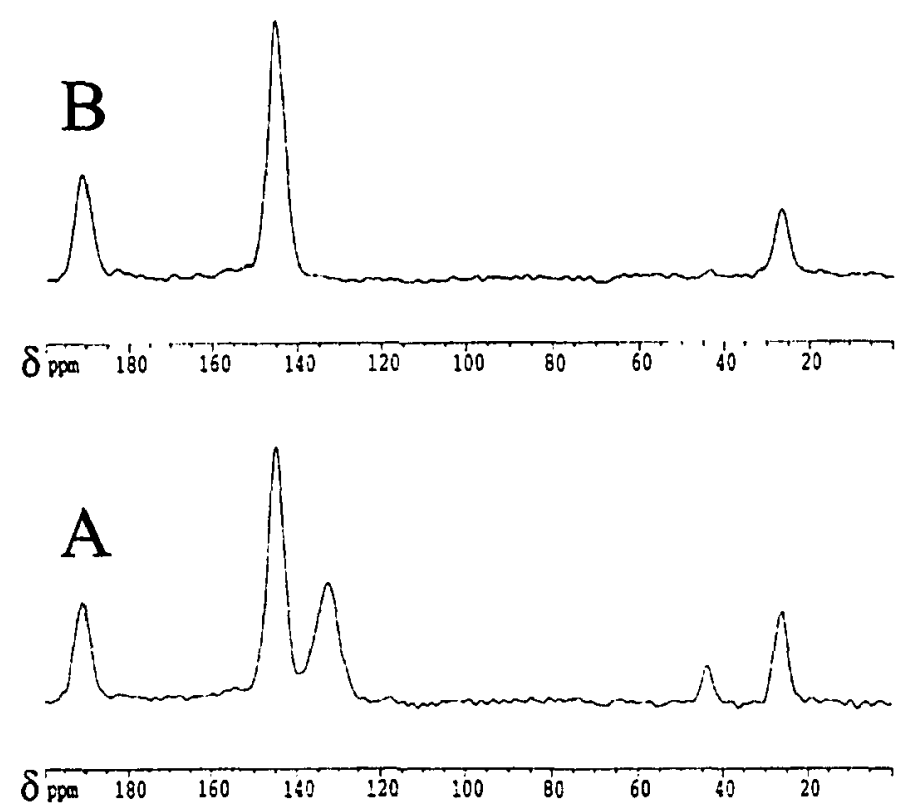

Figure VIII-2: (A) ${ }^{13} \mathrm{C}$ CP/MAS NMR spectrum of guest-free TATM.

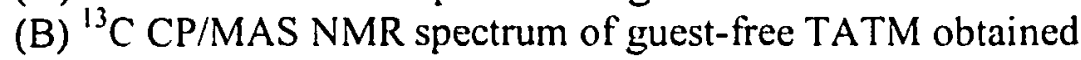
with the dipolar dephasing pulse sequence.

The ${ }^{13} \mathrm{C}$ CP/MAS NMR spectrum of ethanol/TATM appears in Figure VIII-3. The two signals at $16.9 \mathrm{ppm}$ and $56.3 \mathrm{ppm}$ arise from the guest's $\mathrm{CH}_{3}$ and $\mathrm{CH}_{2}$ groups, respectively. The signals from the host's acetyl group (carbonyl and methyl) both show a multiplicity of three. In both cases, all three signals are resolved. In accordance with the published crystal structure, [11] this implies that there is one TATM molecule in the asymmetric unit. This is because there are three methyl groups and three carbonyl groups in one TATM molecule, thus giving a one to one correspondence between carbons in the host molecule and signals in the ${ }^{13} \mathrm{C} C P / M A S$ NMR spectrum. The multiplicity of the methine carbon appears to be one, which provides further support. The signals from the carbons of the thienyl ring are more complex. The multiplicity of the high field signal is six ( 4 single, 1 double), as is that of the low field signal ( 3 single, 1 triple). Since there are six quaternary and six tertiary thienyl ring carbons in one TATM molecule (three thienyl rings), we again see a one to one correspondence between carbons in the host and signals in the NMR spectrum. The high field resonance has a doubly degenerate signal, while the low field resonance has a triply degenerate signal. 


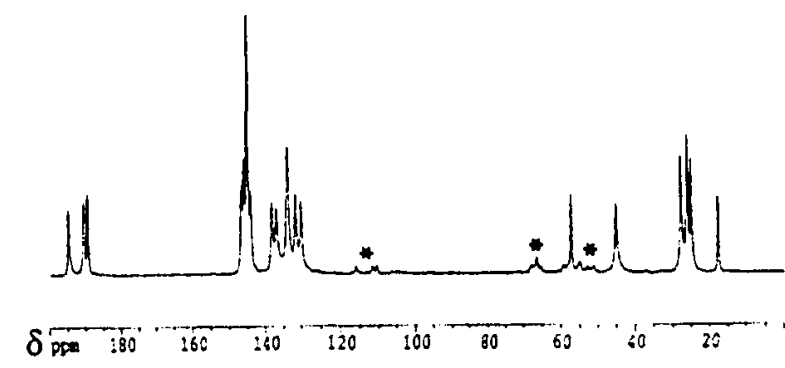

Figure VIII-3: ${ }^{13} \mathrm{C} \mathrm{CP} / \mathrm{MAS}$ NMR spectrum of the ethanol/TATM inclusion compound. Spinning sidebands are indicated with an asterisk. Expansions appear in the insets from left to right as: (1) 197-187 ppm (host carbonyl region) (2) 155-125 ppm (host thienyl region) and (3) 32-22 ppm (host methyl region)

The ${ }^{13} \mathrm{C}$ CP/MAS NMR spectrum of cyclohexane/TATM appears in Figure VIII-4. In the neat liquid, the chemical shift of cyclohexane appears as a singlet at about $27 \mathrm{ppm}$. This occurs in the region of the methyl group signal for TATM. The assignment of the guest signal in the cyclohexane/TATM CP/MAS NMR spectrum is not assisted by dipolar dephasing, because all signals in that region remain, as cyclohexane is reorienting in the TATM cavity at room temperature, and the methyl groups are performing internal methyl rotation. The cyclohexane/TATM inclusion compound is known to exist [12] as a polymorph, the $\mathbf{A}$ form having one TATM molecule in the asymmetric unit, the $\mathbf{B}$ form having two. By observing the TATM carbonyl signal in Figure VIII-4, it appears that we have prepared the B form with our crystallization procedure, as all six resonances of the two TATM molecules in the asymmetric unit are resolved. The TATM methyl group resonances appear as a triplet (one of the signals comes from the guest cyclohexane). The relative intensities may be interpreted as being $1,4,1$, consistent with two TATM molecules in the asymmetric unit. The TATM methine signal appears as a singlet, although in theory it should be a doublet. The thienyl carbon signals, which each should have a multiplicity of twelve, may be assigned the following relative intensities (from lowest to highest field): TATM quaternary $2,1,2,1,4,2$; TATM tertiary $1,1,1,3,3,1$, 1,1 . 

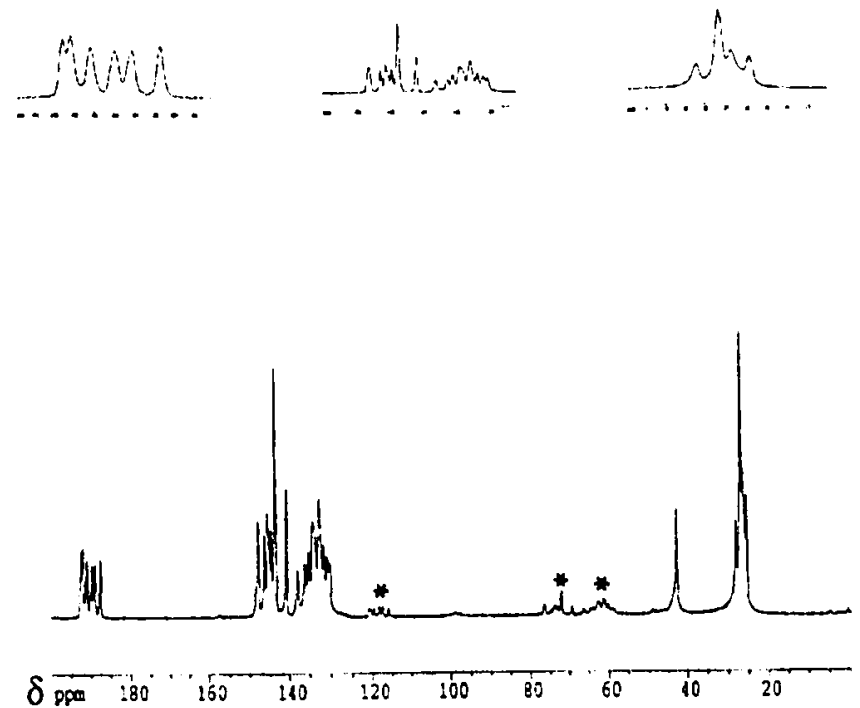

Figure VIII-4: ${ }^{13} \mathrm{C}$ CP/MAS NMR spectrum of the cyclohexane B/TATM inclusion compound. Spinning sidebands are indicated with an asterisk. Expansions appear in the insets from left to right as: (1) $195-185 \mathrm{ppm}$ (host carbonyl region) (2) $155-125 \mathrm{ppm}$ (host thienyl region) and (3) 32-22 ppm (host methyl region)

The ${ }^{13} \mathrm{C}$ CP/MAS NMR spectrum of $n$-hexane/TATM appears in Figure VIII-5. The signals at $13.0 \mathrm{ppm}\left(\mathrm{CH}_{3}\right), 24.2 \mathrm{ppm}\left(\alpha-\mathrm{CH}_{2}\right)$ and $33.1 \mathrm{ppm}\left(\beta-\mathrm{CH}_{2}\right)$ come from the nhexane guest. According to the literature crystal structure [13] $n$-hexane/TATM is an atypical TATM clathrate in that it forms a complex with a host:guest ratio of $3: 1$, and there are three TATM molecules in the crystallographic asymmetric unit. In the NMR spectrum, the carbonyl TATM signal shows a multiplicity of nine. From lowest to highest field, the individual multiplicities are 2, 1, 4, 2. The methyl TATM signal also shows a multiplicity of nine, the individual multiplicities are $2,1,1,5$. This again illustrates a one-to-one correspondence between carbons in the asymmetric unit and signals in the ${ }^{13} \mathrm{C} \mathrm{CP} / \mathrm{MAS}$ NMR spectrum. Theoretically, the thienyl tertiary carbon and the thienyl quaternary carbon signals should each show multiplicities of 18 , however there is extensive signal overlap in the NMR spectrum, and a detailed analysis is not possible. Finally, the TATM central methine carbon $\mathrm{CH}$ signal at $44 \mathrm{ppm}$ shows a splitting, one weak signal (1) at low field and a stronger signal (2) at high field, for a multiplicity of three, which is expected for three TATM molecules in the asymmetric unit. 


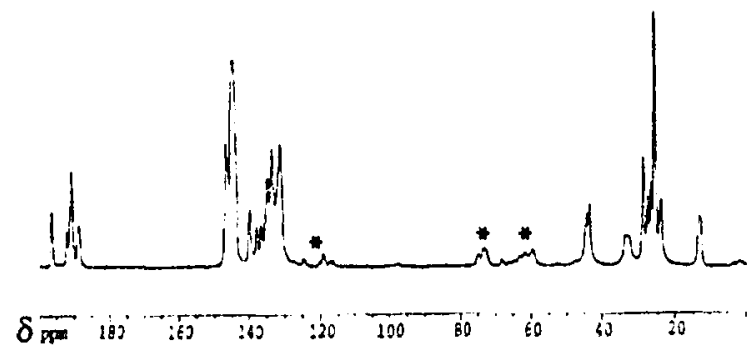

Figure VIII-5: ${ }^{13} \mathrm{C}$ CP/MAS NMR spectrum of the $n$-hexane/TATM inclusion compound Spinning sidebands are indicated with an asterisk. Expansions appear in the insets from left to right as: (1) 199-186 ppm (host carbonyl region) (2) $155-125 \mathrm{ppm}$ (host thienyl region) and (3) 32-22 ppm (host methyl region)

We screened a variety of TATM inclusion compounds using ${ }^{13} \mathrm{C}$ CP/MAS NMR spectroscopy to determine the number of TATM molecules in the asymmetric unit for each complex. For example, in Figure VIII-6, we present the ${ }^{13} \mathrm{C}$ CP/MAS NMR spectra of 2-chlorobutane/TATM, 2-bromobutane/TATM and 2-iodobutane/TATM. In all three cases, the multiplicity of the TATM carbonyl signal is six, and the multiplicity of the TATM methyl signal is also six. This indicates that there are two TATM molecules in the crystallographic asymmetric unit for all three inclusion complexes. Similar results for the other TATM inclusion compound families appear in Table VIII-1. At first glance, it seems that TATM forms inclusion compounds with the same number of TATM molecules in the asymmetric unit within each of the five groups. More interestingly, TATM forms complexes with one TATM molecule in the asymmetric unit for the smaller guest molecules such as the methylene halides \{Figure VIII-7 ( 1 carbon)\} and 1,3dihalopropanes \{Figure VIII-8 (3 carbons)\}, while there are two TATM molecules in the asymmetric unit for the complexes with larger guests such as the 2-halobutanes \{Figure VIII-6 (4 carbons)\}, cyclohexyl halides \{Figure VIII-9 (6 carbons)\} and monohalobenzenes \{Figure VIII-10 (6 carbons)\}. Thus we see the properties of molecular recognition of the TATM host for differently sized guests, of which further details are discussed next. 


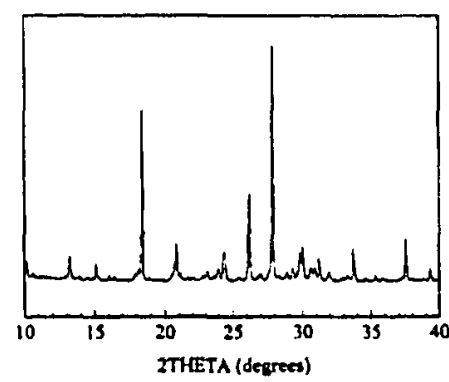

C: $\left(\mathrm{C}_{4} \mathrm{H}_{9}\right) \mathrm{I}$
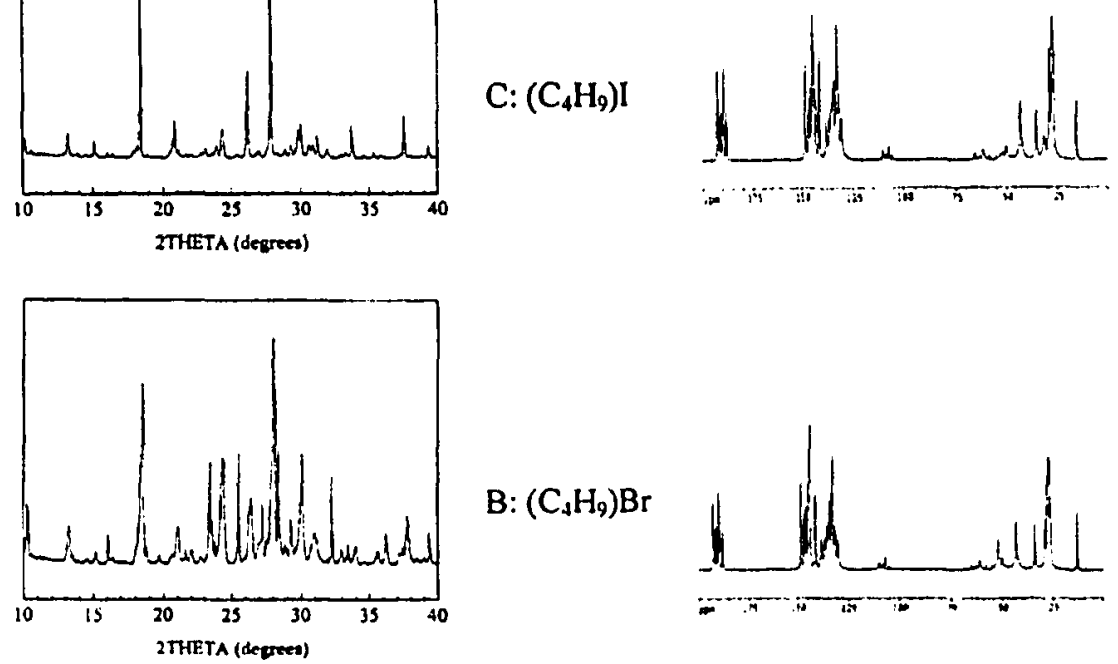

B: $\left(\mathrm{C}_{s} \mathrm{H}_{9}\right) \mathrm{Br}$
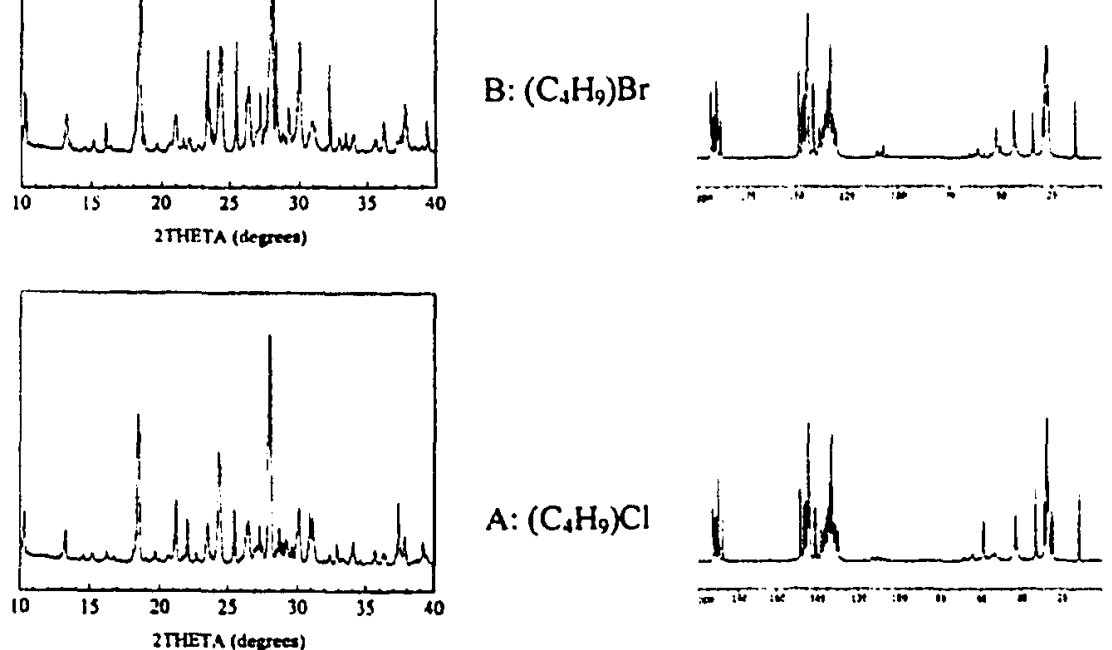

A: $\left(\mathrm{C}_{4} \mathrm{H}_{9}\right) \mathrm{Cl}$

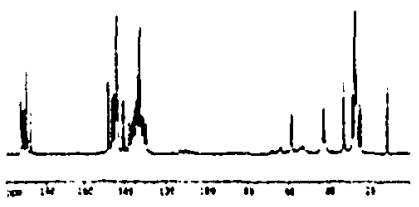

Figure VIII-6: Powder diffraction patterns (left) and ${ }^{13} \mathrm{C} \mathrm{CP/MAS} \mathrm{NMR} \mathrm{spectra} \mathrm{(right)}$ for the three 2-halobutane guests in TATM: (A) 2-chlorobutane/TATM (B) 2-bromobutane/TATM and (C) 2-iodobutane/TATM

(B) Powder X-ray diffraction (PXRD)

There are a multitude of interpretations in the literature as to what exactly is "molecular recognition". Here we offer one more interpretation, in essence the recognition by the host, during the crystallization process, of a physical property or properties of potential guest molecules. Perhaps the two simplest possibilities include the recognition of guest size and shape. Shape recognition may be thought of as the host forming isostructural complexes with a number of different guests that have the same hydrocarbon framework, but differing substituents. Size distinction (by a host) in this context could be the crystallization, by a host, of inclusion complexes of different structures with guests that have the same hydrocarbon framework, but substituents at selected positions that differ in size. 
Table VIII-1: A table of TATM guest families indicating the number of TATM Molecules in the respective asymmetric units of each, determined from ${ }^{13} \mathrm{C} \mathrm{CP} / \mathrm{MAS}$ NMR spectroscopy. Additionally, isostructural TATM inclusion compounds are indicated by a common superscript.

\begin{tabular}{|c|c|}
\hline GUEST & $\begin{array}{c}\text { Number of TATM molecules in the } \\
\text { asymmetric unit }\end{array}$ \\
\hline methylene chloride* $^{*}$ & 1 \\
\hline methylene bromide* $^{*}$ & 1 \\
\hline methylene iodide* $^{*}$ & 1 \\
\hline 1,3-difluoropropane & 1 \\
\hline 1,3 -dichloropropane & 1 \\
\hline 1,3-dibromopropane & 1 \\
\hline & 2 \\
\hline 2-chlorobutane & \\
\hline 2-bromobutane & \\
\hline 2-iodobutane & 2 \\
\hline & 2 \\
\hline benzene $^{+}$ & 2 \\
\hline fluorobenzene $^{+}$ & 2 \\
\hline chlorobenzene $^{\circ}$ & 2 \\
\hline bromobenzene $^{\circ}$ & 2 \\
\hline iodobenzene $^{\circ}$ & 2 \\
\hline cyclohexyl chloride $^{\mathrm{x}}$ & 2 \\
\hline cyclohexyl bromide $^{\mathrm{x}}$ & 2 \\
\hline cyclohexyl iodide $^{\mathrm{x}}$ & 2 \\
\hline
\end{tabular}

This definition is somewhat different than the more conventional view of molecular recognition in solution, which usually involves selective binding of one potential guest in a mixture of two or more potential guests. However, crystallization is a non-equilibrium process. In order to ensure that the inclusion mixture is indeed the equilibrium material, a selective inclusion experiment must be performed by stirring the solid host in the guest mixture, for an extended period of time (days or weeks) at the appropriate temperature. For inclusion compounds that exist as a definite entity in the dissolved state (e.g. cyclodextrins), this is not a problem, as equilibrium is achieved much more rapidly, however, the selectivity in the solid and solution can be expected to be very different because of packing effects and long-range interactions. 
As an example of shape recognition, TATM forms isostructural complexes with benzene, fluorobenzene, chlorobenzene and bromobenzene, as depicted in Figure VIII-7. This demonstrates that TATM "recognizes" the $\mathrm{C}_{6} \mathrm{H}_{5}$ phenyl functional group, regardless of whether the substituent is $\mathrm{H}, \mathrm{F}, \mathrm{Cl}$ or $\mathrm{Br}$. The same group of guests also illustrate size distinction, as the powder X-ray diffractograms for the benzene and monohalobenzene guests listed above are in stark contrast to the diffractogram for iodobenzene / TATM (see Figure VIII-7). This shows that TATM can distinguish between the smaller $\mathrm{H}, \mathrm{F}, \mathrm{Cl}$ and $\mathrm{Br}$ substituents on one hand, and the larger iodide substituent on the other. The structure of benzene / TATM has been published in the literature, [14] existing as a triclinic lattice in the $\mathrm{P} T \overrightarrow{\mathrm{l}}$ space group. By comparing the powder $\mathrm{X}$-ray diffractograms of benzene and the monohalobenzene $(\mathrm{X}=\mathrm{F}, \mathrm{Cl}, \mathrm{Br})$ guests in TATM with the powder $\mathrm{X}$ ray diffractograms of other TATM inclusion compounds (in Figures VIII-6 and VIII-8 to VIII-10), it is apparent that the former group's diffractograms contain many more reflections than the latter's. This suggests that the benzene and monohalobenzene ( $X=F$, $\mathrm{Cl}, \mathrm{Br}$ ) TATM inclusion compounds may exist as mixture of at least two polymorphs, as was also observed for the cyclohexane / TATM inclusion compound [12]. The powder $\mathrm{X}$-ray diffractogram for iodobenzene / TATM contains many fewer reflections, so it is possible that it crystallizes in a lattice of monoclinic or higher symmetry.

Based on a detailed analysis of the powder X-ray diffractograms of TATM inclusion complexes with guests cyclohexyl chloride, cyclohexyl bromide and cyclohexyl iodide (see Figure VIII-8), we have concluded that all three inclusion compounds are isostructural. This demonstrates molecular shape recognition by TATM of the cyclohexyl $\mathrm{C}_{6} \mathrm{H}_{11}$ functional group, regardless of the substituents present. Although the diffractograms do not appear to be identical at first glance, they do share many common

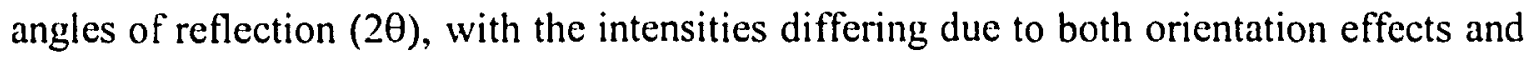
a moderate difference in guest electron density for the three different inclusion compounds. Additionally, the powder X-ray diffractograms of the three methylene halide guests appear in Figure VIII-9. On the basis of their appearance we conclude that methylene chloride / TATM, methylene bromide / TATM and methylene iodide / TATM are isostructural. This demonstrates molecular shape recognition by TATM of the methylene $\mathrm{CH}_{2}$ functional group, regardless of the halogen substituents. 

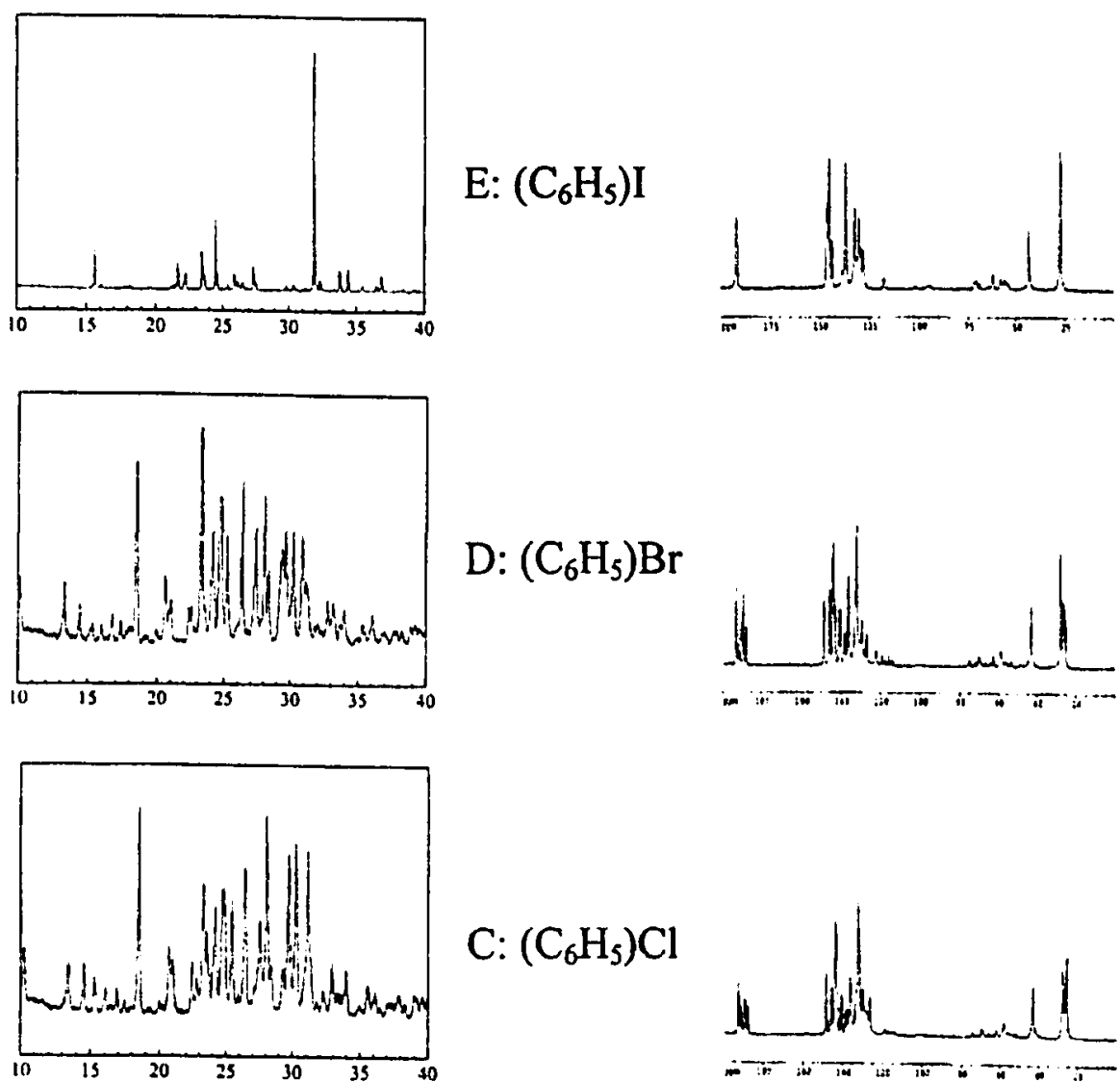

$\mathrm{C}:\left(\mathrm{C}_{6} \mathrm{H}_{5}\right) \mathrm{Cl}$
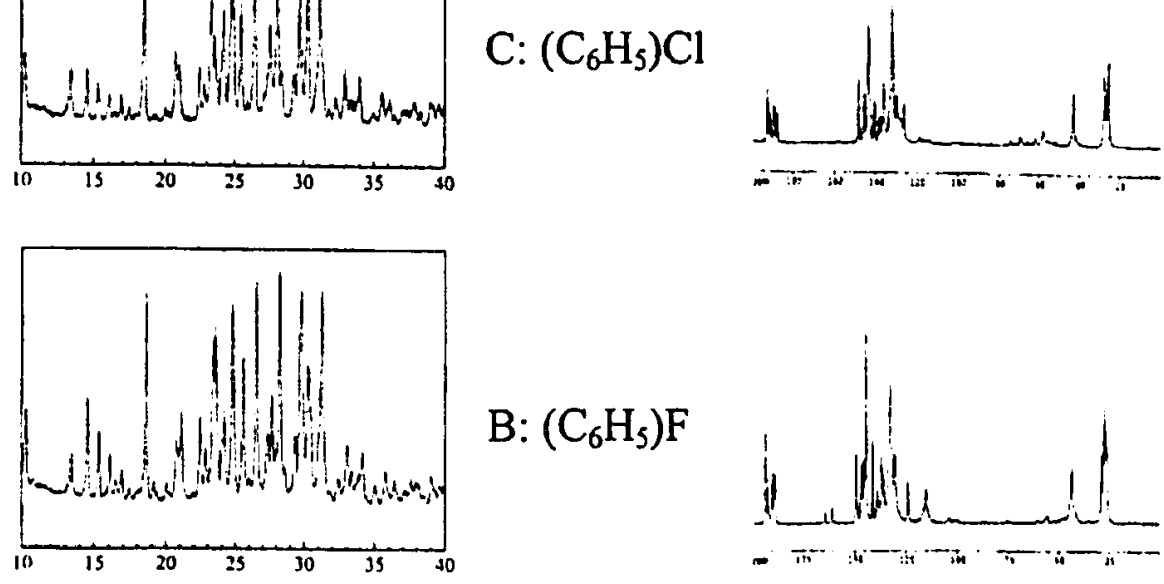

B: $\left(\mathrm{C}_{6} \mathrm{H}_{5}\right) \mathrm{F}$
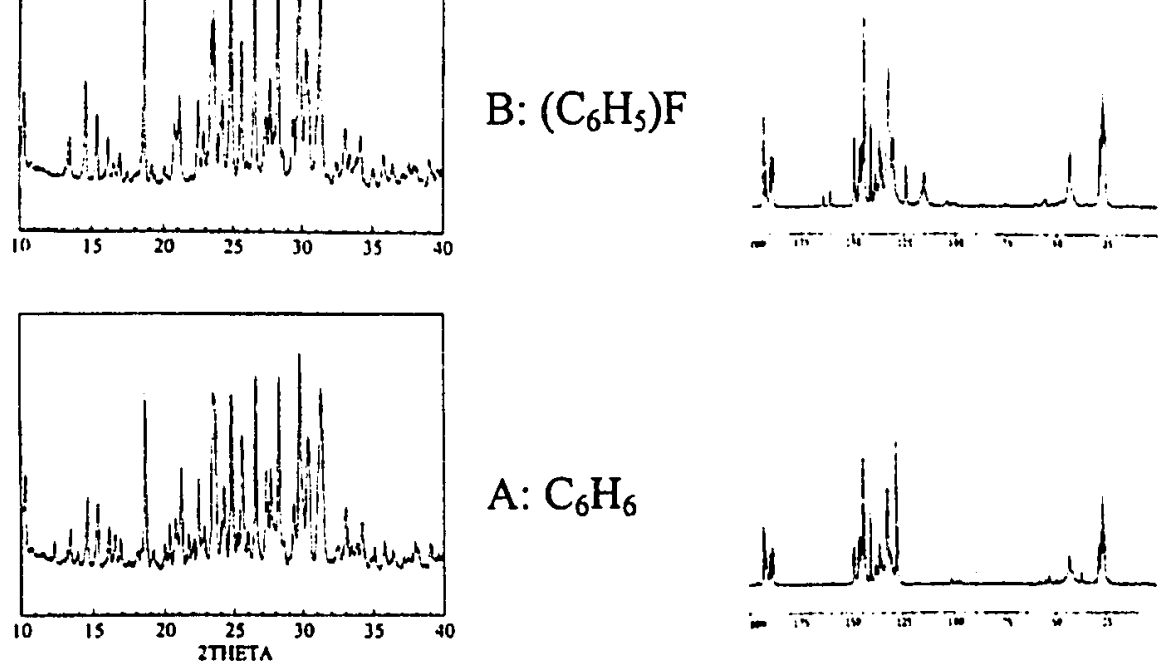

\section{A: $\mathrm{C}_{6} \mathrm{H}_{6}$}

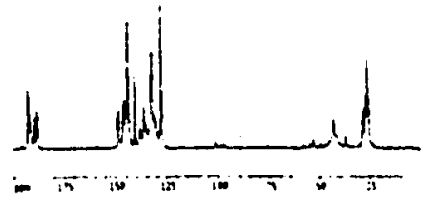

Figure VIII-7: Powder X-ray diffraction patterns (left) and ${ }^{13} \mathrm{C}$ CP/MAS NMR spectra (right) for benzene and the four monohalobenzene guests in TATM.

(A) benzene / TATM (B) fluorobenzene / TATM (C) chlorobenzene / TATM

(D) bromobenzene / TATM (E) iodobenzene / TATM 


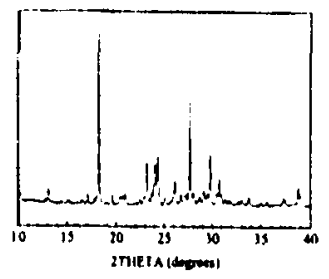

C: $\left(\mathrm{C}_{6} \mathrm{H}_{11}\right) \mathrm{I}$
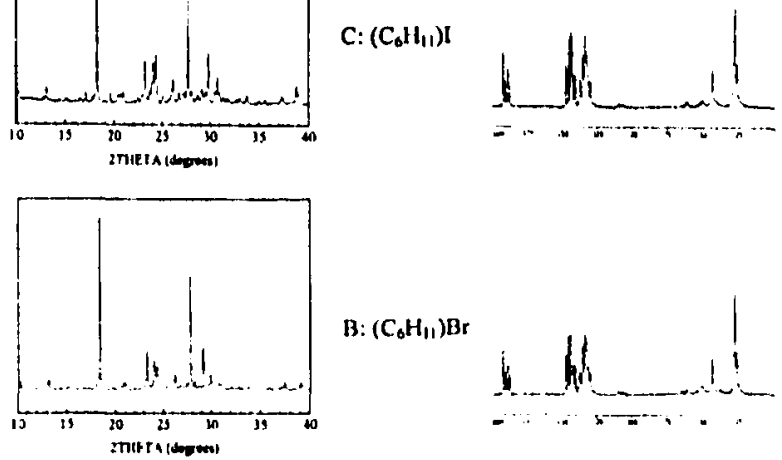

B: $\left(\mathrm{C}_{6} \mathrm{H}_{11}\right) \mathrm{Br}$
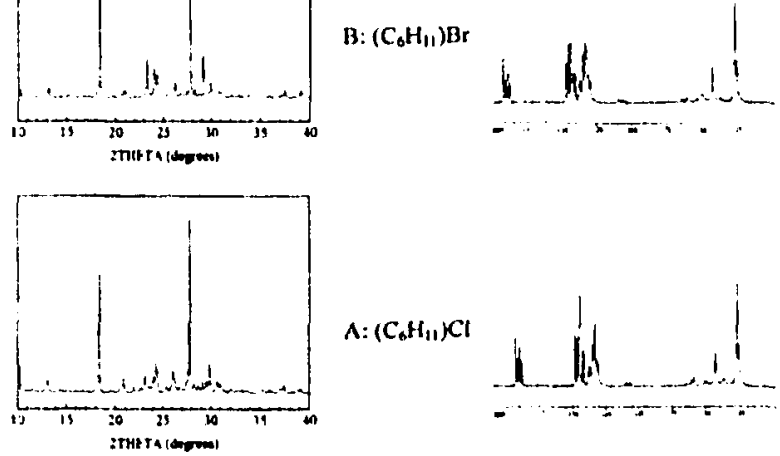

$A:\left(\mathrm{C}_{6} \mathrm{H}_{11}\right) \mathrm{Cl}$

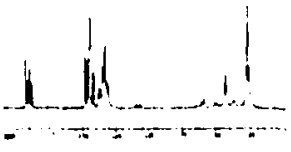

Figure VIII-8: Powder X-ray diffraction patterns (left) and ${ }^{13} \mathrm{C}$ CP/MAS NMR spectra (right) for the three cyclohexyl halide guests in TATM: (A) cyclohexyl chloride/TATM (B) cyclohexyl bromide/TATM and (C) cyclohexyl iodide / TATM

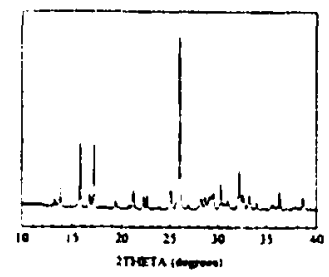

C: $\mathrm{CH}_{2} \mathrm{l}_{2}$
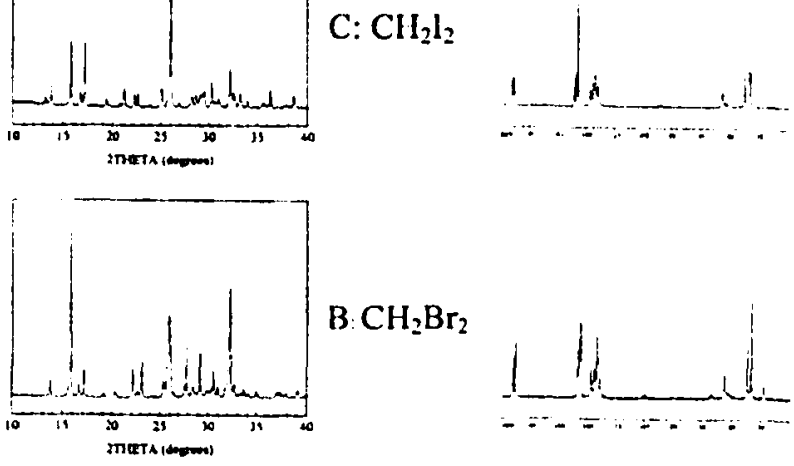

B: $\mathrm{CH}_{2} \mathrm{Br}_{2}$
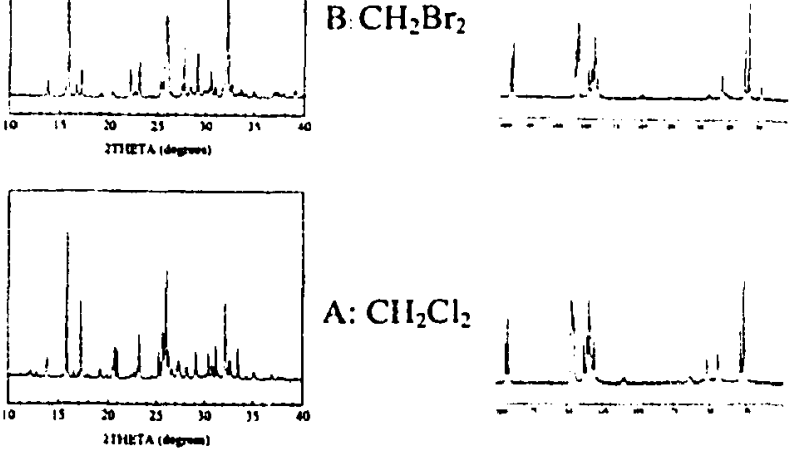

A: $\mathrm{Cl}_{2} \mathrm{Cl}_{2}$

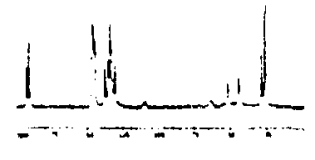

Figure VIII-9: Powder X-ray diffraction patterns (left) and ${ }^{13} \mathrm{C} C P /$ MAS NMR spectra (right) for the three methylene halide guests in TATM: (A) methylene chloride/TATM (B) methylene bromide / TATM and (C) methylene iodide / TATM 
Finally, we present the powder X-ray diffractograms for the 2-halobutane and 1,3dihalopropane inclusion compounds in Figures VIII- 6 and VIII-10 respectively. By a detailed analysis of the spectra we have concluded that 2-chlorobutane / TATM and 2bromobutane / TATM are isostructural, but that 2-iodobutane / TATM is of a different structure (see Figure VIII-6). Similarly, 1,3-dichloropropane / TATM and 1,3dibromopropane / TATM are isostructural, but 1,3-difluoropropane / TATM is not isostructural with the first two (see Figure VIII-10). It is also to be noted that, according to the literature crystal structures, [12] cyclohexane B/TATM, cycloheptane/TATM and cyclooctane/TATM are all isostructural, possibly demonstrating that TATM "senses" all three guests, during the crystallization process, as being of similar shape and size.

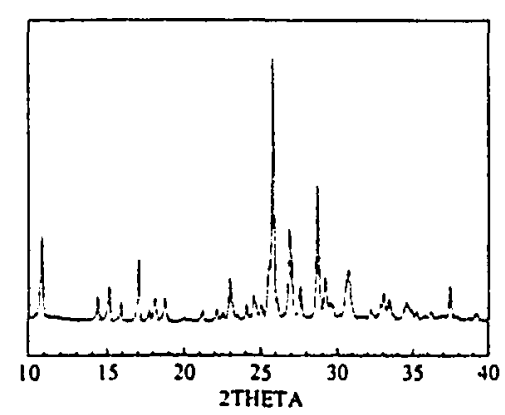

$\mathrm{C}: \mathrm{Br}\left(\mathrm{CH}_{2}\right)_{3} \mathrm{Br}$
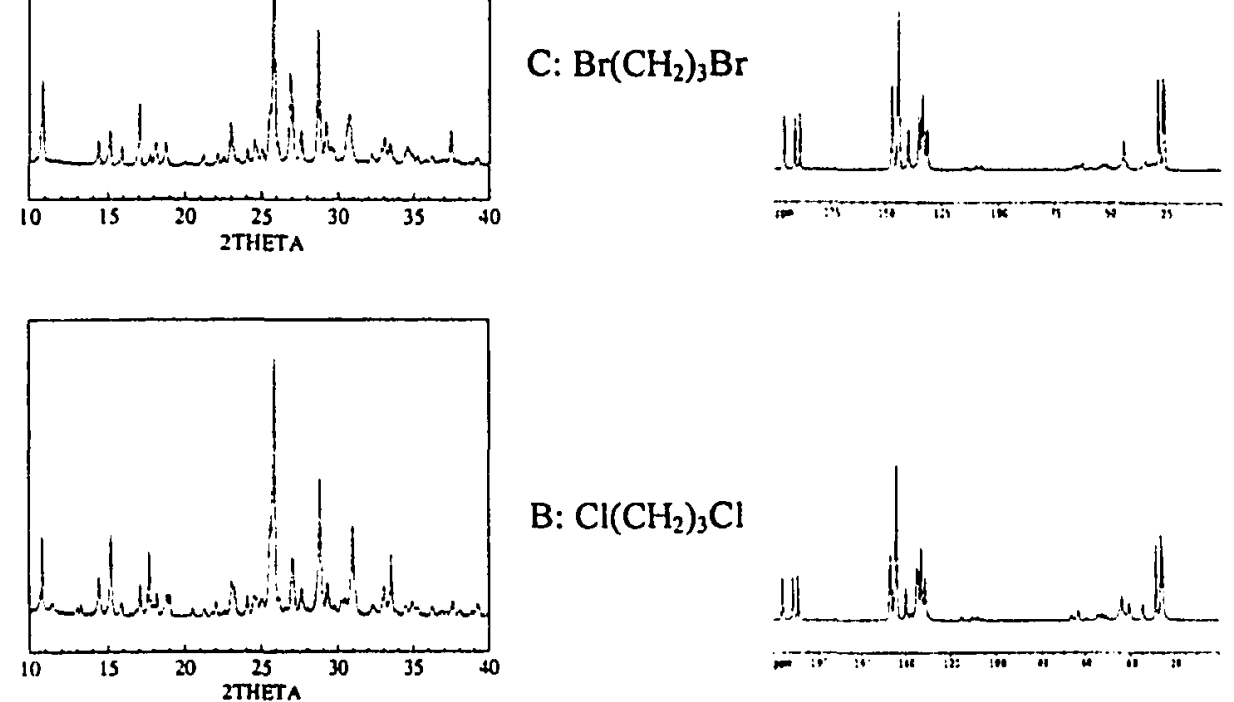

B: $\mathrm{Cl}\left(\mathrm{CH}_{2}\right)_{3} \mathrm{Cl}$
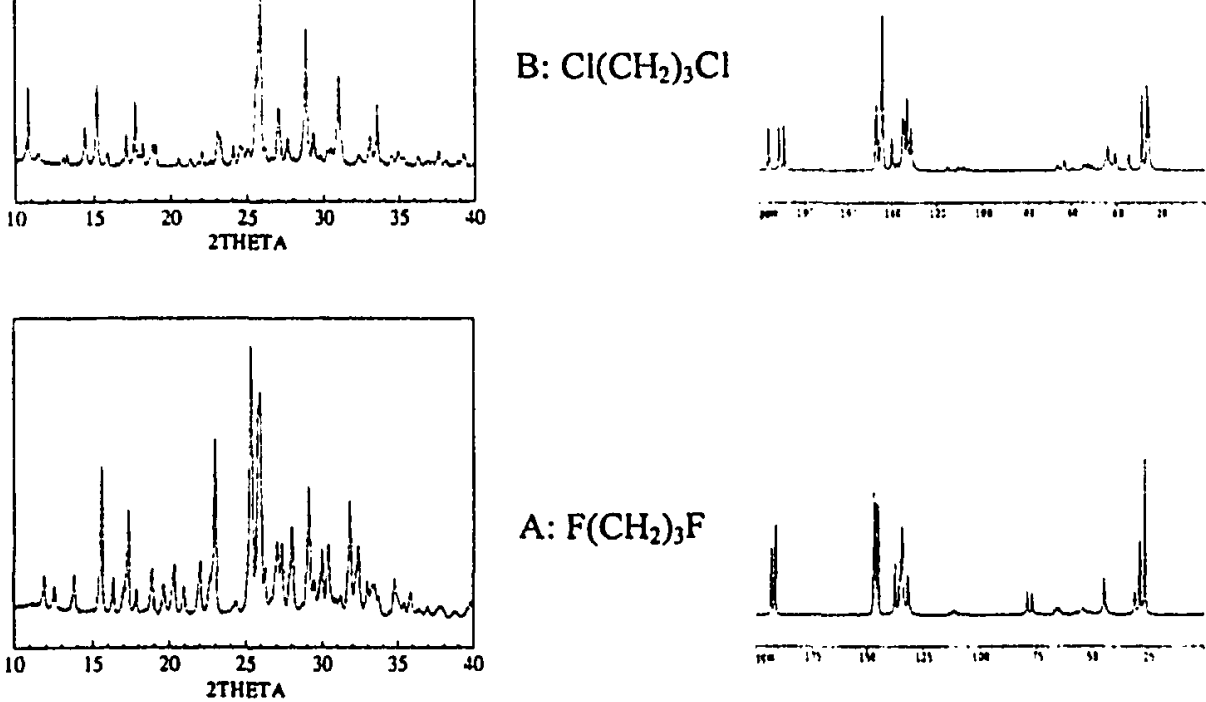

A: $\mathrm{F}\left(\mathrm{CH}_{2}\right)_{3} \mathrm{~F}$

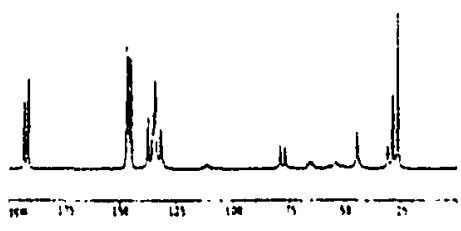

Figure VIII-10: Powder X-ray diffraction patterns (left) and ${ }^{13} \mathrm{C} C \mathrm{CP} / \mathrm{MAS}$ NMR spectra (right) for the three 1,3-dihalopropane guests in TATM: (A) 1,3-difluoropropane / TATM (B) 1,3-dichloropropane / TATM and (C) 1,3-dibromopropane / TATM 


\section{(C) Differential Scanning Calorimetry (DSC)}

We determined the melting points of TATM inclusion compounds using two different techniques. The first involved visual inspection of the samples (ca. $1 \mathrm{mg}$ ) while they were being heated at a fixed rate in an open capillary tube. These melting points, reported in the $3^{\text {rd }}$ column of Table VIII-2 under the heading " $T_{\text {m }}$ (MPA)" (MPA being short for melting point apparatus), are tabulated as a range, the lower temperature being that corresponding to the first appearance of liquid, and the upper temperature being that at which the last particles of solid enter the liquid phase. The second technique entailed differential scanning calorimetry (DSC), the details of which are listed in the experimental section. It should be noted that the DSC samples were heated in hermetically sealed pans, specified as capable of withstanding pressures of up to five atmospheres. The melting endotherms of all of the TATM complexes measured were single sharp endotherms, as can be seen in Figure VIII-1I for the case of the cyclohexyl halides. From lowest to highest temperature the endotherms come from TATM / cyclohexyl iodide $\left(T_{\text {onset }}=96.4^{\circ} \mathrm{C}\right), \mathrm{TATM} /$ cyclohexyl bromide $\left(T_{\text {onset }}=107.3^{\circ} \mathrm{C}\right)$ and TATM / cyclohexyl chloride $\left(T_{\text {onset }}=111.4^{\circ} \mathrm{C}\right)$. No other phase transitions were observed for any of the TATM inclusion compounds studied in the temperature range from room temperature to $250^{\circ} \mathrm{C}$. The melting points listed in the $4^{\text {th }}$ column of Table VIII-2 are onset temperatures, the temperature corresponding to the intersection of two tangents, one to the baseline immediately before the thermal event, the other to the steepest part of the leading edge of the thermal event itself. In theory, the onset temperature should be independent of the heating rate used in DSC experiments; we used a heating rate of $5 \mathrm{~K} / \mathrm{min}$.

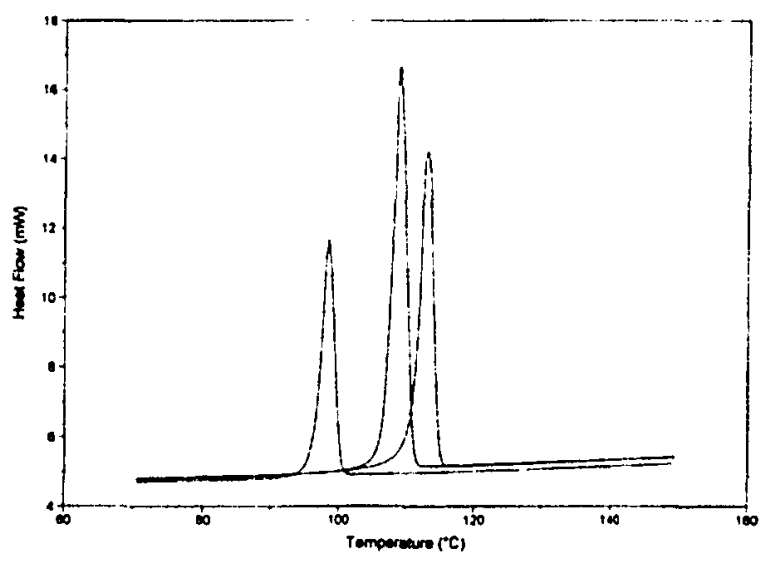

Figure VIII-11: DSC thermograms of cyclohexyl halide / TATM inclusion compounds. From lowest to highest temperature, the traces correspond to: (A) cyclohexyl iodide $\left(T_{\mathrm{m}}=96.4^{\circ} \mathrm{C}\right.$, $\Delta_{\text {ius }} H=31 \mathrm{~kJ} \mathrm{~mol}^{-1}$ )

(B) cyclohexyl bromide $\left(T_{\mathrm{m}}=107.3^{\circ} \mathrm{C}\right.$, $\Delta_{\text {ius }} H=35 \mathrm{~kJ} \mathrm{~mol}^{-1}$ )

(C) cyclohexyl chloride $T_{\mathrm{m}}=111.4^{\circ} \mathrm{C}$, $\Delta_{\text {tus }} H=37 \mathrm{~kJ} \mathrm{~mol}^{-1}$ ) 
Table VIII-2: A table of TATM guest families melting point data.

\begin{tabular}{|c|c|c|c|c|}
\hline GUEST & $\begin{array}{c}\text { Guest } \\
\mathrm{BP}\left( \pm{ }^{\circ} \mathrm{C}\right)\end{array}$ & $\begin{array}{c}T_{\mathrm{m}} \mathrm{MPA} \\
\left( \pm 1^{\circ} \mathrm{C}\right)\end{array}$ & $\begin{array}{c}\text { DSC onset } T_{\mathrm{m}} \\
\left( \pm 0 .{ }^{\circ} \mathrm{C}\right)\end{array}$ & $\begin{array}{c}T_{\mathrm{MPA}}-T_{\mathrm{DSC}} \\
\left({ }^{\circ} \mathrm{C}\right)\end{array}$ \\
\hline 2-Chlorobutane & 68 & $108-110$ & 108.6 & -0.6 \\
\hline 2-Bromobutane & 91 & $104-105$ & 107.1 & -3.1 \\
\hline 2-Iodobutane & 119 & $102-103$ & 100.8 & +1.2 \\
\hline Fluorobenzene & 85 & $106-110$ & 129.8 & -23.8 \\
\hline Chlorobenzene & 132 & $116-119$ & 128.5 & -12.5 \\
\hline Bromobenzene & 156 & $128-130$ & 126.0 & +2.0 \\
\hline lodobenzene & 188 & $131-132$ & 134.9 & -3.9 \\
\hline & & & & -14.9 \\
\hline Methylene chloride & 40 & $111-113$ & 125.9 & -18.7 \\
\hline Methylene bromide & 96 & $115-117$ & 133.7 & -0.9 \\
\hline Methylene iodide & 181 & $118-119$ & 118.9 & \\
\hline Cyclohexyl chloride & 142 & $108-109$ & 111.4 & -3.4 \\
\hline Cyclohexyl bromide & 167 & $106-107$ & 107.3 & -1.3 \\
\hline Cyclohexyl iodide & $180($ dec $)$ & $95-96$ & 96.4 & -1.4 \\
\hline & & & & \\
\hline 1,3-dichloropropane & 120 & $98-99$ & 103.2 & -5.2 \\
\hline 1,3 -dibromopropane & 167 & $101-102$ & 102.4 & -1.4 \\
\hline 1,3-diiodopropane & 192 & $114-115$ & 114.7 & -0.7 \\
\hline
\end{tabular}

Column 2: $\quad$ The boiling point of the pure guest solvent in ${ }^{\circ} \mathrm{C}$

Column 3: The melting point of the TATM inclusion compound determined using a hot stage melting point apparatus with the sample in an open capillary tube.

Column 4: The onset melting point temperature of the TATM inclusion compound determined using differential scanning calorimetry experiments with the sample in a hermetically sealed aluminum pan.

Column 5: The difference between the melting points from column 3 and column 4 .

By inspection of the data in Table VIII-2, it can be seen that almost all of the melting points determined with the melting point apparatus are lower than those of the same inclusion compound determined using DSC. The reason for the low melting points lies in the fact that as the temperature is raised, volatile guests escape from the inclusion compound if the material isn't confined. Although the detailed phase diagrams are not known, it is likely that the (hypothetical) empty host lattice is unstable without a guest present, and may have a low melting point (however a crystalline dense guest-free material, with a fairly high melting point, is reported in Chapter XV). Notably, for the more volatile guests such as fluorobenzene (bp $85^{\circ} \mathrm{C}$ ), chlorobenzene (bp $132^{\circ} \mathrm{C}$ ), methylene chloride (bp $40^{\circ} \mathrm{C}$ ) and methylene bromide (bp $96^{\circ} \mathrm{C}$ ), the differences are quite 
large, as one would expect low boiling guests to disappear from the lattice more easily (i.e. at a lower temperature) than the higher boiling guests, with a concomitant lower melting point in the open capillary tube. (On the other hand, for the fairly volatile guest 2-chlorobutane (bp. $68^{\circ} \mathrm{C}$ ), there is little difference between the two melting points $\left.\left(\Delta_{\mathrm{MP}}=-0.6^{\circ} \mathrm{C}\right)\right)$. The only inclusion compounds for which the onset melting point from DSC is lower are 2-iodobutane/TATM $\left(+1.2^{\circ} \mathrm{C}\right)$ and bromobenzene/TATM $\left(+2.0^{\circ} \mathrm{C}\right)$. Both guests are fairly involatile relative to the ones listed above. The differences for the rest of the complexes are small and negative, no greater than $-4^{\circ} \mathrm{C}$. These results suggest that in order to obtain correct melting points for inclusion compounds with volatile guests, it is necessary to use differential scanning calorimetry with hermetically sealed pans, as inclusion compounds in open capillary tubes may show a melting point depression.

Similar results have been published by McAdie $[15-18]$ who studied the thermal decomposition of urea adducts. For a number of urea inclusion compounds with evennumbered $n$-paraffins, the melting temperatures determined from hot stage microscopy, in which the samples were "spread out in a thin layer", were considerably lower than those determined by differential thermal analysis, in which "a narrow cylinder of compressed sample offers considerable impedance to diffusion of the n-paraffin guest molecules". The difference in temperature was more pronounced with the smaller, more volatile guest molecules, as is also observed in our study with TATM inclusions.

The enthalpy of melting and the entropy of melting for the present TATM inclusion compounds are listed in Table VIII-3. The enthalpy can be measured directly from the DSC melting endotherm by integration, while the entropy is calculated by dividing the enthalpy by the onset temperature $\left(\Delta_{\text {tus }} G=0\right)$. As can be seen from Table VIII-3, for all five groups of guests, the enthalpy of melting is consistently larger for smaller guests within one group, and decreases as the size of the halogen substituent increases.

The entropy of melting within each group is also consistently larger for smaller guests, and decreases as the size of the halogen substituent increases. Again, if one assumes that entropy of the liquid melt is similar for all complexes within one group, this suggests that the absolute entropy of the solid inclusion complex is smaller for the 
smaller guests, or that they are more ordered. Intuitively, this is what one may expect based on the guest's dipole moments. The more polar fluorides and chlorides would likely interact more strongly with the TATM host and show less orientational disorder compared to the less polar bromides and iodides. This may also suggest why TATM forms more strongly bound complexes with more polar guests, based on the $\Delta_{\text {fus }} H$ values given in Table VIII-3, that is, TATM may distinguish on the basis of polarity rather than on the basis of size. We wish to remark that the $\Delta_{\text {lus }} \mathrm{S}$ values in Table VIII-3 (73-113 J $\mathrm{mol}^{-1} \mathrm{~K}^{-1}$ ) fall in the general range of $90 \mathrm{~J} \mathrm{~mol}^{-1} \mathrm{~K}^{-1}$, remarked to be a typical" $\Delta_{\text {fus }} \mathrm{S}$ value for molecular crystals by Brown and Brown [19]. Finally, we state that the TATM melting process is not reversible, as samples cooled from high temperature to not display an exotherm corresponding to inclusion compound formation.

Table VIII-3: A table of TATM guest families enthalpy and entropy of fusion data.

\begin{tabular}{|c|c|c|c|}
\hline GUEST & $\begin{array}{c}\Delta_{\text {fus }} H \\
\left( \pm 1 \mathrm{~J} \mathrm{~g}^{-1}\right)\end{array}$ & $\begin{array}{c}\Delta_{\text {fus }} H( \pm 1 \mathrm{~kJ} \mathrm{~mol} \\
\text { TATM })\end{array}$ & $\begin{array}{c}\Delta_{\text {fus }} S( \pm 2 \mathrm{~J} \\
\left.\mathrm{mol}^{-1} \mathrm{~K}^{-1}\right)\end{array}$ \\
\hline 2-Chlorobutane & 111 & 43 & 113 \\
\hline 2-Bromobutane & 94 & 36 & 96 \\
\hline 2-lodobutane & 84 & 33 & 87 \\
\hline Fluorobenzene & & & 109 \\
\hline Chlorobenzene & 113 & 44 & 103 \\
\hline Bromobenzene & 107 & 42 & 97 \\
\hline Iodobenzene & 100 & 39 & 88 \\
\hline & 93 & 36 & 106 \\
\hline Methylene chloride & & & 94 \\
\hline Methylene bromide & 109 & 42 & 81 \\
\hline Methylene iodide & 98 & 38 & 96 \\
\hline Cyclohexyl chloride & 82 & 32 & 91 \\
\hline Cyclohexyl bromide & & & 84 \\
\hline Cyclohexyl iodide & 95 & 37 & 97 \\
\hline
\end{tabular}

Column 2: The enthalpy of fusion for the TATM inclusion compounds, in joules per gram. determined from DSC experiments.

Column 3: The enthalpy of fusion for the TATM inclusion compounds, in kilojoules per mole of unit cell, calculated from $\Delta_{\text {fus }} H$ in column 2 .

Column 4: The entropy of fusion for TATM inclusion compounds, in joules per mole Kelvin, calculated from $\Delta_{\text {fus }} H$ in column 3 . 


\section{DISCUSSION}

(D) Solid-solution model

An assumption which is generally made with respect to thermodynamic properties of inclusion compounds is that the host lattice behaves as if it is a static, weakly interacting framework. Therefore, each thermodynamic property may be split into contributions from the host and guest. Support for this assumption comes from the success of the theories for which independence of the contributions is invoked, such as decomposition pressures, enthalpies of clathration and heat capacities [20]. Therefore, for an isostructural series of inclusion compounds (with one host and different guests), one would expect the host lattice to contribute a constant and set amount to the enthalpy of melting. Consideration of the "solid solution" model [21] for clathrates allows us to set up a framework to consider this in more detail. This model assumes that: (a) a hypothetical empty lattice $\left(\beta\right.$-form, $S_{\beta}{ }_{\beta}$ ) exists that is metastable with respect to the stable, dense guest-free $\alpha$-form, $S_{\mu}$, (b) the $S_{\beta}^{0}$-lattice is stabilized by the presence of guest molecules, (c) the $S_{\beta}^{o}$-lattice does not depend on the nature of the guest, and (d) guest-guest interactions are absent. At this stage, little is known about the TATM clathrates regarding the validity of these assumptions. However, as described in Chapter $\mathrm{XV}$, we were able to prepare a stable dense guest-free $\left(\mathrm{S}_{\alpha}\right)$ TATM phase (a) and its structure does not depend on the nature of the complex from whence it was prepared (c).

Clathrate formation from the stable $S_{\alpha}$ form and gaseous guest $G_{g}$ can be decomposed into the following steps:

$$
\begin{aligned}
& S_{\alpha} \rightarrow S_{\beta}^{0} \\
& S_{\beta}^{0}+G_{g} \rightarrow S_{\beta}
\end{aligned}
$$

where Equation VIII-1 represents the activation of the stable $S_{\alpha}$ form to the metastable empty $S_{\beta}^{o}$ form. The heat of inclusion can be understood in terms of the sum of two contributions:

$$
\Delta H_{\text {inclusion }}=\Delta H\left(\mathrm{~S}_{\alpha} \rightarrow \mathrm{S}_{\beta}^{\circ}\right)+\Delta H\left(\mathrm{~S}_{\beta}^{\circ}+\mathrm{G}_{g} \rightarrow \mathrm{S}_{\beta}\right)
$$


If the guest material is liquid or solid, an extra step must be taken into account in order to produce gaseous guest, $\mathrm{G}_{\mathrm{l}} \rightarrow \mathrm{G}_{\mathrm{g}}$, or $\mathrm{G}_{\mathrm{s}} \rightarrow \mathrm{G}_{\mathrm{g}}$, introducing the heats of vaporization or sublimation to account for the fact that guest-guest cohesive interactions in the pure phases are lost during the clathration process. Fusion then involves the steps:

$$
\begin{aligned}
& \mathrm{S}_{\beta} \rightarrow \mathrm{S}_{\beta}{ }+\mathrm{G}_{\mathrm{g}} \\
& \mathrm{S}_{\beta}^{\mathrm{o}} \rightarrow \mathrm{S}_{\alpha} \\
& \mathrm{S}_{\alpha} \rightarrow \mathrm{L} \\
\text { net: } & \mathrm{S}_{\beta} \rightarrow \mathrm{G}_{\mathrm{g}}+\mathrm{L}
\end{aligned}
$$

and the corresponding heat of fusion is:

$$
\Delta_{\text {fus }} H=-\Delta H_{\text {inclusion }}+\Delta H\left(\mathrm{~S}_{\mathrm{u}} \rightarrow \mathrm{L}\right)
$$

Appropriate terms must be added if there is a change of phase of the guest material. Also, in this "zero order" approximation it is assumed that no corrections are needed for the fact that formation and fusion do not take place at the same temperature. In this model, differences in the enthalpy of fusion for the TATM clathrates must then be accounted for by differences in the enthalpy of inclusion. The two contributions in Equation VIII-3 are due to the activation of the dense form to the hypothetical empty lattice, and the enthalpy due to the interaction of the the empty lattice with the isolated guest. Since there are different structural "polymorphs" of the empty $S_{\beta}^{\circ}$ form (as deduced from the groupings in Table VIII-1), these can be labelled by an additional number, $n$, as in $\mathrm{S}_{\beta \mathrm{n}}^{\mathrm{O}}$. $\Delta H$ for this step therefore is constant within each isostructural group $n$, and the second contribution will depend on the nature of the guest.

There are a number of well-known correlations of enthalpies of fusion for inclusion compounds. For instance, for $n$-paraffins and monosubstituted $n$-paraffins in urea there is an incremental increase in the enthalpy of decomposition with guest chain length $[15,16]$. Two publications by McAdie deal with differential thermal analysis studies of the thermal decomposition of $\beta$-quinol inclusion compounds $[17,18]$. Decomposition temperatures and enthalpy of decomposition for the guests nitrogen, carbon dioxide, methanol and methyl cyanide, $\mathrm{HCl}, \mathrm{HBr}$, methyl chloride and methyl bromide were made. In general two trends were found. For small molecules presumed not to distort the quinol cavity the decomposition enthalpies increase with the size of the guest, whereas for the larger guests the decomposition enthalpies decrease with 
increasing guest size. The first instance can be explained by attractive guest-host interactions becoming more effective as the guest fills the cavity more efficiently. Once an optimum guest size has been reached, further increase in guest size stretches the cavity (albeit minimally), thus decreasing the net enthalpy of the inclusion material. We note that for such measurements to have value, knowledge of the composition of the clathrate is essential, as the guest-host interaction energy will depend on the number of filled cavities. Stretching of the cavity, as witnessed by changes in crystallographic parameters, clearly would place the materials beyond description by the simple solid-solution model.

This trend is also seen with the TATM inclusion compounds if we assume that all of the guests are considered on the large side: smaller guests (e.g. fluorides and chlorides) form TATM inclusions with greater $\Delta_{\text {fus }} H$ than larger guests (e.g. bromides and iodides). Truly small guests have not been studied at all. Whether cage stretching occurs will have to be determined from more complete structural work. Similar trends were noted for determinations of decomposition enthalpies for chloro- and bromo- substituted $\mathrm{C}_{12}$ paraffins in urea, with the lauryl chloride compound having a larger value than that of the lauryl bromide [22].

Differences in average enthalpies of fusion among the different groups of isostructural clathrates may be in part to the first term in Equation VIII-3. The enthalpy required for organizing the stable $S_{12}$ form to the differing hypothetical empty $S_{\beta n}^{\prime}$ forms would be expected to be relatively small and positive, and seems to be the smallest for the substituted aromatics and largest for the 1,3-dihalopropanes. It is clear that the hydrocarbon fragment rather than the halogen is the key element in determining the structural grouping. As a final remark, we note that differences in the composition of the inclusion compounds when they melt (a measured from TGA) may also affect the enthalpy of fusion. It is likely that a lower guest content at melting (due to a higher guest volatility) may lower $\Delta_{\text {fus }} H$.

(E) Cyclodextrins

A recent study [23] reported substituent effects of the guest on the driving force for inclusion complexation of $\alpha$-cyclodextrin and $\beta$-cyclodextrin ( $\alpha-C D$ and $\beta-C D)$ with a large number of monosubstituted benzene derivatives. Among other guests, they 
measured association constants in aqueous solution $\left(K_{\mathrm{a}}\right)$ for monohalobenzene guests $(\mathrm{PhF}, \mathrm{PhCl}, \mathrm{PhBr}$ and $\mathrm{Phl})$, by means of UV-vis absorption and fluorescence spectroscopy (see Table VIII-4). From this table, it can be seen that for both $\alpha-C D$ and $\beta-\mathrm{CD}$, the order of association constant follows: $\mathrm{PhI}>\mathrm{PhBr}>\mathrm{PhCl}>\mathrm{PhF}$, which is exactly the opposite as that found in our DSC studies of TATM inclusion compounds with the same guests, for which $\Delta_{\text {fus }} H$ follows the order: $\mathrm{PhI}<\mathrm{PhBr}<\mathrm{PhCl}<\mathrm{PhF}$.

Table VIII-4: Association constants $\left(\mathrm{L} \mathrm{mol}^{-1}\right)$ for the inclusion complexation of $\alpha-\mathrm{CD}$ and $\beta-C D$ with monohalobenzenes in $\mathrm{H}_{2} \mathrm{O}$ at $25^{\circ} \mathrm{C}$ [23]:

\begin{tabular}{lll}
\hline Guest & $K_{:}(\alpha-\mathrm{CD})$ & $K_{3}(\beta-\mathrm{CD})$ \\
\hline fluorobenzene & $39.7 \pm 1.6$ & $90.9 \pm 20$ \\
chlorobenzene & $112 \pm 10$ & $186 \pm 12$ \\
bromobenzene & $540 \pm 170$ & $322 \pm 15$ \\
iodobenzene & $1200 \pm 20$ & $846 \pm 20$ \\
\hline
\end{tabular}

The authors noted that several driving forces have been postulated for the inclusion complexation of cyclodextrin with guest compounds [24,25], namely: (1) van der Waals forces [26], (2) hydrophobic interactions [27], (3) hydrogen bonding [28], (4) release of distortional energy of the cyclodextrin host by binding guest, and (5) extrusion of "high energy" water molecules from the cyclodextrin upon inclusion compound formation. As far as TATM inclusions with the alkyl halide guests are concerned, perhaps the most important driving forces are weaker van der Waals forces (esp. alkyl bromides and iodides) and stronger dipolar forces (esp. alkyl fluorides and chlorides).

The authors noted that the absorption and fluorescence intensities of the benzene derivatives in an aqueous environment changed significantly upon addition of $\alpha-C D$ or $\beta$ CD. For all 24 monosubstituted benzene guests, they found a somewhat direct relationship between the association constant of the cyclodextrin inclusion compound and the substituent molar refraction $\left(R_{\mathrm{m}}\right)$ of the guest compound, similar to an earlier finding on the inclusion of substituted phenyl acetates in cyclodextrins [29]. The substituent molar refraction can be used as a parameter reflecting the volume and polarizability of the guest substrate. A larger $R_{\mathrm{m}}$ is associated with a larger molecular volume and thus a higher polarizability of the guest, suggestive of van der Waals interactions as a major 
driving force in the complexation of these monosubstituted benzene derivatives with $\alpha$ $\mathrm{CD}$ or $\beta-\mathrm{CD}$. On the other hand, stronger dipole-dipole forces seem to govern TATM / alkyl halide materials, as explained in detail below.

The binding constant $\left(K_{\mathrm{a}}\right)$ of the halobenzene guests with $\alpha-C D$ is more dependent on the molar refraction $\left(R_{\mathrm{m}}\right)$ than with $\beta$-CD (see Figure VIII-12), suggesting that the varying halogen substituent interacts more strongly with the inner surface $\alpha-C D$, while the invariant phenyl ring of the guest interacts more strongly with the internal surface of $\beta-C D$. In other words, for entry from the wider rim (halogen first), the depth of inclusion is greater for $\beta-C D$ than it is for $\alpha-C D$ (see Figure VIII-13). As far as TATM is concerned, it seems likely that the halogen substituent (rather than the alkyl backbone) interacts strongly with the host substructure, which manifests itself in the significant variation of $\Delta_{\text {fus }} H$ with the halogen atom(s) of the guest molecule within each guest family (Table VIII-3).

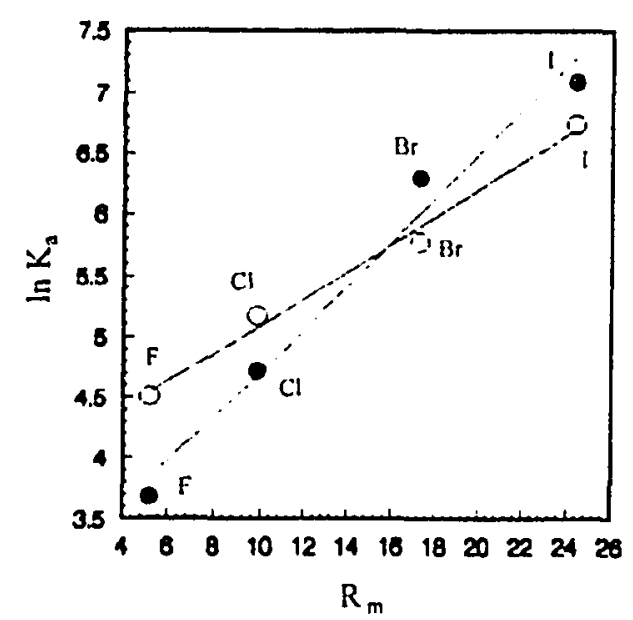

Figure VIII-12: Plots of $\ln K_{\mathrm{a}}$ vs molar refraction $\left(R_{\mathrm{m}}\right)$ of the halogen atoms for the inclusion of $\alpha-C D(\bullet)$ and $\beta-C D(0)$ with halobenzenes [23]
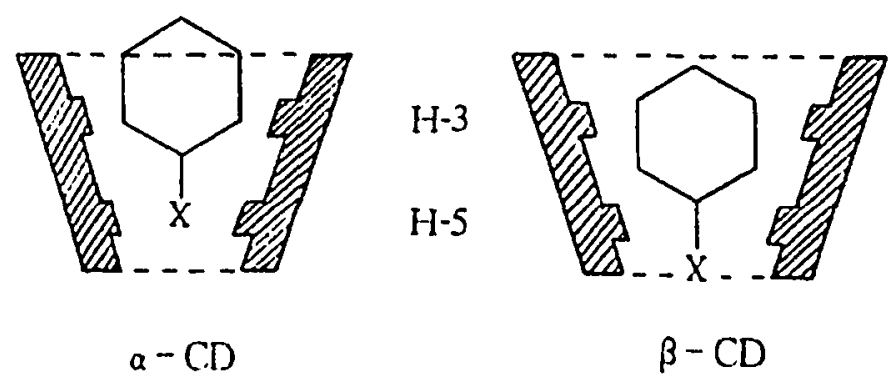

Figure VIII-13: Schematic illustration of the features of orientation and location of the halosubstituted benzenes in the cavities of $\alpha-C D$ and $\beta-C D$. The H-3 and H-5 atoms (of the cyclodextrin host) pointing into the cavity are shown in cross-section [23]. 
Interestingly, $K_{\mathrm{a}}$ values for the inclusion complexes of $\beta-\mathrm{CD}$ with $\mathrm{PhF}$ and $\mathrm{PhCl}$ are significantly larger than those of the corresponding $\alpha-C D$ complexes, whereas the $K_{\mathfrak{a}}$ values for $\beta-\mathrm{CD} / \mathrm{PhBr}$ and $\beta-\mathrm{CD} / \mathrm{PhI}$ are smaller than those of the corresponding $\alpha-\mathrm{CD}$ complexes. This result may be explained by a consideration of the van der Waals interaction. The effective van der Waals radii of $\mathrm{F}, \mathrm{Cl}, \mathrm{Br}$ and $\mathrm{I}$ in halobenzenes are $1.47 \AA, 1.77 \AA, 1.92 \AA$ and $2.06 \AA$, respectively [30]. Since the substituent locates near to the narrower rim of the $\mathrm{CD}$ cavity, the $K_{\mathrm{a}}$ values are directly dependent on the radii of the halogen atoms. The difference in slope in Figure VIII-12 between $\alpha-C D$ and $\beta-C D$ complexes indicates that the interactions of halogen atoms, especially $\mathrm{Br}$ and $\mathrm{I}$ with $\alpha$ $\mathrm{CD}$, are much stronger because the host molecule and guest substituent approximate to van der Waals contact. On the other hand, with $\beta-C D$ they are somewhat weak, since the halogen atoms are far from the internal surface of the $\beta-C D$ cavity in the inclusion complexes. As a result, both $\mathrm{PhBr}$ and $\mathrm{PhI}$ match $\alpha-\mathrm{CD}$ better than $\beta$-CD.

One can speculate on the difference in the order of $K_{\mathrm{a}}$ (or $\Delta_{\mathrm{fus}} H$ ) between monohalobenzene guests in cyclodextrin and TATM. For cyclodextrin, the authors noted that the principal driving force for complexation with these monosubstituted guests is van der Waals (e.g. dispersive) forces. Thus the more polarizable $\mathrm{PhI}$ and $\mathrm{PhBr}$ form more strongly bound complexes (as opposed to $\mathrm{PhF}$ and $\mathrm{PhCl}$ ) with $\mathrm{CD}$ because they are more polarizable. On the other hand, it seems likely that the principal driving force for these guests with TATM is electrostatic (e.g. dipole-dipole) interactions. This perhaps leads to TATM complexes with $\mathrm{PhF}$ and $\mathrm{PhCl}$ guests that have a greater $\Delta_{\text {fus }} H$ than either $\mathrm{PhBr}$ or PhI.

Finally, results on a number of 1,4-disubstitued halogenated benzenes included in $\alpha-\mathrm{CD}$ display similar results [31]. For I,4-sym-dihalobenzenes (halo $=\mathrm{Cl}, \mathrm{Br}, \mathrm{I}$ ), 4halophenols (halo $=\mathrm{Cl}, \mathrm{Br}, \mathrm{I}$ ) and 4-halo-fluorobenzenes (halo $=\mathrm{Cl}, \mathrm{Br}, \mathrm{I}$ ), the same trend is observed, namely that $K_{\mathrm{a}}$ of the inclusion compound follows the order I $>\mathrm{Br}>\mathrm{Cl}$ (see Table VIII-5), and the results may be explained in the same way as for the monohalobenzenes above, namely that $\alpha$-CD prefers more polarizable guests, reflecting the predominance of van der Waals interactions between host and guest in determining the strength of host-guest interactions in this system. $\beta-C D$ inclusion complexes show 
similar behaviour [31]. The order for 1,4-sym-disubstituted halogenated benzene guests follow the order $\mathrm{I}>\mathrm{Br}>\mathrm{Cl}$, while the 4-halophenols similarly follow the order $\mathrm{I}>\mathrm{Br}>$ $\mathrm{Cl}$ (see Table VIII-5).

Interestingly, if one compares $K_{\mathrm{a}}$ values in Table VIII-4 (monohalobenzenes) and Table VIII-5 (p-sym-dihalobenzenes), one sees a uniform increase in $K_{\mathrm{a}}$ on going from $\mathrm{PhX}$ to $p-\mathrm{XPhX}$, for both $\alpha-\mathrm{CD}$ and $\beta-\mathrm{CD}$, suggesting that the extra halogen atom of the guest gives rise to a stronger bond between host and guest. Finally, in Table VIII-5, one observes that the substitution of $\mathrm{Cl}, \mathrm{Br}$ or I ( $p$-sym-XPhX - first group in TableVIII-5) with fluorine ( $p-\mathrm{FPhX}$ - second group in the table) leads to a significant reduction in the association constant of the $\alpha-C D$ inclusion compound, suggestive of van der Waals forces governing these systems, with dipolar forces of secondary importance.

Table VIII-5: Association constants $\left(K_{\mathrm{a}}\right)$ for inclusion complexation of $\alpha-\mathrm{CD}$ and $\beta-\mathrm{CD}$ with 1,4-disubstituted benzene guests [31]:

\begin{tabular}{lll}
\hline Guest & $K_{i}(\alpha-\mathrm{CD})$ & $K_{a}(\beta-\mathrm{CD})$ \\
\hline$p-\mathrm{Cl}\left(\mathrm{C}_{6} \mathrm{H}_{4}\right) \mathrm{Cl}$ & 226 & 320 \\
$p-\mathrm{Br}\left(\mathrm{C}_{6} \mathrm{H}_{4}\right) \mathrm{Br}$ & 1022 & 944 \\
$p-\mathrm{I}\left(\mathrm{C}_{6} \mathrm{H}_{4}\right) \mathrm{I}$ & 4188 & 1495 \\
& & \\
$p-\mathrm{F}\left(\mathrm{C}_{6} \mathrm{H}_{4}\right) \mathrm{Cl}$ & 64.7 & - \\
$p-\mathrm{F}\left(\mathrm{C}_{6} \mathrm{H}_{4}\right) \mathrm{Br}$ & 250 & - \\
$p-\mathrm{F}\left(\mathrm{C}_{6} \mathrm{H}_{4}\right) \mathrm{I}$ & 982 & - \\
& & \\
$p-\mathrm{HO}\left(\mathrm{C}_{6} \mathrm{H}_{4}\right) \mathrm{Cl}$ & 273 & 257 \\
$p-\mathrm{HO}\left(\mathrm{C}_{6} \mathrm{H}_{4}\right) \mathrm{Br}$ & 706 & 446 \\
$p-\mathrm{HO}\left(\mathrm{C}_{6} \underline{H}_{4}\right) \mathrm{I}$ & 2322 & 953 \\
\hline
\end{tabular}

In 1990, a study performed independently from the one above was concerned [32] with the association of monohalobenzenes with cyclodextrins in an aqueous medium. In contrast to the similar study above, the authors measured association constants based on the principle that the rate of transfer of a volatile solute (i.e. halobenzene) from the aqueous phase into the gaseous phase decreases with its increasing association with nonvolatile solutes (i.e. cyclodextrin) present in the aqueous medium. The authors noted that the monohalobenzenes have some regularity in their physicochemical properties, such as their van der Waals radius, electronegativity, and hydrophobicity, and that it is interesting to examine how the nature of $\mathrm{CD}$ complex formation changes with different halocarbon 
guests, allowing one to determine the major factor(s) governing the association of guests with the CD hosts.

The association constant $\left(K_{\mathrm{a}}\right)$ for monohalobenzene guests with $\alpha-\mathrm{CD}, \beta-\mathrm{CD}$ and $\gamma$-CD appear in Table VIII- 6 . With the exception of iodobenzene $/ \gamma-C D$, a universal trend of increasing $K_{\mathrm{a}}$ with increasing size of halogen substituent (for all three hosts) is observed. The authors suggested that hydrogen bonding is not important, as the only guest capable of participating in H-bonding, fluorobenzene, has the universally lowest stability constant (see Table VIII-6). On the other hand, the hydrophobicity of the guest (partition coefficient) increases in the order $\mathrm{PhF}<\mathrm{PhCl}<\mathrm{PhBr}<\mathrm{Phl}$, so this driving force may be partly applicable to these systems. Furthermore, the authors suggested that van der Waals forces, in part, seem to govern these guest-host systems. Indeed, as the surface area (and polarizability) of the guest increases $\mathrm{PhF}<\mathrm{PhCl}<\mathrm{PhBr}<\mathrm{Phl}$, the stabilities of the respective $C D$ inclusion compounds also increase. Finally, we wish to make note of the fact that these association constants agree very well (for both $\alpha-C D$ and $\beta$-CD) with those depicted in Table VIII-4, as measured by UV-VIS fluorescence spectroscopy.

Table VIII-6: Association constants $\left(K_{\mathrm{a}}\right)$ for complexation of $\alpha-C D, \beta-C D$ and $\gamma-C D$ with monohalobenzene guests at aqueous solution at $25^{\circ} \mathrm{C}[32 \mid$.

\begin{tabular}{llll}
\hline Guest & $K_{a}(\alpha-C D)$ & $K_{i}(\beta-C D)$ & $K_{i}(\gamma-C D)$ \\
\hline fluorobenzene & $34 \pm 1$ & $70 \pm 30$ & $14 \pm 6$ \\
chlorobenzene & $100 \pm 10$ & $160 \pm 10$ & $33 \pm 2$ \\
bromobenzene & $510 \pm 10$ & $310 \pm 20$ & $39 \pm 8$ \\
iodobenzene & $1100 \pm 100$ & $800 \pm 100$ & $30 \pm 410$ \\
\hline
\end{tabular}

One year later, the same research group presented results on the association of 1,4-symdihalobenzene guests with cyclodextrins in aqueous solution [33]. The authors hoped to compare association constants from the previous study on monohalobenzene guests with the present results to elucidate the mechanisms and driving forces of halobenzene / CD complexation. The authors used the same experimental technique, as in the study above [32], to measure association constants, namely the variation in volatility of a halobenzene guest in an aqueous solution, as a function of cyclodextrin concentration. The present data appear in Table VIII-7. From this table, one observes that the association constants, 
for all three cyclodextrins $(\alpha-C D, \beta-C D$ and $\gamma-C D)$, follow the order 1,4-difluorobenzene $<$ 1,4-dichlorobenzene $<$ 1,4-dibromobenzene $<$ 1,4-diiodobenzene. Furthermore, for each guest, the order of stability constant (except for $\mathrm{F}\left(\mathrm{C}_{6} \mathrm{H}_{4}\right) \mathrm{F} / \alpha-\mathrm{CD}$ and $\mathrm{Cl}\left(\mathrm{C}_{6} \mathrm{H}_{4}\right) \mathrm{Cl} / \alpha$ $C D$ ), follows the order $\alpha-C D>\beta-C D>\gamma-C D$, suggesting that $\alpha-C D$ has the most suitably sized cavity for maximal van der Waals interactions between host and (at least) the larger guests.

Similarly to the monohalobenzene guests above, the authors found, for the 1,4sym-dihalobenzene guests, a direct relationship between $\mathrm{CD}$ association constant and the total surface area of the 1,4-dihalobenzene guest, for all three host systems, again suggesting the predominance of van der Waals forces in determining the strength of intermolecular interactions in this system. Furthermore, increases in guest polarizability $(\mathrm{I}>\mathrm{Br}>\mathrm{Cl}>\mathrm{F})$ are also associated with increases in cyclodextrin association constants. This is in contrast to TATM materials, where a smaller total surface areas and lower polarizabilities of the guests may lead to the observed increase in $\Delta_{\text {fus }} H$. Finally, as was the case with monohalobenzene guests, there is a direct relationship between the cyclodextrin association constant and the hydrophobicity of the free guest molecule, suggesting that hydrophobicity is at least partially important as a driving force in these systems. On the other hand, hydrophobicity is not a significant driving force for TATM materials.

Table VIII-7: Association constants $\left(K_{\mathrm{a}}\right)$ for complexation of $\alpha-\mathrm{CD}, \beta-\mathrm{CD}$ and $\gamma-\mathrm{CD}$ with sym-1,4-dihalobenzene guests at aqueous solution at $25^{\circ} \mathrm{C}$ [33]. Note similarity of $K_{\mathrm{a}}$ values with those quoted in Table VIII-5, for both $\alpha-\mathrm{CD}$ and $\beta-\mathrm{CD}$.

\begin{tabular}{llll}
\hline Guest & $K_{2}(\alpha-C D)$ & $K_{i}(\beta-C D)$ & $K_{:}(\gamma-C D)$ \\
\hline$p$-difluorobenzene & $19.6 \pm 0.3$ & $40 \pm 10$ & $28 \pm 9$ \\
$p$-dichlorobenzene & $225 \pm 8$ & $320 \pm 20$ & $43 \pm 1$ \\
$p$-dibromobenzene & $1020 \pm 60$ & $940 \pm 20$ & $48 \pm 8$ \\
$p$-diiodobenzene & $4200 \pm 900$ & $1500 \pm 200$ & $81 \pm 8$ \\
\hline
\end{tabular}

Stability constants $\left(K_{\mathrm{s}}\right)$ for complex formation between $\alpha$-cyclodextrin and 4-substituted phenols were measured in aqueous solution at $25^{\circ} \mathrm{C}$, by potentiometric and competitive spectrophotometric methods $[34]$. The authors found that complex stability constant increases with increases in electron density and polarizability of the 4-substituent. 
Indeed, the order of $K_{\mathrm{s}}$ for 4-substituted phenols in $\alpha$-cyclodextrin follows the order phenol $\left(10.9 \mathrm{M}^{-1}\right)<4$-fluorophenol $\left(15.6 \mathrm{M}^{-1}\right)<4$-chlorophenol $\left(487.9 \mathrm{M}^{-1}\right)<4$ bromophenol $\left(1221 \mathrm{M}^{-1}\right)<4$-iodophenol $\left(3955 \mathrm{M}^{-1}\right)$. The authors rationalized this data on the basis of increasing attractive van der Waals interactions between host and guest as the polarizability of the halogen substituent increases. As a final point, we wish to make note of the fact that these stability constants are significantly different than the values for the same $\alpha-C D$ inclusion compounds quoted in Table VIII- 5 , as measured by UV-VIS fluorescence spectroscopy.

The following year, binding constants of $\alpha$-cyclodextrin with 1,4-symdihalobenzene guests in aqueous solution were measured by the same research group [35]. Again, the order of stability constant for $\alpha$-cyclodextrin guest follows: 1,4dichlorobenzene $\left(232 \mathrm{M}^{-1}\right)<1$,4-dibromobenzene $\left(913 \mathrm{M}^{-1}\right)<1$,4-diiodobenzene (5060 $\mathrm{M}^{-1}$ ). These values agree quite well with those listed in Table VIII-5 (UV-VIS fluorescence spectroscopy) and VIII-7 (vapor pressure measurements). The authors explained these results, in part, on the basis of explanation of the results obtained above for 4-substituted phenol guests in $\alpha$-cyclodextrin. Furthermore, empirical correlations of complex stability constant were found with (1) guest solubility, (2) binding site dipole moment and (3) guest partition coefficient. Indeed, the complex stability constant and the partition coefficient (hydrophobicity) of the guest both increase in the order $\mathrm{ClPhCl}<$ $\mathrm{BrPhBr}<\mathrm{IPhI}$. On the other hand, as the binding constant of the complex increases: $\mathrm{ClPhCl}<\mathrm{BrPhBr}<\mathrm{IPhI}$, the solubility of the guest decreases: $\mathrm{ClPhCl}(3.99 \mathrm{M})>\mathrm{BrPhBr}$ $(0.56 \mathrm{M})>\mathrm{IPhl}(0.031 \mathrm{M})$.

Finally, we wish to make note of the fact that these cyclodextrin studies are performed in aqueous solution, while the TATM materials are measured in the solid phase (actually melting). The driving forces of hydrophobic interactions, release of distortional energy and extrusion of high energy water molecules are thus not likely to govern host-guest binding in TATM materials.

\section{(F) Cholic Acid}

The crystal structures of inclusion compounds of cholic acid (CA) with halobenzene guests have been systematically investigated [36]. All four crystals ( $\mathrm{PhF}, \mathrm{PhCl}, \mathrm{PhBr}$ and 
$\mathrm{PhI}$ guests) belong to the monoclinic space group $\mathrm{P} 2$, and have bilayer structures with one-dimensional molecular channels that include the guests. The host framework is characterized by the conformation and stacking mode of the host compound. The size and shape of the host cavities are essentially the same for all four guests, regardless of the halogen substituent and the steric dimensions of the guest. In all four structures, the guest in the cavity is disordered over two symmetry equivalent positions. In comparison, the guests benzene, $\mathrm{PhF}, \mathrm{PhCl}$ and $\mathrm{PhBr}$ form isostructural TATM inclusions (as determined by PXRD), while PhI forms a different structure.

\section{(G) Gossypol}

In 1994, Ibragimov and co-workers measured crystal structures of the gossypol host with various halogenomethane guests [37]. The crystal parameters appear in Table VIII-8. The guests include tetrahalomethane $\left(\mathrm{CCl}_{4}\right)$, trihalomethanes $\left(\mathrm{CHCl}_{3}, \mathrm{CHBr}_{3}\right)$ and dihalomethanes $\left(\mathrm{CH}_{2} \mathrm{Cl}_{2}, \mathrm{CH}_{2} \mathrm{Br}_{2}\right.$ and $\left.\mathrm{CH}_{2} \mathrm{I}_{2}\right)$.

Table VIII-8: Crystallographic parameters of complexes of gossypol with halogeno methane derivatives [37].

\begin{tabular}{|c|c|c|c|c|c|c|}
\hline$\overline{\text { Lattice type }}$ & clathrate & \multicolumn{3}{|c|}{ intercalates } & \multicolumn{2}{|c|}{ channels } \\
\hline Guest & $\mathrm{CCl}_{4}$ & $\mathrm{CHCl}_{3}$ & $\mathrm{CHBr}_{3}$ & $\mathrm{CH}_{2} \mathrm{l}_{2}$ & \multirow{2}{*}{\multicolumn{2}{|c|}{$\underset{\text { monoclinic }}{\mathrm{CH}_{2}}$}} \\
\hline Lattice & triclinic & \multicolumn{3}{|c|}{ monoclinic } & & \\
\hline S.G. & PI & $\mathrm{C} 2 / \mathrm{c}$ & $\mathrm{C} 2 / \mathrm{c}$ & $\mathrm{C} 2 / \mathrm{c}$ & $\mathrm{C} 2 / \mathrm{c}$ & $\mathrm{C} 2 / \mathrm{c}$ \\
\hline $\mathrm{H}: \mathrm{G}$ SR & $1: 1$ & $1: 1$ & $1: 1$ & $1: 1$ & $1: 1$ & $1: 1$ \\
\hline$a(\AA)$ & $8.8+7(2)$ & $28.46+4(4)$ & $28.54+4(3)$ & $28.676(8)$ & $21.320(4)$ & $21.439(9)$ \\
\hline$b(\AA)$ & $1+.30+(6)$ & $8.948(1)$ & $8.987(2)$ & $9.16+(4)$ & $19.199(6)$ & $19.087(8)$ \\
\hline$c(\AA)$ & $13.395(5)$ & $26.480(4)$ & $26.759(6)$ & $26.158(6)$ & $15.765(2)$ & $16.049(8)$ \\
\hline$\alpha\left({ }^{\circ}\right)$ & $102.54(3)$ & 90 & 90 & 90 & 90 & 9) \\
\hline$\beta\left({ }^{\circ}\right)$ & $69.53(3)$ & $108.93(2)$ & $109.42(2)$ & $108.21(2)$ & $113.05(2)$ & $113.68(4)$ \\
\hline$\gamma\left({ }^{\circ}\right)$ & $91.12(3)$ & 90 & 90 & 90 & 90 & 90 \\
\hline$V\left(\AA^{3}\right)$ & 1547 & 6380 & 6474 & 6529 & 5916 & 6015 \\
\hline$\underline{Z}$ & 2 & 8 & 8 & 8 & 8 & 8 \\
\hline
\end{tabular}

The authors found that gossypol forms a clathrate-type inclusion with $\mathrm{CCl}_{4}$, in which the guest molecules are situated in closed cages. Decreasing the number of $\mathrm{Cl}$ atoms by one $\left(\mathrm{CHCl}_{3}\right)$ gives an intercalate-type inclusion, in which the chloroform guest molecules are localized between layers of host molecules (see Figure VIII-14). A further decrease in the number of $\mathrm{Cl}$ atoms $\left(\mathrm{CH}_{2} \mathrm{Cl}_{2}\right)$ results in a channel-type inclusion. 


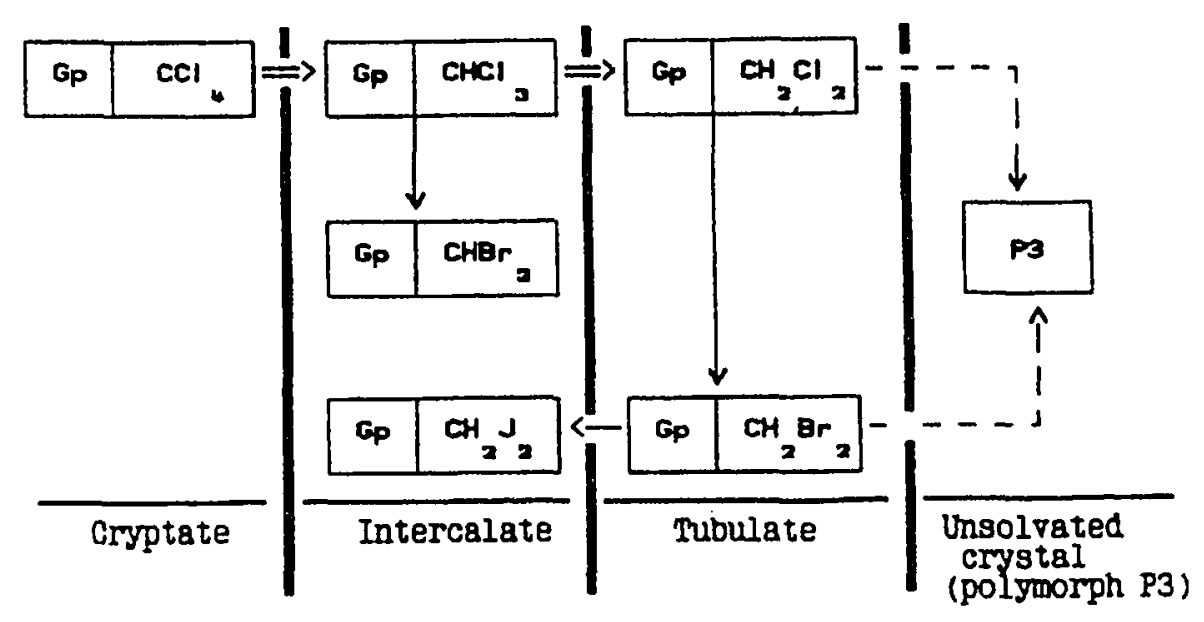

Figure VIII-14: Morphological transitions and desolvation $(\rightarrow)$ of gossypol clathrates with halogeno derivatives of methane during the change of the number $(\Rightarrow)$ and type $(\rightarrow)$ of the halogen atoms in the guest molecule [37].

Substitution of the $\mathrm{Cl}$ atoms with $\mathrm{Br}$ atoms $\left(\mathrm{CHCl}_{3} \rightarrow \mathrm{CHBr}_{3}\right.$ and $\left.\mathrm{CH}_{2} \mathrm{Cl}_{2} \rightarrow \mathrm{CH}_{2} \mathrm{Br}_{2}\right)$ does not change the resulting gossypol crystal structure (see Table VIII-8). However, methylene iodide $\left(\mathrm{CH}_{2} \mathrm{I}_{2}\right)$ is too large to be accommodated in the tubulate-like channels, and a transition to an intercalate-type structure occurs (Table VIII-8). For TATM, the $\mathrm{CH}_{2} \mathrm{Cl}_{2}$ and $\mathrm{CH}_{2} \mathrm{Br}_{2}$ inclusions are also isostructural, while, similarly, $\mathrm{CH}_{2} \mathrm{I}_{2}$ forms a different structure. On the other hand, most all TATM inclusions are generally composed of (albeit sometimes bottleneck) channels.

(H) Other Host Systems

(1) 1,2,3,4,5-penta-O-benzoyl-6-O-tosyl myo-inositol

A myo-inositol derivative (racemic 1,2,3,4,5-penta- $O$-benzoyl-6-O-tosyl myo-inositol), 1 , was found to assemble around a crystallographic 2-fold axis, selectively accommodating dihalomethane guests $\left(\mathrm{CH}_{2} \mathrm{Cl}_{2}, \mathrm{CH}_{2} \mathrm{Br}_{2}\right.$ and $\left.\mathrm{CH}_{2} \mathrm{I}_{2}\right)$ having $\mathrm{C}_{2}$ symmetry [38]. The formation of highly stable host-guest complexes was attributed to $\mathrm{C}-\mathrm{X} \cdots \mathrm{O}$ and $\mathrm{C}-\mathrm{H} \cdots \mathrm{O}$ interactions. The host compound was found to resist crystallization in most common solvents, such as chloroform, ethyl acetate, ethanol etc., but readily yielded good quality crystals when crystallized from $\mathrm{CH}_{2} \mathrm{X}_{2}$ guests $(\mathrm{X}=\mathrm{Cl}, \mathrm{Br}$ or I). All other attempts at 
crystallization (with other solvents) were unsuccessful. (This is in contrast to TATM, for which an unsuitable solvent has yet to be found.) The authors noted that the remarkable specificity of the myo-inositol host for methylene halide guests may have applications in the separation of halocarbon compounds, or in removal of traces of methylene halide impurities from other organic compounds or solvents. It was also found that $\mathrm{CH}_{2} \mathrm{Cl}_{2}$ and $\mathrm{CH}_{2} \mathrm{Br}_{2}$, as judged by their unit cell parameters, form isostructural inclusion compounds with this host. (This is similar, as noted in the previous section, with TATM, in that $\mathrm{CH} 2 \mathrm{Cl} 2 / \mathrm{TATM}$ and $\mathrm{CH} 2 \mathrm{Br} 2 / \mathrm{TATM}$ are isostructural.) The crystal data for these two myo-inositol inclusions appear in Table VIII-9.

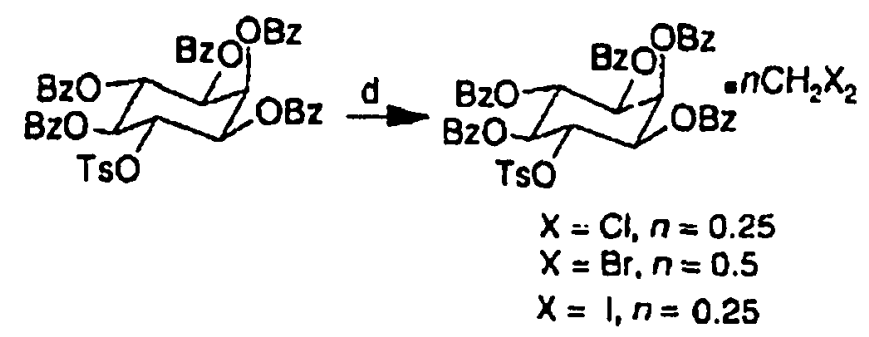

Scheme 1: Host myo-inositol derivative with $\mathrm{CH}_{2} \mathrm{X}_{2}$ guests

Table VIII-9: Crystallographic parameters of complexes of 1,2,3,4,5-penta- $O$-benzoyl-6$O$-tosyl myo-inositol with $\mathrm{CH}_{2} \mathrm{Cl}_{2}$ and $\mathrm{CH}_{2} \mathrm{Br}_{2}$ guests [38].

\begin{tabular}{lll} 
Guest & $\mathrm{CH}_{2} \mathrm{Cl}_{2}$ & $\mathrm{CH}_{2} \mathrm{Br}_{2}$ \\
\hline $\mathrm{H}: \mathrm{G} \mathrm{SR}$ & $4: 1$ & $2: 1$ \\
MolWt (amu) & 876.08 & 941.77 \\
Lattice & monoclinic & monoclinic \\
S.G. & $\mathrm{C} 2 / \mathrm{c}$ & $\mathrm{C} 2 / \mathrm{c}$ \\
$a(\AA)$ & $26.771(4)$ & $26.927(17)$ \\
$b(\AA)$ & $11.608(3)$ & $11.716(8)$ \\
$c(\AA)$ & $30.783(6)$ & $30.874(2)$ \\
$\beta\left({ }^{\circ}\right)$ & $105.176(6)$ & $105.998(10)$ \\
$V\left(\AA^{3}\right)$ & $9232(3)$ & $9362(11)$ \\
$Z$ & 8 & 8 \\
\hline
\end{tabular}

(2) 2,4,6-tris(4-halophenoxy)-1,3,5-triazine

In 2001, crystal structures of the 2,4,6-tris(4-halophenoxy)-1,3,5-triazine host (halo $=\mathrm{Cl}$ or $\mathrm{Br}$ ) with 1,3,5-trihalobenzene guests (halo $=\mathrm{Br}$ or $\mathrm{l}$ ), 2, were reported [39]. All four structures crystallized in the hexagonal, non-centrosymmetric $P G_{3}$ space group, with a 1:1 host:guest stoichiometry. Furthermore, all four complexes are isostructural. The 
structures of the two hosts with a wide variety of other guests, such as benzene, hexamethylbenzene, hexachlorobenzene, etc., all crystallize in the centrosymmetric space group $P 6_{3} / m$ [40]. This indicates the self-assembly of chiral crystals from achiral components only for the 1,3,5-trihalobenzene guests. This behaviour may have applications in the process of spontaneous resolution during crystallization. (Most all TATM materials so far investigated are centrosymmetric, either triclinic or monoclinic lattices, resulting in achiral crystals). These four inclusion compounds were found to be a robust example of supramolecular assembly via $C-X \cdots \pi$ and $C-H \cdots \pi$ interactions between host and guest (see Figure VIII-15). On the other hand, TATM materials investigated to this point seem to be dominated by weak $\mathrm{C}-\mathrm{H} \cdots \mathrm{O}$ hydrogen bonding and $\pi-\pi$ thienyl ring interactions.

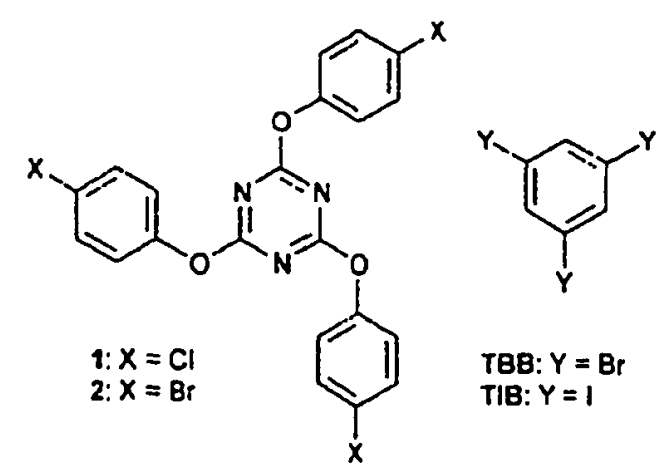

Scheme 2: 2,4,6-tris(4-halophenoxy)-1,3,5-triazine host compound

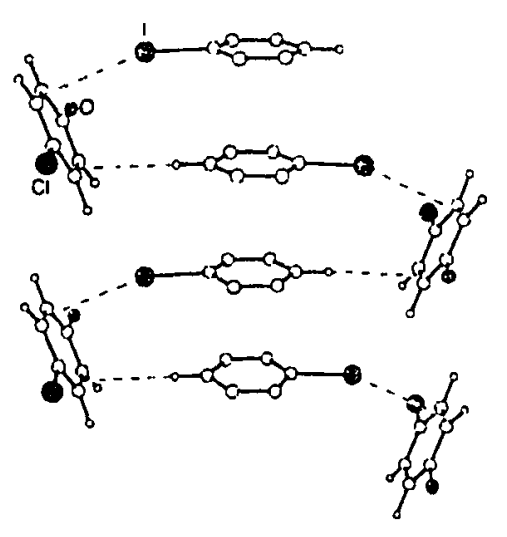

Figure VIII-15: Sinusoidal chain of C-X $\cdots \pi$ and $\mathrm{C}-\mathrm{H} \cdots \pi$ interactions that result in polar crystallization. Three such motifs rotated by $120^{\circ}$ stabilize the host-guest channel architecture. [39]

(3) catena-(1,2-diaminopropane)cadmium(II) tetra- $\mu$-cyanonickelate(II)

Two different host structures of catena-(1,2-diaminopropane)cadmium(II) tetra- $\mu$ cyanonickelate(II), $\left[\mathrm{Cd}(p n) \mathrm{Ni}(\mathrm{CN})_{4}\right]$ are known [41-43], both of which are made up of 
one-dimensional tunnels. For the inclusion compounds containing $n$-pentane, $n$-hexane, $n$-heptane, diethyl ether, ethylene glycol, 1-bromobutane or 1-bromopentane guests, the structure is monoclinic (space group $A 2 / a$ ), whereas for the inclusion compounds containing 1,1,2,2-tetrachloroethane, 2-chlorobutane, 1,2-dichloropropane, 2-propanol, 1,2-dichloroethane, 1,2-dibromoethane or 1,3-dichloropropane guests, the structure is orthorhombic (space group Pnma). The two types of structures are substantially similar $\left(13.8 \AA<a<14.2 \AA, 26.7 \AA<b<27.2 \AA, 7.5 \AA<c<7.7 \AA, \gamma(\right.$ monoclinic $\left.)=90^{\circ} \pm 2^{\circ}\right)$.

Both structures contain tunnels parallel to the $c$ axis. The essential difference between the monoclinic and orthorhombic structures lies in the relative orientations of the 1,2-diaminopropane ligands. In the monoclinic structure, pairs of 1,2-diaminopropane ligands are related across a center of symmetry lying on the tunnel axis. In the orthorhombic structure, each pair of 1,2-diaminopropane moieties are related by a mirror plane lying within the axis of the channel. From Table VIII-10, taken from references [41-43] (single crystal structures) and [44] (powder X-ray diffraction), one can see that the substitution of chlorine atoms for bromine atoms of the alkyl halide guest has little influence on the structure of the resulting inclusion compound, for at least two of the three pairs (structural data on the 2,3-dibromobutane inclusion was not available due to difficulty in obtaining a suitable sample). This behaviour is similar to TATM materials, where in most cases substitution of chlorine atoms with bromine atoms of the guest has little influence on the nature of the resulting TATM complex.

Table VIII-10: Lattice type for various alkyl halide guests incorporated into the $\mathrm{Cd}(p n) \mathrm{Ni}(\mathrm{CN})_{4}$ host material. All orthorhombic lattices of inclusion compounds with this host (so far encountered) are isostructural $|41-44|$.

\begin{tabular}{|c|c|c|}
\hline Guest & Lattice type & Measuring technique \\
\hline 2-chlorobutane & orthorhombic $P_{n m a}$ & SCXRD, PXRD \\
\hline 2-bromobutane & orthorhombic $P_{n m a}$ & PXRD \\
\hline 1,2-dichloroethane & orthorhombic $P_{n m a}$ & SCXRD, PXRD \\
\hline 1,2-dibromoethane & orthorhombic $P_{n m a}$ & SCXRD \\
\hline 2,3-dichlorobutane & orthorhombic $P_{n m a}$ & PXRD \\
\hline 2,3-dibro & & - \\
\hline
\end{tabular}




\section{CONCLUDING REMARKS}

For these TATM inclusion compounds, differences in the strength of host-guest binding may be attributed to differences in the interaction of individual guest molecules with the channel walls, arising not only from differences in the chemical nature of the various guest species, but also from differences in the fraction of available channel volume occupied by each guest. The strength of host-guest intermolecular interactions of some of these complexes also depends, among other factors, upon the initial distortion of host void space required to accommodate a particular guest.

Decomposition of a TATM inclusion compound requires (a) overcoming guestchannel wall interactions and (b) an opening of sufficient size to permit liberation of the guest. It has been well established that many guest species are capable of a considerable degree of motion within the host lattice, even at low temperatures, depending in part upon the closeness of fit between host and guest. This motion may be expected to increase with temperature, accompanied by a decrease in the extent to which the complex lattice is stabilized by interactions with the guest molecules. When the guest has attained a certain amount of energy, the contribution of the guest molecules to the stability of the inclusion compound is virtually lost. The combination of the loss of this stabilizing influence of the guest upon the complex, plus increased overall lattice vibrations, results in destruction of the inclusion compound, accompanied by liberation of the guest molecules.

In this contribution, we have used three different physical techniques to characterize TATM inclusion compounds with alkyl halide guest families. The relationship between the nature of the guest and the resulting TATM structural type were measured within each guest family, using powder X-ray diffraction and ${ }^{13} \mathrm{C} C P / M A S$ NMR. The nature of the halogen substituent(s) on a given hydrocarbon guest backbone generally has little effect on the nature of the subsequent TATM inclusion compound. Furthermore, the DSC results show that TATM inclusions display a heat of fusion ranking with guests that have substituents in the order $\mathrm{F}>\mathrm{Cl}>\mathrm{Br}>\mathrm{I}$. These results are valuable in drawing correlations to other systems where perhaps only a single crystal structure is available. 
(1) J.M. Thomas and K.D.M. Harris. In Organic Solid State Chemistry, G.R. Desiraju, Ed. Elsevier: Amsterdam, 1987.

(2) M. Nishikawa. Chem. Pharm. Bull. 11, 977 (1963).

(3) K. Fukushima. J. Mol. Struct. 34, 67 (1976).

(4) A. Allen, V. Fawcett and D.A. Long. J. Raman Spectrosc. 4, 285 (1976).

(5) G.R. Desiraju. Crystal Engineering: The Design of Organic Solids. Elsevier: Amsterdam, 1989.

(6) J.M. McBride, S.B. Bertman, D.Z. Cioffi, B.E. Segmuller and B.A. Weber. Mol. Cryst. Liq. Cryst. Inc. Nonlin. Opt. 161, I (1988).

(7) J.S. Chickos, D.G. Hesse, J.F. Liebman and S.Y. Panshin. J. Org. Chem. 53, 3424 (1988).

(8) N. Hartley and N.G. Thomas. J. Chem. Soc. 89, 1013 (1906).

(9) L. Bin Din and O. Meth-Cohn. J. Chem. Soc. Chem. Commun. 21. 741 (1977).

(10) S.J. Opella and M.H. Frey. J. Am. Chem. Soc. 101, 5855 (1979).

(11) J.L.M. Dillen and H.M. Roos. Acta Cryst. C48, 2229 (1992).

(12) L. Pang and F. Brisse. Can. J. Chem. 72, 2318 (1994).

(13) H.M. Roos and J.L.M. Dillen. Acta Cryst. C48, 1882 (1992).

(14) P.H. van Rooyen and H.M. Roos. Acta Cryst. C47, 2718 (1991).

(15) H.G. McAdie. Can. J. Chem. 40, 2195 (1962).

(16) H.G. McAdie. Can. J. Chem. 41, 2144 (1963).

(17) H.G. McAdie. Can. J. Chem. 41, 2137 (1963).

(18) H.G. McAdie. Can. J. Chem. 44, 1373 (1966).

(19) R.J.C. Brown and R.F.C. Brown. J. Chem. Ed. 77, 724 (2000).

(20) N.G. Parsonage and L.A.K. Staveley, Thermodynamic Studies of Clathrates and Inclusion Compounds. In Inclusion Compounds J.L. Atwood, J.E.D. Davies and D.D. MacNicol Eds. Volume 3, Chapter I Academic Press: New York, 1984.

(21) Y.A. Dyadin, G.N. Chekova and N.P. Sokolova. J. Inclus. Phenom. 5, 187 (1987).

(22) O. Redlich, C.M. Gable, A.K. Dunlop and R.M. Millar. J. Am. Chem. Soc. 72. 4153 (1950).

(23) Q.X. Guo, S.H. Luo and Y.C. Liu. J. Inclus. Phenom. Mol. Recognit. Chem. 30, 173 (1998).

(24) M.L. Bender and M. Komiyama. Cyclodextrin Chemistry. Springer Verlag: Berlin, 1978.

(25) K. Kano, Y. Tawiya and S. Hashimoto. J. Inclus. Phenom. 3, 287 (1992).

(26) I. Tabushi, Y. Kiyosuke, T. Sugimoto and R. Yamamura. J. Am. Chem. Soc. 100. 916 (1978).

(27) M. Komiyama and M.L. Bender. J. Am. Chem. Soc. 100, 2259 (1978).

(28) W. Blokzijl and J.F. Engberts. Angew. Chem. Int. Ed. Engl. 32, 1545 (1993).

(29) R.L. van Etten, J.F. Sebastian, G.A. Clowes and M.L. Bender. J. Am. Chem. Soc. 89, 3242 (1967).

(30) A. Bondi. J. Phys. Chem. 68, 441 (1964).

(31) L. Liu and Q.X. Guo. J. Phys. Chem. B. 103, 3461 (1999).

(32) T. Takuma, T. Deguchi and I. Sanemasa. Bull. Chem. Soc. Jpn. 63(4), 1246 (1990). 
(33) T. Takuma, T. Deguchi and I. Sanemasa. Bull. Chem. Soc. Jpn. 64(2), 480 (1991).

(34) S.F. Lin and K.A. Connors. J. Pharm. Sci. 72(11), 1333 (1983).

(35) K. A. Connors and D.D. Pendergast. J. Am. Chem. Soc. 106, 7607 (1984).

(36) K. Nakano, K. Sada, Y. Kurozumi and M. Miyata. Chem. Eur. J. 7(1), 209 (2001).

(37) B.T. Ibragimov, S.A. Talipov and T.F. Aripov. J. Inclus. Phenom. Mol. Recognit. Chem. 17, 317 (1994).

(38) K.M. Sureshan, R.G. Gonnade, M.S. Shashidhar, V.G. Puranik and M.M. Bhadbhade. Chem. Commun. 881 (2001).

(39) R.K.R. Jetti, A. Nangia, F. Xue and T.C.W. Mak. Chem. Commun. 919 (2001).

(40) R.K.R. Jetti, F. Xue, T.C.W. Mak and A. Nangia. Cryst. Eng. 2, 215 (1999).

(41) K.M. Park, M. Hashimoto, T. Kitazawa and T. Iwamoto. Chem. Lett. 1701 (1990).

(42) K.M. Park and T. Iwamoto. J. Chem. Soc. Chem. Commun. 72 (1992).

(43) K.M. Park and T. Iwamoto. J. Chem. Soc. Dalton Trans. 1875 (1993).

(44) M.W. Jones, K.D.M. Harris, G. Sankar, T. Maschmeyer and J.M. Thomas. J. Chem. Soc. Faradav Trans. 92(6), 1043 (1996). 


\section{CHAPTER IX}

\section{Tris (5-acetyl-3-thienyl) methane inclusion compounds with alkyl halide guest families. Competitive inclusion experiments}

$\begin{array}{lr}\text { Abstract } & 156 \\ \text { Introduction } & 157 \\ \text { Experimental } & 159 \\ \text { Results } & 160 \\ \text { Discussion } & \\ \text { (A) } \beta \text {-quinol } & 165 \\ \text { (B) Dianin's compound } & 168 \\ \text { (C) 1,1-bis(4-hydroxyphenyl)cyclohexane } & 172 \\ \text { (D) Cyclophane } & 174 \\ \text { (E) 2,5-bis(9-hydroxyfluoren-9-yl)thieno[2,3-b]thiophene } & 176 \\ \text { Concluding remarks } & 176 \\ \text { References } & 177 \\ \text { Supplemental Material } & 179\end{array}$




\section{ABSTRACT}

In this work we perform competitive inclusion experiments of the tris (5-acetyl-3-thienyl) methane (TATM) host with alkyl halide and aryl halide guest families. The guest families are the methylene halides, the 1,3-dihalopropanes, the 2-halobutanes, the cyclohexyl halides and the monohalobenzenes. Within each group, the halide substituents include chloride, bromide and iodide. Competitive inclusion experiments were performed within each guest family, with three combinations in each group $(\mathrm{Cl}-\mathrm{Br}$, $\mathrm{Cl}-\mathrm{I}, \mathrm{Br}-\mathrm{I})$. A universally observed result is that for all five guest families, the alkyl iodides are the least preferred guest (in comparison to alkyl chlorides and alkyl bromides). As far as the chlorides and bromides are concerned, for the smaller sized guests (methylene halides ( 1 carbon) and 1,3-dihalopropanes ( 3 carbons)), bromides are preferred over chlorides. However for the larger sized guests (2-halobutanes (4 carbons) and cyclohexyl halides (6 carbons)), the opposite result occurs, namely that chlorides are preferred over bromides. For the monohalobenzenes, alkyl chlorides and alkyl bromides are equally preferred. These results are compared to heat of fusion results of the same guest families in a previous paper. In order to rationalize and reconcile these results, one may reason that since the alkyl iodide guests are so large, they may fit only with great difficulty into the TATM cavity, resulting in a lower preference. Indeed, in the previous study, TATM inclusion compounds with alkyl iodide guests always had the lowest heat of decomposition of the alkyl halide / TATM inclusions within each of the five guest families. As far as the chlorides and bromides are concerned, the smaller guests may be able to accommodate a larger substituent (i.e. bromides), without excessive destabilization, while the larger guests may prefer a smaller halogen substituent (i.e. chlorides) because they are already large to begin with. The smaller guests may prefer more polarizable bromides to maximize stabilizing van der Waals interactions between host and guest, while the larger guests may prefer the smaller chloride substituents to minimize destabilizing TATM cage stretching forces. 


\section{INTRODUCTION}

Selective enclathration is the quintessential example of molecular recognition, as it depends on the strengths and the directions of the non-covalent interactions that occur between host and guest molecules [1,2]. This is an important concept in industry, particularly for the resolution of optical isomers, which is of great interest to the pharmaceutical industry [3]. The separation of a given isomer from a mixture of close isomers (e.g. $o-, m$ - and $p$-xylene $[4,5]$ ) by formation of a host-guest compound is industrially appealing in that the procedure is simple and efficient, and is not energy intensive [6]. The process generally requires the crystallization of the targeted isomer in the presence of the host. This can be done either directly from a mixture of liquid guests, or in an added inert solvent (for solid guests). The harvested clathrate crystals are then filtered, and the guest is released by gentle warming. This procedure allows a recycling of the host material [7]. The efficiency of the process varies, but a high purity sample of the required isomer (e.g. 95\%) can usually be achieved after three or four successive recrystallizations $[8,9]$.

The authors believe that one of the most straightforward ways to investigate guest-property relationships for TATM inclusion compounds is to choose guests that have the same hydrocarbon backbone, but with different halogen substituents at selected positions on that backbone. Accordingly, we have chosen five families of guest molecules, as depicted in Figure VIII-1 (p. 121), with associated halogen substituents $(\mathrm{Cl}$, $\mathrm{Br}, \mathrm{I})$. The guest families are the methylene halides, the 1,3-dihalopropanes, the 2halobutanes, the cyclohexyl halides and the monohalobenzenes. By choosing guests that are very similar in structure, we hope to eliminate extraneous complications in the interpretation of our data, the only two significant differences within any one group of guests being the size and electronegativity of the halogen substituents. 
In a previous study (see chapter VIII), we examined structural type and thermal properties of TATM inclusion compounds with the same guest families examined in this study. The results of interest to this study are the differential scanning calorimetric (DSC) data, where we measured the onset melting temperature and heat of decomposition for these TATM inclusion compounds. The melting endotherms of all of the TATM complexes measured were single sharp endotherms, as can be seen in Figure IX-1 for the case of the cyclohexyl halides. From lowest to highest temperature the endotherms come from TATM/cyclohexyl iodide $\left(T_{\text {onset }}=96.4^{\circ} \mathrm{C}\right), \mathrm{TATM} /$ cyclohexyl bromide $\left(T_{\text {onset }}=\right.$ $\left.107.3^{\circ} \mathrm{C}\right)$ and cyclohexyl chloride $\left(T_{\text {onset }}=111.4^{\circ} \mathrm{C}\right)$. No phase transitions, other than the melting endotherms, were encountered for any of the TATM inclusion compounds studied in the temperature range from $25^{\circ} \mathrm{C}$ to $250^{\circ} \mathrm{C}$. In Table IX-1 we list the onset DSC melting temperatures, the heat of decomposition in Joules per gram and the heat of decomposition in kilojoules per mole of unit cell. As can be seen from Table IX-1, for all five guest families, the enthalpy of melting is consistently larger for TATM inclusion compounds with smaller guests (within each group), and monotonically decreases as the size of the halogen substituent of the guest increases, which suggests that TATM may perhaps preferentially incorporate smaller guests from a mixture.

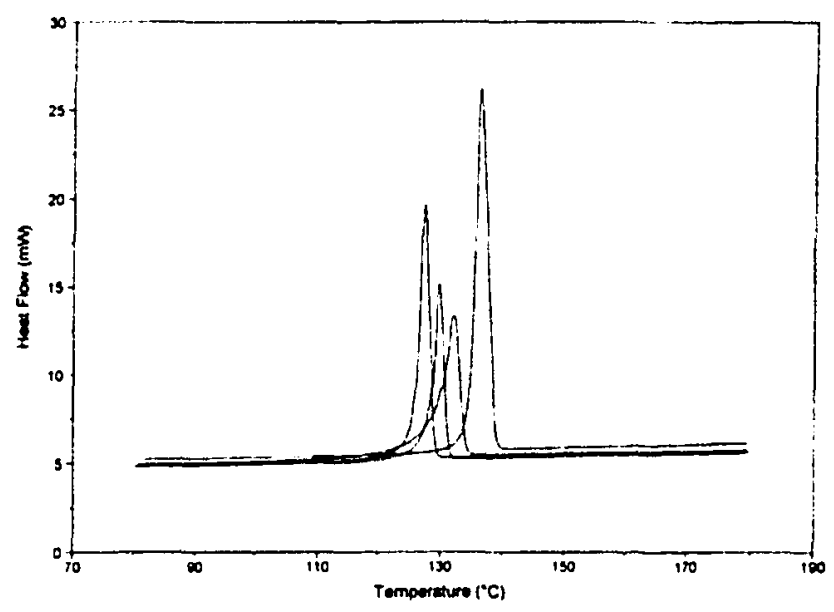

Figure IX-1: DSC traces for monohalobenzene / TATM inclusion compounds. From lowest to highest temperature the traces correspond to: (A) $\mathrm{PhBr}\left(T_{\mathrm{m}}=126.0^{\circ} \mathrm{C}, \Delta_{\text {fus }} H=39\right.$ $\left.\mathrm{kJ} \mathrm{mol}{ }^{-1}\right)$, (B) PhCl $\left(T_{\mathrm{m}}=128.5^{\circ} \mathrm{C}, \Delta_{\text {fus }} H=42 \mathrm{~kJ} \mathrm{~mol}^{-1}\right)$, (C) $\mathrm{PhF}\left(T_{\mathrm{m}}=129.8^{\circ} \mathrm{C}, \Delta_{\text {fus }} H=44 \mathrm{~kJ}\right.$ $\left.\mathrm{mol}^{-1}\right)$, (D) $\operatorname{Phl}\left(T_{\mathrm{m}}=134.9^{\circ} \mathrm{C}, \Delta_{\text {fus }} H=36 \mathrm{~kJ} \mathrm{~mol}^{-1}\right)$. 
Table IX-1: DSC onset melting point and heat of melting data for four TATM guest alkyl halide families (see chapter VIII).

\begin{tabular}{llll}
\hline Guest & $T_{\text {In }}\left( \pm 0.1^{\circ} \mathrm{C}\right)$ & $\Delta_{\text {fus }} H\left( \pm 1 \mathrm{~J} \mathrm{~g}^{-1}\right)$ & $\Delta_{\text {fux }} H\left( \pm 1 \mathrm{~kJ} \mathrm{~mol}^{-1} \mathrm{TATM}\right)$ \\
methylene chloride & 125.9 & 109 & 42 \\
methylene bromide & 133.7 & 98 & 38 \\
methylene iodide & 118.9 & 82 & 32 \\
& & & \\
1,3-dichloropropane & 103.2 & 94 & 37 \\
1,3-dibromopropane & 102.4 & 83 & 32 \\
1,3-diiodopropane & 114.7 & 73 & 28 \\
2-chlorobutane & 108.6 & 111 & 43 \\
2-bromobutane & 107.1 & 94 & 36 \\
2-iodobutane & 100.8 & 84 & 33 \\
& & & 37 \\
cyclohexyl chloride & 111.4 & 95 & 35 \\
cyclohexyl bromide & 107.3 & 89 & 31 \\
cyclohexyl iodide & 96.4 & 80 & 44 \\
& & & 42 \\
fluorobenzene & 129.8 & 113 & 39 \\
chlorobenzene & 128.5 & 107 & 36 \\
bromobenzene & 126.0 & 100 & \\
iodobenzene & 134.9 & 93 &
\end{tabular}

\section{EXPERIMENTAL}

The competitive inclusion experiments were performed by adding equimolar proportions of two guests (total volume $1.00 \mathrm{~mL}$ ) into a covered $2 \mathrm{~mL}$ vial that contained approximately $200 \mathrm{mg}$ ethanol / TATM and a magnetic stir bar. The vials were sealed with parafilm to prevent guest evaporation, and then heated to dissolve the TATM solid into the guest mixture. Following this, the vials were placed on a magnetic stir plate and stirred at $600 \mathrm{rpm}$ for approximately two weeks (at room temperature) to allow equilibrium conditions to be established. Then the contents of the vials were filtered under suction, and after drying overnight, the solid inclusion compound mixtures were each dissolved in $\mathrm{CDCl}_{3}$ and their ${ }^{\prime} \mathrm{H}$ solution NMR spectra were acquired on a commercial Bruker DRX $400 \mathrm{MHz}$ high resolution NMR spectrometer. 
A $5 \mathrm{~mm}$ multinuclear probehead was used, at a frequency of $400.132 \mathrm{MHz}$ for ${ }^{1} \mathrm{H}$. A $90^{\circ}$ pulse length of $9.20 \mu$ s was employed, with 32 scans for each sample, a spectral width of $8224 \mathrm{~Hz}$, an acquisition time of $1.9923 \mathrm{~s}$ and a relaxation delay of $4.0 \mathrm{~s}$. Following Fourier transformation of the free induction decay, the spectra were calibrated to the ${ }^{\prime} \mathrm{H}$ signal of the residual chloroform solvent, and are tabulated relative to tetramethylsilane at $0 \mathrm{ppm}$. The molar ratio of guests incorporated from each equimolar guest mixture was then determined by direct integration of the ${ }^{1} \mathrm{H}$ NMR resonances.

Because of extensive second order effects in the ${ }^{1} \mathrm{H}$ NMR spectra of the monohalobenzenes, we employed ${ }^{13} \mathrm{C}$ solution NMR with inverse gated decoupling to obtain guest ratios. In this pulse sequence, the ${ }^{1} \mathrm{H}$ decoupler is left off between scans to minimize NOE distortions. The data were obtained at a ${ }^{13} \mathrm{C}$ frequency of $100.62 \mathrm{MHz}$, with a $\pi / 2$ pulse length of $7.20 \mu \mathrm{s}, 500$ to 1000 scans per sample and a relaxation delay of $10 \mathrm{~s}$. The relative intensities of the guest's signals were determined by measuring peak heights, as all of the signals were very sharp.

\section{RESULTS}

The competitive inclusion results for all four alkyl halide / TATM guest families are listed in Table IX-2. All experiments were performed in duplicate, using separately prepared samples. The average values of each of the two individual determinations are indicated in bold. The absolute uncertainty for each average value is calculated as onehalf of the difference between the two individual determinations. In all twelve cases, the absolute uncertainty is less than the difference in stoichiometric ratio between the two guests in the TATM inclusion mixture (e.g. for methylene chloride and methylene bromide, $0.27<(1.65-1.00)$ ). Furthermore, in 11 of the 12 cases, the preference for one guest over the other is the same for both determinations. The only case where this does not occur is the mixture of 2-chlorobutane and 2-bromobutane. However, the conflicting preference is very small, $1.00 \mathrm{Cl}: 1.05 \mathrm{Br}$. The other preference is much more substantial, $1.85 \mathrm{Cl}: 1.00 \mathrm{Br}$. In all 11 other cases, there is no conflict between each of the two determinations. In summary, these two factors suggest that the size and nature of the experimental uncertainty is small enough to conclude that the experimental trends in preferential inclusion are in fact valid. 
Table IX-2: Results of competitive inclusion experiments of alkyl halide guest families with TATM. In each case an equimolar mixture was used, and the molar ratio in the product mixture was determined from ${ }^{\mathrm{H}} \mathrm{H}$ NMR.

A) methylene halides

methylene chloride

methylene bromide

1.00

1.38

1.00

1.91

1.00

$1.65( \pm 0.27)$

methylene chloride

methylene iodide

8.50

1.00

4.98

1.00

$6.74( \pm 1.76)$

1.00

methylene bromide

6.10

methylene iodide

4.48

1.00

$5.29( \pm 0.81)$

1.00

1.00

B) 1,3-dihalopropanes

1,3-dichloropropane

1.00

1,3-dibromopropane

1.00

1.38

1.00

1.05

$1.22( \pm 0.17)$

1,3-dichloropropane

1,3-diiodopropane

2.35

1.00

3.02

1.00

$2.68( \pm 0.34)$

1.00

1,3-dibromopropane

1.31

1,3-diiodopropane

1.96

1.00

$1.63( \pm 0.32)$

1.00

1.00 
Table IX-2: (continued)

C) 2-halobutanes

2-chlorobutane

2-bromobutane

1.00

1.05

1.85

1.00

$1.77( \pm 0.43)$

1.00

2-chlorobutane

2-iodobutane

1.33

1.00

1.49

1.00

$1.41( \pm 0.08)$

1.00

2-bromobutane

2-iodobutane

1.33

1.00

1.82

1.00

$1.57( \pm 0.25)$

1.00

D) cyclohexyl halides

cyclohexyl chloride

3.23

cyclohexyl bromide

1.25

1.00

1.00

$2.24( \pm 0.99)$

1.00

cyclohexyl chloride

9.53

cyclohexyl iodide

1.00

2.45

1.00

$5.99( \pm 3.54)$

1.00

cyclohexyl bromide

cyclohexyl iodide

2.18

1.00

2.04

1.00

$2.11( \pm 0.07)$

1.00 
On first glance at Table IX-2, it appears that the competitive inclusion results for all four alkyl halide guest families are consistently the same, in that alkyl iodides are the least preferred TATM guest, while the alkyl chlorides and alkyl bromides are more preferred. As far as the alkyl iodide result is concerned, large guests may be less preferred by TATM, because they stretch the TATM cavity beyond its most preferred size. On the other hand, alkyl chlorides and alkyl bromides may fit into the TATM cavity more comfortably, resulting in the greater preference observed in this work.

In addition, it appears that there is a relationship (qualitatively speaking) between the size of the hydrocarbon backbone of the guest and the relative preference of alkyl chlorides and alkyl bromides. For the larger alkyl halides (cyclohexyl halides and 2halobutanes), chlorides are preferred over bromides. This may be because the large size of these hydrocarbon backbones may lead to a preference for smaller halogen substituents (i.e. $\mathrm{Cl}$ ), as the larger substituents (i.e. $\mathrm{Br}$ ) may lead to excessive guest size, which would run into the same problem encountered with the alkyl iodides. On the other hand, for the smaller gucsts (methylene halides and 1,3-dihalopropanes), we see the opposite result, namely that bromides are preferred over chlorides. The smaller guests may prefer a larger and more polarizable halogen substituent (i.e. $\mathrm{Br}$ ) to maximize stabilizing van der Waals interactions between host and guest, yet the hydrocarbon backbone is still small enough to minimize destabilizing cage stretching forces encountered with the larger guests above.

Another factor that may be of relevance is the competition (quantitatively speaking) between alkyl chlorides and alkyl bromides. For the two smaller sized guest families (methylene halides and 1,3-dihalopropanes), the selectivity of bromides over chlorides (1.64:1.00 and 1.22:1.00 respectively) is less pronounced than the selectivity of chlorides over bromides for the larger sized guest families (2-halobutanes and cyclohexyl halides $-1.78: 1.00$ and 2.24:1.00 respectively). This result suggests, that for these TATM inclusion compounds, cage stretching forces, which may destabilize TATM inclusion compounds with 2-bromobutane and cyclohexyl bromide, are more important in determining the strength of attractive host-guest interactions of a TATM inclusion compound than the lack of extensive van der Waals interactions between host and guest, which may serve to destabilize TATM inclusion compounds with methylene chloride and 
1,3-dichloropropane. Furthermore, the fact that alkyl chlorides are universally preferred over alkyl iodides lends support to the conclusion reached above that cage stretching forces (which may serve to destabilize TATM / alkyl iodides) are more important in determining the strength of attractive intermolecular interactions of a TATM inclusion compound than lack of extensive van der Waals interactions between host and guest (which may serve to destabilize TATM / alkyl chlorides).

From Table IX-2, one observes that the TATM preference of chlorides over iodides and bromides over iodides is very pronounced for the methylene halides, and generally much less pronounced for the other three alkyl halide guest families. This result may be rationalized by the relative increase in size and change in shape on going from chloride to bromide to iodide. For the methylene halides, there are two halogen atoms for each carbon atom. Thus the relative increase in size on going from chloride to bromide to iodide is very pronounced, while it is generally much smaller for the other three guest families. Therefore, one might expect that for the methylene halide guest family, it may be easier for the TATM host to distinguish one of these guests from a mixture of two. Indeed, for the two mixtures involving iodide, the selectivity is very high $(6.74 \mathrm{Cl}: 1.00 \mathrm{I}$ and $5.29 \mathrm{Br}: 1.00 \mathrm{l})$, while for the other three alkyl halide guest families, it is in most cases much smaller (see Table IX-2).

Finally, in Table IX-3, we present the competitive inclusion results for the monohalobenzenes. A summary of the results is tabulated in Table IX-4. From this table, one sees that chlorobenzene and bromobenzene are the most preferred TATM guests (5 points each), next comes fluorobenzene/TATM ( 2 points) and finally iodobenzene/TATM ( 0 points). So again we see that chlorides and bromides are the most preferred guests. Interestingly, fluorobenzene is preferred over iodobenzene. This provides further and definite proof that cage-stretching forces (which may destabilize alkyl iodide/TATM inclusions) are more important in determining the host-guest binding of a TATM inclusion compound than lack of extensive van der Waals interactions (which may destabilize alkyl fluoride/TATM inclusions). In addition, the results for this guest family demonstrates agreement with experiments on $\beta$-quinol (see below) $[10,11]$ in that intermediate size guests are most suitably sized for inclusion formation, while larger and smaller guests are less suitably sized, be it $\beta$-quinol or TATM. 
Table IX-3: Results of competitive inclusion experiments $( \pm 10 \%)$ of monohalobenzene guest families with TATM. In each case an equimolar mixture was used, and the molar ratio in the product mixture was determined from inverse gated decoupling ${ }^{13} \mathrm{C} N M R$.

$\begin{array}{ll}\text { fluorobenzene } & \text { chlorobenzene } \\ 1.00 & 2.25 \\ \text { fluorobenzene } & \text { bromobenzene } \\ 1.00 & 1.75 \\ \text { fluorobenzene } & \text { iodobenzene } \\ 1.23 & 1.00 \\ \text { chlorobenzene } & \text { bromobenzene } \\ 1.00 & 1.00 \\ \text { chlorobenzene } & \text { iodobenzene } \\ 2.00 & 1.00 \\ & \\ \text { bromobenzene } & \text { iodobenzene } \\ 2.10 & 1.00\end{array}$

Table IX-4: Summary of results for competitive inclusion experiments involving monohalobenzenes:

\begin{tabular}{lllll} 
Guest & preferred & not preferred equally preferred & points \\
\hline fluorobenzene & 1 & 2 & 0 & 2 \\
chlorobenzene & 2 & 0 & 1 & 5 \\
bromobenzene & 2 & 0 & 1 & 5 \\
iodobenzene & 0 & 3 & 0 & 0 \\
\hline
\end{tabular}

\section{DISCUSSION}

\section{(A) $\quad \beta$-quinol}

These results may be compared with those obtained by H. G. McAdie (see Table IX-5) on a large group of $\beta$-quinol inclusion compounds $[10,11]$. McAdie separated the guests into two groups, the first group being smaller guests that do not distort the $\beta$-quinol cavity (as measured by the crystallographic $c$ parameter), such as argon, nitrogen, 
methane and carbon monoxide. The second group was comprised of larger guests that do in fact distort the $\beta$-quinol cavity (again as measured by the crystallographic $c$ parameter), such as ethane, methanol, carbon dioxide and acetonitrile. In Table IX-5, the guests become larger and larger in the downward direction of the table.

For the first group of smaller guests, the decomposition enthalpies, as measured by differential thermal analysis (DTA), increase with increasing size of guest (see Table IX-5). McAdie believed that this was because of differences in the interaction of these guest molecules with the cavity walls, arising from differences in the fraction of available cavity volume occupied by each guest. As can be seen in Table IX-5 (for the first group of smaller guests), the heat of decomposition increases approximately linearly with the polarizability $(\alpha)$ of the guest. McAdie believed that the strength of host-guest binding depends on the interactions of the guest with the cavity walls, imparting stability to the entire lattice structure. McAdie believed that these interactions were mainly of the van der Waals type, with other effects such as dipole-dipole interactions relatively less important because most of the guest molecules studied were relatively nonpolar.

The second group of larger guests that do in fact distort the $\beta$-quinol cavity displayed the opposite behaviour. As depicted in Table IX-5, McAdie showed that there is a trend toward decreasing enthalpy of decomposition with increasing molecular size of the guest. For these guests, increasing molecular size is generally accompanied by increasing distortion of the cavity along its $c$-axis. Therefore, the decomposition enthalpy of the $\beta$-quinol inclusion compound decreases as the initial distortion required to accommodate the gucst increases.

Furthermore, for the markedly unstable $\mathrm{CH}_{3} \mathrm{CN}$ and $\mathrm{CH}_{3} \mathrm{NC}$ clathrates with $\beta$ quinol (larger guests), which rapidly lose the guest in air [12], the mean $\mathrm{O} \cdots \mathrm{O}$ hydrogen bond lengths, $2.778 \AA$ and $2.790 \AA$ respectively, are significantly longer than the corresponding distances of $2.696 \AA, 2.690 \AA$ and $2.716 \AA$, found in the relatively stable $\beta$ quinol inclusions with smaller guests, namely $\mathrm{H}_{2} \mathrm{~S}, \mathrm{HCl}$ and $\mathrm{CH}_{3} \mathrm{OH}$ respectively [11]. Thus, we can use either the length of the crystallographic $c$ direction or the $\mathrm{O} \cdots \mathrm{O}$ hexamer hydrogen bond length as a measure of the stretching of the $\beta$-quinol cage. As an aside, it is intriguing that $\mathrm{CH}_{3} \mathrm{CN}$ and $\mathrm{CH}_{3} \mathrm{NC}$, which are isoelectronic, yield $\beta$-quinol clathrates that are not isostructural. 
Table IX-5: Thermal and structural properties of $\beta$-quinol adducts with increasing size of guest molecules (taken from reference [11])

\begin{tabular}{lllll} 
& & \multicolumn{4}{l}{ Thermal properties of smaller guests } \\
\cline { 3 - 5 } Guest & $c(\AA)^{4}$ & $T_{d}\left({ }^{\circ} \mathrm{C}\right)^{13}$ & $\left.\Delta H_{d}(\mathrm{~kJ} \mathrm{~mol})^{-1}\right)^{(}$ & $\alpha \times 10^{24}\left(\mathrm{~cm}^{2}\right)^{1)}$ \\
\hline argon & - & 127.1 & 26.4 & 1.68 \\
nitrogen & - & 128.4 & 28.4 & 1.76 \\
carbon monoxide & - & 126.6 & 28.9 & 1.98 \\
methane & - & 129.4 & 39.7 & 2.60 \\
hydrogen chloride & 5.46 & 139.9 & 41.8 & 2.63 \\
hydrogen bromide & 5.48 & 151.0 & 35.1 & 3.36 \\
hydrogen sulfide & 5.49 & 150.9 & 43.5 & 3.65 \\
\hline
\end{tabular}

\begin{tabular}{|c|c|c|c|c|}
\hline \multirow[b]{2}{*}{ Guest } & \multicolumn{4}{|c|}{ Thermal properties of larger guests } \\
\hline & $c(\AA)^{A}$ & $T_{4}\left({ }^{\circ} \mathrm{C}\right)^{13}$ & $\Delta H_{4}\left(\mathrm{~kJ} \mathrm{~mol}^{-1}\right)^{\mathrm{c}}$ & $b(\mathrm{cc} \mathrm{mol})^{-1}$ \\
\hline acetylene & 5.46 & 131.6 & 39.7 & 0.0514 \\
\hline ethane & - & 115.1 & 31.4 & 0.0638 \\
\hline ethylene & - & 108.5 & 47.3 & 0.0571 \\
\hline methanol & 5.55 & 122.3 & 38.5 & 0.0670 \\
\hline sulfur dioxide & 5.60 & 134.5 & 43.5 & 0.0564 \\
\hline formic acid & 5.65 & 135.7 & 36.4 & - \\
\hline carbon dioxide & 5.82 & 103.3 & 29.7 & 0.0427 \\
\hline nitrous oxide & - & 119.2 & 26.8 & 0.0442 \\
\hline methyl chloride & - & 105.5 & 47.3 & 0.0648 \\
\hline methyl bromide & - & 96.5 & 26.8 & 0.0928 \\
\hline acetonitrile & 6.24 & 100.8 & 31.8 & 0.1168 \\
\hline
\end{tabular}

the crystallographic $c$ direction, notice a steady increase with size of guest.

${ }^{B}$ the onset decomposition temperature, obtained from differential thermal analysis.

'the enthalpy of decomposition. as measured by integrating the DTA endotherm

Dmolar polarizability $(\alpha)$, a measure of molecular volume for the smaller guests

van der Waals covolume (b), a measure of molecular volume for the larger guests

These results may be compared with the conclusions obtained in the present study on TATM inclusions. Alkyl chlorides are in some cases less preferred than alkyl bromides because alkyl chlorides may give rise to less extensive stabilizing van der Waals interactions between host and guest. On the other hand, alkyl iodides are less preferred than alkyl chlorides and alkyl bromides because alkyl iodides stretch the TATM cavity beyond its most preferred size. 


\section{(B) Dianin's compound}

A paper from 1971 [13] was concerned with selective enclathration of hydrocarbons by Dianin's compound. Under comparable conditions of crystallization (e.g. rate of cooling, etc.), studies of binary guest blends with different mole fraction compositions showed that the ratio of components in the ternary inclusion compound is proportional (but not equal) to the ratio of components in the initial binary guest mixture from which enclathration occurred. With paraffinic hydrocarbons, the selective enclathration results were in accord with those expected based on the shape and dimensions of the host cage. The experimental results are depicted in Table IX-6. On the other hand, the aromatic hydrocarbons follow a more complicated pattern, and are not discussed here.

Based on the known dimensions of the cage [14], difficulty in inclusion compound formation would be expected for hydrocarbons having lengths significantly in excess of $11 \AA$, or containing bulky substituents that interfere sterically with the methyl groups of the host, that form the "waist" of the hourglass shaped cage. Results with nparaffins show that $n$-pentane and $n$-hexane are enclathrated with ease, but at higher carbon numbers, steric repulsion progressively increases, until with $n$-decane, in which case well over $90 \%$ of the cages remain unfilled. The branched 3-ethylpentane is less readily included than the isomeric $n$-heptane, while the bulky $2,2,3$-trimethylbutane is almost totally rejected.

The enclathration selectivity for 3-methylhexane/n-heptane and 2,4dimethylpentane $/ n$-heptane were measured as $0.22: 1$ and $0.40: 1$ respectively, showing a distinct preference for linear hydrocarbons with these $\mathrm{C}_{7} \mathrm{H}_{16}$ isomeric guests. The 3methylhexane guest shows the lowest preference likely due to the presence of a 3-methyl group precisely at the most constricted point of the host cage, while the 2,4dimethylpentane isomer is less sterically hindered in this regard.

Indeed, the reported results (see Table IX-6) [13] show that the linear $n$-paraffins $\mathrm{C} 5, \mathrm{C} 6$, and $\mathrm{C} 7$ are most readily preferred, while hydrocarbons containing one or more methyl groups in the middle of the guest molecule (e.g. 3,3-dimethylpentane) are almost completely rejected.

The long dimension (height) of the cage is about $11 \AA$, and is similar to the chainextended length of $n$-hexane. The $n$-hexane molecule may be accommodated into the 
host cage without invoking any appreciable strain, and enclathration would appear to be relatively easy. Similarly, $n$-pentane is also readily complexed, while the $n$-butane guest likely leads to a reduction in the attractive interaction energy between guest and host, which may account for its lower selectivity when compared with $n$-hexane. On the other hand, the longer $n$-heptane guest brings the terminal methyl groups of the guest into close association with the top and bottom of the host cage, and suggests that $n$-heptane may be accommodated only if it is slightly twisted in shape. The $n$-octane guest requires more extensive coiling, and the authors noted that it seems unlikely that this guest could be included without incurring appreciable repulsion between host and guest molecules. The observed significant decrease in selectivity on passing from n-hexane to n-octane therefore results principally from increased steric repulsion. These results on competitive inclusions in Dianin's compound correlate with those on TATM if it is assumed that the alkyl halide guests are all on the large size. In this case, alkyl iodides fit into the cavity with only great difficulty, resulting in a lower preference. In some cases, smaller alkyl chlorides are most preferred because they fit into the TATM more comfortably than the larger guests. On the other hand, it is likely that TATM is more "accommodating" for larger guests, as it is generally more flexible than the invariant Dianin`s compound structure, which is reflected in the significantly greater preference of Dianin`s compound for more suitably sized guests (see Table IX-6) than the TATM preference (Tables IX-2 and (X-4)

The selectivity among branched hydrocarbons follows a particularly interesting pattern that again may be accounted for in terms of the hourglass structure of the cage. Among the dimethylpentane isomers, 3,3-dimethylpentane has the lowest relative selectivity, likely because inclusion in this case would invoke severe steric repulsion between the 3-methyl substituents of the guest and the waist-forming methyl groups of the cage. Furthermore, 2,3-dimethylpentane is also included only with difficulty, while for similar reasons, 3-ethylpentane and 2,2,3-trimethylbutane also have low selectivity. The presence of methyl substituents at the $\mathrm{C}-2$ and $\mathrm{C}-4$ positions along the pentane backbone incur appreciably less strain during enclathration, and (apart from 2methylhexane), 2,4-dimethylpentane is the most readily accommodated branched heptane isomer. 
Table IX-6: Relative accommodation factors for paraffinic isomers (C5 - C8) in Dianin's compound [13].

\begin{tabular}{lll}
\hline Guest & Boiling point $\left({ }^{\circ} \mathrm{C}\right)$ & Relative accommodation factor \\
\hline Pentanes & & \\
2-methylbutane & 27.85 & 0.23 \\
n-pentane & 36.07 & 4.5 \\
Hexanes & & \\
2-methylpentane & 60.27 & 1.4 \\
3-methylpentane & 63.28 & 0.1 \\
2,2-dimethylbutane & 49.74 & $<0.01$ \\
2,3-dimethylbutane & 57.99 & $<0.01$ \\
n-hexane & 68.74 & 9.0 \\
& & \\
Heptanes & & \\
2-methylhexane & 90.05 & 2.5 \\
3-methylhexane & 91.85 & 0.22 \\
2,2-dimethylpentane & 79.20 & 0.05 \\
2,3-dimethylpentane & 89.78 & $<0.01$ \\
2,4-dimethylpentane & 80.50 & 0.40 \\
3,3-dimethylpentane & 86.06 & $<0.01$ \\
2,2,3-trimethylbutane & 80.68 & $<0.01$ \\
3-ethylpentane & 93.48 & $<0.01$ \\
n-heptane & 98.43 & 1.00 \\
& & \\
Octanes & & \\
2-methylheptane & 117.65 & 0.20 \\
2,2-dimethylhexane & 106.84 & 0.43 \\
2,4-dimethylhexane & 109.43 & 0.07 \\
2,5-dimethylhexane & 109.10 & 0.80 \\
2,2,4-trimethylpentane & 99.24 & 0.06 \\
n-octane & 125.66 & 0.06 \\
\hline
\end{tabular}

Finally, the authors noted that Dianin's compound may allow facile separations of isomeric hydrocarbons that have very similar boiling points, for whose separation would be extremely difficult using more conventional methods. A particularly attractive example is the separation of 2-methylhexane (bp $90.05^{\circ} \mathrm{C}$ ) from 2,3-dimethylpentane (bp $89.78^{\circ} \mathrm{C}$ ). These two isomers can only be separated by fractional distillation with several hundred cycles. The preference for 2-methylhexane in Dianin's compound is over 200 times greater than that of 2,3-dimethylpentane. As a result, 99\% pure 2-methylhexane can be recovered from an equimolar mixture by a single enclathration. This is in contrast to TATM, where the enclathration is considerably less selective, which would make it a less suitable host system for effecting separations. Other possible applications for Dianin's compound noted by the authors include selective enclathration of impurities, 
storing unstable or expensive chemicals (some hydrocarbon clathrates have remained completely unchanged in the author's laboratory for over 10 years) [13], while their stability and ease of handling would make them convenient for preparing reference standards in analytical work (e.g. gas chromatography).

A more recent study [15] was concerned with the structural properties of the Dianin's compound host with a carboxylic acid guest family, namely formic acid $\rightarrow$ octanoic acid, plus dimethylacetic acid and trifluoroacetic acid. The smaller guests (formic, acetic and trifluoroacetic acids) occupy any one host cavity in pairs, while the rest of the larger acids were mono-occupied. Formic and acetic acid each formed hydrogen bonded dimers in any one cage. Heptanoic and octanoic acid adopt gauche conformations to allow them to fit into the host cavity with only slight distortion of the cage.

For the intermediate sized guests (propionic acid $\rightarrow$ hexanoic acid), which each occupy the host cavity individually, the X-ray derived map of electron density is seen to be spread (disordered) along the whole length of the cavity. This smearing of electron density is approximately to the same extent irrespective of the acid chain length. The carboxylic acid functionality of the guest forms a hydrogen bond to the hexagon of $\mathrm{O}-\mathrm{H}$ bonds of the host lattice. These four acid guests are present as fully extended all-trans straight chains, with all carbon and oxygen atoms of each guest lying in one plane.

The heptanoic acid and octanoic acid guests form a limited number of gauche conformations to fit the constraints of the host cavity more easily. It has been estimated [16] that a single gauche conformation involves an energy of approximately $4 \mathrm{~kJ} \mathrm{~mol}^{-1}$ greater that that of the all trans fully extended conformation. The authors suggested that this increase in intramolecular conformational energy may be offset during clathrate formation by favourable hydrogen bonding between host and guest. From the single crystal structures [15], the heptanoic acid inclusion was constructed and refined with one gatche conformation, while the octanoic acid was found to have two gauche conformations, one at each end of the guest. On the other hand, it is likely that larger guests do not have to undergo considerable distortion when in the TATM lattice, due the "plasticity" of the TATM host lattice, which may go a long way towards explaining 
TATM's very versatile host properties, as compared to Dianin's compound, which limits the guest's length to $\sim 11 \AA$.

(C) 1,1-bis(4-hydroxyphenyl)cyclohexane

A series of four publications [17-20] by Nassimbeni and co-workers dealt with separation of close isomers by the host 1,1-bis(4-hydroxyphenyl)cyclohexane, 1. This host forms inclusion compounds with a wide variety of guests, and the crystal structures with water and with ethanol as guests have been published [21].

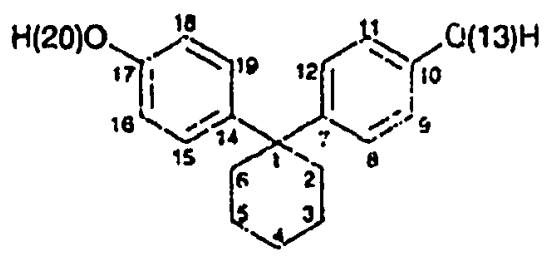

Scheme 1: 1,1-bis(4-hydroxyphenyl)cyclohexane host structure

In the first publication [17], the competitive inclusion experiments showed that paraphenylenediamine $\left(\mathrm{H}_{2} \mathrm{~N}_{-} \mathrm{C}_{6} \mathrm{H}_{4}-\mathrm{NH}_{2}\right)$ is preferentially incorporated over orthophenylenediamine. Indeed, the host gives $\sim 100 \%$ selectivity for para-phenylenediamine mole fractions in the mother solution of $0.1<X_{\text {para }}<1$. The authors rationalized this behaviour on the basis of guest-host interactions. Namely, each para-phenylenediamine guest molecule engages in four intermolecular host-guest hydrogen bonds, while each ortho-phenylenediamine guest is stabilized by only two host-guest hydrogen bonds. TATM generally does not form host-guest hydrogen bonds, so this feature is not applicable in its case.

The second publication dealt with the separation of hydroquinone (benzenediol: HO- $\left.\mathrm{C}_{6} \mathrm{H}_{4}-\mathrm{OH}\right)$ isomers by this host [18]. Again, the guests were para- and orthosubstituted benzene rings. The authors found that the para-hydroquinone guest is preferentially incorporated over ortho-hydroquinone. Furthermore, each parahydroquinone guest participates in four hydrogen bonds with the host, while each orthohydroquinone guest molecule participates in only three hydrogen bonds with the host 
framework. The authors used lattice energy calculations for these two inclusion compounds to compare with the competitive inclusion results. The potential energy of each lattice was calculated by the method of atom-atom potentials. Calculations revealed that the lattice energy for the para-hydroquinone inclusion compound of $-264 \mathrm{~kJ} \mathrm{~mol}^{-1}$, while that for the ortho-hydroquinone inclusion was only $-250 \mathrm{~kJ} \mathrm{~mol}^{-1}$. Thus both the extent of host-guest hydrogen bonding and the lattice energy calculations can be used to explain the competitive inclusion results. In these cases, there is a definite correlation between guest selectivity and calculated lattice energy. In contrast, for TATM / alkyl halide systems, the correlation between $\Delta_{\text {fus }} H$ and guest selectivity is not as strong. For example, the alkyl chloride inclusions investigated have a universally highest enthalpy of fusion, while in a number of cases, alkyl bromides are preferred over alkyl chlorides.

The same year (1997), a third publication was disseminated that described competitive inclusions of the same host with isomers of picoline (2-3- and 4- methyl pyridine) [19]. From the single crystal structures, 3-picoline and 4-picoline each engage in two hydrogen bonds with the host. Suitable single crystals of the 2-picoline inclusion compound could not be prepared. In the competitive inclusion experiments, both 3picoline and 4-picoline were strongly favored over 2-picoline. On the other hand, no significant selectivity preference was observed between the 3-picoline / 4-picoline mixture. This result agrees with the lattice energy calculations in that the 3-picoline inclusion $\left(-261.9 \mathrm{~kJ} \mathrm{~mol}^{-1}\right)$ and the 4-picoline inclusion $\left(-261.4 \mathrm{~kJ} \mathrm{~mol}^{-1}\right)$ had almost the same lattice energy. An approximate lattice energy for the 2-picoline inclusion (-198.6 $\mathrm{kJ} \mathrm{mol}^{-1}$ ) was obtained by constructing an idealized 2-picoline molecule, and superimposing it (as a rigid structure) on the difference electron density map calculated from the structure factors derived from the host alone. The more positive lattice energy for this 2-picoline inclusion compound agrees with the competitive inclusion results described above. The activation energies for guest release, calculated from variable heating rate TGA curves, were as follows:

$$
\begin{aligned}
& 77 \mathrm{~kJ} \mathrm{~mol}^{-1}<E_{\mathrm{a}}(3 \text {-picoline })<81 \mathrm{~kJ} \mathrm{~mol}^{-1} \\
& 79 \mathrm{~kJ} \mathrm{~mol}^{-1}<E_{\mathrm{i}}(4 \text {-picoline })<82 \mathrm{~kJ} \mathrm{~mol}^{-1}
\end{aligned}
$$


The authors noted that the similarities in activation energies agree well with the results for this system listed above. The correlation between guest selectivity and activation energy (from TGA) for monohalobenzene / TATM systems is also not as strong as the present host system. For TATM, $E_{\mathrm{a}}$ follows the guest order $\mathrm{PhF}>\mathrm{PhCl}>$ $\mathrm{PhBr}>\mathrm{PhI}$ (unpublished material), however $\mathrm{PhF}$ is less preferred by TATM than both $\mathrm{PhCl}$ and $\mathrm{PhBr}$.

The fourth (and final) paper of this series [20] dealt with the same host system selectively including four aliphatic alcohols (methanol, ethanol, isopropanol and $n$ butanol). The binary competitive results showed no significant selectivity between $\mathrm{MeOH}$ and $\mathrm{EtOH}$, while the selectivity between the other binary mixtures is as follows: $\mathrm{Bu}^{\mathrm{n}} \mathrm{OH}>\mathrm{MeOH}, \mathrm{Pr}^{\mathrm{i}} \mathrm{OH}>\mathrm{MeOH}, \mathrm{Bu}^{\mathrm{n}} \mathrm{OH}>\mathrm{EtOH}, \mathrm{Pr}^{\mathrm{i}} \mathrm{OH}>\mathrm{EtOH}, \mathrm{Pr}^{\mathrm{i} O H}>\mathrm{Bu}^{\mathrm{n}} \mathrm{OH}$. The single crystal structures indicated that all four inclusion compounds are isostructural, with two host-guest hydrogen bonds for each inclusion compound. The calculated lattice energies follow the order $-268 \mathrm{~kJ} \mathrm{~mol}^{-1}\left(\mathrm{Pr}^{\mathrm{i}} \mathrm{OH}\right)>-258 \mathrm{~kJ} \mathrm{~mol}^{-1}(\mathrm{Bu} \mathrm{n} \mathrm{OH})>-216 \mathrm{~kJ} \mathrm{~mol}^{-1}$ $(\mathrm{MeOH}) \approx-215 \mathrm{~kJ} \mathrm{~mol}^{-1}(\mathrm{EtOH})$, which agrees well with the competitive inclusion results. The crystal structures of $\mathrm{MeOH} / \mathrm{TATM}$ and EtOH / TATM (see Chapter XV) are suggestive of no intermolecular host-guest hydrogen bonds.

(D) Cyclophane

In 2002, a paper [22] was published that was concerned with structure and guest selectivity of a cyclophane-type host (2) with picoline (3) and lutidine (4) isomeric guests. Individually, 2-picoline and 3-picoline form isostructural inclusion complexes with 2. Perhaps as a result, there was no significant guest selectivity from a binary mixture of 2-picoline and 3-picoline (i.e. 50:50 incorporated molar ratio). This is in contrast to TATM, where guests that form isostructural TATM inclusion compounds in many cases show significantly greater selectivity (e.g. the cyclohexyl halide guest family). The guest 4-picoline was more favored than both 2-picoline and 3-picoline. Indeed, lattice energy calculations suggest that the 4-picoline inclusion has the lowest lattice energy $\left(-160.7 \mathrm{~kJ} \mathrm{~mol}^{-1}\right)$, followed by inclusions of 2 with 2-picoline and 3picoline $\left(-132.1 \mathrm{~kJ} \mathrm{~mol}^{-1}\right.$ and $-130.3 \mathrm{~kJ} \mathrm{~mol}^{-1}$ respectively). So we see another example of the relationship between lattice energy and guest selectivity. As a final note, for 
TATM, we measured experimental $\Delta_{\mathrm{fu}} H$ values to compare with guest selectivity, while in the current case the guest selectivity is compared to calculated lattice energies, and this difference must be taken into account.

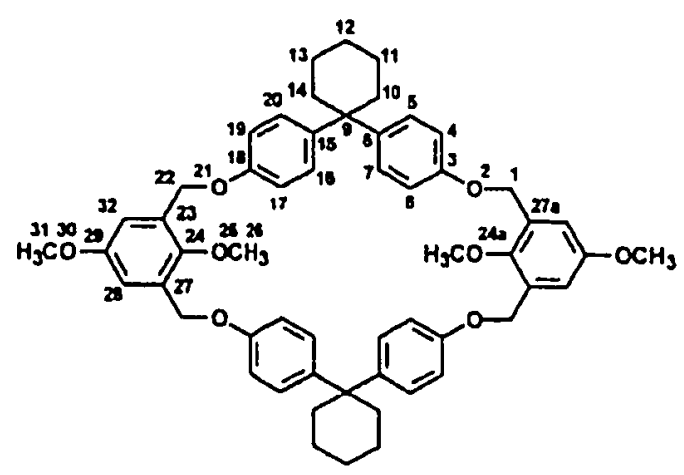

Scheme 2: Cyclophane-type host compound<smiles>Cc1ccccn1</smiles><smiles>Cc1cccnc1</smiles><smiles>Cc1ccncc1</smiles>

Scheme 3: Picoline guest isomers<smiles>Cc1cccc(C)n1</smiles><smiles>Cc1cncc(C)c1</smiles><smiles>Cc1ccnc(C)c1</smiles>

Scheme 4: Luditine guest isomers

As far as the lutidine inclusions with $\mathbf{2}$ are concerned, the authors found that 2,4-lutidine was most preferred from the competitive inclusion experiments. The authors suggested that this is the case because the cyclophane host 2 prefers a methyl group para to the ring nitrogen, since 4-picoline (see above) is most preferred. The other guests, 2,6-lutidine and 3,5-lutidine do not contain a para methyl group, so perhaps this is why they are less preferred. The fact that the 2,4-lutidine and 4-picoline guests are more preferred may perhaps be explained by a set of favourable hydrogen bonding schemes in combination with the methoxy groups of the cyclophane 2 host molecules, 
(E) 2,5-bis(9-hydroxyfluoren-9-yl)thieno[2,3-b]thiophene

In 1998, a publication [23] appeared that dealt with ternary systems comprised of the macrocyclic host 2,5-bis(9-hydroxyfluoren-9-yl)thieno[2,3-b]thiophene, 5, along with $n$ propanol and isopropanol guests. The authors found that both guests individually form isostructural inclusion compounds with 5, both in a $1 \mathrm{H}: 2 \mathrm{G}$ stoichiometric ratio. On the basis of this result, they suggested that the host $\mathbf{5}$ should show insignificant guest selectivity from mixtures of $n$-propanol and isopropanol. Indeed, their selective inclusion experiments using various relative proportions of these two guests indicate that 5 cocrystallizes with the two guests in approximately the same molar ratio as that of the mother solution mixture. On the other hand, in the case of TATM, guests that individually form isostructral inclusion compounds generally do show a significant selectivity for one of the two guests from the initial equimolar mixture (e.g. the monohalobenzene guest family).

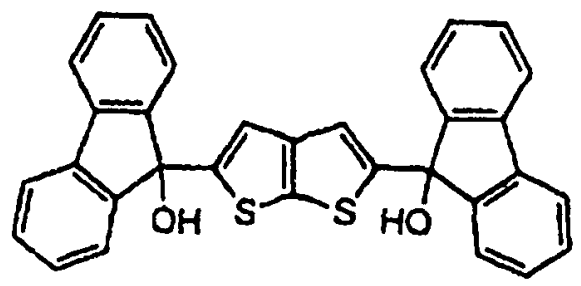

Scheme 5: 2,5-bis(9-hydroxyfluoren-9-yl)thieno[2,3-b]thiophene host material

\section{CONCLUDING REMARKS:}

One of the important applications of inclusion chemistry is the separation of close isomers by selective enclathration. This involves the choice of a suitable host compound, which, when exposed to a mixture of potential guests, combines selectively with a particular guest to form a crystalline inclusion compound. The product is then filtered, and the guest is released by gentle warming, enabling the host material to be recycled. The selectivity of this process depends on the efficiency of molecular recognition between the host and targeted guest, and subsequent enclathration cycles may be used to increase the efficiency of the separation. Selective enclathration is an excellent illustration of molecular recognition, because differences in the strengths and directions 
of host-guest intermolecular forces may be sufficiently large for a given host molecule to encapsulate one particular isomer from a mixture of two or more potential guests.

Selective enclathration by a host compound for a given guest may be studied at the molecular level by mapping the interactions between host and guest in a solid inclusion compound. The resulting non-bonded interaction energies allow a calculation of lattice energies, which are a measure of the relative stabilities of the host-guest networks, and may thus explain the preferential enclathration of a particular guest by a given host compound. The separation of close isomers by clathrate formation is industrially attractive because it is simple, efficient and is not energy intensive. The process is chemically attractive because it does not involve the breaking or formation of covalent bonds, but instead relies on shape recognition of the guest by the host.

In this work we have investigated guest selectivity for TATM inclusion compounds with the same alkyl halide guest families as in chapter VIII. At first, based on the DSC results of chapter VIII, it was tentatively proposed that the order of preference may perhaps go as: chlorides $>$ bromides $>$ iodides. Indeed, in the present study, the alkyl iodides were the least preferred. On the other hand, however, alkyl bromides were in some cases preferred over alkyl chlorides. We reasoned this selectivity on the basis of a balance of destabilizing forces of cage stretching for larger guests (i.e. alkyl iodides) and lack of extensive van der Waals interactions between host and guests for smaller guests (i.e. alkyl chlorides). The alkyl bromides brought about a balance of these two destabilizing forces to become the most preferred guest. This result agrees with literature data on $\beta$-quinol inclusions $[10,11]$.

CHAPTER IX

REFERENCES

(1) T. Kobayashi, S. Isoda and K. Kubono Cyclophosphazines: Structures and Molecular Selectivities In Comprehensive Supramolecular Chemistry J.L. Atwood, J.E.D. Davies, D.C. MacNicol and F. Vogtle, Eds. Volume 6, Chapter 13 Elsevier: Oxford, 1996.

(2) M.R. Caira and L.R. Nassimbeni Phase Transformations in Inclusion Compounds: Kinetics and Thermodynamics of Enclathration In Comprehensive Supramolecular Chemistry J.L. Atwood, J.E.D. Davies, D.C. MacNicol and F. Vogtle, Eds. Volume 6, Chapter 25 Elsevier: Oxford, 1996. 
(3) F. Toda Diol, Bisphenol and Diamide Host Compounds In Comprehensive Supramolecular Chemistry J.L. Atwood, J.E.D. Davies, D.C. MacNicol and F. Vogtle, Eds. Volume 6, Chapter 15 Elsevier: Oxford, 1996.

(4) K. Beketov, E. Weber, J. Seidel, K. Kohnke, K. Makhkamov and B. Ibragimov Chem. Commun. 91 (1999).

(5) C.H. Kim, S.I. Nishikiori and T. Iwamoto Chem. Lett. 409 (1995).

(6) C.G. Moyers Jr. and R.W. Rousseau Handbook of Separation Process Technology R.W. Rousseau, Ed. Chapter 11 Wiley: New York, 1987.

(7) F. Toda. Top. Curr. Chem. 140, 43 (1987).

(8) S. Apel, S. Nitsche, K. Beketov, W. Seichter, J. Seidel and E. Weber J. Chem. Soc. Perkin Trans. 21212 (2001).

(9) E. Weber, T. Hens, Q. Li and T.C.W. Mak Eur. J. Org. Chem. 1115 (1999).

(10) H.G. McAdie Can. J. Chem. 41, 2137 (1963).

(11) H.G. McAdie Can. J. Chem. 44, 1373 (1966).

(12) T.L. Chan and T.C.W. Mak J. Chem. Soc. Perkin Trans. 777 (1983).

(13) A. Goldup and G.W. Smith Separation Science 6, 791 (1971).

(14) H.M. Powell and B.D.P. Wetters Chem. Ind (London) 256 (1955).

(15) R.H.W. Small Acta Cryst. B59, 141 (2003).

(16) H. Morawetz Molecules in Solution Interscience: New York, 1965

(17) M.R. Caira, A. Horne, L.R. Nassimbeni, K. Okuda and F. Toda J. Chem. Soc. Perkin Trans. 21063 (1995).

(18) M.R. Caira, A. Horne, L.R. Nassimbeni and F. Toda J. Chem. Soc. Perkin Trans 2 1717 (1997).

(19) M.R. Caira, A. Horne, L.R. Nassimbeni and F. Toda J. Mater: Chem. 7(10) 2145 (1997).

(20) M.R. Caira, A. Horne, L.R. Nassimbeni and F. Toda J. Mater: Chem. 8(6) 1481 (1998).

(21) D.R. Bond and F. Toda Acta Cryst. C47 348 (1991).

(22) S. Apel, M. Lennartz, L.R. Nassimbeni and E. Weber Chem. Eur. J. 8(16) 3678 (2002).

(23) N. Hayashi, K. Kuruma, Y. Mazaki, T. Imakubo and K. Kobayashi J. Am. Chem. Soc. 120, 3799 (1998). 


\section{CHAPTER IX}

(Supplemental Material)

\section{Tris (5-acetyl-3-thienyl) methane inclusion compounds with alkyl halide guests. Competitive inclusion experiments}


Table IX(S)-1: Volumes of each guest solvent $( \pm 1 \mu \mathrm{L})$ used to prepare the equimolar ratio of two guests from which TATM was recrystallized (total volume $=1 \mathrm{~mL}$ )

A) methylene halides:

methylene chloride methylene bromide

$477 \mu \mathrm{L}$

$523 \mu \mathrm{L}$

methylene chloride methylene iodide

$443 \mu \mathrm{L}$

$557 \mu \mathrm{L}$

methylene bromide

methylene iodide

$466 \mu \mathrm{L}$

$534 \mu \mathrm{L}$

B) 1,3-dihalopropanes

1,3-dichloropropane

$483 \mu \mathrm{L}$

1,3-dibromopropane

$517 \mu \mathrm{L}$

1,3-dichloropropane

$453 \mu \mathrm{L}$

1,3-diiodopropane

$547 \mu \mathrm{L}$

1,3-dibromopropane

1,3-diiodopropane

$469 \mu \mathrm{L}$

$531 \mu \mathrm{L}$

C) 2-halobutanes

2-chlorobutane

$493 \mu \mathrm{L}$

2-bromobutane

$507 \mu \mathrm{L}$

2-chlorobutane

$479 \mu \mathrm{L}$

2-iodobutane

$521 \mu \mathrm{L}$

2-bromobutane

$487 \mu \mathrm{L}$

2-iodobutane

$513 \mu \mathrm{L}$ 
Table IX(S)-I (continued):

D) cyclohexyl halides

$\begin{array}{ll}\text { cyclohexyl chloride } & \text { cyclohexyl bromide } \\ 491 \mu \mathrm{L} & 509 \mu \mathrm{L} \\ & \\ \text { cyclohexyl chloride } & \begin{array}{l}\text { cyclohexyl iodide } \\ 478 \mu \mathrm{L}\end{array} \\ & 522 \mu \mathrm{L} \\ \text { cyclohexyl bromide } & \text { cyclohexyl iodide } \\ 487 \mu \mathrm{L} & 513 \mu \mathrm{L}\end{array}$

E) monohalobenzenes

$\begin{array}{ll}\begin{array}{ll}\text { fluorobenzene } \\ 480 \mu \mathrm{L}\end{array} & \begin{array}{l}\text { chlorobenzene } \\ 520 \mu \mathrm{L}\end{array} \\ \text { fluorobenzene } & \text { bromobenzene } \\ 471 \mu \mathrm{L} & 529 \mu \mathrm{L} \\ \text { fluorobenzene } & \text { iodobenzene } \\ 456 \mu \mathrm{L} & 544 \mu \mathrm{L} \\ \text { chlorobenzene } & \text { bromobenzene } \\ 491 \mu \mathrm{L} & 509 \mu \mathrm{L} \\ \text { chlorobenzene } & \text { iodobenzene } \\ 476 \mu \mathrm{L} & 524 \mu \mathrm{L} \\ & \text { iodobenzene } \\ \text { bromobenzene } & 515 \mu \mathrm{L} \\ 485 \mu \mathrm{L} & \end{array}$


Table IX(S)-2: Integrals from ${ }^{1} \mathrm{H}$ NMR (alkyl halides), along with peak heights from ${ }^{13} \mathrm{C}$ NMR (monohalobenzenes). The integrals are estimated to be accurate within $5 \%$, while the peak heights are estimated to be accurate to within $10 \%$.

A) methylene halides $\left(\mathrm{C}_{1}\right)$ :

$\begin{array}{ll}\text { methylene chloride } & \text { methylene bromide } \\ 1.00 & 1.38 \\ 1.00 & 1.91\end{array}$

methylene chloride methylene iodide

8.50

1.00

4.98

1.00

methylene bromide

methylene iodide

6.10

1.00

4.48

1.00

B) 1,3-dihalopropanes $\left(C_{1,3}\right)$ :

$\begin{array}{ll}\text { 1,3-dichloropropane } & \text { 1,3-dibromopropane } \\ 1.00 & 1.38 \\ 1.00 & 1.05\end{array}$

1,3-dichloropropane

1,3-diiodopropane

2.35

1.00

3.02

1.00

1,3-dibromopropane

1.31

1,3-diiodopropane

1.96

1.00

1.00 
Table IX(S)-2 (continued):

C) 2-halobutanes $\left(\mathrm{C}_{2}\right)$ :

2-chlorobutane

2-bromobutane

1.00

1.05

1.85

1.00

2-chlorobutane

2-iodobutane

1.33

1.00

1.49

1.00

2-bromobutane

2-iodobutane

1.33

1.00

1.82

1.00

D) cyclohexyl halides $\left(C_{1}\right)$

cyclohexyl chloride

3.22

1.25

cyclohexyl bromide

1.00

1.00

cyclohexyl chloride

9.53

2.45

cyclohexyl iodide

1.00

1.00

cyclohexyl bromide

2.18

cyclohexyl iodide

2.04

1.00

1.00 
Table IX(S)-2 (continued):

E) monohalobenzenes

$\begin{array}{lll} & \text { fluorobenzene } & \text { chlorobenzene } \\ \mathrm{C}_{4} & 0.45 \mathrm{~cm} & 1.20 \mathrm{~cm} \\ \mathrm{C}_{2.6} & 1.10 \mathrm{~cm} & 2.50 \mathrm{~cm} \\ \mathrm{C}_{3.5} & 1.10 \mathrm{~cm} & 2.30 \mathrm{~cm}\end{array}$

fluorobenzene bromobenzene

$\begin{array}{lll}\mathrm{C}_{4} & 0.40 \mathrm{~cm} & 1.30 \mathrm{~cm}\end{array}$

$\begin{array}{lll}\mathrm{C}_{2,6} & 1.70 \mathrm{~cm} & 2.60 \mathrm{~cm}\end{array}$

$\mathrm{C}_{3,5} \quad 1.40 \mathrm{~cm} \quad 2.20 \mathrm{~cm}$

$\begin{array}{lll} & \text { fluorobenzene } & \text { iodobenzene } \\ \mathrm{C}_{4} & 0.40 \mathrm{~cm} & 0.30 \mathrm{~cm} \\ \mathrm{C}_{2,6} & 0.95 \mathrm{~cm} & 0.75 \mathrm{~cm} \\ \mathrm{C}_{3.5} & 0.90 \mathrm{~cm} & 0.75 \mathrm{~cm}\end{array}$

chlorobenzene bromobenzene

$\mathrm{C}_{4} \quad 1.00 \mathrm{~cm} \quad 0.90 \mathrm{~cm}$

$\begin{array}{lll}\mathrm{C}_{2.6} & 2.20 \mathrm{~cm} & 2.10 \mathrm{~cm}\end{array}$

$\mathrm{C}_{3.5} \quad 2.10 \mathrm{~cm} \quad 2.15 \mathrm{~cm}$

$\begin{array}{lll} & \text { chlorobenzene } & \text { iodobenzene } \\ \mathrm{C}_{4} & 1.30 \mathrm{~cm} & 0.60 \mathrm{~cm} \\ \mathrm{C}_{2.6} & 2.60 \mathrm{~cm} & 1.20 \mathrm{~cm} \\ \mathrm{C}_{3.5} & 2.10 \mathrm{~cm} & 1.35 \mathrm{~cm} \\ & & \\ & & \text { bromobenzene } \\ \mathrm{C}_{4} & 3.30 \mathrm{~cm} & \text { iodobenzene } \\ \mathrm{C}_{2.6} & 6.90 \mathrm{~cm} & 1.50 \mathrm{~cm} \\ \mathrm{C}_{3.5} & 6.40 \mathrm{~cm} & 3.10 \mathrm{~cm} \\ & & 3.30 \mathrm{~cm}\end{array}$


Table IX(S)-3: Chemical shifts for ${ }^{1} \mathrm{H}$ solution NMR of alkyl halide guests $( \pm 0.01 \mathrm{ppm})$ and for ${ }^{13} \mathrm{C}$ solution NMR of monohalobenzene guests $( \pm 0.1 \mathrm{ppm})$.

A) methylene halides:

methylene chloride $\quad \delta_{\mathrm{H}}=5.32 \mathrm{ppm}$

methylene bromide $\delta_{\mathrm{H}}=4.95 \mathrm{ppm}$

methylene iodide $\quad \delta_{H}=3.86 \mathrm{ppm}$

B) 1,3-dihalopropanes

1,3-dichloropropane $\delta_{H}\left(C_{1,3}\right)=3.72 \mathrm{ppm}$

1,3-dibromopropane $\delta_{H}\left(C_{1,3}\right)=3.57 \mathrm{ppm}$

1,3-diiodopropane $\quad \delta_{H}\left(C_{1,3}\right)=3.29 \mathrm{ppm}$

C) 2-halobutanes

2-chlorobutane $\quad \delta_{\mathrm{H}}\left(\mathrm{C}_{2}\right)=3.98 \mathrm{ppm}$

2-bromobutane $\quad \delta_{H}\left(C_{2}\right)=4.12 \mathrm{ppm}$

2-iodobutane $\quad \delta_{\mathrm{H}}\left(\mathrm{C}_{2}\right)=4.17 \mathrm{ppm}$

D) cyclohexyl halides

cyclohexyl chloride $\delta_{H}\left(C_{1}\right)=4.01 \mathrm{ppm}$

cyclohexyl bromide $\delta_{\mathrm{H}}\left(\mathrm{C}_{1}\right)=4.20 \mathrm{ppm}$

cyclohexyl iodide $\quad \delta_{\mathrm{H}}\left(\mathrm{C}_{1}\right)=4.37 \mathrm{ppm}$

E) monohalobenzenes

$\begin{array}{ll}\text { fluorobenzene } & \delta_{C}\left(C_{4}\right)=124.2 \mathrm{ppm} \\ & \delta_{C}\left(C_{2.6}\right)=115.5 \mathrm{ppm} \\ \text { chlorobenzene } & \delta_{C}\left(C_{3.5}\right)=130.1 \mathrm{ppm} \\ & \delta_{C}\left(C_{4}\right)=126.6 \mathrm{ppm} \\ & \delta_{C}\left(C_{2.6}\right)=128.8 \mathrm{ppm} \\ \text { bromobenzene } & \delta_{C}\left(C_{3.5}\right)=129.7 \mathrm{ppm} \\ & \delta_{C}\left(C_{4}\right)=127.1 \mathrm{ppm} \\ & \delta_{C}\left(C_{2.6}\right)=131.5 \mathrm{ppm} \\ & \delta_{C}\left(C_{3.5}\right)=130.2 \mathrm{ppm} \\ & \delta_{C}\left(C_{4}\right)=127.6 \mathrm{ppm} \\ & \delta_{C}\left(C_{2,6}\right)=137.5 \mathrm{ppm} \\ & \delta_{C}\left(C_{3.5}\right)=130.3 \mathrm{ppm}\end{array}$




\section{CHAPTER X}

\section{Tris (5-acetyl-3-thienyl) methane inclusion compounds with alkyl halide guest families. \\ The nature of inclusion mixtures}

Abstract

Introduction

188

Experimental

191

Results

(A) 1,3-Dihalopropane / TATM pure phases 192

(1) Powder X-Ray Diffraction 192

(2) ${ }^{13} \mathrm{C}$ CP/MAS NMR 195

(3) Single Crystal Structures 198

(a) 1,3-difluoropropane/TATM 199

(b) 1.3-dichloropropane/TATM 201

(c) 1.3-dibromopropane/TATM 201

(B) Nature of Inclusion Mixtures 204

(4) Methylene Halide Guests 205

(5) 1,3-Dihalopropane Guests 207

(6) 2-Halobutane Guests 209

(7) Cyclohexyl Halide Guests 211

Discussion 213

(C) $\quad\left[\mathrm{N}\left(\mathrm{CH}_{3}\right)_{4} \cdot \mathrm{xG}\right]\left[\mathrm{Cd}_{3}(\mathrm{CN})_{7}\right] \quad 216$

(D) 1,1,2,2-tetraphenylethane-1,2-diol 217

(E) Cholic acid 218

Concluding remarks 221

References 223 


\begin{abstract}
In this work, we report crystal structures of tris (5-acetyl-3-thienyl) methane (TATM) inclusion compounds with 1,3-dihalopropane guests (1,3-difluoropropane, 1,3dichloropropane and 1,3-dibromopropane). Crystals of 1,3-difluoropropane / TATM form a triclinic $\mathrm{P} \overline{\mathrm{l}}$ structure, and the TATM host lattice is comprised of linear channels along the $a$ axis into which the guests reside. In contrast, 1,3-dichloropropane and 1,3dibromopropane form isostructural TATM inclusion compounds that crystallize in the monoclinic $\mathrm{C} 2 / \mathrm{c}$ space group. For both structures, the guests reside in zig - zag channels parallel to the $c$ axis, and are also characterized by infinitely $\pi-\pi$ stacked host thienyl rings. In addition, we used powder X-ray diffraction to characterize the nature of the inclusion mixtures for four alkyl halide / TATM guest families, including the 1,3dihalopropanes. We found that the presence of isostructuralism among the pure phases within each guest family was dependent on the size of the hydrocarbon backbone of the guest, smaller guests showing relatively less isostructuralism (e.g. methylene halides), with larger guest families displaying extensive isostructuralism (e.g. cyclohexyl halides). In addition to this, the TATM competitive inclusion experiments generally indicated the formation of new phases for binary mixtures of the 2-halobutane inclusions exclusively, while the PXRD patterns of the recrystallized mixtures were very similar to those of the respective mechanical mixtures for each of the other three TATM guest families, which may be related to the fact that the 2-halobutane guests are the only ones that are chiral. These competitive inclusion results allow us to contemplate on the nature of the crystallization process and molecular recognition as it occurs at the molecular level.
\end{abstract}




\section{INTRODUCTION}

Crystalline inclusion complexes, designated as clathrates, are usually composed of both host and guest species, and their supramolecular design has been investigated extensively [1]. On the other hand, there have been relatively few structural studies of threecomponent clathrates [2-8]. In the context of host-guest-guest clathrate chemistry, two guest compounds of similar molecular shape and size may be incorporated with random distribution in the host cavity to form a ternary clathrate [e.g. 9,10]. The X-ray crystallographic characterization of such three-component crystalline complexes has attracted little attention, since selective inclusion has been much more of a central problem than random incorporation [e.g. 11,12].

There has been growing interest in crystal engineering of nano-porous organic crystalline materials as an area of supramolecular chemistry [13-24]. Open host frameworks formed by self-assembly of host molecules often allow specific enclathration of a particular guest compound from guest mixtures. Selective guest recognition, including optical resolution of racemic mixures, has been well documented [25-39], and might be a promising separation method due to its high efficiency, saving of energy, production of less waste, and easy operation. However, screening for host-guest combinations with high specificity is challenging, due to difficulty in the prediction and control of crystal structures, and most examples have been discovered either by trial and error or by accident. Elucidation of molecular recognition from X-ray crystallographic studies may provide access to the design of selective enclathration, and might also offer insights into the nature of mixed crystals.

Recrystallization from mixtures of two guest compounds generally yields two types of inclusion phenomena: selective enclathration and non-selective enclathration. In the former case, the host-guest crystals formed by recrystallization selectively include only one of the two guests, while the other acts only as a solvent. The crystal structure is identical to that of the authentic sample prepared from the pure guest solvent (I in Figure $\mathrm{X}-1 \mathrm{a})$, and the molecular recognition is explained by differences in preference between the two forms. In non-selective enclathration, both guest compounds are included in the host cavities, and this is sub-categorized by the following two mechanisms. The first is the formation of mixtures of the host-guest crystals that each include one of the guest 
compounds selectively (II in Figure X-1a), while the other is a homogeneous inclusion crystal that randomly includes both guest compounds in the host cavities (III in Figure X1a). These two possibilities, however, are barely distinguishable because of the similarities of the host frameworks with slightly different guest compositions, so less attention has been paid to the elucidation of less efficient (non-selective) molecular recognition through the use of host-guest crystals [40-43].

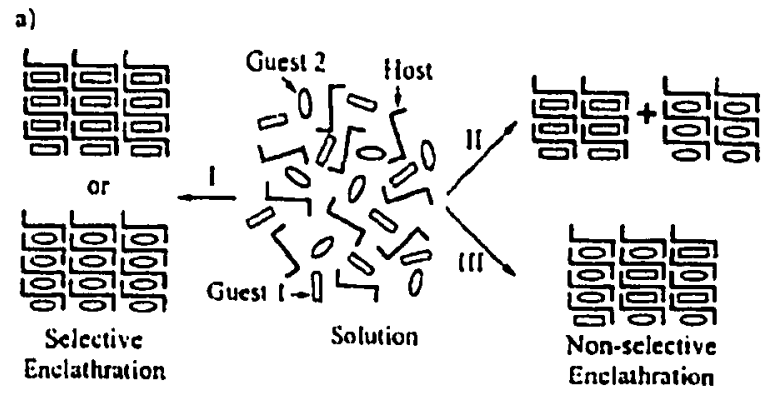

b)
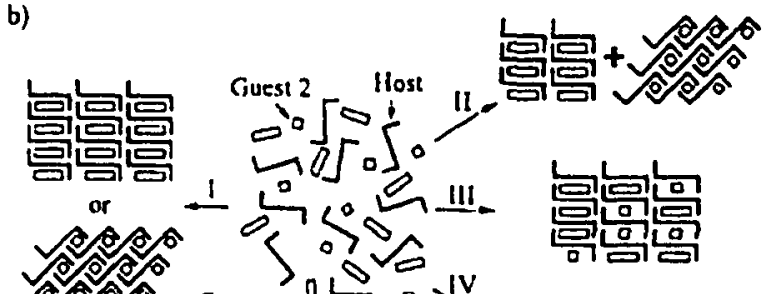

Solution

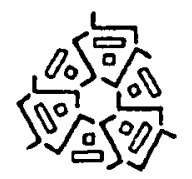

Selective

Enclathration
Figure X-1: Mechanisms of selective and unselective enclathration under competitive conditions: (a) the guest compounds are included in the same host framework (b) the guest compounds are included in different host frameworks [53].

More recently however, extensive structural studies of organic inclusion compounds have suggested that the nature of the host framework may be at least partially dependent upon the functional groups and steric dimensions of the included guest [44-52]. In other words, the host compounds offer many types of open host frameworks, and these vary in response to the guest components through "induced-fit" mechanisms. In such cases, inclusion phenomena of mixed guest compounds are classified into the following four possible types, when the guests are included in different host frameworks:

(1) One guest compound is selectively included, and the crystal structure is the same as that of an authentic crystal recrystallized from the pure guest component. The other component is not included at all, and acts only as a solvent (I in Figure X-lb). 
(2) Both guest compounds are included in the host cavities, and the crystals are mixtures of the individual inclusion crystals (II in Figure X-1b). Each guest compound is included in its respective host framework, identical to those crystallized from each pure guest compound.

(3) The host compound includes both guest compounds without any selectivity. The host framework is the same as that of the pure crystal recrystallized from only one of the two guests. The two guest compounds are statically disordered in the host cavities (III in Figure $\mathrm{X}-1 \mathrm{~b})$.

(4) Both of the guest compounds are included, and the host framework is completely different from those of the two inclusion crystals recrystallized from each of the two pure guests (IV in Figure $\mathrm{X}-\mathrm{lb}$ ).

The last three mechanisms (II, III and IV) provide non-selective enclathration of the two guest compounds. X-ray crystallographic studies offer possible explanations of the mechanisms of either high or low selectivity, and give the atomic coordinates of the host frameworks for comparison of stabilities between them. In spite of this, there have been few reports about the mechanisms of selective and non-selective enclathration in relation to the isomerization of the flexible host frameworks.

As an example, a recent publication [53] presented results on selective and nonselective enclathration of inclusion compounds of cholic acid (CA) with isomeric xylene mixtures. All three guests (ortho-, meta- and para-xylene) give lattice inclusion crystals with $\mathrm{CA}$, and crystallographic studies reveal that they are included in different open host frameworks. Ortho-xylene crystallizes with $\mathrm{CA}$ in two true polymorphic modifications, depending on the recrystallization temperature. Competitive recrystallization from mixtures of xylenes resulted in either selective enclathration or the formation of mixed crystals (non-selective enclathration). Namely, in the presence of an equimolar amount of ortho-xylene, CA selectively includes either meta-xylene or para-xylene in the host framework, the structures of which are identical to those obtained by crystallization of CA from pure meta-xylene or para-xylene, respectively. The lower affinity of orthoxylene was explained in terms of weaker host-guest intermolecular interactions, as judged by the ratio of the volume of guest compound compared to that of the host cavity 
$\left(P C_{\text {cavity }}\right)$. On the other hand, mixtures of meta-xylene and para-xylene yield inclusion crystals that accommodate both guests. All of these mixed crystals have the same framework as that of $\mathrm{CA} /$ meta-xylene, and the guests are statically disordered in the host cavities. The proportions of meta- and para-xylene components in the single crystals are similar to those in both the bulk products and the original recrystallization mixtures, which suggests that CA forms mixed crystals with $m$ - and $p$-xylene. The authors noted that this non-selective nature of crystallization could perhaps be attributed to the similar stabilities of $\mathrm{CA} / m$-xylene and $\mathrm{CA} / p$-xylene, due to the similar extent of space filling of the $C A$ host cavities by both guests $\left(P C_{\text {cavity }}\right)$.

In Part I of this work (chapter IX) we performed competitive inclusion experiments of TATM inclusion mixtures with five alkyl halide guest families, as depicted in Figure VIII-1 (p. 121). We found that alkyl iodides are the universally least preferred guest, while alkyl chlorides and alkyl bromides are more preferred. We reconciled these observations by proposing a balance of forces that result from TATM void stretching for large guests (that may destabilize alkyl iodides) and less than optimal van der Waals interactions between host and guest (that may destabilize alkyl chlorides). The alkyl bromides may bring about a balance of these two forces that result in their generally being the most preferred guests. In Part II of this work (the present chapter), we wish to characterize the nature of the products of TATM crystallized from a mixture of two guests (both guests from any one of the guest families pictured in Figure VIII-1). Specifically, is a new phase formed, when compared to a mechanical mixture of the two pure phase TATM inclusion compounds? These compounds are investigated with the PXRD bulk characterization technique. First, however, we report the crystal structures of pure phase TATM inclusion compounds with 1,3-dihalopropane guests (1,3difluoropropane, 1,3-dichloropropane and 1,3-dibromopropane)

\section{EXPERIMENTAL}

Samples for single crystal X-ray diffraction (1,3-difluoropropane, 1,3-dichloropropane and 1,3-dibromopropane guests) were prepared by dissolving $\sim 300 \mathrm{mg}$ TATM in about 2 $-3 \mathrm{~mL}$ of guest solvent by heating, then allowing the covered samples to cool to room 
temperature on their own. After allowing sufficient time ( $1-2$ days) for the crystals to attain a suitable size, the crystals were harvested and subjected to X-ray analysis.

Samples for powder $\mathrm{X}$-ray diffraction and ${ }^{13} \mathrm{C} \mathrm{CP} / \mathrm{MAS}$ NMR analysis (1,3dihalopropane pure phases, alkyl halide mechanical mixtures and alkyl halide recrystallized mixtures) were prepared as follows: The 1,3-dihalopropane pure phase samples were prepared by carefully grinding the crystals used for single crystal X-ray analysis. Alkyl halide mechanical mixtures were prepared by mixing approximately equal amounts of two of the pure powdered solid TATM inclusion compounds in a 10 $\mathrm{mL}$ vial with a spatula. Alkyl halide recrystallized mixtures were prepared by dissolving 500 mg TATM in an equimolar mixture of two guests by heating (in a covered vial), followed by stirring on a magnetic stirrer for at least two weeks. Following this, the mixtures were filtered under vacuum and then dried overnight, before being subjected to powder X-ray diffraction analysis.

\section{RESULTS}

(A) 1,3-Dihalopropane / TATM pure phases

(1) Powder X-ray diffraction of 1,3-dihalopropane / TATM pure phases:

In this section, we characterize the pure phase TATM inclusion compounds with 1,3dihalopropane guests, namely 1,3-difluoropropane, 1,3-dichloropropane and 1,3dibromopropane (see Figure X-2), using single crystal and powder X-ray diffraction, in addition to solid state ${ }^{13} \mathrm{C} C P / M A S ~ N M R$.
A) 1,3-difluoropropane $\mathrm{F}-\mathrm{CH}_{2}-\mathrm{CH}_{2}-\mathrm{CH}_{2}-\mathrm{F}$

B) 1,3-dichloropropane<smiles>ClCCCCl</smiles>

C) 1,3-dibromopropane<smiles>BrCCCBr</smiles>

Figure X-2: The structure of the three 1,3-dihalopropane compounds focused on as pure phase TATM inclusion compounds. 
The powder X-ray diffraction patterns for the three pure phase 1,3-dihalopropane / TATM inclusion compounds appear in Figure X-3. In the top row of the figure appears the calculated powder $\mathrm{X}$-ray diffraction patterns based on the room temperature single crystal unit cell determination. In the bottom row appears the experimental powder X-ray diffraction patterns obtained at ambient temperature by grinding up the crystals used for single crystal X-ray diffraction analysis. For all three inclusion compounds, there is excellent agreement between the calculated and experimental powder $\mathrm{X}$-ray diffraction patterns. This suggests that the crystals used for single crystal $\mathrm{X}$-ray analysis were representative of the bulk product.

A: 1,3-difluoropropane / TATM
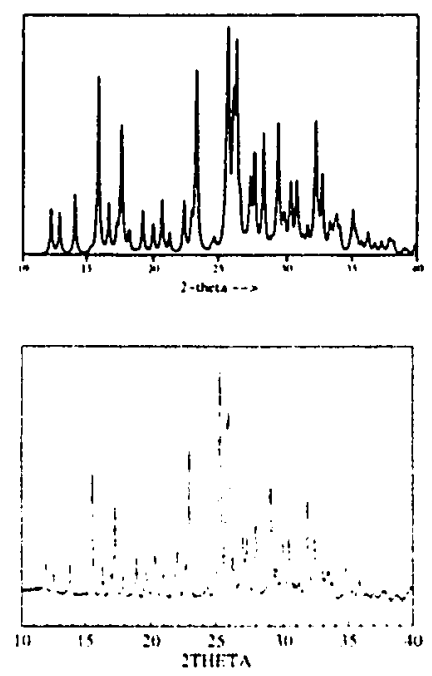

B: 1,3-dichloropropane / 'TA'TM
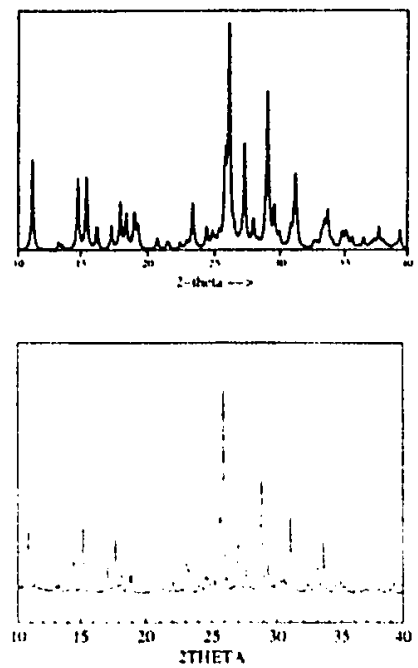

C: 1,3-dibromopropane / TATM
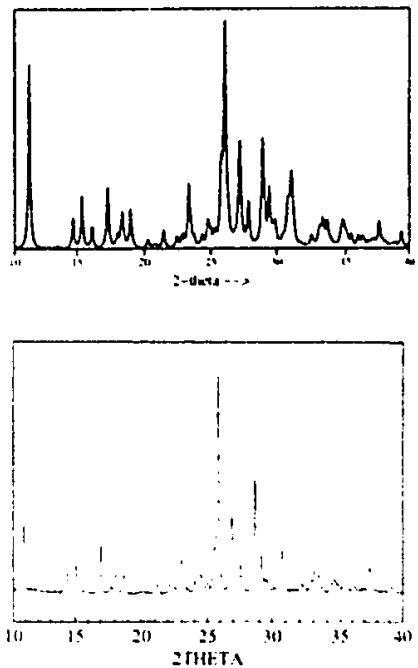

Figure X-3: Powder X-ray diffraction patterns for the three pure phase 1,3dihalopropane/TATM inclusion compounds. Calculated powder X-ray diffraction patterns, based on the single crystal unit cell determination at room temperature, appear in the top row. Experimental powder X-ray diffraction patterns, obtained at room temperature, appear in the bottom row.

We measured the single crystal unit cell parameters at room temperature, from which the calculated patterns of Figure X-3 were obtained (top row). Then we fit the peak positions of these calculated diffraction patterns to the peak positions of the experimental room temperature powder diffraction patterns (also in Figure X-3 - bottom row), and subsequently fit the two data sets for each inclusion compound by a least squares routine that minimized the sum of squares of the differences between observed and calculated $2 \theta$ peak positions, using the unit cell parameters as variables. The unit cell parameters for 
each of the three 1,3-dihalopropane / TATM inclusion compounds, as determined from single crystal analysis and powder X-ray diffraction, appear in Table X-1, and one sees from this table excellent agreement between the unit cell parameters determined by these two techniques. The unit cell dimensions $(a, b, c)$ and volume $(V)$ are slightly (but consistently) smaller for the powder X-ray diffraction determination, compared to the single crystal determination. This is perhaps because the powder X-ray diffraction stage is exposed to cooling water, and its temperature is significantly below room temperature (e.g. $5^{\circ} \mathrm{C}$ ) when compared to the single crystal. The PXRD stage is generally cool to the touch, and particularly in the winter, which is when these patterns were acquired. Thus we believe that this difference in temperature leads to thermal contraction of the unit cell for the PXRD determination.

The three experimental powder X-ray diffraction patterns of Figure X-3 (bottom row) suggest that 1,3-dichloropropane (Figure X-3B) and 1,3-dibromopropane (Figure $\mathrm{X}$ 3C) form isostructural TATM inclusion compounds, as their respective powder X-ray diffraction patterns are very similar in appearance. This agrees with the determination of the unit cell parameters for these two inclusion compounds at both room temperature and $173 \mathrm{~K}$, as measured by single crystal X-ray diffraction analysis. In contrast, 1,3difluoropropane / TATM (Figure X-3A) has both a unique powder X-ray diffraction pattern and unique unit cell parameters (see Table X-1). In supplemental material, we have tabulated experimental and calculated PXRD $2 \theta$ values, $l i k l$ lattice planes for each reflection and relative percent signal intensities for all three of these pure phase TATM inclusion compounds.

Table X-1: Room temperature unit cell parameters for the three 1,3-dihalopropane / TATM inclusion compounds, determined from single crystal (esd in brackets) and powder X-ray diffraction (within $0.003 \%, 0.009 \%$ and $1.7 \%$ for unit cell dimensions, angles and volume, respectively)

\begin{tabular}{lllllll}
\multicolumn{2}{c}{ 1,3-diffuoropropane } & \multicolumn{2}{c}{ 1,3-dichloropropane } & \multicolumn{2}{c}{ 1,3-dibromopropane } \\
& SC & POW & SC & POW & SC & POW \\
\hline$a(\AA)$ & $8.411(2)$ & 8.4105 & $17.680(2)$ & 17.623 & $17.748(5)$ & 17.730 \\
$b(\AA)$ & $10.461(3)$ & 10.452 & $18.445(3)$ & 18.424 & $18.438(5)$ & 18.407 \\
$c(\AA)$ & $12.812(3)$ & 12.795 & $15.220(2)$ & 15.165 & $15.274(4)$ & 15.258 \\
$\alpha\left({ }^{\circ}\right)$ & $95.64(1)$ & 95.74 & 90 & 90 & 90 & 90 \\
$\beta\left({ }^{\circ}\right)$ & $110.26(1)$ & 110.41 & $118.71(1)$ & 118.83 & $118.80(1)$ & 118.90 \\
$\gamma\left(\left(^{\circ}\right)\right.$ & $95.73(1)$ & 95.78 & 90 & 90 & 90 & 90 \\
$V\left(\AA^{3}\right)$ & $1041.7(4)$ & 1037.7 & $4353.5(10)$ & 4313.5 & $4379.9(19)$ & 4359.4 \\
\hline
\end{tabular}


(2) ${ }^{13} \mathrm{C}$ CP/MAS NMR of 1,3-dihalopropane / TATM pure phases

The ${ }^{13} \mathrm{C}$ CP/MAS solid-state NMR spectra for the three pure phase 1,3-dihalopropane / TATM inclusion compounds appear in Figures $\mathrm{X}-4 \mathrm{~A}$ to $\mathrm{X}-4 \mathrm{C}$. Spinning sidebands are indicated by asterisks. Two conclusions reached on the basis of single crystal $X$-ray diffraction (see further down) and powder X-ray diffraction (see above) experiments are confirmed here. First, as mentioned in the introduction, the ${ }^{13} \mathrm{C} C \mathrm{CP} / \mathrm{MAS}$ NMR spectrum allows one to measure the contents of the crystallographic asymmetric unit. The NMR signals from both the carbonyl carbons of the host at about $192 \mathrm{ppm}$ and the methyl carbons of the host at about $27 \mathrm{ppm}$ each have a multiplicity of three for all three inclusion compounds. Since there are three carbonyl carbons and three methyl carbons in one TATM molecule, this conclusively shows that the asymmetric units of each of the three 1,3-dihalopropane / TATM inclusion compounds each contain only one host molecule, in agreement with their respective single crystal structures (see below). Furthermore, the ${ }^{13} \mathrm{C}$ CP/MAS NMR spectra for both 1,3-dichloropropane / TATM (Figure X-4B) and 1,3-dibromopropane / TATM (Figure X-4C) are almost exactly identical in appearance, as far as the host splitting patterns are concerned, indicating that they are isostructural. In contrast, 1,3-difluoropropane / TATM (Figure X-4A) shows a

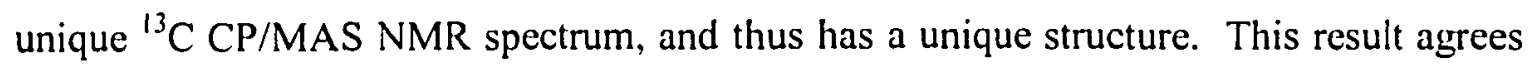
with the conclusions obtained from both single crystal and powder $\mathrm{X}$-ray diffraction

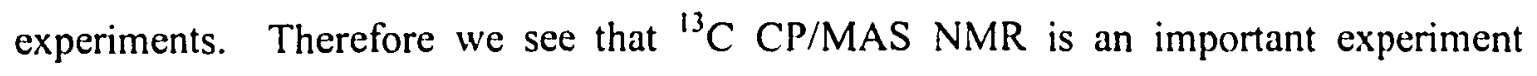
providing information that is complementary in nature to the techniques of single crystal

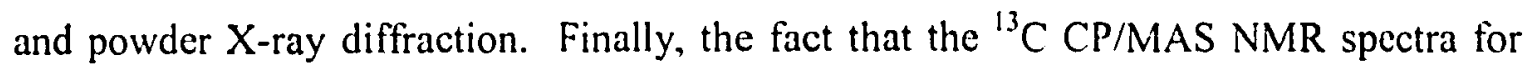
1,3-dichloropropane / TATM (Figure X-4B) and 1,3-dibromopropane / TATM (Figure X4C) are practically identical, as far as the TATM host splitting patterns are concerned, while their powder $\mathrm{X}$-ray diffraction patterns (Figures $\mathrm{X}-3 \mathrm{~B}$ and $\mathrm{X}-3 \mathrm{C}$ ) show some slight differences in appearance, suggests that powder $X$-ray diffraction is very sensitive to small differences in structure (such as the considerable difference in guest electron

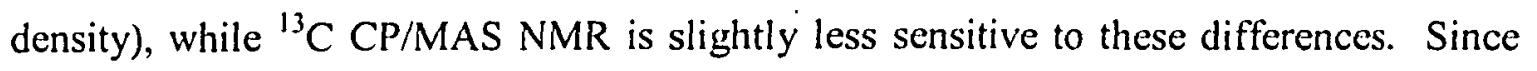
most of the intense peaks in the ${ }^{13} \mathrm{C}$ CP/MAS NMR spectrum of most TATM inclusion compounds generally come from the host, this suggests that the ${ }^{13} \mathrm{C} C P / M A S ~ N M R$ spectrum may be more sensitive to the packing motif of the host lattice, while powder X- 
ray diffraction may be more sensitive to a combination of both the packing motif of the host lattice and the electron density of the guest. In other words, PXRD is more sensitive to small differences in structure between two isostructural TATM inclusion compounds, while ${ }^{13} \mathrm{C} C P / M A S ~ N M R$ is less sensitive to these differences.

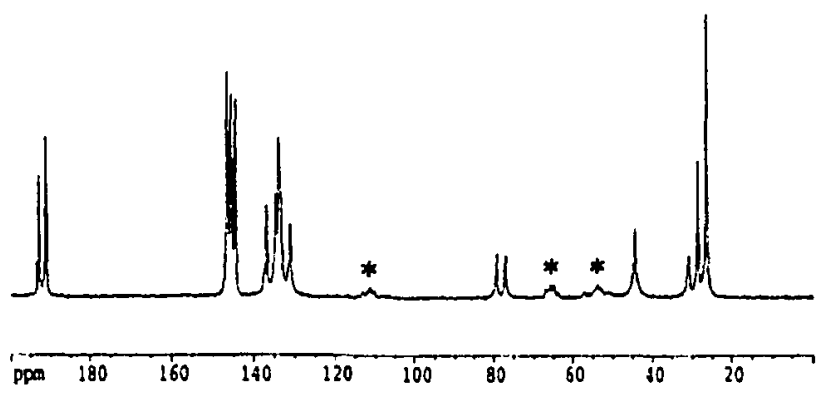

A: 1,3-difluoropropane / TATM

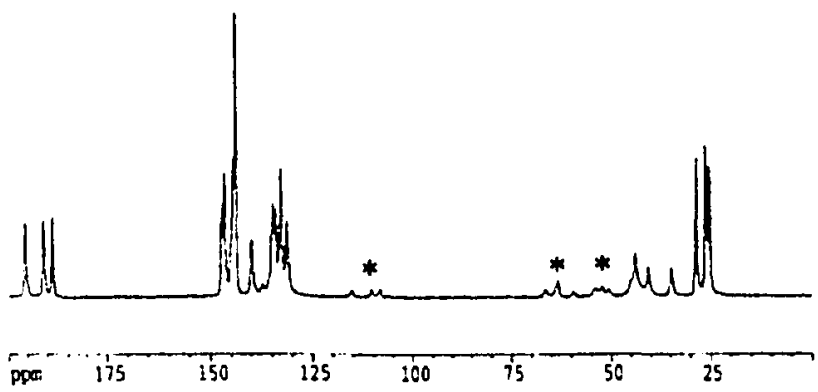

B: 1,3-dichloropropane / TATM

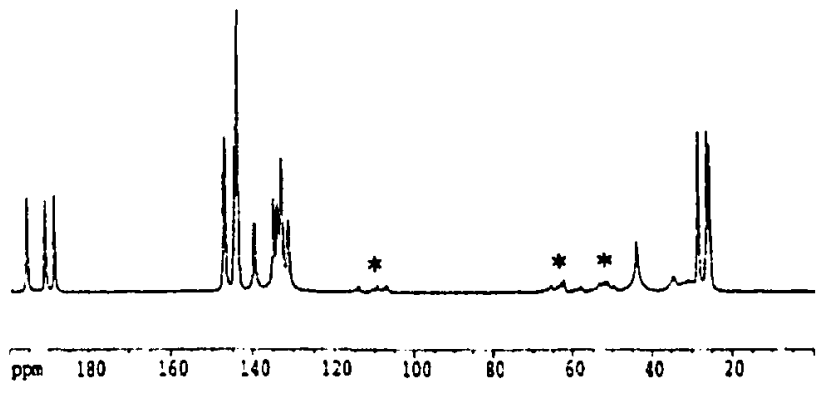

C: 1,3-dibromopropane / TATM

Figure X-4: $\quad{ }^{13} \mathrm{C} \mathrm{CP} / \mathrm{MAS}$ solid-state powder NMR spectra for the three pure phase 1,3dihalopropane/TATM inclusion compounds. Spinning sidebands are marked by asterisks 
Table X-2: Crystal data, data collection and final refinement parameters for TATM inclusion compounds with the three 1,3-dihalopropane guests.

\section{Crystal Data}

Guest

Molecular formula

Molecular weight (amu)

$T(\mathrm{~K})$

Crystal system

Space group

$a(\AA)$

$b(\AA)$

$c(\AA)$

$\alpha\left(^{\circ}\right)$

$\beta\left({ }^{\circ}\right)$

$\gamma\left({ }^{\circ}\right)$

$V\left(\AA^{3}\right)$

$Z$

$d_{\text {calc }}\left(\mathrm{g} \mathrm{cm}^{-3}\right)$

$d_{\text {meas }}\left(\mathrm{g} \mathrm{cm}^{-3}\right)$

$F(000)$

$\mu(\mathrm{MoK} \alpha)\left(\mathrm{mm}^{-1}\right)$

Wavelength $(\AA)$

$2 \theta$ range $\left(^{\circ}\right)$

\section{Data Collection}

Crystal size (mm)

Index ranges

Reflections collected

Independent reflections

$R$ (int)

Completeness to $2 \theta_{\max }$

Scan mode

Absorption correction

Maximum transmission

Minimum transmission

1,3-difluoropropane
$2 \mathrm{C}_{19} \mathrm{H}_{10} \mathrm{O}_{3} \mathrm{~S}_{3}: \mathrm{C}_{3} \mathrm{H}_{6} \mathrm{~F}_{2}$
857.08
173
triclinic
$\mathrm{P} \overline{\mathrm{l}}$

$8.203(2)$

$10.599(3)$

$12.547(3)$

96.334(5)

109.040(5)

95.493(5)

$1014.8(4)$

1

1.402

1.338

446

0.393

0.71073

$1.74<2 \theta<29.69$

$\begin{array}{ll}\text { 1,3-dichloropropane } & \text { 1,3-dibromopropane } \\ 2 \mathrm{C}_{10} \mathrm{H}_{16} \mathrm{O}_{3} \mathrm{~S}_{3} \quad \mathrm{CC}_{3} \mathrm{H}_{6} \mathrm{Cl}_{2} & 2 \mathrm{C}_{10} \mathrm{H}_{16} \mathrm{O}_{3} \mathrm{~S}_{3} \quad \mathrm{CC}_{3} \mathrm{H}_{6} \mathrm{Br}_{2} \\ 889.98 & 978.90 \\ 173 & 173 \\ \text { monoclinic } & \text { monoclinic } \\ \mathrm{C} 2 / \mathrm{c} & \mathrm{C} 2 / \mathrm{c} \\ 17.500(2) & 17.581(5) \\ 18.465(3) & 18.401(5) \\ 14.982(2) & 15.008(4) \\ 90 & 90 \\ 119.428(2) & 119.352(5) \\ 90 & 90 \\ 4216.6(10) & 4232.1(19) \\ 4 & 4 \\ 1.402 & 1.536 \\ 1.363 & 1.471 \\ 1848 & 1992 \\ 0.497 & 2.257 \\ 0.71073 & 0.71073 \\ 1.73<2 \theta<29.69 & 1.73<2 \theta<29.99\end{array}$

$0.30 \times 0.20 \times 0.20$

$-24 \leq h \leq 24$

$-25 \leq k \leq 25$

$-19 \leq l \leq 20$

26324

5965

0.0492

$99.5 \%$

omega

multiscan

0.862

0.742

$0.35 \times 0.25 \times 0.12$

$-24 \leq h \leq 24$

$-25 \leq k \leq 25$

$-20 \leq l \leq 20$

26338

5988

0.0631

$97.0 \%$

omega

multiscan

0.801

0.484

\section{Final Refinement}

Refinement method

Data/restraints/params

Goodness of fit on $F^{2}$

Final $R$ indices $[1>2 \sigma(I)]$

$$
R I
$$

$w R 2$

$R$ indices (all data)

$$
R 1
$$

$w R 2$

Highest peak (e $\AA^{-3}$ )

Deepest hole $\left(\mathrm{e} \AA^{-3}\right)$ full matrix least squares

$5646 / 38 / 315$

1.000

0.0541

0.1314

0.0861

0.1475

0.650

$-0.395$ full matrix least squares $5965 / 26 / 305$

1.015

0.0489

0.1151

0.0879

0.1286

0.507

$-0.344$ full matrix least squares $5988 / 38 / 311$

0.939

0.0530

0.1283

0.1013

0.1456

0.735

$-0.423$ 
(3) Single crystal structures of 1,3-dihalopropane / TATM pure phases

For each of the three 1,3-dihalopropane / TATM inclusion compounds, crystal data, data collection and final refinement parameters for the single crystal structures at $173 \mathrm{~K}$ appear in Table X-2. A schematic representation of each asymmetric unit, including thermal ellipsoids and the atomic numbering scheme, appear in Figures X-5 to X-7.

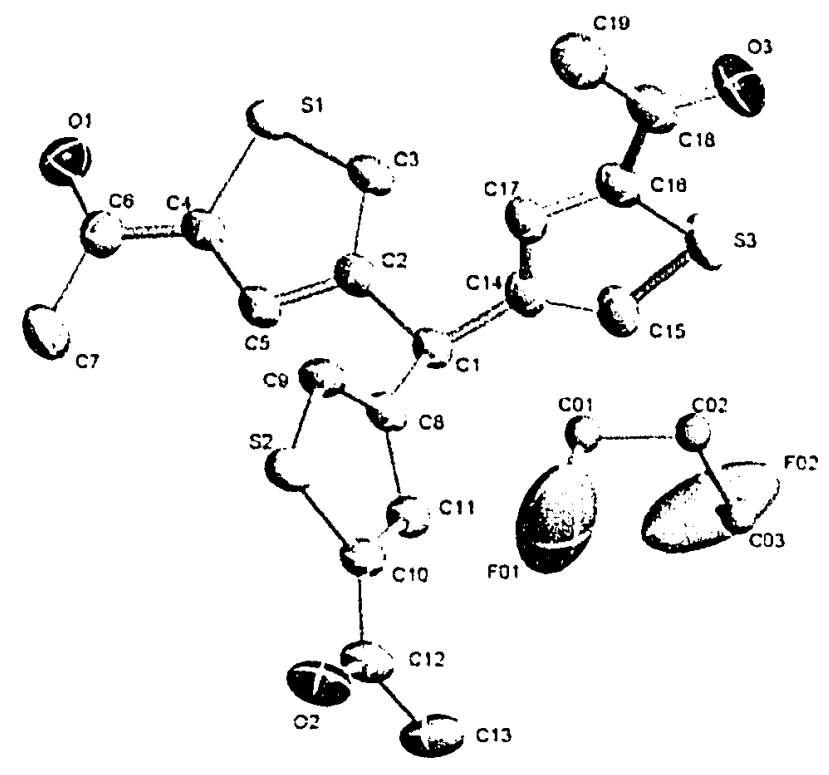

Figure X-5: A schematic depiction of the asymmetric unit of 1,3-difluoropropane/TATM at $173 \mathrm{~K}$, showing the atomic numbering scheme.

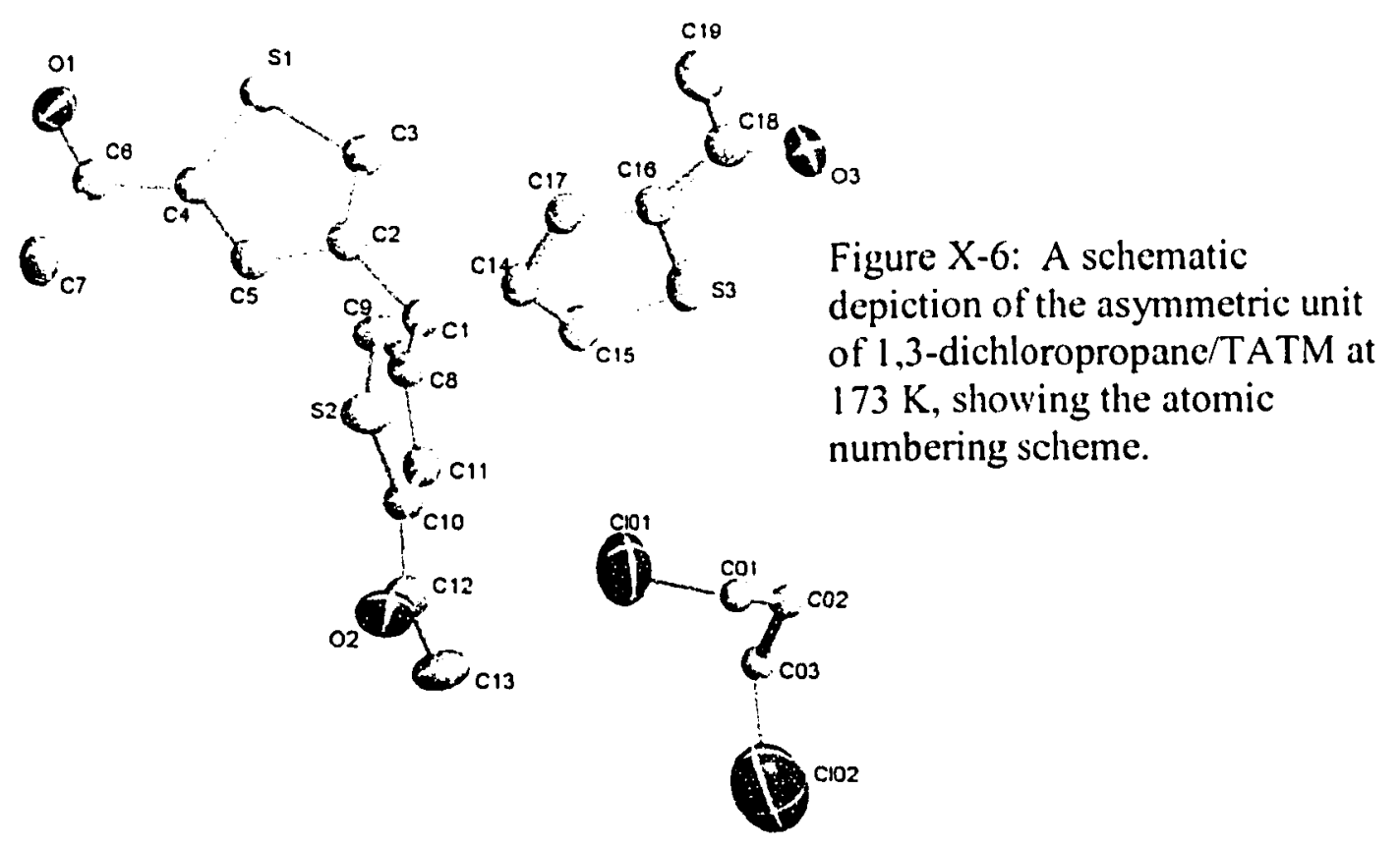




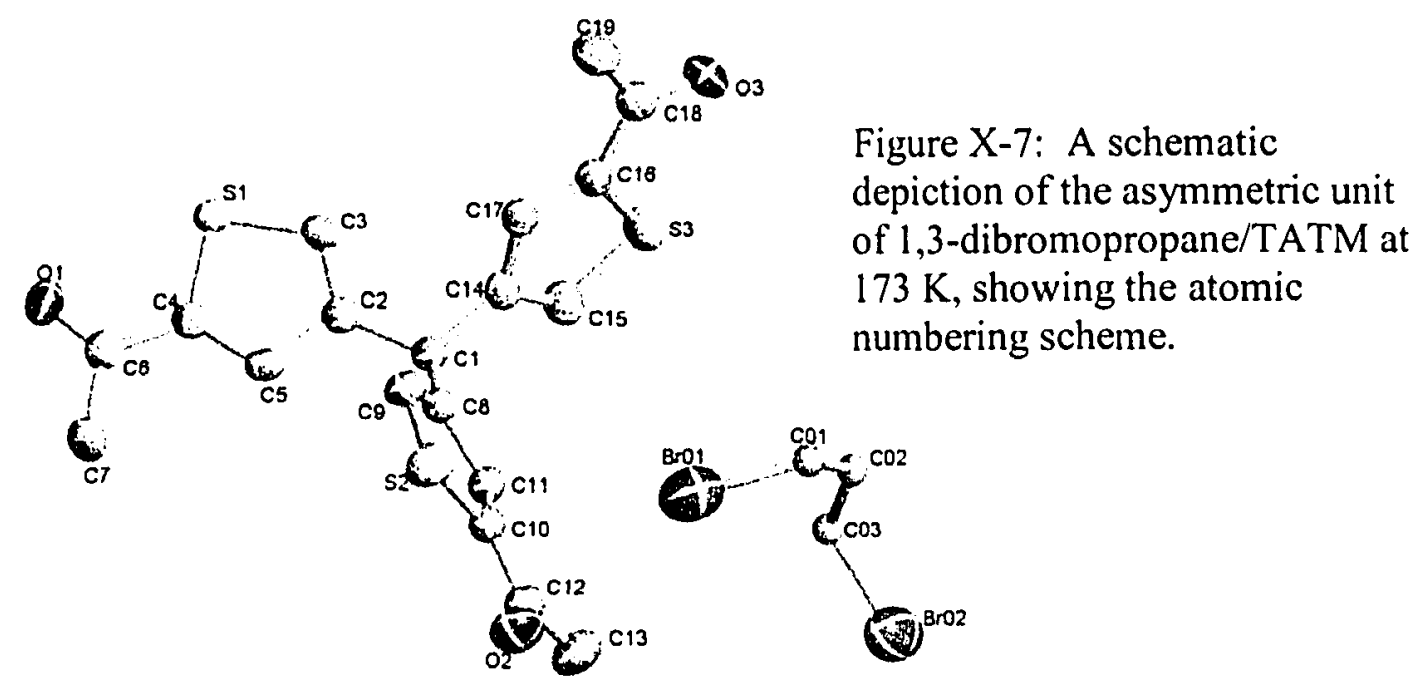

(a) 1,3-difluoropropane / TATM

In Figures X-8 and X-9 we present selected packing diagrams for the single crystal structure of 1,3-difluoropropane / TATM. The TATM host lattice forms channels along the $a$ axis in which the guests reside. The long axis of the 1,3-difluoropropane guest is aligned parallel to the channel axis. Figure X-8 is a view down the channel axis, in the $b c$ plane. Figure X-9 is a view in the ac plane, and the channel axis in this figure runs in the horizontal direction.

As can be seen in Figure $X-8$, the lattice is comprised of endless ribbons running in the [011] direction (along the $S W$ to $N E$ diagonal). Two of the three thienyl rings of each host molecule participate in the formation of the host channel, while the third thienyl ring links up with another non-participating thienyl ring of the host molecule in a neighboring unit cell. In a previous section concerning 1,2-dichloroethane / TATM (see chapter VII), these two thienyl rings are linked via $\pi-\pi$ stacking. In the present structure, however, the two non-participating thienyl rings are too far apart to form this type of bond, as their internuclear distance is approximately $4.3 \AA$, while their sum of van der Waals radii is about $3.5 \AA$. 


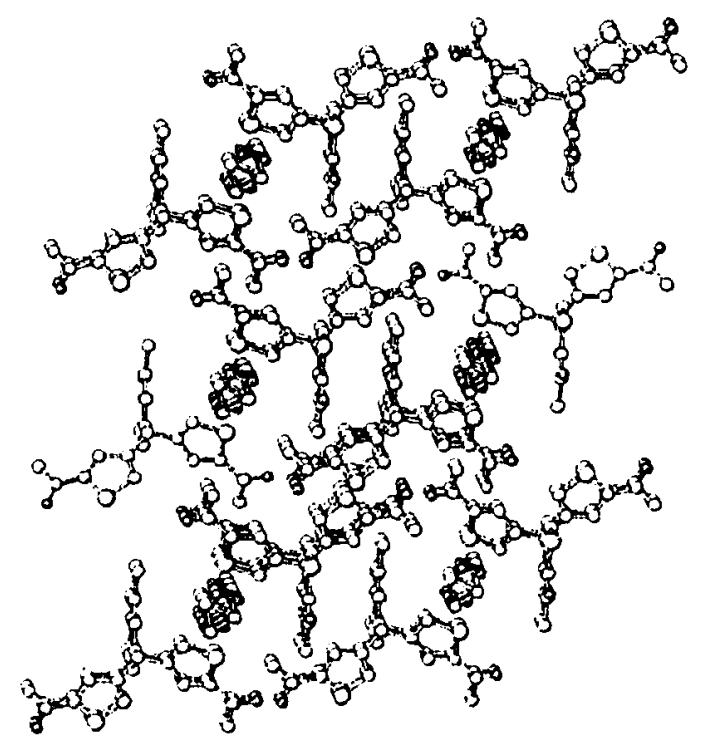

Figure X-8: Packing diagram for TATM with 1,3-difluoropropane guest in the $b c$ plane The view is down the channel axis ( $a$ direction)

In Figure X-9, the channel axis runs in the horizontal direction. In this figure, it appears that there are two host thienyl rings between guest sites that are blocking the channel. However, this figure is misleading, as one thienyl ring is above the channel, while the other thienyl ring is below the channel. However, these two thienyl rings, while not directly blocking the channel, give rise to a significant constriction of the channel between guest sites that keep the 1,3-difluoropropane guest molecules from translating from one guest site to the next.

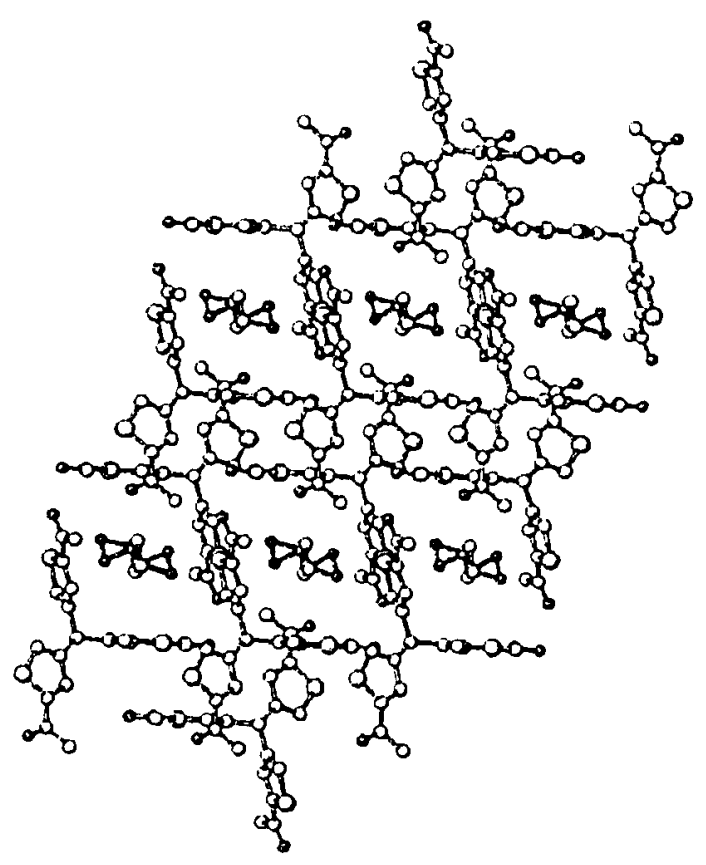

Figure X-9: Packing diagram for TATM with 1,3-difluoropropane guest in the $a c$ plane. The channel axis is in the horizontal direction in the plane of the figure. 
There are no strong hydrogen bonds present in this structure, as there are no hydrogen bond donors in the compound. However there are numerous aromatic $\mathrm{C}-\mathrm{H} \cdots \mathrm{O}$ interactions that serve to help stabilize the structure. Finally, there is considerable empty space in the host channel, perhaps because 1,3-difluoropropane is a much smaller guest than either 1,3-dichloropropane or 1,3-dibromopropane. This empty space is most easily seen in Figure X-9, and a packing diagram employing van der Waals radii (not shown) confirms this. This suggests that there may be extensive guest dynamics (the dipolar dephasing ${ }^{13} \mathrm{C} \mathrm{CP} / \mathrm{MAS}$ NMR spectrum is consistent with this), and that this guest may be more easily lost than those TATM inclusion compounds where there is little or no empty room in the host cavity.

\section{(b) 1,3-dichloropropane / TATM and (c) 1,3-dibromopropane / TATM}

As judged by their unit cell parameters and crystal packing, we have concluded that these two TATM inclusion compounds are virtually isostructural. Thus, we need only to present packing diagrams for one of the two inclusion compounds, and any conclusions reached with respect to packing for one inclusion compound may easily be applied to the other. We present packing diagrams for 1,3-dichloropropane / TATM in Figures X-10 to $\mathrm{X}-12$. The first view, Figure $\mathrm{X}-10$, is down the channel axis ( $c$ direction). The $a$ axis is in the plane of the figure and horizontal in direction. From this figure, one sees that, similarly to 1,2-dichloroethane B / TATM (see chapter VII), the lattice is made up of ribbons that run in the $a$ direction. The ribbons are laid side by side to form sheets in the $a b$ plane, and the sheets are stacked in the $c$ direction. Two of the three thienyl rings of each host molecule participate in the formation of the channel, while the third thienyl ring links via $\pi-\pi$ stacking to the non-participating thienyl ring of a neighboring TATM host molecule. We determined that the distance between the $\pi-\pi$ stacked thienyl rings is about $3.5 \AA$ to $3.6 \AA$, which is very comparable to the sum of van der Waals radii $(\sim 3.5 \AA)$. On the other hand, as previously mentioned, the distance between these thienyl rings for 1,3difluoropropane / TATM is larger, about $4.3 \AA$. In Figure X-10, one may see that each host cavity / guest molecule is surrounded in all four directions by a pair of $\pi-\pi$ stacked thienyl rings, while each pair of $\pi-\pi$ stacked thienyl rings is surrounded in all four directions by a host cavity / guest molecule. 


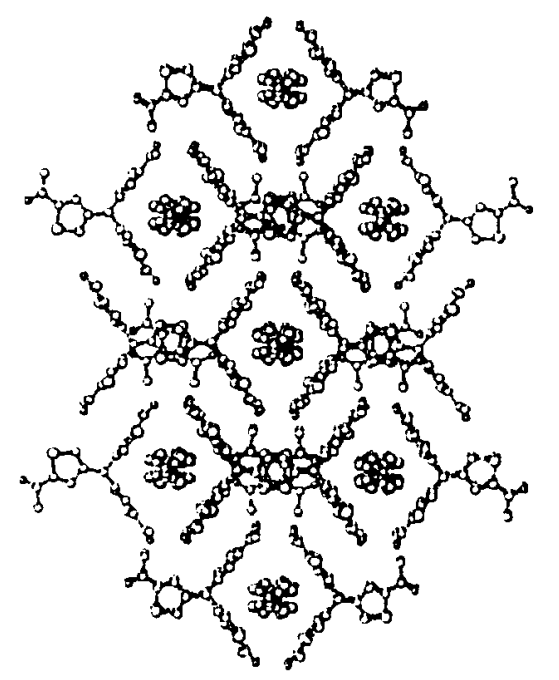

Figure X-10: Packing diagram for TATM with 1,3-dichloropropane guest in the $a b$ plane.

The view is down the channel axis ( $c$ direction). Ribbons run parallel to the $a$ (horizontal) direction.

In Figure X-11 is presented a view of 1,3-dichloropropane / TATM in the ac plane. One immediately notices that the $\pi-\pi$ stacked thienyl rings are infinitely stacked. The other conclusion that may be reached from this figure is that the channels are of a zig - zag type, since the chlorine atoms of the guest alternately point into and out of the plane of the figure as the channel is traversed. Finally, one may see, as was previously observed for 1,3-difluoropropane / TATM, that the long axis of the guest is aligned along the channel axis. In Figure $\mathrm{X}-12$ is presented the third packing diagram for 1,3dichloropropane / TATM, in the $b c$ plane. The channel axis ( $c$ direction) is in the plane of the figure and horizontal in direction. In this figure one again easily sees that the long axis of the guest is approximately co-linear with the channel axis, and also that the chlorine atoms of adjacent guests alternately point up and down, which gives rise to the zig - zag type channel. This suggests that every alternate layer (or sheet) is isostructural. In other words, even numbered layers are isostructural, and odd numbered layers are isostructural. Both of these inclusion compounds exist in the monoclinic $\mathrm{C} 2 / \mathrm{c}$ space group, neighboring layers are related by a $c$ glide plane, and ribbons in adjacent layers run in parallel directions. 

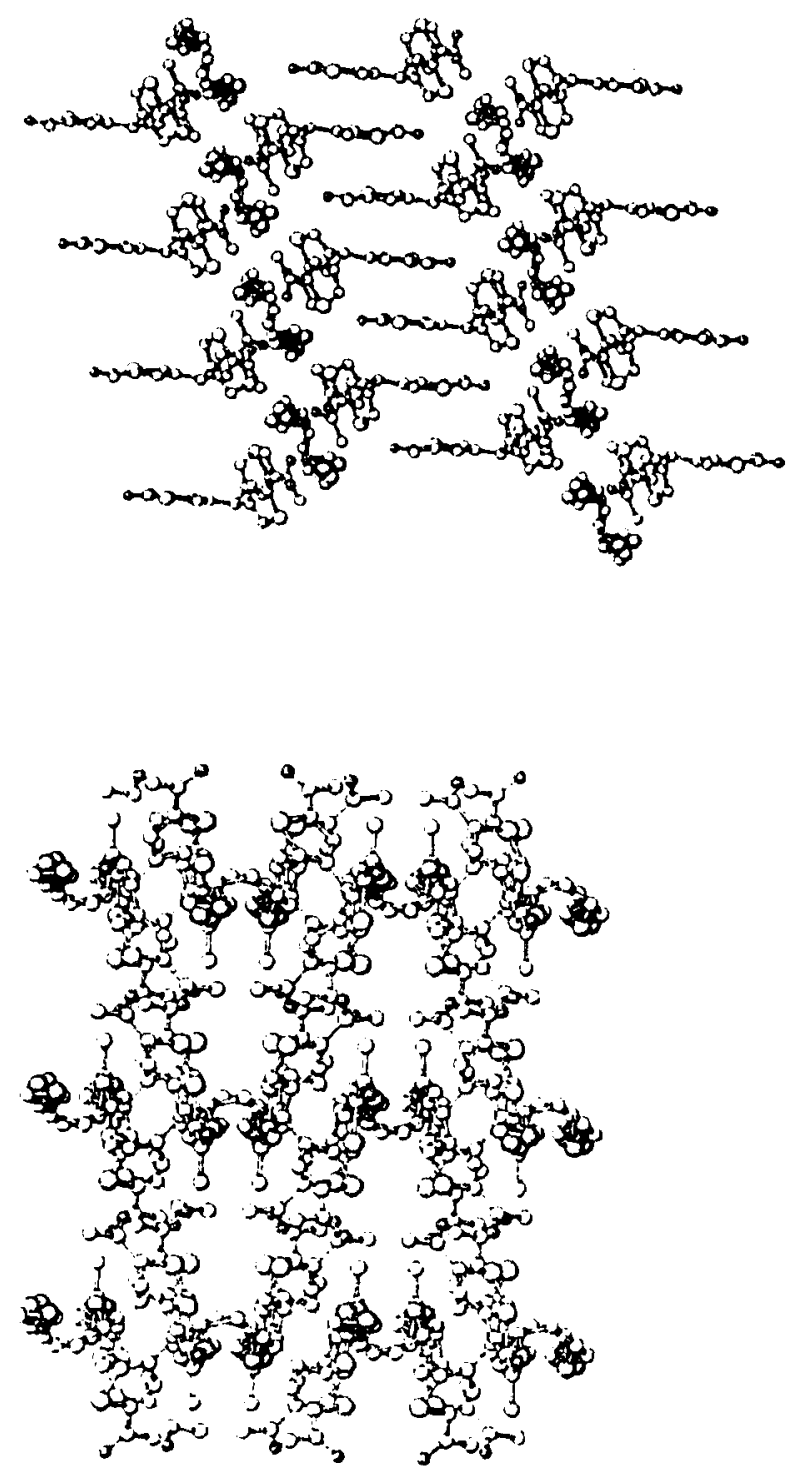

Figure X-11: Packing diagram for TATM with 1,3-dichloropropane guest in the ac plane. Notice the infinitely $\pi-\pi$ stacked host thienyl rings, and a zig - zag type channel.
Figure $\mathrm{X}-12$ : Packing diagram for TATM with 1,3-dichloropropane guest in the $b c$ plane. Notice how the chlorine atoms of the guest alternately point up and down.

Finally, we wish to comment on the extent of disorder of the three guests. The asymmetric unit for 1,3-difluoropropane / TATM appears in Figure X-5. In this figure one can see easily see very large thermal ellipsoids for the fluorine atoms of the guest. In Figure X-6 (1,3-dichloropropane / TATM), the thermal ellipsoids of the chlorine atoms of the guest are smaller. Finally, the thermal ellipsoids for the bromine atoms of the 1,3dibromopropane guest (Figure X-7) are smaller still. This suggests two things, that 1,3difluoropropane may be the most weakly held guest, and also that the 1,3difluoropropane guest may show the greatest degree of thermal motion, compared to the larger guests 1,3-dichloropropane and 1,3-dibromopropane. 
(B) Nature of inclusion mixtures

In this part of the study, we wish to investigate the properties of molecular recognition during crystallization of TATM from a mixture of two guests. We hope to do this by comparing the PXRD pattern of a mechanical mixture of two TATM pure phase inclusion compounds with the PXRD pattern of TATM recrystallized from an equimolar mixture of the same two liquid guests within any one of the four guest families depicted in Figure VIII-1 (p. 121). The specific experimental techniques for preparing the mechanical and recrystallized mixtures appear in the experimental section of this chapter. The advantage of using the PXRD technique is that it characterizes the bulk product, rather than a single selected crystal. We do note, however, that even with two weeks of stirring at room temperature, the "equilibrium product" may not have been obtained. Once something has crystallized, there must be a significant driving force in order to convert it. If there is little difference in free energy between one guest being included versus another, the driving force for conversion may be very low. In that case, whatever crystallizes first will predominate. This may result in a particularly large scatter in the results for duplicate experiments. On the other hand, if duplicate experiments agree well, it may indicate a larger driving force for conversion.

The results to come may allow us to contemplate on the nature of the crystallization process at the molecular level. For the mechanical mixtures, there are no mixed crystals. It would seem reasonable that if the recrystallized mixture PXRD pattern was at least superficially similar to its corresponding mechanical mixture PXRD pattern, then one might conclude that the recrystallized mixture contains few or no mixed crystals. If there were a significant proportion of mixed crystals, one might expect that a new phase would be formed, resulting in novel recrystallized mixture PXRD patterns.

First, for each guest family, we present the powder X-ray diffractograms and ${ }^{13} \mathrm{C}$ CP/MAS NMR spectra of the pure phases, and then move on to the PXRD patterns of the inclusion mixtures. 
(4) Methylene halides

The PXRD and solid-state ${ }^{13} \mathrm{C}$ NMR spectra of the three pure phase methylene halide / TATM inclusion compounds appear in Figure $\mathrm{X}-13$. This PXRD patterns tentatively suggest that none of the three the guests $\left(\mathrm{CH}_{2} \mathrm{Cl}_{2}, \mathrm{CH}_{2} \mathrm{Br}_{2}\right.$ and $\left.\mathrm{CH}_{2} \mathrm{I}_{2}\right)$ form isostructural TATM inclusion compounds. On the other hand, the ${ }^{13} \mathrm{C} \mathrm{CP} / \mathrm{MAS} N M R$ spectra do in fact suggest that $\mathrm{CH}_{2} \mathrm{Cl}_{2}$ and $\mathrm{CH}_{2} \mathrm{Br}_{2}$ form isostructural TATM inclusions. These conflicting results may be explained on the basis of the nature of these guests. The difference in electron density between each of these three guests is quite large, as there are two halogen atoms for each carbon atom. This alone would contribute significantly to the observed differences between the PXRD patterns of the $\mathrm{CH}_{2} \mathrm{Cl}_{2}$ and $\mathrm{CH}_{2} \mathrm{Br}_{2}$ inclusions, even if the host lattice packing is the same. Indeed, the host splitting patterns in the ${ }^{13} \mathrm{C}$ CP/MAS NMR spectra for the $\mathrm{CH}_{2} \mathrm{Cl}_{2}$ /TATM and $\mathrm{CH}_{2} \mathrm{Br}_{2}$ /TATM inclusion compounds are almost identical (see Figure $\mathrm{X}-13$ ). So perhaps the ${ }^{13} \mathrm{C} C P / M A S ~ N M R$ spectrum is more sensitive to the host packing motif (most of the NMR signals come from the host), while the PXRD pattern is more dependent on a combination of host packing and the guest electron density.

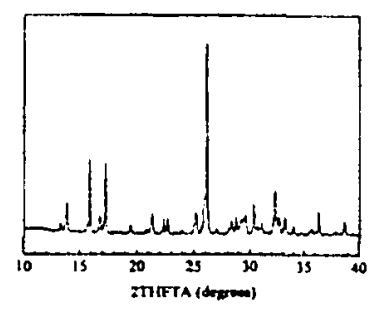

C: $\mathrm{CH}_{2} \mathrm{I}_{2}$
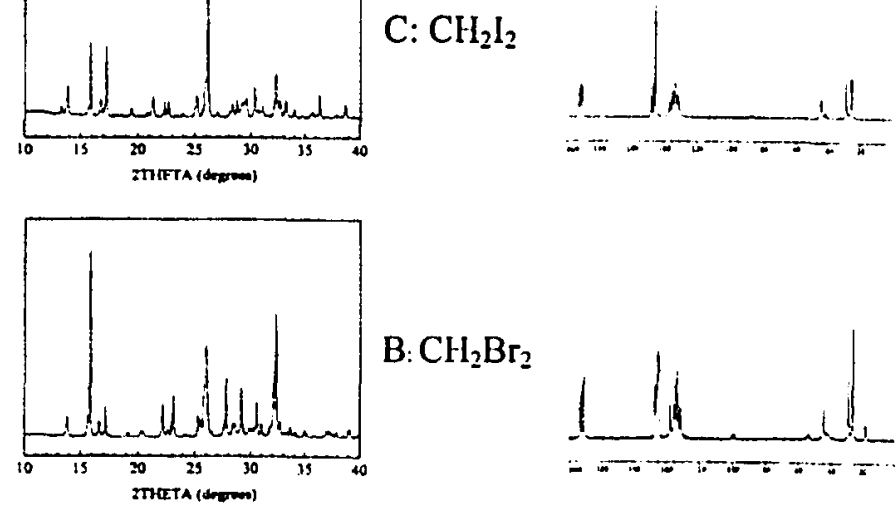

B. $\mathrm{CH}_{2} \mathrm{Br}_{2}$
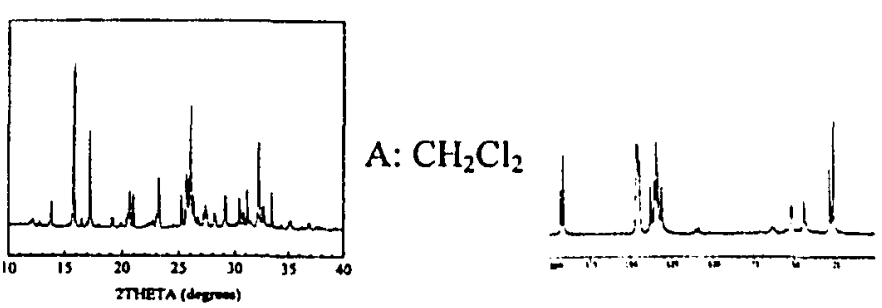

Figure $X-13$ : PXRD patterns (left) and ${ }^{13} \mathrm{C}$ CP/MAS NMR spectra (right) for the three methylene halide guests in TATM:

(A) methylene chloride / TATM

(B) methylene bromide / TATM

(C) methylene iodide / TATM 
The PXRD patterns for the three possible methylene halide / TATM guest mixture combinations appear in Figure $\mathrm{X}-14$. We also indicate the relative composition of the two guests in each recrystallized inclusion mixture, as determined from ${ }^{1} \mathrm{H}$ solution NMR. From Figure X-14, it seems apparent that all three recrystallized combination PXRD patterns match up (at least adequately) with their respective mechanical mixture PXRD patterns. This behaviour was expected (see the introductory section of this chapter) for the $\mathrm{CH}_{2} \mathrm{Cl}_{2} / \mathrm{CH}_{2} \mathrm{Br}_{2}$ combination, but was perhaps not expected for the other two combinations, for which the pure phase inclusions are not isostructural (see Figure $\mathrm{X}-13$ ). Interestingly, the recrystallized mixture $\mathrm{PXRD}$ patterns for the $\mathrm{CH}_{2} \mathrm{Cl}_{2} / \mathrm{CH}_{2} \mathrm{I}_{2}$ and $\mathrm{CH}_{2} \mathrm{Br}_{2} / \mathrm{CH}_{2} \mathrm{I}_{2}$ are very similar in appearance (see Figure $\mathrm{X}-14$ ). This suggests that no matter whether the accompanying guest for $\mathrm{CH}_{2} \mathrm{I}_{2}$ is either $\mathrm{CH}_{2} \mathrm{Cl}_{2}$ or $\mathrm{CH}_{2} \mathrm{Br}_{2}$, the same recrystallized mixture structure is formed, which perhaps is expected.

$\mathrm{CH}_{2} \mathrm{Cl}_{2} / \mathrm{CH}_{2} \mathrm{Br}_{2}$ $1.00 \mathrm{Cl}: 1.90 \mathrm{Br}$
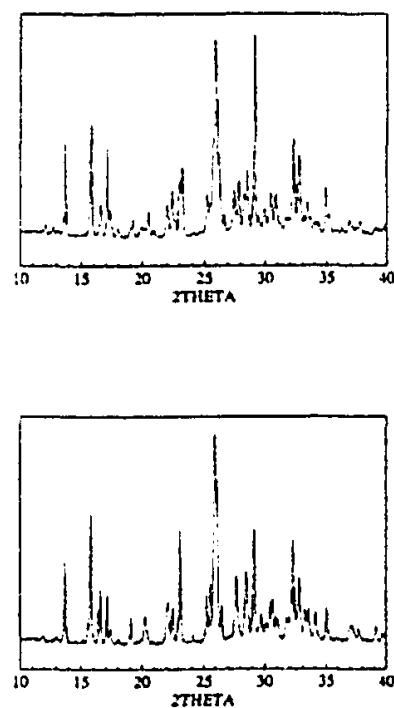

$\mathrm{CH}_{2} \mathrm{Cl}_{2} / \mathrm{CH}_{2} \mathrm{I}_{2}$

$4.98 \mathrm{Cl}: 1.00 \mathrm{l}$
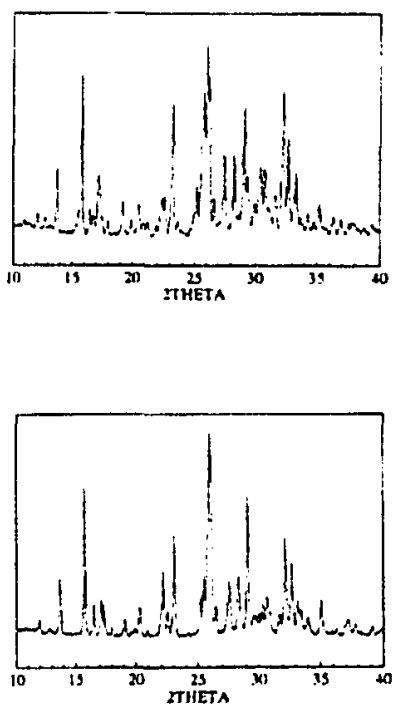

$\mathrm{CH}_{2} \mathrm{Br}_{2} / \mathrm{CH}_{2} \mathrm{I}_{2}$

$4.48 \mathrm{Br}: 1.00 \mathrm{I}$
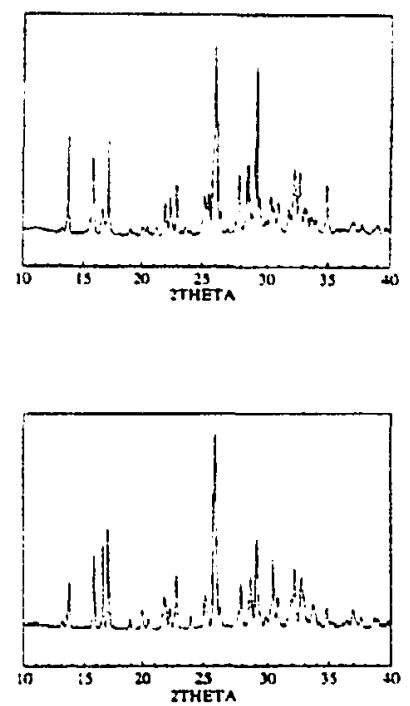

Figure X-14: Powder X-ray diffraction patterns for the methylene halide guest family mixtures. The mechanical mixtures appear in the top row, while the recrystallized mixtures appear in the bottom row: (A) $\mathrm{CH}_{2} \mathrm{Cl}_{2}$ and $\mathrm{CH}_{2} \mathrm{Br}_{2}$ (left) (B) $\mathrm{CH}_{2} \mathrm{Cl}_{2}$ and $\mathrm{CH}_{2} \mathrm{I}_{2}$ (centre) and (C) $\mathrm{CH}_{2} \mathrm{Br}_{2}$ and $\mathrm{CH}_{2} \mathrm{I}_{2}$ (right). 
(5) 1,3-dihalopropanes

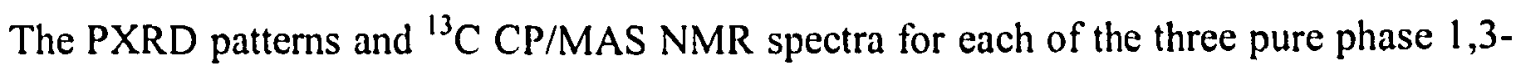
dihalopropane / TATM inclusion compounds appear in Figure X-15. Both the PXRD patterns and ${ }^{13} \mathrm{C}$ CP/MAS NMR spectra for the 1,3-dichloropropane and 1,3dibromopropane inclusion compounds are practically identical, while 1,3difluoropropane apparently forms a unique structure with TATM. Perhaps TATM cannot distinguish 1,3-dichloropropane and 1,3-dibromopropane during crystallization, so we see unmistakable isostructuralism.

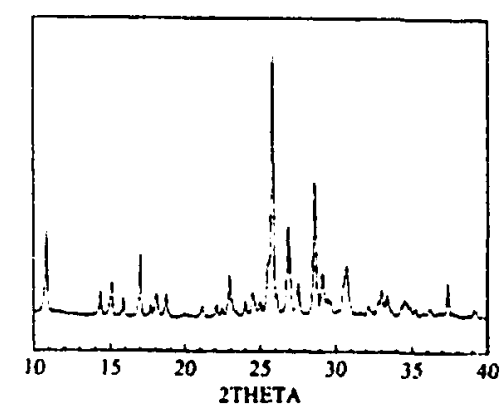

$\mathrm{C}: \mathrm{Br}\left(\mathrm{CH}_{2}\right)_{3} \mathrm{Br}$
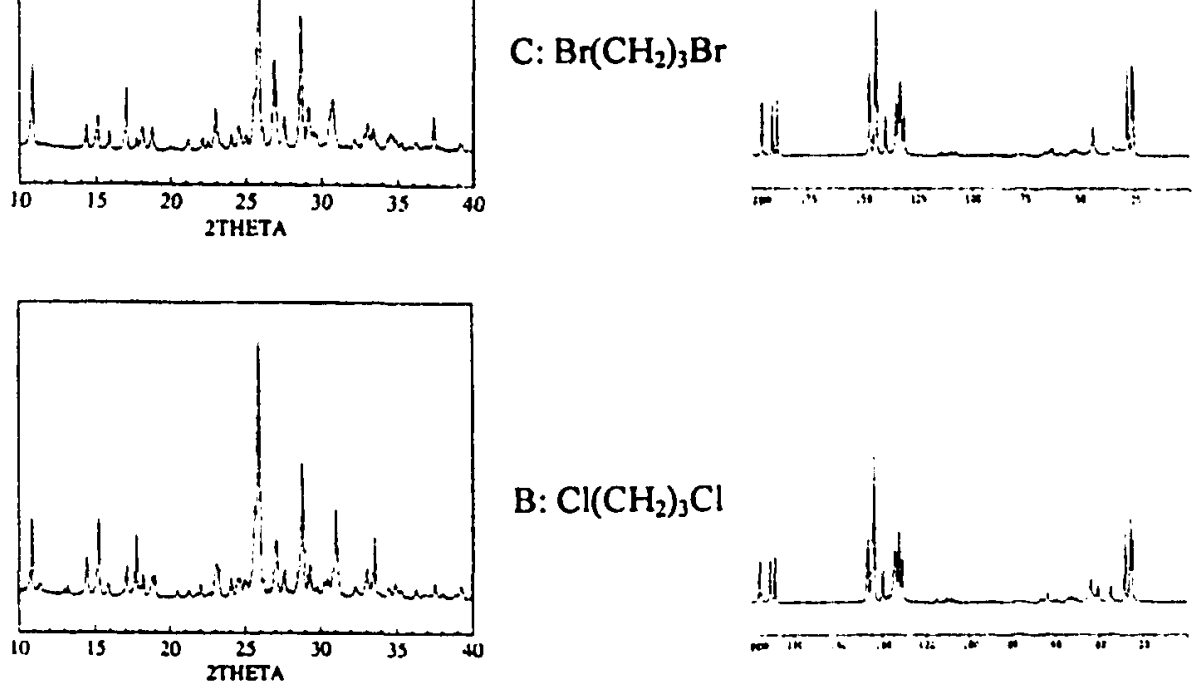

B: $\mathrm{Cl}\left(\mathrm{CH}_{2}\right)_{3} \mathrm{Cl}$
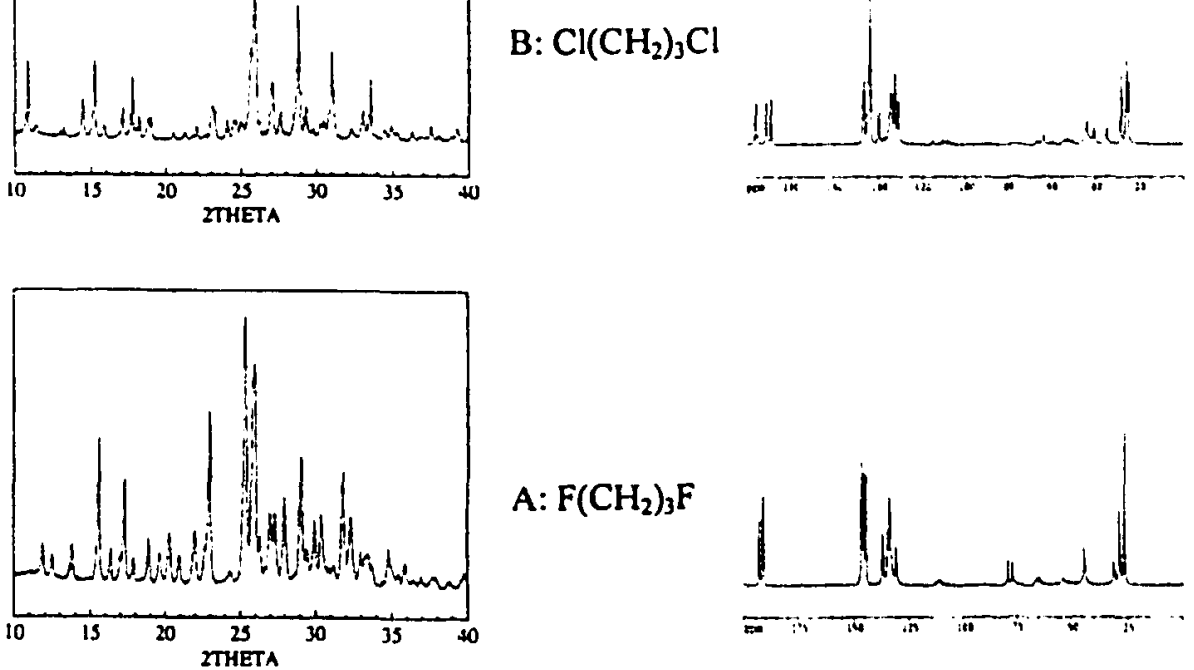

A: $\mathrm{F}\left(\mathrm{CH}_{2}\right)_{3} \mathrm{~F}$

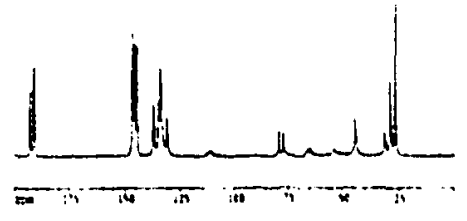

Figure X-15: Powder X-ray diffraction patterns (left) and ${ }^{13} \mathrm{C}$ CP/MAS NMR spectra (right) for the three TATM/1,3-dihalopropane complexes: (A) 1,3-difluoropropane/ TATM (B) 1,3-dichloropropane / TATM and (C) 1,3-dibromopropane / TATM 
In Figure X-16 is depicted the recrystallized and mechanical mixture PXRD patterns for each of the three 1,3-dihalopropane / TATM binary guest mixtures. As was the case for the methylene halide guest family, for all three binary combinations of 1,3dihalopropane guests, each of the three recrystallized mixture PXRD patterns match up quite well with their respective mechanical mixture PXRD patterns. Again, this was expected for the 1,3-dichloropropane/1,3-dibromopropane combination, but perhaps not for the other two binary mixtures. Similarly to the methylene halide guest family, the PXRD patterns suggest that no matter whether the accompanying guest for 1,3difluoropropane is either 1,3-dichloropropane or 1,3-dibromopropane, the same recrystallized mixture is formed, again perhaps as was expected.

$\mathrm{F}\left(\mathrm{CH}_{2}\right)_{3} \mathrm{~F} / \mathrm{Cl}\left(\mathrm{CH}_{2}\right)_{3} \mathrm{Cl}$ $1.00 \mathrm{~F}: 7.02 \mathrm{Cl}$
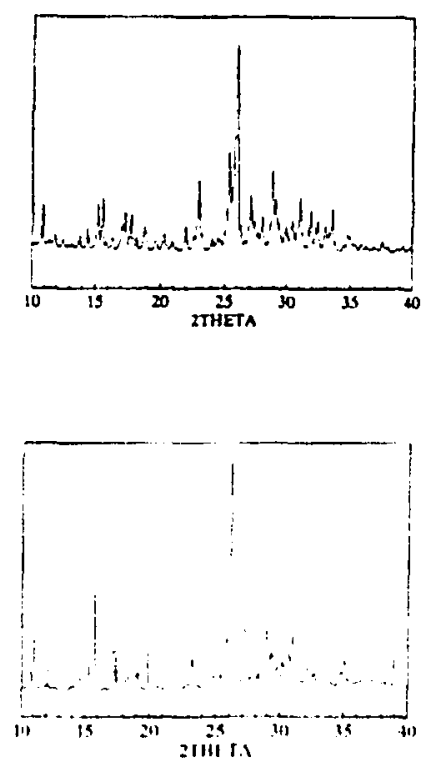

$\mathrm{F}\left(\mathrm{CH}_{2}\right)_{3} \mathrm{~F} / \mathrm{Br}\left(\mathrm{CH}_{2}\right)_{3} \mathrm{Br}$ $1.00 \mathrm{~F}: 2.41 \mathrm{Br}$
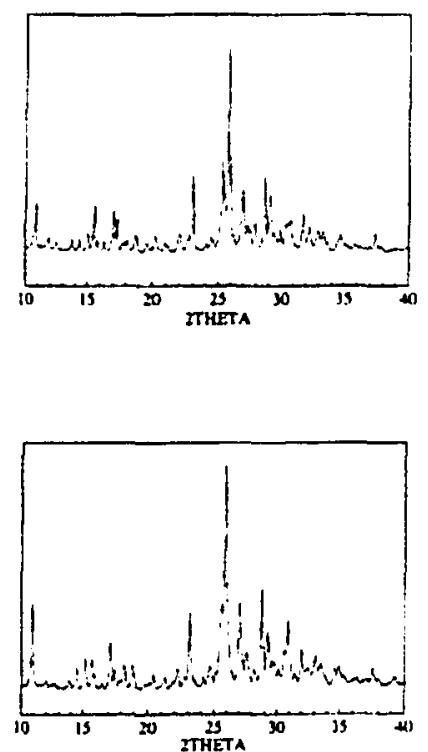

$\mathrm{Cl}\left(\mathrm{CH}_{2}\right)_{3} \mathrm{Cl} / \mathrm{Br}\left(\mathrm{CH}_{2}\right)_{3} \mathrm{Br}$ $1.00 \mathrm{Cl}: 1.09 \mathrm{Br}$
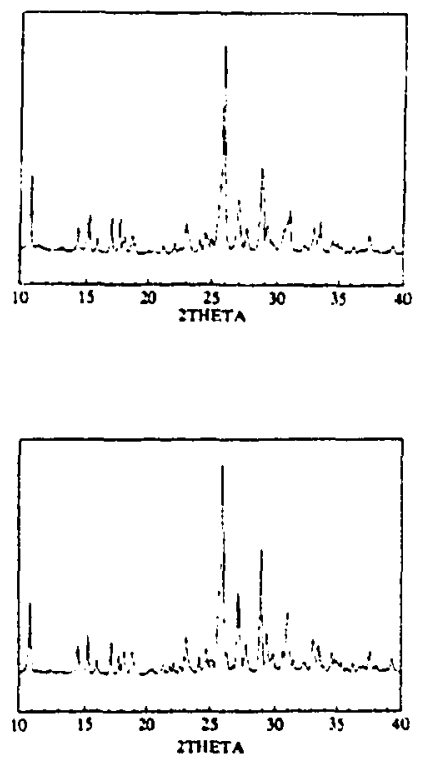

Figure X-16: Powder X-ray diffraction patterns for the 1,3-dihalopropane guest family mixtures. The mechanical mixtures appear in the top row, while the recrystallized mixtures appear in the bottom row: (A) $\mathrm{C}_{3} \mathrm{H}_{6} \mathrm{~F}_{2}$ and $\mathrm{C}_{3} \mathrm{H}_{6} \mathrm{Cl}_{2}$ (left) (B) $\mathrm{C}_{3} \mathrm{H}_{6} \mathrm{~F}_{2}$ and $\mathrm{C}_{3} \mathrm{H}_{6} \mathrm{Br}_{2}$ (centre) and (C) $\mathrm{C}_{3} \mathrm{H}_{6} \mathrm{Cl}_{2}$ and $\mathrm{C}_{3} \mathrm{H}_{6} \mathrm{Br}_{2}$ (right) 
(6) 2-halobutanes

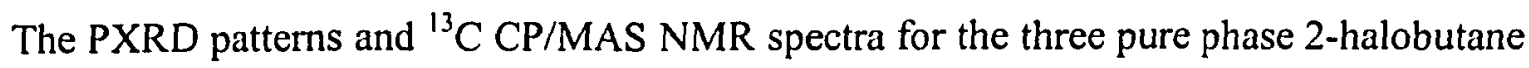
/ TATM inclusion compounds appear in Figure X-17. At first glance, the ${ }^{13} \mathrm{C} C P / M A S$ NMR spectra suggest that all three guests form isostructural TATM inclusion compounds. However, a closer glance at the PXRD patterns reveals that 2-iodobutane / TATM forms a different structure compared to the other two. On the other hand, it is evident that 2-chlorobutane and 2-bromobutane form isostructural TATM inclusion compounds. The respective peak intensities are not exactly the same, however most of the peak positions match up very well.

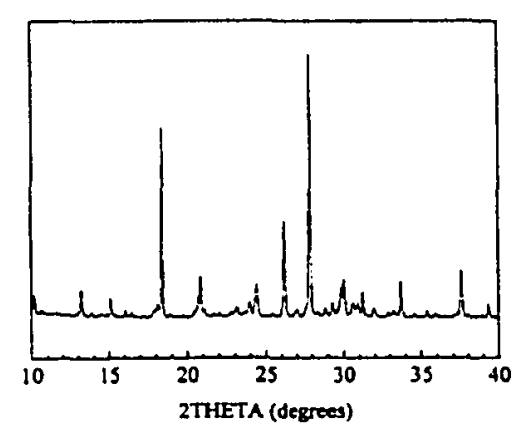

C: $\left(\mathrm{C}_{4} \mathrm{H}_{9}\right) \mathrm{I}$
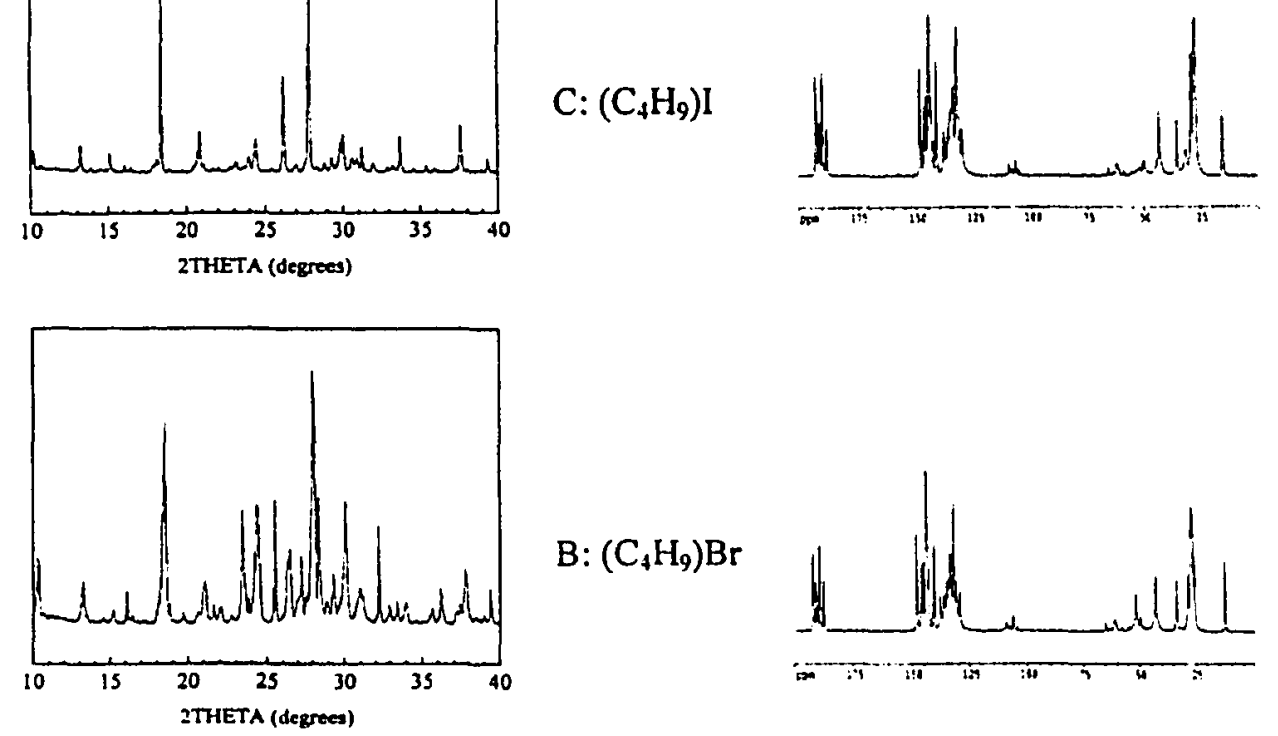

B: $\left(\mathrm{C}_{4} \mathrm{H}_{9}\right) \mathrm{Br}$
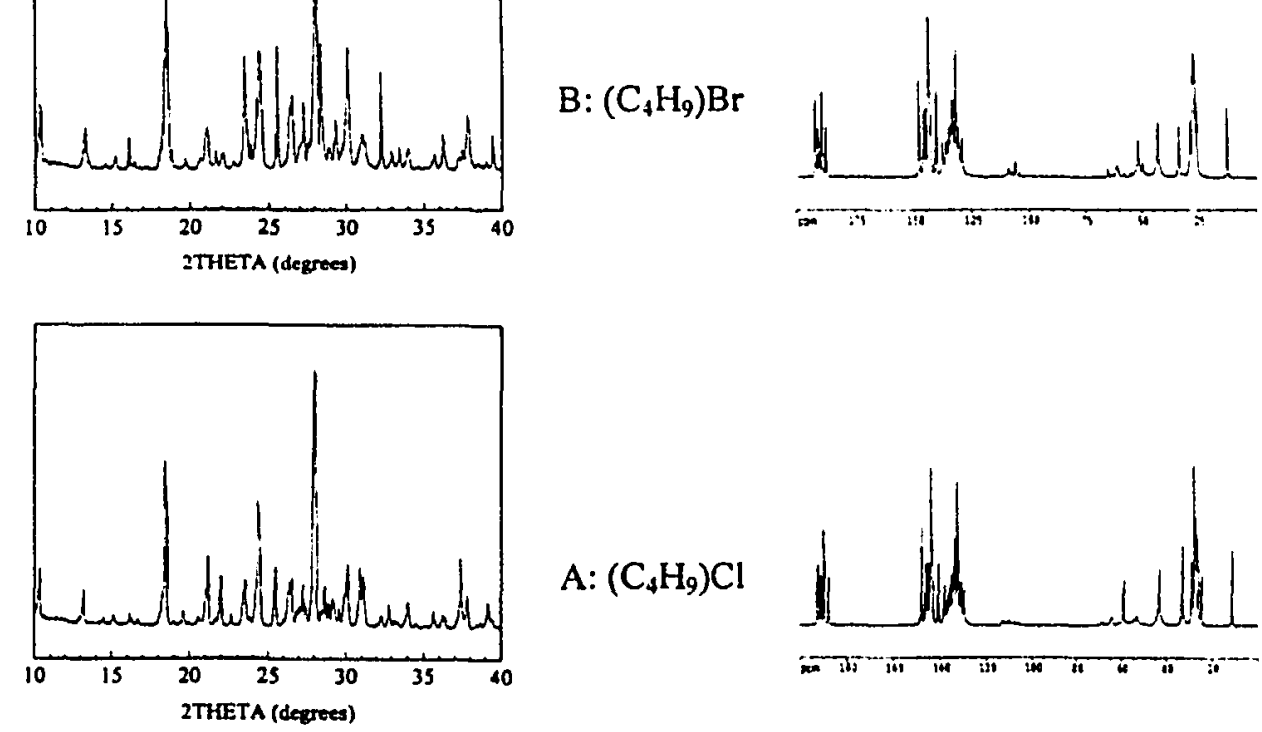

A: $\left(\mathrm{C}_{4} \mathrm{H}_{9}\right) \mathrm{Cl}$

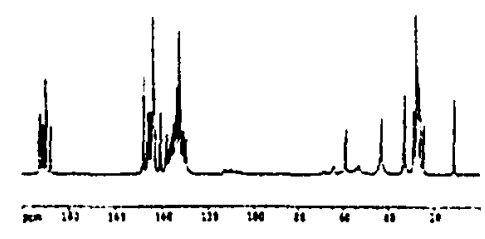

Figure X-17: Powder X-ray diffraction patterns (left) and ${ }^{13} \mathrm{C}$ CP/MAS NMR spectra (right) for the three 2-halobutane guests in TATM: (A) 2-chlorobutane / TATM (B) 2-bromobutane / TATM and (C) 2-iodobutane / TATM 
The inclusion mixture PXRD patterns for the three 2-halobutane combinations appear in Figure X-18. Perhaps unexpectedly, for the chloride/bromide mixture, a new phase is formed, despite the fact that they separately form isostructural TATM inclusion compounds. The same can also be said for the other two combinations. Remarkably however, the recrystallized mixture PXRD patterns for these other two combinations (chloride/iodide and bromide/iodide) are practically identical in appearance. This suggests that no matter whether the accompanying guest for 2-iodobutane is either 2chlorobutane or 2-bromobutane, the same novel phase is formed. This is perhaps expected however, as 2-chlorobutane/TATM and 2-bromobutane/TATM are isostructural. So we have seen three separate examples, where if two guests form isostructural TATM inclusion compounds, they give the same inclusion mixture structure when combined with a non-isostructural TATM guest within the same guest family. For the first two guest families (methylene halides and 1,3-dihalopropanes), all four recrystallized mixture PXRD patterns matched up with their respective mechanical mixture patterns, while for the present guests, in both cases, a new phase is formed.

$\left(\mathrm{C}_{4} \mathrm{H}_{9}\right) \mathrm{Cl} /\left(\mathrm{C}_{4} \mathrm{H}_{9}\right) \mathrm{Br}$ $1.85 \mathrm{Cl}: 1.00 \mathrm{Br}$
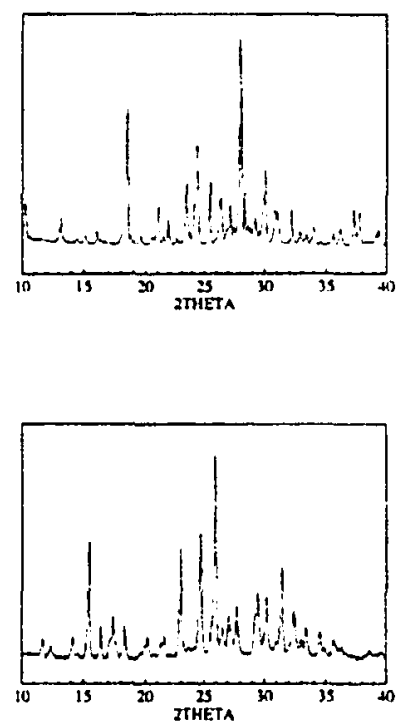

$\left(\mathrm{C}_{4} \mathrm{H}_{9}\right) \mathrm{Cl} /\left(\mathrm{C}_{4} \mathrm{H}_{9}\right) \mathrm{I}$

$1.49 \mathrm{Cl}: 1.001$
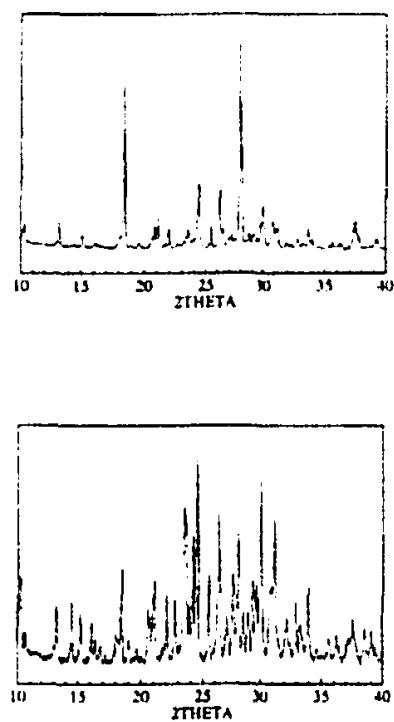

$\left.\left(\mathrm{C}_{4} \mathrm{H}_{9}\right) \mathrm{Br} /\left(\mathrm{C}_{4} \mathrm{H}_{9}\right)\right)$

$1.82 \mathrm{Br}: 1.00 \mathrm{I}$
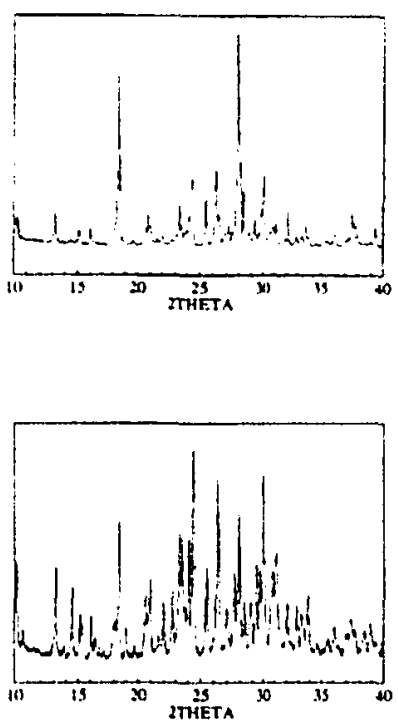

Figure X-18: PXRD patterns for the 2-halobutane guest family mixtures. The mechanical mixtures appear in the top row, while the recrystallized mixtures appear in the bottom row: (A) $\mathrm{C}_{4} \mathrm{H}_{9} \mathrm{Cl}$ and $\mathrm{C}_{4} \mathrm{H}_{9} \mathrm{Br}$ (left) (B) $\mathrm{C}_{4} \mathrm{H}_{9} \mathrm{Cl}$ and $\mathrm{C}_{4} \mathrm{H}_{9} \mathrm{I}$ (centre) and (C) $\mathrm{C}_{4} \mathrm{H}_{9} \mathrm{Br}$ and $\mathrm{C}_{4} \mathrm{H}_{9} \mathrm{I}$ (right) 
(7) cyclohexyl halides

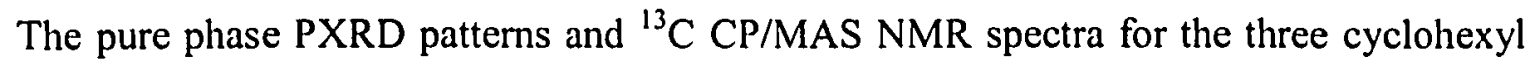
halide / TATM inclusion compounds appear in Figure X-19. The PXRD patterns and NMR spectra both suggest that all three form isostructural TATM inclusion compounds. This result is perhaps expected, as there is only one halogen atom with six carbon atoms in each guest molecule. Thus, the relative increase in size and change in shape on going from chloride to bromide to iodide is not as great as that, for example, as the methylene halide, 1,3-dihalopropane and 2-halobutane guest families.

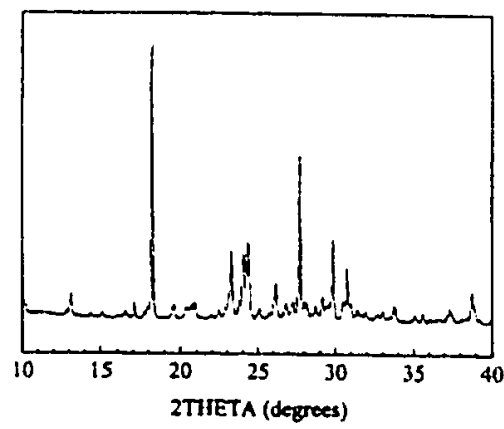

C: $\left(\mathrm{C}_{6} \mathrm{H}_{11}\right) \mathrm{I}$
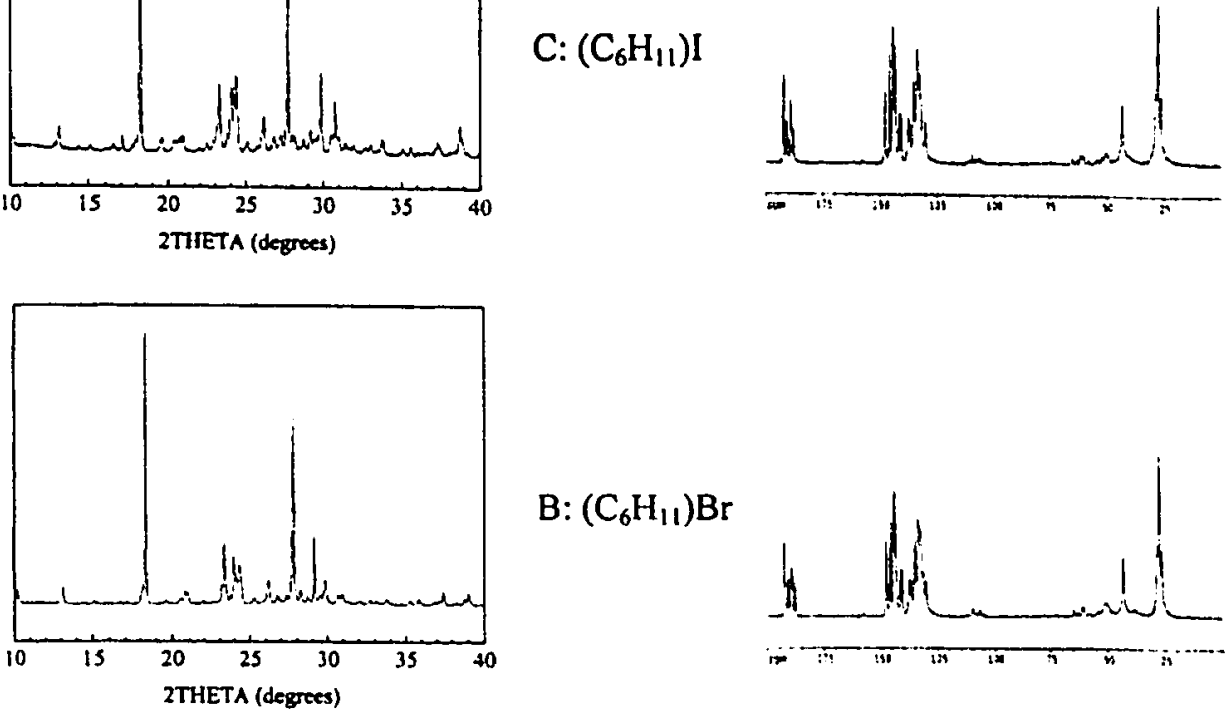

B: $\left(\mathrm{C}_{6} \mathrm{H}_{11}\right) \mathrm{Br}$
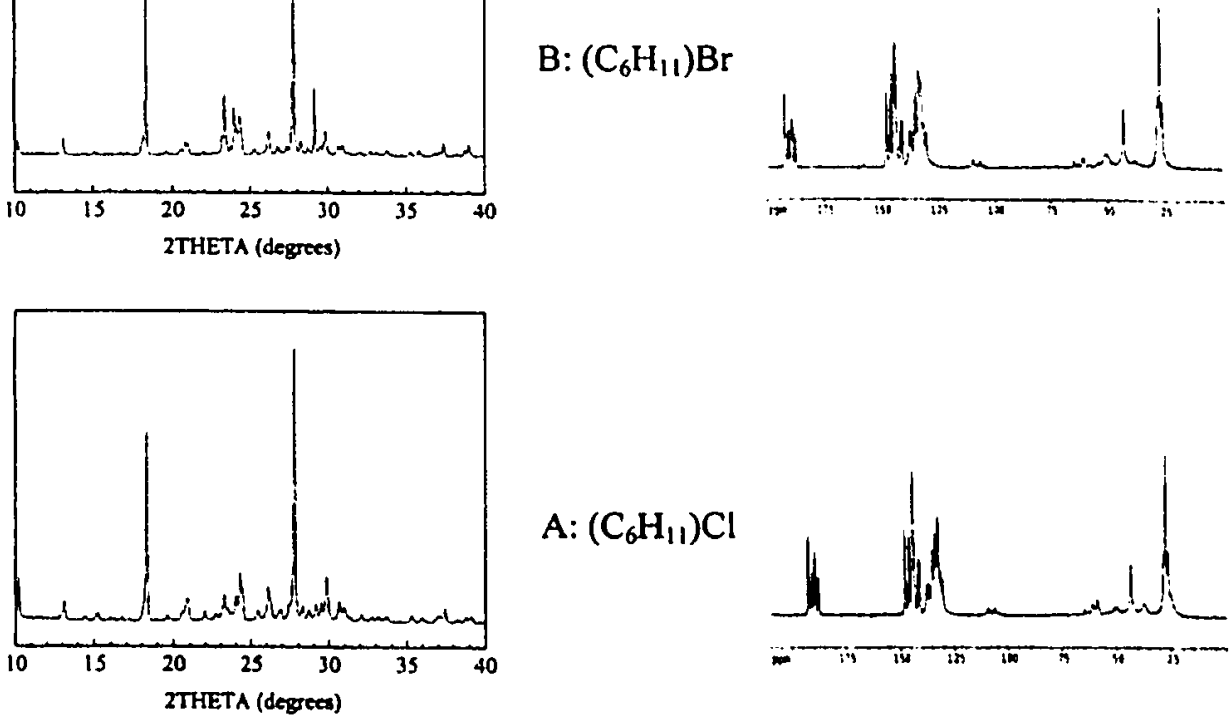

A: $\left(\mathrm{C}_{6} \mathrm{H}_{11}\right) \mathrm{Cl}$

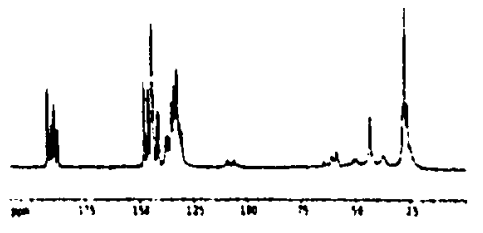

Figure X-19: PXRD patterns (left) and ${ }^{13} \mathrm{C} C \mathrm{CP} / \mathrm{MAS}$ NMR spectra (right) for the three cyclohexyl halide guests in TATM: (A) cyclohexyl chloride / TATM (B) cyclohexyl bromide / TATM and (C) cyclohexyl iodide / TATM 
Indeed, the PXRD patterns and ${ }^{13} \mathrm{C}$ CP/MAS NMR spectra for the monohalobenzene guests (see Figure X-20) suggest that benzene, fluorobenzene, chlorobenzene and bromobenzene all form isostructural TATM inclusion compounds. As is the case for the cyclohexyl halides, for these guests there is only one halogen atom for six carbon atoms, so again the progression of relative increase in size is not very large. However, for this guest family, a critical size limit is reached on going from bromobenzene to iodobenzene, as we see a completely different structure for iodobenzene / TATM (see Figure X-20)

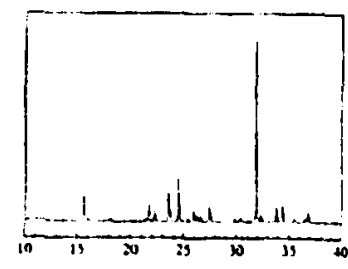

E: $\left(\mathrm{C}_{6} \mathrm{H}_{5}\right) \mathrm{I}$
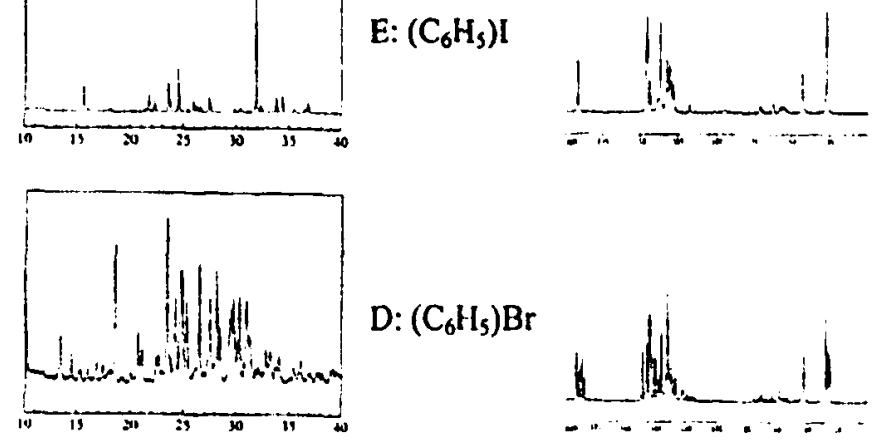

$\mathrm{D}:\left(\mathrm{C}_{6} \mathrm{H}_{5}\right) \mathrm{Br}$
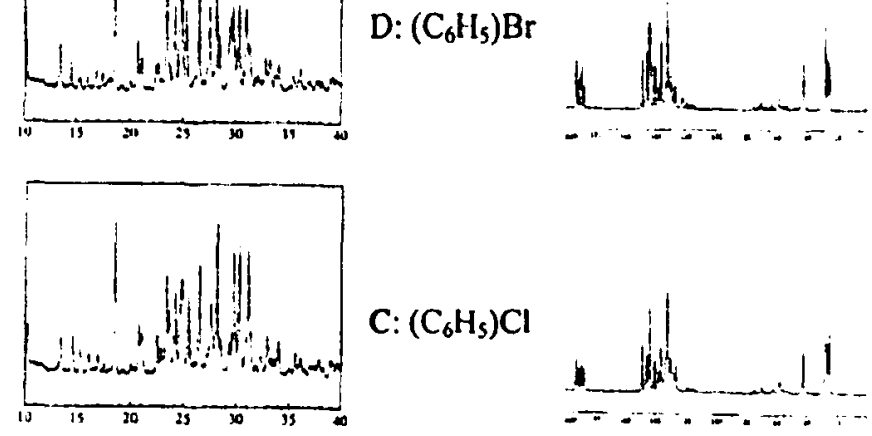

$\mathrm{C}:\left(\mathrm{C}_{6} \mathrm{H}_{5}\right) \mathrm{Cl}$
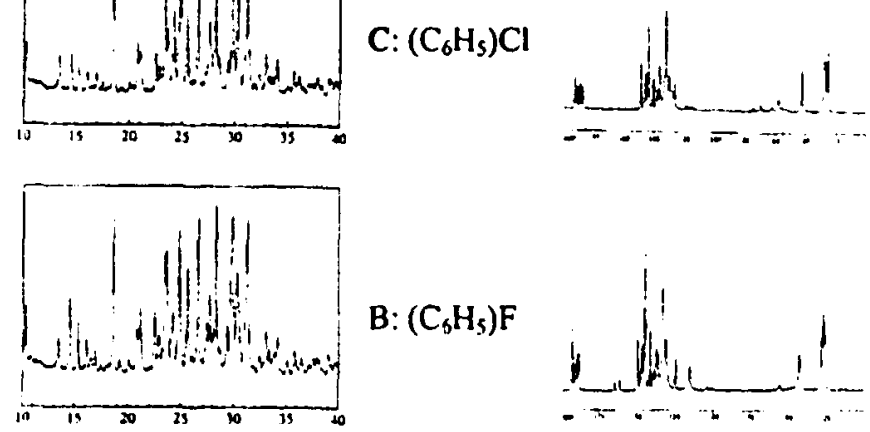

$B:\left(\mathrm{C}_{6} \mathrm{H}_{5}\right) \mathrm{F}$
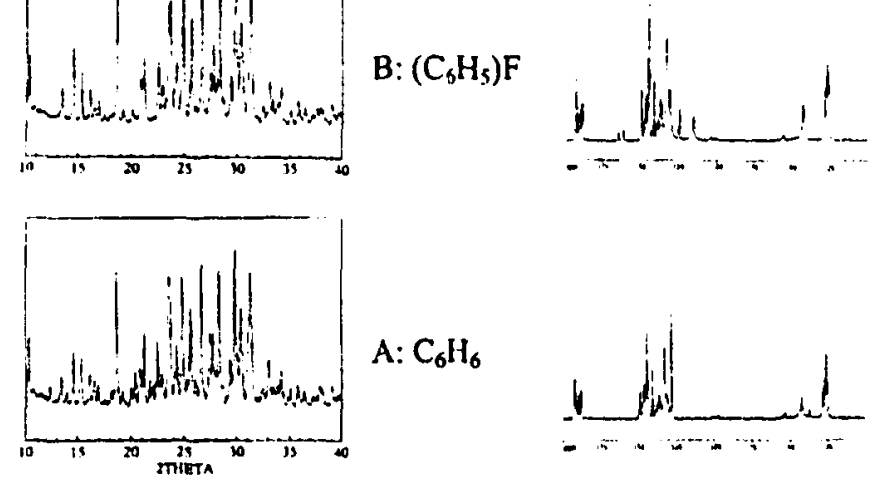

$A: \mathrm{C}_{6} \mathrm{H}_{6}$

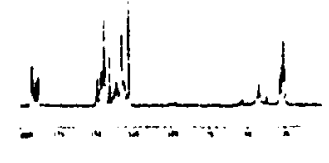

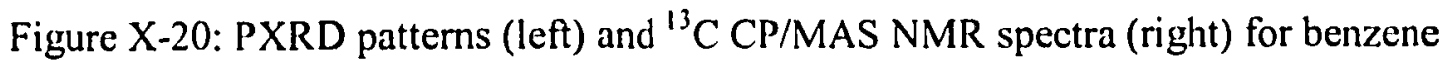
and the four monohalobenzene guests in TATM: (A) benzene (B) fluorobenzene (C) chlorobenzene (D) bromobenzene and (E) iodobenzene 
So we see a theme of minimal isostructuralism for smaller guests, and more and more as the hydrocarbon backbone increases in size. These results may be placed alongside the competitive inclusion results of the previous work (see chapter IX) where the methylene halides had the greatest selectivity of all four guest families, while the larger guests had a lower selectivity. TATM shows a stronger preference among the smaller guest families (such as the methylene halides) as the changing part (2 halogen atoms), is much larger than the invariant part ( 1 carbon atom), so during crystallization from a mixture, the preference for one guest over the other is much greater, as it easier for the TATM host to distinguish them from a mixture.

In Figure X-21 are depicted the mechanical and recrystallized mixture PXRD patterns for the three possible cyclohexyl halide / TATM binary combinations. For the mechanical mixtures (top row), the $y$-axis (signal intensity) is expanded to display the weaker signals more conspicuously. It is easy to see that for each of the three combinations, the mechanical mixture and recrystallized mixture PXRD patterns are practically identical. Another remarkable result is that all three recrystallized mixture combinations give rise to an isostructural phase (bottom row). Since, as described above, the relative increase in size and shape of the guest on going from chloride to bromide to iodide is not very large, the TATM host cannot distinguish them, either as a pure phase or as part of a binary mixture, so we see the same structure for all three recrystallized mixtures.

\section{DISCUSSION}

These results now allow us to contemplate on the nature of the crystallization process, as it occurs at the molecular level. For the mechanical mixtures, there are no mixed crystals. For example, if one considers the cyclohexyl halides (see Figure X-21), in the mechanical mixture, each crystallite is either, for example, cyclohexyl chloride/TATM or cyclohexyl bromide/TATM, but there are no ternary crystals (i.e. two guests). The fact that the recrystallized mixture PXRD patterns for most all ( 9 out of 12) present TATM combinations are very similar to those of the mechanical mixtures suggests that the recrystallized mixtures also contain few or no mixed crystals. 

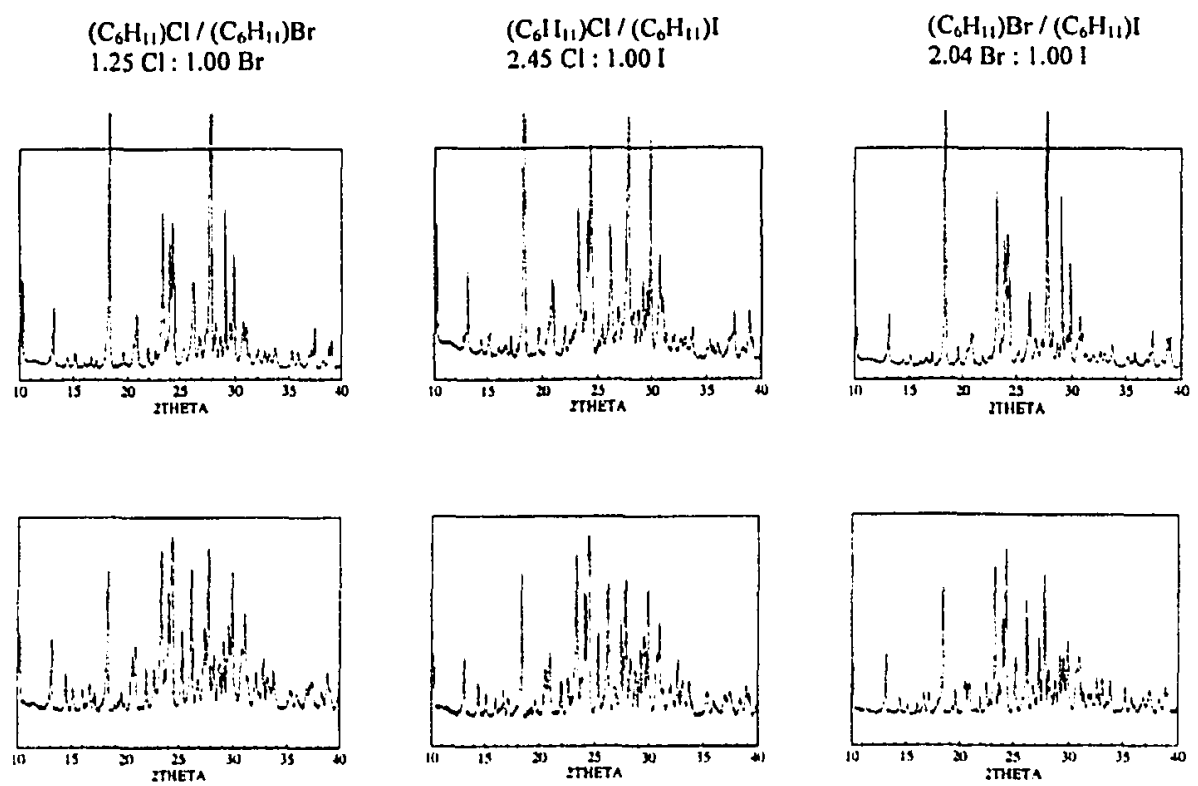

Figure X-21: PXRD patterns for the cyclohexyl halide guest family mixtures. The mechanical mixtures appear in the top row, while the recrystallized mixtures appear in the bottom row: (A) $\mathrm{C}_{6} \mathrm{H}_{11} \mathrm{Cl}$ and $\mathrm{C}_{6} \mathrm{H}_{11} \mathrm{Br}$ (left) (B) $\mathrm{C}_{6} \mathrm{H}_{11} \mathrm{Cl}$ and $\mathrm{C}_{6} \mathrm{H}_{11} \mathrm{I}$ (centre) and (C) $\mathrm{C}_{6} \mathrm{H}_{11} \mathrm{Br}$ and $\mathrm{C}_{6} \mathrm{H}_{11} \mathrm{I}$ (right)

If, on the other hand, there were a significant proportion of mixed crystals, one might expect that a new phase would be formed (e.g. all three 2-halobutane combinations), resulting in novel recrystallized mixture PXRD patterns. This suggests that during the crystallization process of TATM from a mixture of, for example, two cyclohexyl halide guests, each TATM crystallite is seeded by either cyclohexyl chloride or cyclohexyl bromide, but once a crystal is established with that guest, it does not take up the other guest as it grows.

An alternative explanation for the observed behaviour is that there are mixed crystals, however the structure of the ternary crystal is determined by the guest that seeds that particular crystal. Considering the 1,3-dihalopropanes, if a TATM crystal is seeded by 1,3-dichloropropane, the structure of the crystal, as it grows, is the same as that of the pure 1,3-dichloropropane/TATM inclusion compound, regardless of whether it subsequently incorporates 1,3-dichloropropane or 1,3-dibromopropane (or both) as it grows. 
A final possible explanation for the observed behavior is that there are mixed crystals, but the two guests are the "same" as far as molecular recognition of TATM for the guest(s) are concerned. However, this theory is refuted by the experimental data in that many cases (especially methylene halides and 1,3-dihalopropanes) where the recrystallized mixture PXRD patterns matched the mechanical mixture PXRD patterns occurred when the two guests by themselves (individually) did not form isostructural TATM inclusion compounds. For example, $\mathrm{CH}_{2} \mathrm{Br}_{2}$ and $\mathrm{CH}_{2} \mathrm{I}_{2}$ individually do not form isostructural TATM inclusion compounds, however the recrystallized mixture PXRD pattern matched the mechanical mixture PXRD pattern quite well (see Figure X-14). If this theory held, one would not expect this to occur, since, as judged by their different respective pure inclusion compound TATM structures, TATM should be able to distinguish them from a binary mixture, and subsequently a new phase would be formed (i.e. TATM "molecular recognition" should take hold in this recrystallized mixture).

One can speculate on the reasons why only one of the four guest families (the 2halobutanes) gave novel phases for all three mixtures, while the other three guest families universally gave the same phase as the mechanical mixture. It could be possibly due to the fact that this guest family contains an asymmetric carbon atom (i.e. is chiral). Since the TATM molecule is chiral, it may be possible that there are four types of complexes in the ternary mixture (e.g. (R) and (S) 2-chlorobutane, $(R)$ and $(S)$ 2-bromobutane). Perhaps the TATM host can form more than one type of crystal from the binary guest mixture, which perhaps are diastereotopic, and may result in novel inclusion mixtures. An example of diastereoism among inclusion compounds, namely the enantiomeric triortho-thymotide host with 2-chloro-, 2-bromo- and 2-iodobutane guests, have been investigated intensively [54-58]

Another possibility is that for TATM, the guest family for which new phases are formed, the 2-halobutanes, displays the largest variation in $\Delta_{\text {fus }} H$ on going from chloride to bromide to iodide, $30 \mathrm{~J} \mathrm{~g}^{-1}$ (see Table VIII-3). The other three alkyl halide guest families, for which new phases are not formed, have smaller variations, 15, 17 and 21 $\mathrm{J}^{-1}$. Thus, for the 2-halobutanes, one may see new phases because the individual inclusion compounds differ so greatly in enthalpy of decomposition, when compared to the other three guest familes, for which new phases are not formed. 
(C) $\left[\mathrm{N}\left(\mathrm{CH}_{3}\right)_{4} \cdot x \mathrm{G}\right]\left[\mathrm{Cd}_{3}(\mathrm{CN})_{7}\right]$ host system

There are other examples in the literature where the inclusion compounds formed by a given host and two or more isomeric guests are structurally very different. A recent publication [59] examined inclusion selectivity of the "zeolite mimic" host clathrate $\left[\mathrm{N}\left(\mathrm{CH}_{3}\right)_{4} \cdot x \mathrm{G}\right]\left[\mathrm{Cd}_{3}(\mathrm{CN})_{7}\right]$ with guests ethylbenzene, $o$-xylene, $m$-xylene and $p$-xylene. For the pure guest clathrates, ethylbenzene, $o$-xylene and $m$-xylene all form isostructural inclusion compounds (Pnam, $Z=4$ ), while $p$-xylene forms a unique structure $\left(P 6_{3} / m m c\right.$, $Z=2$ ). From competitive inclusion experiments, the authors found that ethylbenzene is always the most preferred guest (from all types of binary, tertiary and quaternary mixtures). On the other hand, $p$-xylene is the second most preferred guest, while $m$ xylene and $o$-xylene are the least preferred guests.

The authors found $[59]$ that the powder X-ray diffraction patterns for all mixed complexes may be assigned to the Pnam structural type, except for those of the ortholpara, metalpara and ortho/metalpara mixtures (for all of which para-xylene is most enriched). As shown in Figure X-22, the PXRD patterns of the single guest ethyl-, ortho- and meta- inclusions are similar to one another (owing to the same space group (Pnam) and similar unit cell parameters), to which the PXRD patterns of the e-o-m-pquaternary mixture (see Figure X-22), as well as those of the ethylbenzene enriched mixtures, show a close similarity. On the other hand, the PXRD pattern observed for the $p$-xylene single guest clathrate shows fewer reflections owing to the higher symmetry hexagonal $P 6_{3} / m m c$ space group. The PXRD diffraction patterns observed for the three para-enriched mixed guest clathrates (not shown) are similar to that of the p-xylene single guest clathrate. The common pattern that seems to be emerging is that the structure of the mixed inclusion compound is determined by the "most preferred" guest, in the order ethyl- > para- $>$ meta- $\approx$ ortho-. In this case, a new phase is not formed. All inclusion mixtures are isostructural to one of the associated pure phase crystals from whence it came. This behaviour is similar to TATM systems in that for the non-para combinations, not only are the individual inclusions isostructural, but the mixtures are also of the same phase, to both each other and the individual one-guest inclusions. On the other hand, when a guest forms a structurally unique type of crystal (i.e. para-xylene), we see a novel mixture, similar to the para-xylene pure inclusion crystal (only when 
ethylbenzene is not present). For all TATM mixtures, except for the 2-halobutanes, a new phase is not formed, in that the recrystallized mixture PXRD pattern is very similar to the mechanical mixture pattern which suggests that in this case, there are both types (for binary mixtures) of pure phase crystal present.

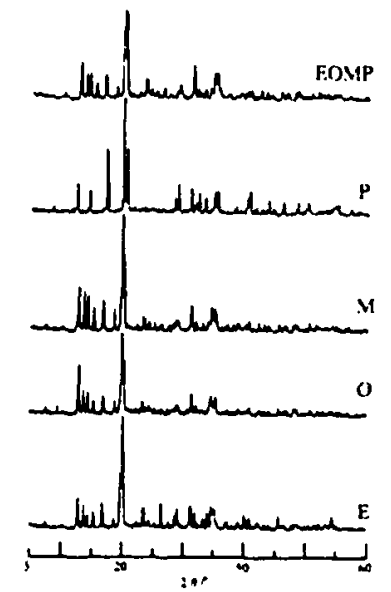

Figure X-22: Powder X-ray diffraction patterns of single guest and mixed guest clathrates of the $\left[\mathrm{N}\left(\mathrm{CH}_{3}\right)_{4} \cdot x \mathrm{G}\right]\left[\mathrm{Cd}_{3}(\mathrm{CN})_{7}\right]$ host system with alkylbenzene guests: ( $c=$ ethylbenzene, $o=$ ortho-xylene, $m=$ meta-xylene, $p=$ para-xylene, comp $=$ quaternary guest inclusion mixture) [59].

\section{(D) 1,1,2,2-tetraphenylethane-1,2-diol host system}

Another example is the 1,1,2,2-tetraphenylethane-1,2-diol / lutidine host-guest system [60]. The inclusion compounds formed with 2,6-lutidine and 3,5-lutidine guests have different host-guest stoichiometric ratios and different crystal packings. It was shown from competitive inclusion experiments that 3,5-lutidine is preferentially included. At compositions where the molar percent of 3,5-lutidine in the mother crystallization mixture is $50 \%$ or more, 3,5 -lutidine is $\sim 100 \%$ incorporated into the host lattice. This is in contrast to TATM, where the preference is generally much less selective. Powder Xray diffraction experiments showed that only two phases are possible for the inclusion compound mixtures, which correspond to the two pure inclusion compounds. For $X_{3,5-\text { lutidine }}=0-0.3$, the phase is that of the 2,6-lutidine inclusion compound, while for $X_{3,5-\text { lutidine }}=0.4-1$, the phase is isostructural to the 3,5-lutidine inclusion compound. This suggests that a new phase is not formed from a mixture of two guests, which agrees with TATM, except for the 2-halobutane guest family. On the other hand, the TATM inclusion mixtures are (macroscopic?) mixtures of the two pure phases, whereas in the present case, the structure of the mixed crystal is identical to the more dominant pure 
phase inclusion compound from which it came, perhaps the same case as in the $\left[\mathrm{N}\left(\mathrm{CH}_{3}\right)_{4} \cdot x \mathrm{G}\right]\left[\mathrm{Cd}_{3}(\mathrm{CN})_{7}\right]$ host system in the previous section.

(E) Cholic acid host system

As mentioned in the introduction, cholic acid (CA) is known to form inclusion compounds with a wide variety of organic substances [61-86]. X-ray crystallographic studies have revealed that $\mathrm{CA}$ can form at least nine host frameworks, depending on the nature of the included guest. Recently, it was discovered that the volume of the guest plays a crucial role in determining the nature of the host framework and host:guest stoichiometric ratio for CA inclusion compounds with monosubstituted benzenes [52]. Furthermore, competitive inclusion studies revealed that the nature of selective enclathration (see Figure $\mathrm{X}-1$ ) of $\mathrm{CA}$ with monosubstituted benzene guests was dependent on which of the nine types of $\mathrm{CA}$ lattice was formed when both guests were included [65]. To further investigate this area, the authors reported single crystal structures of not only the three pure phase xylene/CA inclusion compounds, but also the mixed crystals [53] (crystal data for both the pure inclusion compounds and the mixed crystals appear in Supplemental Material). To the best of our knowledge, this is the first (and only) report of systematic single crystal X-ray structural analysis of varyingcomposition mixed-guest inclusion compound crystals. This pioneering study provides insight into the selective and non-selective enclathration processes in the crystalline state.

The authors found that the packing coefficients of the xylene guest in the CA host cavity ( $P C_{\text {cavity }}$ - the volume ratio of guest molecule to host cavity), is useful in prediction of relative preferences of CA solid-state host-guest compounds [53]. Orthoxylene $\left(P C_{\text {cavily }}=44 \%\right)$ is not included in mixtures with the other two xylenes due to its low packing coefficient, while meta-xylene (58\%) and para-xylene (65\%), with larger packing coefficients, are both preferentially included over ortho-xylene. These results indicate that steric suitability of shape and volume between guest molecules and the host cavity play an important role for the selection of host framework types by mixtures of guest molecules. As previously mentioned, for TATM, alkyl iodides may be less suitable because they stretch the cavity, while alkyl chlorides may be less suitable because they have less extensive van der Waals interactions between host and guest. It would be 
interesting to measure the volume ratio of guest molecule to host cavity for these systems (the single crystal structures of 1,3-dihalopropane / TATM inclusions appear in this chapter). One might expect that 1,3-dibromopropane has the largest $P C_{\text {cavity }}$, followed by 1,3-dichloropropane and 1,3-diiodopropane (in that order).

Competitive recrystallization of $\mathrm{CA}$ from mixtures of xylenes results in characteristic molecular recognition [53]. Recrystallization from 1:1 mixtures of (1) $o$ xylene and $m$-xylene or (2) $o$-xylene and $p$-xylene yielded pure (1) $\mathrm{CA} / m$-xylene and (2) $\mathrm{CA} / p$-xylene respectively. In these two cases, ortho-xylene acts as a solvent, and is not included in the CA host framework at all. As a result, this corresponds to selective enclathration of type I in Figure X-l b. Furthermore, a 1:1:1 mixture of all three isomeric xylenes yielded a 1:1 inclusion mixture with $m$-xylene and $p$-xylene, without $o$-xylene. On the other hand, TATM inclusion mixtures may generally follow types II and III nonselective enclathrations in Figure $\mathrm{X}-1 \mathrm{~b}$, while the 2-halobutanes may adhere to type IV non-selective enclathration.

On the other hand, recrystallization of CA from a 1:1 mixture of $m$-xylene and $p$ xylene yielded a measured single crystal that included both guest components at a ratio similar to that in the bulk sample. X-ray crystallography confirmed that that both $\mathrm{m}$ xylene and $p$-xylene were present in the host cavities. For this starting equimolar mixture, the X-ray occupancy factors indicated an incorporated stoichiometric ratio of $0.38(3)$ for $m$-xylene to $0.62(6)$ for $p$-xylene. This indicated that both guests are included in the single crystals with static and location disorder in the host cavities. The mixed crystal was isostructural to pure $m$-xylene/CA. For TATM, we are uncertain whether both guests are included in each crystallite (type III in Figure X-lb). It is possible that each crystallite is a pure phase TATM inclusion compound (type II), at least for guests other than the 2-halobutanes.

As the next step, the authors investigated crystal structures and guest selectivities for a range of $m$-xylene $/ p$-xylene feed mixtures, namely $m$-xylene $/ p$-xylene $=3: 1,2: 1$, $1: 2,1: 3,1: 4,1: 5$ ). All seven mixed crystals (including 1:1 above) gave host frameworks isostructural to that of pure $\mathrm{CA} / m$-xylene, irrespective of the isomer ratios of the recrystallization mixtures. Again, the ratio $m$-xylene / $p$-xylene in the single crystals was evaluated by refinement of the occupancy factors, and the results are depicted in Table X- 
3 , confirming that both guests were present in the measured single crystals, and that the included ratio was somewhat similar to that of the feed mixture. The bulk product ratios were evaluated by gas-liquid chromatography (see Table X-4). They indicated that the mole fractions ( $m$-xylene / $p$-xylene) in the CA mixed single crystals were is most cases very similar to those in the bulk products. These results indicate that both $m$-xylene and $p$-xylene are included together in the single crystals, and that the bulk crystals consist of homogeneous single crystals. Therefore, these non-selective enclathrations are attributed to type III in Figure X-lb. We wish, at some point, to investigate TATM inclusion mixtures in a similar manner, by obtaining single crystal structures of the mixed-guest inclusion compounds.

Table X-3: Occupancies of meta-xylene $(\mathbf{m X})$ and para-xylene $(\mathbf{p X})$ in single crystals of cholic acid inclusion mixtures, as determined by single crystal X-ray diffraction [53].

\begin{tabular}{lll}
\hline $\begin{array}{l}\text { Percent of } \mathbf{m X} \\
\text { in feed mixture }\end{array}$ & $\mathbf{m X}$ occupancy & $\mathbf{p X}$ occupancy \\
\hline 75 & $65(2)$ & $34(2)$ \\
67 & $56(2)$ & $45(2)$ \\
50 & $38(3)$ & $62(3)$ \\
33 & $25(1)$ & $75(1)$ \\
25 & $20(2)$ & $79(2)$ \\
20 & $17(2)$ & $82(2)$ \\
17 & $12(1)$ & $87(1)$ \\
\hline
\end{tabular}

Table X-4: Percent occupancy, as determined by gas-liquid chromatography, of metaxylene $(\mathbf{m X})$ in the bulk inclusion mixtures of meta-xylene and para-xylene with cholic acid [53].

\begin{tabular}{ll}
\hline $\begin{array}{l}\text { Percent of } \mathbf{m X} \\
\text { in feed mixture }\end{array}$ & $\begin{array}{l}\mathbf{m X} \text { occupancy } \\
\text { in bulk samples }\end{array}$ \\
\hline 91 & 88 \\
83 & 78 \\
80 & 73 \\
75 & 66 \\
67 & 54 \\
50 & 35 \\
33 & 16 \\
25 & 9 \\
20 & 7 \\
17 & 7 \\
9 & 4 \\
\hline
\end{tabular}


These results shed light on inclusion phenomena involving mixed guest compounds included in different host frameworks of the same host. When the two pure inclusion crystals have markedly different stabilities (e.g. $o$-xylene and $m$-xylene inclusions), the host selectively includes the guest from the inclusion with greater $P C_{\text {cavity }}$ ( $m$-xylene) from a mixture of the two, and the host framework is the same as that of the pure inclusion with the favored guest ( $m$-xylene). On the other hand, similar stabilities (e.g. $m$-xylene and $p$-xylene inclusions) lead to non-selective enclathrations. When only one of the two host frameworks has sufficiently large voids to accommodate both guests (e.g. $\mathrm{CA} / m$-xylene), the host framework of the mixed crystal will be the same as that of this pure inclusion compound ( $\mathrm{CA} / m$-xylene), and not the other one ( $\mathrm{CA} / p$-xylene). This is the type of non-selectivity depicted as type III in Figure X-lb. Conversely, when each guest cannot be included in the other host framework, the resulting product is a mixture of the two individual pure inclusion compounds, depicted as type II in Figure $\mathrm{X}-1 \mathrm{~b}$. The inclusion phenomenon of non-selective enclathration therefore depends on the accessibility of the guest compounds to the host cavities. As a result, the steric dimensions of the host cavities play an important role in non-selective recognition. In other words, inclusion crystals of mixed guest compounds without any selectivity may select the host framework with larger host cavities (c.f. CA/meta-xylene). As mentioned above, for TATM, the guest family for which new phases are formed, the 2-halobutanes, $\Delta_{\text {lus }} H$ displays the largest variation on going from chloride to bromide to iodide (see Table VIII-3), namely $30 \mathrm{~J} \mathrm{~g}^{-1}$. The other three alkyl halide guest families, for which new phases are not formed, have smaller variations, 15,17 and $21 \mathrm{~J} \mathrm{~g}^{-1}$. These results agree with the results on the present cholic acid host system.

\section{CONCLUDING REMARKS}

We hope that these results will shed further light upon inclusion phenomena involving mixed guest compounds included in different open host frameworks (Figure X-lb). When the two inclusion crystals have markedly different stabilities, the host may selectively include only one of the guest compounds from the mixture, and the host framework is the same as that obtained from the pure guest. Similar stabilities of the pure inclusion crystals will likely lead to non-selective (competitive) enclathration. In 
this case, when only one of the two host frameworks has a channel or cavity large enough to accommodate both guests, they would likely be randomly distributed in this particular framework (Figure X-1b, type III). On the other hand, when each guest compound cannot be included in the other host framework, the resulting crystals are likely to be macroscopic mixtures of pure crystals (Figure X-lb, type II). The inclusion phenomenon of less selective enclathration therefore would perhaps depend on the accessibility of the guest molecules to the host cavities. The steric dimensions of the host cavities thus play an important role for non-selective recognition. In other words, inclusion crystals of mixed guest compounds without any selectivity may involve guests selecting the host framework with larger cavities, in order to accommodate both guests.

In this contribution we have reported single crystal structural characterization of TATM inclusion compounds with 1,3-dihalopropane guests, in addition to bulk characterization techniques (PXRD and ${ }^{13} \mathrm{C} \mathrm{CP} / \mathrm{MAS} \mathrm{NMR}$ ) to show that the selected single crystals are characteristic of the bulk product. Furthermore, we have used these same bulk characterization techniques to characterize the similarities and differences between mechanical mixtures of two pure phase TATM inclusion compounds and TATM recrystallized from a mixture of the same two guests. With exception of the 2-halobutane guest family, we found that the structural type of the recrystallized mixture was always very similar to that of the mechanical mixture, indicating that a new phase was not formed. This suggests that within each of these three guest families, TATM cannot distinguish the two guests from the mixture (i.e. they are identical as far as molecular recognition of host for guest is concerned). On the other hand, for the 2-halobutanes, in all three cases, a novel phase was formed. We suggested that this may arise out of the fact that the 2-halobutane guests each contain an asymmetric carbon atom (they are chiral), as opposed to the other three guest families, which do not possess chirality. A second possibility arises from the largest variation in $\Delta_{\text {fus }} H$ for the 2-halobutane inclusions $\left(30 \mathrm{~J} \mathrm{~g}^{-1}\right)$, while it is considerably smaller for the other three alkyl halide guest family inclusions $\left(15,17\right.$ and $\left.21 \mathrm{~J} \mathrm{~g}^{-1}\right)$. 
(1) Solid-State Supramolecular Chemistry: Crystal Engineering. In Comprehensive Supramolecular Chemistry, J.L. Atwood, J.E.D. Davies, D.D. MacNicol and F. Vogtle, Eds. Volume 6, D.D. MacNicol, F. Toda and R. Bishop, Volume Eds. Pergamon / Elsevier: Oxford, 1996.

(2) E. deVries, L.R. Nassimbeni and H. Su. Eur. J. Org. Chem. 1887 (2001).

(3) M.R. Caira, A. Horne, L.R. Nassimbeni and F. Toda. J. Mater Chem. 7(10), 2145 (1997).

(4) M.R. Caira, L.R. Nassimbeni, F. Toda and D. Vujovic. J. Am. Chem. Soc. 122, 9367 (2000).

(5) E. Weber, K. Skobridis, A. Wierig, L.J. Barbour, M.R, Caira and L.R. Nassimbeni. Chem. Ber. 126, 1141 (1993).

(6) I. Goldberg, Z. Stein, A. Kai and F. Toda. Chem. Lett. 1617 (1987).

(7) F. Toda, Y. Tagami and T.C.W. Mak. Chem. Lett. 1909 (1986).

(8) D. Dvorak, J. Zavada, M.C. Etter and J.H. Loehlin. J. Org. Chem. 57, 4839 (1992).

(9) T. Kobayashi, S. Isoda, and K. Kubono. Cyclophosphazenes: Structures and Molecular Selectivities In Comprehensive Supramolecular Chemistry, J.L. Atwood, J.E.D. Davies, D.D. MacNicol and F. Vogtle, Eds. Volume 6, Chapter 13, Pergamon / Elsevier: Oxford, 1996.

(10) J Szejtli. Inclusion of Guest Molecules, Selectivity and Molecular Recognition by Cyclodextrins In Comprehensive Supramolecular Chemistry, J.L. Atwood, J.E.D. Davies, D.D. MacNicol and F. Vogtle, Eds. Volume 3, Chapter 5, Pergamon / Elsevier: Oxford, 1996.

(11) J. Snopek and E. Smolkova-Keulemansova. Cyclodextrins in Analytical Separation Methods In Comprehensive Supramolecular Chemistry, J.L. Atwood, J.E.D. Davies, D.D. MacNicol and F. Vogtle, Eds. Volume 3, Chapter 18, Pergamon / Elsevier: Oxford, 1996.

(12) R.M. Izatt, J.S. Bradshaw, R.L. Bruening, B.J. Tarbet and M.L. Bruening. Selective Separation Using Supported Devices In Comprehensive Supramolecular Chemistry, J.L. Atwood, J.E.D. Davies, D.D. MacNicol and F. Vogtle, Eds. Volume 10, Chapter 1, Pergamon / Elsevier: Oxford, 1996.

(13) D.D. MacNicol, J.J. McKendrick and D.R. Wilson. Chem. Soc. Rev. 7, 66 (1978).

(14) J.E.D. Davies, W. Kemula, H.M. Powell and N.O. Smith. J. Incl. Phenom. 1, 3 (1983).

(15) Topics In Current Chemistry. Volumes 140, 149, E. Weber, Ed. Springer-Verlag: Berlin, 1987, 1988.

(16) Inclusion Compounds Volumes 1-5, J.L. Atwood, J.E.D. Davies and D.D. MacNicol, Eds. Academic Press: London, 1984 and Oxford Press: Oxford, 1991

(17) R. Bishop, Chem. Soc. Rev: 311 (1996).

(18) P. Brunet, M. Simard and J.D. Wuest. J. Am. Chem. Soc. 119, 2737 (1997).

(19) R. Bhyrappa, S.R. Wilson and K.S. Suslick J. Am. Chem. Soc. 119, 8492 (1997).

(20) K. Kobayashi, M. Koyanagi, K. Endo and Y. Aoyama. Chem. Eur. J. 4, 417 (1998). 
(21) K.Biradha, D. Dennis, V.A. MacKinnon, C.V.K. Sharma and M.J. Zaworotko. J. Am. Chem. Soc. 120, 11894 (1998).

(22) C.C. Evans, L. Sukarto and M.D. Ward. J. Am. Chem. Soc. 121, 320 (1999).

(23) K. Kobayashi, T. Shirasaka, A. Sato, E. Horn and N. Furukawa. Angew. Chem. Int. Ed. Engl. 38, 3483 (1999).

(24) S.V. Kolotuchin, P.A. Thiessen, E.E. Fenlon, S.R. Wilson, C.J. Loweth and S.C. Zimmerman. Chem. Eur. J. 2537 (1999).

(25) F. Toda, In Topics in Current Chemistry Vol 140, E. Weber, Ed. Springer-Verlag: London, 1987.

(26) F. Toda, In Advances in Supramolecular Chemistry Vol 2, G.W. Gokel, Ed. JAI Press: London, 1992.

(27) G. Kaupp. Angew. Chem. Int. Ed. Engl. 33, 728 (1994).

(28) A. Mravik, Z. Boscskei, K. Simon, F. Elekes and Z. Izsaki. Chem. Eur. J. 4, 1621 (1998).

(29) M. Gdaniec, M.J. Milewska and T. Polonski. Angew. Chem. Int. Ed. Engl. 38, 392 (1999).

(30) A.D.U. Hardy, D.D. MacNicol, S. Swanson and D.R. Wilson. J. Chem. Soc. Perkin Trans. 2. 999 (1980).

(31) D.H. Brown, R.J. Cross, P.R. Mallinson and D.D. MacNicol. J. Chem. Soc. Perkin Trans. 2. 993 (1980).

(32) Y. Aoyama, K. Endo, K. Kobayashi and H. Masuda. Supramol. Chem. 4, 229 (1995).

(33) K. Endo, T. Sawaki, M. Koyanagi, K. Kobayashi, H. Masuda and Y. Aoyama. $J$. Am. Chem. Soc. 117, 8341 (1995).

(34) K. Endo, T. Ezuhara, M. Koyanagi, H. Masuda and Y. Aoyama. J. Am. Chem. Soc. 119, 499 (1997).

(35) M. Caira, A. Horne. L.R. Nassimbeni and F. Toda. J. Mater. Chem. 8, 1481 (1998).

(36) E. Weber, I. Csoeregh, B. Stensland and M. Czugler. J. Am. Chem. Soc. 106, 3297 (1084).

(37) E. Weber, I. Csoeregh, J. Ahrendt, S. Finge and M. Czugler. J. Org. Chem. 5831 (1988).

(38) S.A. Bourne, L.R. Nassimbeni and F. Toda. J. Chem. Soc. Perkin Trans. 2. 1335 (1991).

(39) A.M. Pivovar, K.T. Holman and M.D. Ward. Chem. Mater. 13, 3018 (2001).

(40) J. Allemand and R. Gerdil. Acta Cryst. B38, 1473 (1982).

(41) J. Allemand and R. Gerdil. Acta Cryst. B38, 2312 (1982).

(42) R. Arad-Yellin, B.S. Green, M. Knossow and G. Tsoucaris. J. Am. Chem. Soc. 105, 4561 (1983).

(43) P. Briozzo, T. Kondo, M. Miyata and K. Miki. Acta Cryst. B52, 728 (1996).

(44) D. Lawton and H.M. Powell. J. Chem. Soc. 2339 (1958).

(45) E. Weber, I. Csoregh and B.M. Czugler. J. Am. Chem. Soc. 106, 3297 (1984).

(46) M.P. Bym, C.J. Curtis, Y. Hsiou, S.I. Khan, P.A. Sawin, S.K. Tendick, A. Terzis and C.E. Strouse. J. Am. Chem. Soc. 115, 9480 (1993).

(47) M.D. Hollingsworth, D.B. Santarsiero and K.D.M. Harris. Angew. Chem. Int. Ed. Engl. 33, 649 (1994). 
(48) A.T. Ung, D. Gizachew, R. Bishop, M.L. Scudder, I.G. Dance and D.C. Craig. J. Am. Chem. Soc. 117, 8745 (1995).

(49) J. A. Swift, A.M. Pivovar, A.M. Reynolds and M.D. Ward. J. Am. Chem. Soc. 120, 5887 (1998).

(50) M. Gdaniec, B.T. Ibragimov and S.A. Talipov. Gossypol. In Comprehensive Supramolecular Chemistry, J.L. Atwood, J.E.D. Davies, D.D. MacNicol and F. Vogtle, Eds. Volume 6, Chapter 5. Pergamon / Elsevier: Oxford, 1996.

(51) R. Gerdil. Tri-o-Thymotide and Related Hosts. In Comprehensive Supramolecular Chemistry, J.L. Atwood, J.E.D. Davies, D.D. MacNicol and F. Vogtle, Eds. Volume 6, Chapter 8. Pergamon / Elsevier: Oxford, 1996.

(52) K. Nakano, K. Sada, Y. Kurozumi and M. Miyata. Chem. Eur. J. 7, 209 (2001).

(53) K. Nakano, E. Mochizuki, N. Yasui, K. Morioka, Y. Yamauchi, N. Kanehisa, Y. Kai, N. Yoswathananont, N. Tohnai, K. Sada and M. Miyata. Eur. J. Org. Chem. 2428 (2003).

(54) Glenn Facey, PhD Thesis, Carleton University, Ottawa, Canada, 1991.

(55) R. Gerdil. Tri-o-thymotide Clathrates. In Topics in Current Chemistry. E. Weber, Ed., Volume 140, pp. 71-105. Springer-Verlag: Berlin, 1987.

(56) R. Arad-Yellin, B.S. Green, M. Knossow and G. Tsoucaris. J. Am. Chem. Soc. 105, 4561 (1983).

(57) R. Arad-Yellin, B.S. Green and M. Knossow. J. Am. Chem. Soc. 102, 1157 (1980)

(58) R. Gerdil and J. Allemand. Helv. Chim. Acta. 63, 1750 (1980).

(59) C.H. Kim, S.I. Nishikiori and T. Iwamoto. Chem. Lett. 409 (1995).

(60) S.A. Bourne, L.R. Nassimbeni and F. Toda. J. Chem. Soc. Faraday Trans. 2. $1335(1991)$.

(61) M. Miyata and K. Sada. Deoxycholic Acid and Related Hosts. In Comprehensive Supramolecular Chemistry, J.L. Atwood, J.E.D. Davies, D.D. MacNicol and F. Vogtle, Eds. Volume 6, Chapter 6. Pergamon / Elsevier: Oxford, 1996.

(62) K. Nakano, K. Sada and M. Miyata. J. Chem. Soc. Chem. Commun. 953 (1995).

(63) K. Nakano, K. Sada and M. Miyata. J. Chem. Soc. Chem. Commun. 989 (1996).

(64) K. Nakano, K. Sada and M. Miyata. Progr. Colloid Polym. Sci. 106, 249 (1997).

(65) N. Yoswathananont, K. Sada, M. Miyata, S. Akita and K. Nakano Org. Biomol. Chem. 1, 210 (2003).

(66) E. Giglio. Inclusion Compounds of Deoxycholic Acid In Inclusion Compounds, Volume 2, J.L. Atwood, J.E.D. Davies and D.D. MacNicol, Eds. Academic Press: London, 1984.

(67) P.L. Johnson and J.P. Schaefer. Acta Cryst. B28, 3083 (1972).

(68) E.L. Jones and L.R. Nassimbeni. Acta Cryst. B46, 399 (1990).

(69) M. Miyata, M. Shibakami, W. Goonewardena and K. Takemoto Chem. Lett. 605 (1987).

(70) K. Miki, A. Masui, N. Kasai, M. Miyata, M. Shibakami and K. Takemoto J. Am. Chem. Soc. 110, 6594 (1988).

(71) K. Miki, N. Kasai, M. Shibakami, K. Takemoto, M. Miyata J. Chem. Soc. Chem. Commun. 1757 (1991).

(72) K. Nakano, K. Sada and M. Miyata Chem. Lett. 137 (1994).

(73) K. Nakano, K. Sada and M. Miyata J. Chem. Soc. Chem. Commum. 953 (1995).

(74) K. Nakano, K. Sada and M. Miyata Mol. Cryst. Liq. Cryst. 276, 129 (1996). 
(75) M. Shibakami and A. Sekiya. J. Chem. Soc. Chem. Commun. 429 (1994).

(76) M. Shibakami and A. Sekiya. J. Inclus. Phenom. 18, 397 (1994).

(77) M. Shibikami, M. Tamura and A. Sekiya. J. Inclus. Phenom. 22, 155 (1995).

(78) M. Shibikami, M. Tamura and A. Sekiya. J. Inclus. Phenom. 22, 299 (1995).

(79) M. Shibikami, M. Tamura and A. Sekiya. J. Am. Chem. Soc. 117, 4499 (1995).

(80) M.R. Caira, L.R. Nassimbeni and J.L. Scott. J. Chem. Soc. Chem. Commun. 612 (1993).

(81) M.R. Caira, L.R. Nassimbeni and J.L Scott. J. Chem. Soc. Perkin Trans. 2. 623 (1994).

(82) M.R. Caira, L.R. Nassimbeni and J.L. Scott. J. Chem. Soc. Perkin Trans. 2. 1403 (1994).

(83) J.L. Scott. Supramol. Chem. 7, 201 (1996).

(84) M. Gdaniec and T. Polonski. J. Am. Chem. Soc. 120, 7353 (1998).

(85) M. Gdaniec, M. Milewska and T. Polonski. Angew. Chem. Int. Ed. Engl. 38, 392 (1999).

(86) P. Dastidar. Cryst. Eng. Commun. 8 (2000). 


\section{CHAPTER XI}

Physicochemical characterization of tris (5-acetyl-3thienyl) methane inclusion compounds with $\alpha, \omega-$ dichloroalkane guests ( $n=1$ to 10$)$ : A combined TGA, ${ }^{13} \mathrm{C}$ CP/MAS NMR, powder X-ray diffraction and DSC study.

Abstract

Introduction

Experimental Results
(A) Thermogravimetric Analysis
(B) ${ }^{13} \mathrm{C}$ CP/MAS NMR
(C) Powder X-Ray Diffraction
(D) Differential Scanning Calorimetry
231
234
237
242

Discussion

(E) Urea

(F) Cyclodextrins

(G) 2,2,6,6-tetramethyl-4-oxopiperidine-1-oxyl 256

(H) Graphite

Concluding remarks

References
228

228

230

244

256

258

260

261 


\begin{abstract}
Various physicochemical characterization techniques were used to study tris (5-acetyl-3thienyl) methane (TATM) inclusion compounds with $\alpha, \omega$-dichloroalkane guests ( $n=1$ to 10). Thermogravimetric analysis (TGA) experiments allowed a determination of host guest stoichiometric ratios. We determined that the smallest size guests $(n=1$ to 4$)$ form 2H:1G TATM inclusion compounds, the intermediate size guests $(n=5$ to 6$)$ form $3 \mathrm{H}: 1 \mathrm{G}$ TATM inclusion compounds, while the largest size guests $(n=8$ to 10$)$ form 4H:1G TATM inclusion compounds. From the multiplicity of the host signals in the ${ }^{13} \mathrm{C}$ CP/MAS NMR spectrum, we were able to determine the number of host molecules in the asymmetric unit of each inclusion compound. For $n=1$ to 4 , there is 1 TATM molecule in the asymmetric unit, for $n=5$ to 6 , there are 3 TATM molecules in the asymmetric unit, while for $n=8$ to 10 , there are 2 TATM molecules in the asymmetric unit. Powder $\mathrm{X}$-ray diffraction experiments were conducted to determine if any of the nine guests form isostructural TATM inclusion compounds. Only 1,9-dichlorononane and 1,10dichlorodecane form isostructural TATM inclusion compounds. This behaviour is compared with isostructuralism amongst previously studied TATM - alkyl halide guest systems. DSC experiments were performed to determine the relationship between the size of guest and the thermal properties of the inclusion compound. We found a direct relationship between the melting temperature and the heat of fusion.
\end{abstract}

\title{
INTRODUCTION
}

Intermolecular interactions are fundamentally responsible for the formation and crystallization of a particular supramolecular assembly, and are generally found between atoms or molecules that are not directly bonded or linked by a conjugated framework [13]. Some of these interactions define the shapes and sizes of molecules, and are therefore of great significance in supramolecular construction. It is well known that almost all intermolecular forces in organic crystals are electronically mediated. Examples include: (1) non-directional (e.g. dispersion) forces [4-6] - They constitute a major proportion of the crystal energy because all portions of the molecule(s) are involved in them. (2) directional forces $[7-11]$ - The most important directional interaction responsible for supramolecular assembly is strong hydrogen bonding (e.g. $\mathrm{O}-\mathrm{H} \cdots \mathrm{O}, \mathrm{N}-\mathrm{H} \cdots \mathrm{O}$ etc.) 
(3) weakly directional forces - Examples include weak hydrogen bonding [12-14] (e.g. $\mathrm{C}-\mathrm{H} \cdots \mathrm{O})$, halogen atom interactions $[15-17]$, sulfur atom interactions [18-20], etc.

Thus, it must be emphasized that real supramolecular crystal structures are the result of compromises between interactions of different strengths, directionalities and distance dependent characteristics $[\mathbf{2 1 , 2 2 ]}$. While the broad features of a crystal structure may be attributed to a single type of interaction, be it van der Waals, hydrogen bonding or whatever, a significant control of the secondary and tertiary features of a structure requires one to be able to simultaneously manipulate strong and weak intermolecular interactions $[23,24]$. This is a difficult and subtle task, difficult since the present knowledge in the area of weak interactions is still not quite satisfactory or complete, and subtle because the balancing of these interactions is more of an art rather than a science $[25,26]$.

In a previous work (see chapter VIII) we performed a similar physicochemical characterization of TATM inclusion compounds with alkyl halide guests, again using a multi-technique approach. The strategy employed was to study guests with the same hydrocarbon backbone but with different halogen substituents $(\mathrm{F}, \mathrm{Cl}, \mathrm{Br}, \mathrm{I})$ at selected sites on that backbone. From the multiplicities of the host signals in the ${ }^{13} \mathrm{C} C P / \mathrm{MAS}$ NMR spectra, the number of TATM molecules in the crystallographic asymmetric unit was determined. Powder X-ray diffraction experiments allowed us to investigate structural isomorphism of TATM inclusion compounds with different guests. In most cases, TATM forms isomorphic structures with guests that have the same hydrocarbon backbone, but with different halogen substituents. This was illustrated in the case of cyclohexyl halide and methylene halide guests. On the other hand, TATM will form one type of structure with a guest that has smaller halogen substituents (e.g. $\mathrm{F}, \mathrm{Cl}, \mathrm{Br}$ ), but a completely different structure with a guest that has larger halogen substituents (e.g. I). This was illustrated in the case of monohalobenzene, 2-halobutane and 1,3-dihalopropane guests. Differential scanning calorimetry experiments allow one to investigate thermal stabilities of inclusion compounds with different guests. The enthalpy of decomposition data show that TATM forms inclusion complexes that have greater $\Delta_{\text {fus }} H$ with guests that have smaller, more electronegative halogen substituents (for a given hydrocarbon backbone), and $\Delta_{\text {fus }} H$ decreases as the size of the halogen substituent increases. 
In this study, we chose to perform a systematic study of guests that contain two chlorine atoms at either end of a hydrocarbon chain, with the length of the chain increasing from 1 carbon (dichloromethane) to 10 carbons (1,10-dichlorodecane). The nine guests studied appear in Figure XI-1. We wish to see how the properties of the TATM inclusion compounds change as the length of the hydrocarbon chain of the guest increases. By choosing guests that are similar in structure, we hope to eliminate extraneous complications in the interpretation of our data, a notable difference between the guests being the length of the hydrocarbon chain between the chlorine atoms.

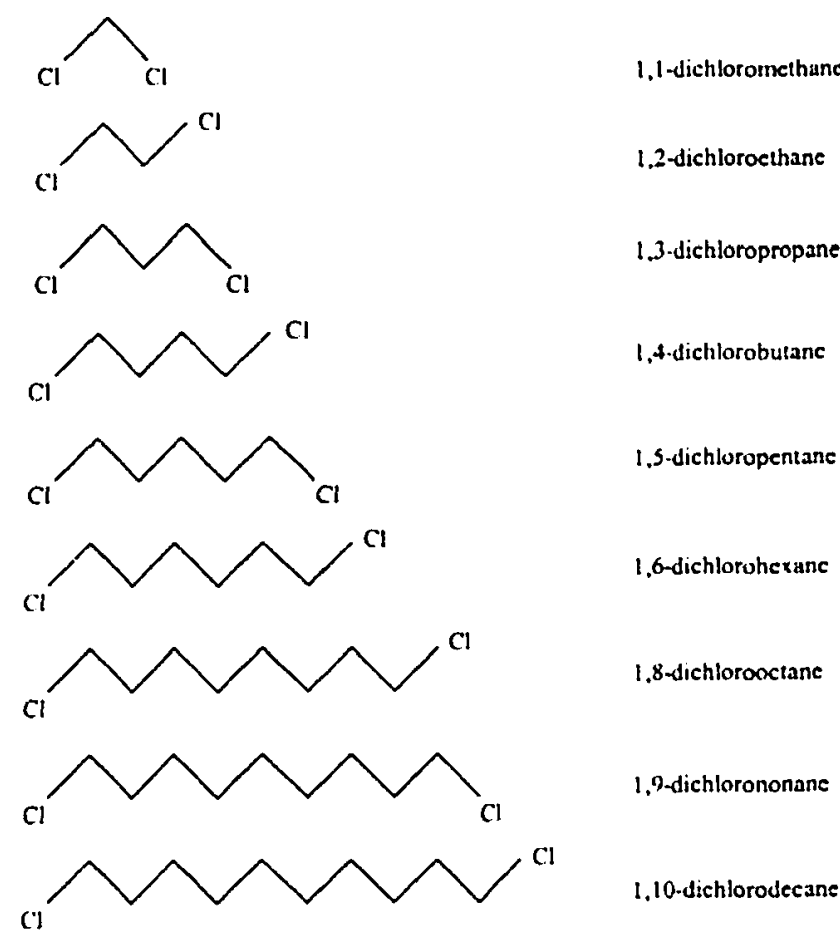

\section{EXPERIMENTAL}

Commercially available reagants (Aldrich) were used as received. (To the best of our knowledge, 1,7-dichloroheptane is not commercially available.) The inclusion compounds were obtained by slow cooling (recrystallization) of hot solutions of $500 \mathrm{mg}$ of the TATM / ethanol inclusion compound in approximately $5 \mathrm{~mL}$ of guest solvent, followed by vacuum filtration. 


\section{RESULTS}

(A) Thermogravimetric Analysis (TGA)

TGA experiments were performed on TATM inclusion compounds with the nine different guests depicted in Figure XI-1. We wished to determine the stoichiometric ratio (host:guest) for each inclusion compound based on the percent guest loss between room temperature and approximately $225^{\circ} \mathrm{C}$. At higher temperatures, the host TATM residue starts to decompose. The TGA curves for the four TATM inclusion compounds with the smallest size guests ( $n=1$ to 4 ) appear in Figure XI-2. In these plots, the percent guest loss between room temperature and $225^{\circ} \mathrm{C}-250^{\circ} \mathrm{C}$ is indicated.
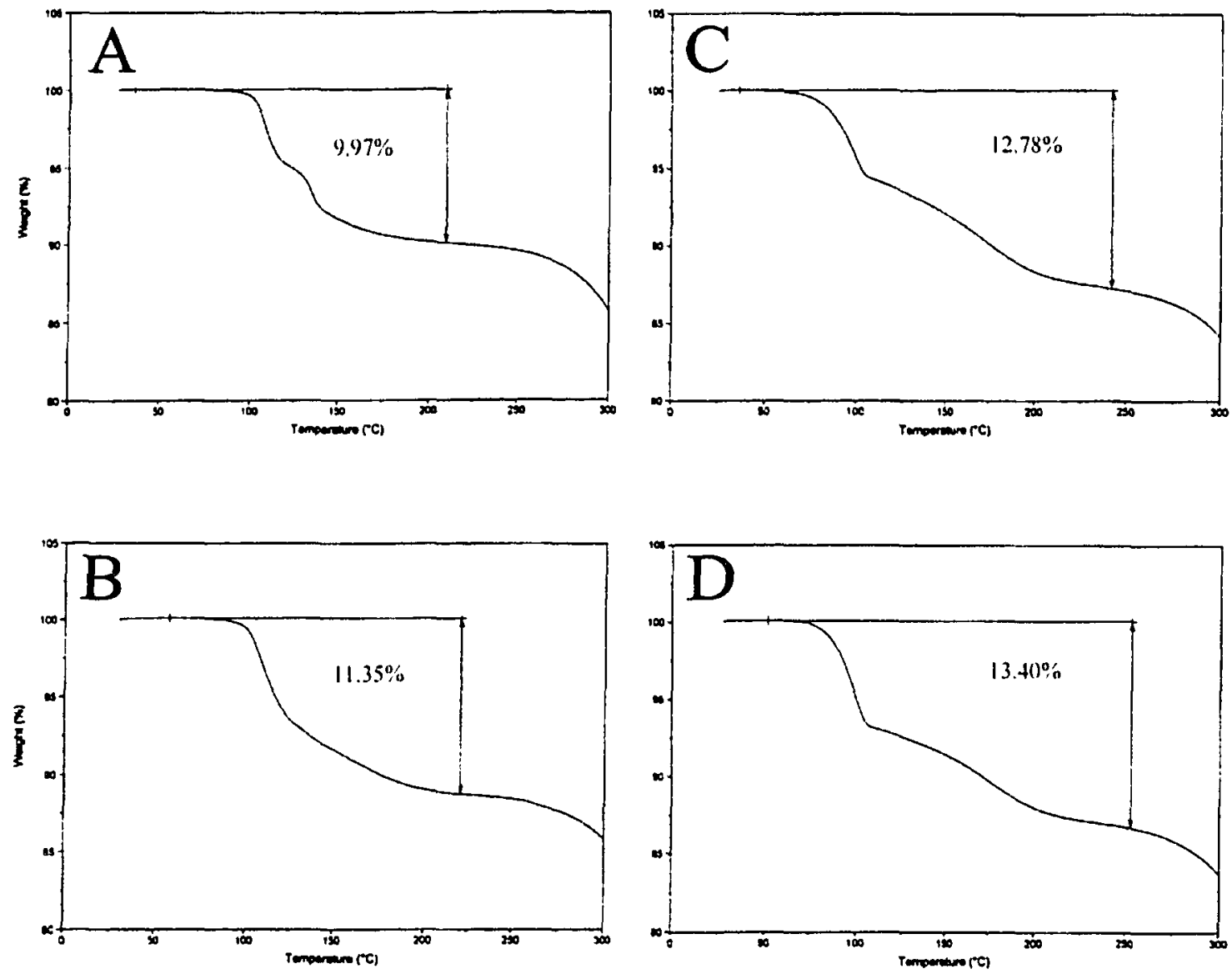

Figure XI-2: TGA curves of the four smallest size $\alpha, \omega$-dichloroalkane/TATM guests. The percent guest loss between room temperature and $\sim 250^{\circ} \mathrm{C}$ is indicated on each TGA curve: (A) 1,1-dichloromethane (9.97\%) (B) 1,2-dichloroethane (11.35\%) (C) 1,3-dichloropropane (12.78\%) and (D) 1,4-dichlorobutane (13.40\%) 
In these plots, one can see two stages of thermal guest loss. Taking 1,3dichloropropane / TATM (Figure XI-2C) as an example, the guest starts to desorb at about $60^{\circ} \mathrm{C}$. An initially rapid mass loss with respect to temperature, characterized by a steep slope $(\mathrm{dM} / \mathrm{dT})$, occurs between $75^{\circ} \mathrm{C}$ and about $100^{\circ} \mathrm{C}$. Then, at about $100^{\circ} \mathrm{C}$, there is an inflection point in the curve, followed by a much slower mass loss at higher temperatures, characterized by a smaller slope $(\mathrm{dM} / \mathrm{dT})$. We believe that this behaviour is related to the change in surface area of the sample when it melts. Before the sample melts at about $100^{\circ} \mathrm{C}$, it exists as a polycrystalline powder, with a large effective surface area, so there are more extensive desorption pathways. This results in a large rate of guest loss. On the other hand, after the sample melts, its effective surface area decreases dramatically, as it exists as a molten mass. Therefore the rate of guest loss at higher temperatures occurs much more slowly. This hypothesis, which would be supported by particle size studies, is also strengthened by the fact that the TGA inflection point temperatures for all nine of the present inclusion compounds (not tabulated here) correspond very well with the melting temperatures determined by DSC (see section D). For example, the TGA inflection point temperature of 1,3-dichloropropane / TATM is about $100^{\circ} \mathrm{C}$, while its DSC melting temperature is about $106^{\circ} \mathrm{C}$. The slight difference may be due to the fact that a slight loss of guest in the open TGA pan lowered the melting point below that of the stoichiometric compound.

In Table XI-I we have compiled the TGA data from all nine inclusion compounds. The experimental percent guest loss for each inclusion compound appears in column 2. In the next five columns, we calculated the expected mass loss for five different host:guest stoichiometric ratios, from $1 \mathrm{H}: 1 \mathrm{G}$ to $5 \mathrm{H}: 1 \mathrm{G}$, based on the molecular weights of the guests and TATM. As can be seen in the table, the experimental guest loss for the first four guests ( $n=1$ to 4 ) correlate very well with $2 \mathrm{H}: 1 \mathrm{G}$, with no correlation with either $1 \mathrm{H}: 1 \mathrm{G}$ or $3 \mathrm{H}: 1 \mathrm{G}$. For the two intermediate size guests $(n=5$ to 6$)$ the experimental guest loss agrees well with that calculated for a $3 \mathrm{H}: 1 \mathrm{G}$ stoichiometric ratio. Finally, for the largest size guests ( $n=8$ to 10 ), their experimental guest losses correlate with that calculated for $4 \mathrm{H}: 1 \mathrm{G}$, with no correlation with either $3 \mathrm{H}: 1 \mathrm{G}$ or $5 \mathrm{H}: 1 \mathrm{G}$. 
Alternatively, working backwards, in the final column of Table XI-1, we tabulate the exact $H: G$ stoichiometric ratio for each of the nine inclusion compounds to three significant figures, based on the experimental guest loss tabulated in the second column of the the table. As can be seen in Table XI-1, the three smallest size guests (dichloromethane, 1,2-dichloroethane and 1,3-dichloropropane) are all within 1\% of being exactly $2 \mathrm{H}: 1 \mathrm{G}$. For 1,4-dichlorobutane, however, there is a slight excess of host above and beyond being exactly $2 \mathrm{H}: 1 \mathrm{G}$, about $5 \%$. The two intermediate size guests (1,5-dichloropentane and 1,6-dichlorohexane) form TATM inclusion compounds with a stoichiometric ratio of almost exactly $3 \mathrm{H}: 1 \mathrm{G}$, within $0.3 \%$ or less. Finally, the three largest size guests (1,8-dichlorooctane, 1,9-dichlorononane and 1,10-dichlorodecane) form TATM inclusion compounds with a slight excess of guest (between $3.0 \%$ and $5.5 \%$ ), which may perhaps be because these guests, as pure compounds, are fairly involatile. A small amount of guest may be tenaciously sitting freely on the surface of the crystal, refusing to evaporate.

Table XI-1: Thermogravimetric data for the nine TATM inclusion compounds with the $\alpha, \omega$-dichloroalkane guests depicted in Figure XI-1.

\begin{tabular}{llllllll} 
Guest & \% Guest $\mathrm{loss}^{\mathrm{n}}$ & $1 \mathrm{H}: 1 \mathrm{G}^{\mathrm{b}}$ & $2 \mathrm{H}: 1 \mathrm{G}^{\mathrm{b}}$ & $3 \mathrm{H}: 1 \mathrm{G}^{\mathrm{b}}$ & $4 \mathrm{H}: 1 \mathrm{G}^{\mathrm{b}}$ & $5 \mathrm{H}: 1 \mathrm{G}^{\mathrm{b}}$ & S.R : \\
\hline 1,1-dichloromethane & 9.97 & 17.94 & $\underline{\mathbf{9 . 8 5}}$ & 6.79 & & & $1.97: 1$ \\
1.2-dichloroethane & 11.35 & 20.30 & $\underline{\mathbf{1 1 . 3 0}}$ & 7.83 & & & $1.99: 1$ \\
1,3-dichloropropane & 12.78 & 22.53 & $\underline{\mathbf{1 2 . 6 9}}$ & 8.84 & & & $1.98: 1$ \\
1,4-dichlorobutane & 13.40 & 24.64 & $\underline{\mathbf{1 4 . 0 5}}$ & 9.83 & & & $2.11: 1$ \\
& & & & & & & \\
1,5-dichloropentane & 10.76 & & 15.36 & $\underline{\mathbf{1 0 . 7 9}}$ & 8.32 & & $3.01: 1$ \\
1,6-dichlorohexane & 11.74 & & 16.64 & $\underline{\mathbf{1 1 . 7 4}}$ & 9.07 & & $3.00: 1$ \\
& & & & & & & \\
1,8-dichlorooctane & 10.84 & & & 13.58 & $\underline{\mathbf{1 0 . 5 4}}$ & 8.61 & $3.88: 1$ \\
1,9-dichlorononane & 11.63 & & & 14.47 & $\underline{\mathbf{1 1 . 2 6}}$ & 9.21 & $3.86: 1$ \\
1,10-dichlorodecane & 12.56 & & & 15.34 & $\mathbf{1 1 . 9 6}$ & 9.80 & $3.78: 1$ \\
\hline
\end{tabular}

a The experimental guest loss $( \pm 0.01 \%)$ between room temperature and about $250^{\circ}$ for each inclusion compound, in percent of weight at room temperature.

b Calculated percent guest loss $( \pm 0.01 \%)$ for five different stoichiometric ratios, based on the molecular weights of guest and TATM. The closest agreement between calculated and experimental guest loss appears in bold.

c The exact stoichiometric ratio \{to three significant figures and $( \pm 0.01)\}$ of each inclusion compound, based on the experimental guest loss in column 2. 
Thus we see the properties of molecular recognition of host for guest during the crystallization process. The smallest size guests $(n=1$ to 4$)$ require only two TATM molecules to form the inclusion compound. The intermediate size guests $(n=5$ to 6$)$ require three TATM molecules to form the clathrate, while the largest size guests $(n=8$ to 10) require four TATM molecules to form the inclusion compound. These properties of molecular recognition are further elaborated upon in the next section.

${ }^{13} \mathrm{C}$ CP/MAS NMR

The ${ }^{13} \mathrm{C}$ CP/MAS NMR spectra of three selected TATM inclusion compounds appear in Figure XI-3. The guests are 1,3-dichloropropane (Figure XI-3A), 1,5-dichloropentane (Figure XI-3B) and 1,9-dichlorononane (Figure XI-3C). For 1,3-dichloropropane / TATM (Figure XI-3A), the multiplicity of the host carbonyl signal at $190 \mathrm{ppm}$ is three (three singlets), as is the multiplicity of the host methyl signal at $26 \mathrm{ppm}$ (also three singlets). As there are three carbonyl carbons and three methyl carbons in one host TATM molecule, this implies that there is one TATM molecule in the asymmetric unit. Since the stoichiometric ratio for this inclusion compound is $2 \mathrm{H}: 1 \mathrm{G}$, if one assumes that there are two host molecules and one guest molecule in the unit cell, the two host molecules in the unit cell may be related by a symmetry element (perhaps a centre of symmetry), to give one host molecule in the asymmetric unit. Although not presented in this work, the three other $2 \mathrm{H}: 1 \mathrm{G}$ inclusion compounds also show a multiplicity of three for the host carbonyl and host methyl signals, again suggesting there is one host in the asymmetric unit for these inclusion compounds.

For 1,5-dichloropentane / TATM (Figure XI-3B), the multiplicity of the host carbonyl signal at $190 \mathrm{ppm}$ is nine. From lowest to highest field, the individual multiplicities are 2, 1, 4, 2, for a total of nine. The multiplicity of the host methyl signal at $26 \mathrm{ppm}$ is also nine. The individual multiplicities, from lowest to highest field, are 3 , 1, 5, for a total of nine. Therefore, these splitting patterns suggest that there are three host molecules in the asymmetric unit of this inclusion compound. The ${ }^{13} \mathrm{C} \mathrm{CP} / \mathrm{MAS}$ NMR spectrum of the other $3 \mathrm{H}: 1 \mathrm{G}$ TATM inclusion compound (1,6-dichlorohexane guest - not included in the paper) also indicates that there are three host molecules in the asymmetric unit of this inclusion compound. One can envisage a situation for these two 
inclusion compounds where there are three hosts and one guest in the unit cell $(3 \mathrm{H}: 1 \mathrm{G})$, with no symmetry elements present. That is, the contents of the unit cell and the contents of the asymmetric unit are equivalent.
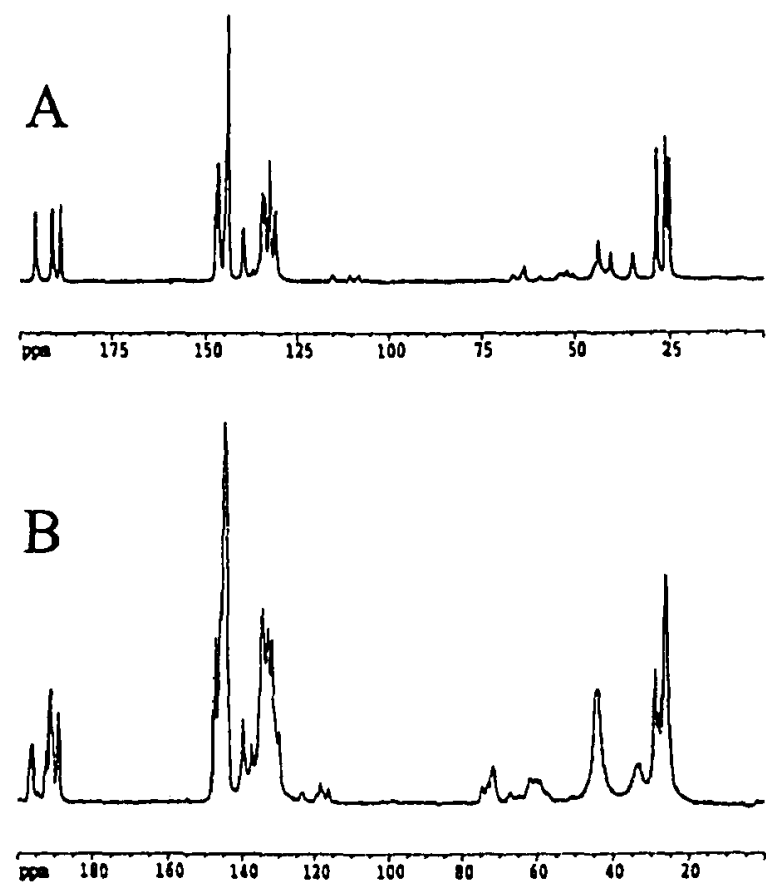

Figure XI-3: ${ }^{13} \mathrm{C}$ CP/MAS NMR spectra of TATM inclusion compounds with three different $\alpha, \omega$-dichloroalkane guests:

(A) 1,3-dichloropropane

(B) 1,5-dichloropentane

(C) 1,9-dichlorononane

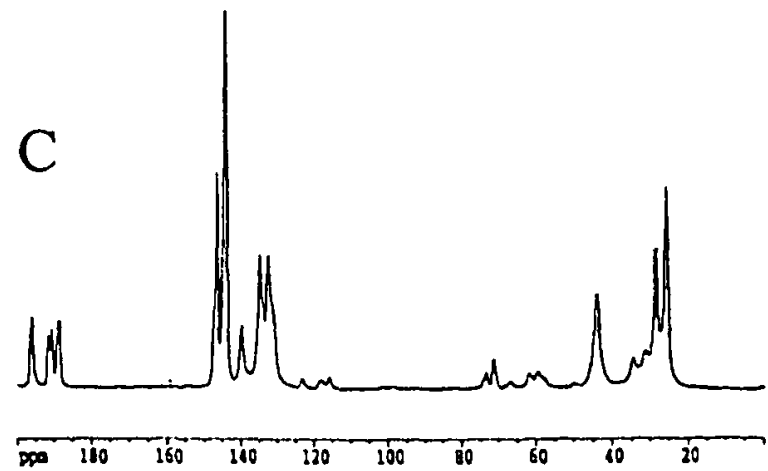

Finally, we present the ${ }^{13} \mathrm{C}$ CP/MAS NMR spectrum of one of the $4 \mathrm{H}: 1 \mathrm{G}$ inclusion compounds, with 1,9-dichlorononane guest (Figure XI-3C). In this case the multiplicity of the host carbonyl signal at $190 \mathrm{ppm}$ is six. From lowest to highest field, the individual multiplicities are 2, 1, 1,2, for a total of six. The multiplicity of the host methyl signal at $26 \mathrm{ppm}$ is also six, from lowest to highest field the individual multiplicities are 2,4 , for a total of six. Therefore, these splitting patterns suggest that there are two host molecules 
in the asymmetric unit of this inclusion compound. Although not depicted in this work, the ${ }^{13} \mathrm{C}$ CP/MAS NMR spectra of 1,8-dichlorooctane / TATM and 1,10-dichlorodecane / TATM again suggest that there are two host molecules in the asymmetric unit for both of these inclusion compounds. If there are four hosts and one guest in the unit cell for these three inclusion compounds, one can imagine a situation where there are two pairs of hosts in the unit cell, and each of these two pairs are related by a symmetry element to give two host molecules in the asymmetric unit. In other words, the content of the asymmetric unit is one-half that of the unit cell.

In Table XI-2 we have compiled the ${ }^{13} \mathrm{C} C \mathrm{CP} / \mathrm{MAS}$ NMR data for all nine inclusion compounds studied. On inspection of this table, we again see the properties of molecular recognition of host for guest during the crystallization process. All four of the smallest size guests $(2 \mathrm{H}: 1 \mathrm{G})$ crystallize with the same number of host molecules in the asymmetric unit, namely one. In addition, the two intermediate size guests $(3 \mathrm{H}: 1 \mathrm{G})$ both crystallize with three host molecules in the asymmetric unit. Finally, all three of the largest size guests $(4 \mathrm{H}: 1 \mathrm{G})$ all crystallize with two host molecules in the asymmetric unit. Thus we see an important correlation between stoichiometric ratio and the contents of the asymmetric unit for TATM inclusion compounds.

Table XI-2: ${ }^{13} \mathrm{C}$ CP/MAS NMR data for the nine TATM inclusion compounds with the $\alpha,(\omega$-dichloroalkane guests depicted in Figure XI-1.

\begin{tabular}{llcc} 
Guest & H:G S.R. & Host carbonyl multiplicity & Number of host in A.U. \\
\hline 1,1-dichloromethane & $2 \mathrm{H}: 1 \mathrm{G}$ & 3 & 1 \\
1,2-dichloroethane & $2 \mathrm{H}: 1 \mathrm{G}$ & 3 & 1 \\
1,3-dichloropropane & $2 \mathrm{H}: 1 \mathrm{G}$ & 3 & 1 \\
1.4-dichlorobutane & $2 \mathrm{H}: 1 \mathrm{G}$ & 3 & 1 \\
& & & 3 \\
1,5-dichloropentane & $3 \mathrm{H}: 1 \mathrm{G}$ & 9 & 3 \\
1.6-dichlorohexane & $3 \mathrm{H}: 1 \mathrm{G}$ & 9 & 2 \\
& & & 2 \\
1,8-dichlorooctane & $4 \mathrm{H}: 1 \mathrm{G}$ & 6 & 2 \\
1,9-dichlorononane & $4 \mathrm{H}: 1 \mathrm{G}$ & 6 & 6 \\
1.10-dichlorodecane & $4 \mathrm{H}: 1 \mathrm{G}$ & 6 & \\
\hline
\end{tabular}

"Host : guest stoichiometric ratio determined from TGA experiments

${ }^{b}$ The multiplicity of the TATM host carbonyl signal in each of the nine ${ }^{13} \mathrm{C} C \mathrm{CP} / \mathrm{MAS}$ NMR spectra (see Figure XI-3). This signal appears at approximately $190 \mathrm{ppm}$.

c The number of host molecules in the asymmetric unit of each TATM inclusion compound, determined from the multiplicity of the host carbonyl signal in column 3 


\section{(C) Powder X-Ray Diffraction}

Our principal goal in performing powder X-ray diffraction studies on these inclusion compounds was to determine if any of the nine inclusion compounds were isostructural. After we performed our TGA and ${ }^{13} \mathrm{C}$ CP/MAS NMR studies on these inclusion compounds, our first inclination was that within each of the three groupings of guest (based on the stoichiometric ratio) we expected to see extensive isostructuralism. This expectation was further fuelled by the fact that within each group, there was the same number of host molecules in the asymmetric unit of each inclusion compound. However, as we will see, these expectations did not bear fruit.

In Figure XI-4, we present the powder X-ray diffraction patterns for the four 2H:IG TATM inclusion compounds ( $n=1$ to 4 ). Although some of the patterns share a few common reflections, a detailed analysis of the peak positions indicates that none of the four powder patterns are identical. In other words, none of the four guests form isostructural TATM inclusion compounds. In Figure XI-5 is depicted the powder diffraction patterns for the TATM inclusion compounds with two intermediate size guests ( $n=5$ to 6). Again, a detailed analysis of peak positions suggests they are not isostructural. An interesting aside is that, as we previously noted, these two inclusion compounds have three host molecules in the asymmetric unit. This is reflected in the much greater number of reflections for these two inclusion compounds (see Figure XI-5) than for either of the other two groups, which have only one or two TATM molecules in the asymmetric unit (see Figures XI-4 and XI-6 respectively). Thus we see correlation

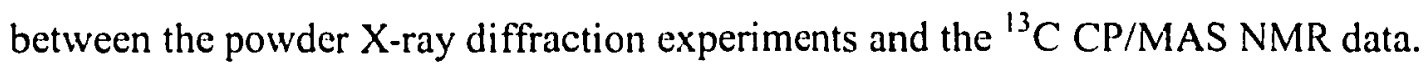

Finally, we present in Figure XI- 6 the powder diffraction patterns for the three largest size guests, which form $4 \mathrm{H}: 1 \mathrm{G}$ inclusion compounds. The diffraction patterns for 1,9-dichlorononane / TATM and 1,10-dichlorodecane / TATM are practically identical, with respect to both peak positions and peak intensities. On the other hand, 1,8dichlorooctane / TATM forms a different type of structure. Thus, for all nine guests, the only ones that are isostructural are 1,9-dichlorononane / TATM and 1,10-dichlorodecane / TATM. 

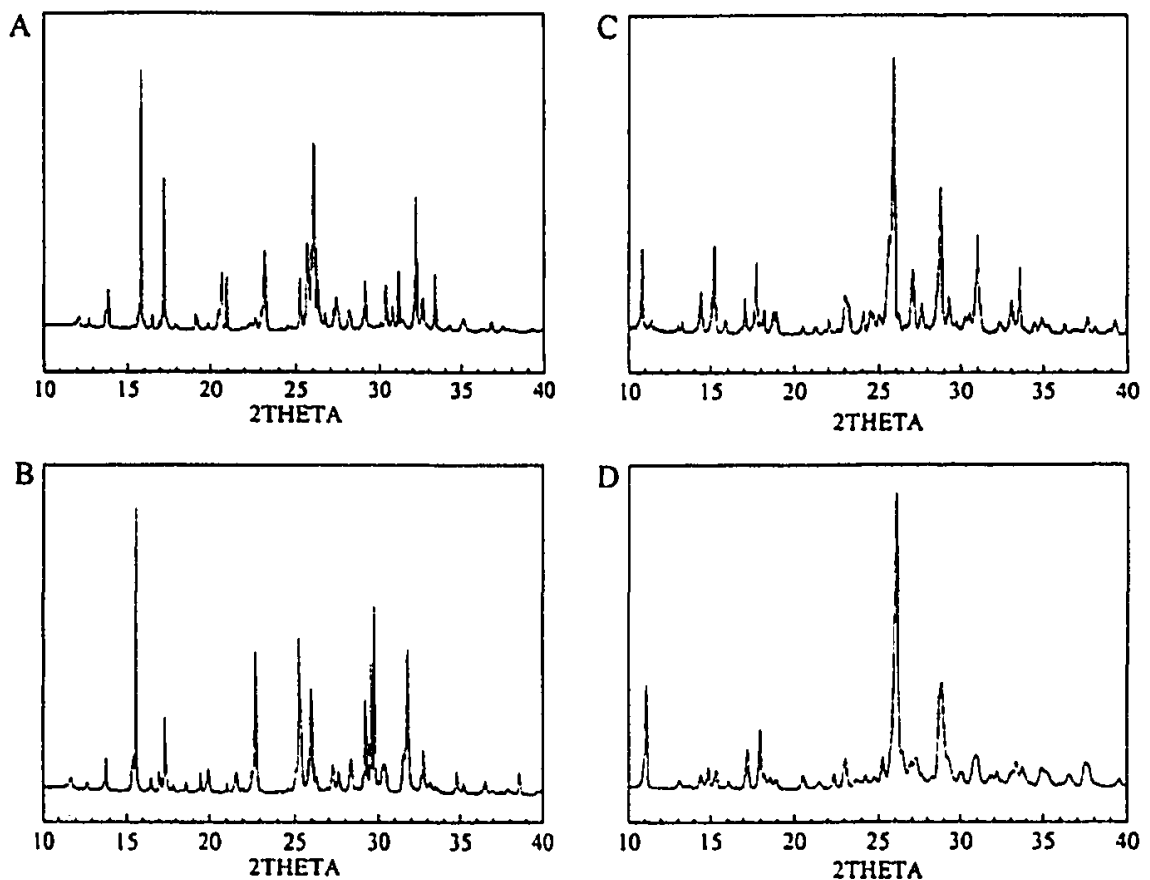

Figure XI-4: PXRD patterns of TATM inclusion compounds with the four smallest size $\alpha$,(1)-dichloroalkane guests: (A) 1,1-dichloromethane (B) 1,2-dichloroethane (C) 1,3dichloropropane and (D) 1,4-dichlorobutane

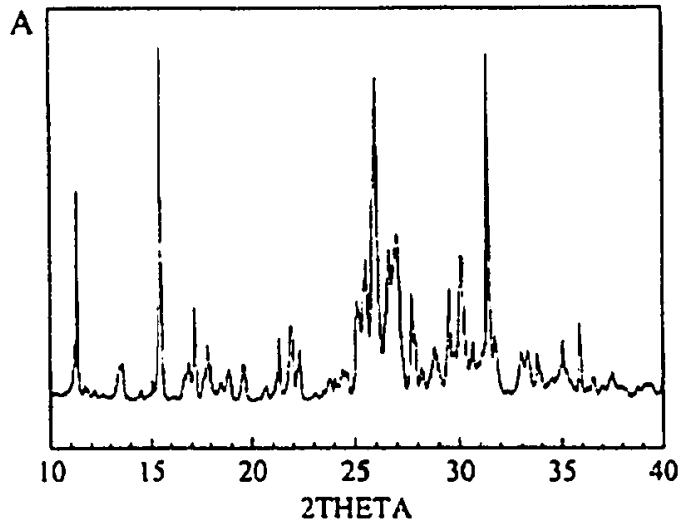

Figure XI-5: PXRD patterns of TATM inclusion compounds with the two intermediate size $\alpha, \omega$-dichloroalkane guests

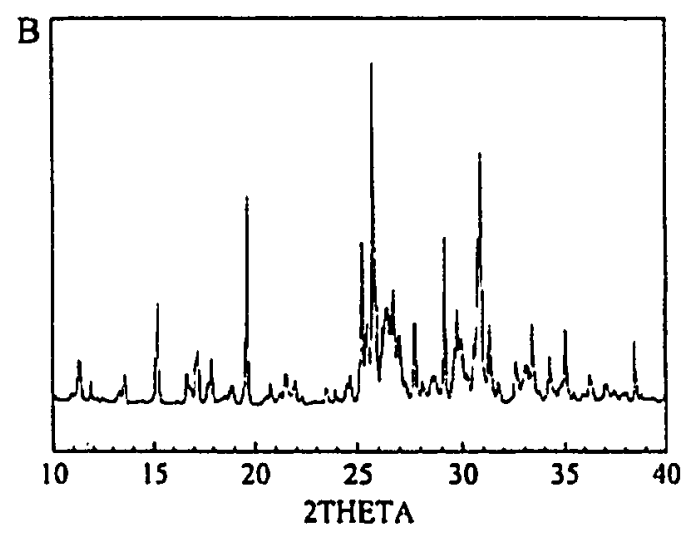
(A) 1,5-dichloropentane
(B) 1,6-dichlorohexane 

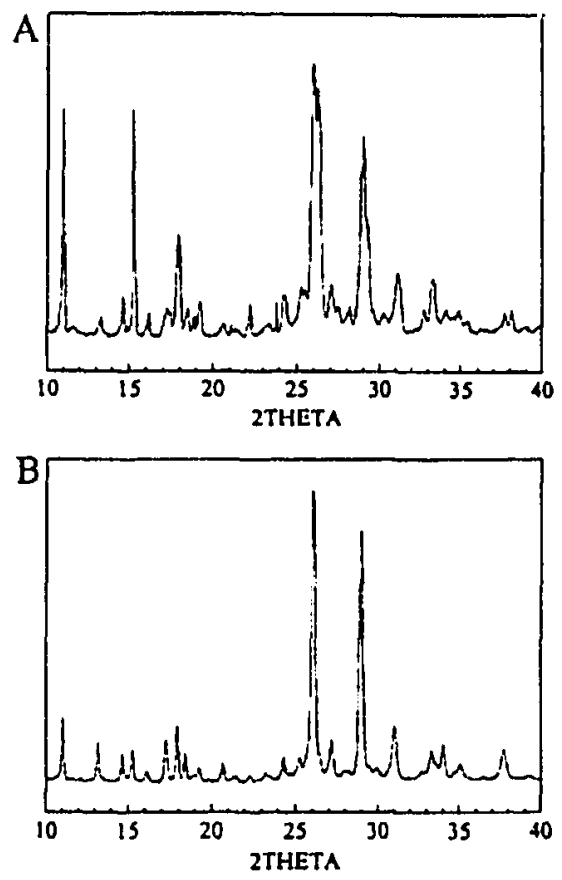

Figure XI-6: PXRD patterns of TATM inclusion compounds with the three largest sized $\alpha, \omega$ dichloroalkane guests
(A) 1,8-dichlorooctane
(B) 1,9-dichlorononane
(C) 1,10-dichlorodecane

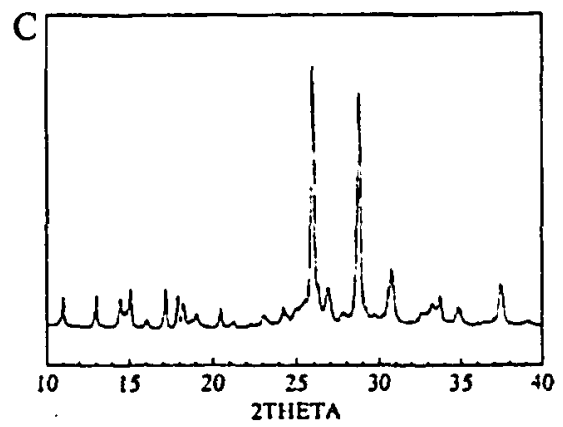

This behaviour may be rationalized by considering the relative percent change in size of the guest on going from a $n$-carbon chain to an $n+1$ carbon chain. In Table XI-3 we tabulate this data, for guests that have the same host:guest stoichiometric ratio. For example, on going from dichloromethane to 1,2-dichloroethane, we are adding one methylene group to a guest that has three backbone atoms ( $2 \mathrm{Cl}$ and $1 \mathrm{C}$ ), so the change in size is $1 / 3 \times 100$, or $33 \%$. For this calculation, we assume that a methylene group and a chlorine atom are roughly similar in size. On the other hand, on going from 1,2dichloroethane to 1,3-dichloropropane, we are adding one methylene group to a guest that has four backbone atoms, so the relative percent increase in size is $1 / 4 \times 100$, or $25 \%$. At the other extreme, on going from 1,9-dichlorooctane to 1,10-dichlorodecane, we are adding a methylene group to a guest that 11 backbone atoms, so the relative percent increase in size in $1 / 11 \times 100$, or $9.1 \%$. 
By considering this approach in conjuction with the powder X-ray diffraction results, we can suggest that there is a minimum percent relative increase in size (in our case $9.1 \%$ ), above which the TATM host can distinguish between two guests that differ in length by one carbon, and subsequently form different structures with the two guests. On the other hand, for percent relative increases in size equal to, or below this value (9.1\%), the TATM host cannot distinguish the difference between the size of the two guests, and subsequently forms isostructural TATM inclusion compounds.

Table XI-3: Relative percent increase in size of the guest $( \pm 1 \%)$ on going from an $n$ carbon chain to an $n+1$ carbon chain, for guests that have the same stoichiometric ratio.

\begin{tabular}{ll} 
Guest & Relative percent size change \\
\hline 1,1-dichloromethane & $33 \%$ \\
1,2-dichloroethane & $25 \%$ \\
1.3-dichloropropane & $20 \%$ \\
1,4-dichlorobutane & \\
1.5-dichloropentane & $14 \%$ \\
1,6-dichlorohexane & \\
1,8-dichlorooctane & $10 \%$ \\
1,9-dichlorononane & $9.1 \%$ \\
1,10-dichlorodecane &
\end{tabular}

This data can be compared with our previous work on TATM with alkyl halide guests (see chapter VIII). In this work, we investigated isostructuralism, again using powder Xray diffraction, for these guests. The guest families, depicted in Figure VIII-1 (p. 121), are the following: methylene halides, 1,3-dihalopropanes, 2-halobutanes, cyclohexyl halides and monohalobenzenes. We systematically varied the halogen substituent(s) ( $F$, $\mathrm{Cl}, \mathrm{Br}, \mathrm{I}$ ) on each hydrocarbon backbone to see whether the change in halogen substituent(s) would prompt the TATM host to form a different structure. As summarized in chapter VIII, there is extensive isostructuralism within each guest family. 
Table XI-4 compiles this data. Guests that form isostructural TATM inclusion compounds are labelled with a common superscript letter.

Now we can compare the powder X-ray diffraction data for the present study and the previous study. In the previous study (chapter VIII), our approach was to choose an invariant hydrocarbon backbone, with varying halogen substituents on that backbone. In the present study, we chose an opposite approach, namely invariant halogen substituents (Cl) with a varying hydrocarbon backbone. There is extensive isostructuralism for the first group, but practically no isostructuralism for the second group. This suggests that during the crystallization process, as far as molecular recognition is concerned, the TATM host cannot distinguish between many of the first group of guests (chapter VIII), but can recognize a difference between the guests of the present study. In other words, the effect of systematically changing the size of the hydrocarbon chain has a much greater effect on the size and shape of the guest than does the effect of changing a halogen substituent on a given hydrocarbon framework.

Table XI-4: A table of TATM guest families indicating the number of TATM molecules in the respective asymmetric units of each, determined from ${ }^{13} \mathrm{C} \mathrm{CP} / \mathrm{MAS}$ NMR spectroscopy; additionally, isostructural TATM inclusion compounds are indicated by a common superscript letter.

\begin{tabular}{|c|c|}
\hline Guest & \# of TATM molecules in the asymmetric unit \\
\hline methylene chloride ${ }^{a}$ & 1 \\
\hline methylene bromide" & $i$ \\
\hline methylene iodide & 1 \\
\hline 1,3-difluoropropane & 1 \\
\hline 1,3-dichloropropane ${ }^{b}$ & 1 \\
\hline 1,3-dibromopropane ${ }^{b}$ & $i$ \\
\hline 2-chlorobutane $e^{c}$ & 2 \\
\hline 2-bromobutane ${ }^{c}$ & 2 \\
\hline 2-iodobutane & 2 \\
\hline benzene $^{d}$ & 2 \\
\hline fluorobenzene $e^{d}$ & 2 \\
\hline chlorobenzene $\mathrm{d}^{\mathrm{d}}$ & 2 \\
\hline bromobenzene $\mathrm{d}^{\mathrm{d}}$ & 2 \\
\hline iodobenzene & 2 \\
\hline cyclohexyl chloride ${ }^{e}$ & 2 \\
\hline cyclohexyl bromide $^{e}$ & 2 \\
\hline cyclohexyl iodide ${ }^{c}$ & 2 \\
\hline
\end{tabular}


(D) Differential Scanning Calorimetry (DSC)

DSC experiments were performed on TATM inclusion compounds with the nine different guests depicted in Figure XI-1. We wished to learn how the melting temperature and the enthalpy of decomposition depend on the size of the guest's methylene spacer unit between the two chlorine atoms. The DSC data appears in Table XI-5. A depiction of the melting endotherms for the TATM inclusion compounds with the four smallest size guests appear in Figure XI-7.

Table XI-5: Melting temperatures, $\Delta_{\text {lus }} H$ and $\Delta_{\text {lus }} S$ for the nine TATM inclusion compounds with $\alpha, \omega$-dichloroalkane guests, determined from DSC experiments.

\begin{tabular}{|c|c|c|c|c|}
\hline Guest & $T_{110}\left( \pm 0.1^{\circ} \mathrm{C}\right)$ & $\Delta_{\operatorname{lu}} \underline{H\left( \pm 1 \mathrm{~J} \mathrm{~g}^{-1}\right)}$ & $\Delta_{\text {fus }} H\left( \pm 1 \mathrm{~kJ} \mathrm{~mol}^{-1}\right)$ & $\Delta_{\mathrm{fu}} S\left( \pm 2 \mathrm{~J} \mathrm{~mol}^{-1} \mathrm{~K}^{-1}\right)$ \\
\hline 1,1-dichloromethane & 134.6 & 107 & 42 & 102 \\
\hline 1,2-dichloroethane & 130.2 & 103 & 40 & 99 \\
\hline 1,3-dichloropropane & 106.5 & 83 & 32 & 85 \\
\hline 1,4-dichlorobutane & 109.7 & 89 & 34 & 91 \\
\hline 1,5-dichloropentane & 117.6 & 91 & 35 & 90 \\
\hline 1,6-dichlorohexane & 122.1 & 92 & 36 & 91 \\
\hline 1,8-dichlorooctane & 126.8 & 95 & 37 & 92 \\
\hline 1,9-dichlorononane & 127.1 & 96 & 37 & 93 \\
\hline 1,10-dichlorodecane & 130.9 & 97 & 38 & 94 \\
\hline
\end{tabular}

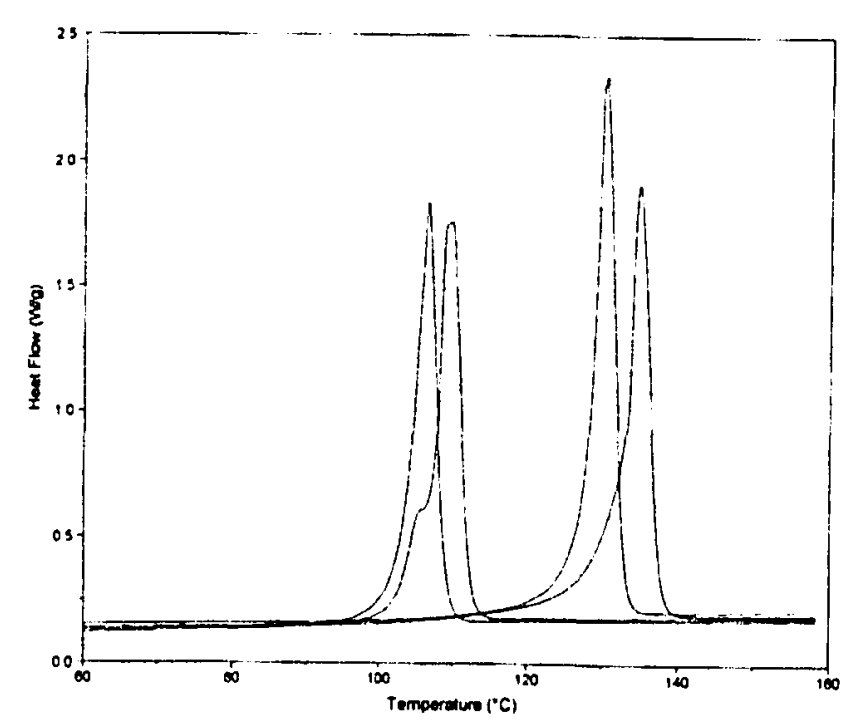

Figure XI-7: DSC melting endotherms for TATM inclusion compounds with the four smallest size $\alpha, \omega$-dichloroalkane guests. From lowest to highest temperature, the TATM guests are: $\{\mathrm{A}\}$ 1,3-dichloropropane $\left(106.5^{\circ} \mathrm{C}\right)\{\mathrm{B}\}$ 1,4-dichlorobutane $\left(109.7^{\circ} \mathrm{C}\right)$, $\{\mathrm{C}\}$ 1,2-dichloroethane $\left(130.2^{\circ} \mathrm{C}\right)\{\mathrm{D}\} 1,1$-dichloromethane $\left(134.6^{\circ} \mathrm{C}\right)$ 
The melting temperature is highest for dichloromethane / TATM $\left(134.6^{\circ} \mathrm{C}\right)$, rapidly decreases to a minimum for 1,3 -dichloropropane $\left(106.5^{\circ} \mathrm{C}\right)$, and then gradually increases with the size of guest to reach a local maximum for 1,10-dichlorodecane / TATM $\left(130.9^{\circ} \mathrm{C}\right)$. A graph of the dependence of the melting temperature on the carbon chain length of the guest appears in Figure XI-8.

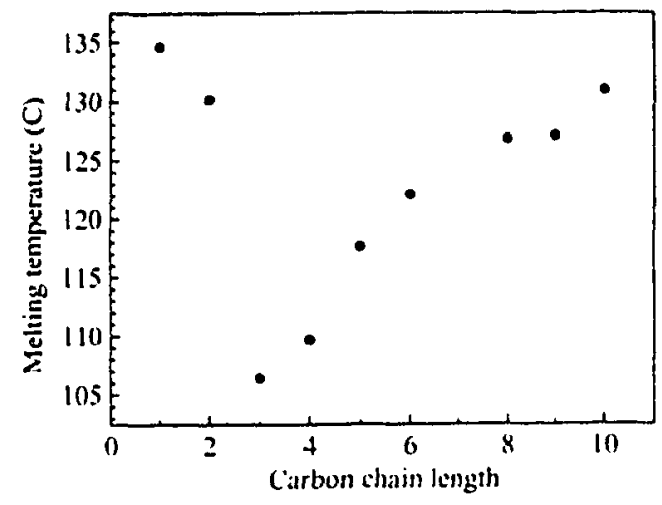

Figure XI-8: A plot of melting temperature against guest carbon chain length for $\alpha, \omega-$ dichloroalkane/TATM inclusion compounds.

The heat of fusion data shows a behaviour similar to that of the melting temperature. It is highest for dichloromethane / TATM $\left(42 \mathrm{~kJ} \mathrm{~mol}^{-1}\right)$, decreases rapidly to a minimum for 1,3-dichloropropane / TATM ( $\left.32 \mathrm{~kJ} \mathrm{~mol}^{-1}\right)$, and then gradually increases with the size of the guest to reach a local maximum for 1,10-dichlorodecane/TATM (38 kJ mol $\left.{ }^{-1}\right)$. A graph of the dependence of the heat of fusion on the guest's carbon chain length appears in Figure XI-9.

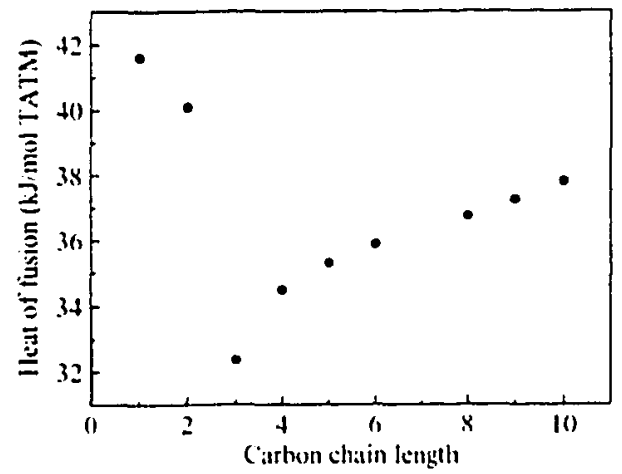

Figure XI-9: A plot of heat of fusion against guest carbon chain length for $\alpha, \omega$ dichloroalkane/TATM inclusion compounds.

In Figure XI-10 is a graph of the heat of fusion plotted against the melting temperature, with a best fit linear regression line. From this graph, one may see that the TATM inclusion compounds with a low melting temperature have a low heat of fusion, and that TATM inclusion compounds with a high melting temperature have a high heat of fusion. This may be perhaps because of the insignificant variation in $\Delta_{\text {tus }} S$, which varies 
from 85-102 $\mathrm{J} \mathrm{mol}^{-1} \mathrm{~K}^{-1}$, as depicted in Table XI-5. These $\Delta_{\text {fus }} S$ values fall in the "typical" range of $\Delta_{\text {fus }} S$ for molecular crystals of $90 \mathrm{~J} \mathrm{~mol}^{-1} \mathrm{~K}^{-1}$ as quoted by Brown and Brown [27]. Finally, we state that the TATM melting process is not reversible, as samples cooled from high temperature to not display an exotherm corresponding to inclusion compound formation.

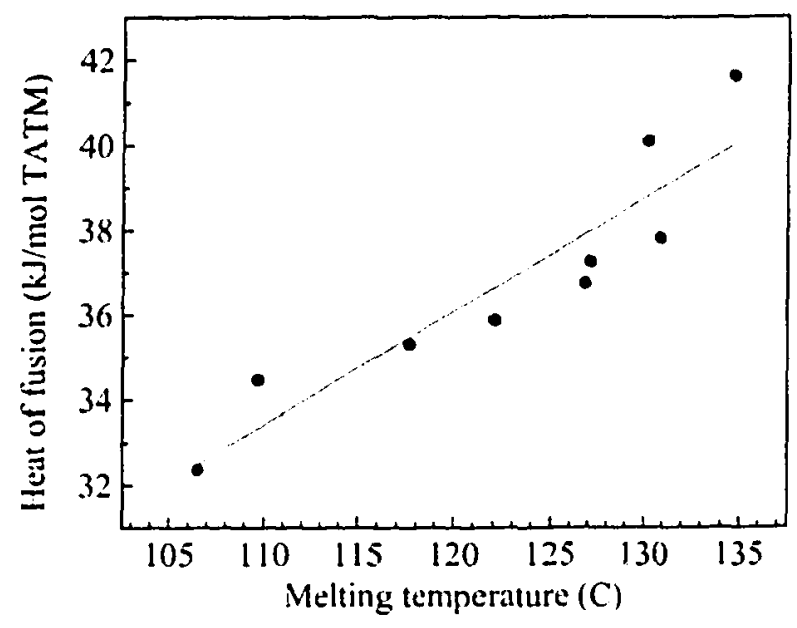

Figure XI-10: A plot of heat of fusion against melting temperature for $\alpha, \omega$ dichloroalkane/TATM inclusion compounds, with a best-fit linear regression line.

\section{DISCUSSION}

(E) Urea

In "conventional" urea inclusion compounds [28-30], the urea molecules form a hydrogen-bonded crystal structure (see Figure XI-11), which contains linear, parallel tunnels. The guest molecules are densely packed along these tunnels. The host structure is hexagonal $(P 6,22$ or $P 6522: a=b \sim 8.2 \AA, c \sim 11.0 \AA)$ at room temperature, and the effective tunnel diameter ranges from $5.5 \AA$ to $5.8 \AA$ at various points in the tunnel [31]. The urea tunnel structure is formed only with guest molecules based on a sufficiently long $n$-alkane chain, with only a limited degree of substitution of the chain permitted. Examples of suitable guests include n-alkanes, $n$-haloalkanes, $n$-alcohols, carboxylic acids, etc [32]. In general, molecules containing benzene rings or cyclohexane rings are not appropriate as guest molecules in urea. The urea tunnel collapses if the guest is removed, and recrystallizes as "pure" solid urea, the structure of which does not contain empty tunnels $[33,34]$. 

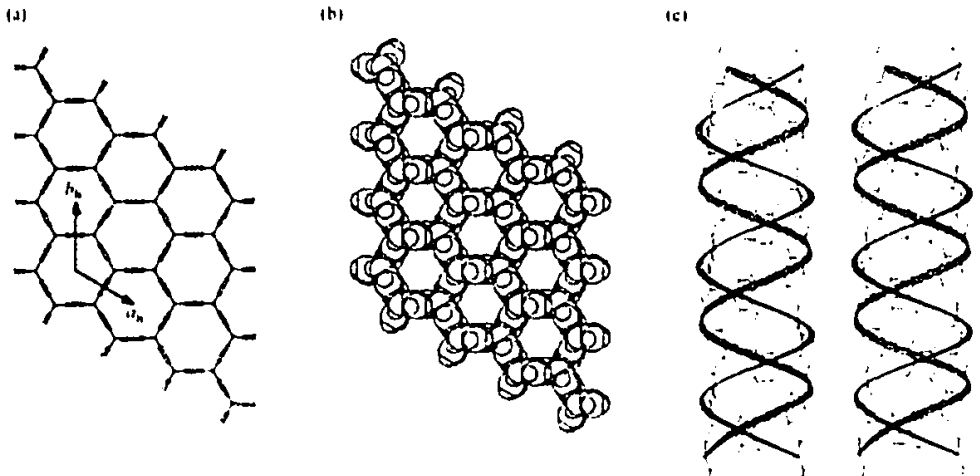

Figure XI-11: (a) Stick representation of the host structure of a conventional urea inclusion compound viewed down the channel axis. (b) An equivalent view showing van der Waals radii of the host molecules. (c) A view showing the ribbon structure of the host channel, which repeats every six urea molecules [28].

The results presented above can be compared with a publication [35] concerning DSC studies on urea - fatty acid inclusion complexes $(\mathrm{C} 10-\mathrm{C} 30)$. In this study, the authors measured host:guest stoichiometric ratios, melting temperatures and heats of fusion for these urea complexes. Their data appear in Table XI-6. As can be seen in the table, the host:guest stoichiometric ratio increases steadily with the size of guest. They determined the guest acid content by titrating the inclusion compounds against a standard $\mathrm{NaOH}$ solution. This data correlates well with our data, in that more host molecules are needed to form the inclusion compound as the size of the guest increases.

Table XI-6: Host-guest stoichiometric ratios, melting temperatures and heat of decomposition data for urea - fatty acid inclusion compounds (from ref. [35] )

\begin{tabular}{llll} 
Acid guest & $\mathrm{H}: \mathrm{G}$ ratio & $T_{\mathrm{m}}\left( \pm 1^{\circ} \mathrm{C}\right)$ & $\left.\Delta_{\mathrm{fu}} \underline{H( \pm 1 \mathrm{~kJ} \mathrm{~mol}}{ }^{-1}\right)$ \\
\hline decanoic acid (C10) & $8.18: 1$ & 105 & 163 \\
dodecanoic acid (C12) & $9.76: 1$ & 113 & 206 \\
tetradecanoic acid (C14) & $11.0: 1$ & 121 & 260 \\
hexadecanoic acid (C16) & $12.2: 1$ & 125 & 307 \\
heptadecanoic acid (C17) & $13.8: 1$ & 126 & 302 \\
octadecanoic acid (C18) & $14.2: 1$ & 129 & 307 \\
eicosanoic acid (C20) & $14.5: 1$ & 130 & 325 \\
docosanoic acid (C22) & $15.6: 1$ & 131 & 365 \\
tricosanoic acid (C23) & $17.1: 1$ & 133 & 414 \\
tetracosanoic acid (C24) & $18.8: 1$ & 135 & 433 \\
octacosanoic acid (C28) & $23.7: 1$ & 135 & 436 \\
triacontanoic acid (C30) & $25.4: 1$ & 136 & 490 \\
\hline
\end{tabular}


The melting temperature and heat of fusion for the urea inclusion compounds both increase steadily with the size of guest, going from a minimum for $\mathrm{Cl} 0$ - decanoic acid $\left(T_{\mathrm{m}}=105^{\circ} \mathrm{C}, \Delta_{\mathrm{fus}} H=163 \mathrm{~kJ} \mathrm{~mol}^{-1}\right)$ to a maximum for $\mathrm{C} 30-$ triacontanoic acid $\left(T_{\mathrm{m}}=\right.$ $136^{\circ} \mathrm{C}, \Delta_{\text {fus }} H=490 \mathrm{~kJ} \mathrm{~mol}^{-1}$ ). This agrees partially with the results of our study, with the exception of the two smallest size guests dichloromethane and 1,2-dichloroethane. In Figure XI-12 we plot the heat of fusion against the melting temperature for the urea fatty acid complexes, taken from reference [35]. We see a very similar behaviour compared to our results, in that urea inclusion compounds with lower melting temperatures have a smaller heat of fusion, while urea inclusion compounds with higher melting temperatures have a larger heat of fusion.

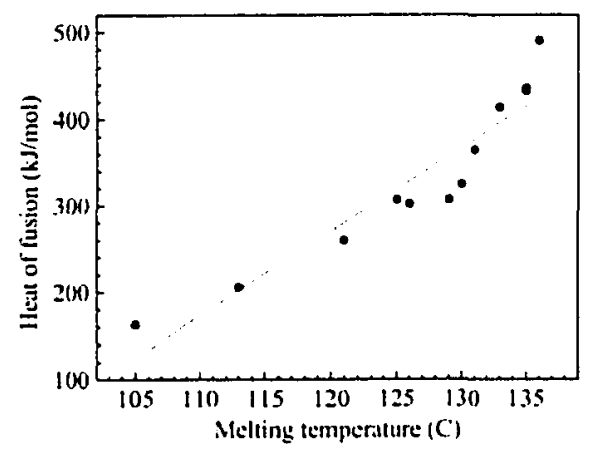

Figure XI-12: A plot of heat of fusion against melting temperature for urea/long chain fatty acid inclusion compounds, with a best-fit linear regression. The data were taken from reference [35].

In 1950, O. Redlich and co-workers published a study on host-guest stoichiometry and increase in heat content upon decomposition for a number of urea inclusion compounds with a wide variety of guest families [36]. They noted that urea complexes with nparaffins and their various derivatives are of considerable interest for various reasons, including analytical and technical applications [37]. Their approach was an attempt at securing a systematic description that could furnish the basic data required for various applications.

The authors determined $\Delta H$ for each inclusion compound by measuring the temperature dependence of the equilibrium constant, $K_{\text {eq. }}$. Their data appear in Table XI7. Their methods for measurement of $K_{\text {eq }}$ include: (1) aqueous reaction (2) non-aqueous reaction and (3) dew point method. The first two techniques involve measurements of concentration of reactants and products in water and guest solvent respectively, while the third technique is based on measurement of vapor pressure over a solid inclusion compound, and its variation with temperature. 
Table XI-7: Enthalpy of decomposition and host:guest stoichiometric ratio for urea inclusion compounds with five guest families [36].

\begin{tabular}{llc} 
Guest & $\Delta H\left(\mathrm{kcal}^{\text {mol }}{ }^{-1}\right)$ & Host:guest molar ratio \\
\hline$n$-heptane & 7.3 & 6.1 \\
$n$-octane & 9.7 & 6.7 \\
$n$-nonane & 11.8 & 7.4 \\
$n$-decane & 13.1 & 8.1 \\
$n$-dodecane & 16.1 & 9.3 \\
$n$-hexadecane & 21.0 & 11.9 \\
& & \\
1-octene & 7.3 & 6.3 \\
1-decene & 11.6 & 7.8 \\
1-undecene & 14.4 & 8.8 \\
1-dodecene & 12.6 & 9.3 \\
1-hexadecene & 23.5 & 12.0 \\
octanoic acid & 10.0 & 6.6 \\
tetradecanoic acid & 22.5 & 11.1 \\
& & 6.7 \\
1-octanol & 7.3 & 9.5 \\
1-dodecanol & 15.6 & 10.6 \\
& & 10.4 \\
1-dodecylchloride & 16.3 & \\
1-dodecylbromide & 15.2 &
\end{tabular}

For most of the urea inclusion compounds studied, a plot of $\log K_{\mathrm{eq}}$ vs inverse temperature yielded fairly linear relationships. Furthermore, (at least for the n-paraffin guests), a plot of $\Delta H$ vs number of carbons of the guest was also linear within experimental error. The authors noted that the increase in heat of inclusion may be crudely interpreted as due mainly to the heat of fusion plus the difference in energy of the hydrogen bonds between urea in the inclusion complex and pure urea.

With few exceptions, the authors noted that linear relationships hold between log $K_{\mathrm{tq}}$ and the host:guest stoichiometric ratio, and also between the heat of inclusion and the host:guest stoichiometric ratio. From these plots (not shown here), the following empirical relationships have been derived for $n$-paraffin guests ( $m=\mathrm{H}: \mathrm{G}$ molar ratio):

$$
\begin{array}{ll}
\log K_{\mathrm{eq}}=2.20-0.403 \mathrm{~m} & \text { (at } 25^{\circ} \mathrm{C} \text { ) } \\
\Delta H=-6.5+2.37 \mathrm{~m} & \text { (in kcal mol }{ }^{-1} \text { ) }
\end{array}
$$

As far as the stoichiometric ratio is concerned, the experimental data for $n$-paraffin guests yield the following equation: 


$$
m=0.653 n+1.51 \quad(7 \leq n \leq 16)
$$

where $n$ is the number of carbon atoms in the $n$-paraffin guest molecule. These results agree with our results on TATM, in that longer alkyl chains of the guest cause a progressive increase in the enthalpy of decomposition.

In 1962, H.G. McAdie performed a study [38] concerned with thermal decomposition of urea / $n$-paraffin inclusion complexes, using differential thermal analysis (DTA) and thermogravimetric analysis (TGA). The decomposition temperatures from hot stage microscopy, TGA and DTA (onset and peak) all appear in Table XI-8 (10 $\leq n \leq 28$ ). As can be seen, there is a steady and continuous increase in decomposition temperature with the length of the $n$-paraffin guest molecule. From the experimental DTA traces (see Figure $\mathrm{XI}-13$ ), the gradual progression of melting temperature asymptotically approaches the endotherm arising from the melting of pure urea, with the latter providing an internal calibration standard for $T_{\mathrm{m}}$ and $\Delta_{\text {fus }} H$. The enthalpy and entropy of decomposition of these urea / $n$-paraffin inclusion compounds appears in Table XI-9. One observes (similarly to $T_{\text {in }}$ ) a steady increase in both $\Delta_{\text {fus }} H$ and $\Delta_{\text {lis }} S$ with the length of the included $n$-paraftin guest molecule. These results correlate nicely with TATM melting temperatures and enthalpy of fusion in Table XI-5.

Table XI-8: Decomposition temperatures $\left({ }^{\circ} \mathrm{C}\right)$ of urea/n-paraffin complexes [38].

\begin{tabular}{lllll}
\hline Carbon chain length $(n)$ & Visual & TGA & DTA-onset & DTA-peak \\
\hline 10 & $46.1 \pm 1.6$ & $56 \pm 1.0$ & $72.8 \pm 1.2$ & $81.5 \pm 1.1$ \\
12 & $60.0 \pm 0.5$ & $73 \pm 1.0$ & $85.3 \pm 0.3$ & $91.2 \pm 1.7$ \\
14 & $79.4 \pm 0.0$ & $86 \pm 1.0$ & $96.2 \pm 0.7$ & $101.7 \pm 2.3$ \\
16 & $96.3 \pm 0.3$ & $95 \pm 0.5$ & $103.0 \pm 1.3$ & $108.1 \pm 0.7$ \\
18 & $113.8 \pm 0.0$ & $104 \pm 0.0$ & $110.0 \pm 2.0$ & $114.2 \pm 1.5$ \\
20 & $118.4 \pm 0.2$ & $113 \pm 5.0$ & $113.9 \pm 0.9$ & $117.9 \pm 0.4$ \\
22 & $121.7 \pm 0.5$ & $120 \pm 3.0$ & $119.3 \pm 0.7$ & $123.2 \pm 0.8$ \\
24 & $124.3 \pm 0.6$ & 123 & $121.4 \pm 0.1$ & $125.3 \pm 1.6$ \\
26 & $126.2 \pm 0.7$ & 123 & $123.3 \pm 0.2$ & $130.0 \pm 0.1$ \\
28 & - & - & 125.3 & 131.2 \\
\hline
\end{tabular}


Table XI-9: Enthalpy and entropy of decomposition for urea/n-paraffin complexes [38].

\begin{tabular}{lcc}
\hline $\begin{array}{l}\text { Carbon chain } \\
\text { length }(n)\end{array}$ & $\begin{array}{c}\Delta_{\text {lus }} H \\
\text { kcal mole }\end{array}$ & $\begin{array}{c}\Delta_{\text {ius }} S \\
\text { paraffin }\end{array}$ \\
\hline 10 & 9.9 & cal mole $\mathrm{K}^{-1}$ paraffin \\
12 & 13.4 & 33.5 \\
14 & 18.7 & 40.7 \\
16 & 22.9 & 47.6 \\
18 & 23.3 & 54.8 \\
20 & 23.5 & 61.6 \\
22 & 29.6 & 68.7 \\
\hline
\end{tabular}

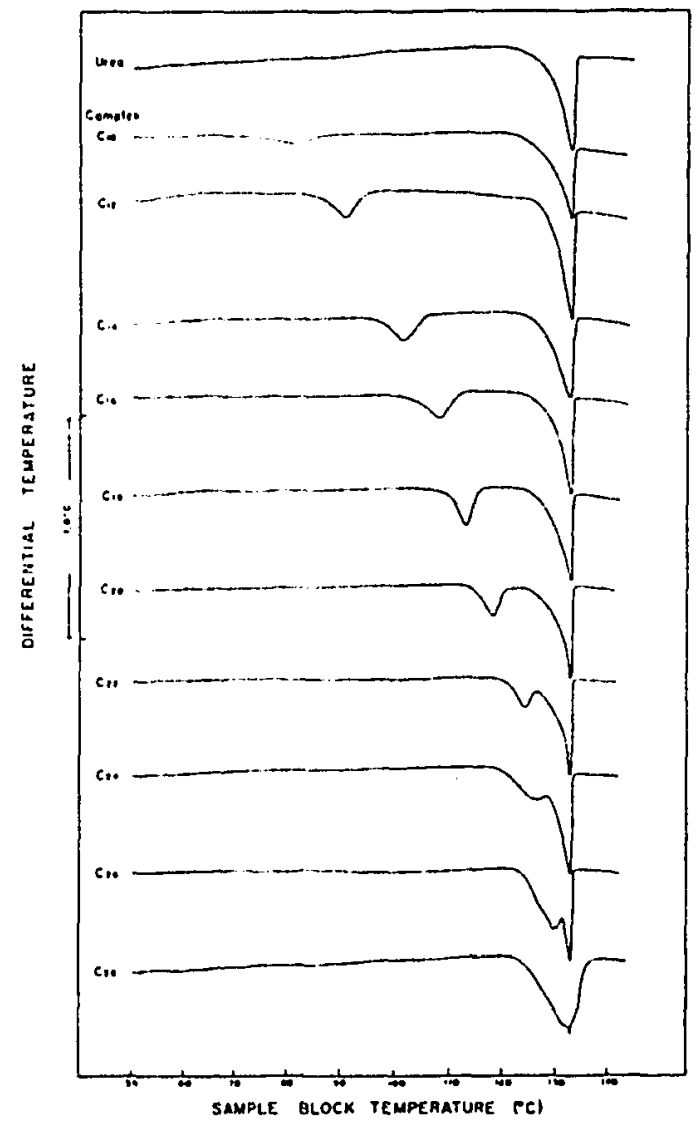

Figure XI-13: DTA thermograms of urea and a series of urea $/ n-$ paraffin complexes [38]

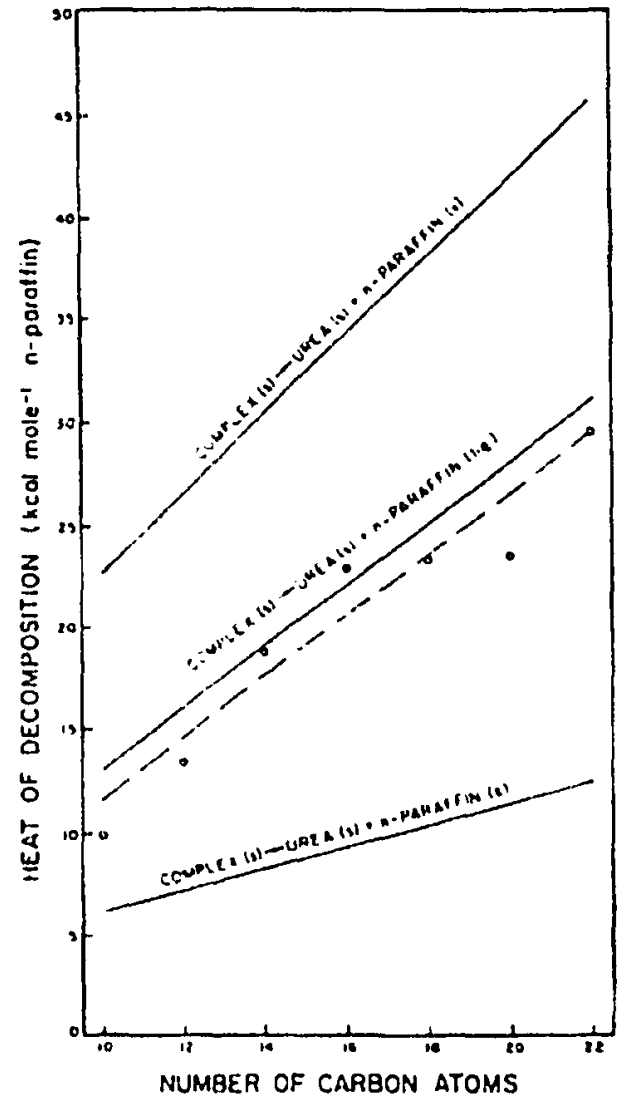

Figure XI-14: $\Delta_{\text {fus }} H$ for urea $/ n$ paraffin complexes as a function of guest chain length: solid lines $=$ calculated values, broken line $=$ least squares fit to expt'l data [38] 
A comparison of the measured $\Delta_{\text {fus }} H$ with heats of reaction for three possible decomposition processes (liberated guest emerging in either gas, liquid or solid phase), estimated from equilibrium data in the literature $[39,40]$, as depicted in Figure XI-14, suggests that the decomposition process is energetically equivalent to:

$$
\mathrm{UIC}(\mathrm{s}) \rightleftharpoons \mathrm{urea}(\mathrm{s})+n \text {-paraffin(liq) }
$$

The similarity in shape between the urea complex decomposition temperature curve (as a function of length of $n$-paraffin guest), and the melting point curve for the pure $n$ paraffins (see Figure XI-15), provides further support for the suggested decomposition mechanism, in that the n-paraffin guest is liberated at temperatures significantly above its melting point, but below its boiling point. Thus, the released guest emerges in a state energetically equivalent to that of a liquid. On the other hand, TATM guests are in many cases liberated at temperatures above their boiling point, so they are released as a gas. In other cases, for less volatile guests, they are liberated in the liquid phase.

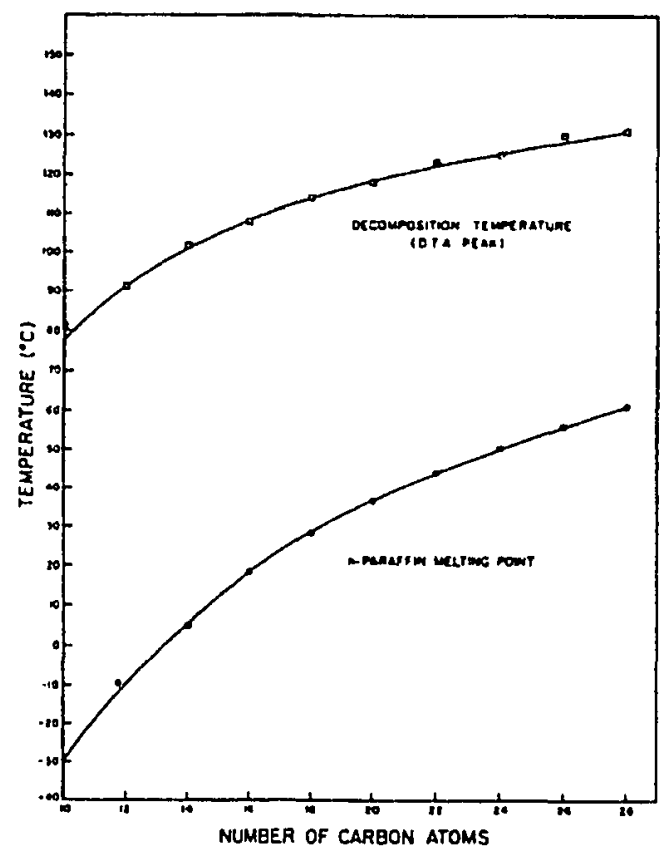

Figure XI-15: A comparison of the decomposition temperature of urea / $n$-paraffin complexes with the melting points of pure $n$-paraffins as a function of chain length [38] 
The TGA curves (see Figure XI-16), correlated with the enthalpy of decomposition data, in that the urea complexes with smaller $n$-paraffin guests (e.g. $n$ decane) lost their guests more easily when heated, while the complexes with larger guests held onto their guest more strongly when heated. A steady progression in this behaviour from $\mathrm{C}_{10} \rightarrow \mathrm{C}_{24}$ is observed (see Figure XI-16). This correlates with the DTA data, in that more strongly held guests (from TGA - Figure $\mathrm{Xl}-16$ ) have a higher melting temperature and a higher enthalpy of decomposition (from DTA - see Tables XI-8 and $\mathrm{XI}-9)$

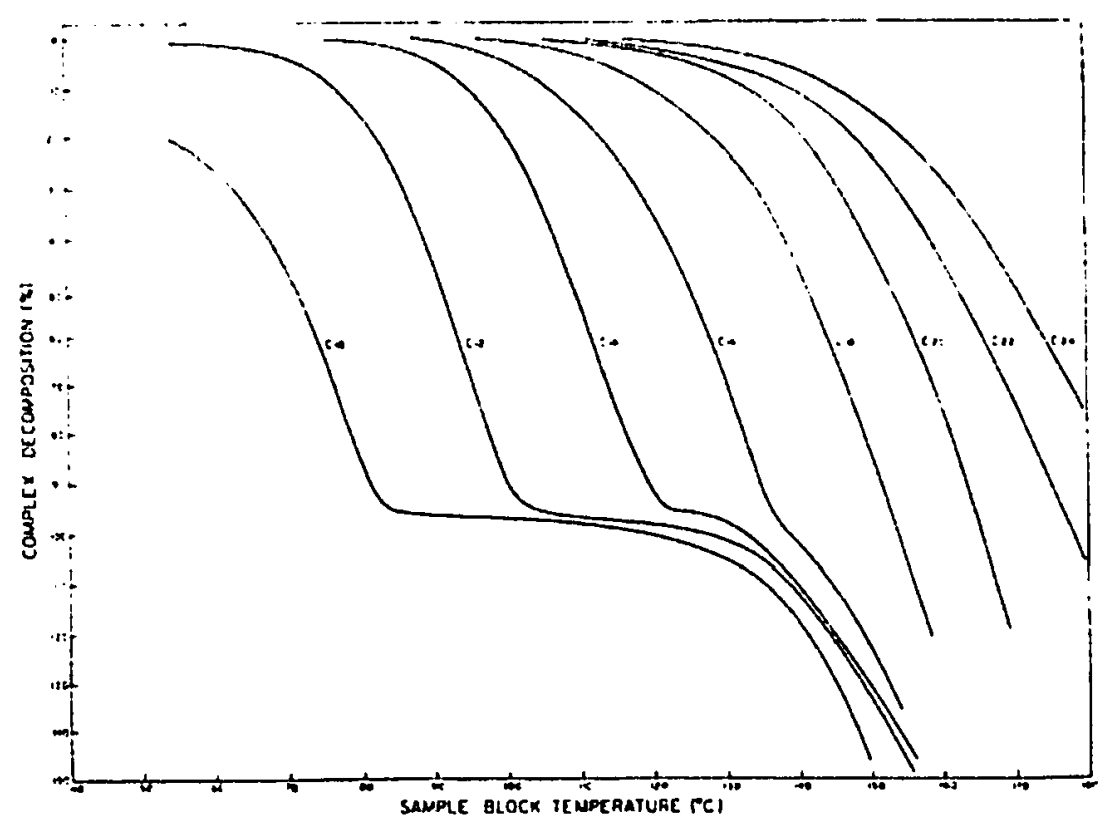

Figure XI-16: TGA curves for a series of urea / $n$-paraffin inclusion compounds (from left to right, $n=10,12,14,16,18,20,22,24)$ [38]

One year later, in 1963 [41], McAdie further elaborated upon his previous results by examining thermal decomposition processes of urea inclusion compounds with nparaffins and $n$-substituted paraffins, including n-alcohols, n-alkylamines, n-alkyl bromides and $n$-carboxylic acids $(10 \leq n \leq 20)$. McAdie noted that the strength of intermolecular interactions of the urea complex should be influenced by the size of the terminal substituent of the guest, and its ability to associate with other guest molecules, either through dipole-dipole interactions or hydrogen bonding, as two neighboring guest molecules may be mutually associated within the urea canal. 
The DTA traces for urea inclusion compounds of all five guest families display a lower temperature endotherm, corresponding to complex decomposition, which progressively approaches the higher temperature urea fusion endotherm as the carbon chain length of the guest molecule increases. The decomposition temperatures of the various complexes are given in Table XI-10, as the peak temperature of the decomposition endotherm. The enthalpy of decomposition, as measured by DTA, for all five guest families, appears in Table XI-11. For both the melting temperature and enthalpy of decomposition, one observes a steady increase with carbon chain length of guest.

The order of decomposition enthalpy, for a particular carbon chain length, generally follows the order $n$-paraffin $<n$-bromide $<n$-alcohol $<n$-alkylamine $<n$ carboxylic acid. McAdie believes that the lower decomposition enthalpy of urea / paraffins is likely due to only weak dispersion forces between neighboring guests in the same channel. The alkyl bromides may interact more strongly through dipole - dipole forces, while the last three guest families interact through much stronger hydrogen bonding. Indeed, the highest enthalpy of decomposition for the carboxylic acid guests may arise from two hydrogen bonds for each pair of guests, while alcohols and amines form only one hydrogen bond.

For all five guest families, McAdie noted that the enthalpy changes compared favorably with those obtained from equilibrium data $[39,40]$ for the process:

$$
\mathrm{UIC}(\mathrm{s})-\cdots-\mathrm{urea}(\mathrm{s})+\text { guest(liq) }
$$

Energetically, the decomposition follows a process that yields the guest molecule in the liquid state, as expected since the decomposition temperature of the complexes all lie considerably above the melting points of the pure guest species.

As was noted above for $n$-paraffin / urea inclusion compounds, the TGA curves for the urea / $n$-substituted paraffins show that the inclusion compounds with smaller guests (e.g. $\left.\mathrm{C}_{10}\right)$ lose their guest more easily when heated, and as the carbon chain length of the guest increases, the guest is held progressively more and more strongly while being heated (see Figure XI-17 for TGA curves of urea $n$-alcohol adducts), which agrees well with the enthalpies of decomposition from DTA. 


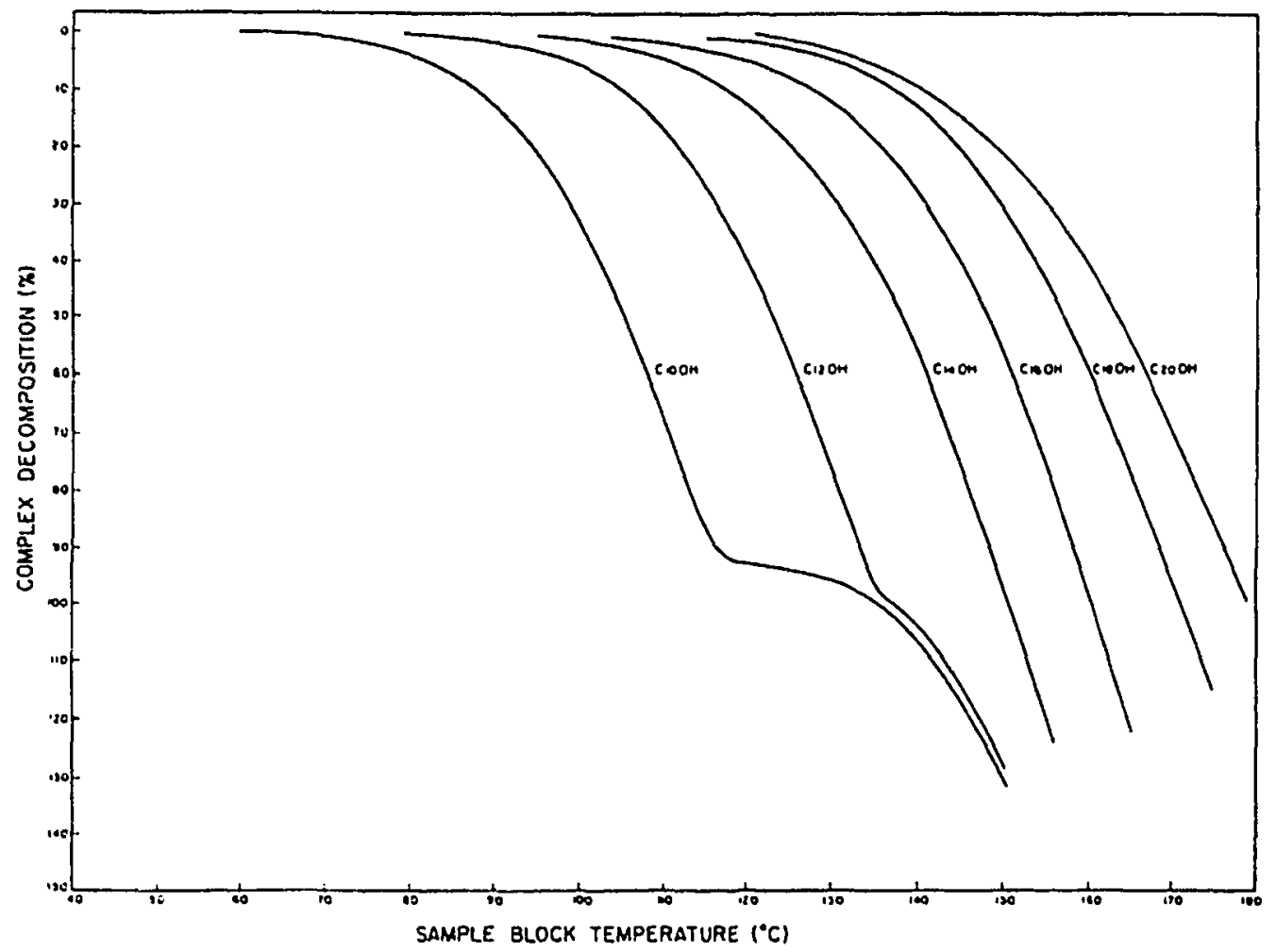

Figure XI-17: TGA curves for urea $/ n$-alcohol complexes (from left to right, $n=$ $10,12,14,16,18,20)[41]$

McAdie remarked that the $n$-alkyl bromide complexes decompose at higher temperatures than the same length of guest $n$-paraffin complexes. He rationalized this on the basis of the difference in volume of a terminal bromine atom, compared to that of a hydrogen atom, which increases the strength of host-guest binding of the urea complex for the former guests by occupying a larger portion of the available channel cross-section.

McAdie also suggested that the n-alcohol, n-alkylamine and n-carboxylic acid guests are capable of extensive intermolecular association through the formation of guestguest hydrogen bonds. In their urea complexes, it is known that the n-carboxylic acids exist as dimers within the canal, such that the guest molecules lie alternately head-to-head and tail-to-tail [42], such that terminal methyl group interactions alternate with hydrogenbonded carboxylic acid groups along a given channel. It was suggested that $n$-alcohols and $n$-alkylamines may also associate within the urea channels in this manner. 
In general, the melting points [42] of the pure guest species, for a given carbon chain length, increase in the same order as for the decomposition temperatures of their respective urea complexes, which suggests that many of the molecular properties responsible for the sequence of melting points of the pure solids may be may be retained in the urea canal.

McAdie suggested that these results appear to be consistent with the mechanism for complex decomposition, in that the decomposition temperature of a urea complex is determined by the temperature at which the guest molecule becomes sufficiently mobile to pass frequently over the potential barriers in the channel, and possesses sufficient thermal energy for translational diffusion out of the canal. The larger the end group, or the stronger its interaction with a neighboring guest molecule, the higher will be the energy requirement for the guest to achieve adequate freedom to diffuse out of the host channel.

Table XI-10: Decomposition temperatures of urea inclusion compounds $\left({ }^{\circ} \mathrm{C}\right)[41\}$

\begin{tabular}{llllll} 
Chain length & n-paraffin & n-alkyl bromide & n-alcohol & n-alkvlanine & n-carboxylic acid \\
\hline 10 & 82.4 & 92.4 & 87.2 & 100.0 & 107.6 \\
12 & 92.5 & 102.1 & 102.7 & 109.3 & 114.3 \\
14 & 102.4 & 108.1 & 113.6 & 119.2 & 120.8 \\
16 & 108.4 & 113.5 & 121.7 & 125.7 & 127.2 \\
18 & 113.9 & 117.5 & 127.1 & 129.0 & 130.5 \\
20 & 117.9 & - & 129.4 & - & 131.8 \\
\hline
\end{tabular}

Table XI-11: Enthalpy of decomposition of urea inclusion compounds $\left(\mathrm{kcal} \mathrm{mol}^{-1}\right)$ [41]

\begin{tabular}{llllll} 
Chain length & n-paraffin & n-alkyl bromide & n-alcohol & n-alkylamine & n-carboxylic acid \\
\hline 10 & 11.3 & 9.6 & 9.4 & 11.9 & 12.6 \\
12 & 13.8 & 12.8 & 12.1 & 14.7 & 16.3 \\
14 & 16.6 & 16.5 & 15.0 & 16.6 & 20.5 \\
16 & 19.5 & 20.7 & 18.4 & 19.0 & 25.2 \\
18 & 22.6 & 25.3 & 22.0 & 22.7 & - \\
20 & 26.0 & - & - & - & - \\
\hline
\end{tabular}

On the basis of these results, one might expect that for TATM inclusion compounds, $\alpha,(\omega$-dichloroalkanes would have significantly different thermal properties than $\alpha, \omega$-dibromoalkanes. We have obtained DSC data for TATM inclusion compounds with $\mathrm{Cl}\left(\mathrm{CH}_{2}\right)_{n} \mathrm{Cl}$ (see Table XI-5 above) and $\mathrm{Br}\left(\mathrm{CH}_{2}\right)_{n} \mathrm{Br}$ (unpublished material) $(n=1$ to 
6) guest families. The data appears in Table XI-12. All $\Delta_{\text {fus }} H$ and all but one $T_{\mathrm{m}}$ value are in most cases significantly higher for $\alpha, \omega$-dichloroalkane / TATM inclusions when compared to $\alpha, \omega$-dibromoalkane / TATM inclusions. In addition, 1-dodecylchloride/urea has a higher enthalpy of decomposition $\left(16.3 \mathrm{kcal} \mathrm{mol}^{-1}\right)$ than 1-dodecylbromide/TATM (15.2 $\mathrm{kcal} \mathrm{mol}^{-1}$ ), as depicted in Table XI-7 [36]. We do note that it has been previously suggested that the order of decomposition enthalpy in urea inclusion compounds (for $\mathrm{X}\left(\mathrm{CH}_{2}\right)_{\mathrm{n}} \mathrm{X}$ guests $(\mathrm{X}=\mathrm{Cl}, \mathrm{Br}$ or $\mathrm{I})$ should follow the order $\mathrm{I}>\mathrm{Br}>\mathrm{Cl}$ [43]. However our results for the alkyl halide guest families in TATM (chapter VIII) suggest that the order should go in the opposite direction, as do the results for the $\mathrm{C}_{12} \mathrm{X} /$ urea inclusion compounds in Table XI-7 [36].

Table XI-12: DSC data for $\alpha,(1)$-dichloroalkane / TATM and $\alpha,(\omega$-dibromoalkane / TATM inclusion compounds (Table $\mathrm{XI}-5(\mathrm{Cl})$ and unpublished material $(\mathrm{Br})$ )

\begin{tabular}{lllll} 
& \multicolumn{2}{c}{$\mathrm{Cl}\left(\mathrm{CH}_{2}\right)_{n} \mathrm{Cl}$ guests } & \multicolumn{2}{c}{$\mathrm{Br}\left(\mathrm{CH}_{2}\right)_{n} \mathrm{Br}$ guests } \\
\cline { 2 - 4 }$T_{\mathrm{m}}\left({ }^{\circ} \mathrm{C}\right)$ & $\Delta_{\text {rus }} \mathrm{H}\left(\mathrm{J} \mathrm{g} \mathrm{g}^{-1}\right)$ & 128.1 & 74.7 \\
\hline 1 & 134.6 & 107.1 & 102.6 & 65.1 \\
2 & 130.2 & 103.2 & 104.5 & 73.9 \\
3 & 106.5 & 83.3 & 104.2 & 76.5 \\
4 & 109.7 & 88.7 & 117.0 & 82.3 \\
5 & 117.6 & 90.8 & 127.1 & 85.5 \\
6 & 122.1 & 92.3 & $\left.\mathrm{~J} \mathrm{~g} \mathrm{~g}^{-1}\right)$ \\
\hline
\end{tabular}

In 1998, a publication by M.A. White [44] described the melting behavior of urea inclusion compounds with $n$-paraffin guests (decane, dodecane and hexadecane). It was discovered that both $T_{\mathrm{m}}$ and $\Delta_{\mathrm{as}} G$ follow the order urea/decane $<$ urea/dodecane $<$ urea/hexadecane. All three guests form incommensurate urea inclusion complexes and melt incongruently. In all three cases, the inclusion compounds were found to be thermodynamically stable with respect to their component species up to their respective melting points. The $T_{\mathrm{m}}$ and $\Delta_{\mathrm{as}} G$ values go as follows (1) urea/decane: $335 \mathrm{~K},-0.95 \mathrm{~kJ}$ $\mathrm{mol}^{-1}$ urea, (2) urea/dodecane: $364 \mathrm{~K},-1.06 \mathrm{~kJ} \mathrm{~mol}^{-1}$ urea, (3) urea/hexadecane: $384 \mathrm{~K}$, $1.34 \mathrm{~kJ} \mathrm{~mol}^{-1}$ urea. 
(F) Cyclodextrins

The association $\left(K_{\mathrm{n}}\right)$ of anionic surfactants (sodium alkyl sulfates: $\mathrm{C}_{n} \mathrm{H}_{2 n+1} \mathrm{OSO}_{3} \cdot \mathrm{Na}, n=$ 5-10,12) with $\beta$-cyclodextrins (in aqueous solution) have been measured by ${ }^{1} \mathrm{H}$ NMR spectroscopy [45]. More specifically, $K_{\mathrm{a}}$ was calculated from the observation of changes in ${ }^{\prime} \mathrm{H}$ chemical shift of both host and guest molecules as a function of the guest to host molar ratio in the solution, according to a modified Hildebrand-Benesi equation $[46,47]$. $K_{\mathrm{a}}$ was obtained by measuring the evolution of chemical shift of $\mathrm{H}-5$ of $\beta-\mathrm{CD}$ with added surfactant. Table XI-13 lists these calculated standard free energy changes $\left(-\Delta G^{\circ}\right)$ and association constants $\left(K_{\mathrm{a}}\right)$ of sodium alkyl sulfates with $\beta-\mathrm{CD}$. The results indicate that both $-\Delta G^{\circ}$ and $K_{\mathrm{a}}$ increase steadily with the length of the hydrocarbon chain of the guest, in agreement with $\Delta_{\text {fus }} H$ data on $\alpha,(\omega)$-dichloroalkane / TATM materials as depicted in Table XI-5.

Table XI-13: Standard free energy changes $\left(-\Delta G^{0}\right)$ and association constants $\left(K_{\mathrm{a}}\right)$ for the inclusion of $\beta$-cyclodextrin with sodium alkyl sulfates: $\mathrm{C}_{n} \mathrm{H}_{2 \mathrm{n}+1} \mathrm{OSO}_{3} \cdot \mathrm{Na}|\mathbf{4 5}|$

\begin{tabular}{lll}
\hline $\begin{array}{l}\text { Surfactant } \\
\text { chain length }(n)\end{array}$ & $-\Delta G^{0}\left(\mathrm{~kJ} \mathrm{~mol}^{-1}\right)$ & $K_{a}\left(\mathrm{~L} \mathrm{~mol}^{-1}\right)$ \\
\hline 5 & 11.34 & $101 \pm 10$ \\
6 & 12.31 & $144 \pm 37$ \\
7 & 14.19 & $307 \pm 70$ \\
8 & 14.54 & $343 \pm 11$ \\
9 & 17.59 & $1210 \pm 290$ \\
10 & 19.11 & $2240 \pm 150$ \\
12 & 20.74 & $4320 \pm 670$ \\
\hline
\end{tabular}

\section{(G) 2,2,6,6-tetramethyl-4-oxopiperidine-1-oxyl (tano)}

The nitroxide radical 2,2,6,6-tetramethyl-4-oxopiperidine-1-oxyl $\left(\mathrm{C}_{9} \mathrm{H}_{16} \mathrm{NO}_{2}\right)$, 1, referred to as "tano", forms stable crystalline inclusion compounds with, among others, linear nparaffin guests $\mathrm{C}_{n} \mathrm{H}_{2 n+2}$, for $n$ ranging from 7 to at least 44 [48-52]. The tano molecules form an organic host matrix containing parallel one-dimensional channels approximately $5 \AA$ in diameter, into which the linear $n$-alkanes pack end-to-end. At room temperature, all tano inclusion compounds are monoclinic $(C 2 / c, Z=24)$, with similar unit cell parameters $\left(a \sim 36 \AA, b \sim 5.95 \AA, c \sim 35.5 \AA, \beta \sim 120^{\circ}\right)$, revealing a relatively invariant 
organization of the tano host matrix [53]. (This is in contrast to TATM, which is generally much more "plastic", or accommodating, for differently sized guests.) For tano, in one unit cell, there are four channels parallel to $b, 18 \AA$ apart and $5 \AA$ in diameter [48]. These systems exhibit a wide variety of structural, dynamical and thermodynamic properties that have been studied by X-ray diffraction [48,50,54], DSC [55], infrared and Raman spectroscopies $|56|$ and incoherent quasielastic neutron scattering (IQNS) $\{51,53\}$.

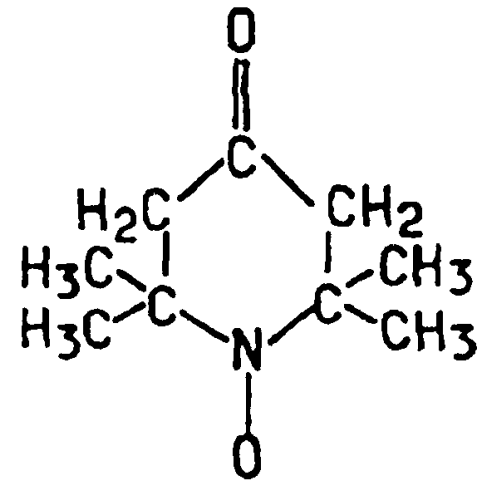

Scheme 1: 2,2,6,6-tetramethyl-4-oxopiperidine- 1 -oxyl host radical (tano)

For $n$-paraffin guests, the DSC melting temperatures $\left(T_{\mathrm{m}}\right)$ and enthalpies of decomposition $\left(\Delta_{\text {fus }} H\right)$ generally increase with the length of the n-paraffin guest [49] (see Table XI-14). Melting temperatures of these complexes, higher than that for pure tano $[57,58 \mid(309 \mathrm{~K})$, increase with the number $n$ of carbons in the hydrocarbon chain, from $320 \mathrm{~K}$ for tano-n-heptane to asymptotically approach $340 \mathrm{~K}$ for very long guest chain lengths (e.g. tano-n-hexadecane: $n=16$ ). In addition, the melting temperatures and melting enthalpies of pure $n$-paraffins also increase with the length of the hydrocarbon chain, in a similar manner as their respective tano adducts. The least stable compound of those listed in Table XI-14 is the complex with $n$-heptane, it can be kept for only a few days at room temperature. On the other hand, the compound with $n$-hexadecane undergoes no change for months. This illustrates the importance of guest size on the stability of the resulting tano complex, due, in part, to the relative invariance of the host matrix, and the stronger van der Waals interactions between host and guest (with both TATM and tano) for longer and longer alkyl chains of the guest. 
Table XI-14: Melting temperatures $\left(T_{\text {in }}\right)$ and melting enthalpies $\left(\Delta_{\text {lus }} H\right)$ for tano $/ n$-alkane inclusion compounds [49]

\begin{tabular}{lll}
\hline Guest $(n)$ & $T_{\text {nU }}(\mathrm{K})$ & $\Delta_{\text {fu }} H\left(\mathrm{~J} \mathrm{~g}^{-1}\right)$ \\
\hline$n$-heptane (7) & 320 & 84 \\
n-octane (8) & 329 & 96 \\
$n$-nonane (9) & 329 & 79 \\
$n$-decane (10) & 330 & 87 \\
n-undecane (11) & 330 & 92 \\
n-dodecane (12) & 331 & 100 \\
$n$-tridecane (13) & 335 & 100 \\
n-tetradecane (14) & 337 & 92 \\
n-hexadecane (16) & 339 & 100 \\
\hline
\end{tabular}

(H) Graphite

A study published in 1997 [59| was concerned with thermodynamic properties of $n$ alkanes confined in the pores of delaminated graphite, as measured by DSC. The authors noted that melting phase transitions are generally shifted to slightly lower temperatures when compounds are confined in porous matrices $[\mathbf{6 0}, 61 \mid$. The melting points of pure $n$ paraffins, as a function of $n$, are plotted in Figure XI-18. One observes a progressive increase in $T_{\text {in }}$ with the length of the $n$-paraffin compound. Interestingly, $n$-even and $n$ odd $n$-paraffins show slightly different melting temperatures, more noticeably for the shorter chain molecules, as a result of different packing forces.

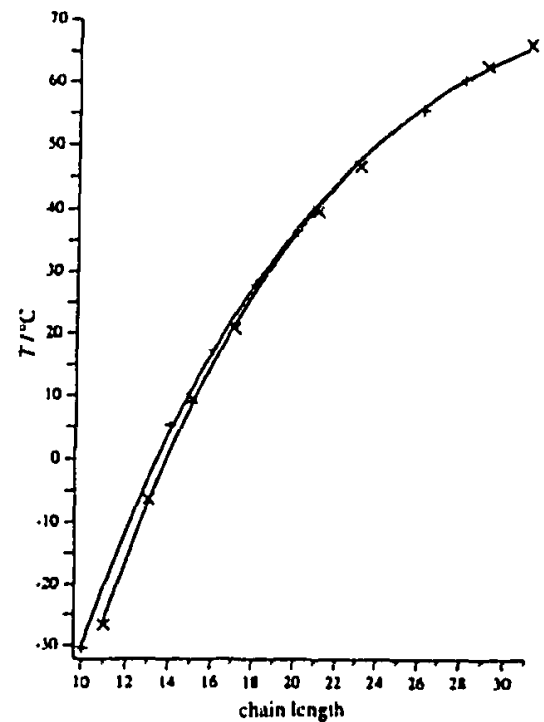

Figure XI-18: Melting temps of $(+)$ even-numbered and $(x)$ oddnumbered pure $n$-alkanes [59]

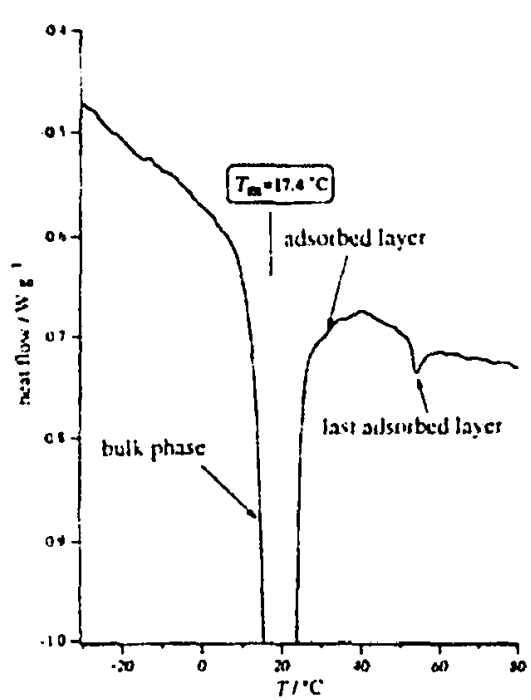

Figure XI-19: DSC signal of $n$-hexadecane / graphite in the melting region [59] 
Two endotherms were encountered close to the melting point of the incorporated $n$-paraffins. A strong intensity endotherm (e.g. $17.4^{\circ} \mathrm{C}$ for $n$-hexadecane/graphite) corresponds to the melting of the $n$-paraffin that is intercalated in the graphite pore structure (see Figure XI-19). A significantly weaker endotherm at slightly higher temperature (e.g. $54.4^{\circ} \mathrm{C}$ for $n$-hexadecane/graphite), that corresponds to melting of $n$ paraffin physically adsorbed on the external surface of the graphite crystals. Both endotherms steadily increase in temperature with the length of the $n$-paraffin molecule incorporated in the graphite lattice (see Figure XI-20). Despite the point made above concerning melting point depression in porous materials, the authors found little evidence of melting point depression on incorporation of the n-paraffins into the graphite pore structure, at least for the larger guests $(n>14)$. This behaviour is similar to 1bromoalkane / TATM inclusions with longer guests $(\mathrm{Br} 10-\mathrm{Br} 15)$, for which there may be a solid-solid transition at temperatures slightly below the "true" melting point.

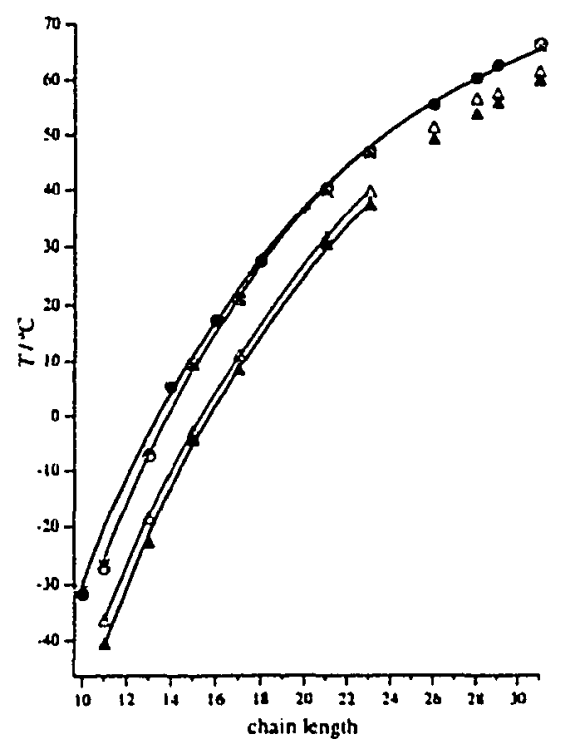

Figure XI-20: Solid-solid and solid-liquid transitions: $(\Delta)$ solid-solid transition of free alkanes, $(+)$ melting of the free "even-numbered" alkanes, $(x)$ melting of the free "odd-numbered" alkanes, $(\mathbf{A})$ solid-solid transition of alkanes in the graphite pores, $(\bullet)$ melting of "even-numbered" alkanes in the graphite pores, $(0)$ melting of "oddnumbered" alkanes in the graphite pores [59] 


\section{CONCLUDING REMARKS}

The variety of molecular complexes now known provides the means for examining many molecules of different sizes and shapes in an environment more or less isolated from others of their own kind. Within the channels that penetrate the structures of TATM crystals, long-chain guest molecules are longitudinally isolated from one another by the channel walls, but may be permitted end-group interactions between successive chains, affording a means for comparing these interactions in different guest families through their contribution to the stability of the complex. For the 1-bromoalkane guests of chapter XIII, a maximum of two guest molecules may be mutually associated within the TATM channel, while for the $\alpha, \omega$-dichloroalkane guests of the present study, the number of guest molecules consecutively associated within the TATM channel may be considerably greater.

The decomposition temperature of these TATM complexes (at least for the medium and large sized guests) is determined by the temperature at which the $\alpha, \omega$ dichloroalkane guest molecule becomes sufficiently mobile to pass frequently over the rotational potential barriers in the channel, and possesses a sufficient amount of energy (an activation energy) for translational diffusion out of the canal. The larger (or less volatile) the guest molecule, the higher will be the energy requirement for the guest molecule to achieve adequate freedom within the canal

In this work we have investigated a suite of TATM inclusions by changing the length of the methylene chain in between two invariant chlorine atoms (of the guest) from 1 to $10-\mathrm{CH}_{2}$ - groups. In a previous study (chapter VIII) we adopted the opposite approach, namely a consistent hydrocarbon backbone and varying halogen substitiuents. By drawing correlations between the two groups of TATM inclusion compounds, we were able to determine the nature of the process of inclusion compound formation of TATM with various guests, and the ability of TATM to "recognize" similarities or differences within each of the different groups of guests. 
(1) P. Hobza and R. Zahradnik. Intermolecular Complexes: The Role of van der Waals Systems in Physical Chemistry and in the Biodisciplines Elsevier: Amsterdam, 1988.

(2) B. Pullman. Intermolecular Interactions - From Diatomics to Biopolymers Wiley: Chichester, 1978.

(3) P. Schuster. Angew. Chem. Int. Ed. Engl. 20, 546 (1981).

(4) G.R. Desiraju and A. Gavezzotti. J. Chem. Soc. Chem. Commun. 621 (1989).

(5) C.A. Hunter and J.K.M. Sanders. J. Am. Chem. Soc. 112, 5525 (1990).

(6) O. Ermer. Helv. Chim. Acta 74, 1339 (1991).

(7) P. Schuster, G. Zundel and C. Sandorfy. The Hydrogen Bond: Recent Developments in Theory and Experiments Elsevier: Amsterdam, 1976.

(8) G.A. Jeffrey and S. Takagi. Acc. Chem. Res. 11, 264 (1978).

(9) P. Dauber and A.T. Hagler. Acc. Chem. Res. 13, 105 (1980).

(10) R. Taylor and O. Kennard. Acc. Chem. Res. 17, 320 (1984).

(11) C.E. Dykstra. Acc. Chem. Res. 21, 355 (1988).

(12) G.R. Desiraju. Acc. Chem. Res. 24, 290 (1991).

(13) G.A. Jeffrey and W. Saenger. Hydrogen Bonding in Biological Structures Springer: Berlin, 1991.

(14) J.W. Steed and J.L. Atwood. Supramolecular Chemistry John Wiley and Sons: New York, 2002.

(15) G.R. Desiraju and R. Parthasarathy. J. Am. Chem. Soc. 111, 8725 (1989).

(16) G.M.J. Schmidt. Pure Appl. Chem. 27, 647 (1971).

(17) D.E. Williams and L.Y. Hsu. Acta Cryst. A41, 296 (1985).

(18) G.R. Desiraju. Crystal Engineering: The Design of Organic Solids Elsevier: Amsterdam, 1989.

(19) J.M. Williams, M.A. Beno, H.H. Wang, P.C.W. Leung, T.J. Emge, U. Geiser and K.D. Carlson. Acc. Chem. Res. 18, 261 (1985).

(20) A.D.U. Hardy, J.J. McKendrick, D.D. MacNicol and D.R. Wilson. J. Chem. Soc. Perkin Trans. 2729 (1979).

(21) J.M. Lehn. Angew: Chem. Int. Ed. Engl. 29, 1304 (1990).

(22) D.J. Cram. Angew. Chem. Int. Ed. Engl. 27. 1009 (1988).

(23) J.D. Dunitz. Pure Appl. Chem. 63, 177 (1991).

(24) A.I. Kitaigorodsky. Molecular Crystals and Molecules Academic Press: New York, 1973.

(25) C.V.K. Sharma, K. Panneerselvam, T. Pilati and G.R. Desiraju. J. Chem. Soc. Chem. Commun. 832 (1992).

(26) K. Biradha, C.V.K. Sharma, K. Panneerselvam, L. Shimoni, H.R. Carrell, D.E. Zacharias and G.R. Desiraju. J. Chem. Soc. Chem. Commun. 1473 (1993).

(27) R.J.C. Brown and R.F.C. Brown. J. Chem. Ed. 77, 724 (2000).

(28) M.D. Hollingsworth and K.D.M. Harris. Urea, Thiourea and Selenourea In Comprehensive Supramolecular Chemistry, J.L. Atwood, J.E.D. Davies, D.D. MacNicol and F. Vogtle, Eds. Volume 6, Chapter 7. Pergamon / Elsevier: Oxford, 1996.

(29) A.E. Smith. Acta Cryst. 5, 224 (1952).

(30) K.D.M. Harris and J.M. Thomas. J. Chem. Soc. Faraday Trans. 86, 2985 (1990). 
(31) K.D.M. Harris. Phase Transitions 76(3), 205 (2003).

(32) K. Takemoto and N. Sonada. Inclusion Compounds of Urea. Thiourea and Selenourea In Inclusion Compounds. J.L. Atwood, J.E.D. Davies and D.D. MacNicol, Eds. Volume 2, Chapter 2. Academic Press: London, 1984.

(33) H.G. McAdie and G.B. Frost. Can. J. Chem. 36, 635 (1958).

(34) K.D.M. Harris. J. Phys. Chem. Solids 53, 529 (1992).

(35) J. Ahmad, A.J. Freestone and A. Hussain. J. Inclus. Phenom. Mol. Recognit. Chem. 18, 115 (1994).

(36) O. Redlich, C.M. Gable, A.K. Dunlop and R.W. Millar. J. Am. Chem. Soc. 72, 4153 (1950).

(37) W.J. Zimmerschied, R.A. Dinerstein, A.W. Weitkamp and R.F. Marschner. J. Am. Chem. Soc. 71, 2947 (1949).

(38) H.G. McAdie. Can. J. Chem. 40, 2195 (1962).

(39) E. Terres and S. Nath Sur. Brennstoff Chem. 38, 7 (1957).

(40) F.D. Rossini, K.S. Pitzer, R.L. Arnett, R.M. Brown and C.C. Pimentel. Selected Values of Physical and Thermodynamic Properties of Hydrocarbons and Related Compounds Carnegie Press: Pittsburgh, 1953.

(41) H.G. McAdie. Can. J. Chem. 41, 2144 (1963).

(42) L.C. Fetterley. PhD Thesis. University of Washington, Seattle, 1950.

(43) S.P. Smart, A. El Baghdadi, F. Guillaume and K.D.M. Harris. J. Chem. Soc. Faraday Trans. 90, 1313 (1994).

(44) M.A. White. Can. J. Chem. 76, 1695 (1998).

(45) Q.X. Guo, Z.Z. Li, T. Ren, X.Q. Zhu and Y.C. Liu. J. Inclus. Phenom. Mol. Recognit. Chem. 17, 149 (1994).

(46) R. Bergeron, M.A. Channing, G. Gibeily and D.M. Pillor. J. Am. Chem. Soc. 99, 5146 (1977).

(47) H.A. Benesi and J.H. Hildebrand. J. Am. Chem. Soc. 71, 2703 (1949).

(48) M.L Bars-Combe and J. Lajzerowicz. Acta Cryst. B43, 386 (1987).

(49) M.L Bars-Combe and J. Lajzerowicz. Acta Cryst. B43, 393 (1987).

(50) P.A. Albouy, J.L. Bonneteau and M.L. Bars-Combe. J. Physique 51, 1213 (1990).

(5I) M. Bee, A. Renault, J.L. Bonneteau and M.L. Bars-Combe. J. Chem. Phys. 97. 7730 (1992).

(52) M. Bee, J.L. Bonneteau and A. Renault. Physica B 180/181, 653 (1992).

(53) J. Combet, N.D. Morelon, M. Fernand, M. Bee, N. Djurado, G. Commandeur and J.M. Castejon J. Phys. Condens. Matter 9, L403 (1997).

(54) M.L. Bars-Combe. PhD Thesis. Universite Joseph Fournier. Grenoble, France, 1985.

(55) M.L. Bars-Combe and J. Lajzerowicz. J. Phys. C: Solid State Phys. 17, L669 (1984).

(56) J. Combet. PhD Thesis. Universite Joseph Fournier. Grenoble, France, 1997.

(57) D. Bordeaux and J. Lajzerowicz. Acta Cryst. B30, 790 (1974).

(58) M.L. Bars-Combe. Acta Cryst. B38, 2749 (1982).

(59) P. Espeau and J.W. White. J. Chem. Soc. Faraday Trans. 93(17), 3197 (1997).

(60) C.L. Jackson and G.B. McKenna. J. Chcm. Phys. 93, 9002 (1990).

(61) D.D. Awschalom and J. Warnock. Phys. Rev. B 35, 6779 (1987). 


\section{CHAPTER XII}

\section{A tris (5-acetyl-3-thienyl) methane inclusion}

compound with 1,3-dichloropropane that crystallizes in at least five different forms. A combined single crystal and powder X-ray diffraction, ${ }^{13} \mathrm{C} \mathrm{CP} / \mathrm{MAS}$ solid-state NMR, density measurement and thermal analysis study.

Abstract 264

Introduction 265

Experimental

Results

(A) Powder Diffraction and ${ }^{13} \mathrm{C}$ CP/MAS NMR 268

(1) Forms 1 and $3 \quad 269$

(2) Form 2

(3) Forms 4 and $5 \quad 273$

(B) Thermogravimetric Analysis 275

(C) Differential Scanning Calorimetry 276

(D) Polycrystalline Densities 281

(E) Single Crystal X-Ray Diffraction 281

(4) Form 1

(5) Form $2 \quad 284$

(6) Form $3 \quad 286$

(7) Form $4 \quad 288$

(8) Form 5

Discussion

(F) Conformational preferences $\quad 292$

(G) Polymorphism in other systems 299

(9) Dianilinegossypol 299

(10) 5-methyl-2-[(2-nitrophenyl)amino]-3-thiophenecarbonitrile $\quad 301$

Concluding remarks 304

References $\quad 305$ 


\section{ABSTRACT}

In this work, we describe the preparation and structural properties of five polymorphs of the 1G:2H 1,3-dichloropropane / TATM inclusion compound. By employing six different synthetic techniques, we were able to obtain products whose structures were solved by single crystal X-ray diffraction. In addition, solid-state ${ }^{13} \mathrm{C}$ NMR spectroscopy and powder X-ray diffraction (PXRD) were used for bulk phase identification. On the basis of the unit cell parameters as determined from single crystal diffraction, the PXRD results were used to resolve the products as being from either pure or mixed phases. Four of the six polycrystalline products exhibited PXRD patterns that were each fit to one type of crystal (form 2-fast cool, anneal $50^{\circ}$ ) and (form 5-stir 4D RT, stir while cooling). On the other hand, the experimental PXRD pattern for the sample prepared by slow cooling may be fit to a combination of the calculated patterns for single crystal forms 1 and 3 , while the sample prepared by slow evaporation is made up of a mixture of forms $\mathbf{4}$ and 5 . One of the five types of single crystal $(1)$ is triclinic $(P \overline{1})$, while the other four forms are monoclinic (various space groups). In four of the five forms, the host molecules have similar torsional conformations and share a common layered motif. The guest molecules are disordered, and reside in cages or channels that are formed from the stacking of these layers. In form 2 , the conformation of the TATM host molecules differ significantly, and the disordered guests reside in a pronounced zig-zag channel. Apparently, slightly different conformations for the host and the flexile guest give rise to structures that differ little in energy. Thermogravimetric analysis (TGA) and differential scanning calorimetry (DSC) experiments allowed us to speculate on the thermal stabilities of the products of the six synthetic techniques. We found a direct correlation between rate of guest loss as it is heated (TGA) and decomposition enthalpy (as determined by DSC). Furthermore, both increasing crystal densities and pronounced zig-zag channels give rise to greater $\Delta_{\text {fus }} H$. These results suggest that minor variations in synthetic procedures can have a profound effect on the structure of the resulting crystalline product. Some of the structures presented here are closely related to previously reported TATM inclusion structures, suggesting that this may be a particularly rich area for studying polymorphism. 


\section{INTRODUCTION}

Polymorphism, the ability of the same compound to appear in different crystal forms $[1,2]$, is a phenomenon of both fundamental significance and industrial concern [3-5]. Therapeutically active substances (i.e. pharmaceuticals) frequently crystallize in different polymorphic forms as well as different solvated forms. Whereas the majority of synthetic organic compounds reported in the literature may be crystallized only once or twice during their purification, pharmaceuticals are generally recrystallized several times during the manufacturing process. The opportunities for detecting new forms are therefore more abundant for these materials, and the pharmaceutical literature abounds with reports of new polymorphs and solvates. A 1990 article lists nearly 500 pharmaceuticals for which crystalline polymorphism has been reported |6|. The importance of this phenomenon in the pharmaceutical industry cannot be underestimated, since the different crystal forms of a drug substance generally have different physical properties [7]. In particular, the solubilities of two polymorphs or two solvates of a drug substance may differ by as much as an order of magnitude, leading to significantly different systemic blood levels when the same dosages are employed. Identification and characterization of drug polymorphs and solvates, together with studies of their thermodynamic stabilities, are also important for predicting possible phase changes (e.g. hydration, polymorphic transition) during drug storage. Reviews on these aspects of crystal polymorphism have been published $[8,9]$.

Polymorphism is closely related to the inclusion phenomenon. as many versatile host compounds exist in two or more polymorphic forms [10,11]. Extensive polymorphism might be expected for the molecular crystals of inclusion compounds, as the existence of several energetically similar forms is an intrinsic feature of supramolecular materials based on weak interactions [12]. Surprisingly, very little is known about true polymorphism of inclusion compounds (the existence of different crystal modifications with the same host, guest and host:guest stoichiometry). Only two examples were reported for dianilinegossypol $[13,14]$ and another two for the title host (e.g. see chapter VII and reference [15]), with only two polymorphs being isolated in each of the four cases. Here we report data on the preparation and crystal structures of as many as five polymorphs of the title inclusion compound. 
TATM is a much-studied tripodal host molecule [16-30] related to the triphenylmethane group of hosts [31]. In a preliminary study [17], inclusion compounds resulted for every solvent from which the host was crystallized. TATM guest-host materials show a variety of stoichiometries and structural motifs [18-24,29,30]. Somewhat surprisingly, only three general distinct conformations of the substituted thienyl rings have been encountered among the reported structures |32|. Upon guest removal by vacuum sublimation, the host material becomes an amorphous solid, which has a broad melting point range of $50^{\circ}-58^{\circ} \mathrm{C}$. To this point in time, a crystalline form of the guest-free host has yet to be reported (however see Chapter XV below). Likely reasons are that the host molecules lack the ability to interact with each other via strong directional interactions, as there are only acetyl oxygens and weakly acidic hydrogens. In addition, despite the small number of observed conformations in the solid state [32], it is likely that a wide variety of conformational isomers are present in the liquid or vapour, thus making efficient packing difficult upon solidification.

For these reasons, molecules such as TATM may well be good candidates for finding multiple polymorphic forms, and indeed for two guests dimorphs are known. ([15], chapter VII) It is widely accepted that polymorphism provides a unique opportunity to study the effects of molecular structure upon bulk properties [33-37], conformational preferences [38-40], the ability to influence the crystallization process [41-44], and crystal engineering applications $[45,46]$.

As dimorphic forms have previously been encountered, it is apparent that in any investigation of TATM guest-host materials, it is important to verify that the bulk crystalline product is indeed characteristic of that selected for single-crystal $\mathrm{X}$-ray diffraction analysis. During the course of such an investigation it was discovered that a variety of crystal forms could be obtained with 1,3-dichloropropane as guest. Ultimately, five different crystal forms were identified for the $2 \mathrm{H}: \mathrm{lG}$ inclusion compound that were sufficiently stable for studying their structural properties in detail. This level of polymorphism is unusual, as evident from a recent survey: For compounds containing $C$, $\mathrm{H}, \mathrm{N}, \mathrm{O}, \mathrm{F}, \mathrm{Cl}$ and $\mathrm{S}$, there were 291 dimorphic compounds, 27 trimorphic, 3 were tetramorphic, while one of the survey papers reported a hexamorphic system [47]. Thus, it is clear that the structural diversity of the present pentamorphic system is quite unusual. 


\section{EXPERIMENTAL}

When it became apparent that multiple forms of the 1,3-dichloropropane / TATM inclusion compound existed, six different techniques were used to prepare either single crystals and/or polycrystalline powders of the 1,3-dichloropropane/TATM inclusion compound as indicated in Table XII-1:

Table XII-1: Summary of preparation techniques used to produce the six different products of 1,3-dichloropropane / TATM. We always use $500 \mathrm{mg}$ TATM and $2 \mathrm{~mL}$ of 1,3-dichloropropane.

Anneal $50^{\circ} \mathrm{C}$

Fast cooling

Slow cooling

Slow evaporation

Stir 4 days at RT

Stir while cooling
Dissolve $500 \mathrm{mg}$ TATM in $2 \mathrm{~mL} 1,3$-dichloropropane, seal in a 20 $\mathrm{mL}$ screw cap vial, place in an oven at $50^{\circ} \mathrm{C}$ and allow to anneal for 2 weeks, then cool to room temperature at a rate of $5^{\circ} \mathrm{C}$ per day.

Seal same mixture in a $20 \mathrm{~mL}$ screw cap vial, heat on a hot plate until the TATM solid dissolves, remove from the heat and allow to cool to room temperature on its own, over the course of $1-2$ hours.

Seal same mixture in a $20 \mathrm{~mL}$ screw cap vial, dissolve TATM on a hot plate, then place in a programmable temperature controlled oven, and allow to cool to room temperature at $1.2^{\circ} \mathrm{C}$ per hour $(3-4$ days).

Dissolve same mixture in a $20 \mathrm{~mL}$ vial at elevated temperature, allow to cool to room temperature while covered, then remove cap and allow excess guest to evaporate over the course of about 1 week.

Dissolve same mixture in a $20 \mathrm{~mL}$ vial, with a magnetic stir bar, and stir (covered) at room temperature for 4 days, then filter off excess solvent.

Dissolve same mixture, stir on a stir plate (covered) as it cools to room temperature ( $\sim 1$ hour), then filter.

The products were analyzed by a combination of techniques, including single crystal and powder X-ray diffraction, and ${ }^{13} \mathrm{C}$ CP/MAS NMR spectroscopy. Initially, if possible, several crystals from each batch were mounted on the single-crystal diffractometer and screened in order to obtain unit cell parameters and provide initial information on phase 
purity. The bulk products were identified both from the distinct multiplicities of the lines in the ${ }^{13} \mathrm{C}$ solid NMR spectrum (arising from the differing contents of the respective asymmetric units), and from the nature of non-overlapping reflections in the PXRD patterns. Once single-crystal structural data became available for the five different forms, the PXRD patterns for the polycrystalline products obtained from the various preparative procedures were indexed.

Using six different preparation techniques (see Table XII-1) to prepare the 1,3dichloropropane / TATM inclusion compound (as single crystals and/or as a polycrystalline powder), single crystal X-ray diffraction experiments yielded five different lattice types. The results are briefly summarized in Table XII-2. Form 1 is triclinic, space group $P \bar{T}$, while the other four forms $(2-5)$ are monoclinic, of various space groups.

\section{RESULTS}

(A) Powder X-ray diffraction and ${ }^{13} \mathrm{C}$ CP/MAS NMR:

Table XII-2: Preparation techniques and single crystal X-ray diffraction unit cell data for the five different forms of the 1,3-dichloropropane/TATM inclusion compound.

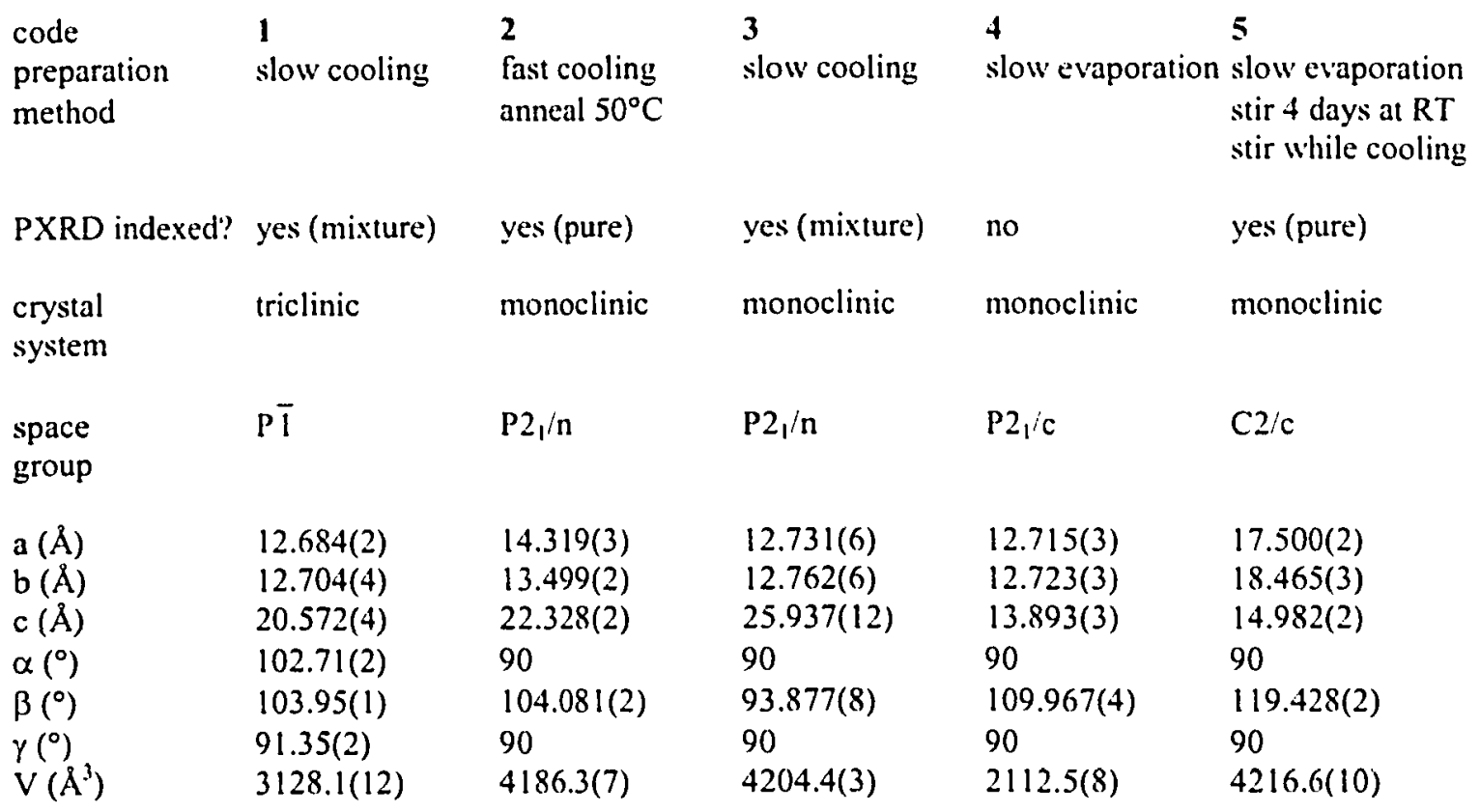


(1) Forms 1 and $\mathbf{3}$ (slow cooling):

The first and third forms ( 1 and 3 ) were prepared (as a mixture) by slow cooling (the details of the crystallization procedures are given in Table XII-1). This preparation technique yielded single crystals, and after single crystal X-ray diffraction experiments were completed, the crystals were ground into a fine powder and subjected to additional experiments (e.g. PXRD and ${ }^{13} \mathrm{C} C \mathrm{C} / \mathrm{MAS} N \mathrm{NM}$ ). The ${ }^{13} \mathrm{C}$ CP/MAS NMR spectrum of the slow cooling sample suggests that there are three host molecules in the asymmetric unit. This agrees with form 1 ( 3 host in A.U.), but not form 3 ( 2 host in A.U.). Despite this, the experimental PXRD pattern for the slow cooling sample may be fit to a combination of the calculated patterns for single crystal structures 1 and 3 . From the ${ }^{13} \mathrm{C}$ solid NMR and our observations of the crystals, we have come to the conclusion that form 1 is the major component of the slow cooling sample, and form 3 is the minor component. In Figure XII-1A, we present the experimental powder X-ray diffraction pattern for the slow cooling sample, along with a schematic depiction of the calculated patterns for forms 1 and 3. Additionally, in the supplemental material, we provide a peak-by-peak breakdown of the comparison between the calculated and experimental PXRD reflections. Some of the observed reflections may be accounted by form 1 , others by form 3, and others still by both 1 and 3 . There are only three reflections in the experimental pattern that cannot be accounted for by those calculated by either form 1 and/or form 3. Because the agreement between calculated and experimental PXRD patterns perhaps leaves something to be desired, we prepared the slow cooling sample three separate times, and the same experimental PXRD pattern was faithfully reproduced each time. For sake of brevity, we presented only one of the three patterns. Finally, the ${ }^{13} \mathrm{C}$ CP/MAS solid-state NMR spectrum of the slow cooling sample is presented in Figure XII-1B. By comparing the ${ }^{13} \mathrm{C}$ CP/MAS NMR spectrum and the PXRD pattern of the slow cooling sample with those of the other five products (see Figures XII-2 and XII-3), it is quite apparent that the slow cooling preparation technique produces a product that is unique in nature, at least when compared to the other 1,3-dichloropropane / TATM products of this study. Finally, as mentioned above, the multiplicity of the carbonyl carbon of the TATM host (at about $192 \mathrm{ppm}$ ) shows a multiplicity of 9 (from lowest to highest field, the relative intensities are 2,1,4,2), which implies that there are three TATM 
host molecules in the asymmetric unit, which agrees with the single crystal structure of form 1 , but not form 3 . Form 1 is novel in that the asymmetric unit is made up of 3 host molecules and $1 \frac{1}{2}$ guest molecules.

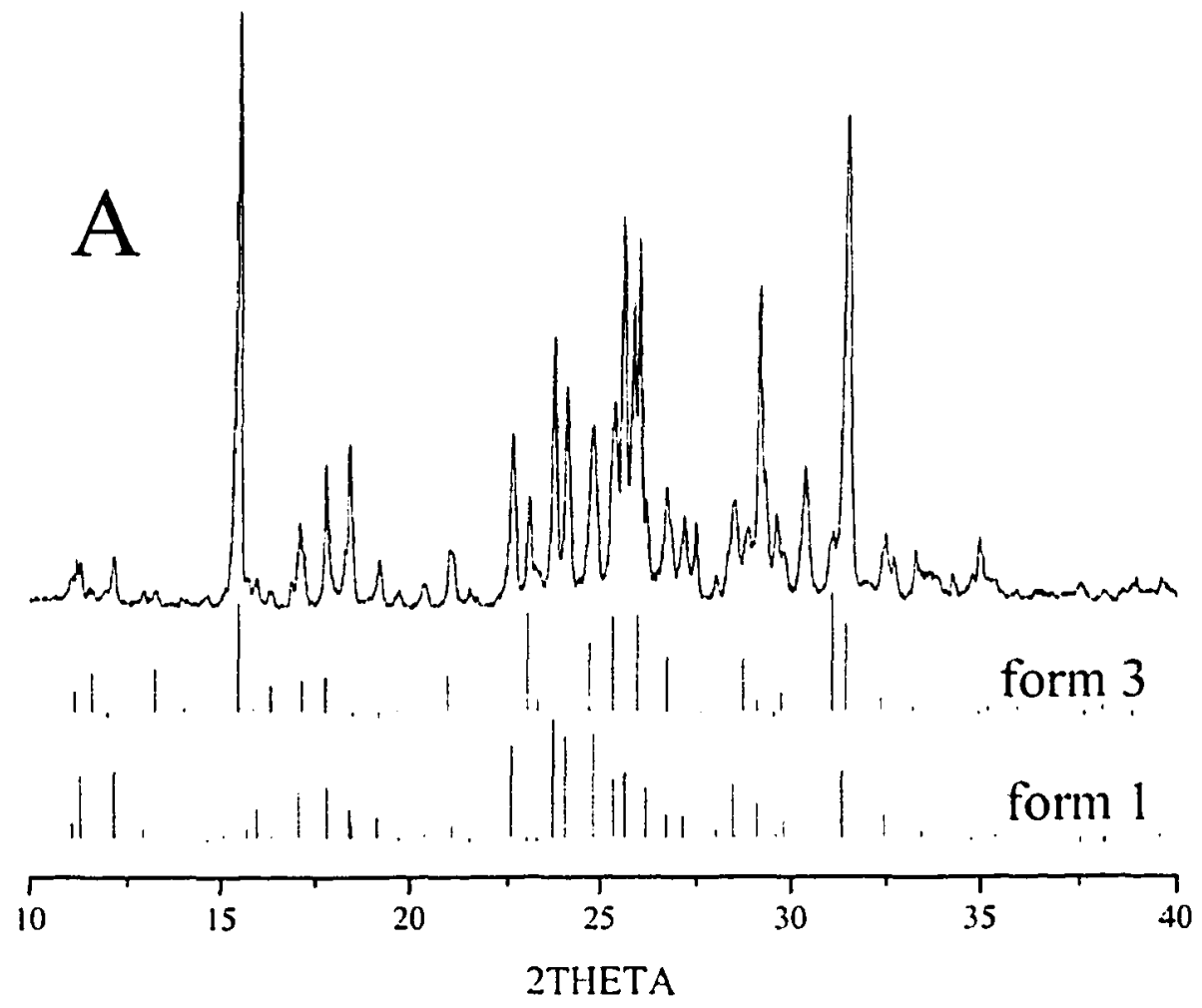

Figure XII-1A: Experimental PXRD pattern (above) of the TATM/1,3-dichloropropane inclusion compound obtained by slow cooling, along with calculated patterns (below) based on the single crystal unit cell parameters for form 1 and form 3 . Note correlation with both calculated patterns, suggesting the sample contains a mixture of both forms.

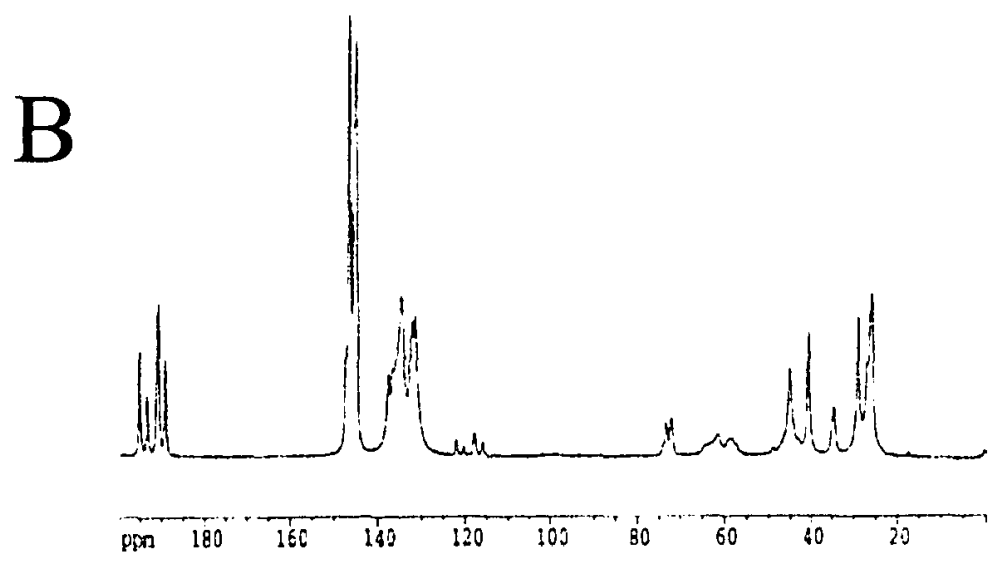

Figure XII-1B: ${ }^{13} \mathrm{C}$ CP/MAS NMR spectrum of the TATM/1,3-dichloropropane complex obtained by slow cooling. The multiplicity of the carbonyl signal of the host at $192 \mathrm{ppm}$ is nine, suggesting three host molecules in the asymmetric unit, in agreement with form 1 , but not form 3 . 
(2) Form 2 (fast cooling and annealing at $50^{\circ} \mathrm{C}$ )

Form 2 (see Table XII-2) was prepared independently by two different techniques, fast cooling and annealing at $50^{\circ} \mathrm{C}$. The sample used for single crystal X-ray diffraction was made from the fast cooling procedure, however, when each batch of crystals were ground into a fine powder, both samples gave experimental PXRD patterns that could be matched, at least to some degree, to calculated patterns for form 2. The calculated spectrum for form 2 , based on the single crystal unit cell parameters at room temperature, along with the experimental powder $\mathrm{X}$-ray diffraction patterns from samples prepared by fast cooling and annealing at $50^{\circ} \mathrm{C}$, appear in Figure XII-2. (In addition, we present the ${ }^{13} \mathrm{C}$ CP/MAS NMR spectra of these two products in the same figure). It is immediately

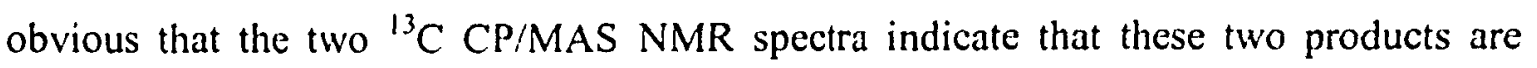
isostructural. The host splitting patterns at $192 \mathrm{ppm}, 140 \mathrm{ppm}$ and $28 \mathrm{ppm}$ are almost identical between the two spectra. On the other hand, the PXRD patterns in the same figure, although superficially similar, show some differences. This shows that PXRD is a much more sensitive technique to elucidate small differences in unit cell parameters, while ${ }^{13} \mathrm{C} \mathrm{CP} / \mathrm{MAS}$ NMR is less sensitive to these differences. However when one examines the two PXRD patterns very carefully, one sees that most of the correlating reflections are present, only the intensities are different. This may arise if the samples are not sufficiently ground. We were hesitant to grind the samples in a mortar and pestle, since in our experience, it leads to the buildup of static electricity, making it practically unusable. Regardless, both experiments suggest that these two preparation techniques produce the same product, and also that the two experimental PXRD patterns fit, more or less, with the calculated XRD spectrum for form 2.

By indexing the reflections of the two experimental patterns, we were able to calculate the room temperature unit cell parameters of both samples, using the room temperature single crystal unit cell parameters of form 2 as a starting point. The three sets of unit cell parameters appear Table XII-3. In this table we see very good agreement between single crystal and powder unit cell parameters, despite the fact that the fit between the three powder patterns in Figure XII-2 is only adequate. 

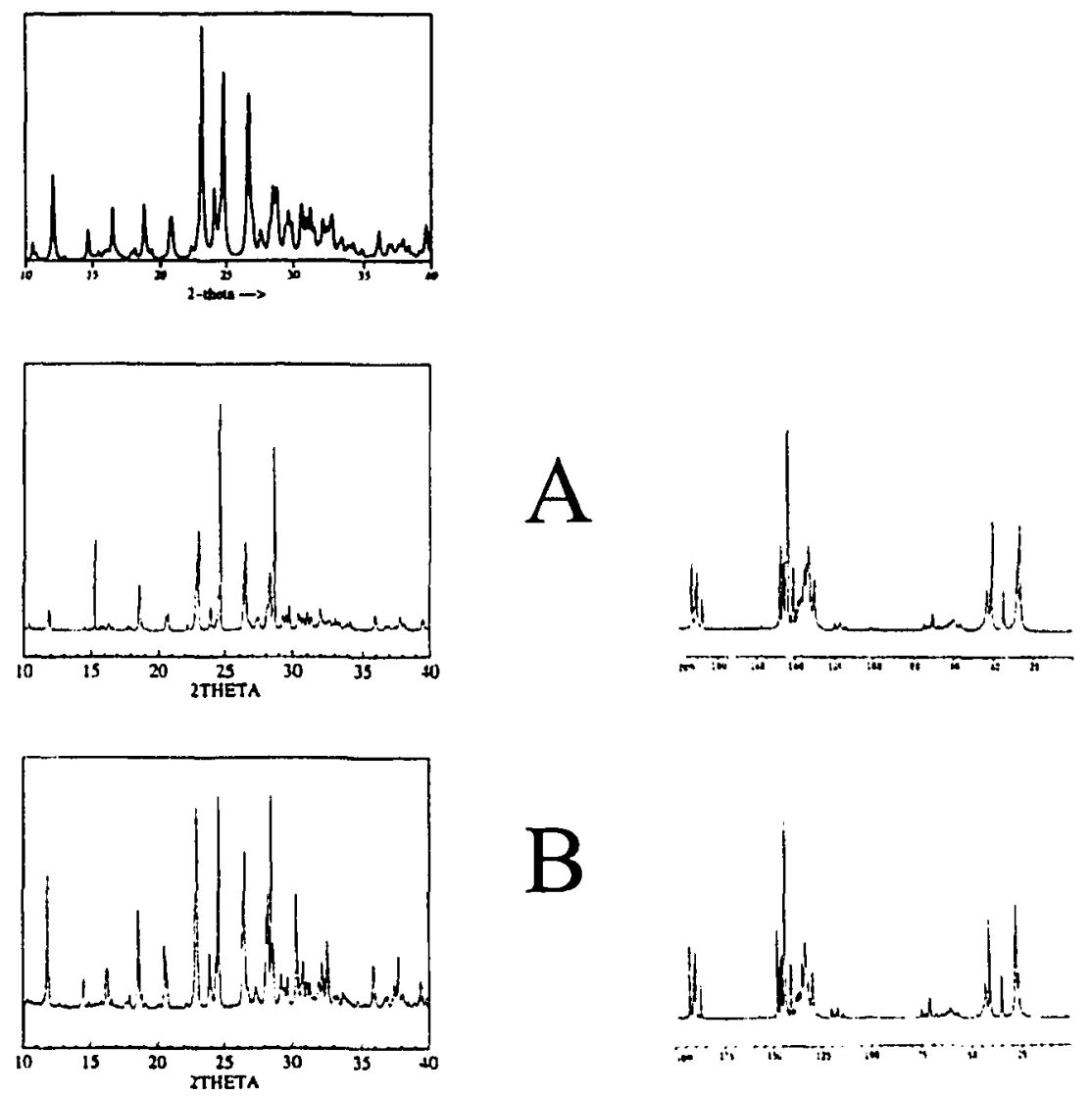

B

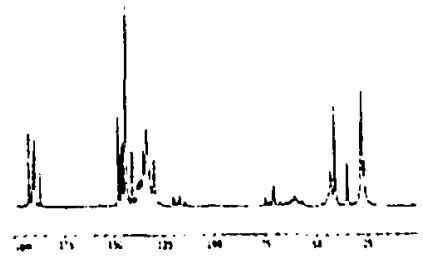

Figure XII-2: PXRD patterns (left) and ${ }^{13} \mathrm{C}$ CP/MAS NMR spectra (right) of the 1,3dichloropropane/TATM inclusion compounds prepared by (A) fast cooling and (B) annealing at $50^{\circ} \mathrm{C}$. The calculated PXRD pattern for form 2 is also depicted (at the top of the left column). Note the similarity of both experimental PXRD patterns with each other and also with the calculated pattern, and the near identical appearance of both experimental ${ }^{13} \mathrm{C}$ CP/MAS NMR spectra.

Table XII-3: Unit cell parameters for the 1,3-dichloropropane / TATM inclusion compound determined from single crystal X-ray diffraction (esd in brackets) at room temperature (for form 2), along with unit cell parameters calculated from the PXRD patterns (within $0.003 \%, 0.009 \%$ and $1.7 \%$ for unit cell dimensions, angles and volume, respectively) at room temperature for products from fast cooling and annealing at $50^{\circ} \mathrm{C}$

\begin{tabular}{llll} 
& form 2 (SC) & fast cooling (POW) & anneal $50^{\circ} \mathrm{C}(\mathrm{POW})$ \\
\hline$a(\AA)$ & $14.421(3)$ & 14.381 & 14.399 \\
$b(\AA)$ & $13.611(2)$ & 13.587 & 13.605 \\
$c(\AA)$ & $22.526(2)$ & 22.513 & 22.539 \\
$\alpha\left({ }^{\circ}\right)$ & 90 & 90 & 90 \\
$\beta\left(^{\circ}\right)$ & $103.840(2)$ & 103.81 & 103.78 \\
$\gamma\left({ }^{\circ}\right)$ & 90 & 90 & 90 \\
$V\left(\AA^{3}\right)$ & $4292.9(7)$ & 4271.80 & 4287.95 \\
\hline
\end{tabular}


(3) Forms 4 and 5 (slow evaporation, stir 4 days at RT, stir while cooling)

Single crystals of forms $\mathbf{4}$ and $\mathbf{5}$ were prepared by slow evaporation. Form $\mathbf{4}$ is the minor component, while form 5 is the major component, since we were able to fit the experimental PXRD pattern of the slow evaporation sample to the calculated pattern based on the crystal structure $\mathbf{5}$, but could not match it to the calculated pattern for form 4. In addition, we employed two more preparation techniques to yield polycrystalline samples of form 5 , namely stir while cooling and stir 4 days at room temperature (see Table XII-1). It should be noted that these last two preparation techniques yielded only polycrystalline powders, not single crystals.

In Figure XII-3 is depicted the calculated PXRD pattern based on the room temperature single crystal structure for form $\mathbf{5}$, along with the three experimental PXRD patterns and three ${ }^{13} \mathrm{C} C P / M A S ~ N M R$ spectra for samples prepared by slow evaporation, stir 4 days at room temperature and stir while cooling. As can be seen in Figure XII-3, the visual agreement between calculated and experimental PXRD patterns is excellent for all three samples. In Table XII-4 are listed the room temperature unit cell parameters for the three products obtained by correlating the calculated and experimental patterns, using the unit cell parameters of form 5 as a starting point. The numerical agreement between single crystal and powder unit cell parameters is also excellent. Also, the three experimental ${ }^{13} \mathrm{C} \mathrm{CP} / \mathrm{MAS}$ spectra are almost identical in appearance, and furthermore suggest that there is only one TATM molecule in the asymmetric unit, which agrees with the single crystal structure of the slow evaporation product. 

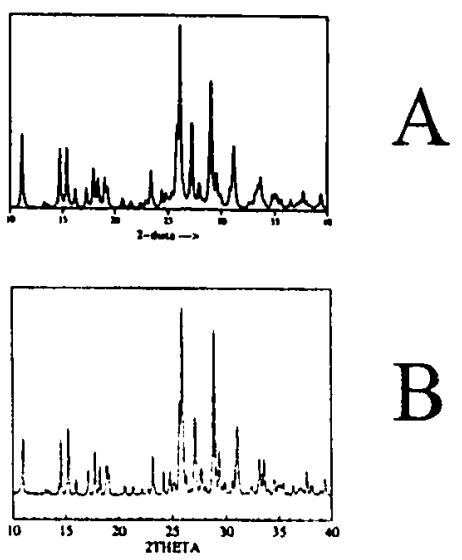

B
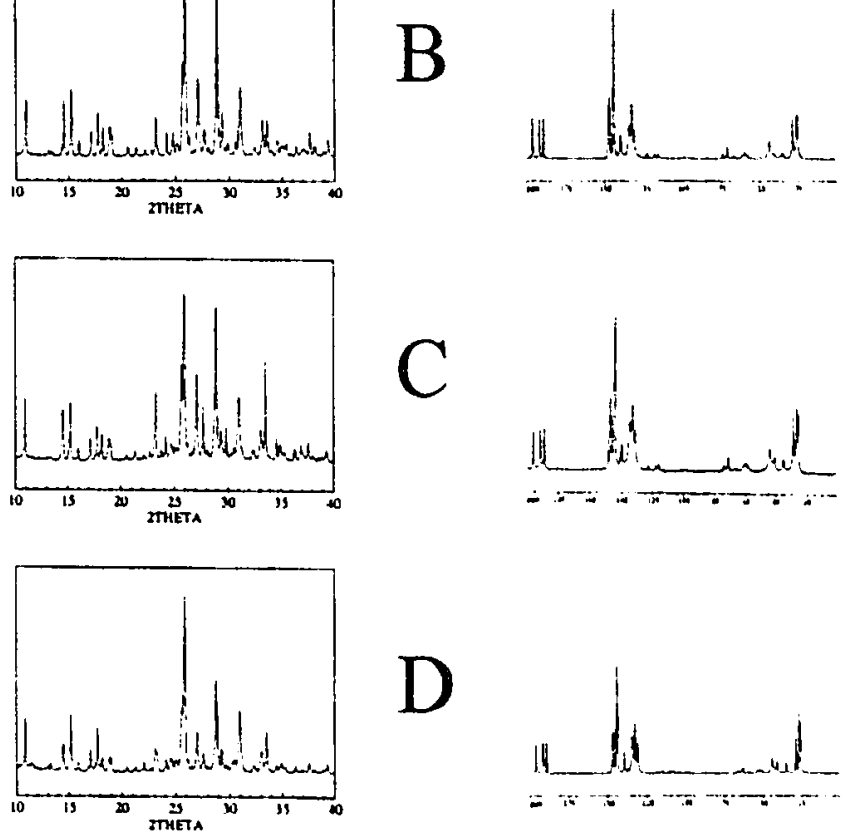

Figure XII-3: PXRD patterns (left) and ${ }^{13} \mathrm{C} C \mathrm{CP} / \mathrm{MAS}$ NMR spectra (right) of the 1,3dichloropropane/TATM inclusion compounds prepared by (B) stir 4 days at room temperature, (C) slow evaporation and (D) stir while cooling. The calculated PXRD pattern (A) for form 5 is depicted (at the top of the left column). Note the similarity of all three experimental PXRD patterns with each other and also with the calculated pattern,

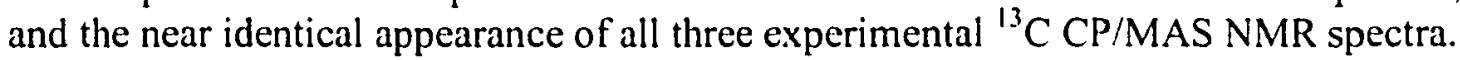

Table XII-4: Unit cell parameters for the 1,3-dichloropropane / TATM inclusion compound determined from single crystal X-ray diffraction (esd in brackets) at room temperature (for form 5), along with unit cell parameters calculated from the PXRD patterns (within $0.003 \%, 0.009 \%$ and $1.7 \%$ for unit cell dimensions, angles and volume, respectively) at room temperature for products from slow evaporation, stir 4 days at room temperature, and stirring while cooling.

\begin{tabular}{lllll} 
& form 5 (SC) & slow evap (POW) & stir 4 days RT (POW) & stir while cooling (POW) \\
\hline$a(\AA)$ & $17.680(2)$ & 17.629 & 17.677 & 17.623 \\
$b(\AA)$ & $18.445(3)$ & 18.471 & 18.487 & 18.424 \\
$c(\AA)$ & $15.220(2)$ & 15.177 & 15.206 & 15.165 \\
$\alpha\left({ }^{\circ}\right)$ & 90 & 90 & 90 & 90 \\
$\beta\left({ }^{\circ}\right)$ & $118.710(2)$ & 118.84 & 119.02 & 118.83 \\
$\gamma\left({ }^{\circ}\right)$ & 90 & 90 & 90 & 90 \\
$V\left(\AA^{3}\right)$ & $4353.5(10)$ & 4328.86 & 4345.70 & 4313.53 \\
\hline
\end{tabular}


(B) Thermogravimetric analysis (TGA)

We wish to use TGA in this study to measure the relative thermal stabilities of the 5 crystal forms (or more specifically the 6 products that correspond to the six preparation techniques in Table XII-1). One might expect that TATM inclusions with higher $\Delta_{\text {fus }} H$ may hold onto their guest molecules more tenaciously than TATM inclusions with lower $\Delta_{\text {fus }} H$ as the samples are heated above room temperature.

The TGA curves for the polycrystalline products of the six different preparation methods appear in Figure XII-4. In these graphs, one observes a rapid loss of guest at lower temperatures (up to the inflection point at about $90^{\circ} \mathrm{C}-100^{\circ} \mathrm{C}$ ), characterized by a steep slope of the TGA curve. At temperatures above the inflection point, guest loss occurs much more slowly. From our experience with TGA of TATM inclusion compounds, we interpret the temperature of the inflection point as corresponding to the melting temperature of the inclusion compound. At lower temperatures, the loss of guest occurs rapidly due to the large surface area of the polycrystalline powder. Above the inflection point, the rate of guest loss is much smaller, as the sample is basically a molten mass, with a much smaller surface area. Therefore, we are only concerned with TGA data at temperatures below the inflection point, since at higher temperatures, the integrity of the structure is lost.

From Figure XII-4, one immediately notices that some of the compounds, namely anneal at $50^{\circ} \mathrm{C}$, fast cooling and slow cooling (refer to Table XII-1), do not lose the guest as easily upon heating. As is clearly seen in Figure XII-4, when the slow cooling sample melts, it had lost only about $3 \%$ of its original mass, while fast cooling and anneal at $50^{\circ} \mathrm{C}$ samples each lose about $3.5 \%$ of their original mass before they melt. These three samples correspond to forms $\mathbf{1}-\mathbf{3}$ (see Table XII-2), with larger $\Delta_{\text {fus }} H$.

On the other hand, the other three preparation techniques give rise to forms of 1,3dichloropropane / TATM that do indeed lose guest more easily on heating, as is also depicted in Figure XII-4. The slow evaporation sample has lost about $4.5 \%$ of its mass when it melts, while stir 4D RT and stir while cooling (refer to Table XII-1) have each lost about $6 \%$ to $6.5 \%$ of their original masses when they melt. These three samples have a smaller $\Delta_{\text {fus }} H$. So we see an interesting correlation between the DSC and TGA experiments. 


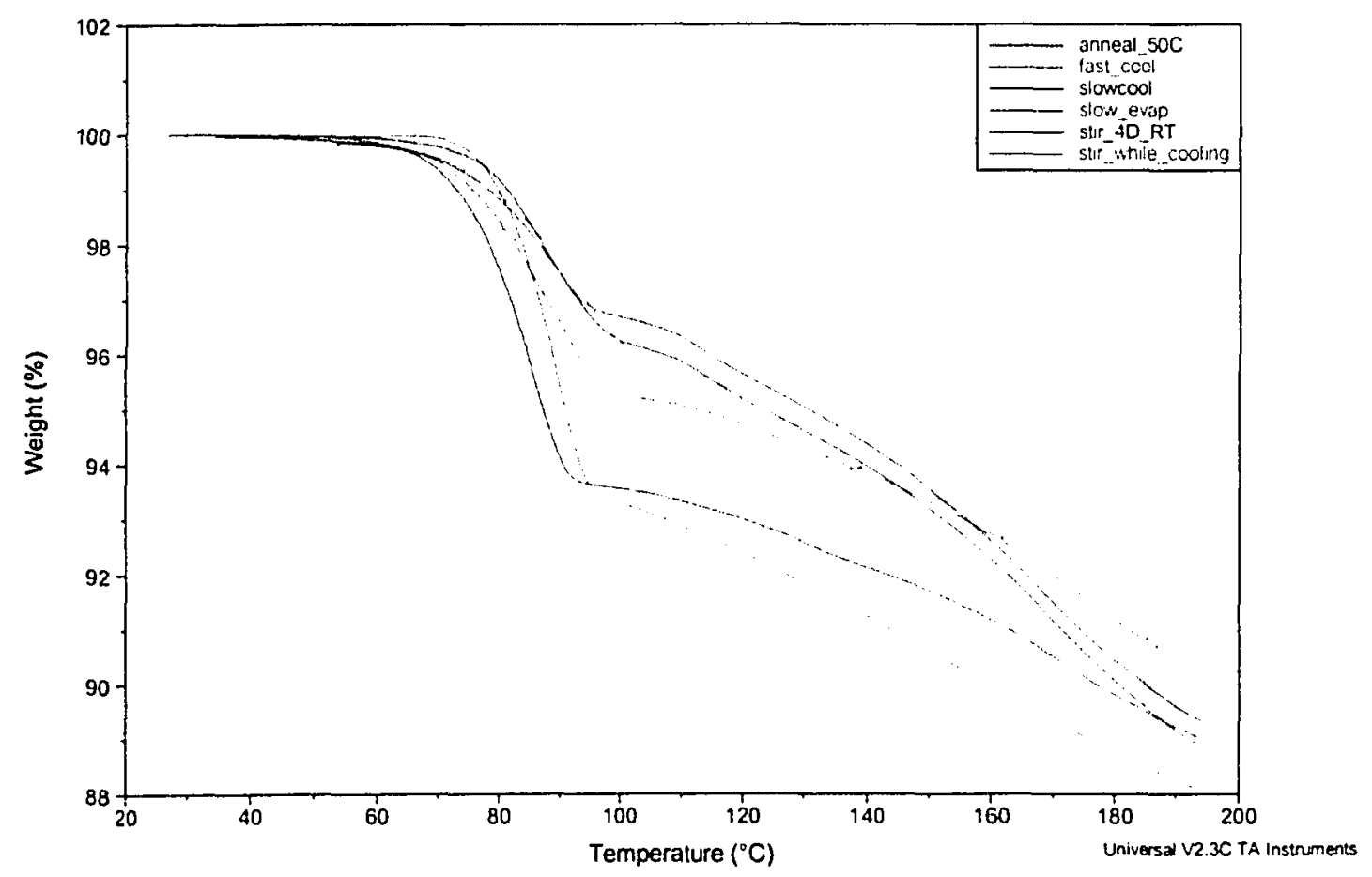

Figure XII-4: Thermogravimetric traces of the six 1,3-dichloropropane/TATM inclusion compounds prepared according to procedures listed in Table XII-1. The labeling scheme of the six traces is given in the figure legend. Note that the products prepared by annealing at $50^{\circ} \mathrm{C}$, fast cooling and slow cooling (forms 1,2 and 3 ) hold onto their guests more tenaciously as heated, while the products slow evaporation, stir 4 days at room temperature and stir while cooling (forms $\mathbf{4}$ and $\mathbf{5}$ ) lose their guests more easily as they are heated above room temperature.

(C) Differential scanning calorimetry (DSC):

We wish to employ DSC studies as a complementary technique to TGA in terms of inferring relative thermal stabilities of the different forms of the 1,3-dichloropropane / TATM inclusion compound. By heating the samples from room temperature to about $200^{\circ} \mathrm{C}$, we pass through the melting endotherm of each form. The DSC data for the six different preparation techniques (polycrystalline samples) appear in Table XII-5. In this table we list the peak melting temperature and the enthalpy of decomposition for each sample. 
Table XII-5: DSC and polycrystalline density (at RT) data for the 1,3-dichloropropane / TATM inclusion compounds prepared according to the six different techniques outlined in Table XII-1.

\begin{tabular}{lllll} 
Preparation method & $\begin{array}{l}T_{\mathrm{m}} \\
\left( \pm 0.1^{\circ} \mathrm{C}\right)\end{array}$ & $\begin{array}{l}\Delta_{\text {fus }} \mathrm{H} \\
\left( \pm 1 \mathrm{~kJ} \mathrm{~mol}^{-1}\right)\end{array}$ & $\begin{array}{l}d_{\text {meas }}\left(\mathrm{g} \mathrm{cm}^{-3}\right) \\
(\mathrm{pycnometer})\end{array}$ & $\begin{array}{l}d_{\text {calc }}( \pm 0.001 \mathrm{~g} \\
\left.\mathrm{cm}^{-3}\right)(\mathrm{crystal})\end{array}$ \\
\hline $\begin{array}{l}\text { strongly held guest } \\
\text { anneal 50 }\end{array}$ & 107.6 & 30 & $1.374( \pm 0.002)$ & 1.377 (form 2) \\
fast cooling & 104.6 & 30 & $1.359( \pm 0.002)$ & 1.377 (form 2) \\
slow cooling & 104.6 & 32 & $1.364( \pm 0.003)$ & $1.370($ form 3) \\
& & & & \\
weakly held guest & & & & \\
slow evaporation & 106.2 & 27 & $1.362( \pm 0.001)$ & 1.359 (form 4) \\
stir while cooling & 106.5 & 27 & $1.371( \pm 0.003)$ & 1.358 (form 5) \\
stir 4 days at RT & 107.3 & 29 & $1.363( \pm 0.002)$ & 1.358 (form 5)
\end{tabular}

At this point, it is possible to speculate about the correlation of results between the TGA and DSC experiments. As we saw in the previous section (TGA), the six samples may be broken down into two groups of three. The anneal at $50^{\circ} \mathrm{C}$, fast cooling and slow cooling samples hold onto their guests quite strongly as they are heated, while the other three samples, namely slow evaporation, stir 4 days RT and stir while cooling, lose guest more readily upon heating. By comparing these results (TGA) with the DSC results (see Table XII-5), we see the same type of grouping. The three samples that hold onto their guest quite strongly (TGA) have a higher enthalpy of decomposition (DSC), ranging from 30 $\mathrm{kJ} \mathrm{mol}^{-1}$ to $32 \mathrm{~kJ} \mathrm{~mol}^{-1}$ (see Table XII-5). On the other hand, the three forms that release guest more easily on heating (TGA), have a smaller enthalpy of decomposition (DSC), varying from $27 \mathrm{~kJ} \mathrm{~mol}^{-1}$ to $29 \mathrm{~kJ} \mathrm{~mol}^{-1}$. We believe that these differences are larger than can be attributed to experimental error. However, while the heat of decomposition results (DSC) show a correlation with the TGA studies, the melting temperatures of each sample fail to show the same correlation, ranging from $104.6^{\circ} \mathrm{C}$ to $107.6^{\circ} \mathrm{C}$ for the first group (strongly held guest), and $106.2^{\circ} \mathrm{C}$ to $107.3^{\circ} \mathrm{C}$ for the second group (less strongly held guest). 
The melting endotherms for the six products appear in Figures XII-5 and XII-6. The baseline of each endotherm was manually offset to allow an easier comparison. For the first set of products that hold the guest more strongly (Figure XII-5), the melting endotherms for the slow cooling and fast cooling products appear almost identical, although the fast cooling product endotherm is slightly broader. The relative broadness of the two peaks suggests that these two products each consist of perhaps two forms (e.g. 1,2 or 3 - see Table XII-2). On the other hand, the endotherm of the product from annealing at $50^{\circ} \mathrm{C}$ occurs at a significantly higher temperature, and is much more narrow. These results suggest that, as far as the DSC results are concerned, the rate of cooling does not have a significant effect on the nature of the product, and also that these two products may each consist of more than one form. Additionally, the sharpness of the endotherm for the product from annealing at $50^{\circ} \mathrm{C}$ suggests that this product consists of only one form (form 2 - see Table XII-2)

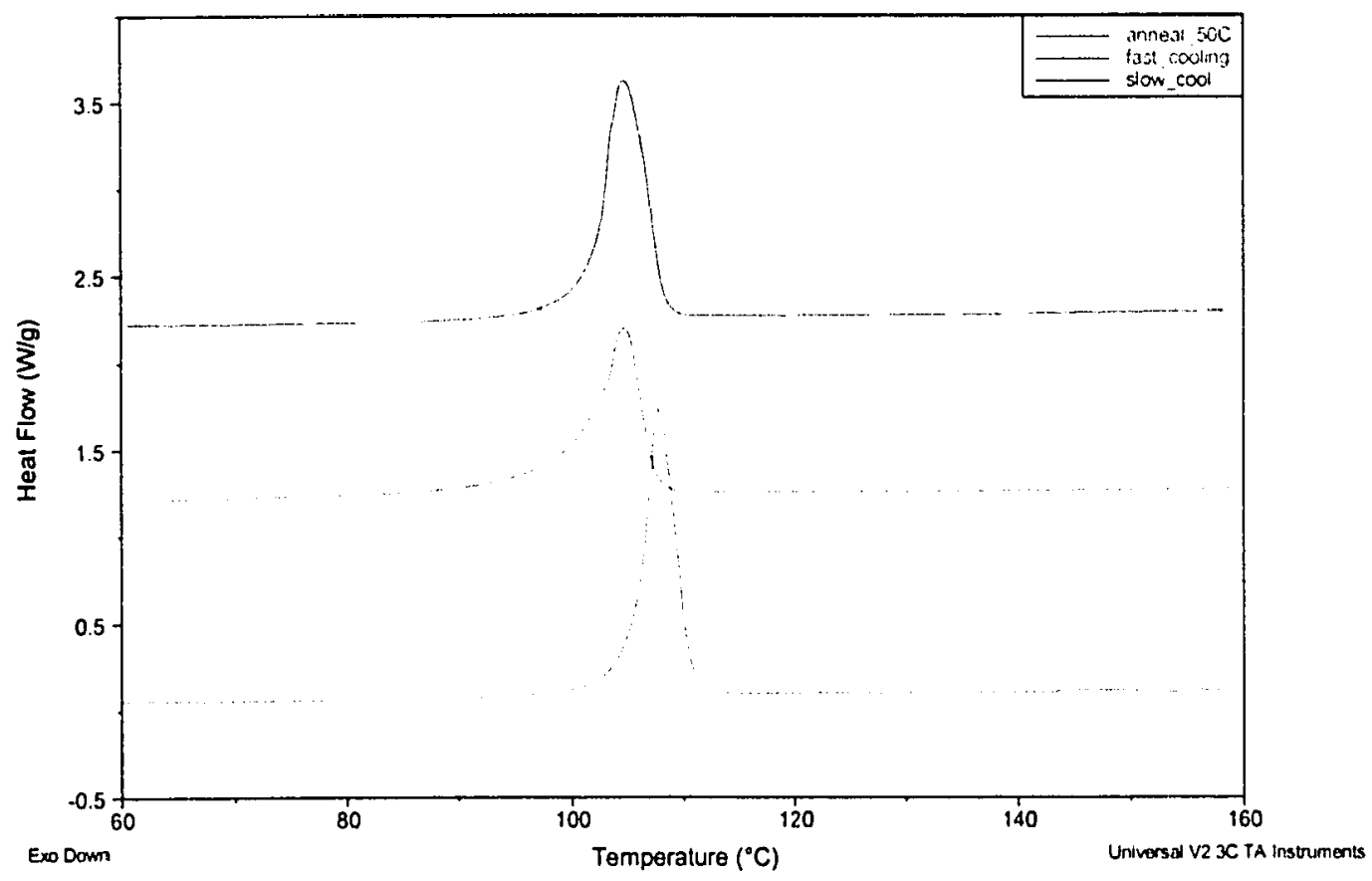

Figure XII-5: DSC traces for 1,3-dichloropropane/TATM inclusion compounds prepared by annealing at $50^{\circ} \mathrm{C}$, fast cooling and slow cooling. Melting temperatures and enthalpies of decomposition derived from the traces are given in Table XII-5. 
In Figure XII- 6 are depicted the melting endotherms for the three products that lose guest more easily on heating. The melting endotherms from the products stir while cooling and stir 4 days at RT are almost identical in appearance. Both show a fairly sharp melting endotherm, and also a very weak signal for each at about $115^{\circ} \mathrm{C}$. This suggests that the process of stirring is a decisive factor in determining the structure of these two forms, whether it is a short process (stir while cooling) or a long process (stir 4 days at RT). Additionally, a weak peak at about $115^{\circ} \mathrm{C}$ for both stirred samples implies that there is perhaps a minor component for each of these products, possibly one of the three minor components 1,3 or 4 - see Table XII-2. Finally, the slow evaporation product displays a melting endotherm that is very broad, much broader than that of the other five products. This suggests that this product consists of at least two (namely 4 and 5) or more forms. This endotherm was faithfully reproduced in two separate samples.

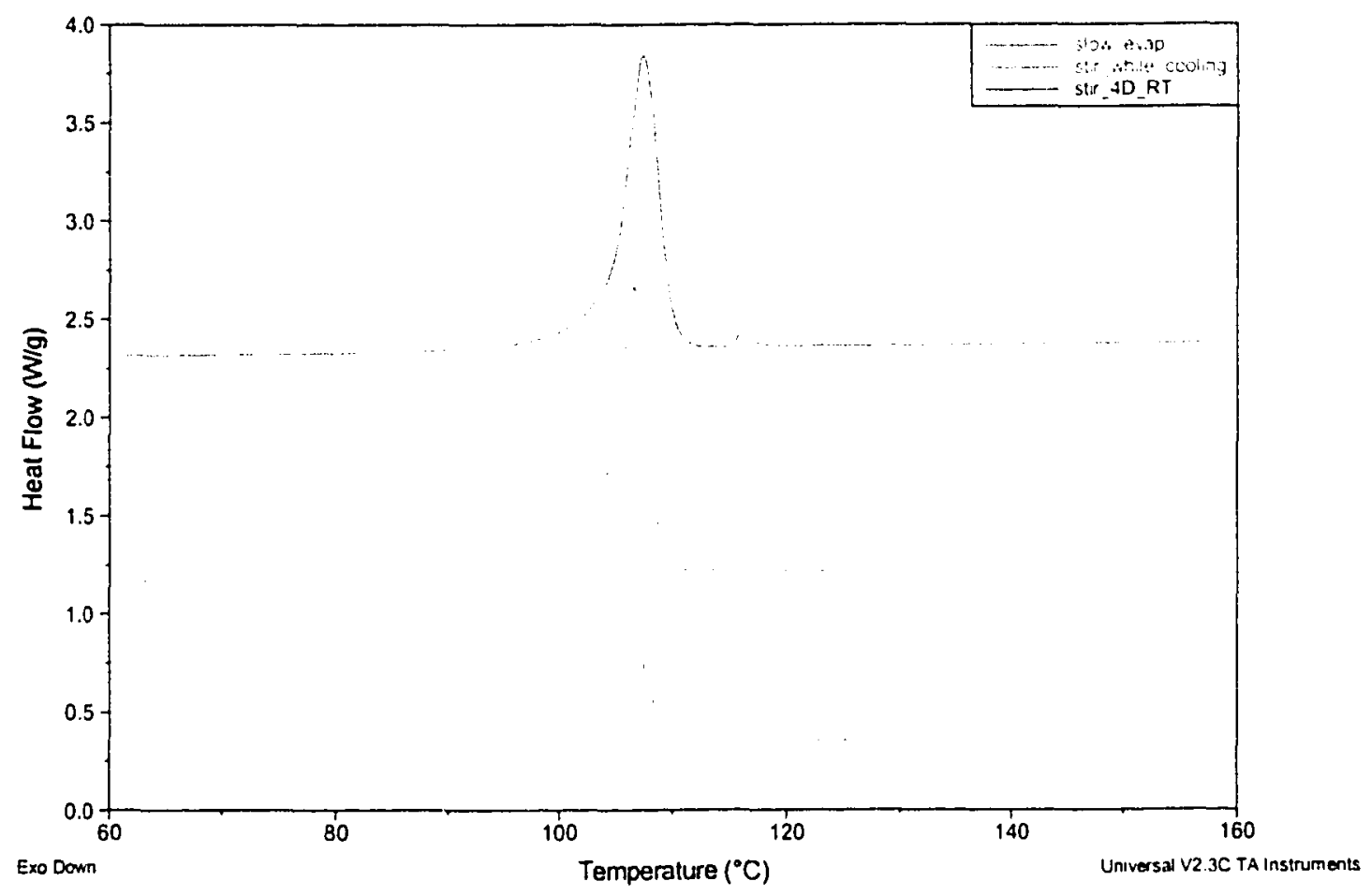

Figure XII-6: DSC traces for 1,3-dichloropropane/TATM inclusion compounds prepared by slow evaporation, stir while cooling and stir 4 days at room temperature. Melting temperatures and enthalpies of decomposition derived from the traces are given in Table XII-5. 
A publication from 1999 [48] reported inclusion compounds of ortho-, meta- and para-xylene with the host 1,1'-binaphthyl-2,2'-carboxylic acid, 1 . The authors found a direct relationship between the two measures of thermal properties, namely the heat of decomposition (DSC) and the rate of guest loss with an increase in temperature (TGA). Their experiments show that $\Delta H_{\mathrm{d}}$ for inclusions with 1 rank as follows: $1 / p$-xylene $58 \mathrm{~kJ}$ $\mathrm{mol}^{-1}, 1 / o$-xylene $49 \mathrm{~kJ} \mathrm{~mol}^{-1}$ and $1 / m$-xylene $30 \mathrm{~kJ} \mathrm{~mol}^{-1}$. This is reflected in the rate of guest loss with increasing temperature (see Figure XII-7). The $p$-xylene inclusion holds onto its guest most strongly, followed by the $o$-xylene inclusion. Finally, the $m$ xylene inclusion holds onto its guest the least strongly. Thus we see another example of the complementary information that can be obtained by using both DSC and TGA.<smiles>O=C(O)c1ccc2ccccc2c1-c1c(C(=O)O)ccc2ccccc12</smiles>

Scheme 1: 1,1'-binaphthyl-2,2'-carboxylic acid host material

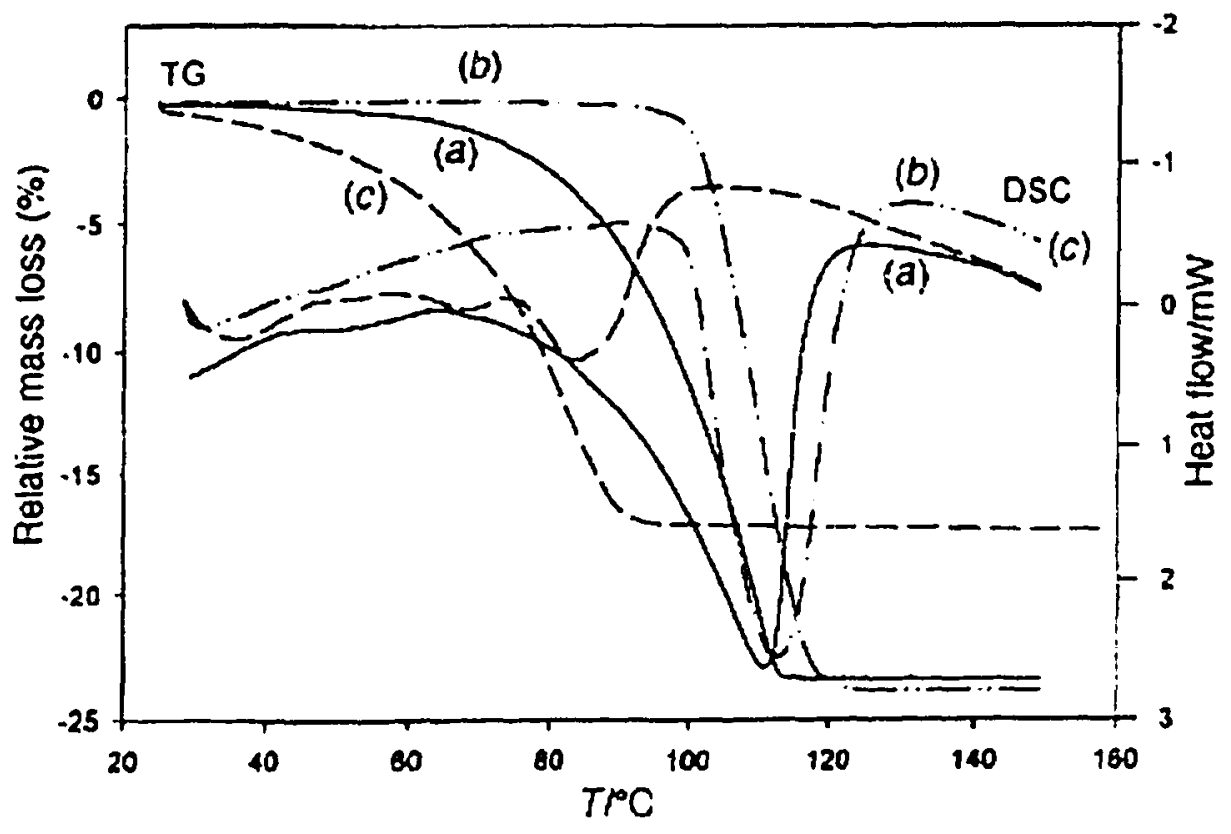

Figure XII-7: TGA and DSC traces for the 1,1'-binaphthyl-2,2'-carboxylic acid host with xylene guests (a) ortho-xylene, (b) para-xylene, (c) meta-xylene [48] 
(D) Polycrystalline densities (gas pycnometer):

In Table XII-5, along with the DSC results, is depicted the density measurement results for the six samples prepared according to Table XII-1. Both experimental polycrystalline measurements $\left(d_{\text {meas }}\right)$ and calculated single crystal values $\left(d_{\text {calc }}\right)$ are tabulated. All values correspond to densities at room temperature. Initially, one might expect that the three samples with more strongly held guests (anneal $50^{\circ} \mathrm{C}$, fast cooling and slow cooling), as determined from TGA, would have a higher density, due to more extensive attractive intermolecular interactions. On the other hand, the other three samples (slow evaporation, stir while cooling and stir 4 days at RT) might be expected to have a lower density. On examination of the values in Table XII-5, this expectation is fulfilled for the $d_{\text {calc }}$ (single crystal) values, but not the $d_{\text {meas }}$ (powder) measurements. As far as $d_{\text {calc }}$ is concerned, the three samples with more strongly held guests (from TGA) have values ranging from $1.377 \mathrm{~g} / \mathrm{mL}$ (form 2) to $1.370 \mathrm{~g} / \mathrm{mL}$ (form 3), while the other three samples have $d_{\text {calc }}$ values ranging from $1.359 \mathrm{~g} / \mathrm{mL}$ (form 4) to $1.358 \mathrm{~g} / \mathrm{mL}$ (form 5). On the other hand, the experimental polycrystalline density measurements ( $\left.d_{\text {meas }}\right)$ fail to show a similar correlation with thermal stabilities.

(E) Single crystal $X$-ray diffraction:

The crystal data, data collection and refinement parameters for the five forms are summarized in Table XII- 6 . The $C$-centered cell of form 5 can be reduced to a primitive unit cell with $a=12.718 \AA, b=12.722 \AA, c=14.982 \AA, \alpha=109.707^{\circ}, \beta=109.791^{\circ}, \gamma=$ 93.074 . (transformation matrix $-1 / 2,-1 / 2,0,-1 / 2,1 / 2,0,0,0,-1$ ). Upon examination of all of the structural parameters, it becomes clear that forms 1, 3, 4 and 5 all share a common planar motif characterized by $a \sim b \sim 12.70 \AA$, with an included angle $\gamma$ close to $90^{\circ}$. In all of these structures, the host TATM molecules are arranged in layers similar to those depicted in Figure XII-8 (form 1). In general, one thienyl ring from each host molecule $\pi$-stacks with a thienyl ring from a neighboring host to form TATM dimers. The remaining two thienyl rings combine with those from a neighboring dimer (in the other direction) to form a cage or channel that encloses the included guest molecules, thus forming endless ribbons of TATM dimers that are aligned along a diagonal in $a+b$ or $a-b$ 
directions. Adjacent ribbons are connected by weak aromatic $\mathrm{C}-\mathrm{H} \cdots \mathrm{O}$ interactions. Subtle differences in the structural motifs are discussed below.

Table XII-6: Crystal data, data collection and final refinement parameters for the five crystal forms of 1,3-dichloropropane / TATM

\begin{tabular}{|c|c|c|c|c|c|}
\hline \multicolumn{6}{|l|}{ Crystal Data } \\
\hline Form code & 1 & 2 & 3 & $t$ & 5 \\
\hline Empirical lonmula & $\begin{array}{l}2 \mathrm{C}_{14} \mathrm{H}_{16} \mathrm{O}_{4} \mathrm{~S}_{3} \\
1 \mathrm{C}_{2} \mathrm{H}_{4} \mathrm{Cl}_{2}\end{array}$ & $\begin{array}{l}2 \mathrm{C}_{34} \mathrm{H}_{1 \mathrm{r}} \mathrm{O}_{1} \mathrm{~S}_{3} \\
\mid \mathrm{C}_{2} \mathrm{H}_{4} \mathrm{Cl}_{2}\end{array}$ & $\begin{array}{l}2 \mathrm{C}_{14} \mathrm{H}_{11,} \mathrm{O}_{3} \mathrm{~S}_{3} \\
1 \mathrm{C}_{2} \mathrm{H}_{4} \mathrm{Cl}_{2}\end{array}$ & $\begin{array}{l}2 \mathrm{C}_{11} \mathrm{H}_{16} \mathrm{O}_{3} \mathrm{~S}_{3} \\
1 \mathrm{C}_{2} \mathrm{H}_{4} \mathrm{Cl}_{2}\end{array}$ & $\begin{array}{l}2 \mathrm{C}_{14} \mathrm{H}_{16} \mathrm{O}_{3} \mathrm{~S}_{3} \\
1 \mathrm{C}_{2} \mathrm{H}_{4} \mathrm{Cl}_{2}\end{array}$ \\
\hline Fornula weighı & 889.97 & 889.97 & 889.97 & 889.97 & 889.97 \\
\hline Temperature $(K)$ & $125(2)$ & $173(2)$ & $173(2)$ & $173(2)$ & $173(2)$ \\
\hline Crystal system & triclinic & monoclinic & monoclinic & monoclinic & monoclinic \\
\hline Space Group & $\mathrm{P} \bar{l}$ & $\mathrm{P} 2_{1} / \mathrm{n}$ & $\mathrm{P} 2_{1} / \mathrm{n}$ & $\mathrm{P} 2, / \mathrm{c}$ & $\mathrm{C} 2 / \mathrm{c}$ \\
\hline$a(\AA)$ & $12.68+(2)$ & $14.319(3)$ & $12.731(6)$ & $12.715(3)$ & $17.500(2)$ \\
\hline$b(A)$ & $12.704(4)$ & $13.499(2)$ & $12.762(6)$ & $12.723(3)$ & $18.465(3)$ \\
\hline$c(\bar{A})$ & $20.572(4)$ & $22.328(2)$ & $25.937(12)$ & $13.893(3)$ & $14.982(2)$ \\
\hline$\alpha\left({ }^{\circ}\right)$ & $102.71(2)$ & 90 & 90 & 90 & 90 \\
\hline$\beta\left({ }^{\circ}\right)$ & $103.95(1)$ & $104.081(2)$ & $93.877(8)$ & $109.967(4)$ & $119.428(2)$ \\
\hline$y\left({ }^{\circ}\right)$ & $91.35(2)$ & 90 & 90 & 90 & 90 \\
\hline$V\left(A^{\prime}\right)$ & $3128.1(12)$ & $4186.3(7)$ & $+204.4(3)$ & $2112.5(8)$ & $4216.6(10)$ \\
\hline 7 & 3 & 4 & 4 & 2 & 4 \\
\hline$d_{\text {Lalc }}\left(\mathrm{g} \mathrm{cm}^{-1}\right)$ & 1.417 & 1.412 & 1.406 & 1.399 & 1.402 \\
\hline$F(000)$ & 1386 & 1848 & 1848 & 924 & 1848 \\
\hline$\mu\left(\operatorname{MoK}(\ell)\left(\mathrm{nm}^{-1}\right)\right.$ & 0.502 & 0.501 & 0.498 & 0.496 & 0.497 \\
\hline$\lambda(A)$ & 0.71073 & 0.71073 & 0.71073 & 0.71073 & 0.71073 \\
\hline 20 range $\left({ }^{\circ}\right)$ & $1.05^{\circ}<20<29.59^{\circ}$ & $1.54^{\circ}<20<29.62^{\circ}$ & $1.57^{\circ}<20<25.68^{\circ}$ & $1.70^{\circ}<20<26.46^{\circ}$ & $1.73^{\circ}<20<29.69^{\circ}$ \\
\hline \multicolumn{6}{|l|}{ Data Collection } \\
\hline Crystal dimensions & $0.40 \times 0.30 \times 0.25$ & $0.20 \times 0.20 \times 0.35$ & $0.08 \times 0.35 \times 0.45$ & $0.15 \times 0.15 \times 0.23$ & $0.15 \times 0.20 \times 0.30$ \\
\hline \multirow[t]{3}{*}{ Index runges } & $-17 \leq h \leq 17$ & $-19 \leq h \leq 19$ & $-15 \leq h \leq 15$ & $-15 \leq h \leq 15$ & $-24 \leq h \leq 24$ \\
\hline & $-17 \leq k \leq 16$ & $-18 \leq k \leq 18$ & $-15 \leq k \leq 15$ & $-15 \leq k \leq 15$ & $-25 \leq k \leq 25$ \\
\hline & $-28 \leq 1 \leq 28$ & $-31 \leq 1 \leq 30$ & $-31 \leq l \leq 31$ & $-15 \leq 1 \leq 17$ & $-19 \leq 1 \leq 20$ \\
\hline Reflections measured & 39057 & 52066 & 39069 & 16361 & 26324 \\
\hline Unique reflections & 17221 & $117+2$ & 7971 & 4331 & 5965 \\
\hline$R(\mathrm{int})$ & 0.0269 & 0.0803 & 0.0769 & 0.0770 & 0.0492 \\
\hline Completeness to $20_{\mathrm{nux}}$ & $97.9 \%$ & $99.5 \%$ & $99.9 \%$ & $99.3 \%$ & $99.5 \%$ \\
\hline Scan mode & omega & omega & omega & omega & omega \\
\hline Absorption correction & multiscan & multiscan & multiscan & multiscan & multiscan \\
\hline \multicolumn{6}{|l|}{ Final Refinement } \\
\hline \multirow[t]{2}{*}{ Refinement method } & full-matrix & full-matrix & full-matrix & full-matrix & full-matrix \\
\hline & least squares & least squares & least squares & least squares & least squares \\
\hline Data' restraints params & $17221: 21: 876$ & $11742: 38: 596$ & $7971: 14: 586$ & $4331 \cdot 14318$ & 596514318 \\
\hline Goodness of fit on $F^{2}$ & 1.011 & 0.918 & 1.028 & 0.932 & 1.012 \\
\hline $\begin{array}{l}\text { Final } R \text { indices } \\
(1>2 \sigma(I))\end{array}$ & $\begin{array}{l}R_{1}=0.0483 \\
w R_{2}=0.1228\end{array}$ & $\begin{array}{l}R_{1}=0.0559 \\
w R_{1}=0.1235\end{array}$ & $\begin{array}{l}R_{1}=0.0569 \\
w R_{1}=0.1289\end{array}$ & $\begin{array}{l}R_{1}=0.0522 \\
w R_{1}=0.1036\end{array}$ & $\begin{array}{l}R_{1}=0.0483 \\
w R_{2}=0.1119\end{array}$ \\
\hline $\mathrm{R}$ indices (all data) & $\begin{array}{l}R_{1}=0.0672 \\
w R_{2}=0.1351\end{array}$ & $\begin{array}{l}R_{1}=0.1252 \\
w R_{1}=0.1490\end{array}$ & $\begin{array}{l}R_{1}=0.0966 \\
w R_{1}=0.1503\end{array}$ & $\begin{array}{l}R_{1}=0.1127 \\
w R_{1}=0.1217\end{array}$ & $\begin{array}{l}R_{1}=0.0875 \\
w R_{1}=0.1251\end{array}$ \\
\hline Highest peak (c $\left.A^{-1}\right)$ & 0.752 & 0.541 & 0.412 & 0.265 & 0.449 \\
\hline Deepest hole (e $\left.A^{-1}\right)$ & -0.868 & -0.451 & -0.585 & -0.296 & -0.310 \\
\hline
\end{tabular}


(4) Form 1

A
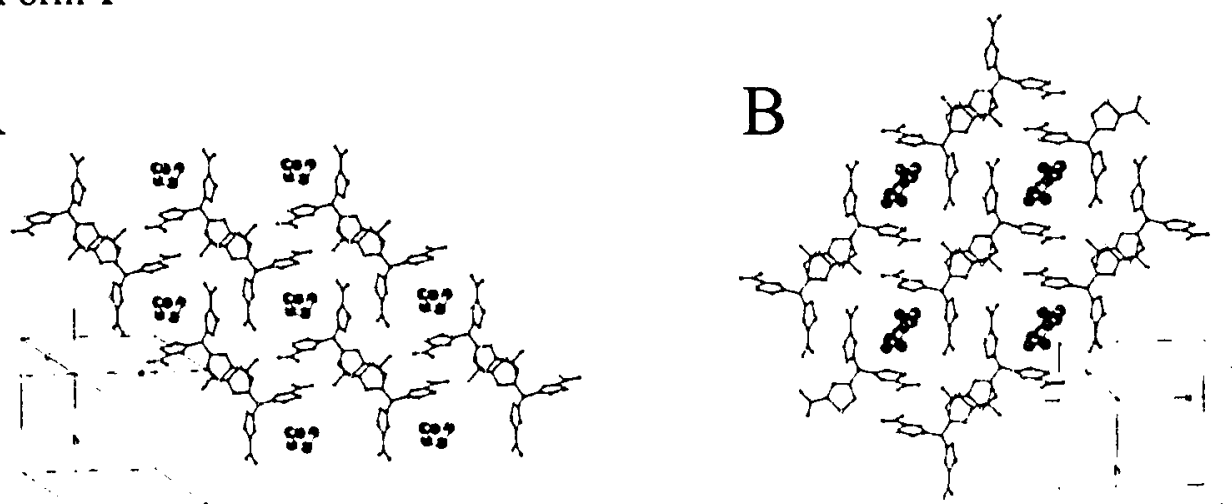

Figure XII-8: Packing diagrams of form 1 of TATM/1,3-dichloropropane obtained by single crystal $\mathrm{X}$-ray diffraction:

(A) A view down the $c^{*}$ direction. Ribbons in this figure run in the NW-SE (110) direction. The channels are parallel to (001). The 1,3-dichloropropane guests in this layer are disordered over two inequivalent positions (doubly disordered DD).

(B) Another view down the $c^{*}$ direction, however this view is of the layer adjacent to that in Figure XII-8A. Note how the ribbons now run in a direction perpendicular to those in IX-7A, namely the NE-SW (1-10) direction. In addition, in this layer the guests are disordered over two equivalent positions (symmetry disordered $\mathrm{SD})$.

Figure XII-8 depicts two packing diagrams of form 1 , which is triclinic, space group $\mathrm{P} \overline{\mathrm{l}}$. The principal axes of the unit cell are displayed in both diagrams, to show that the viewing perspective remains the same. Figure XII-8A shows a view down the $c^{*}$ direction. The lattice is made up of endless ribbons running in the NW-SE (110) direction. Channels, formed by the alignment of cages in adjacent layers, run perpendicular to the plane of the figure, namely the (001) direction. The 1,3dichloropropane guests, located in the $\left(a b^{1 / 3}\right)$ and $\left(a b^{2 / 3}\right)$ layers (Figure XII-8A), are disordered over two inequivalent positions. Only the majority position (occupancy = 0.87 ) is depicted. Figure XII-8B displays another view down the $c^{*}$ direction, however this view is of layers at $(a b 0)$ and $(a b 1)$. Note how the ribbons now run in a direction perpendicular to those in Figure XII-8A, namely the NE-SW (1-10) direction. Within this layer, the guests lie across an inversion center and are disordered over two equivalent positions. 
A unique feature of this structure is that the layer-repeat unit consists of three layers, rather than the more commonly encountered one or two layers. The repeat unit is composed of two layers with doubly disordered guest (DD - Figure XII-8A), followed by one layer with symmetry disordered guest (SD - Figure XII-8B). That is, the layers go as: SD-DD-DD-SD-DD-DD-SD...etc.

Finally, the host framework packing motif of form 1 is similar to that reported for $n$-hexane / TATM [21]. Both inclusion compounds crystallize in the triclinic $\mathrm{P} \overline{1}$ space group, and the dimensions of the respective unit cells are almost identical, namely $a(\AA)=$ $12.684(12.647), b(\AA)=12.704(12.694), c(\AA)=20.572(20.604)$, and $V\left(\AA^{3}\right)=3128$ (3173). However, the unit cell angles do not agree, as those for form 1 are, by convention, all above $90^{\circ}$, while those for $n$-hexane / TATM are all below $90^{\circ}$, and a unit cell transformation, such as $\left(\begin{array}{lllllllll}-1 & 0 & 0 & 0 & 1 & 0 & -1 & 0 & -1\end{array}\right)$ will make the comparison more reasonable. Finally, the contents of the asymmetric unit are slightly different for each. Form 1 contains three host molecules and 1.5 guest molecules, while the asymmetric unit of $n$-hexane / TATM contains three host molecules, but only one guest molecule. The $n$ hexane guests are located in channels between inequivalent guest layers.

\section{(5) Form 2}

Figure XII-9 depicts two packing diagrams for form 2. This structure is of the monoclinic $\mathrm{P} 2 / \mathrm{n}$ space group. The host lattice consists of pronounced zig - zag channels in the $a$ direction. From Figure XII-9B, a view in the $a b$ plane, one sees that the chlorine atoms of 1,3-dichloropropane guests alternately point left and right as the channel is traversed in the $a$ direction (the vertical direction in the figure). There are intermolecular close contacts (about $3.6 \AA-3.8 \AA$ ) between the chlorine atoms of neighboring guests in the host channel, which may serve to enhance the stability of the complex. The guest molecules are disordered over two inequivalent sites.

The guest molecules from neighboring layers are displaced from one another, as depicted in Figure XII-9A, a view down the channel axis (a direction). We note, however, that Figure XII-9A may be misleading, as it appears there is a large amount of empty space in the channel. When the guests' atoms are displayed as van der Waals radii 
(not shown), it is readily apparent that there is very little empty space in the host channel, despite neighboring guests being markedly displaced from one another.
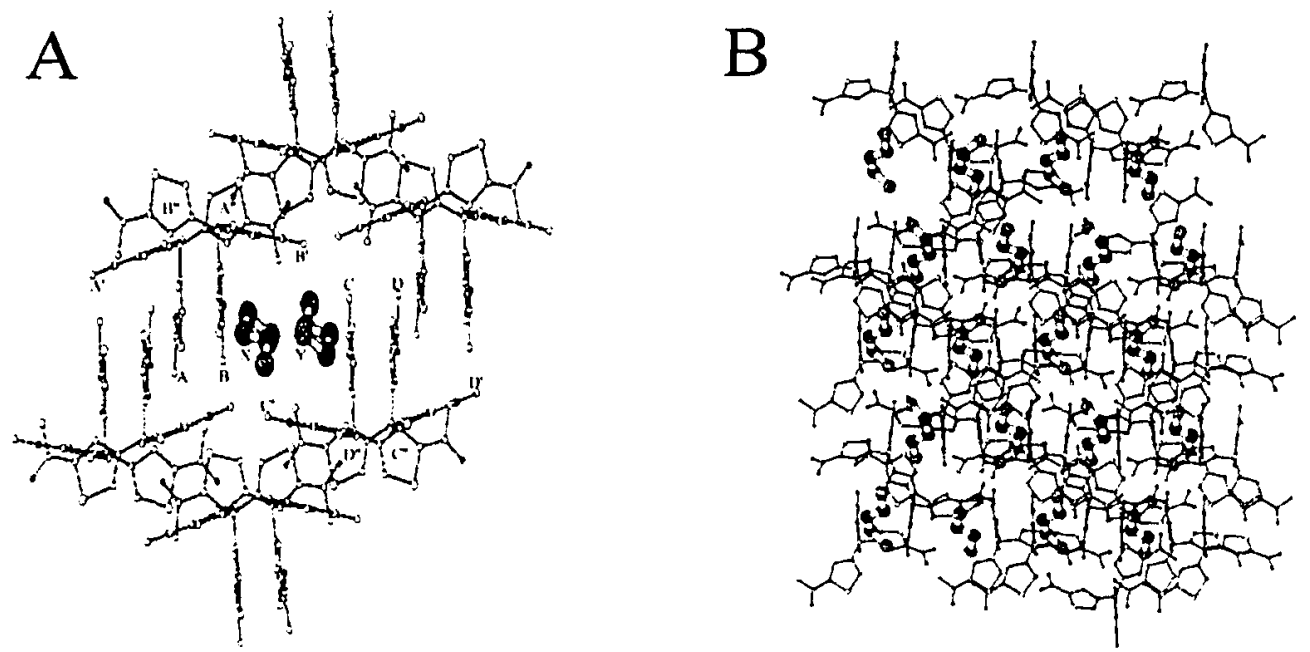

Figure XII-9: Packing diagrams of form 2 of TATM/1,3-dichloropropane obtained by single crystal $\mathrm{X}$-ray diffraction:

(A) A view in the $b c$ plane. The channel axis is perpendicular to the plane of the figure (the $a$ direction). Notice how neighboring guests in the channel are markedly displaced from one another, giving rise to a pronounced zig-zag type channel. Selected thienyl rings of the host are labeled. Refer to the text for details.

(B) A view in the $a b$ plane. The channel axis is in the plane of the figure and vertical in direction. Notice clearly that the guests are markedly displaced from one another as one traverses the channel.

In contrast to form 2, if one considers form $\mathbf{5}$ (see below), adjacent guests in the channel are practically superimposed upon one another, despite pointing in opposite directions as the channel is traversed. These observations suggest that zig - zag of the form $\mathbf{2}$ channel is more pronounced than the zig - zag of the form $\mathbf{5}$ channel. Indeed, as one can see in Figure XII-9A (form 2), there are four thienyl rings between guest sites (rather than the more commonly encountered two rings). From this figure (with labeled thienyl rings), two of the four thienyl rings (labeled as rings $A$ and $C$ in the figure) help to enclose the guest in the figure labeled as $\mathrm{X}$, while the thienyl rings $\mathrm{B}$ and $\mathrm{D}$ (one layer down) help to enclose the guest labeled as $Y$. In one layer, thienyl ring $B^{\prime}$ helps to enclose guest $X$. In the adjacent layer, thienyl ring $C^{\prime}$ helps to enclose guest $Y$. In addition, the acetyl functionalities of thienyl rings $A$ " and $D$ " also participate in the formation of the channel. 
The cyclohexane B/TATM inclusion compound [15] and others that are isostructural to it, such as TATM inclusions with benzene, [22] $\mathrm{CCl}_{4},[23]$ cycloheptane, [15] and cyclooctane $[15]$ bear some similarity to the present structure. These structures are all triclinic (space group $P \overline{1}$ ), and have unit cell dimensions that can be related to those of form 2. In particular, they have $b \sim 13.7 \AA$ and $c \sim 14.2 \AA$, with an included angle $(\alpha)$ of $\sim 90^{\circ}$ (compares with $b, a$ and $\gamma$, respectively, of form 2 ), while the $a$ axis ( $11.6 \AA)$ is approximately half the $c$ axis dimension of form $2(\sim 22.3 \AA)$. The packing motifs are similar, with guests located in pronounced zig-zag channels along the $\sim 14.2 \AA$ axes.

In addition, we can correlate the single crystal structure with the thermal analysis data presented above. Form 2 was prepared by two methods, namely fast cooling and annealing at $50^{\circ} \mathrm{C}$. From the TGA curves presented above, both of these samples released their guest less readily than slow evaporation, stir 4 days RT and stir while cooling (forms 4 and 5). From the single crystal structures, we saw that the channel of form 2 consisted of a pronounced zig - zag morphology, while the channel of form 5 (see below) is almost linear in nature. Thus one would expect that form $\mathbf{5}$ would lose its guest more easily on heating, while form $\mathbf{2}$ would hold onto its guest more strongly, which was readily observed in the TGA curves presented above (see Figure XII-4). This trend was also observed in the DSC experiments, where the annealing at $50^{\circ} \mathrm{C}$ and fast cooling samples (form 2) had a higher enthalpy of decomposition $\left(77.03 \mathrm{~J} \mathrm{~g} \mathrm{~g}^{-1}\right.$ and $76.74 \mathrm{~J} \mathrm{~g}^{-1}$ respectively) while the stir 4 days RT, stir while cooling and slow evaporation samples (forms 4 and 5) had a lower enthalpy of decomposition $\left(75.26 \mathrm{~J} \mathrm{~g}^{-1}, 69.70 \mathrm{~J} \mathrm{~g}^{-1}\right.$ and 70.01 $\mathrm{J} \mathrm{g}^{-1}$ respectively) - see Table XII-5.

\section{(6) Form 3}

Form 3 has an unusual bilayer motif. The asymmetric unit consists of two host molecules and one guest that is disordered (69:31) over two inequivalent positions (although one terminal $\mathrm{Cl}$ atom site is shared). As in most of the other forms, ribbons of TATM dimers are aligned along the $a+b$ and $a-b$ directions. Figure XII-10A shows the layer at $(a, b$, $1 / 8)$. Inversion centers in the $(a, b, 0)$ plane produce a second layer at $(a, b,-1 / 8)$. In the resulting bilayer, centered about $(a, b, 0)$, the cages partially overlap, producing an 
extended cage (along $c$ ) that contains a pair of guest molecules, as in Figure XII-10B. The closest $\mathrm{Cl}-\mathrm{Cl}$ distance is $4.12 \AA$. The $n$ glide produces a related bilayer centered about $(a, b, 1 / 2)$, in which the ribbons now run in the $a-b$ direction. To the best of our knowledge, this is the first reported TATM inclusion compound with this bilayer motif.

Finally, these observations concur with the thermal analysis (TGA and DSC) studies presented above, where it was seen that the form 3 sample (prepared by slow cooling) holds onto its guest more tenaciously than any of the other five samples, as determined from TGA experiments (see Figure XII-4). This would be expected since the lattice for form 3 is made up of isolated bilayer cavities (more or less) compared to the other forms, made up of either zig - zag channels or linear channels. Also, as was seen above, the slow cooling sample has the highest enthalpy of decomposition of the six different samples, namely $81.53 \mathrm{~J} \mathrm{~g}^{-1}$ (see Table XII-5). Thus we see a very interesting correlation between structure and thermal properties.
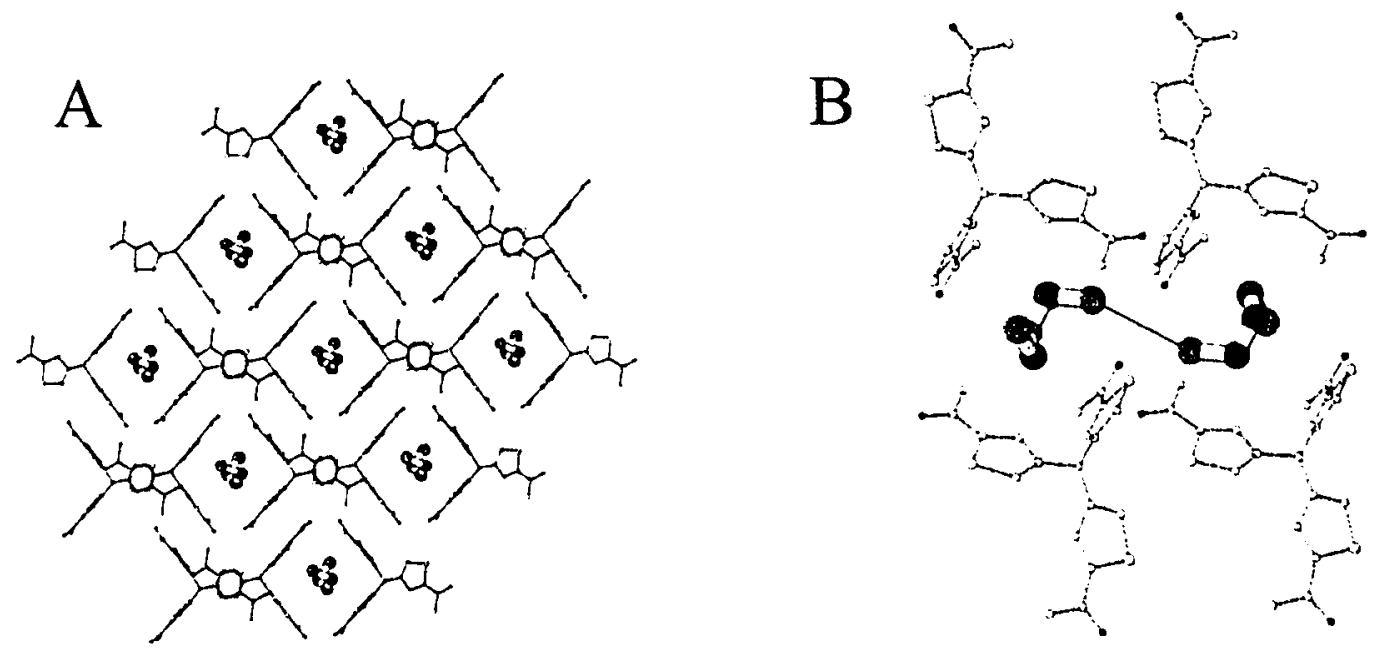

Figure XII-10: Packing diagrams of form 3 of TATM/1,3-dichloropropane obtained by single crystal $\mathrm{X}$-ray diffraction:

(A) A view down the (001) direction, showing one layer. The ribbons run in the plane of the figure, in the horizontal (110) direction. The channels run perpendicular to the plane of the figure, the (001) direction.

(B) Cages of adjacent layers partially overlap forming an extended cavity containing two guest molecules. A Cl- $\mathrm{Cl}$ approach distance of $4.12 \AA$ is indicated (solid line). 
Form 4 is similar to form 3 , but without the bilayer motif. The asymmetric unit consists of one TATM molecule and half a guest molecule. The guest molecule lies across an inversion center and is further disordered (58:42) over two inequivalent positions. The layer at $(a, b, 0)$, in Figure XII-11 A, is made up of ribbons (of cages and TATM dimers) aligned along the $a+b$ direction, while a $c$ glide produces a layer at $(a, b, 1 / 2)$, as depicted in Figure XII-IIB, with ribbons now along $a-b$. The cages containing the guest molecules are isolated and do not form channels. This structure is isostructural to the previously reported 1,2-dichloroethane / TATM monoclinic P2, / s structure (see chapter VII). The unit cell parameters for the 1,2-dichloroethane (and form 4) inclusions are as follows: $a(\AA)=12.690(12.715), b(\AA)=12.743(12.723), c(\AA)=13.737(13.893), \beta\left({ }^{\circ}\right)=$ $109.05(109.97), V\left(\AA^{3}\right)=2100$ (2175). Both materials were determined to be minority components of their respective polymorphic mixtures, as estimated by ${ }^{13} \mathrm{C} \mathrm{CP} / \mathrm{MAS}$ NMR, to be about $1 \%-10 \%$ abundant in our samples.
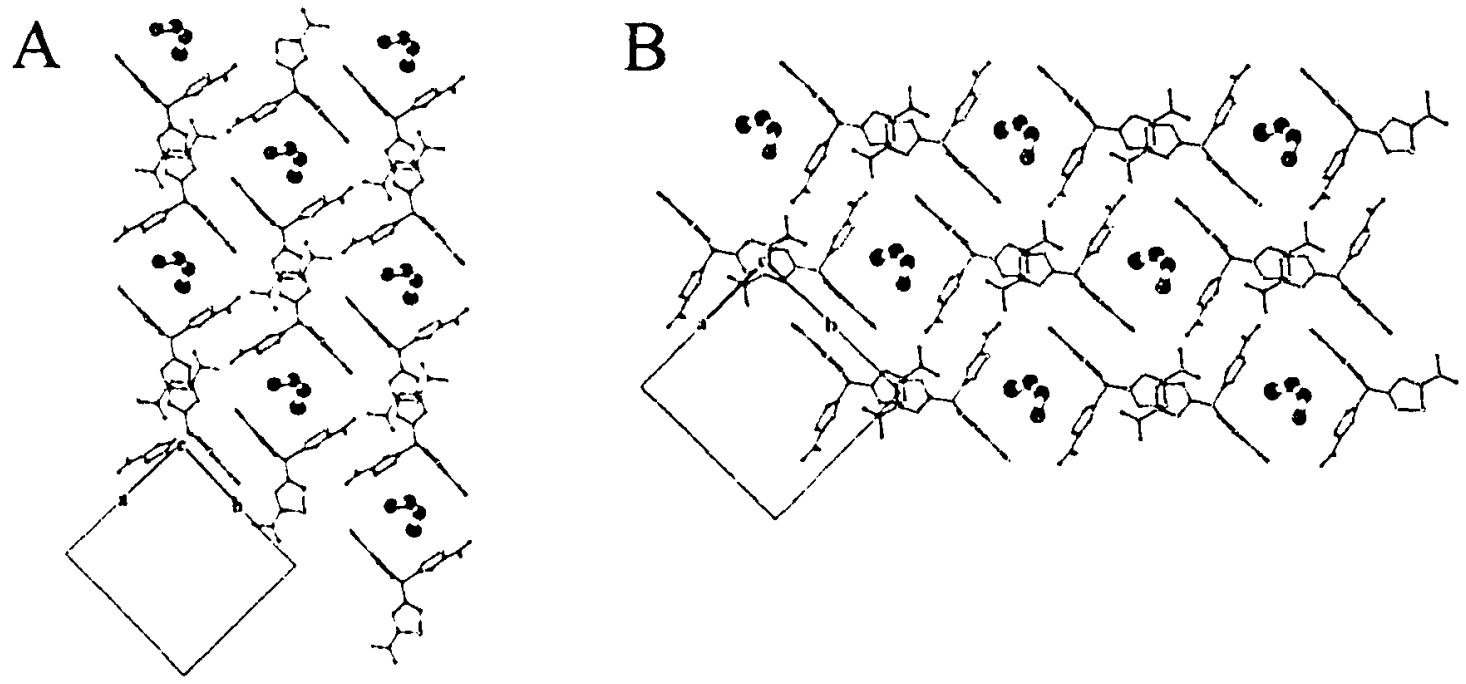

Figure XII-11: Packing diagrams of form 4 of 1,3-dichloropropane / TATM obtained by single crystal $\mathrm{X}$-ray diffraction:

(A) A view down $c$ (the channel axis). Note how the ribbons run in the vertical (110) direction.

(B) Another view in the $a b$ plane, but of a layer adjacent to that in Figure XII-11A. Now the ribbons run in the horizontal $(1-10)$ direction 


\section{(8) Form 5}

We present two packing diagrams for form 5 of 1,3-dichloropropane/TATM in Figure XII-12. The first view, Figure XII-12A, is down the channel axis ( $c$ direction). The $a$ axis is in the plane of the figure and horizontal in direction. From the figure, one sees that the lattice is made up of ribbons running in this direction. The ribbons are laid side by side to form layers in the $a b$ plane. From Figure XII-12A, it is also apparent that within each layer, each host cavity / guest molecule is surrounded in all four directions by a pair of $\pi$-stacked thienyl rings, while each pair of $\pi$-stacked thienyl rings is surrounded in all four directions by a host cavity / guest molecule. Again, two of the three thienyl rings of each host molecule participate in the formation of the channels. These channels contain guest molecules that lie across a twofold axis and are further disordered (68:32) over two inequivalent positions. The major difference between the layer motif observed in form 5 and those observed in the similar forms 1,3 and 4 is that the large angle ( $\beta$ $119.43^{\circ}$ ) between the ribbon direction $(a)$ and the $c$ axis tilts the thienyl rings so that they are directed towards an adjacent layer. The guest is located between layers within the channels formed by pairs of TATM molecules in adjacent layers. Also unique to form $\mathbf{5}$ is that the adjacent layers are arranged such that the cages overlap and $\pi$-stacked thienyl rings overlap, forming infinite channels and infinite $\pi$-stacked columns along $c$, respectively. Figure XII-12B, a view in the ac plane, shows these channels and infinite $\pi$-stacked columns. The other conclusion that may be reached from this figure is that the channels are of a slight zig - zag type. This is in contrast to form 2 (see above), where the zig-zag of the channel is much more pronounced. In the present structure, as the channels are traversed in the vertical (c) direction, the chlorine atoms of the guest alternately point toward and away from the viewer (see Figure XII-12B). The guest molecule's long axis extends approximately along the channel axis with $\mathrm{Cl}-\mathrm{Cl}$ distances between neighboring guests of 3.65-3.82 $\AA$. Form 5 is isostructural to the unpublished structure of 1,3-dibromopropane / TATM (see chapter X). They both crystallize in the monoclinic $\mathrm{C} 2 / \mathrm{c}$ space group, and the unit cell parameters for 1,3-dibromopropane / TATM (and form 5 of 1,3-dichloropropane / TATM) are as follows: $a(\AA)=17.581$ $(17.500) ; b(\AA)=18.401(18.465) ; c(\AA)=15.008(14.982) ; \beta\left(^{\circ}\right)=119.35(119.43) ; V\left(\AA^{3}\right)$ $=4232(4217)$. 
A

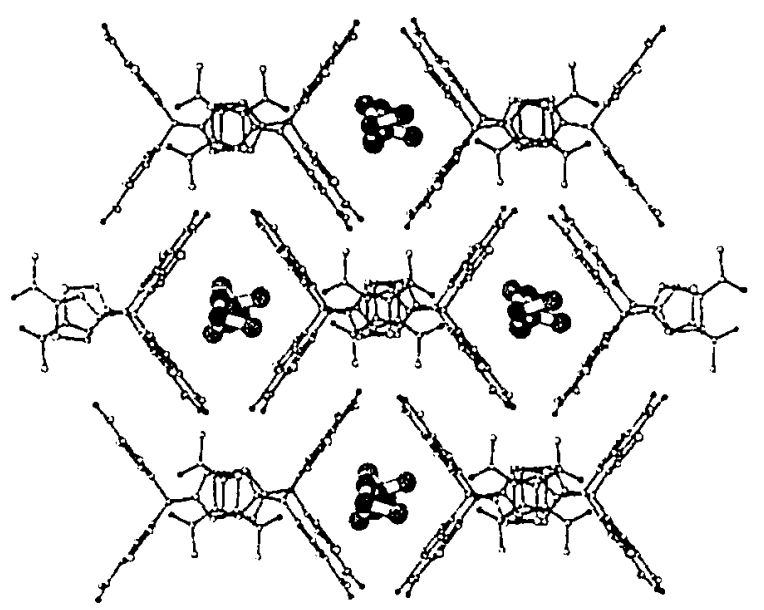

B

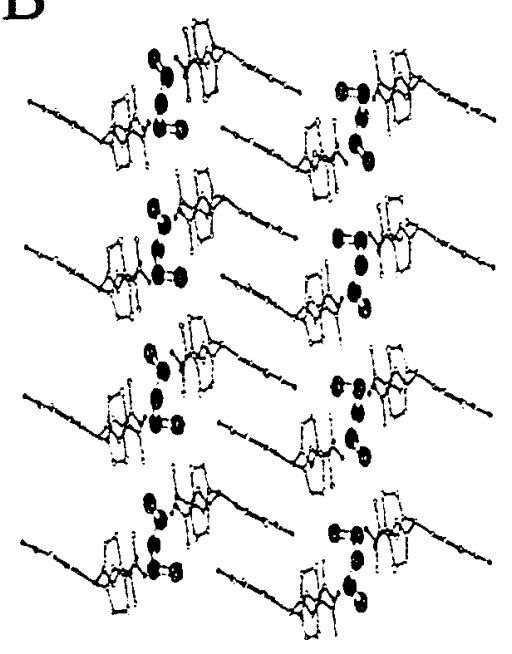

Figure XII-12: Packing diagrams of form 5 of 1,3-dichloropropane / TATM obtained by single crystal $\mathrm{X}$-ray diffraction:

(A) A perspective view in the $a b$ plane. The channels run in the $c$ direction. Note the minimal staggering of guests as the channel is traversed (compare with form $\mathbf{2}$ (Figure XII-9)).

(B) A view in the $a c$ plane. Notice clear evidence of infinite $\pi$-stacked thienyl rings running in the $c$ (vertical) direction. The chlorine atoms of the guest alternately point toward and away from the viewer as the channel is traversed in the $c$ direction.

Finally, one may speculate on the nature of the channel and the degree of constriction between guest sites. For structure 2 (see above) the zig - zag of the channel is very pronounced. Neighboring guests in the channel do not lie directly above one another. A schematic is depicted in Figure XII-13 (bottom). Since the constriction between guest sites as depicted in this schematic is very pronounced, translation of the guest from one site to the next is very difficult. On the other hand, for form $\mathbf{5}$, neighboring guests lie almost directly on top of one another. Thus, one would expect the constriction between guest sites for form 5 to be much less pronounced, as depicted in Figure XII-13 (top). This agrees with the TGA and DSC results above, where form 2 (made by fast cooling and annealing at $50^{\circ} \mathrm{C}$ ) held onto its guest much more strongly than form $\mathbf{5}$, and form $\mathbf{2}$ also had a higher heat of decomposition than form 5 (see Table XII-5). 


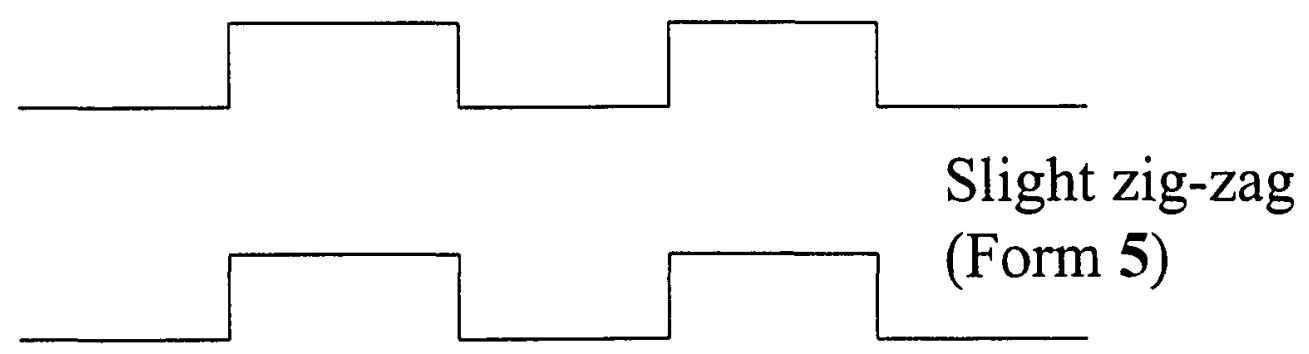

Figure XII-13: Schematic illustration of the nature of the channels of forms 2 and $\mathbf{5}$ of the 1,3-dichloropropane / TATM inclusion compound. The channels run in the horizontal direction. Notice that the zig-zag nature of channels of form $\mathbf{2}$ are much more pronounced than the zig-zag nature of the channels of form $\mathbf{5}$, which correlate with their respective thermal stabilities.

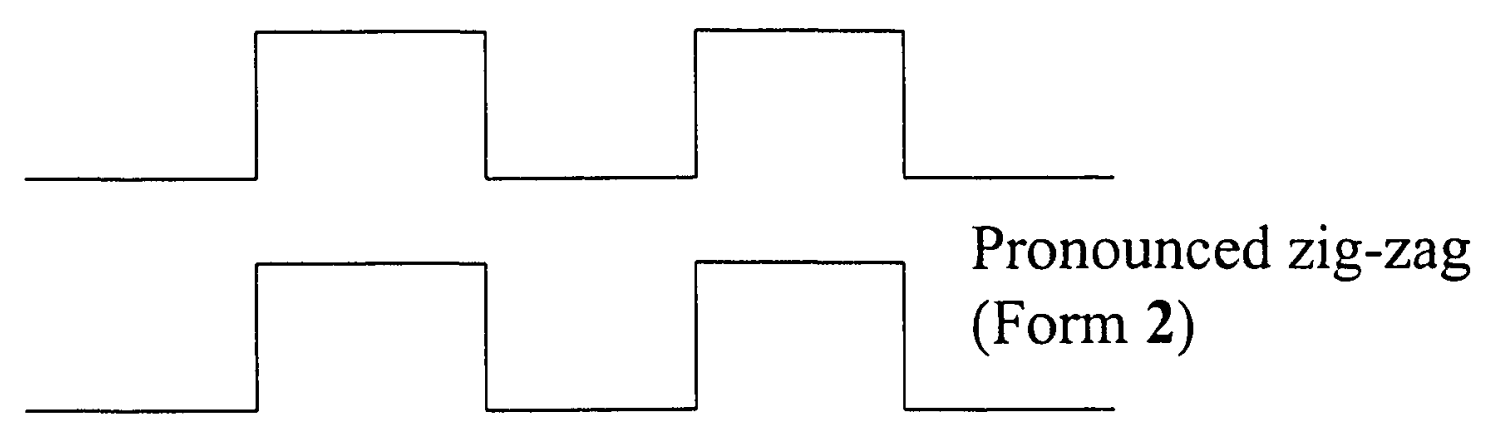




\section{DISCUSSION}

(F) Conformational preferences of TATM and 1,3-dichloropropane

Table XII-7 summarizes the torsion angles for the nine crystallographically independent TATM molecules from the five crystal structures of the present work. The TATM molecules are individually chiral, however all of the presently reported structures are centrosymmetric. As there was no attempt to ensure that the asymmetric units contained TATM molecules of the same "chirality", in tabulating the data it was necessary to check the torsion angles of both the TATM molecules in the asymmetric unit and those related through an inversion center. The atomic numbering scheme and torsion angle ordering are as discussed by Herbstein [32]. In Table XII-7, one may notice that for forms 1, 3, 4 and 5 , the conformations of each of the three thienyl rings about the central methine $\mathrm{C}-\mathrm{H}$ bond are each very similar. For these four forms, the thienyl ring that is approximately coplanar to the central $\mathrm{C}-\mathrm{H}$ bond has torsion angles ranging from $-6.2^{\circ}$ to $7.1^{\circ}$, the next largest torsion angle varies from $100.5^{\circ}$ to $106.8^{\circ}$, while the largest angle varies from $154.4^{\circ}$ to $161.0^{\circ}$. Furthermore, all four of these structures suggest that the universal orientation of the carbonyl bond of the acetyl groups is toward the direction of the sulfur atom of its associated thienyl ring (i.e. all 7 structures are SSS, where $S$ denotes $s y n$ ).

On the other hand, form $\mathbf{2}$ displays unique torsion angles as compared to the other four structures of the present study. Furthermore, both of the crystallographically unique TATM molecules of form $\mathbf{2}$ have torsion angles that are again very similar to each other, and both indicate that one of the three carbonyl groups of each host molecule points away from its associated thienyl ring sulfur ( $S S A$, where $A$ means anti). This behavior is not surprising, because form 2 exhibits a different layer structure when compared to the other four forms. 
Table XII-7: Torsion angles for TATM host molecules of the present study.

\begin{tabular}{|c|c|c|c|}
\hline \multirow{10}{*}{$\frac{\text { Form }}{1}$} & Fragment & Torsion angle $\left( \pm 0.1^{\circ}\right)$ & Orientation of $\mathrm{C}=\mathrm{O}$ wrto ring $\mathrm{S}$ \\
\hline & $\mathrm{H} \mid-\mathrm{Cl}-\mathrm{C} 2 \cdot \mathrm{C} 3$ & -1.2 & $S$ \\
\hline & $\mathrm{HI}-\mathrm{Cl}-\mathrm{C} 8-\mathrm{Cg}$ & 103.7 & ${ }_{S}^{S}$ \\
\hline & $\mathrm{HI}-\mathrm{Cl}-\mathrm{Cl} 4 \mathrm{-Cl5}$ & 154.8 & $S$ \\
\hline & $\mathrm{H} 1^{\prime}-\mathrm{Cl}^{\prime} \cdot \mathrm{C}^{\prime} \cdot \mathrm{C}^{\prime}$ & -6.2 & $S$ \\
\hline & $\mathrm{H} l^{\circ}-\mathrm{Cl}^{\circ} \cdot \mathrm{C} 8^{\prime}-\mathrm{C} 9^{\prime}$ & 100.9 & $S$ \\
\hline & $\mathrm{HI} 1^{\circ}-\mathrm{Cl} \cdot \mathrm{Cl} 4^{\circ} \cdot \mathrm{Cl} 5^{\circ}$ & 159.3 & $S$ \\
\hline & $\mathrm{HI}{ }^{*}-\mathrm{Cl} 1_{-}^{*} 2^{*}-\mathrm{C} 3^{*}$ & 1.1 & $s$ \\
\hline & $\mathrm{H} 1^{*}-\mathrm{Cl}^{*}-\mathrm{C} 8^{*}-\mathrm{C} 9^{*}$ & 100.5 & $S$ \\
\hline & $\mathrm{H}^{*}-\mathrm{Cl}^{*}-\mathrm{Cl} 4^{*}-\mathrm{C} 15^{*}$ & 154.8 & $S$ \\
\hline \multirow[t]{6}{*}{2} & $\mathrm{HI}-\mathrm{Cl}-\mathrm{C} 2-\mathrm{C} 3$ & -11.9 & $s$ \\
\hline & HI-Cl-C8-C9 & 131.0 & $S$ \\
\hline & $\mathrm{HI}-\mathrm{Cl}-\mathrm{Cl}+\mathrm{Cl}$ & 145.0 & $A$ \\
\hline & $\mathrm{H} 1^{\prime}-\mathrm{Cl} l^{\prime}-\mathrm{C} 2^{\prime}-\mathrm{C} 3^{\prime}$ & -11.4 & $S$ \\
\hline & $\mathrm{H} l^{\prime}-\mathrm{Cl}^{\prime}-\mathrm{Cl}^{\prime}-\mathrm{C} 9^{\prime}$ & 130.7 & $S$ \\
\hline & $\mathrm{H} 1^{\circ}=\mathrm{Cl}^{\circ}-\mathrm{Cl} 4^{\circ}-\mathrm{Cl} 5^{\circ}$ & $1+8.0$ & $d$ \\
\hline \multirow[t]{6}{*}{3} & $\mathrm{H} 1-\mathrm{Cl}-\mathrm{C} 2-\mathrm{C} 3$ & -2.7 & $S$ \\
\hline & $\mathrm{H} \mid-\mathrm{Cl}-\mathrm{C} 8-\mathrm{C} 9$ & 101.4 & $S$ \\
\hline & $\mathrm{HI}-\mathrm{Cl}-\mathrm{Cl} 4-\mathrm{Cl} 5$ & 161.0 & $S$ \\
\hline & $\mathrm{HI}-\mathrm{Cl}^{\prime}-\mathrm{C}^{\prime}-\mathrm{C} 3^{\circ}$ & -0.2 & $s$ \\
\hline & $\mathrm{HI}^{\prime}-\mathrm{Cl}^{\prime}-\mathrm{C}^{\circ}-\mathrm{C}^{\circ}$ & 101.7 & $S$ \\
\hline & $\mathrm{HI}-\mathrm{Cl}^{\prime}-\mathrm{C} 14^{\circ}-\mathrm{C} 15^{\circ}$ & 156.7 & $S$ \\
\hline \multirow[t]{3}{*}{4} & $\mathrm{H} 1-\mathrm{Cl}-\mathrm{C} 2-\mathrm{C} 3$ & -0.9 & $S$ \\
\hline & HI-CI-C8-C9 & 102.2 & $S$ \\
\hline & $\mathrm{HI}-\mathrm{Cl}-\mathrm{Cl} 4-\mathrm{Cl} 15$ & 154.9 & $s$ \\
\hline \multirow[t]{3}{*}{5} & $\mathrm{H} 1-\mathrm{Cl}-\mathrm{C} 2-\mathrm{C} 3$ & 7.1 & $S$ \\
\hline & $\mathrm{H} 1-\mathrm{Cl}-\mathrm{C} 8-\mathrm{C} 9$ & 106.8 & $s$ \\
\hline & $\mathrm{Hl}-\mathrm{Cl}-\mathrm{Cl} 4-\mathrm{Cl} 5$ & 154.4 & $S$ \\
\hline
\end{tabular}

Next it is interesting to compare our torsion angles with those reported in 1997 by Herbstein [13]. On the basis of the crystal structures of TATM inclusion compounds that were available at the time $[15,18-24]$, he classified the structures according to the torsional angles of the thienyl rings about the central methine $\mathrm{C}-\mathrm{H}$ bond of the host. The data indicated that there were only three general types of host torsional conformation (see Table XII-8). They were as follows: (conformation \#1) $\tau_{1} \sim 0^{\circ}, \tau_{2} \sim 105^{\circ}, \tau_{3}-160^{\circ}$, SSS; (conformation \#2) $\tau_{1} \sim 0^{\circ}, \tau_{2} \sim 130^{\circ}, \tau_{3} \sim 150^{\circ}, S S A$ and (conformation \#3) $\tau_{1} \sim 0^{\circ}, \tau_{2} \sim 100^{\circ}$, $\tau_{3} \sim 15^{\circ}$, SSS. In the present study, we see similar results. Forms $1,3,4$ and 5 have 
torsional angles that are similar to those of Herbstein's conformation \#1, while form 2 possesses torsions that are similar to Herbstein's conformation \#2. Herbstein noted that the range of torsion angles within each of the three conformational groups did not exceed $10^{\circ}$, while in our case, the largest variation is $13.3^{\circ}$ (for the smallest torsion angle). The range for the other two torsion angles are both less than $10^{\circ}$. Finally, conformation $\# 3$ was found by Herbstein to occur only for the cyclononanone/TATM inclusion compound [24]. Cyclononanone is a very large guest, forms a 1:1 stoichiometry with TATM, and is a solid at room temperature. These reasons may be why cyclononanone forms a unique structure with TATM, at least when compared to the other TATM inclusion compounds reported in the literature. A recently reported water/TATM inclusion [30] displays similar torsional angles $\left(-3.5^{\circ}, 99.6^{\circ},-23.8^{\circ}, S S S\right)$, and may have structural features in common with cyclononanone / TATM.

Table XII-8: Torsion angles $\left( \pm 0.1^{\circ}\right)$ for TATM host molecules as reported in the literature [32].

\begin{tabular}{|c|c|c|c|c|c|}
\hline Guest & Host Molecule & $\tau_{1}\left({ }^{\circ}\right)$ & $\tau_{2}\left({ }^{\circ}\right)$ & $\tau_{3}\left({ }^{\circ}\right)$ & Orientation of $\mathrm{C}=\mathrm{O}$ wrto $\mathrm{S}$ \\
\hline ethyl acetate & A & 7.0 & 102.9 & 157.0 & $S S S$ \\
\hline ethanol & $\mathrm{A}$ & -3.2 & 110.7 & 160.0 & SSS \\
\hline cyclohexane A & A & -12.6 & 109.4 & 162.8 & SSS \\
\hline \multirow[t]{2}{*}{ benzene } & A & -16.2 & 126.2 & 148.2 & $S S . A$ \\
\hline & B & -10.1 & 124.2 & 148.7 & $S S A$ \\
\hline \multirow[t]{2}{*}{ carbon tetrachloride } & A & -20.0 & 129.0 & 148.3 & $S S A$ \\
\hline & B & -10.3 & 131.6 & 156.7 & $S S A$ \\
\hline \multirow[t]{2}{*}{ cyclohexane B } & A & -11.1 & 129.5 & $151: 0$ & $S S A$ \\
\hline & B & -18.7 & 128.6 & 149.4 & $S S A$ \\
\hline \multirow[t]{2}{*}{ cycloheptane } & $A$ & -12.2 & 130.4 & 150.2 & $S S A$ \\
\hline & B & -17.1 & 129.6 & 148.0 & SS.t \\
\hline \multirow[t]{2}{*}{ cyclooctane } & $A$ & 16.6 & 130.1 & 146.5 & SSA \\
\hline & B & -13.9 & 132.2 & 151.9 & $S S . A$ \\
\hline \multirow[t]{3}{*}{$n$-hexane } & A & 2.4 & 108.2 & 156.8 & SSS \\
\hline & B & 0.6 & 110.1 & 157.6 & SSS \\
\hline & C & 1.6 & 110.5 & 155.4 & SSS \\
\hline cyclononanone & A & -7.3 & 101.8 & -15.2 & SSS \\
\hline
\end{tabular}


In Herbstein's work, he found approximately equal occurrences of SSA (10) and SSS (7). In the present study, we found only 2 occurrences of SSA, while 7 TATM molecules have an SSS conformation. Herbstein also suggested that when there is more than one TATM molecule in the asymmetric unit (for guests benzene [22], carbon tetrachloride [23], cyclohexane B [15], cycloheptane [15], cyclooctane [15] and $n$-hexane [21]), the two or three host molecules (within each structure) have approximately the same conformation. We found the same behaviour in the present study. For the 1,3dichloropropane / TATM structures that have more than one host molecule in the asymmetric unit (forms 1, 2 and 3), one observes nearly identical TATM torsional angles within each form. Finally, as reported by Herbstein, within each of the five types of TATM structures (based on the crystallographic unit cell parameters), the same TATM conformation is observed in each group.

As an illustration of this, form 1 is has similar unit cell parameters to $n$-hexane / TATM [21], and both belong to Herbstein`s conformation group \#1. Although they have different unit cell parameters, form 2 has a packing motif that is very similar to both cyclohexane B / TATM [15] and carbon tetrachloride / TATM [23]. All three inclusion compounds belong to Herbstein's conformational group \#2. Form 4 of the present study is isostructural to 1,2-dichloroethane B / TATM [29], and as expected, both have very similar torsional angles $\left(\tau_{1} \sim 1.34^{\circ}, \tau_{2} \sim 105.33^{\circ}, \tau_{3} \sim 156.17^{\circ}\right.$, SSS for the latter structure). Finally, form 5 is isostructural to the 1,3-dibromopropane / TATM inclusion compound (see chapter X). Both also have nearly identical host torsions, those for the latter structure are as follows: $\tau_{1} \sim 8.2^{\circ}, \tau_{2} \sim 105.3^{\circ}, \tau_{3} \sim 153.7^{\circ}$, SSS. These observations lend further support to Herbstein`s conclusion that TATM inclusion compounds with similar unit cell parameters (or similar packing motifs) will display similar torsional angles for the TATM host molecules.

The other manner in which one can correlate the torsion results is to see if each TATM polymorph (identical guests that crystallize in more than one form) have similar torsions and unit cell parameters. There are three TATM polymorphs reported so far, namely cyclohexane (2 forms) [15], 1,2-dichloroethane (2 forms - see chapter VII) and 1,3-dichloropropane ( 5 forms). For cyclohexane, each of the two forms have both different unit cell parameters and TATM torsions. For 1,2-dichloroethane, the two forms 
have different unit cell parameters but very similar host torsional angles. In this case, the triclinic cell in the standard setting does not appear at first glance to contain an $a b$ layer similar to that observed in the monoclinic form. However, the $\left(\begin{array}{lll}-1 & 1 & 0\end{array}\right)$ reciprocal lattice plane of the triclinic form is indeed similar to the $a b$ plane of the monoclinic form. For the guest of the present study, each of the five forms has unique unit cell parameters, while four of the five forms have very similar host torsion angles. However, these four forms $(1,3,4$ and 5$)$ have very similar dimensions of the $a b$ plane, as discussed above. So for cyclohexane, there is a strong dependence of host torsion on structural parameters, as different structural types have different TATM torsions. On the other hand, for 1,2dichloroethane and 1,3-dichloropropane, structural types with common properties do indeed have similar host torsions.

There are four possible conformations for 1,3-dichloropropane, namely $G G$ (gauche-gauche), TG (trans-gauche), TT (trans-trans) and GG' (gauche-gauche'). It is well accepted [49-55] that the relative stabilities in the gas phase, as estimated by electron diffraction [50], follow the order $G G(73 \%)>T G(24 \%)>T T(<10 \%)>G G^{\circ}$ $(0 \%)$. In the gas phase electron diffraction study, the authors found that the energy of the $T G$ conformer lies approximately $4.6 \mathrm{~kJ} \mathrm{~mol}^{-1}$ above that of the $G G$ conformer, with the $T T$ conformer being at an undetermined amount higher in energy than the $T G$ conformer. For the $G G^{\prime}$ conformer, the prime signifies counter-rotation of the $-\mathrm{CH}_{2} \mathrm{Cl}$ substituents, producing a conformer in which both $\mathrm{C}-\mathrm{Cl}$ bonds are eclipsed. This conformer has never been observed [56].

As far as the pure liquid phase is concerned, wide-angle X-ray scattering experiments [57] suggested only two detectable conformers, namely $G G(-82 \%)$ and $T G$ $(\sim 18 \%)$. The energy of the $T G$ conformer lies about $4.7 \mathrm{~kJ} \mathrm{~mol}^{-1}$ above the energy of the $G G$ conformer. The authors noted that there was no conclusive evidence to support the presence or absence of the TT conformer, and furthermore no non-staggered (eclipsed) conformers appear to exist in the pure liquid phase of 1,3-dichloropropane.

Finally, low temperature pure 1,3-dichloropropane solid has been determined, by infrared and Raman measurements $[49,55,58]$, to exist exclusively in the $G G$ conformation. The authors $[55]$ used $\mathrm{C}-\mathrm{Cl}$ stretching vibrations to measure the presence (or absence) of the different possible conformers. 
Thus, it appears that as one goes from the pure 1,3-dichloropropane vapor to the liquid to the solid, one observes a progressive decrease in the population of the higher energy $T G$ conformer $\{24 \%$ (gas) $\rightarrow 18 \%$ (liquid) $\rightarrow \sim 0 \%$ (solid) $\}$, which further confirms the assignment of $G G$ as the most preferred conformer in the pure material.

As far as the conformations of the 1,3-dichloropropane guests are concerned (as depicted in Table XII-9) four of the five forms (1, 3, 4 and 5$)$ have one guest as gauchegauche and a second guest as gauche-trans. For these four structures, the conformational torsions of the host are all very similar (see above). On the other hand, the polymorph with a unique host conformation (form 2) also has a unique guest conformation, namely both guests as gauche-trans. So we see an interesting relationship between the host conformation and the guest conformation of these inclusion compounds. Furthermore, the presence of a significant amount of the $T G$ conformation in the TATM inclusion compounds suggests that the environment that the guest 1,3-dichloropropane experiences in its TATM cavity is more like that experienced in the pure liquid or the pure gas phases rather than the pure solid.

To date, it has only been possible to produce pure bulk samples of forms $\mathbf{2}$ and $\mathbf{5}$. The three remaining forms have been produced only as mixtures. The morphology of all of the crystal forms appears to be similar. Crystals examined under the microscope all appeared to be rectangular plates or blocks. The color of the crystals was pale yellow / light orange, similar to that encountered for most other TATM inclusions in this laboratory.

In the context of inclusion compounds, extensive polymorphism might be expected, as the existence of several energetically similar forms is an intrinsic feature of supramolecular materials based on weak interactions. Suprisingly, very little is known about polymorphism of inclusion compounds if one excludes pseudopolymorphism, for which the composition is not constant. As mentioned in the introduction, only four examples of true polymorphism have been reported, for dianilinegossypol and the title host. However, it may be another case of the more one looks, the more one will find. 
Table XII-9: Torsion angles for 1,3-dichloropropane guest molecules of the present study.

\begin{tabular}{|c|c|c|c|c|}
\hline Form & Fragment & Torsion angle $\left( \pm 0.1^{\circ}\right)$ & Conformation & Occupancy $( \pm 1 \%)$ \\
\hline \multirow[t]{6}{*}{1} & $\mathrm{CL} 1-\mathrm{Cl} 101-\mathrm{Cl} 102-\mathrm{Cl} 103$ & 56.5 & $G$ & \multirow[t]{2}{*}{87} \\
\hline & $\mathrm{C} 101-\mathrm{C} 102-\mathrm{Cl} 03-\mathrm{CL} 2$ & 57.4 & $G$ & \\
\hline & CL3-C104-C105-Cl06 & 105.3 & intermediate & \multirow[t]{2}{*}{13} \\
\hline & $\mathrm{C} 104-\mathrm{C} 105-\mathrm{Cl} 06-\mathrm{CL} 4$ & 58.4 & G & \\
\hline & CL5-C201-C202-C203 & -69.5 & G & \multirow[t]{2}{*}{50} \\
\hline & $\mathrm{C} 201-\mathrm{C} 202-\mathrm{C} 203-\mathrm{CL} 6$ & 179.3 & $T$ & \\
\hline \multirow[t]{4}{*}{2} & CL2A-C02-C01-C03 & 41.4 & $G$ & \multirow[t]{2}{*}{49} \\
\hline & $\mathrm{C} 02-\mathrm{C} 01-\mathrm{C} 03-\mathrm{CL} 3 \mathrm{~A}$ & -174.5 & & \\
\hline & CL2B-C05-C04-C06 & -176.5 & $T$ & \multirow[t]{2}{*}{51} \\
\hline & $\mathrm{C} 05-\mathrm{C} 04-\mathrm{C} 06-\mathrm{Cl} 13 \mathrm{~B}$ & 65.5 & $G$ & \\
\hline \multirow[t]{4}{*}{3} & CLIA-C101-Cl02-C103 & 64.2 & G & \multirow[t]{2}{*}{69} \\
\hline & $\mathrm{Cl01-C102-C103-CL2A}$ & 52.1 & G & \\
\hline & CLIB-C201-C202-C203 & 55.8 & $G$ & \multirow[t]{2}{*}{31} \\
\hline & C201-C202-C203-CL2B & -165.5 & $T$ & \\
\hline \multirow[t]{4}{*}{4} & $\mathrm{CLO} 1-\mathrm{C} 001-\mathrm{C} 002-\mathrm{C} 003$ & -75.8 & $G$ & \multirow[t]{2}{*}{57} \\
\hline & $\mathrm{C} 001-\mathrm{C} 002-\mathrm{C} 003-\mathrm{CLO} 2$ & -164.0 & $T$ & \\
\hline & CL03-C004-C005-C006 & 77.8 & $G$ & \multirow[t]{2}{*}{43} \\
\hline & C004-C005-C006-CL04 & -47.5 & $G$ & \\
\hline \multirow[t]{4}{*}{5} & $\mathrm{CLO} 1-\mathrm{C} 01-\mathrm{C} 02-\mathrm{CO}$ & 59.4 & $G$ & \multirow[t]{2}{*}{68} \\
\hline & C01-C02-C03-CLO2 & 62.8 & $G$ & \\
\hline & CL03-C04-C05-C06 & -58.6 & $G$ & \multirow[t]{2}{*}{32} \\
\hline & C04-C05-C06-CLO4 & 168.0 & $T$ & \\
\hline
\end{tabular}


(G) Polymorphism in other systems

(9) Dianilinegossypol inclusions

As mentioned in the introductory section of the thesis (and Chapter XV below), gossypol, 2 , is a versatile host material, giving inclusion complexes with an extremely wide variety of organic compounds [59-61]. In a condensation reaction between gossypol and aniline, a Schiff's base type derivative, named dianilinegossypol, 3 , is formed. As is the case for gossypol, dianilinegossypol is also capable of including numerous guest species $[62,63]$, with the exception of alcohols and carboxylic acids. Single crystals of about 30 dianilinegossypol host-guest complexes have been prepared, and their crystallographic parameters have been determined [64|. Moreover, they are capable of forming more than one type of host-guest complex with the same guest, depending on the crystallization conditions.<smiles>Cc1cc2c(C(C)C)c(O)c(O)c(C=O)c2c(O)c1-c1c(C)cc2c(C(C)C)c(O)c(O)c(C=O)c2c1O</smiles>

Scheme 2: Gossypol host structure<smiles>[R]NC=C1C(=O)C(O)=C(C(C)C)c2cc(C)c(-c3c(C)cc4c(c3O)C(=CN[R])C(=O)C(O)=C4C(C)C)c(O)c21</smiles>

Scheme 3: Dianilinegossypol host $(\mathrm{R}=$ phenyl ring)

Most of the dianilinegossypol inclusions are unstable under ambient conditions. In contrast, TATM inclusions, even with very volatile guests, are stable at ambient conditions for excessively long periods of time (i.e. years). In a similar manner as the 1,3-dichloropropane / TATM polymorphs, gossypol and dianilinegossypol host-guest complexation is generally dependent on crystallization parameters such as temperature, concentration and pressure [65]. For instance, at room temperature, a $1 \mathrm{H}: 1 \mathrm{G}$ channeltype inclusion is formed from a solution of dianilinegossypol in ethyl acetate ( $\alpha$-phase), while a $1 \mathrm{H}: 1 \mathrm{G}$ cage-type inclusion compound ( $\beta$-phase) is obtained by increasing the crystallization temperature to $35^{\circ} \mathrm{C}$ under constant concentration and pressure [66]. The two forms possess similar host-host associations. The difference between the two phases is in the packing mode of these associations. 
In these two ethyl acetate inclusions, centrosymmetric dimers of dianilinegossypol molecules formed via strong $\mathrm{O}(5)-\mathrm{H} \cdots \mathrm{O}(3)$ hydrogen bonds are associated into columns by a weak $\mathrm{O}(8)-\mathrm{H} \cdots \mathrm{O}(7)$ hydrogen bond (see Figure XII-14). A difference in the structure of these two phases is in the packing mode of the columns. The angle formed by intersecting host columns is about $126^{\circ}$ for the $\alpha$-phase and $\sim 104^{\circ}$ for the $\beta$-form. Ethyl acetate guest molecules are hydrogen bonded to the host molecules via an $\mathrm{O}(1)-\mathrm{H} \cdots \mathrm{O}(10)$ linkage, and are accommodated into channels in the $\alpha$-phase complex and into cavities in the $\beta$-phase complex. In contrast, TATM inclusions are almost always comprised of (albeit often bottleneck-type) channels.

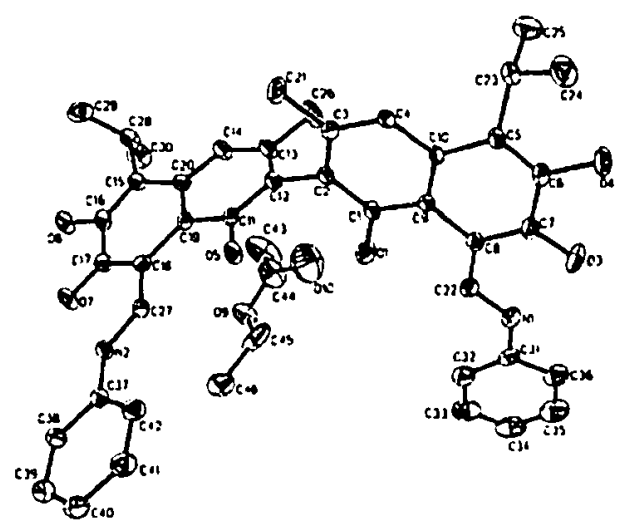

Figure XII-14: ORTEP drawing and atomic labeling scheme for the host and guest molecules in the $\beta$-phase complex of dianilinegossypol with ethyl acetate [66]

Acetone, which like ethyl acetate is an aprotic solvent, yields two ( $1 \mathrm{H}: 2 \mathrm{G})$ complexes with dianilinegossypol [67], each one being related to different crystal families of dianilinegossypol inclusion compound structures. Both of these complexes crystallize in more highly symmetric space groups (monoclinic and orthorhombic) than the ethyl acetate inclusions. The $\alpha$-phase crystallizes at $20^{\circ} \mathrm{C}$, while the $\beta$-phase crystallizes at $30^{\circ} \mathrm{C}$. In both forms, molecules of dianilinegossypol form hydrogen-bonded ribbons via $\mathrm{O}(4)-\mathrm{H} \cdots \mathrm{O}(3)$ linkages (see Figure XII-15). The two phases are characterized by similar structural motifs. In the $\beta$-phase complex, the cages are prismatic in shape, but in the $\alpha$ phase inclusion the cavities undergo a modification by shrinking in two directions, and widening in the other. (The host enclosures of the five forms of 1,3dichloropropane/TATM are also significantly different in size and shape). Molecules of acetone are hydrogen-bonded to the host molecules via an $\mathrm{O}(1)-\mathrm{H} \cdots \mathrm{O}(\mathrm{G})$ bond, and are accommodated in cavities for both types (i.e. both forms are clathrate-type inclusion compounds). 


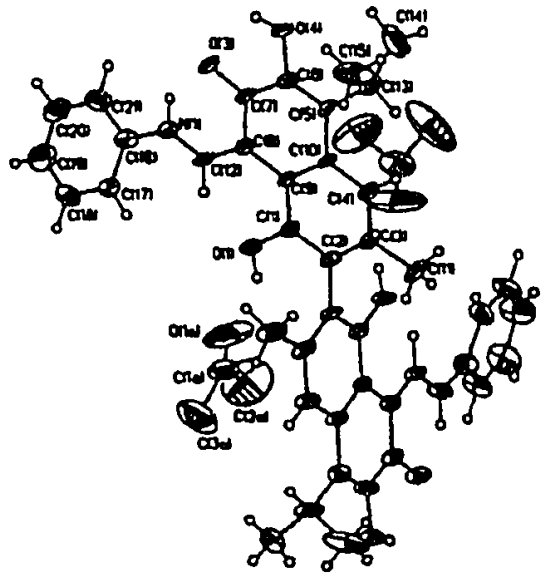

a)

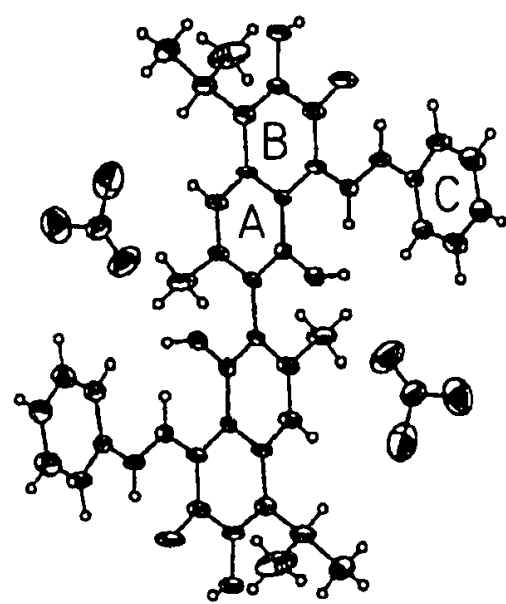

b)

Figure XII-15: The dianilinegossypol / acetone structures in the (a) $\alpha$-phase and (b) $\beta$-phase complexes, along with the atomic numbering scheme [67]

(10) 5-methyl-2-[(2-nitrophenyl)amino]-3-thiophenecarbonitrile

In 2000, a publication appeared [47] that reported a molecular compound, namely 5methyl-2-[(2-nitrophenyl)amino]-3-thiophenecarbonitrile, 4, that crystallizes in six solvent-free polymorphs, which differ in the mode of packing and in molecular conformation. (Except for 1,3-dichloropropane/TATM, this is, to the best of our knowledge, the only study that reports five or more polymorphs of the same compound, be it a pure solid or an inclusion compound.) The conformational difference results principally from the thiophene torsion relative to the ortho-nitroaniline fragment, which may explain the different crystal colors (red, orange and yellow). All six forms were sufficiently stable to permit $\mathrm{X}$-ray structural determination at room temperature. The comparable colors for the five forms of 1,3-dichloropropane/TATM (light orange yellow) suggest that the $\pi$-conjugated system of the TATM host in minimally affected by the nature of the polymorphic structures. Indeed, the six possible torsion angles of each host molecule are minimally different for four of the five polymorphs (forms $1,3,4$ and 5) - see Table XII-7. 


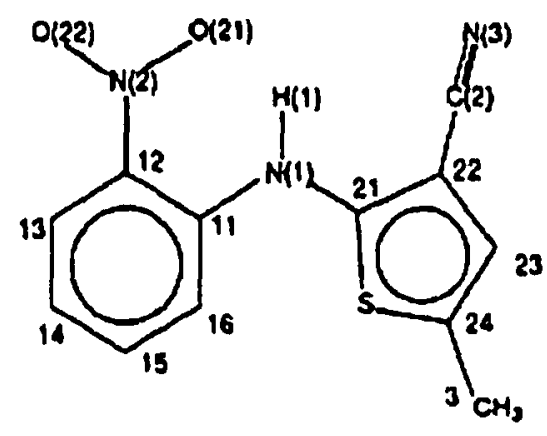

Scheme 4: Hexamorphic 5-methyl-2-[(2nitrophenyl)amino]-3-thiophenecarbonitrile

As mentioned, six solvent-free polymorphs have been identified (Figure XII-16): $\{1\} R-$ red prisms, mp 106.2 C $\{2\} Y$-yellow prisms, $m p 109.8 \mathrm{C}\{3\} O P$-orange plates, $m p$ $112.7 \mathrm{C}\{4\} O N$ - orange needles, mp $114.8 \mathrm{C}\{5\} Y N$-yellow needles $\{6\} O R P-$ orange-red plates. The melting points of $Y N$ and $O R P$ were not measurable due to thermal instability at elevated temperatures.

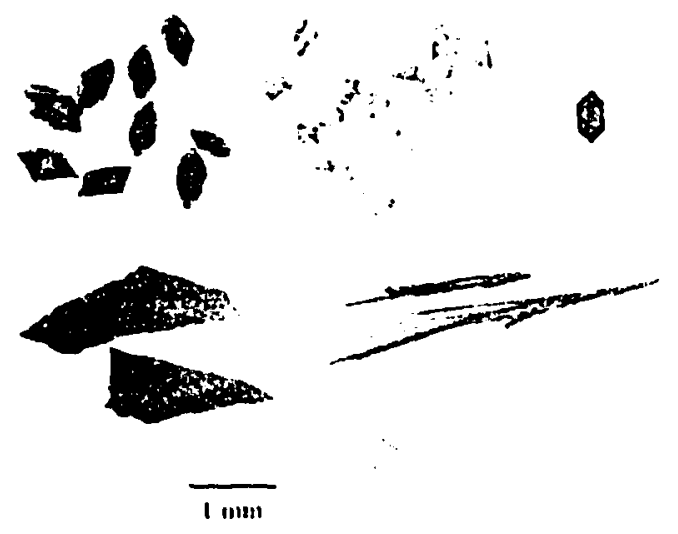

Figure XII-16: Photomicrographs of the crystal polymorphs of 4 . From upper left, clockwise: $R, Y, O P, O N, Y N, O R P$ (see text for details) $|47|$

The authors noted that the crystallization of the title compound showed poor polymorphic selectivity. (On the other hand, TATM generally shows better polymorphic selectivity see Table XII-2.) For example, all polymorphs (and sometimes mixtures of two or more forms) were obtained from methanol solution, while several polymorphs would nucleate simultaneously from a supercooled melt. Because of this poor selectivity, the authors were able to provide only a rough guide for polymorph preparation. $Y$ was produced by solution-mediated (slurry) conversion of any other form at room temperature. Fast crystallization favored the needle-like $O N$ and $Y N$. Although obtainable by heating $R$, solution-grown $O P$ was obtained only with seeding, and tended to crystallize with $O N$. $Y N$ tended to crystallize first as spherulites from a supercooled melt between a slide and a cover glass. 
Table XII-10 summarizes the crystallographic data for the six forms of the title compound. The molecules in the different polymorphs differ significantly in the torsion angle $\mathrm{C}(11)-\mathrm{N}(1)-\mathrm{C}(21)-\mathrm{S}\left(\theta_{\text {thio }}\right)$. (As far as 1,3-dichloropropane/TATM is concerned, for four of the five forms, the host torsion angles are relatively invariant.) In the order $Y$ $\sim Y N>O N>O P>O R P>R, \theta_{\text {thio }}$ changes by $\sim 83^{\circ}$ (see Table XII-11), which brings the thiophene ring from being perpendicular to being nearly coplanar to the amino group. Forms $Y$ and $Y N$ adopt essentially the same conformation. In addition to $\theta_{\text {thio, }}$ smaller changes are observed in the other torsional angles (see Table XII-II). The phenyl torsional angles $\mathrm{C}(21)-\mathrm{N}(1)-\mathrm{C}(11)-\mathrm{C}(16)\left(\theta_{\text {phen }}\right)$ differ by $\sim 25^{\circ}$ among the six forms. Furthermore, the nitro group is twisted $\left(\theta_{\text {nitro }}\right)$ out of its phenyl plane by $\sim 18^{\circ}$ in $R$ and $O P$, but is approximately coplanar in the other four forms.

Table XII-10: Crystal data for the six crystal forms of 5-methyl-2-[(2nitrophenyl)amino]-3-thiophenecarbonitrile [47]

\begin{tabular}{|c|c|c|c|c|c|c|}
\hline Form & $r$ & $O N$ & $Q$ & $R$ & 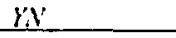 & $O R P$ \\
\hline$r(\mathrm{~K})$ & 293 & 293 & 295 & 293 & 296 & 296 \\
\hline crystal system & monoclinic & monoclinic & monoclinic & triclinic & triclinic & orthorhombic \\
\hline space group & $\mathrm{P} 2 / \mathrm{n}$ & $\mathrm{P} 2, / \mathrm{c}$ & $\mathrm{P} 2, / \mathrm{n}$ & $\mathrm{P} \bar{l}$ & $\mathrm{P} \overrightarrow{1}$ & Pbca \\
\hline crystal type & ycllow prism & orange neculs & onange plate & red prism & yellow needle & orange-red plate \\
\hline radiation & Mo K.. & CuK.. & Cu K.. & $\mathrm{CuK}$ & $M o K_{1}$ & $M o K_{4}$ \\
\hline$i(A)$ & 0.71073 & 1.54184 & 1.54184 & 1.54184 & 0.71073 & 0.71073 \\
\hline$a(A)$ & $8.5001(8)$ & $3.9453(7)$ & $7.9760(9)$ & $7 .+918(5)$ & $4.5918(8)$ & $13.177(3)$ \\
\hline$b(A)$ & $16.413(2)$ & $18.685(1)$ & $13.319(2)$ & $7.7902(5)$ & $11.249(2)$ & $8.0209(18)$ \\
\hline$c(A)$ & $8.5371(5)$ & $16.3948(4)$ & $11.676(1)$ & $11.9110(8)$ & $12.31 .5(2)$ & $22.801(5)$ \\
\hline$\alpha\left({ }^{\circ}\right)$ & 90 & 90 & 90 & $75.494(6)$ & $71.194(3)$ & 90 \\
\hline$\beta\left({ }^{\circ}\right)$ & $91.767(7)$ & $93.830(5)$ & $104.683(8)$ & $77.806(6)$ & $89.852(4)$ & 90 \\
\hline$Y\left({ }^{\circ}\right)$ & 90 & 90 & 90 & $63.617(6)$ & $88.174(3)$ & 90 \\
\hline$V\left(\hat{A}^{\prime}\right)$ & $1190.5(4)$ & $1205.9(3)$ & $1199.9(4)$ & $598.88(7)$ & $601.85(19)$ & $2409.8(9)$ \\
\hline$d_{\text {talk }}\left(g \operatorname{cm}^{-3}\right)$ & 1.447 & 1.428 & 1.435 & 1.438 & 1.431 & 1.429 \\
\hline$Z$ & 4 & 4 & 4 & 2 & 2 & 8 \\
\hline$R$ & 0.033 & 0.042 & 0.049 & 0.037 & 0.050 & 0.051 \\
\hline$R_{\mathrm{w}}$ & 0.044 & 0.055 & 0.122 & 0.050 & 0.100 & 0.106 \\
\hline GOF & 1.415 & 1.392 & 1.000 & 1.428 & 0.839 & 0.757 \\
\hline
\end{tabular}

Although most covalent bond lengths and bond angles do not change significantly among the six forms, some exceptions are observed (see Table XII-11): $\{1\}$ The HN-C (thiophene) bond is significantly longer, and the amino $\mathrm{C}-\mathrm{N}-\mathrm{C}$ bond angle is significantly smaller in $Y$ and $Y N$. \{2\} The $\mathrm{C}-\mathrm{C} \equiv \mathrm{N}$ bond angle deviates from $180^{\circ}$ in several polymorphs, most notably $O N$. $\{3\}$ The amino nitrogen is not always coplanar with the thiophene ring plane (see torsional angle $\mathrm{C}(24)-\mathrm{S}-\mathrm{C}(21)-\mathrm{N}(1)$ ), again most significantly in $O N .\{4\}$ Longer HN-C (thiophene) bond lengths in $Y$ and $Y N$ correlate with weaker $\pi$ conjugation between the thiophene ring and the amino lone pair in these two forms. 
Table XII-11: Selected structural parameters of 5-methyl-2-[(2-nitrophenyl)amino]-3thiophenecarbonitrile polymorphs (see Scheme 4 for atomic labelling scheme) [47]

\begin{tabular}{|c|c|c|c|c|c|c|}
\hline Formi & $\gamma$ & $O N$ & $O P$ & $R$ & $Y N$ & ORP \\
\hline $0_{\text {llai, }}\left\{\mathrm{C}_{11}-\mathrm{N}_{1}-\mathrm{C}_{21}-\mathrm{S}\right\}\left(^{\circ}\right)$ & $104.7(2)$ & $52.6(4)$ & $46.1(4)$ & $21.7(3)$ & $104.1(3)$ & $39.4(5)$ \\
\hline $0_{\left|{ }^{\alpha}\right| 111}\left\{C_{21}-N_{1}-C_{11}-C_{12}\right\}\left(^{\circ}\right)$ & $-175.0(2)$ & $-173.3(2)$ & $-167.3(2)$ & $-150.0(2)$ & $-175.2(2)$ & $-174.3(3)$ \\
\hline $0_{11 t r u}\left\{\mathrm{O}_{21}-\mathrm{N}_{2}-\mathrm{C}_{12}-\mathrm{C}_{11}\right\}\left(^{\circ}\right)$ & $-1.8(3)$ & $-4.4(4)$ & $-18.7(4)$ & $-18.4(3)$ & $-3.6(4)$ & $-3.5(5)$ \\
\hline$C_{2,}-S-C_{21}-N_{1}\left({ }^{\circ}\right)$ & $179.6(2)$ & $172.1(2)$ & $173.9(2)$ & $174.0(2)$ & $179.1(2)$ & $173.8(3)$ \\
\hline$C-C \equiv N$ angle $\left({ }^{\circ}\right)$ & $177.9(2)$ & $176.2(3)$ & $178.6(3)$ & $178.6(2)$ & $178.5(3)$ & $177.4(4)$ \\
\hline Amino $\mathrm{C}-\mathrm{N}-\mathrm{C}$ angle $\left({ }^{\circ}\right)$ & $122.8(2)$ & $126.1(2)$ & $126.2(2)$ & $126.3(1)$ & $123.9(3)$ & $129.6(4)$ \\
\hline HN-C(thiophene) (A) & $1.401(2)$ & $1.386(3)$ & $1.389(3)$ & $1.386(3)$ & $1.401(3)$ & $1.384(4)$ \\
\hline $\mathrm{HN}-\mathrm{C}$ (plenyl) $(\AA)$ & $1.377(2)$ & $1.380(3)$ & $1.380(3)$ & $1.381(2)$ & $1.365(3)$ & $1.371(4)$ \\
\hline $\mathrm{O}, \mathrm{N}-\mathrm{C}$ (nhenyl) $(\mathrm{A})$ & $1 .+46(2)$ & $1.441(3)$ & $1.445(4)$ & $1.446(2)$ & $1.447(3)$ & $1.456(4)$ \\
\hline
\end{tabular}

An intramolecular hydrogen bond exists between the nitro and amino groups in all six polymorphs, as perhaps would be expected. The only good hydrogen bond donor, thus engaged, would lead one to expect no intermolecular hydrogen bonds. This is the case in all but one form, $Y$, for which the amino hydrogen makes a close contact with the cyano group of a neighboring molecule.

All six forms possess centrosymmetry, containing equal numbers of molecules of opposite chirality in the respective unit cells. Apart from the intermolecular hydrogen bond in $Y$, no intermolecular contacts or special packing motifs appear to be significant, which indicates that the primary mode of molecular association in these crystals is van der Waals interactions (i.e. dispersion, dipole-dipole, etc.) This is quite similar behaviour compared to TATM inclusions, where the same two types of interactions are generally the most important in determining complex stability. Furthermore, intermolecular host-guest hydrogen bonds have not yet been encountered for TATM materials.

\section{CONCLUDING REMARKS}

The theory and practice of crystallography have greatly facilitated the development of solid-state supramolecular chemistry. The computing power and algorithms now available are generally sufficient to solve supramolecular structures, provided that experimental data, obtained at atomic resolution, are available. Further advances in the theory and practice of direct and Patterson methods will extend the power of such techniques to larger structures, and may make structure solution possible at lower resolutions. As a result, macromolecular and small-molecule crystallographies have 
converged to some extent, with medium-sized supramolecular structures providing the bridge, and further benefitting from the combination of methods used in both sectors. The application of molecular graphics, originally developed for protein structures, is of particular importance for work on medium-sized structures, where in some cases disordered moieties or solvent regions must be modelled.

As shown by several recently reported results, recording data at the highest possible resolution, using a combination of state-of-the-art detectors, powerful X-ray (e.g. synchrotron) sources, and cryogenic temperatures, will become the standard approach for determining supramolecular structures. Such an approach means that atomic-resolution data can be recorded in a reasonable time, and is no longer the rate-limiting step in such analyses. In addition, such data are now leading to structural models of supramolecular assemblies that are of an accuracy comparable to that expected for small structures, which was not possible some years ago.

This paper presents structural work on five polymorphic modifications of TATM/1,3-dichloropropane, reporting detailed variations in crystal packing and the torsional conformations of both host and guest molecules. These are discussed and compared with earlier results on TATM inclusions as reported by Herbstein. From our results, one may predict that the possibility of producing conformational isomers of both host and guest molecules may well provide a road map for preparing a large number of polymorphs, thus giving unparalleled opportunities for the study of this complex phenomenon.

\section{CHAPTER XII}

\section{REFERENCES}

(1) W.C. McCrone. In Physics and Chemistry of the Organic Solid State. D. Fox, M.M. Labes and A. Weissberger Eds. Volume 2, Interscience: New York, 1965.

(2) T.L. Threlfall. Analyst. 120, 2435 (1995).

(3) J.D. Dunitz and J. Bernstein. Acc. Chem. Res. 28, 193 (1995).

(4) A. Grunenberg. Pharm. Unserer. Zcit. 26, 224 (1997).

(5) A.L. Bingham, D.S. Hughes, M.B. Hursthouse, R.W. Lancaster, S. Tavener and T.L. Threlfall. Chem. Commun. 603 (2001).

(6) L. Borka and J.K. Haleblian. Acta Pharm. Jugosl. 40, 71 (1990).

(7) T. Laird. In Comprehensive Medicinal Chemistry C. Hansch, Ed. Volume 1, Pergamon: Oxford, 1990.

(8) S.R. Bym. Solid-State Chemistry of Drugs Academic Press: New York, 1982 
(9) J.K. Haleblian. J. Pharm. Sci. 64, 1269 (1975).

(10) D.V. Soldatov and J.A. Ripmeester. Chem. Eur. J. 7, 2979 (2001).

(1I) D.V. Soldatov, G.D. Enright, C.I. Ratcliffe, A.T. Henegouwen and J.A.

Ripmeester. Chem. Mater. 13, 4322 (2001).

(12) D.V. Soldatov. J. Inclusion Phenom. 48, 3 (2004).

(13) K.M. Beketov, B.T. Ibragimov, S.A. Talipov, K. Makhkamov and T.F. Aripov. J. Inclusion Phenom. 27, 105 (1997).

(14) K.M. Beketov, B.T. Ibragimov and S.A. Talipov. J. Inclusion Phenom. 28, 141 (1997).

(15) L. Pang and F.Brisse. Can. J. Chem. 72, 2318 (1994).

(16) A.P. Yakubov, Y.K. Sudarushkin, L.I. Belen`kii, L. Gol'dfarb. J. Org. Chem. (USSR) 9, 1549 (1973).

(17) L. Bin Din and O. Meth-Cohn. J. Chem. Soc., Chem. Commun. 74 l (1977).

(18) R.E. Marsh. Acta Crys. B50, 112 (1994).

(19) P.H. van Rooyen and H.M. Roos, H. M. Acta Cryst. C47, 2468 (1991).

(20) J.L.M. Dillen and H.M. Roos. Acta Cryst. C48, 2229 (1992).

(21) H.M. Roos and J.L.M. Dillen. Acta Cryst. C48, 1882 (1992).

(22) P.H. van Rooyen and H.M. Roos. Acta Cryst. C47, 2718 (1991).

(23) L. Pang, R.C. Hynes and M.A. Whitehead. Acta Cryst. C50, 615 (1994).

(24) L. Pang and F. Brisse. Acta Ciyst. C50, 1947 (1994).

(25) P.S. Sidhu and J.A. Ripmeester. J. Supramol. Chem. 1, 63 (2001).

(26) P.S. Sidhu and J.A. Ripmeester. Supramol. Chem. 15(6), 433 (2003).

(27) P.S. Sidhu, J. Bell, G.H. Penner and K.R. Jeffrey. Can. J. Chem. 73, 2196 (1995).

(28) P.S. Sidhu, J. Bell, G.H. Penner and K.R. Jeffrey. Can. J. Chem. 74, 1784 (1996).

(29) P.S. Sidhu, G.D. Enright, J.A. Ripmeester and G.H. Penner. J. Phys. Chem. B 106, 8569 (2002).

(30) P.S. Sidhu, K.A. Udachin and J.A. Ripmeester. Chem. Commun. 12, 1358 (2004).

(31) H. Hartley and N.G. Thomas. J. Chem. Soc. 89, 1013 (1906).

(32) F.H. Herbstein. Acta Cryst. B53, 168 (1997).

(33) A. Gavezotti and G. Fillipini. J. Am. Chem. Soc. 117, 12299 (1995).

(34) J. Bernstein. J. Phys D: Appl. Phys. 26, B67 (1993).

(35) J.D. Dunitz. Acta Cryst. B51, 619 (1995).

(36) J. Jacques, A. Collet and S.H. Wilen. Enantiomers, Racemates and Resolutions Krieger Publishing Company: Malabar, Florida, 1991.

(37) C.P. Brock, W.B. Schweizer and J.D. Dunitz. J. Am. Chem. Soc. 113, 9811 (1991).

(38) J. Bernstein. Conformational Polymorphism. In Organic Solid State Chemistry; G.R. Desiraju, Ed.; Elsevier: Amsterdam, 1987.

(39) D. Buttar, M.H. Charlton, R. Docherty and J. Starbuck. J. Chem. Soc. Perkin Trans. 2. 763 (1998).

(40) J. Starbuck, R. Docherty, M.H. Charlton and D. Buttar. J. Chem. Soc. Perkin Trans. 2. 677 (1999).

(41) E. Staab, L. Addadi, L. Leiserowitz and M. Lahav. Adv. Mater. 2, 40 (1990).

(42) R.J. Davey, N. Blagden, G.D. Potts and R. Docherty. J. Am. Chem. Soc, 119, 1767 (1997).

(43) S. Bonafede and M. Ward. J. Am. Chem. Soc. 117, 7853 (1995). 
(44) J. Bernstein, R.J. Davey and J.O. Henck. Angew. Chem. Int. Ed. 38, 3441 (1999).

(45) A. Gavezzotti. Acc. Chem. Res. 27, 7153 (1994).

(46) G.R. Desiraju. Crystal Engineering: The Design of Organic Solids. Elsevier: New York, 1989.

(47) L. Yu, G.A. Stephenson, C.A. Mitchell, C.A. Bunnell, S.V. Snorek, J.J. Bowyer, T.B. Borchardt, J.G. Stowell and S.R. Byrn. J. Am. Chem. Soc. 122, 585 (2000).

(48) K. Beketov, E. Weber, J. Seidel, K. Kohnke, K. Makhkamov and B. Ibragimov. Chem. Commun. 91 (1999).

(49) A.B. Dempster, K. Price and N. Sheppard. Spectrochim. Acta 25A, 1381 (1969).

(50) S. Grindheim and R. Stolevik. Acta Chem. Scand. 30A, 625 (1976).

(51) S. Madurga, J.C. Paniagua and E. Vilaseca. Chem. Phys. 255, 123 (2000).

(52) M. Kato and Y. Taniguchi. J. Phys. Chem. Ref. Data 98, 2688 (1994).

(53) T. Shimanouchi, H. Matsuura, Y. Ogawa and I. Harada. J. Phys. Chem. Ref. Data 9, $1149(1980)$.

(54) J.E. Gustavsen, P. Klaboe and R. Stolevik. J. Mol. Struct. 15, 61 (1973).

(55) J. Thorbjornsrud, O.H. Ellestad, P. Klaboe and T. Torgrimsen. J. Mol. Struct. 15, 61 (1973).

(56) D.G. Duffy, J. Quenneville, T.M. Baumbaugh, S.A. Kitchener, R.K. McCormick, C.N. Dormady, T.A. Croce, A. Navabi, H.D. Stidham, S.L. Hsu, G.A. Guirgis, S. Deng and J.R. Durig. Spectrochim. Acta 60A, 659 (2004).

(57) D.L. Wertz and A.J. Holder. J. Phys. Chem. 91, 3479 (1987).

(58) J.K. Brown and N. Sheppard. Proc. Roy. Soc. (London) A231, 555 (1955).

(59) B.T. Ibragimov, S.A. Talipov and P.M. Zorky. Supramol. Chem. 3, 147 (1994).

(60) B.T. Ibragimov and S.A. Talipov. J. Inclus, Phenom. 17, 325 (1994).

(61) B.T. Ibragimov, S.A. Talipov and T.F. Aripov. J. Inclus. Phenom. 17, 317 (1994).

(62) N.I. Baram, F.G. Kamaev, K.L. Ziyaev, L. Biktimirov, A.I. Ismailov, G.B. Nazarov and B.T. Ibragimov. Khim. Prir. Soedin 650 (1988).

(63) G.B. Nazarov, B.T. Ibragimov and T.F. Aripov. Khim. Prir. Soedin 661 (1988).

(64) M. Gdaniec, B.T. Ibragimov and S.A. Talipov. Gossypol. In Comprehensive Supramolecular Chemistry, J.L. Atwood, J.E.D. Davies, D.D. MacNicol and F. Vogtle, Eds. Volume 6, Chapter 5 (p142). Pergamon / Elsevier: Oxford, 1996.

(65) B.T. Ibragimov, Z.G. Tiljakov, K.M. Beketov and S.A. Talipov. J. Inclus. Phenom. 27, 99 (1997).

(66) K.M. Beketov, B.T. Ibragimov, S.A. Talipov, K. Makhkamov and T.F. Aripov. J. Inclus. Phenom. Mol. Recognit. Chem. 27, 105 (1997).

(67) K.M. Beketov, B.T. Ibragimov and S.A. Talipov. J. Inclus. Phenom. Mol. Recognit. Chem. 28, 141 (1997). 


\section{CHAPTER XIII}

Physicochemical characterization of tris(5-acetyl-3-thienyl) inclusion compounds with 1-bromoalkane guests $\mathrm{Br}\left(\mathrm{CH}_{2}\right)_{\mathrm{n}} \mathrm{CH}_{3}(n=1$ to 15$)$. A combined powder X-ray diffraction, ${ }^{13} \mathrm{C} \mathrm{CP} / \mathrm{MAS}$ NMR, DSC, TGA and polycrystalline density measurement study.

$\begin{array}{ll}\text { Abstract } & 309 \\ \text { Introduction } & 310 \\ \text { Experimental } & 312 \\ \text { Results } & \\ \text { (A) TGA and 'H Solution NMR } & 312 \\ \text { (B) PXRD and }{ }^{13} \text { C CP/MAS Solid NMR } & 318 \\ \text { (C) }{ }^{13} \text { C CP/MAS NMR with Dipolar Dephasing } 327 \\ \text { (D) Differential Scanning Calorimetry } \\ \text { (E) Polycrystalline Densities } \\ \text { Discussion } & 331 \\ \text { Concluding remarks } & 339 \\ \text { References } & 342\end{array}$




\section{ABSTRACT}

In this work we report results of various physical characterization techniques as applied to TATM inclusion compounds with the 1-bromoalkane guest family $\left(\mathrm{Br}\left(\mathrm{CH}_{2}\right)_{n} \mathrm{CH}_{3}\right.$, $n=1$ to 15 ). Thermogravimetric analysis and ${ }^{1} \mathrm{H}$ solution NMR permitted us to calculate host:guest molar ratios. A steady ramp like (generally non-integral) increase in $\mathrm{H}: \mathrm{G}$ molar ratios is observed for the smaller guests $(\mathrm{Br} 2-\mathrm{Br} 11)$, but then unexpectedly, falls down to approximately between $1: 1$ and 2:1 for the larger guests, suggesting that they perhaps form incommensurate inclusion compounds with TATM. Powder X-ray diffraction and ${ }^{13} \mathrm{C}$ CP/MAS NMR experiments allow a comparison of the different structural types. The smaller guests (i.e. bromoethane, 1-bromopropane and 1bromobutane) do not form isostructural TATM inclusion compounds. On the other hand, for the larger guests, one observes extensive isostructuralism. Indeed all seven guests from $\operatorname{Br} 10(n=9)$ to $\operatorname{Brl6}(n=15)$ form isostructural complexes with TATM. Similarly to previous work (see chapter XI), we rationalized this behaviour on the relative percent increase in size of the guest on going from an $n$ carbon chain to an $n+1$ carbon chain. The ${ }^{13} \mathrm{C}$ CP/MAS NMR spectra confirmed the PXRD results, in that the l-bromoalkane guests that formed isostructural TATM inclusion compounds had the same number of TATM molecules in the asymmetric unit, in addition to very similar splitting patterns for the signals corresponding to the host's atoms. Dipolar dephasing solid-state ${ }^{13} \mathrm{C}$ NMR experiments allow a qualitative determination of the presence or absence of dynamics in the solid state. We found that all of the I-bromoalkane guests, even the largest ones (e.g. $\mathrm{Brl6)}$ are dynamically reorienting at room temperature. On the other hand, for the $\alpha, \omega-$ dichloroalkane guests of chapter $\mathrm{Xl}$, only the smallest guests are dynamic. We reasoned these guest behaviors on the nature of their structure, where the first group is anchored only at one end, while the latter is anchored at both ends. The DSC results show that for the smaller guests $(\mathrm{Br} 3-\mathrm{Br} 9)$, both the melting temperature and decomposition enthalpy increase with size of guest, likely due to increasing van der Waals interactions between host and guest. The enthalpy of decomposition then drops off dramatically with larger guests $(\mathrm{Br} 10-\mathrm{Brl6})$, which again suggests that they are incommensurate inclusion compounds with only weak intermolecular interactions. In a similar manner to the $\alpha,(\omega)-$ dichloroalkane guests of chapter $\mathrm{XI}$, we found a linear relationship between melting 
temperatures and decomposition enthalpy, at least for the smaller guests $(\mathrm{Br} 2-\mathrm{Br} 9)$. Finally, the polycrystalline densities generally decrease with the size of the guest, suggesting that TATM can more easily pack in the crystal lattice with smaller guests, and that the fraction of filled space is smaller for TATM inclusions with the larger guests.

\section{INTRODUCTION}

A very important aspect of the philosophy of scientific research is the necessity of adopting a multidisciplinary approach in tackling problems in solid-state chemistry. There is now a very wide and diverse range of experimental and computational techniques available for probing the properties of solids, each with its own inherent strengths and limitations, and each becoming of increasing power and widening scope with the simultaneous evolution of scientific instrumentation. The prerogative of the solid-state chemist, in attempting to answer (to the best of his/her ability) the important questions of the day, is to identify the combination of techniques, and the experimental strategy, which can best elucidate the information that he/she seeks. The synergistic benefits of adopting a multidisciplinary approach of this type in attempting to derive a detailed fundamental understanding of a family of organic solids, is demonstrated throughout this thesis. Thus, in this chapter we wish first to weave together many of the available strands of evidence on the current TATM materials in order to describe their structural and dynamic properties, in as complete a manner as presently possible. Secondly, we wish to emphasize that the level of understanding that has been acquired for these systems has arisen only through the combined use, and exploitation of, a wide range of experimental approaches. Nevertheless, it is clear that many important fundamental issues relating to these TATM molecules remain to be fully understood, and this limitation gives directions and suggestions for further study.

In this study, we chose to perform a systematic study of guests that contain a terminal $\mathrm{Br}$ atom at the end of a hydrocarbon chain, with the length of the carbon chain increasing from 2 carbons (1-bromoethane) to 16 carbons (1-bromohexadecane). The fifteen TATM guests studied appear in Figure XIII-1. We wish to see how the properties of the TATM inclusion compounds change as the length of the hydrocarbon chain of the guest increases. By choosing guests that are similar in structure, we hope to eliminate 
extraneous complications in the interpretation of our data, a primary difference between the guests being the length of the hydrocarbon chain attached to the bromine atom. In a previous study (see chapter XI), we performed a similar multi-technique characterization on a series of TATM inclusion compounds with $\alpha, \omega$-dichloroalkane guests, namely $\mathrm{Cl}\left(\mathrm{CH}_{2}\right)_{n} \mathrm{Cl}(n=1$ to 10$)$. These nine guests appear in Figure XI-1. For each group of guests, we maintain the same halogen atom(s), while varying the length of the nhydrocarbon chain attached to the halogen(s). Thus, we wish to compare and contrast physicochemical properties both within each guest family, and also between the two guest families.

\begin{tabular}{lll}
\hline $\mathrm{Br} 2$ & $\mathrm{Br}\left(\mathrm{CH}_{2}\right)_{1} \mathrm{CH}_{3}$ & 1-bromoethane \\
$\mathrm{Br} 3$ & $\mathrm{Br}\left(\mathrm{CH}_{2}\right)_{2} \mathrm{CH}_{3}$ & 1-bromopropane \\
$\mathrm{Br} 4$ & $\mathrm{Br}\left(\mathrm{CH}_{2}\right)_{3} \mathrm{CH}_{3}$ & 1-bromobutane \\
$\mathrm{Br} 5$ & $\mathrm{Br}\left(\mathrm{CH}_{2}\right)_{4} \mathrm{CH}_{3}$ & 1-bromopentane \\
$\mathrm{Br} 6$ & $\mathrm{Br}\left(\mathrm{CH}_{2}\right)_{5} \mathrm{CH}_{3}$ & 1-bromohexane \\
$\mathrm{Br} 7$ & $\mathrm{Br}\left(\mathrm{CH}_{2}\right)_{6} \mathrm{CH}_{3}$ & 1-bromoheptane \\
$\mathrm{Br} 8$ & $\mathrm{Br}\left(\mathrm{CH}_{2}\right)_{7} \mathrm{CH}_{3}$ & 1-bromooctane \\
$\mathrm{Br} 9$ & $\mathrm{Br}\left(\mathrm{CH}_{2}\right)_{4} \mathrm{CH}_{3}$ & 1-bromononane \\
$\mathrm{Br} 10$ & $\mathrm{Br}\left(\mathrm{CH}_{2}\right)_{1} \mathrm{CH}_{3}$ & 1-bromodecane \\
$\mathrm{Br} 11$ & $\mathrm{Br}\left(\mathrm{CH}_{2}\right)_{10} \mathrm{CH}_{3}$ & 1-bromoundecane \\
$\mathrm{Br} 12$ & $\mathrm{Br}\left(\mathrm{CH}_{2}\right)_{11} \mathrm{CH}_{3}$ & 1-bromododecane \\
$\mathrm{Br} 13$ & $\mathrm{Br}\left(\mathrm{CH}_{2}\right)_{12} \mathrm{CH}_{3}$ & 1-bromotridecane \\
$\mathrm{Br} 14$ & $\mathrm{Br}\left(\mathrm{CH}_{2}\right)_{13} \mathrm{CH}_{3}$ & 1-bromotetradecane \\
$\mathrm{Br} 15$ & $\mathrm{Br}\left(\mathrm{CH}_{2}\right)_{14} \mathrm{CH}_{3}$ & 1-bromopentadecane \\
$\mathrm{Br} 16$ & $\mathrm{Br}\left(\mathrm{CH}_{2}\right)_{15} \mathrm{CH}_{3}$ & 1-bromohexadecane \\
\hline
\end{tabular}

Figure XIII-1: A list of the fifteen 1-bromoalkane guests $\mathrm{Br}\left(\mathrm{CH}_{2}\right)_{11} \mathrm{CH}_{3}(n=1$ to 15) of the current study, along with the abbreviation convention used in this work 


\section{EXPERIMENTAL}

Commercially available reagants (Aldrich) were used as received. The inclusion compounds were generally obtained by slow cooling (recrystallization) of hot solutions of $500 \mathrm{mg}$ TATM in approximately $3 \mathrm{~mL}$ of guest solvent, followed by vacuum filtration.

The inclusion compounds were each dissolved in $\mathrm{CDCl}_{3}$ and their ${ }^{~} \mathrm{H}$ solution NMR spectra were acquired on a commercial Bruker DRX $400 \mathrm{MHz}$ high resolution NMR spectrometer. A $5 \mathrm{~mm}$ multinuclear probehead was used, at a frequency of 400.132 $\mathrm{MHz}$ for ${ }^{1} \mathrm{H}$. A $90^{\circ}$ pulse length of $9.20 \mu$ was employed, with 32 scans for each sample, a spectral width of $8224 \mathrm{~Hz}$, an acquisition time of $1.9923 \mathrm{~s}$ and a relaxation delay of $4.0 \mathrm{~s}$. Following Fourier transformation of the free induction decay, the spectra were calibrated to the ${ }^{1} \mathrm{H}$ signal of the residual chloroform solvent, and are tabulated relative to tetramethylsilane at $0 \mathrm{ppm}$. The host:guest molar ratios were then determined by integrating appropriate resonances of guest and TATM host.

\section{RESULTS:}

(A) Thermogravimetric analysis (TGA) and 'H solution NMR:

In the present study, we used both TGA and ' $\mathrm{H}$ solution NMR ( $\mathrm{CDCl}_{3}$ solvent) to obtain the host:guest molar ratios of the I-bromoalkane / TATM inclusion compounds. We decided to use both techniques to examine consistency of results between the two techniques. The TGA curves for TATM inclusion compounds with $\mathrm{Br} 2$ and $\mathrm{Br} 3$ guests appear in Figure XIII-2. We used the percentage mass loss between room temperature and $175^{\circ} \mathrm{C}$ to $200^{\circ} \mathrm{C}$ (at higher temperatures, the host residue starts to thermally decompose), along with the molecular weights of TATM and the guest, to determine the host:guest molar ratios. In Table XIII-1 we list these molar ratios. In the second column of Table XIII- 1 is listed the percent mass loss for each inclusion compound between room temperature and $175^{\circ} \mathrm{C}$ to $200^{\circ} \mathrm{C}$, while in the third column of the table is listed the TGA host:guest molar ratios determined from the percent mass losses in the second column of the table, for each of the fifteen inclusion compounds. 

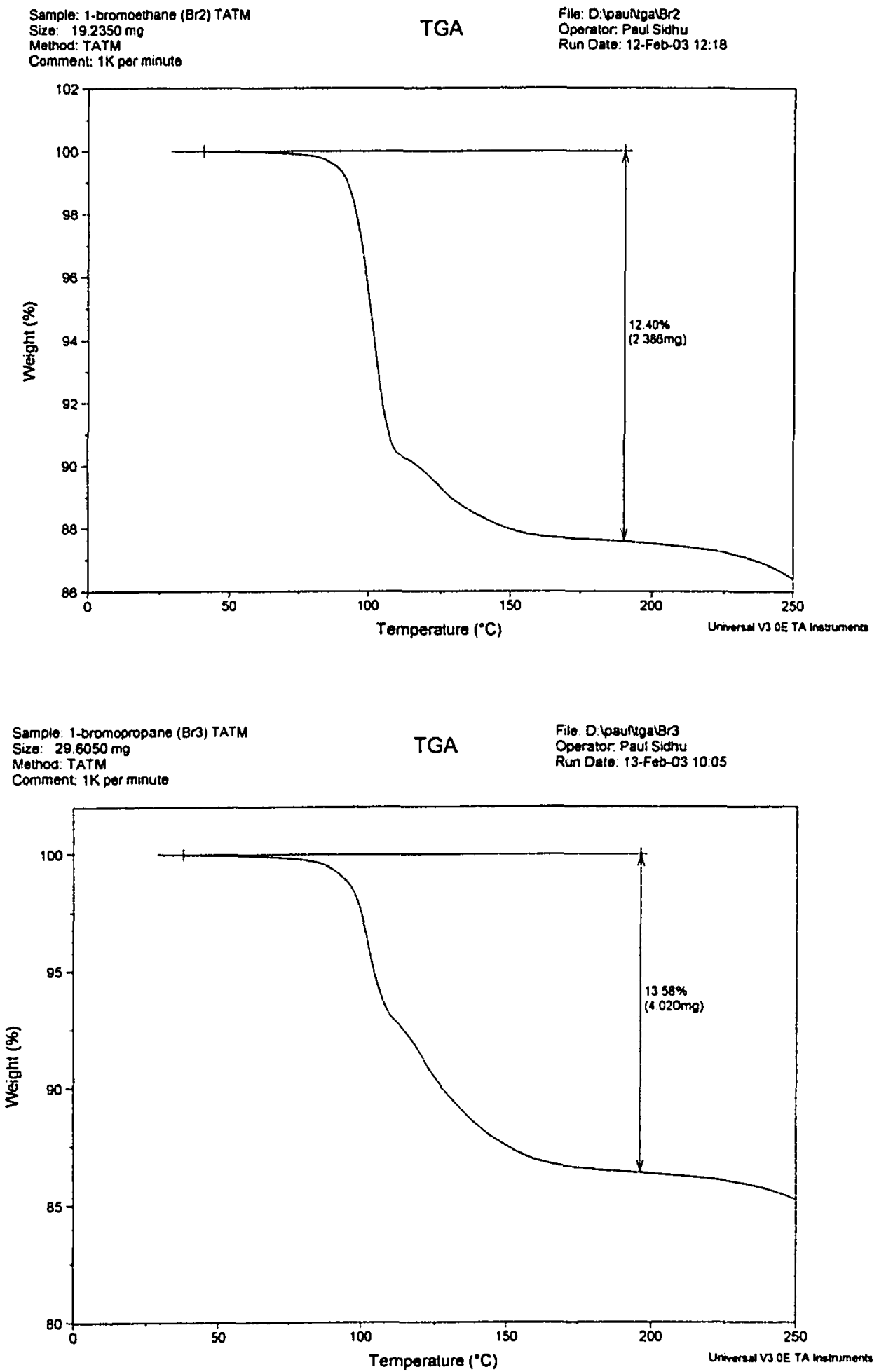

Figure XIII-2: TGA curves for TATM inclusion compounds with $\mathrm{Br} 2$ (top) and $\mathrm{Br} 3$ (bottom) guests. The host:guest stoichiometric ratios were calculated from the percent guest loss between room temperature and $175^{\circ} \mathrm{C}-200^{\circ} \mathrm{C}$ (see Table XIII-1) 


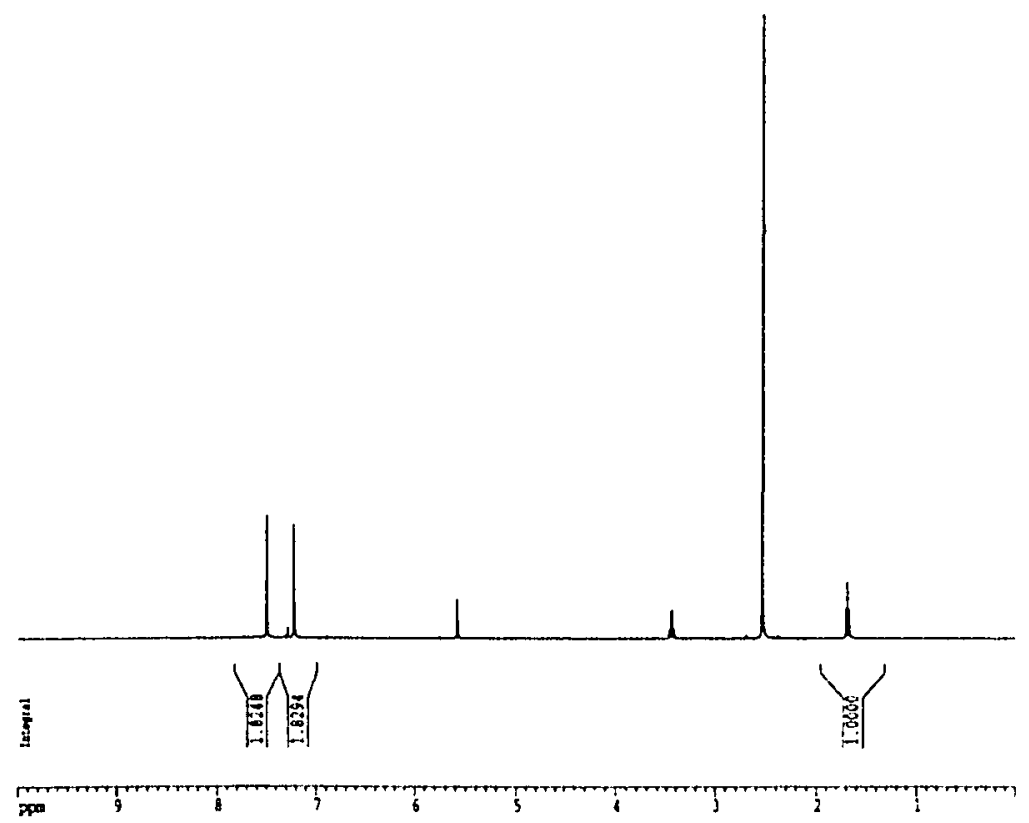

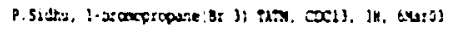

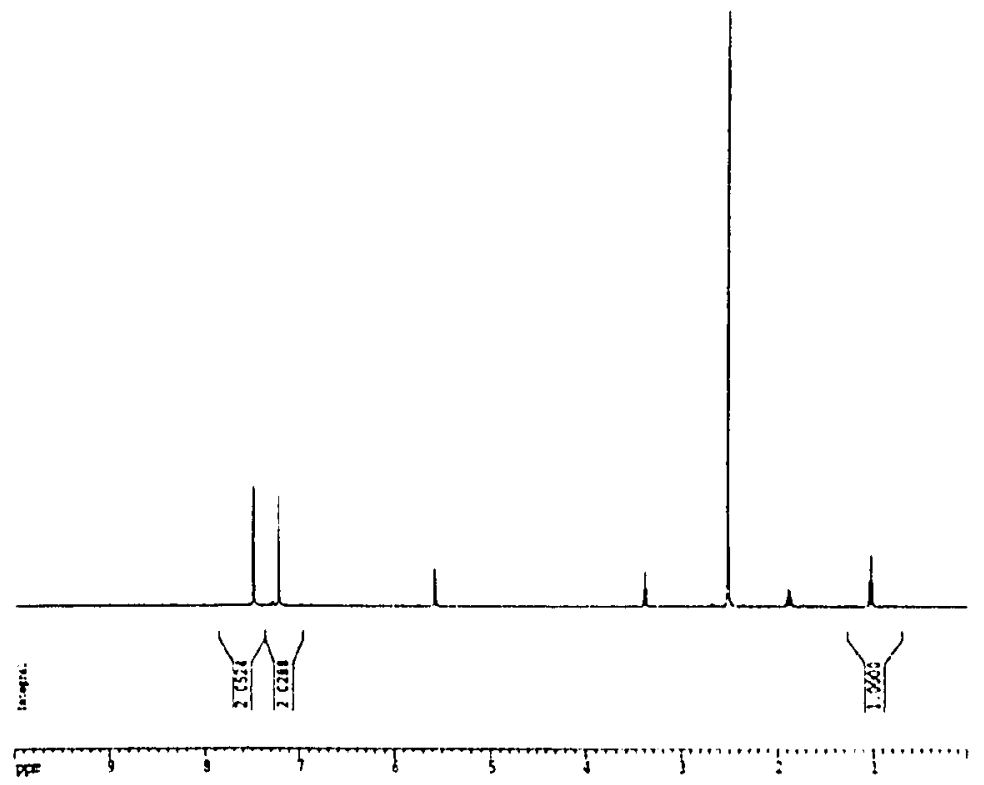

Figure XIII-3: ' $\mathrm{H}$ solution NMR ( $\mathrm{CDCl}_{3}$ solvent) of TATM inclusion compounds with $\mathrm{Br} 2$ (top) and $\mathrm{Br} 3$ (bottom) guests. The host:guest molar ratios were obtained by integration of the guest's methyl signal at about $1.7 \mathrm{ppm}$ and the host's thienyl proton signals at $7.2 \mathrm{ppm}$ and $7.5 \mathrm{ppm}$. 
Table XIII-1: TATM host:guest molar ratios for the 1-bromoalkane guests $\left\{\mathrm{Br}\left(\mathrm{CH}_{2}\right)_{11} \mathrm{CH}_{3}\right.$ $(n=1$ to 15$)\}$, determined from TGA $( \pm 0.01)$ and ${ }^{\prime} \mathrm{H}$ solution NMR $( \pm 0.01)$.

\begin{tabular}{llll}
\hline Guest & TGA \% guest loss $( \pm 0.1)$ & H:G SR (TGA) & H:G SR ('H NMR) \\
\hline $\mathrm{Br} 2$ & 12.4 & $1.98: 1$ & $1.83: 1$ \\
$\mathrm{Br} 3$ & 13.6 & $2.01: 1$ & $2.05: 1$ \\
$\mathrm{Br} 4$ & 12.4 & $2.48: 1$ & $2.49: 1$ \\
$\mathrm{Br} 5$ & 12.0 & $2.84: 1$ & $2.82: 1$ \\
$\mathrm{Br} 6$ & 12.8 & $2.91: 1$ & $2.93: 1$ \\
$\mathrm{Br} 7$ & 11.8 & $3.44: 1$ & $3.44: 1$ \\
$\mathrm{Br} 8$ & 11.4 & $3.87: 1$ & $3.90: 1$ \\
$\mathrm{Br} 9$ & 12.1 & $3.87: 1$ & $3.92: 1$ \\
$\mathrm{Br} 10$ & 12.7 & $3.91: 1$ & $3.85: 1$ \\
$\mathrm{Br} 11$ & 12.4 & $4.26: 1$ & $4.25: 1$ \\
$\mathrm{Br} 12$ & 16.0 & $3.36: 1$ & $3.91: 1$ \\
$\mathrm{Br} 13$ & 38.4 & $1.09: 1$ & $1.18: 1$ \\
$\mathrm{Br} 14$ & 25.1 & $2.13: 1$ & $2.20: 1$ \\
$\mathrm{Br} 15$ & 39.8 & $1.13: 1$ & $1.03: 1$ \\
$\mathrm{Br} 16$ & 31.1 & $1.74: 1$ & $1.59: 1$ \\
\hline
\end{tabular}

We also used ' $\mathrm{H}$ solution NMR of each inclusion compound dissolved in $\mathrm{CDCl}_{3}$ to determine the host:guest molar ratios. The ' $H$ solution NMR spectra for the TATM inclusion compounds with $\mathrm{Br} 2$ and $\mathrm{Br} 3$ guests appear in Figure XIII-3. As can be seen in the figure, for each guest we integrated the guest $\mathrm{s}$ terminal methyl $\left(\mathrm{CH}_{3}\right)$ group signal at approximately $1.7 \mathrm{ppm}$, while for the host we used the two thienyl ring proton signals at about $7.2 \mathrm{ppm}$ and $7.5 \mathrm{ppm}$. All three signals arise from three protons in each molecule (for both guest and host), so the integrals displayed in Figure XIII-3 can be used unchanged to give the host:guest molar ratios. We always assigned the guest 's methyl $\mathrm{CH}_{3}$ signal an integral of 1.00 , so the integral of each of the two thienyl ring proton signals gives the host:guest molar ratios directly. These molar ratios, for each of the fifteen TATM inclusion compounds, as determined by ' $\mathrm{H}$ NMR, appear in the fourth column of Table XIII-1. As can be seen in the table, there is, in most cases, very good consistency in the determination of host:guest molar ratios between the two techniques (TGA and 'H NMR).

Interestingly, one observes that a significant number of these TATM inclusion compounds crystallize in non-integral molar ratios. This was one of the reasons why we decided to use both techniques (TGA and ' $\mathrm{H}$ solution NMR) to confirm the molar ratio 
determination. In Figure XIII-4 we plot the host:guest molar ratios against the carbon chain length of the guest for TATM inclusion compounds with the 1-bromoalkane guests. From Figure XIII-4 one observes a ramp-like increase in host:guest molar ratios with the size of guest for the 1-bromoalkane guests, at least for the smaller size guests, with many of the inclusion compounds displaying non-integral host:guest molar ratios. This may arise for any number of reasons: Firstly, perhaps only a certain proportion of the host cavities are occupied by guest molecules, namely in that some sites in the host lattice do not contain a guest. Secondly (and possibly related to the first point) some of these inclusion compounds (most likely with the larger guests) may be incommensurate. Finally, some of these inclusion compounds may crystallize in polymorphic forms, with different $\mathrm{H}: \mathrm{G}$ molar ratios for the different forms. Two TATM guests, cyclohexane [1] and 1,2-dichloroethane (chapter VII), are each known to crystallize in at least two different forms, while 1,3-dichloropropane crystallizes with TATM in at least five different forms (chapter XII).

These results illustrate the characteristics of molecular recognition of host for guest during the crystallization process, in that as the guests become larger and larger in size, they require more TATM molecules to form the inclusion compound, at least for the smaller size guests. Perhaps unexpectedly, one sees that the TATM host:guest molar ratios for the largest size guests $(\mathrm{Brl} 2-\mathrm{Brl} 6)$ actually decreases significantly from that seen for smaller size guests. This may possibly be because that these guests are so large that they do not form inclusion compounds with TATM in the classical sense of a host enclosing a guest. Instead, they may just coexist in the crystal lattice as a co-solvate, which would perhaps give rise to smaller host:guest molar ratios.

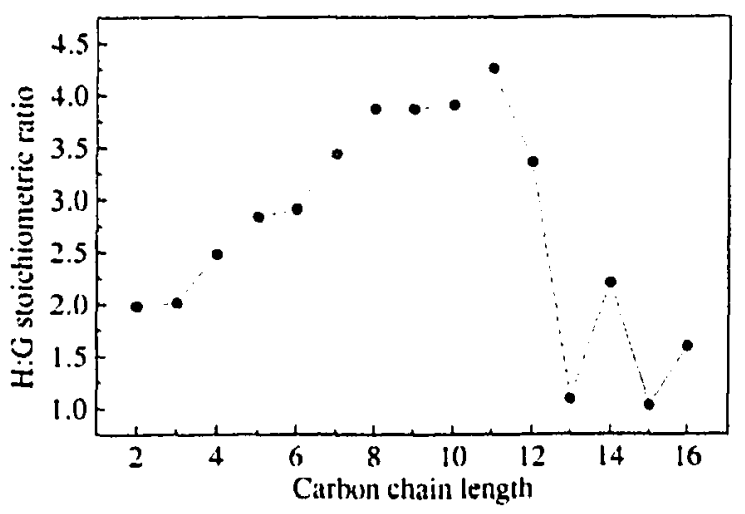

Figure XIII-4: A plot of host:guest stoichiometric ratio for TATM inclusion compounds with I-bromoalkane guests, as a function of the number of carbon atoms in the guest molecule. Notice a ramp like increase in the stoichiometric ratio with size of guest. 
The composition of urea $\left\{\mathrm{H}_{2} \mathrm{~N}(\mathrm{CO}) \mathrm{NH}_{2}\right\}$ inclusion compounds (i.e. the number of urea molecules per guest) may be determined using several differein techniques [2]. In general, however, there seems to be no simple integral stoichiometric relationship between the host:guest molar ratio to the number of carbon atoms of the guest compound to be included. This fact indicates quite clearly that no classical coordination theory can be applied to these materials, and points to their nature as inclusion compounds. The relationship between the number of carbon atoms of the guest molecule with the moles of urea required to form the inclusion compound is shown in Figure XIII-5 for $n$-paraffins and Figure XIII- 6 for carboxylic acids. Both figures show that the number of moles of urea required for the formation of the inclusion compound is directly (but non-integrally) proportional to the chain length of the guest molecule [3].

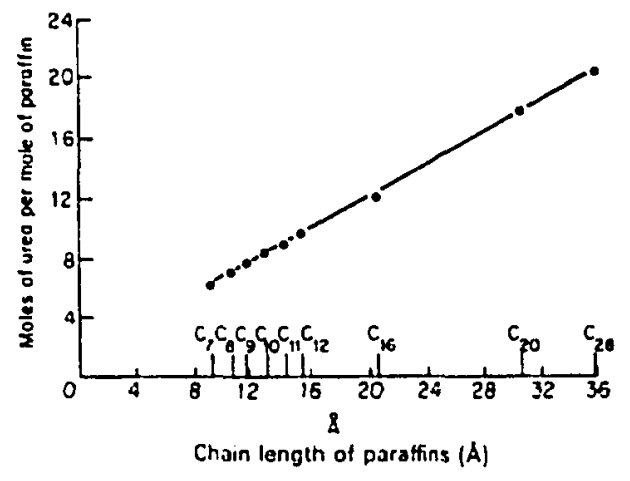

Figure XIII-5: Dependence of host:guest stoichiometric ratio upon guest carbon chain length for urea/nparaffin inclusion compounds $|3|$

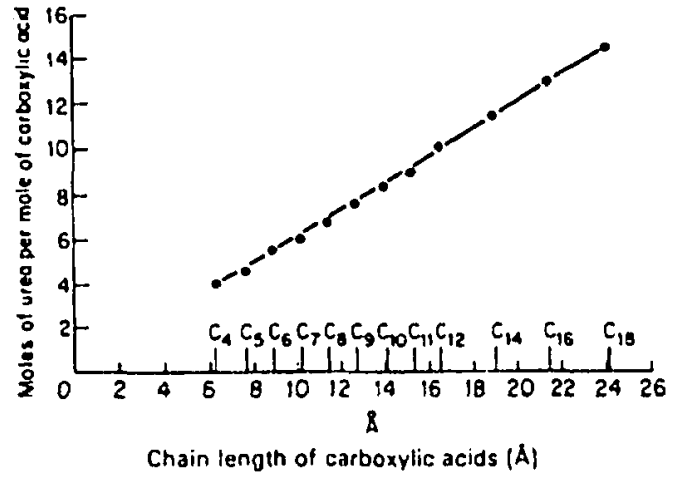

Figure XIII-6: Dependence of host:guest stoichiometric ratio upon guest carbon chain length for urea/carboxylic acid inclusion compounds $|3|$

For the sulfur analogue of urea, namely thiouren $\left\{\mathrm{H}_{2} \mathrm{~N}(\mathrm{CS}) \mathrm{NH}_{2}\right\}$, which forms inclusion compounds with larger and more highly branched guests, it was shown that the length of the highly branched paraffin guests gave a linear relationship to the number of moles of thiourea necessary to form the inclusion compound (see Figure XIII-7) [4]. Thiourea also forms inclusion compounds with the $\alpha, \omega$-dicyclohexyl-paraffin guest family. In this case, the relationship between the length of the guest molecule and the host:guest molar ratio is not exactly linear (see Figure XIII-8), which may be a result of the various ways of fitting the guest molecules (of differing sizes and shapes) into the constraints of the thiourea channel. 


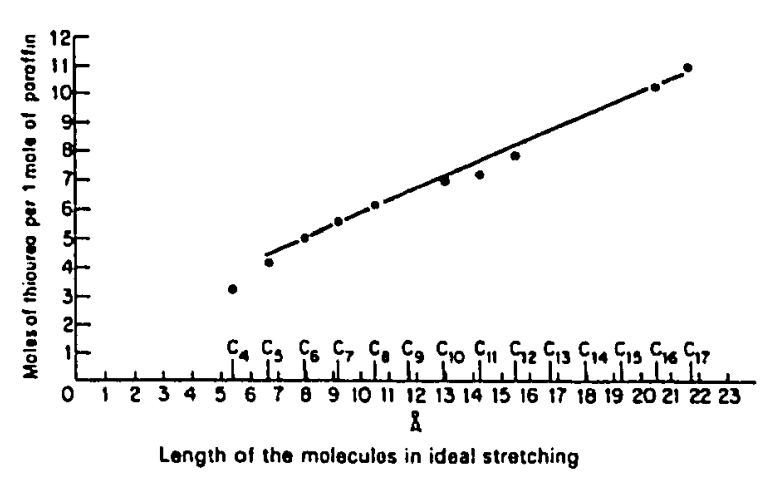

Figure XIII-7: Dependence of host:guest stoichiometric ratio upon guest carbon chain length for thiourea/branched-paraffin inclusion compounds [4]

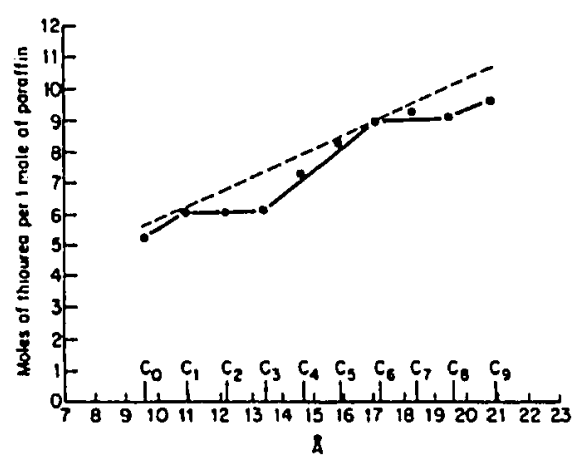

Figure XIII-8: Dependence of host:guest stoichiometric ratio upon guest carbon chain length for thiourea $/ \alpha, \omega$-dicyclohexylparaffin inclusion compounds [4]

(B) ${ }^{13} \mathrm{C} C P / \mathrm{MAS} N \mathrm{NR}$ and powder $\mathrm{X}$-ray diffraction:

The ${ }^{13} \mathrm{C}$ CP/MAS NMR spectra and powder X-ray diffractograms for TATM inclusion compounds with the 15 1-bromoalkane guests appear in Figures XIII-9 to XIII-11. As for the $\alpha, \omega$-dichloroalkanes of a previous study (chapter XI), we wished to determine which guests form isostructural TATM inclusion compounds, using these two techniques. More specifically, we want to know what effect does adding a single methylene unit to the guest have on the structural type of the resulting TATM inclusion compound (i.e. Do two guests that differ in size by one methylene unit form isostructural TATM inclusion compounds?). As we shall see, one may use the more common technique of powder Xray diffraction to detect isostructuralism, but we may also use the less prevalent technique of ${ }^{13} \mathrm{C}$ CP/MAS solid state NMR to obtain more or less the same information. When two compounds are isostructural (i.e. equivalent unit cell parameters), they should in theory both display very similar powder X-ray diffraction patterns and very similar ${ }^{13} \mathrm{C} C P / \mathrm{MAS}$ solid-state NMR spectra. Thus, these two techniques are complementary to each other in the information they provide.

In addition, the ${ }^{13} \mathrm{C} C P / M A S$ solid state NMR spectrum provides a "snapshot" on the contents of the crystallographic asymmetric unit. This is because each crystallographically unique carbon nucleus in the asymmetric unit should in theory give rise to its own resonance. Thus, we may use this technique to determine the number of TATM host molecules in the asymmetric unit. The carbonyl resonance of the host at 
about $190 \mathrm{ppm}$ (see Figures XIII-9 to XIII-11) is the most appropriate signal for this purpose. Since there are three carbonyl carbons in each TATM molecule, a multiplicity of three for this signal implies there is one host molecule in the asymmetric unit. On the other hand, a multiplicity of six for this signal implies that there are two host molecules in the asymmetric unit, and so on.

Finally, another advantage of using these two techniques is that they characterize the bulk product, as opposed to single crystal X-ray diffraction, which characterizes only a single selected crystal. This may be very important for compounds that can crystallize in more than one form (i.e. as a polymorph). If this is the case, one may calculate an Xray diffraction pattern based on the single crystal structure, and compare this calculated pattern with an experimental powder X-ray diffraction for the same batch of crystals that have been ground up. As mentioned above, three TATM guests have been found to form polymorphic inclusion compounds, namely cyclohexane, [1] 1,2-dichloroethane (chapter VII), and 1,3-dichloropropane (chapter XII).

In Figure XIII-9 appears the powder X-ray patterns and ${ }^{13} \mathrm{C}$ CP/MAS solid-state NMR spectra for TATM inclusion compounds with the five smallest sized 1bromoalkane guests $(\mathrm{Br} 2-\mathrm{Br} 6)$. The ${ }^{13} \mathrm{C} C P$ MAS NMR spectra for $\mathrm{Br} 2$ /TATM and $\mathrm{Br} 3 / \mathrm{TATM}$ are practically identical, as far as the host signals are concerned. However, their powder X-ray diffraction patterns are not similar in appearance, which tentatively suggests that $\mathrm{Br} 2$ and $\mathrm{Br} 3$ do not form isostructural TATM inclusion compounds. Similarly, Br4/TATM has a ${ }^{13} \mathrm{C}$ CP/MAS NMR spectrum that is very similar in appearance to $\mathrm{Br} 5 / \mathrm{TATM}$ and $\mathrm{Br6} / \mathrm{TATM}$, but its powder $\mathrm{X}$-ray diffraction pattern is again unique. On the other hand, Br5/TATM and Br6/TATM have both very similar ${ }^{13} \mathrm{C}$ CP/MAS NMR spectra and almost identical powder X-ray diffraction patterns, which suggests unequivocally that they are isostructural. 


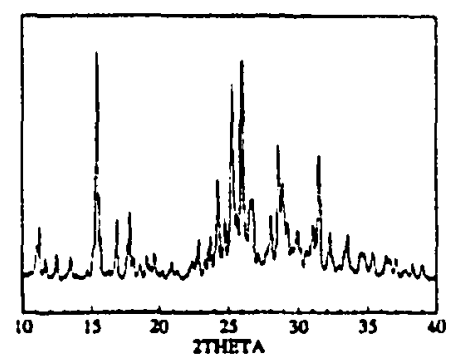

E
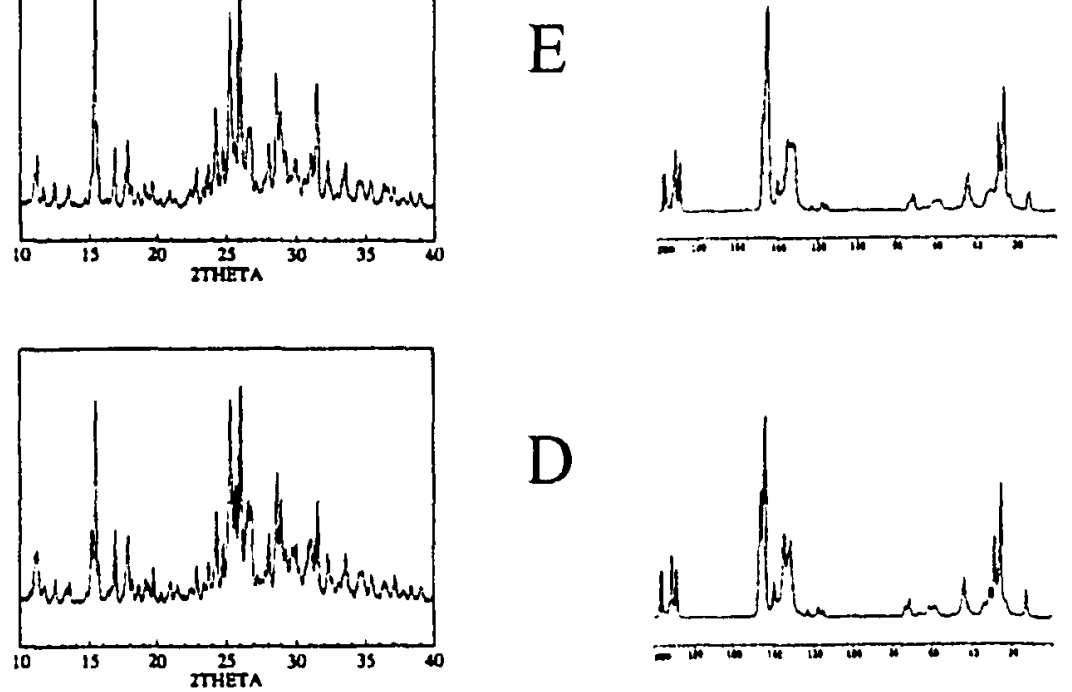

D
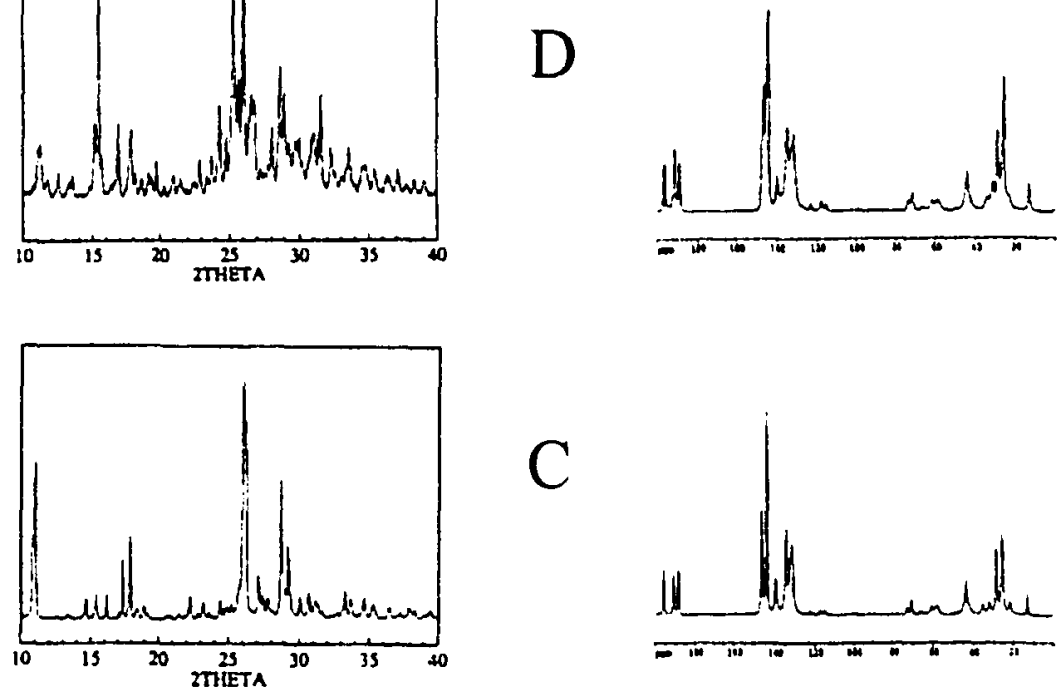

$\mathrm{C}$
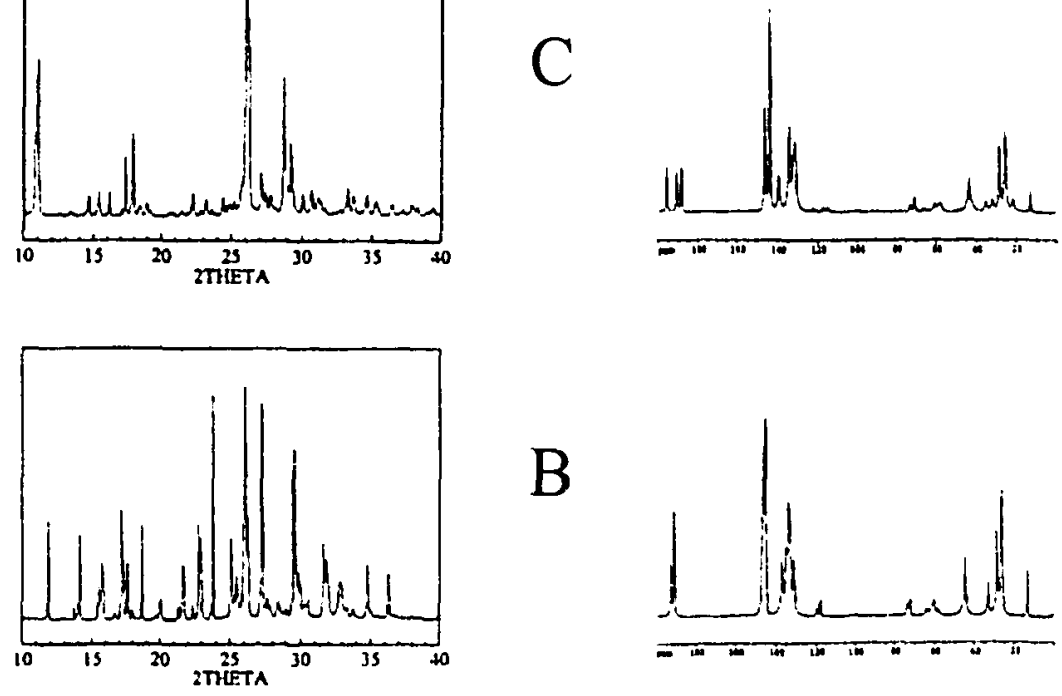

$\mathrm{B}$
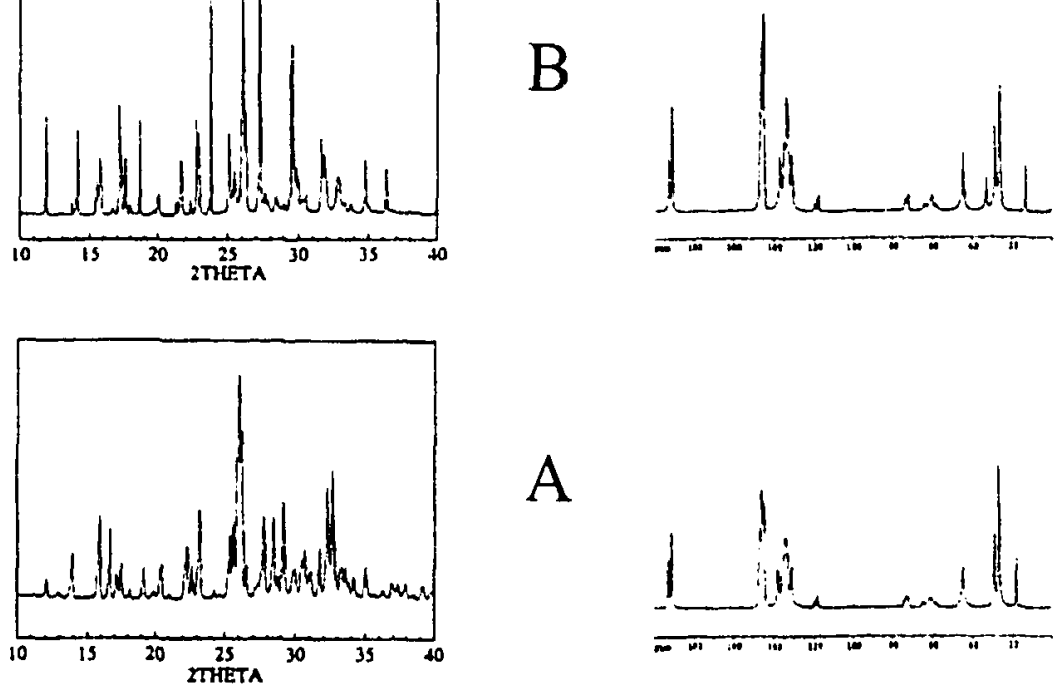

A

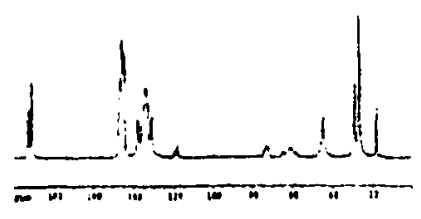

Figure XIII-9: PXRD patterns (left column) and ${ }^{13} \mathrm{C}$ CP/MAS solid-state NMR spectra (right column) for TATM inclusion compounds with the five smallest sized 1-bromoalkane guests: (A) 1-bromoethane/TATM (B) 1-bromopropane/TATM (C) 1-bromobutane/TATM (D) 1-bromopentane/TATM (E) 1-bromohexane/TATM 


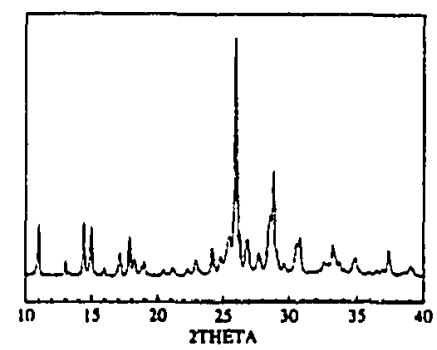

$\mathrm{E}$
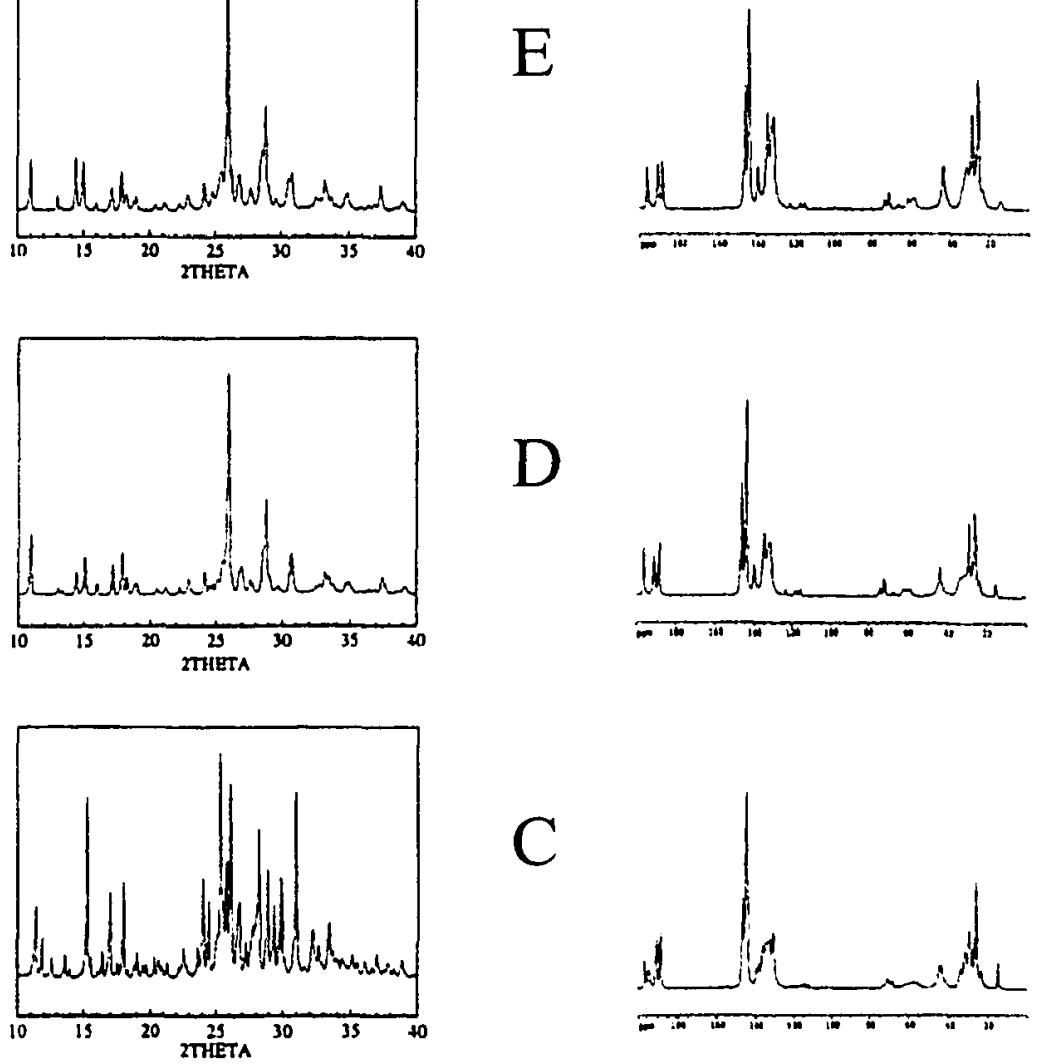

$\mathrm{C}$
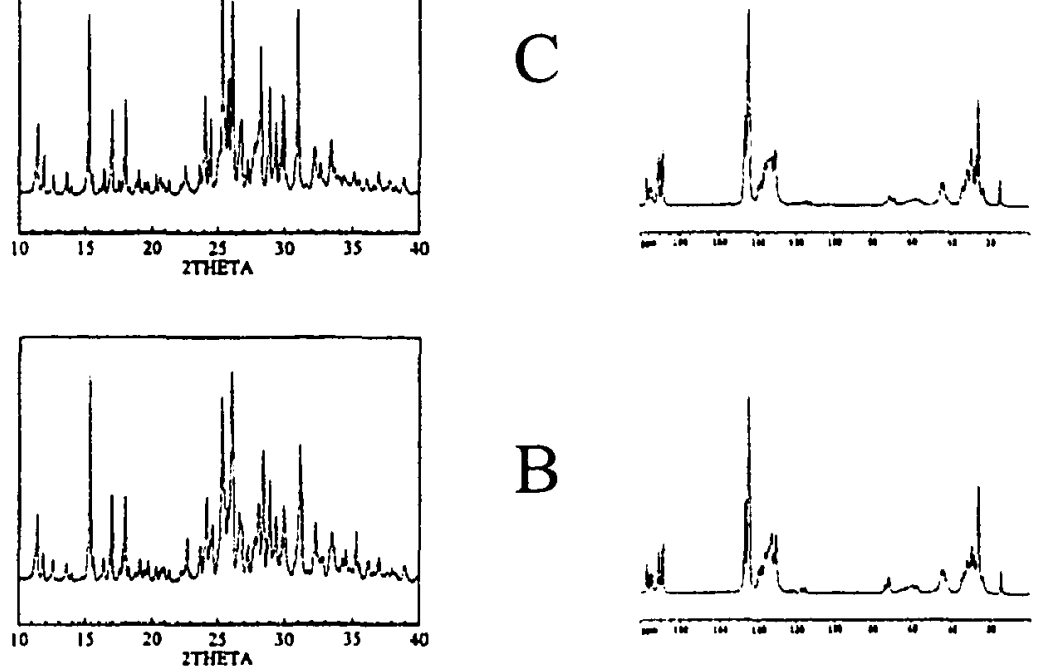

B
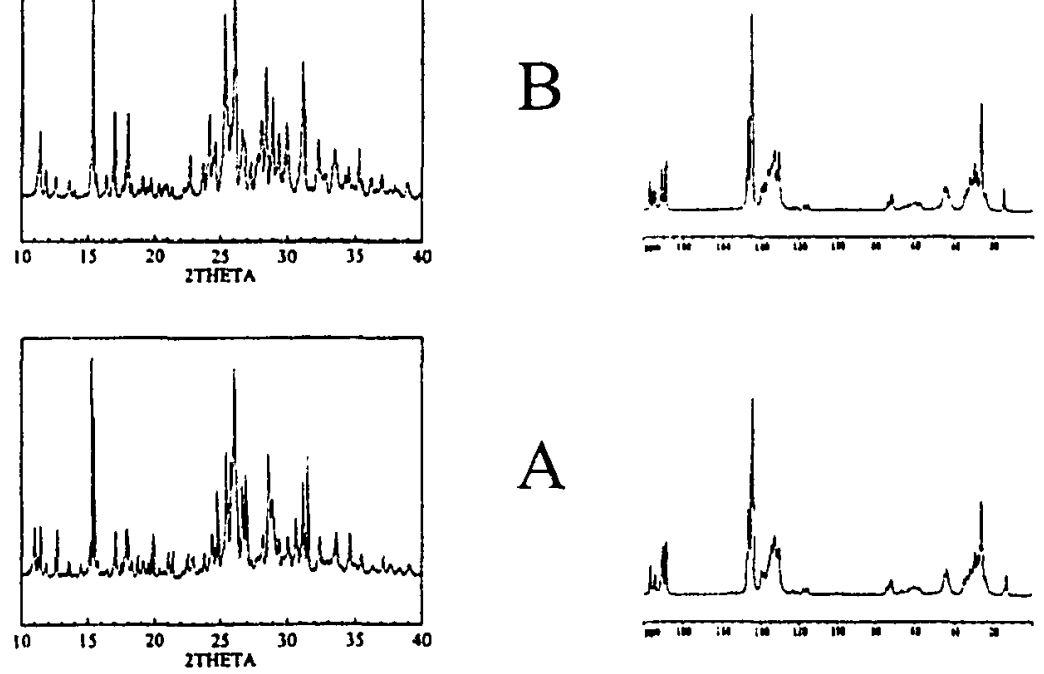

Figure XIII-10: PXRD patterns (left column) and ${ }^{13} \mathrm{C} \mathrm{CP} / \mathrm{MAS}$ solid-state NMR spectra (right column) for TATM inclusion compounds with the five intermediate sized 1-bromoalkane guests: (A) 1-bromoheptane/TATM (B) 1-bromooctane/TATM (C) 1-bromononane/TATM (D) 1-bromodecane/TATM (E) 1-bromoundecane/TATM 


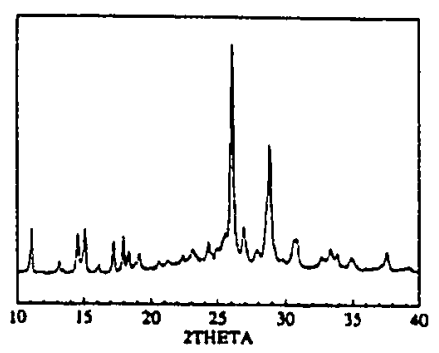

$\mathrm{E}$
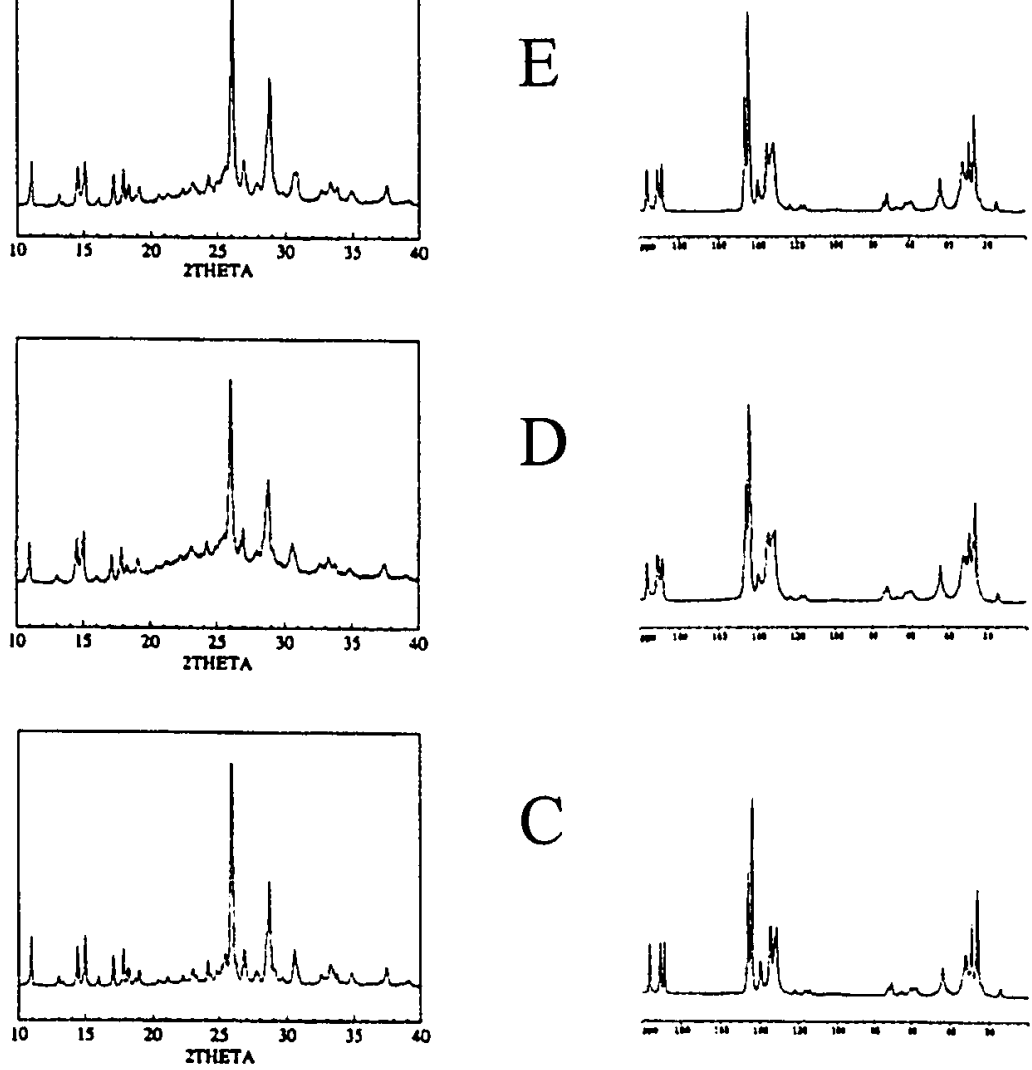

C
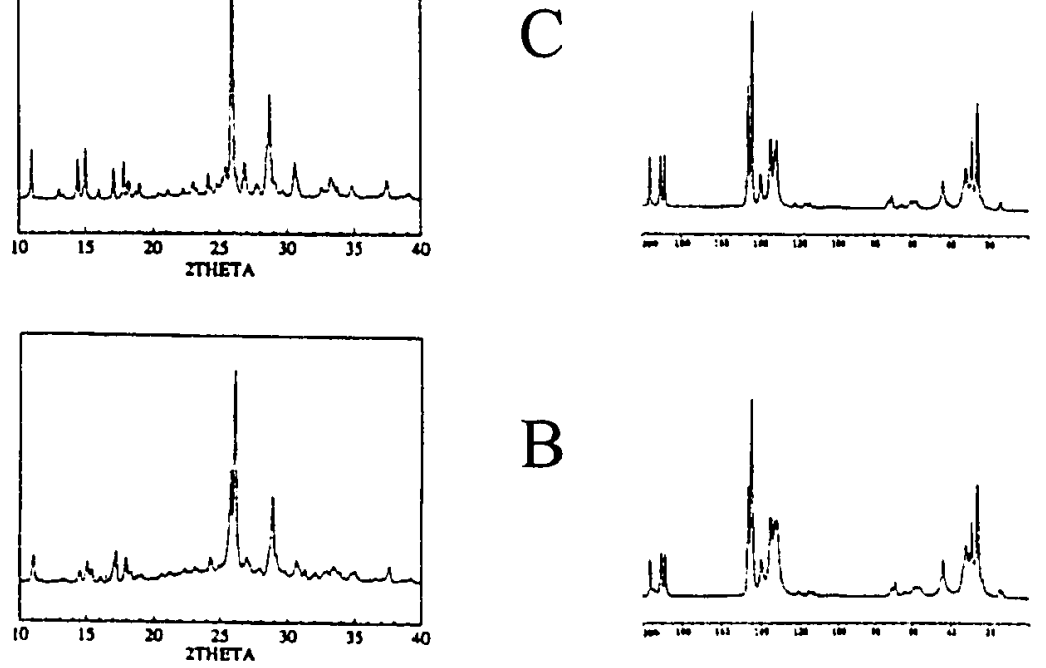

B
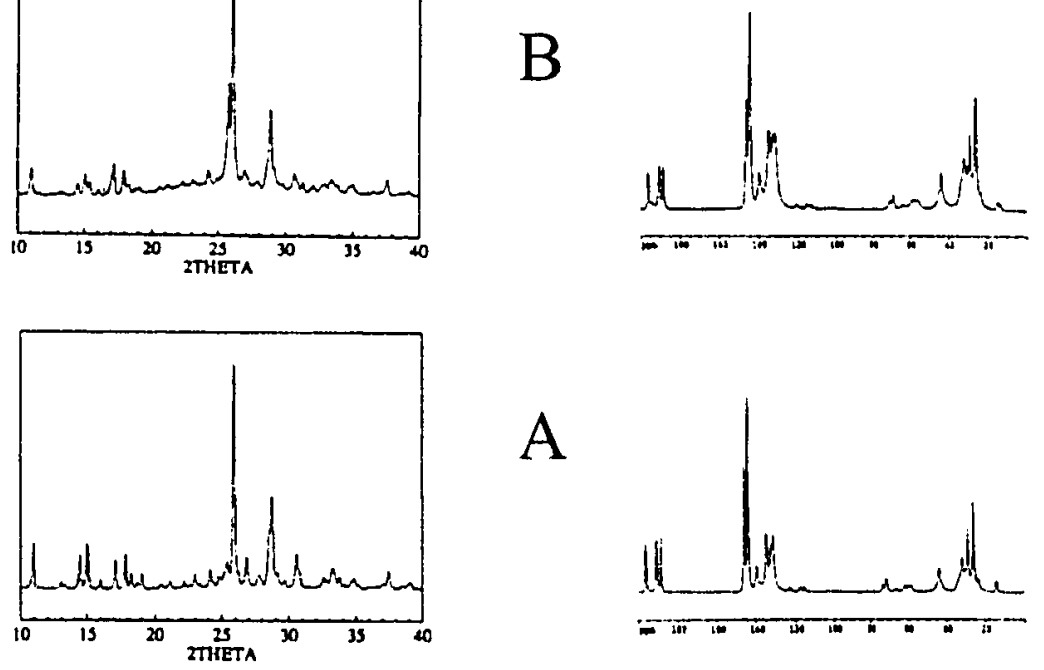

Figure XIII-11: PXRD patterns (left column) and ${ }^{13} \mathrm{C}$ CP/MAS solid-state NMR spectra (right column) for TATM inclusion compounds with the five largest sized 1-bromoalkane guests: (A) 1-bromododecane/TATM (B) 1-bromotridecane/TATM (C) 1-bromotetradecane/TATM (D) 1-bromopentadecane/TATM and (E) 1-bromohexadecane/TATM 
These ${ }^{13} \mathrm{C} \mathrm{CP} / \mathrm{MAS}$ spectra allow us to determine the number of host molecules in the asymmetric unit. For the TATM inclusion compounds with the five smallest size guests, the ${ }^{13} \mathrm{C}$ CP/MAS NMR spectra in Figure XIII-9 display a multiplicity of three for the carbonyl signal of the host (at $\sim 190 \mathrm{ppm}$ ) for all five inclusion compounds. This suggests that all five of these inclusion compounds have one host molecule in the asymmetric unit.

The powder X-ray diffraction patterns and ${ }^{13} \mathrm{C}$ CP/MAS NMR spectra for TATM inclusion compounds with the five intermediate sized 1-bromoalkane guests $(\mathrm{Br} 7-\mathrm{Br} 11)$ appear in Figure XIII-10. The first three inclusion compounds $(\mathrm{Br} 7, \mathrm{Br} 8$ and $\mathrm{Br} 9$ ) have near identical ${ }^{13} \mathrm{C} C \mathrm{CP} / \mathrm{MAS}$ NMR spectra, but the powder $\mathrm{X}$-ray diffraction patterns suggest that only $\mathrm{Br} 8$ and $\mathrm{Br} 9$ form isostructural TATM inclusion compounds, while $\mathrm{Br} 7$ forms a unique TATM structural type. For these inclusion compounds $(\mathrm{Br} 7-\mathrm{Br} 9)$, the ${ }^{13} \mathrm{C}$ CP/MAS NMR spectra suggest that there are two host molecules in the asymmetric unit, as the multiplicity of the carbonyl signal at about $190 \mathrm{ppm}$ is six.

The powder X-ray diffraction patterns and ${ }^{13} \mathrm{C}$ CP/MAS NMR spectra for TATM inclusion compounds with the five largest sized 1-bromoalkane guests $(\mathrm{Br} 12-\mathrm{Br} 16)$ appear in Figure XIII-11. Perhaps unexpectedly, the seven largest sized guests (including $\mathrm{Brl} 10$ and $\mathrm{Brll}$ from Figure XIII-10) form isostructural TATM inclusion compounds. They all have not only near identical ${ }^{13} \mathrm{C} \mathrm{CP} / \mathrm{MAS}$ NMR spectra, but also practically identical powder X-ray diffraction patterns. For all seven of these inclusion compounds, the ${ }^{13} \mathrm{C} C P / M A S$ solid-state NMR spectra show a multiplicity of three for the carbonyl carbon signal of the host at about $190 \mathrm{ppm}$, suggesting that all seven of these TATM inclusion compounds have one host molecule in the asymmetric unit

These results suggest that powder $\mathrm{X}$-ray diffraction is a more sensitive technique than ${ }^{13} \mathrm{C}$ CP/MAS NMR to detect isostructuralism, as we have just seen that two compounds which are not isostructural (as judged by powder X-ray diffraction) may display near identical ${ }^{13} \mathrm{C}$ CP/MAS solid-state NMR spectra. This suggests that the PXRD pattern is more sensitive to the differences in guest electron density that exist between two 1-bromoalkane guests in their respective TATM lattices, while the TATM host resonances of the ${ }^{13} \mathrm{C} C P / M A S ~ N M R$ spectrum may be more sensitive to the packing motif of the TATM host lattice, regardless of the nature of the included guest. 
Table XIII-2: The number of host molecules in the asymmetric unit for TATM inclusion compounds with 1-bromoalkane guests $\mathrm{Br}\left(\mathrm{CH}_{2}\right)_{n} \mathrm{CH}_{3}(n=1$ to 15), as determined from ${ }^{13} \mathrm{C}$ CP/MAS solid-state NMR. Additionally, isostructural TATM inclusion compounds, as determined from powder $\mathrm{X}$-ray diffraction, are denoted with a common superscript letter.

\begin{tabular}{lc} 
Guest & Number of host molecules in the asymmetric unit \\
\cline { 2 - 2 } $\mathrm{Br} 2$ & 1 \\
$\mathrm{Br} 3$ & 1 \\
$\mathrm{Br} 4$ & 1 \\
$\mathrm{Br} 5^{\mathrm{A}}$ & 1 \\
$\mathrm{Br} 6^{\mathrm{A}}$ & 1 \\
$\mathrm{Br} 7$ & 2 \\
$\mathrm{Br} 8^{\mathrm{B}}$ & 2 \\
$\mathrm{Br} 9^{\mathrm{B}}$ & 2 \\
$\mathrm{Br} 10^{\mathrm{C}}$ & 1 \\
$\mathrm{Br} 11^{\mathrm{C}}$ & 1 \\
$\mathrm{Br} 12^{\mathrm{C}}$ & 1 \\
$\mathrm{Br} 13^{\mathrm{C}}$ & 1 \\
$\mathrm{Br} 14^{\mathrm{C}}$ & 1 \\
$\mathrm{Br} 15^{\mathrm{C}}$ & 1 \\
$\mathrm{Br} 16^{\mathrm{C}}$ & 1
\end{tabular}

From Table XIII-2, we see that there is a very substantial degree of isostructuralism among the 1-bromoalkane / TATM inclusion compounds of the present study. One may consider the relative percent increase in size of the guest on going from an $n$ carbon chain to an $n+1$ carbon chain. For the 1 -bromoalkane TATM guests, this data is compiled in Table XIII-3. Again, when the percent relative increase in size is large, (e.g. $\mathrm{Br} 2 \rightarrow \mathrm{Br} 3$ $=33 \%$ ), TATM will form unique structural types with each of the two guests. This behaviour is observed for the three smallest size guests $(\mathrm{Br} 2, \mathrm{Br} 3$ and $\mathrm{Br} 4)$. However $\mathrm{Br} 5$ and $\mathrm{Br} 6$ form isostructural TATM inclusion compounds. The percent relative increase in size on going from $\mathrm{Br} 5$ to $\mathrm{Br} 6$ is a hefty $17 \%$. In the previous study (see chapter XI) on TATM inclusion compounds with $\alpha,(1)$-dichloroalkane guests $\left(\mathrm{Cl}\left(\mathrm{CH}_{2}\right)_{11} \mathrm{Cl} ; n=1\right.$ to 10$)$, (see Figure $\left.\mathrm{XI}-1\right)$ we found that the only guests that form isostructural TATM inclusion compounds were 1,9-dichlorooctane and 1,10dichlorodecane. The percent relative increase in size in this case is only $9.1 \%$.

Indeed, when the percent relative increase in size for the 1-bromoalkane / TATM guests of the present study reaches the same value of $9.1 \%$ - see Table XIII-3), one 
observes very extensive isostructuralism. All seven guests from $\mathrm{Br} 10$ to $\mathrm{Br} 16$ form isostructural TATM inclusion compounds. We may add a full six methylene groups to $\mathrm{Br} 10$ to form Brl6, yet the TATM host cannot distinguish a difference in size or shape of these two guests upon their respective crystallizations, and they subsequently form isostructural TATM inclusion compounds.

Table XIIl-3: Relative percent increase in size $( \pm 1)$ of the guest ( 2 s.f.) on going from an $n$ carbon chain to an $n+1$ carbon chain, for 1-bromoalkane guests.

\begin{tabular}{lc} 
Guest & $\%$ increase in size \\
\hline $\mathrm{Br} 2$ & $33 \%$ \\
$\mathrm{Br} 3$ & $25 \%$ \\
$\mathrm{Br} 4$ & $20 \%$ \\
$\mathrm{Br} 5$ & $17 \%$ \\
$\mathrm{Br} 6$ & $14 \%$ \\
$\mathrm{Br} 7$ & $12 \%$ \\
$\mathrm{Br} 8$ & $11 \%$ \\
$\mathrm{Br} 9$ & $10 \%$ \\
$\mathrm{Br} 10$ & \\
\hline
\end{tabular}

\begin{tabular}{lc} 
Guest & $\%$ increase in size \\
\hline Brl & $10 \%$ \\
Brl0 & $9.1 \%$ \\
Brll & $8.3 \%$ \\
Brl2 & $7.7 \%$ \\
Brl3 & $7.1 \%$ \\
Brl4 & $6.7 \%$ \\
Brl5 & $6.2 \%$ \\
\hline Brl6
\end{tabular}

Perhaps one reason why we see this difference in behaviour between the two groups of guests is because we were unable to form inclusion compounds of the $\alpha,(1)$ dichloroalkane guests of the previous study (chapter XI) that were larger than 1,10dichlorodecane, as larger guests (e.g. 1,11-dichloroundecane) exist as solids at room temperature. Preparation of TATM inclusion compounds with solids is a very challenging task, as it is almost impossible to find a common solvent that will not itself be incorporated into the host lattice. However, one may imagine that if we were able to prepare these $\alpha,(1)$-dichloroalkane / TATM inclusion compounds, one might also see more extensive TATM isostructuralism for these larger guests, as the percent relative increase in size would be getting smaller and smaller, so it would be more difficult for the TATM host to distinguish two guests that differ in size by one methylene unit.

On the other hand, for the 1-bromoalkane guests of the present study, we see isostructuralism among smaller guests. As previously mentioned, $\mathrm{Br} 5$ and $\mathrm{Br} 6$ form 
isostructural TATM inclusion compounds. Also, $\mathrm{Br} 8$ and $\mathrm{Br} 9$ form isostructural TATM inclusion compounds. The percent relative increase in size is $16.7 \%$ for the first pair, and $11.1 \%$ for the latter pair. So despite the fact that we were unable to make TATM inclusion compounds with guests larger than 1,10-dichlorodecane, there are definite suggestions of a difference in the properties of molecular recognition between the two groups of guests, as the 1-bromoalkanes form isostructural TATM inclusion compounds with much smaller guests than the previously studied $\alpha, \omega$-dichloroalkane guests.

One may speculate on reasons for this difference in behaviour. Firstly, the $\alpha, \omega-$ dichloroalkane guests may be anchored, in the TATM lattice, at both ends of the guest molecule. On the other hand, the 1-bromoalkane guests are anchored at only one end. One may imagine that it may be easier for the TATM host to distinguish between two guests that are rod - like in shape (the $\alpha$, ()-dichloroalkanes) and differ in size by one methylene unit, thus forming a smaller number of isostructural TATM inclusion compounds. Conversely, it may be more difficult for the TATM host to distinguish between two guests that are perhaps spherical or globular in shape (the 1-bromoalkanes) and differ in size by one methylene unit, so we observe much more extensive isostructuralism for this group of guests.

Another possibility for this isostructuralism behaviour may arise from the postulate mentioned above, namely that with larger guests, the compound formed between TATM and these guests are incommensurate. The intermolecular interactions between the larger 1-bromoalkanes and the TATM molecules may be much weaker than those for the commensurate TATM inclusion compounds with the smaller size 1-bromoalkane guests. Thus there would be a significantly lower influence by the larger 1-bromoalkane guests upon the packing mode of the TATM host lattice, resulting in extensive isostructuralism for these larger 1 -bromoalkane guests. Indeed, numerous attempts to obtain good quality crystals of TATM with the larger 1-bromoalkane guests met with failure each time, suggesting that the minute aggregates do not assemble into high quality single crystals due to the weakness of intermolecular interactions between the larger 1-bromoalkane guests and TATM host.

Finally, an important question to ask is how two TATM inclusion compounds can be isostructural, yet have vastly different $H: G$ molar ratios. For example, Br12/TATM 
has a $H: G$ molar ratio of 3.36:1, while Br13/TATM has a H:G molar ratio of 1.09:1 (see Table XIII-1), yet both the ${ }^{13} \mathrm{C} \mathrm{CP} / \mathrm{MAS}$ solid state NMR spectra and powder X-ray diffraction patterns (Figure XIII-11) suggest that they are isostructural. Again, for the larger guests, this may be related to the fact that they form incommensurate inclusion compounds and do not crystallize easily, so they may be somewhere between $\{1\}$ crystalline (as is evidenced by the fairly well-resolved signals / peaks in the ${ }^{13} \mathrm{C} C P / \mathrm{MAS}$ NMR spectra and the PXRD patterns for Brl0 to Brl6 guests, see Figures XIII-10 and XIII-11, and relatively high melting points and enthalpies of decomposition) and $\{2\}$ amorphous (changing $\mathrm{H}: \mathrm{G}$ molar ratio has no strong influence on the ultimate structure of the resulting compound, suitable quality single crystals are difficult to prepare, and some of the PXRD patterns consist of a broad baseline "hump", upon which the sharper signals are superimposed, particularly so for $\mathrm{Br} 15$ and $\mathrm{Br} 16$ inclusions).

(C) ${ }^{13} \mathrm{C}$ CP/MAS NMR with dipolar dephasing:

Another difference between these two groups of guests may be the presence (or absence) of reorientational dynamics. One of the easiest and most powerful experimental techniques to qualitatively detect the presence of dynamics in the solid state is ${ }^{13} \mathrm{C}$ CP/MAS solid state NMR with dipolar dephasing [5]. This pulse sequence involves placing a short delay ( $\sim 4 \mu \mathrm{s}$ ), without ${ }^{1} \mathrm{H}$ decoupling, in between the end of ${ }^{1} \mathrm{H} \rightarrow{ }^{13} \mathrm{C}$ cross polarization and the start of ' $H$ decoupled signal acquisition. This technique dephases (removes) signals from the ${ }^{13} \mathrm{C}$ CP/MAS NMR spectrum corresponding to carbons that are strongly dipolar coupled to hydrogen (i.e. carbon atoms with directly bonded protons). The ${ }^{13} \mathrm{C}$ signals from quaternary carbons (i.e. with no directly bonded protons) are not attenuated by this pulse sequence because they are only weakly dipolar coupled to hydrogen. In addition, signals corresponding to methyl $\left(-\mathrm{CH}_{3}\right)$ carbons are not dephased by this experiment because their dipolar coupling to ' $\mathrm{H}$ is almost completely averaged by internal $\mathrm{C}_{3}$ methyl reorientation. Therefore, the signals corresponding to methylene $\left(\mathrm{CH}_{2}\right)$ and methine $(\mathrm{CH})$ carbons may be used to qualitatively detect the presence of reorientation of those regions of the guest molecule. If the $\mathrm{CH}_{2}$ or $\mathrm{CH}$ group is static, it is strongly coupled to hydrogen, and its signal will be eliminated. On the other hand, if that portion of the guest molecule is reorienting in the solid-state, the ${ }^{1} \mathrm{H}-{ }^{13} \mathrm{C}$ 
dipolar coupling will be almost completely averaged to zero, and the corresponding signal will retain at least part of its intensity in the dipolar dephased spectrum

The ${ }^{13} \mathrm{C}$ CP/MAS dipolar dephasing NMR spectra of the fifteen 1-bromoalkane / TATM inclusion compounds appear in Figure XIII-12. To locate the guest resonances in each ${ }^{13} \mathrm{C}$ CP/MAS NMR spectrum, the ${ }^{13} \mathrm{C}$ chemical shifts (in $\mathrm{CDCl}_{3}$ solvent) of each carbon of each of the fifteen guests appears in Table XIII-4 [6]. As an example, for the 1-bromoethane ( $\mathrm{Br} 2)$ / TATM inclusion compound, the terminal $\mathrm{CH}_{3}$ resonance is visible at about $18 \mathrm{ppm}$ (19.4 ppm in $\mathrm{CDCl}_{3}$ solvent), while the $\mathrm{CH}_{2}$ resonance, expected at 27.6 $\mathrm{ppm}$, is buried underneath the methyl signal of the TATM host. By comparing the ${ }^{13} \mathrm{C}$ chemical shift data in Table XIII-4 with the dipolar dephasing NMR spectra of Figure XIII-12, it is evident that even the largest size guests (such as 1-bromohexadecane $\mathrm{Brl6}$ ), are undergoing dynamic reorientation inside the TATM lattice. Most all of the signals retain at least some of their intensities when compared to their respective "normal ${ }^{13} \mathrm{C}$ CP/MAS NMR spectra (Figures XIII-9 to XIII-11). On the other hand, for the $\alpha, \omega$-dichloroalkane guests of the previous study (dipolar dephasing spectra in Figure XIII-13 and ${ }^{13} \mathrm{C}$ chemical shifts in $\mathrm{CDCl}_{3}$ in Table XIII-5), one observes a different behaviour. With the exception of 1,1-dichloromethane, 1,2-dichloroethane and 1,3dichloropropane, there is practically no residual signal intensity, which suggests that the larger sized $\alpha, \omega$-dichloroalkane guests $(\mathrm{Cl} 4 \mathrm{Cl}-\mathrm{Cl} 6 \mathrm{Cl}$ and $\mathrm{Cl} 8 \mathrm{Cl}-\mathrm{Cl} 10 \mathrm{Cl})$ are not reorienting in the TATM lattice.

Table XIII-4: ${ }^{13} \mathrm{C}$ NMR solution chemical shifts $( \pm 0.1 \mathrm{ppm})$ for 1-bromoalkanes [6]. Chemical shifts are given relative to tetramethylsilane (TMS) at zero ppm

\begin{tabular}{|c|c|c|c|c|c|c|c|c|c|c|c|c|c|c|c|c|}
\hline Guest & 1 & 2 & 3 & 4 & 5 & 6 & 7 & 8 & 9 & 10 & 11 & 12 & 13 & 14 & 15 & 16 \\
\hline $\mathrm{Br} 2$ & 27.6 & 19.4 & & & & & & & & & & & & & & \\
\hline $\mathrm{Br} 3$ & 35.6 & 26.4 & 13.0 & & & & & & & & & & & & & \\
\hline $\mathrm{Br} 4$ & 33.3 & 35.0 & 21.4 & 13.2 & & & & & & & & & & & & \\
\hline $\mathrm{Br} 5$ & 34.0 & 30.5 & 32.7 & 22.1 & 14.0 & & & & & & & & & & & \\
\hline Br6 & 33.1 & 33.4 & 28.1 & 31.2 & 22.7 & 14.0 & & & & & & & & & & \\
\hline $\mathrm{Br} 7$ & 33.7 & 33.0 & 28.2 & 28.5 & 31.7 & 22.6 & 14.0 & & & & & & & & & \\
\hline Br8 & 33.1 & 33.1 & 28.4 & 28.9 & 29.3 & 31.9 & 22.7 & 14.1 & & & & & & & & \\
\hline $\mathrm{Br} 9$ & 33.4 & 33.1 & 28.3 & 28.9 & 29.4 & 29.6 & 32.0 & 22.7 & 14.1 & & & & & & & \\
\hline Brlo & 33.5 & 32.9 & 28.2 & 28.8 & 29.5 & 29.5 & 29.3 & 31.9 & 22.7 & 14.1 & & & & & & \\
\hline Brll & 33.4 & 33.1 & 28.3 & 28.9 & 29.7 & 29.7 & 29.7 & 29.7 & 32.0 & 22.8 & 14.2 & & & & & \\
\hline $\mathrm{Br} 12$ & 33.2 & 33.2 & 28.3 & 28.9 & 29.8 & 29.8 & 29.8 & 29.8 & 29.8 & 32.1 & 22.8 & 14.0 & & & & \\
\hline $\mathrm{Br} 13$ & 33.6 & 33.0 & 28.3 & 28.9 & 29.8 & 29.8 & 29.8 & 29.8 & 29.8 & 29.8 & 32.1 & 22.8 & 14.2 & & & \\
\hline $\mathrm{Brl} 14$ & 33.1 & 28.4 & 29.0 & 29.8 & 29.8 & 29.8 & 29.8 & 29.8 & 29.8 & 29.8 & 29.8 & 32.1 & 22.8 & 14.1 & & \\
\hline Br15 & 33.1 & 33.1 & 28.3 & 28.9 & 29.8 & 29.8 & 29.8 & 29.8 & 29.8 & 29.8 & 29.8 & 29.8 & 32.1 & 22.8 & 14.2 & \\
\hline $\mathrm{Br} 16$ & 33.2 & $.33,2$ & 28.3 & 28.9 & 29.8 & 29.8 & 29,8 & 29,8 & 29.8 & 29.8 & 29.8 & 29.8 & 29,8 & 32.1 & 22.8 & 4.2 \\
\hline
\end{tabular}



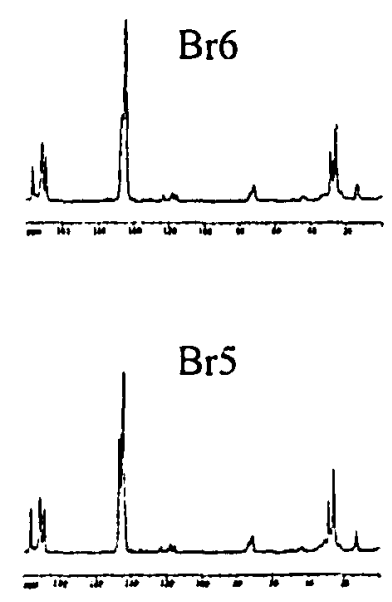

$\mathrm{Br} 4$

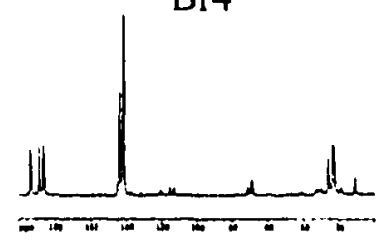

$\mathrm{Br} 3$

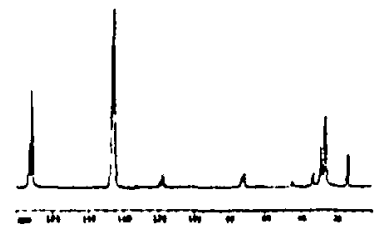

$\mathrm{Br} 2$

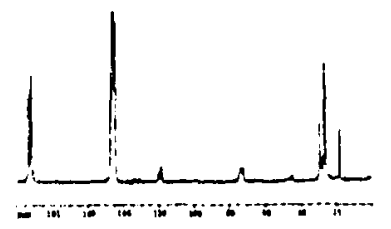

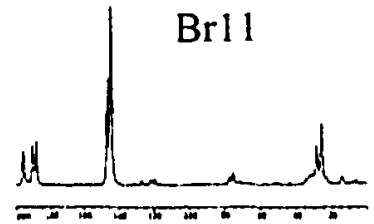
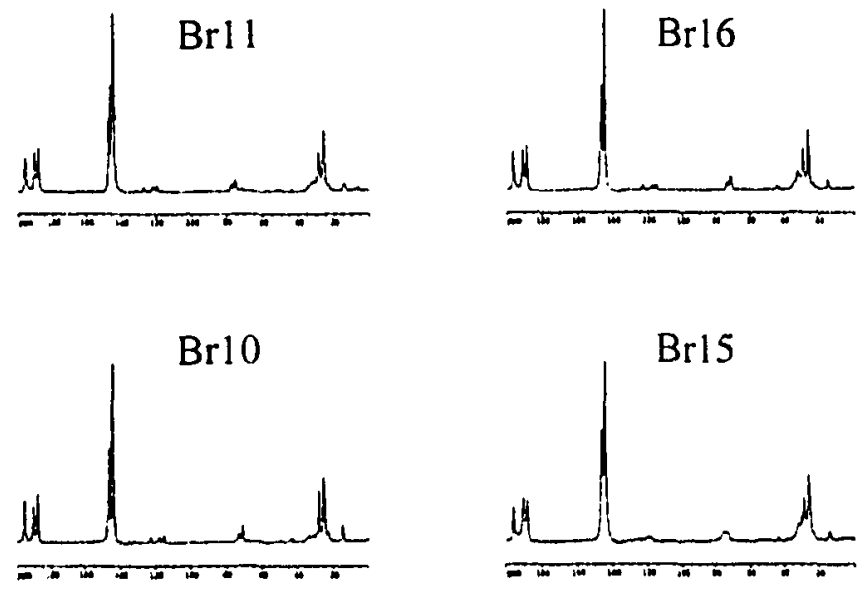

$\mathrm{Br} 9$

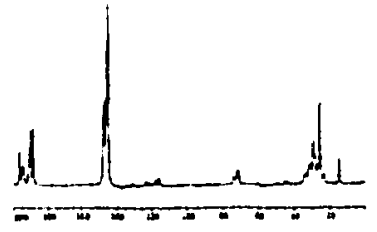

$\mathrm{Br} 8$

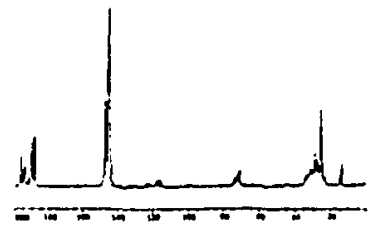

$\mathrm{Br} 7$

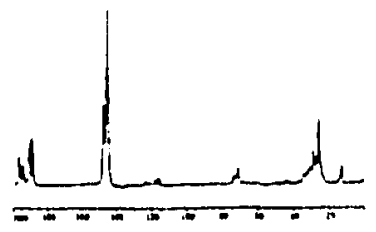

$\mathrm{Br} 15$

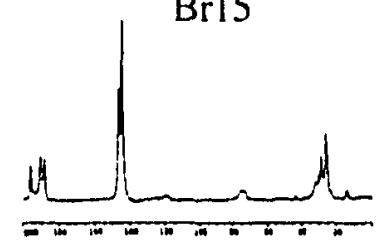

$\mathrm{Br} 14$

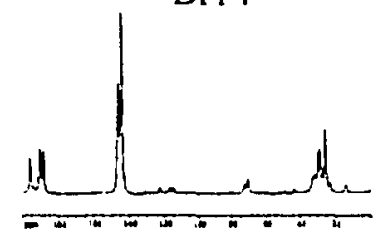

$\mathrm{Br} 13$

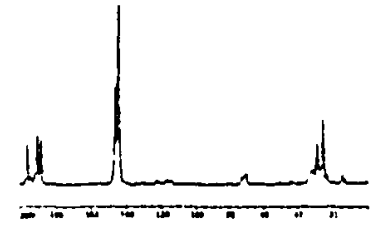

$\operatorname{Br} 12$

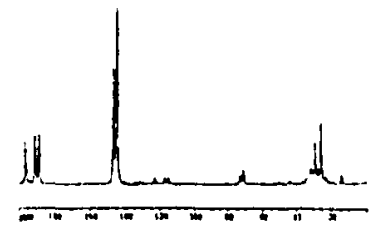

Figure XIII-12: ${ }^{13} \mathrm{C} \mathrm{CP} / \mathrm{MAS}$ solid-state dipolar dephasing NMR spectra of the fifteen TATM inclusion compounds with I-bromoalkane guests. Refer to Figure XIII-1 for our labeling convention. 

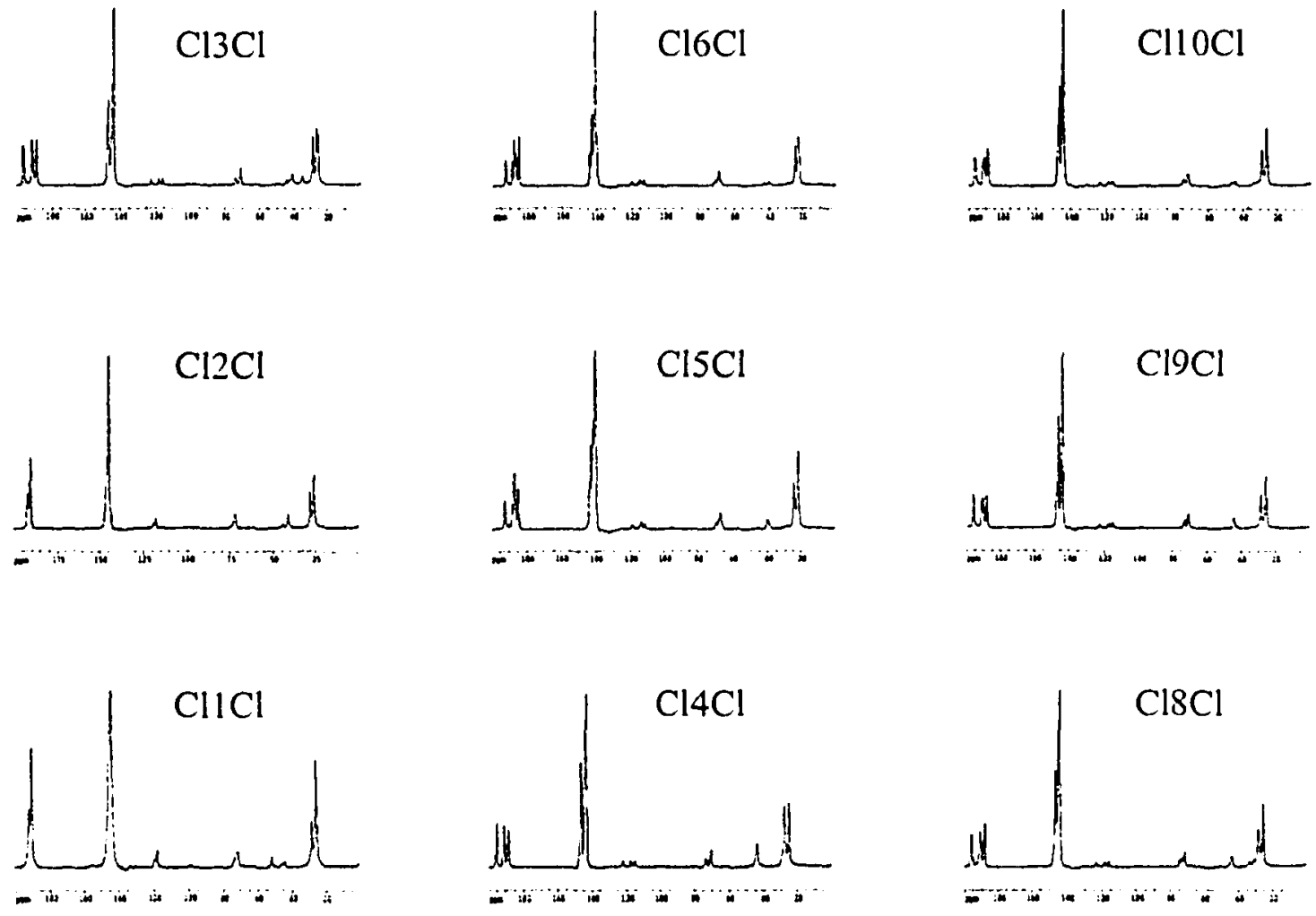

Figure XIII-13: ${ }^{13} \mathrm{C}$ CP/MAS solid-state dipolar dephasing NMR spectra of the nine TATM inclusion compounds with $\alpha, \omega$-dichloroalkane guests of a previous study (Figure $\mathrm{XI}-1$, p. 224).

Table XIII-5: ${ }^{13} \mathrm{C}$ NMR solution chemical shifts $( \pm 0.1 \mathrm{ppm})$ for $\alpha, \omega$-dichloroalkanes [6]. Chemical shifts are given relative to tetramethylsilane (TMS) at zero ppm.

\begin{tabular}{lllllllllll} 
Guest & 1 & 2 & 3 & 4 & 5 & 6 & 7 & 8 & 9 & 10 \\
\hline $\mathrm{Cl} 1 \mathrm{Cl}$ & 53.9 & & & & & & & & & \\
$\mathrm{Cl} 2 \mathrm{Cl}$ & 46.2 & 46.2 & & & & & & & & \\
$\mathrm{Cl3Cl}$ & 42.2 & 35.6 & 42.2 & & & & & & & \\
$\mathrm{Cl} 4 \mathrm{Cl}$ & 44.7 & 30.3 & 30.3 & 44.7 & & & & & & \\
$\mathrm{C} 15 \mathrm{Cl}$ & 44.7 & 32.0 & 24.3 & 32.0 & 44.7 & & & & & \\
$\mathrm{Cl} C \mathrm{Cl}$ & 44.9 & 32.5 & 26.2 & 26.2 & 32.5 & 44.9 & & & \\
$\mathrm{C} 18 \mathrm{Cl}$ & 45.1 & 32.6 & 26.8 & 28.8 & 28.8 & 26.8 & 32.6 & 45.1 & & \\
$\mathrm{C} 19 \mathrm{Cl}$ & 44.9 & 32.7 & 26.9 & 28.8 & 29.3 & 28.8 & 26.9 & 32.7 & 44.9 & \\
$\mathrm{Cl} 110 \mathrm{Cl}$ & 44.9 & 32.7 & 27.0 & 28.9 & 29.4 & 29.4 & 28.9 & 27.0 & 32.7 & 44.9 \\
\hline
\end{tabular}

A possible explanation for this may be found from the structural nature of the two guest families. The $\alpha, \omega$-dichloroalkane guests of the previous study are anchored at both ends, while the 1-bromoalkane guests of the present study are anchored at only one end. Thus, we propose that the $\alpha, \omega$-dichloroalkane guests, in the TATM lattice, are rod - like in shape, in an all trans extended chain conformation, while the 1-bromoalkane guests, in 
the TATM lattice, are perhaps more globular or spherical in shape, with a substantial number of gauche conformers. Perhaps this is why we see extensive dynamics for the latter group of guests (as is evidenced in the dipolar dephasing NMR spectra), in that they are possibly performing internal rotation about the $\mathrm{C}$ - $\mathrm{C}$ bonds due to the loose anchoring to the TATM lattice. On the other hand, the $\alpha, \omega$-dichloroalkane guests are anchored tightly at both ends, preventing internal rotation for the larger guests of this guest family. Indeed, as was concluded in chapter VII, based on the solid-state deuterium NMR spectra of the guest 1,2-dichloroethane- $\mathrm{d}_{4}$ in its TATM lattice, the guest exists exclusively in the trans conformation, and is performing two-fold reorientation about an axis through the center-of-mass of the guest and perpendicular to the $\mathrm{Cl}-\mathrm{C}-\mathrm{C}-\mathrm{Cl}$ plane. There is no evidence for the existence of internal rotation about the $\mathrm{C}-\mathrm{C}$ bond to give rise to gauche conformers. On the other hand, there are a substantial number of gauche conformations of the guest for the five polymorphic modifications of 1,3-dichloropropane / TATM (see chapter XII above), so this line of reasoning may be an oversimplification. Another possibility, mentioned above, may be due to the fact that Br10/TATM - Br16/TATM are incommensurate. The weak intermolecular interactions $[7 \mid$ between TATM and the 1bromoalkanes in these incommensurate structures may allow the guests to reorient in the solid state, despite their relatively large size. Finally, we note that variable temperature ${ }^{2} \mathrm{H}$ solid-state NMR would shed considerable light upon this situation, perhaps allowing detailed models for the motion (or lack thereof) of these guest molecules in their TATM lattices.

(D) Differential scanning calorimetry (DSC)

The DSC traces for the TATM inclusion compounds with six of the fifteen 1bromoalkane guests appear in Figure XIII-14. For Br2/TATM $\rightarrow$ Br10/TATM (and $\mathrm{Br}$ 16/TATM), there is one melting endotherm (primary endotherm), while for $\mathrm{Br} 1$ / TATM to $\mathrm{Br} 15 / \mathrm{TATM}$ there is, in addition to the primary endotherm, a secondary endotherm at a slightly higher temperature. This odd melting behaviour was reproducible for two separate batches of these TATM inclusion compounds, and is thus likely characteristic of the sample itself. The melting temperatures (in ${ }^{\circ} \mathrm{C}$ ), enthalpies of fusion (in $\mathrm{J} \mathrm{g}^{-1}$ and $\mathrm{kJ} \mathrm{mol}^{-1}$ TATM) and entropies of fusion (in $\mathrm{J} \mathrm{mol}^{-1} \mathrm{~K}^{-1}$ ) for each TATM 
inclusion compound (for both primary and secondary endotherms) aprear in Table XIII6. A plot of the first two two parameters, as a function of the number of carbon atoms of the guest, appears in Figure XIII-15. A general trend of increasing melting temperature and increasing decomposition enthalpy ( $T_{\mathrm{m}}$ with almost all guests, and $\Delta_{\mathrm{fus}} H$ at least with the smaller guests) with increasing size of guest is evident, with a break in this trend occurring on going from $\mathrm{Br}$ 9/TATM to $\mathrm{Br}$ 10/TATM. This break coincides with a change in structural type (all seven guests $\mathrm{Br} 10$ to $\mathrm{Br} 16$ forming isostructural TATM inclusion compounds) as perhaps is expected.

Perhaps unexpectedly, Br14/TATM shows a sharp increase in $\Delta H$ when compared to smaller and larger 1-bromoalkane guests on either side of Br14 / TATM (see Figure XIII-15). Why this occurs for $\mathrm{Br} 14$ and not for the other large guests ( $\mathrm{Br} 12-\mathrm{Br} 13$ and $\mathrm{Br} 15-\mathrm{Br} 16)$ may be beyond our ability to explain. Perhaps it has to do with the nature of the secondary melting endotherm (see Table XIII-6). Brl4 / TATM has both the largest secondary melting enthalpy $\left(\Delta H=27.7 \mathrm{~J} \mathrm{~g}^{-1}\right)$ and the highest secondary melting temperature $\left(T_{\mathrm{m}}=137.16^{\circ} \mathrm{C}\right)$, when compared to the other large TATM guests on either side. As far as $\Delta_{\text {lis }} S$ is concerned, one observes a significant range in values among the different 1 -bromoalkane inclusion compounds, from $55-89 \mathrm{~J} \mathrm{~mol}^{-1} \mathrm{~K}^{-1}$, in contrast to the $\alpha, \omega$-dichloroalkane guest family, where the range was only $90-102 \mathrm{~J} \mathrm{~mol}^{-1} \mathrm{~K}^{-1}$, suggesting first that the disorder of the crystal lattices are greater for the 1-bromoalkane inclusions, as would be expected from incommensurate inclusion compounds whose guests are anchored at only one end, and generally engage in reorientational dynamics (see dipolar dephasing NMR spectra above), and second, that the differences in the disorder of the crystal lattices are greater for the different 1-bromoalkane inclusions, perhaps due to the presence of both commensurate and incommensurate inclusions. 

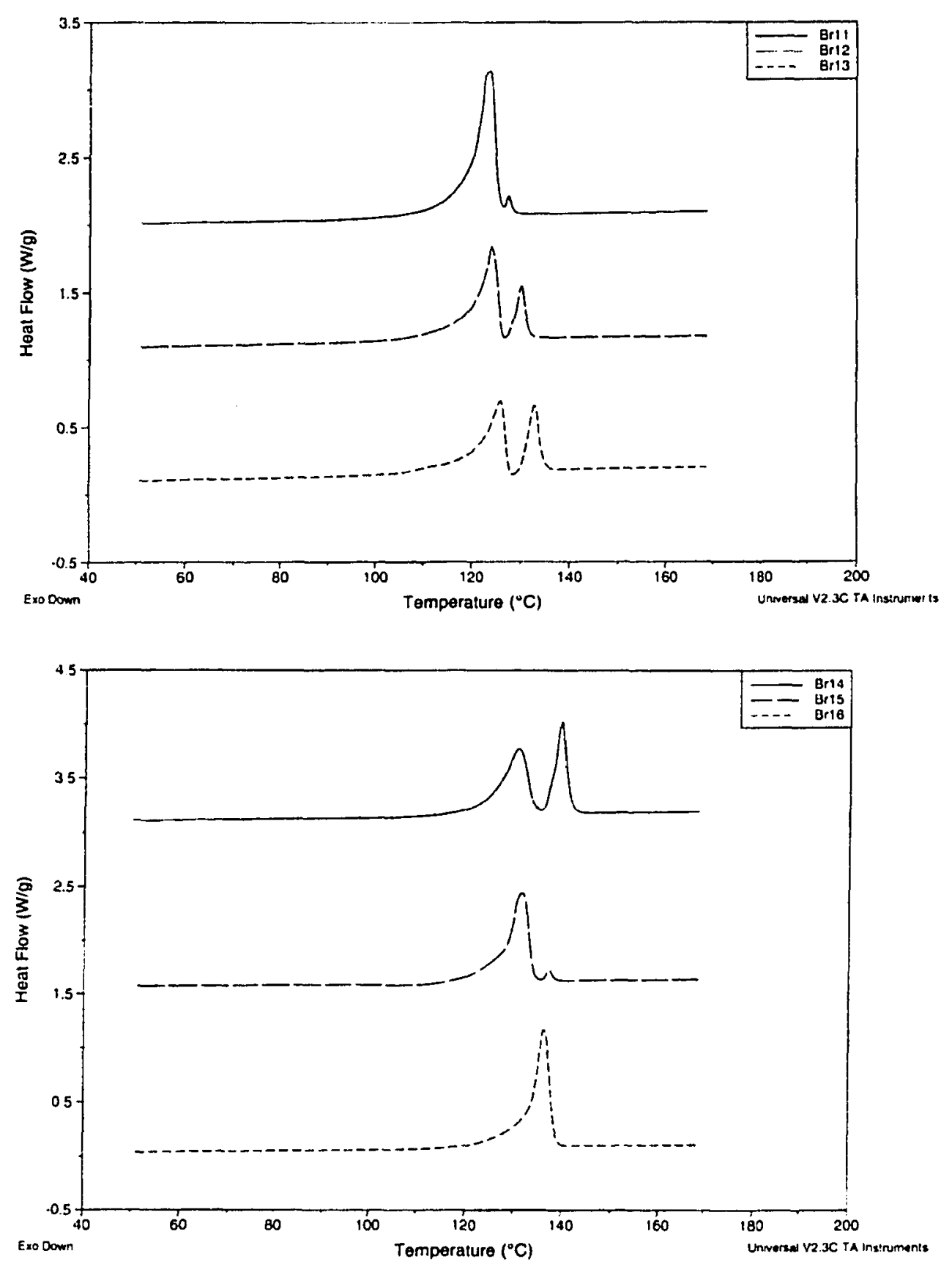

Figure XIII-14: DSC traces for TATM inclusion compounds with the six largest sized1-bromoalkane guests $(\mathrm{Br} 10-\mathrm{Br} 16)$. Note the secondary endotherm at a temperature slightly higher than that of the primary melting temperature for guests $\mathrm{Brll}-\mathrm{Brl}$. All of the DSC traces with smaller guests $(\mathrm{Br} 2-\mathrm{Br} 10)$, although not shown (in addition to $\mathrm{Br} 16)$ show only a single endotherm. 


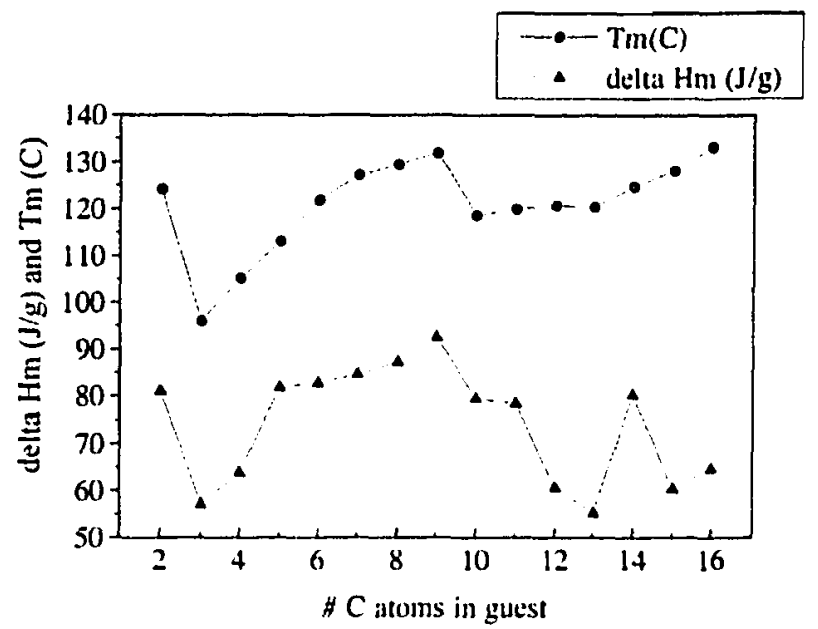

Figure XIII-15: A plot of the primary endotherm DSC melting temperature $\left({ }^{\circ} \mathrm{C}\right.$ - circles) and enthalpy of decomposition $\left(\mathrm{J} \mathrm{g}^{-1}\right.$ - triangles) as a function of the number of carbon atoms of the guest, for TATM inclusion compounds with the 1 -bromoalkane guests of the present study.

Table XIII-6: DSC melting temperature $\left( \pm 0.1^{\circ} \mathrm{C}\right), \Delta_{\text {fus }} H\left( \pm 1 \mathrm{~J} \mathrm{~g}^{-1}\right.$ and $\pm 1 \mathrm{~kJ} \mathrm{~mol}^{-1}$ TATM), and $\Delta_{\text {tus }} S\left( \pm 2 \mathrm{~J} \mathrm{~mol}^{-1} \mathrm{~K}^{-1}\right)$ for TATM inclusion compounds with the fifteen 1-bromoalkane guests. Refer to Figure XIII-14 for the DSC traces.

\begin{tabular}{|c|c|c|c|c|c|c|c|c|}
\hline \multicolumn{5}{|c|}{ Primary endotherm } & \multicolumn{3}{|c|}{ Secondary endotherm } & \multirow[t]{2}{*}{ Visual $\mathrm{MP}\left( \pm 1^{\circ} \mathrm{C}\right)$} \\
\hline$\overline{\text { Guest }}$ & $\begin{array}{c}T_{1 \mathrm{~m}} \\
\left({ }^{\circ} \mathrm{C}\right)\end{array}$ & $\left(\mathrm{Jg}^{-1}\right.$ & $\begin{array}{l}\Delta_{\text {rus }} H \\
)\left(\mathrm{kJmol}^{-1}\right)\end{array}$ & $\begin{array}{c}\Delta_{\text {fus }} S \\
\left(\mathrm{Jmol}^{-1} \mathrm{~K}^{-1}\right)\end{array}$ & $\begin{array}{l}T_{11} \\
\left({ }^{\circ} \mathrm{C}\right)\end{array}$ & $\left(\mathrm{Jg} \mathrm{g}^{-1}\right.$ & $\begin{array}{l}\mathrm{f} f \mathrm{~s} \\
\mathrm{~L}\left(\mathrm{~kJ} \mathrm{~mol}^{-1}\right)\end{array}$ & \\
\hline $\mathrm{Br} 2$ & 124.2 & 81 & 31 & 79 & & & & $105-107$ \\
\hline $\mathrm{Br} 3$ & 95.9 & 57 & 22 & 60 & & & & $98-99$ \\
\hline Br4 & 105.2 & 64 & 25 & 66 & & & & $101 \cdot 103$ \\
\hline $\mathrm{Br} 5$ & 113.1 & 82 & 32 & 82 & & & & $108-110$ \\
\hline Br6 & 121.9 & 83 & 32 & 81 & & & & $121-122$ \\
\hline $\mathrm{Br} 7$ & 127.2 & 85 & 33 & 82 & & & & $125-126$ \\
\hline $\mathrm{Br} 8$ & 129.5 & 87 & 34 & 84 & & & & $130-131$ \\
\hline $\mathrm{Br} 9$ & 131.9 & 92 & 36 & 89 & & & & $124-125$ \\
\hline $\mathrm{Br} 10$ & 118.6 & 79 & 31 & 79 & & & & $121-122$ \\
\hline $\mathrm{Brll}$ & 119.1 & 78 & 30 & 78 & 126.9 & 1.0 & 0.39 & $121-122$ \\
\hline $\mathrm{Br} 12$ & 120.7 & 61 & 24 & 60 & 128.1 & 10 & 4.0 & $120-121$ \\
\hline $\mathrm{Brl} 3$ & 120.4 & 55 & 21 & 55 & 130.1 & 17 & 6.5 & $119-120$ \\
\hline $\mathrm{Brl} 4$ & 124.8 & 80 & 31 & 78 & 137.2 & 28 & 11 & $140-141$ \\
\hline $\mathrm{Br} 15$ & 128.2 & 60 & 23 & 58 & 136.1 & 1.6 & 0.62 & $127-130$ \\
\hline Bri6 & 133.3 & 65 & 25 & 62 & & & & $132-134$ \\
\hline
\end{tabular}

An exception to the general trend of Figure XIII-15 occurs with Br2/TATM. The boiling point of pure 1 -bromoethane is only about $40^{\circ} \mathrm{C}$, much lower than the melting point of its TATM inclusion compound $\left(124.23^{\circ} \mathrm{C}\right)$. Thus, as soon as the $\mathrm{Br} 2$ guest is thermally liberated from its TATM host, it may evaporate, at least partially (since the inclusion compound was hermetically sealed), into the gaseous phase, absorbing heat in the process. This may explain why the enthalpy of decomposition of $\mathrm{Br} 2$ /TATM is quite high $\left(80.98 \mathrm{~J} \mathrm{~g}^{-1}\right)$, at least when compared to $\operatorname{Br} 3 / \operatorname{TATM}\left(57.13 \mathrm{~J} \mathrm{~g}^{-1}\right)$. However, why the melting point of $\mathrm{Br} 2 / \mathrm{TATM}$ is so high may be harder to explain. The shape of the melting endotherm for $\mathrm{Br} 2$ /TATM (see Figure XIII-14) is very broad, with the melting 
starting at temperatures as low as $100^{\circ} \mathrm{C}$, where the deviation of the heat flow from the baseline starts. On the other hand, the DSC endotherms for TATM inclusion compounds with larger 1-bromoalkane guests are generally much sharper in appearance (melting occurs over a smaller temperature interval).

As mentioned above, these observations may be partially explained by the high volatility of the pure 1-bromoethane guest. In a previous study (see chapter VIII) on TATM inclusion compounds with alkyl halide guest families, we found that highly volatile guests such as dichloromethane $\left(\mathrm{bp} 40^{\circ} \mathrm{C}\right.$ ) and 2-chlorobutane (bp $\left.68^{\circ} \mathrm{C}\right)$ form TATM inclusion compounds that have a broad melting point range when visually inspected in a hot-stage melting point apparatus. For these compounds, melting starts (first visual appearance of liquid droplets) at temperatures considerably lower than the end of melting (last appearance of solid). This may be why Br2/TATM of the present study shows anomalous melting behaviour when compared to TATM inclusion compounds with larger 1-bromoalkane guests.

Nevertheless, these observations suggest that (at least for the smaller 1-bromoalkane guests) TATM may form more strongly bound inclusion compounds with guests that have longer hydrocarbon chains, in that more extensive dispersive forces between host and guest are promoted by longer and longer hydrocarbon chains of the guest. Indeed, for the seven inclusion compounds from $\mathrm{Br} 3$ /TATM to Br9/TATM, both the melting temperature and the enthalpy of decomposition steadily increase with the size of the guest, putting this conclusion beyond a reasonable doubt. This result may be compared with our previous conclusions concerning TATM inclusion compounds with alkyl halide guest families (see chapter VIII), where we chose an opposite approach in our survey, namely an invariant hydrocarbon backbone with varying halogen substituent(s). In this case, we found from DSC that TATM forms inclusion compounds with greater $\Delta_{\text {fus }} H$ with guests in the order $\mathrm{F}>\mathrm{Cl}>\mathrm{Br}>\mathrm{I}$. The differences in interactions between host and guest here are primarily dipole-dipole (polar) in nature as one systematically changes the halogen substituent, which suggests that the increasing polar forces of the previous study and the increasing dispersive interactions of the present study may both give rise to increasing interactions between host and guest. 
A further conclusion that may be gleaned from Figure XIII-15 is that, based on their enthalpies of decomposition, the seven isostructural inclusion compounds from Br10/TATM to Br16/TATM have lower thermal stabilities than those of the smaller guests $\mathrm{Br} 5$ /TATM to $\mathrm{Br}$ /TATM. Indeed, the TATM inclusion compounds with $\mathrm{Br} 12$, $\mathrm{Br} 13, \mathrm{Br} 15$ and $\mathrm{Brl} 6$ all have very low decomposition enthalpies (see Figure XIII-15). Despite the large size of these guests, their TATM H:G molar ratios are generally near unity (see Figure XIII-4). As mentioned above, they likely are not true inclusion compounds in the sense of host including guest, rather they are simple solvates in the sense of just co-existing in the same lattice. This may explain why their $\Delta_{\text {fus }} H$ values are quite low. Another reason for this unexpected behaviour may be due to the influence of the complicated melting behaviour on the thermal properties of TATM inclusion compounds with these larger guests. For these inclusion compounds, we added the integrals of both peaks to obtain the total enthalpy of decomposition, however the sum of the two enthalpies is still quite low, as can be seen in Figure XIII-15.

Finally, for the smaller guests $\mathrm{Br}$ /TATM to Br9/TATM, one observes a direct relationship between the melting temperature and the enthalpy of decomposition. A plot of $\Delta_{\text {fus }} H$ vs. $T_{\mathfrak{m}}$ appears in Figure XIII-16. The TATM inclusion compounds with a higher decomposition enthalpy have a higher melting temperature, while those with a lower decomposition enthalpy have a lower melting temperature. The TATM inclusion compounds with larger guests fail to show a similar correlation, perhaps because of the influence of the secondary endotherm for these samples.

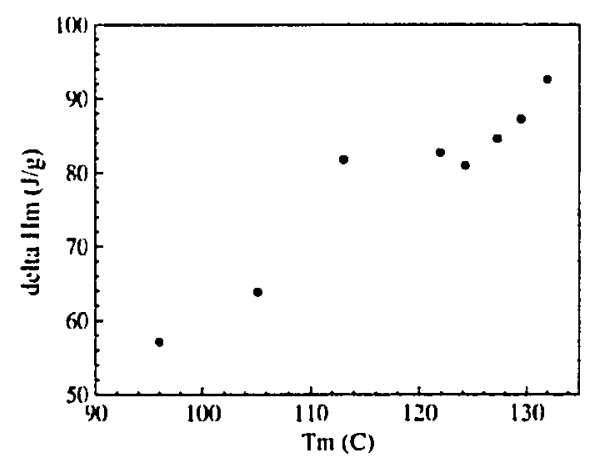

Figure XIII-16: A plot of decomposition enthalpy $\left(\mathrm{J} \mathrm{g}^{-1}\right)$ vs melting temperature for TATM inclusion compounds with the smaller I-bromoalkane guests $(\mathrm{Br} 2-\mathrm{Br} 9)$. Note the direct relationship between the two variables.

As far as the secondary DSC melting endotherm is concerned (see Figure XIII-17) for $\mathrm{Brl1/TATM}$ to $\mathrm{Br}$ 5/TATM, one sees a general increase in intensity and temperature with increasing size of guest, and then a drop-off on going from Br14/TATM to 
Br15/TATM (see Table XIII-6). One also observes from this figure a direct relationship between the secondary enthalpy melting temperature and its corresponding enthalpy. We are unable to definitely explain the nature of this secondary endotherm, but the fact that it only occurs for TATM inclusion compounds with large guests that have near 1:1 H:G molar ratios suggest that it may arise from the co-solvate nature of these five materials, in that they are not "true" inclusion compounds. As mentioned above, the larger 1bromoalkane guests form TATM inclusion compounds that may consist of amorphous regions. The primary endotherms (at slightly lower temperature) may be the melting of the amorphous parts of the sample, while the secondary endotherms (at slightly higher temperature) may be the melting of the crystalline regions. An alternative explanation may be related to possible host-guest associations in the liquid melt, which may be significant only for the larger 1-bromoalkane guests (most significant for $\mathrm{Br} 14$ ). These interactions may be broken up at a temperature slightly above that of the true melting point (i.e. slightly after their formation in the DSC pan), and give rise to the secondary endotherm in the process. Another possibility, as suggested by the visual melting points, is that the lower temperature endotherm may correspond to the decomposition of the solid inclusion compound, while the higher temperature endotherm may arise from the destruction of a mesophase that may form between the guest molecules after their liberation from the host lattice. These 1-bromoalkane guests have a polar head $(\mathrm{Br})$ and a long hydrocarbon tail, and may associate in the molten phase. The DSC data suggest that 1-bromotetradecane $(\mathrm{Brl4})$ may be the optimal size for mesophase aggregation in the liquid melt. Other possibilities for this behaviour include a two-step melting process (pre-melting) or a solid-solid phase transition that occurs slightly below the actual melting temperature. An example of the latter is shown in Figure XI-2l for n-alkanes enclathrated in graphite.

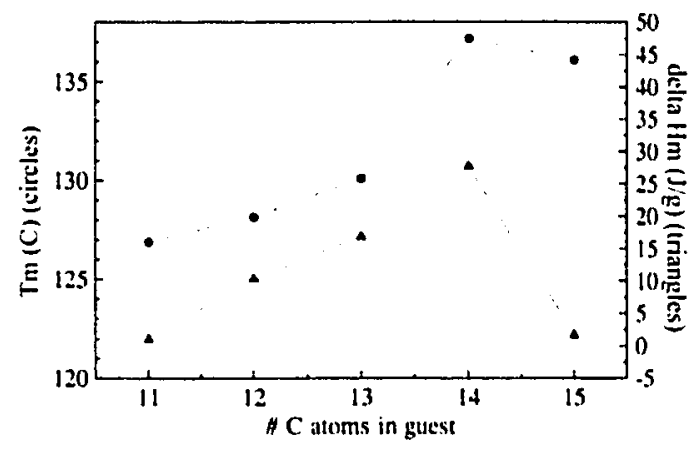

Figure XIII-17: A plot of the secondary endotherm DSC melting temperature $\left({ }^{\circ} \mathrm{C}\right.$ - circles) and enthalpy of decomposition $\left(\mathrm{J} \mathrm{g}^{-1}\right.$ - triangles) as a function of the number of carbon atoms of the guest, for TATM inclusion compounds with the 1-bromoalkane guests $(\mathrm{Br} 11-\mathrm{Br} 15)$ of the present study. 
To investigate this matter further, we measured visual melting points of the 1bromoalkane / TATM inclusion compounds using a hot-stage melting point apparatus. These melting points are listed in Table XIII-6 above, under the heading "Visual MP". They are tabulated as a range, the lower temperature corresponding to the first appearance of liquid and the higher temperature corresponding to the last appearance of solid. All three sets of melting temperatures are plotted in Figure XIII-18. One can see that the visual melting points (circles) generally correspond very well with the primary DSC melting point endotherms (triangles), but are significantly lower than those corresponding to the secondary DSC melting point endotherms (squares), for the guests $\mathrm{Br} 11-\mathrm{Br} 15$. This suggests that for these five TATM inclusion compounds, the visual melting point corresponds to the lower temperature DSC endotherm. An exception to this trend is the melting behaviour of $\mathrm{Br} 14$ / TATM, whose visual melting point is significantly larger than that of the other large guests. This exceptional behaviour is also reflected for this inclusion compound in its DSC enthalpy of decomposition (see Figure XIII-15) and its polycrystalline density (see Figure XIII-19 below), both of which are significantly greater for Brl4 / TATM than for the other 1-bromoalkane / TATM inclusion compounds of similar guest size.

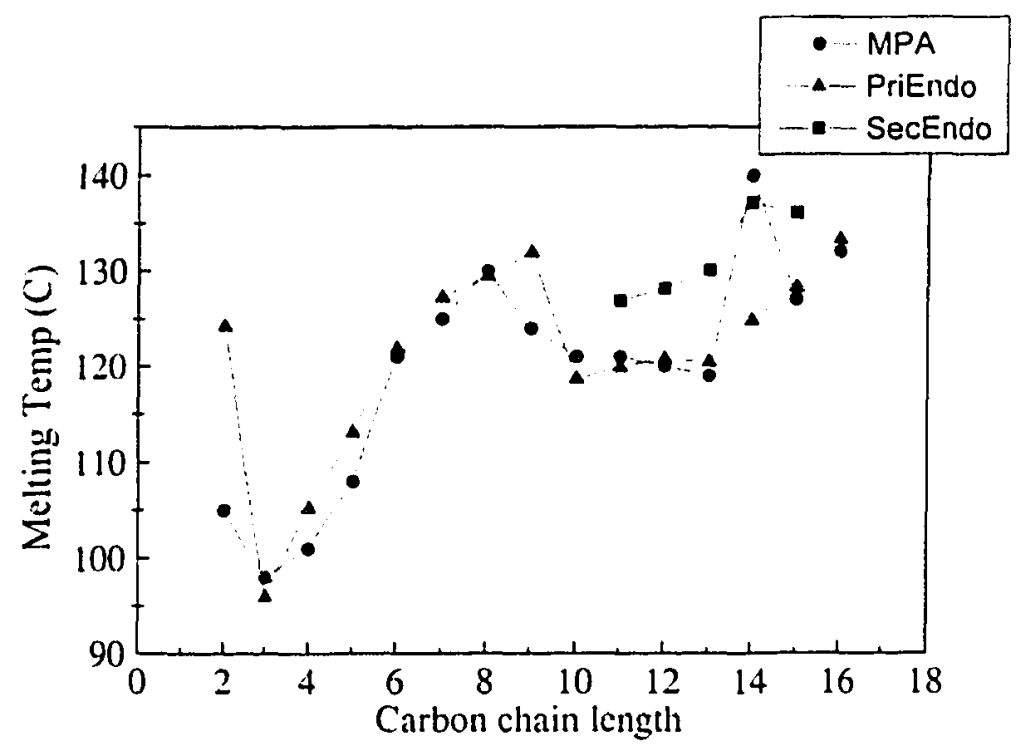

Figure XIII-18: A plot of melting point data for TATM inclusion compounds with 1bromoalkane guests, determined using DSC and a hot-stage visual melting point apparatus. Note the general correlation of visual melting points with the primary DSC endotherms, while the secondary DSC endotherms occur at significantly higher temperatures (except for Br14/TATM). The raw data is given in Table XIII-6. 


\section{(E) Polycrystalline densities (gas pycnometer)}

The experimental bulk densities for TATM inclusion compounds with the fifteen 1bromoalkane guests in Figure XIII-1 are plotted in Figure XIII-19, as a function of the number of carbon atoms in the guest molecule $(\mathrm{C} 2-\mathrm{C} 16)$. For the smaller sized guests $(\mathrm{Br} 2$ to $\mathrm{Br} 11)$, one generally observes a gradual decrease in density with an increasing size of guest (with the exception of $\mathrm{Br} 9 / \mathrm{TATM}$ ). Perhaps TATM is more easily able to closely pack with smaller guests, which would give rise to a higher density. Indeed, for the very large guests $(\mathrm{Br} / 2-\mathrm{Br} 16)$, with the exception of $\mathrm{Br} 14$ /TATM, the polycrystalline density is much lower than that for TATM inclusion compounds with the smaller guests.

In comparing Figures XIII-4 and XIII-19, one may notice that for the smaller size guests $(\mathrm{Br} 2-\mathrm{Brll})$, as the $\mathrm{H}: \mathrm{G}$ molar ratios are steadily increasing (Figure XIII-4), the polycrystalline density is steadily decreasing (Figure XIII-19). Perhaps when there are more and more host molecules in the unit cell, the crystal packing efficiency is gradually decreasing, resulting in a lowering density. On the other hand, for the larger size guests (Brl2 - Br16), the $\mathrm{H}: \mathrm{G}$ molar ratios decreases dramatically $(\mathrm{H}: \mathrm{G}=1: 1$ to $1.5: 1$ ), accompanied by a correspondingly dramatic decrease in polycrystalline density. As was mentioned above, these larger guests may not be true "inclusion compounds" in the sense of host including guest, but rather simple co-solvates. This would perhaps lead to the decrease in density, as the strength of intermolecular interactions are likely not a strong as for "true" TATM inclusion compounds with the smaller guests. Indeed, we made numerous attempts to grow suitable quality single crystals of TATM with these larger guests $\mathrm{Br}\left(\mathrm{CH}_{2}\right)_{n} \mathrm{CH}_{3}, n \geq 9$, but met with failure each time. If the host - guest interactions are very weak, one might expect that it would be difficult for these two compounds to grow into single crystals of suitable size and quality for conventional single crystal X-ray diffraction studies. Furthermore, if some of these larger guests form incommensurate TATM inclusion compounds, there may be a significant amount of empty space in the TATM channel between guest sites, which may give another reason why the larger 1-bromoalkane guests form TATM inclusion compounds with a significantly lower density. 


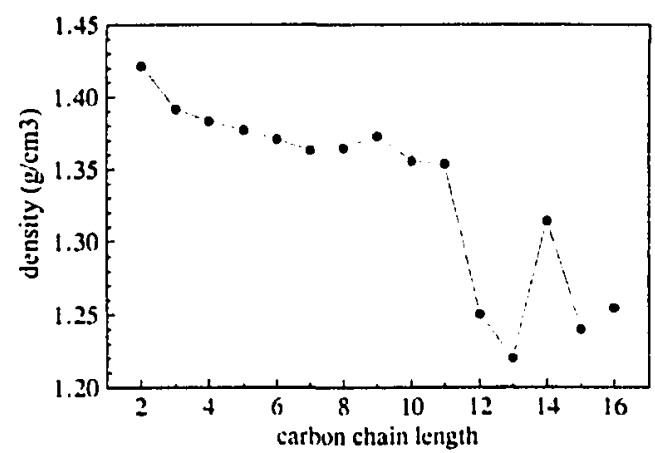

Figure XIII-19: Polycrystalline densities of TATM inclusion compounds with the l-bromoalkane guests pictured in Figure XIII-1, as measured with a gas pycnometer.

It is also interesting to compare the bulk densities with the DSC data (Figure XIII-15) for these 1-bromoalkane/TATM inclusion compounds. In Figure XIII-15, for the guests $\mathrm{Br} 3$ $-\mathrm{Br} 9$, both the melting point and enthalpy of decomposition are steadily increasing with size of guest. This is accompanied, perhaps unexpectedly, by a steady decrease in the bulk density (Figure XIII-19). This suggests that, for these "true" inclusion compounds, lower densities are associated with greater heat of fusion. On the other hand, for the "cosolvate" TATM inclusion compounds with larger guests $(\mathrm{Br} 12-\mathrm{Br} 16)$, a decrease in density is associated with a decrease in heat of fusion, the opposite case compared to the smaller guests. Indeed, as an example, Br14/TATM has a much higher density compared to $\mathrm{Br} 13$ /TATM or Br15/TATM (see Figure XIII-19), and, as perhaps expected, has a much higher enthalpy of decomposition than either Br13/TATM or Br15/TATM (see Figure XIII-15 - triangles). Why this occurs for Br14/TATM and not for TATM inclusion compounds with any of the other large 1-bromoalkane guests may be beyond our ability to explain.

We wish to make a point here that may go a long way towards explaining the physicochemical properties of some of these TATM inclusion compounds. Some of these inclusion compounds (especially with larger guests) may be incommensurate. This means that the repeat distance of one guest molecule (in $\AA$ ) does not form a small number fraction or integer with the repeat distance of the host channel. If this was the case, then all guests would experience a broad distribution of roughly equivalent and weakly (guesthost) interacting environments, which along with other factors, would help to explain the following physicochemical properties of the present family of compounds: 
(1) Why we see a significant drop off in $\mathrm{H}: \mathrm{G}$ molar ratios for larger guests, while those of the commensurate $\alpha, \omega$-dichloroakanes do not drop off with larger guests.

(2) Why there is only one host molecule in the asymmetric unit for the largest size 1bromoalkanes in TATM.

(3) Why the seven PXRD patterns of the Br10/TATM - B16/TATM inclusions have significantly broader peaks (when compared to the smaller guests), and the PXRD patterns of the former group (larger guests) have many fewer reflections than those of the latter group (smaller guests).

(4) Why all seven of the TATM inclusion compounds with larger guests (Br10-Br16) are isostructural, and why the commensurate $\alpha$,(1)-dichloroalkane inclusions have almost no isostructuralism

(5) Why even the largest size guests are dynamically reorienting in the TATM lattice at room temperature, and why the larger $\alpha, \omega$-dichloroalkane guests are static. It is well accepted $[8,9]$ that incommensurate inclusion compounds generally afford the enclathrated guest a significant degree of dynamic freedom.

(6) A significant drop off in both $T_{\mathrm{m}}$ and $\Delta_{\text {fus }} H$ on going from Br9/TATM to BrI0/TATM

(7) The presence of the secondary melting endotherm for all guests from $\mathrm{Br} 11 / \mathrm{TATM}$ to $\mathrm{Br} 15 / \mathrm{TATM}$

(8) Why the enthalpy of decomposition $\left(\Delta_{\text {lus }} H\right)$ for TATM inclusions with the larger guests is generally quite low (when compared to smaller guests)

(9) The generally significant decrease in polycrystalline density for TATM inclusions with the larger 1-bromoalkane guests.

Indeed, we were able to make single crystals with the smaller guests (several of the $\mathrm{Br} 2-$ $\mathrm{Br} 9$ inclusions) that gave good quality data sets. On the other hand, crystals of the larger TATM inclusions ( $\mathrm{BrlO}-\mathrm{Brl6}$ ), although visually acceptable (clear and of sufficient size), did not diffract to high angles, so an attempt to collect their full data sets was not made. The most likely reason for this result is that the larger guests form incommensurate inclusions with TATM. 


\section{DISCUSSION}

The majority of urea inclusion compounds [10] possess the conventional urea structure as described in the introduction of the thesis. The guest molecules are densely packed within the host tunnels, and generally exhibit sufficient positional ordering to allow an average three-dimensional lattice to be defined and measured, despite substantial dynamic disorder of the guest molecules [11,12]. An important structural issue is the relationship between the periodicities $c_{\mathrm{h}}$ and $c_{\mathrm{g}}$ of the host and guest substructures along the tunnel axis, respectively [13]. These inclusion compounds may be denoted as incommensurate if there are no sufficiently small integers $p$ and $q$ such that $p c_{\mathrm{h}} \sim q c_{\mathrm{g}}$, and commensurate if sufficiently small $p$ and $q$ can be found for which $p c_{h} \sim q c_{\mathrm{g}}$. If the host and guest are truly incommensurate, then each guest molecule within a given tunnel is, in principle, in a slightly different environment with respect to the host substructure [14]. On the other hand, if the host and guest are commensurate, the guest molecules sample a much narrower range of host environments [15]. In general, there is much more motional freedom of the guest molecules in an incommensurate urea inclusion compound, as compared to commensurate urea inclusion compounds [16-18]. Within the incommensurate systems, the guests exist in a multitude of roughly equivalent sites [19]. As was evidenced in this chapter, incommensurate host-guest systems are generally characterized by broader peaks in the PXRD patterns, extensive guest dynamics and lower $\Delta_{\text {tus }} H$ when compared to commensurate systems with the same host.

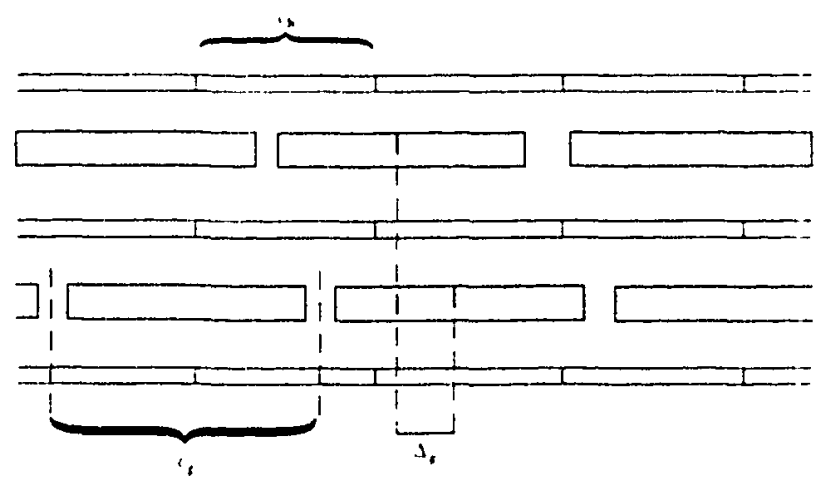

Figure XIII-20: Schematic twodimensional representation of a urea inclusion compound, indicating the definitions of $c_{\mathrm{g}}, c_{\mathrm{h}}$ and $\Delta_{\mathrm{g}}[10\}$.

Urea inclusion compounds with $\alpha, \omega$-dibromoalkane $\left\{\mathrm{Br}\left(\mathrm{CH}_{2}\right)_{n} \mathrm{Br}\right\}$ guests $(n=7$ to 10$)$ display an incommensurate three dimensional ordering (intra- and interchannel) at room temperature, in which the offset of guests in adjacent tunnels $\left(\Delta_{\mathrm{g}}\right)$ is approximately equal to $c_{\mathrm{g}} / 3$, as depicted in Figure XIII-20 [20]. A larger homologous guest $\left\{\mathrm{Br}\left(\mathrm{CH}_{2}\right)_{12} \mathrm{Br}\right\}$ 
shows only one dimensional (intrachannel) ordering at room temperature [21]. The three dimensional (interchannel) ordering is lost for this larger guest.

The ordering pattern observed for $n=7$ to 10 guests is unusual in that the offset $\left(\Delta_{\mathrm{g}}\right)$ between guests in adjacent tunnels depends on the length of the guest molecule. This observation strongly suggests that the offset is controlled by interchannel guestguest interactions, being mediated by intervening urea molecules. Although bromine is considered to have approximately the same size as a methyl group, its interaction with the channel wall is almost certainly much stronger than that of a methyl group. Substantial distortion of the urea host structure in crystals of 1,6-dibromohexane / urea suggest that the bromine atom can interact significantly with the channel wall [22], so it is perhaps not surprising that the terminal bromines may have a strong influence on the interchannel guest-guest interactions of these commensurate inclusion compounds. On the other hand, TATM is more flexible, so perhaps interchannel guest-guest interactions are stronger for TATM than for urea.

It is worth noting that urea inclusion compounds with $\alpha, \omega$-diiodoalkane guests exhibit the same type of interchannel ordering $\left(\Delta_{\mathrm{g}}=c_{\mathrm{g}} / 3\right)$ as that of the $\alpha, \omega$ dibromoalkane guests [201. In contrast, 1,10-dichlorodecane / urea exhibits no intra- or interchannel ordering of the guests [23].

On the other hand, shorter chain $\alpha, \omega$-dihaloalkane complexes $(n \leq 6)$ with urea have commensurate structures, and near integral host:guest molar ratios $[24,25]$. (This shows agreement with TATM/1-bromoalkane systems, in that shorter guest alkyl chains lead to commensurate inclusions, while longer guest alkyl chains $(n \geq 10)$ lead to incommensurate complexes.) Photographic XRD methods were used to solve the structure of 1,4-dichlorobutane / urea, for which $3 c_{h} \sim 4 c_{\mathrm{g}}$ [26]. Urea inclusion compounds with 1,6-dichlorohexane, 1-bromo-6-chlorohexane and 1,6-dibromohexane were all identified as commensurate channel-type inclusion compounds with $\sim 6: 1$ host:guest molar ratios $[22,27]$. All three complexes belong to the monoclinic $\mathrm{P} 2 \mathrm{l} / \mathrm{n}$ space group, and all three guests contain gauche $\mathrm{C}-\mathrm{Cl}$ and / or $\mathrm{C}-\mathrm{Br}$ bonds (see Table XIII-7). By noting that an orthorhombic description of a guest-free hypothetical hexagonal urea channel structure has $a=8.22 \AA, b=14.24 \AA$ and $c=11.01 \AA$ (see Figure XIII-21), one may see that all three guests contract the cross-sectional area of the channel 
(the $a b$ product) [22]. On the other hand, all three guests lengthen the repeat distance of the host channel $(c)$. A careful examination of the structures (Figure XIII-22) and lattice parameters (Table XIII-7) reveal that all three structures are nearly isomorphous. (This is similar to TATM, in that guest substitution of chlorine for bromine generally has little effect on the resulting TATM structural type.) In all three structures, the planes defined by the methylene chains of the guest molecules lie $22^{\circ}-24^{\circ}$ away from the horizontal [001] plane, and the terminal C-X bonds (which are gauche to the rest of the guest chain), point toward the vertices of the channels [28].

Table XIII-7: Crystal structural data for commensurate 1,6-dihaloalkane/urea inclusion compounds [22].

\begin{tabular}{llll}
\hline Guest & 1,6-dichlorohexane & 1-bromo-6-chlorohexane & 1,6-dibromohexane \\
\hline$T(\mathrm{~K})$ & 99 & 102 & 108 \\
space group & $\mathrm{P} 2_{1} / \mathrm{n}$ & $\mathrm{P} 2{ }_{1} / \mathrm{n}$ & $\mathrm{P} 2_{1} / \mathrm{n}$ \\
& & & \\
$a(\AA)$ & $8.509(9)$ & $8.545(1)$ & $8.560(8)$ \\
$b(\AA)$ & $10.860(7)$ & $10.875(1)$ & $10.889(6)$ \\
$c(\AA)$ & $13.435(9)$ & $13.418(2)$ & $13.381(7)$ \\
$\beta\left({ }^{\circ}\right)$ & $92.02(7)$ & $92.44(2)$ & $92.82(6)$ \\
$V\left(\AA^{3}\right)$ & $1240(1)$ & $1245.3(3)$ & $1245(1)$ \\
Comments & 1) gauche $\mathrm{C}-\mathrm{Cl}$ bonds. & 1) gauche C-Cl and C-Br bonds & 1) gauche C-Br bonds. \\
& 2) guests not in contact & 2) guests not in contact & 2) guests not in contact \\
\hline
\end{tabular}
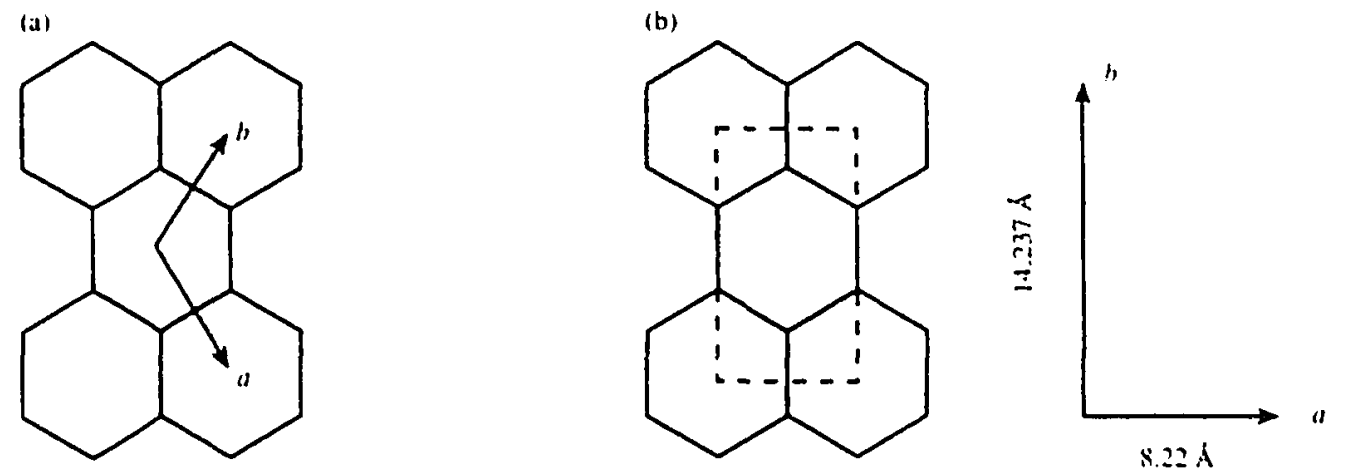

Figure XIII-21: Schematic illustration of (a) hexagonal and (b) orthohexagonal unit cells of undistorted urea inclusion compounds. Deviation from the orthohexagonal values given in (b) describe the distortion of the channel caused by the guest molecules [10] 


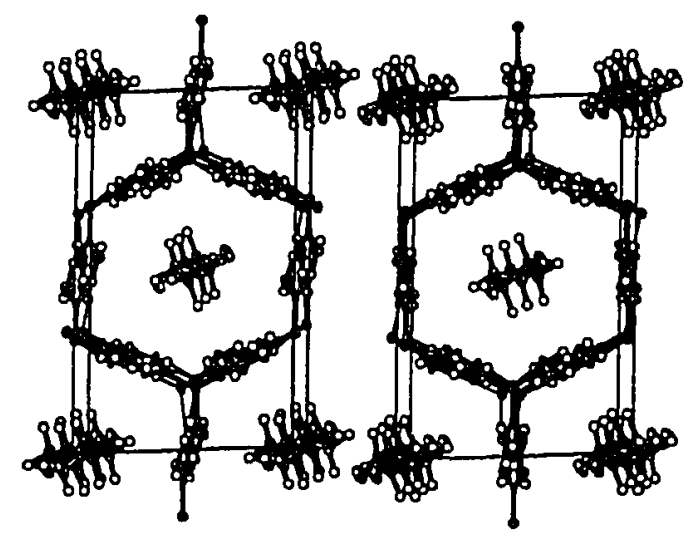

Figure XIII-22: Stereoview of the $\mathrm{Br}\left(\mathrm{CH}_{2}\right)_{6} \mathrm{Br}$ - urea complex down the channel axis. The structures of $\mathrm{Br}\left(\mathrm{CH}_{2}\right)_{6} \mathrm{Cl}$ - urea and $\mathrm{Cl}\left(\mathrm{CH}_{2}\right)_{6} \mathrm{Cl}$ urea are nearly isostructural with that of $\mathrm{Br}\left(\mathrm{CH}_{2}\right)_{6} \mathrm{Br}$ - urea [22]

As mentioned above, all three guests exist in partly unstaggered conformations. For $\mathrm{Br}\left(\mathrm{CH}_{2}\right)_{6} \mathrm{Br}$, the $c_{\mathrm{g}}$ periodicity may be calculated from the incommensurate urea complexes with larger homologous guests (which are generally fully staggered). This extrapolation gives a predicted value for $c_{\mathrm{g}}$ of $12.8 \AA$ for a fully staggered $\mathrm{Br}\left(\mathrm{CH}_{2}\right)_{6} \mathrm{Br}$ guest in urea (see Figure XIII-23) [22]. From this plot, a shortening of the guest by $1.8 \AA$ (from $12.8 \AA$ to $11.0 \AA$ ) may arise primarily as a means by which it can fit into the confines of a single repeat unit of the host substructure $[\mathbf{2 2 , 2 3}$, by a change of the endof-chain conformation from trans to gauche. (The flexibility of the TATM lattice, when compared to urea, may make distortion of the guest unnecessary in this case.) Conformational properties of $\mathrm{Br}\left(\mathrm{CH}_{2}\right)_{6} \mathrm{Br}$ in urea, as measured by Raman and IR spectroscopy [29] reveal that the end groups in the $\mathrm{Br}\left(\mathrm{CH}_{2}\right)_{6} \mathrm{Br}$ guest are gauche, in accord with the results from X-ray diffraction. This is in contrast to the incommensurate urea inclusion compounds with $\mathrm{Br}\left(\mathrm{CH}_{2}\right)_{11} \mathrm{Br}$ guests $(n=7-11)$, where most all end groups are in a trans conformation $[30 \mid$.

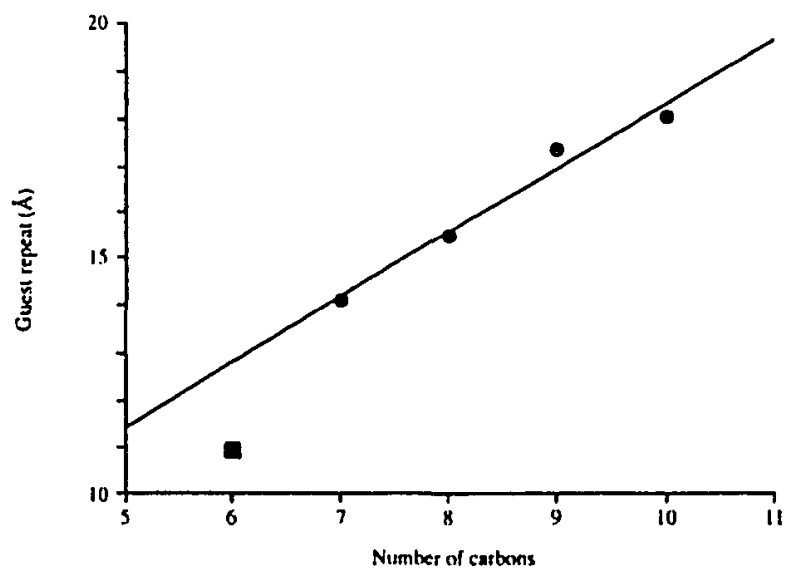

Figure XIII-23: Guest repeat $\left(c_{\mathrm{g}}\right)$ vs. carbon chain length for $\alpha, \omega-$ dibromoalkanes in urea. For $n=6$ $\left\{\mathrm{Br}\left(\mathrm{CH}_{2}\right)_{6} \mathrm{Br}\right\}$, the guest molecules must shrink from their extended conformations by $\sim 1.8 \AA$ to achieve a commensurate relationship with the host $[\mathbf{1 0}]$ 
During the formation of a urea inclusion compound containing an unsymmetric guest $\mathrm{X}\left(\mathrm{CH}_{2}\right)_{\mathrm{n}} \mathrm{Y}$, the guest molecule may be included with either its "head" or "tail" pointing into the bulk of the crystal [31]. As depicted in Figure XIII-24, this gives rise to three different local arrangements of a pair of adjacent end groups from two neighboring guest molecules in one channel: $X \cdots X(\mathrm{HH}), \mathrm{Y} \cdots \mathrm{Y}(\mathrm{TT})$ or $\mathrm{X} \cdots \mathrm{Y}(\mathrm{HT})$. For a random distribution of guest orientations, the ratio $(\mathrm{HH}+\mathrm{TT}) / 2(\mathrm{HT})$ should be close to 1 . Therefore, deviations from unity reveal the nature of the molecular recognition process during crystal formation.

\section{$-\mathrm{X}\left(\mathrm{CH}_{2}\right)_{11} \mathrm{Y} \ldots \mathrm{X}\left(\mathrm{CH}_{2}\right)_{11} \mathrm{Y} \ldots \mathrm{Y}^{\prime}\left(\mathrm{CH}_{2}\right)_{11} \mathrm{X} \ldots \mathrm{X}\left(\mathrm{CH}_{2}\right)_{11} \mathrm{Y}^{\prime} \ldots \mathrm{Y}\left(\mathrm{CH}_{2}\right)_{11} \mathrm{X}-$}

Figure XIII-24: Schematic illustration of the $X \cdots X, X \cdots Y$ and $Y \cdots Y$ end-group interactions for unsymmetric guest molecules of the type $\mathrm{X}\left(\mathrm{CH}_{2}\right)_{\mathbb{n}} \mathrm{Y}$ inside a host tunnel structure [32].

For incommensurate urea inclusion compounds, guest molecules generally pack reproducibly within van der Waals contact of each other, while experiencing a distribution of roughly equivalent host environments [19]. Because the guest molecules undergo dynamic averaging, the space and time averaged structure of a given functional group pair in an incommensurate urea inclusion compound is virtually the same as that for the same functional group pair in another urea inclusion compound containing an analogous guest with a different chain length (i.e. $-\mathrm{CH}_{2}$-), or a guest bearing a different substituent at the other end.

Static and dynamic disorder normally preclude the use of X-ray diffraction to measure the distribution of guest orientations, but the characteristics of these systems make them ideally suited for quantitative studies with high-resolution solid-state NMR [33]. Rapid motions of the guests give rise to sharp spectral lines, so it is possible to use the resonances of the terminal functional groups to identify the HH, TT and HT sites. The high-resolution solid-state NMR spectra of urea complexes with dozens of unsymmetrical guests $\mathrm{X}\left(\mathrm{CH}_{2}\right)_{n} \mathrm{Y}$ show band doubling for terminal and nearest neighbor 
carbons of the guest, but not for internal carbons [34]. The doubling arises from differences in nearest neighbors, and band intensities in carefully measured spectra reflect the distribution of end group interactions. To assign the bands from terminal carbons as HH, TT or HT pairs, one simply measures NMR spectra of the symmetric difunctionalized compounds (i.e. $\mathrm{X}\left(\mathrm{CH}_{2}\right)_{n} \mathrm{X}$ and $\left.\mathrm{Y}\left(\mathrm{CH}_{2}\right)_{n} \mathrm{Y}\right)$ in urea, to give ${ }^{13} \mathrm{C}$ chemical shifts corresponding to the $\mathrm{HH}$ and TT interactions, from which the HT chemical shift can be identified by climination [35|. As far as 1-bromoalkane/TATM inclusions are concerned, the ${ }^{13} \mathrm{C} \mathrm{CP} / \mathrm{MAS}$ NMR is some cases (especially for longer guests) show a broader signal, and in some cases even band doubling, for the signal corresponding to the terminal methyl group. in most cases around 13-14 ppm, as depicted in Figures XIII-9, XIII-10 and XIII-11. This suggests a distribution of end group interactions for some of these 1-bromoalkane/TATM inclusion compounds.

On the basis of ' $H T_{1}$ measurements, early NMR studies of the incommensurate $\mathrm{Br}\left(\mathrm{CH}_{2}\right)_{10} \mathrm{Br} /$ urea- $\mathrm{d}_{4}$ inclusion compound suggested the existence of rapid rotation of the guest molecules, even at low temperatures [36]. At higher temperatures, additional dynamic processes involving segmental motions of the guest were inferred. Dielectric loss spectroscopy experiments [37] of $\mathrm{Br}\left(\mathrm{CH}_{2}\right)_{10} \mathrm{Br} /$ urea-d $\mathrm{d}_{4}$ suggested that the two end groups of the guest molecule undergo independent motions. Deuterium solid-state NMR studies of $\mathrm{Br}\left(\mathrm{CD}_{2}\right)_{10} \mathrm{Br} /$ urea $|16|$ also confirm that there is appreciable molecular motion of the guest molecules in this material. This agrees with the dipolar dephasing studies on the 1-bromoalkane/TATM systems, where even the largest guests were found to be undergoing dynamic reorientation at room temperature (Figure XIII-12)

\section{CONCLUDING REMARKS:}

In solid inclusion compounds with tunnel host structures, the spatial distribution and orientation of the guest molecules is usually highly constrained, allowing different types of guest molecules to be introduced in a geometrically controlled manner within the same host structure. These materials therefore provide a good opportunity for systematic investigations of intermolecular interactions, in which different functional end-groups of neighboring guests can be permitted to interact only in a highly specified manner. Indeed, experimental measurements of the relative numbers of different types of end- 
group interaction between guest molecules in host channel lattices may lead to fundamental information on the relative preferences for interactions between different types of functional groups.

In this contribution, we have employed a wide variety of physical techniques to examine TATM inclusion compounds with the $\mathrm{Br}\left(\mathrm{CH}_{2}\right)_{n} \mathrm{CH}_{3}(n=1$ to 15) 1bromoalkane guest family. The advantage of using as many characterization techniques as possible is that it is sometimes possible to make correlations among the different experiments, perhaps the simplest example being a determination of the host:guest molar ratios using both TGA and ' $\mathrm{H}$ solution NMR. This was important, as many of the present guests crystallize with TATM in non-integral H:G molar ratios, so unequivocal establishment of the $\mathrm{H}: \mathrm{G}$ ratio is critical. Furthermore, another correlation may be made between different guest families, as we did here with a previous study of the $\alpha,(1)-$ dichloroalkane $\left(\mathrm{Cl}\left(\mathrm{CH}_{2}\right)_{n} \mathrm{Cl}: n=1\right.$ to 10$)$ guest family, perhaps the most important being the difference in dynamic behaviour between the two guest families, which we attributed to the difference in their respective structural characteristics.

\section{CHAPTER XIII}

REFERENCES

(1) L. Pang and F. Brisse. Can. J. Chem. 72, 2318 (1994).

(2) W.J. Zimmerschied, R.A. Dinerstein, A.W. Weitkamp and R.F. Marschner. Ind. Eng. Chem. 42, 1300 (1950).

(3) W. Schlenk. Justus Liebigs Ann. Chem. 565, 204 (1949).

(4) W. Schlenk. Justus Liebigs Ann. Chem. 573, 142 (1951).

(5) S.J. Opella and M.H. Frey. J. Am. Chem. Soc. 101, 5854 (1979).

(6) ${ }^{13} \mathrm{C}$ NMR Spectral Data - A "living" COM-microfiche collection of reference material. Wolfgang Bremser, Ludger Ernst and Burghard Franke. VCH Verlagsgesellschaft mbH, D-6940 Weinheim, 1987.

(7) K.D.M. Harris and P. Jonsen. Chem. Phys. Lett. 154, 593 (1989).

(8) H. Serrano-Gonzalez and K.D.M. Harris. J. Mol. Struct. 519, 41 (2000).

(9) F. Guillaume, S.P. Smart, K.D.M. Harris and A.J. Dianoux. J. Phys. Condens. Matter. 2169 (1994).

(10) M.D. Hollingsworth and K.D.M. Harris. Urea, Thiourea and Selenourea. In Comprehensive Supramolecular Chemistry, J.L. Atwood, J.E.D. Davies, D.D. MacNicol and F. Vogtle, Eds. Volume 6, Chapter 7, Pergamon / Elsevier: Oxford, 1996.

(11) J. Schmider and K. Muller. Mol. Cryst. Liq. Cryst. 313, 277 (1998).

(12) H.L. Casal, D.G. Cameron, E.C. Kelusky and A.P. Tulloch. J. Chem. Phys. 81, 4322 (1984).

(13) A.J.O. Rennic and K.D.M. Harris. Proc. R. Soc. A. 430, 615 (1990). 
(14) K.D.M. Harris. J. Solid State Chem. 106, 83 (1993).

(15) A.J.O. Rennie and K.D.M. Harris. J. Chem. Phys. 96, 7117 (1992).

(16) A.E. Aliev, S.P. Smart, I.J. Shannon and K.D.M. Harris. J. Chem. Soc. Faraday Trans. 92(12), 2179 (1996).

(17) E. Gelerinter, Z. Luz, R. Poupko and H. Zimmermann. J. Phys. Chem. 94, 5391 (1990).

(18) A.E. Aliev, S.P. Smart and K.D.M. Harris. J. Mater: Chem. 4(1), 35 (1994).

(19) A.J.O. Rennie and K.D.M. Harris. Chem. Phys. Lett. 188, 1 (1992).

(20) K.D.M. Harris, S.P. Smart and M.D. Hollingsworth. J. Chem. Soc. Faraday Trans. 87(20), 3423 (1991).

(21) Reference 10, p 203.

(22) M.D. Hollingsworth, U. Werner-Zwanziger, M.E. Brown, J.D. Chaney, J.C. Huffman, K.D.M. Harris and S.P. Smart. J. Am. Chem. Soc. 121 (41), 9732 (1999)

(23) S.P. Smart. PhD thesis, University of St. Andrews, 1993.

(24) H.U. Lenne, H.C. Mez and W Schlenk Jr. Justus Liebigs Ann. Chim. 732, 70 (1970).

(25) H.U. Lenne. Z. Krystallogr: 115, 297 (1961).

(26) J. Otto. Acta Cryst. B28, 543 (1972).

(27) L. Elizabe, A. El-Baghdadi, S.P. Smart, F. Guillaume and K.D.M. Harris. J. Chem. Soc. Faraday Trans. 92, 267 (1996).

(28) Reference 10, p 208.

(29) S.P. Smart, A. El-Baghdadi, F. Guillaume and K.D.M. Harris. J. Chem. Soc. Faraday Trans. 90, 1313 (1994).

(30) K.D.M. Harris and J.M. Thomas. J. Chem. Soc. Faraday Trans. 86, 2985 (1990).

(31) Reference 7, p 211.

(32) K.D.M. Harris. Phase Transitions 76(3), 205 (2003).

(33) M.D. Hollingsworth and N. Cyr. Mol. Cryst. Liq. Cryst. 187, 135 (1990).

(34) M.D. Hollingsworth and A.R. Palmer. J. Am. Chem. Soc. 115, 5881 (1993).

(35) A. Nordon, R.K. Harris, L. Yeo and K.D.M. Harris. Chem. Commun. 2045 (1997).

(36) J.D. Bell and R.E. Richards. Trans. Faraday Soc. 65, 2529 (1969).

(37) R.J. Meakins. Trans. Faraday Soc. 51, 953 (1955). 


\title{
CHAPTER XIV
}

\section{Water and tris (5-acetyl-3-thienyl) methane (TATM) assemble into a one-dimensional channel compound.}

$\begin{array}{ll}\text { Abstract } & 350 \\ \text { Introduction } & 351 \\ \text { Experimental } & 352 \\ \text { Results } & 352 \\ \text { References } & 358\end{array}$

\begin{abstract}
In an attempt to produce a crystalline guest-free form of the well-known hydrophobic host tris (5-acetyl-3-thienyl) methane (TATM), a stable crystalline compound was produced in which water assembles with the host molecules into a structure where the water induces long-range order into the TATM host by linking the host acetyl oxygens, resulting in a novel one-dimensional channel structure that contains additional disordered water molecules.
\end{abstract}




\section{INTRODUCTION}

Water transport is a fundamental requirement for all life processes, and its elucidation in biological systems has captured much attention in recent years [1-4]. Two types of pore systems that have been extensively studied are the trans-membrane proteins gramicidin A and the aquaporins, each of which form essentially hydrophobic pore systems capable of varying degrees of specialized functional transport [1-4]. Because of the complexity of these systems, their study is often a difficult and time-consuming process [5]. On the other hand, much may be learned by studying simpler model systems, which may display some degree of related functionality [6,7]. For instance, some work on one-dimensional water channels in simple dipeptides has shown some unusual properties, such as negative thermal expansion related to the ordering of water in the peptide nanotubes [7]. In this work, we show that tris (5-acetyl-3-thienyl) methane (TATM), 1, a well-known host system, is an example of a very simple hydrophobic molecule that assembles with water into a one-dimensional channel system that may also be capable of water transport.

TATM is a much-studied tripodal host molecule $[\mathbf{8 - 2 0}]$ related to the triphenylmethane group of hosts [21]. In an initial study [9], inclusion compounds resulted for every solvent from which the host was recrystallized. TATM guest-host materials show a variety of stoichiometries and structural motifs [12-19], they have also demonstrated some propensity for polymorphism $[17,20]$. Upon removal of the guest by heating, the remaining host material generally becomes an oil or amorphous solid, the latter melting at $\sim 50^{\circ} \mathrm{C}-58^{\circ} \mathrm{C}$. Similarly, vacuum sublimation produces only an amorphous form, and at the time of writing, a crystalline form of the guest-free host has yet to be reported (however, see Chapter XV below). Likely reasons are that the molecules lack the ability to interact with each other via strong directional interactions, as there are only acetyl oxygens and weakly acidic hydrogens. Also, the absence of strongly preferred orientations of the thienyl rings is likely to give a variety of conformational isomers in the liquid or vapor, thus making efficient packing difficult upon solidification. In almost all of the structural studies, the observed host and guest interact weakly through van der Waals forces, so the energy gained by crystallization appears to be significant only when a suitable guest is present to organize the host for efficient packing. During attempts to produce a crystalline guest-free host, we have discovered a novel crystalline 
form of TATM where long-range order is induced by connecting the individual host molecules with strongly directional linkers, namely water molecules. Besides using nonspecific interactions to improve packing, as mentioned above, this represents a second strategy for incorporating long-range order into TATM.

\section{EXPERIMENTAL}

Guest - free amorphous TATM was dissolved in liquid $\mathrm{SO}_{2}$ in a Pyrex tube immersed in a Dewar, which was cooled to $-78^{\circ} \mathrm{C}$ with dry ice. The $\mathrm{SO}_{2}$ was allowed to evaporate in air as the sample warmed to room temperature. Figure XIV-l (top) shows the ${ }^{13} \mathrm{C}$ CP/MAS NMR spectrum of the product, as well as the amorphous material obtained upon vacuum sublimation (bottom). Clearly, the former product is crystalline, as is evident by the sharp lines that show splittings because of the crystallographic inequivalence of various carbons in the asymmetric unit. The material turned out to be a hydrated form of TATM, as was revealed by crystallographic analysis of a suitable single crystal. There was no evidence of $\mathrm{SO}_{2}$ being incorporated into the structure. Apparently, during evaporation of the $\mathrm{SO}_{2}$ solvent, sufficient water was taken up from the atmosphere to give only the hydrated product.

\section{RESULTS}

Crystallographic analysis shows that the material is TATM $\cdot 2 \mathrm{H}_{2} \mathrm{O}$, which has a triclinic $P \bar{I}$ structure. The material is made up of a novel one - dimensional channel structure formed from pairs of TATM host molecules linked through a water molecule that is hydrogen-bonded to a carbonyl group of each host pair. The channel is oriented along the $a$ axis of the structure and contains additional water molecules (Figures XIV-2 and XIV-3). 


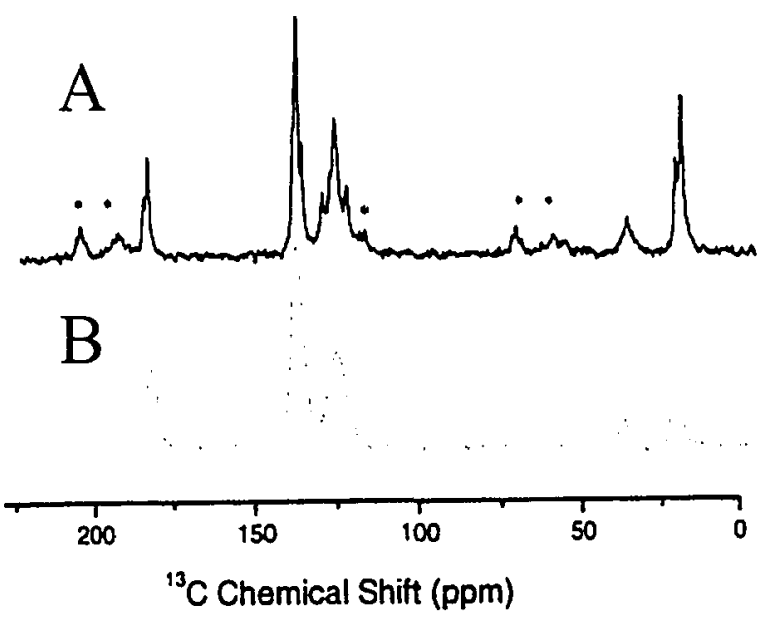

Figure XIV-1: ${ }^{13} \mathrm{C}$ CP/MAS NMR spectrum of TATM.

(A) TATM hydrate.

(B) Vacuum sublimed (amorphous) material.

(Stars mark spinning sidebands)

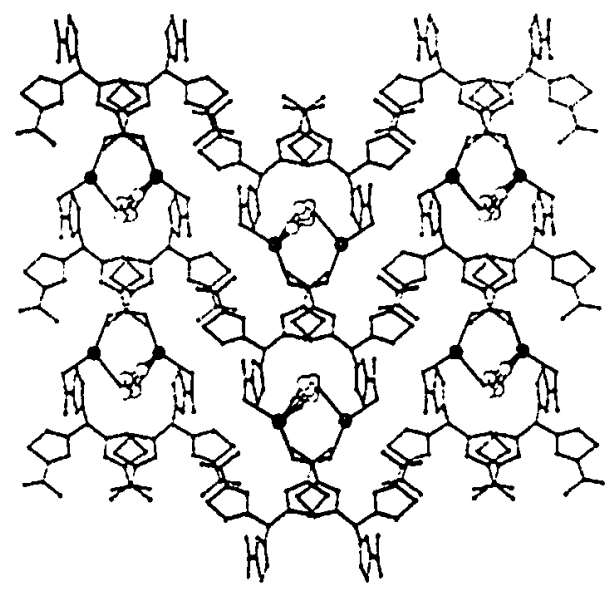

Figure XIV-2: View of $2 \mathrm{H}_{2} \mathrm{O}$ :TATM

approximately along the channel ( $a$ axis).

Ordered water molecules that form part of the channel wall are shown in red circles, disordered water molecules as yellow circles. Hydrogen bonds are shown as blue lines

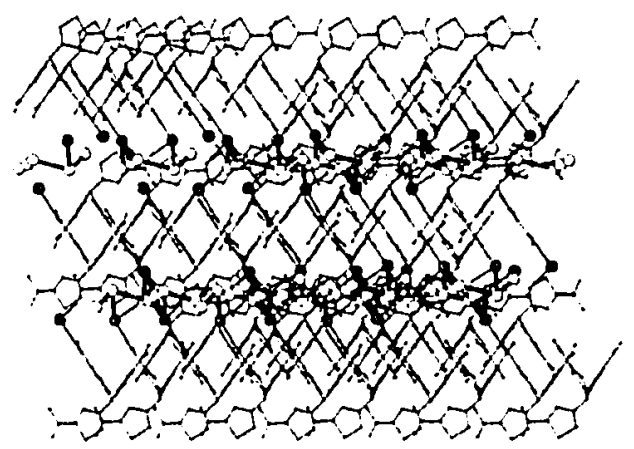

Figure XIV-3: View of $2 \mathrm{H}_{2} \mathrm{O}$ :TATM along the $c$ axis (perpendicular to the channel). Water molecules are marked as red and yellow circles, as in Figure XIV-2. 
There are four water molecules in the asymmetric unit of the structure. Two sites, $\mathrm{O} 1 \mathrm{~A}$ and $\mathrm{O} 2 \mathrm{~A}$, are fully occupied by water molecules and form hydrogen bonds with the oxygen atoms of the TATM acetyl groups ( $\mathrm{O} \cdots \mathrm{O}$ distances are $2.85 \AA$ to $2.88 \AA$ respectively). Two other water molecules are each disordered over two positions with site occupancies of $0.78 / 0.22$ and $0.57 / 0.43$. The disordered water molecules form hydrogen bonds only with the water molecules in the fully occupied positions $(0 \cdots O$ distances are $2.73 \AA-2.82 \AA$ ), but not with TATM.

The crystalline product is stable at room temperature. The material was studied by DSC and TGA, and these experiments show that the material holds on to its water quite tenaciously, considering that two water molecules are bound only through hydrogen bonds to the acetyl oxygens and two reside in an open channel. Rapid water loss starts only on heating to temperatures above $\sim 100^{\circ} \mathrm{C}$ (see Figure XIV-4), and the compound melts at $\sim 128^{\circ} \mathrm{C}$. An isothermal TGA experiment at $100^{\circ} \mathrm{C}$, as depicted in Figure XIV-5, showed that two - thirds of the water was released in $\sim 70$ minutes, with the remainder taking another five hours.

Transport of water is not likely to be very efficient, as the channel is filled with water molecules that are hydrogen bonded to the host and to each other. The TATM bound water molecules are ordered (Figure XIV-3), while the two unbound water molecules are each disordered over pairs of positions because of two possibilities for hydrogen bond formation. Each of the two disordered water molecules has one perfect distance $(2.75 \AA-2.79 \AA)$ for hydrogen bonding to an ordered TATM - bound water molecule. There is a second reasonable hydrogen bonding distance $(3.15 \AA)$ to a disordered water molecule, but too long a distance $(4.3 \AA-4.6 \AA)$ to the secondary position of this disordered water molecule. If we put one fully occupied water position into an intermediate position, all distances, except that to the TATM - bound water molecule, will be too long. So these unbound water molecules are likely distributed over two possible flip - flop positions. Water transport must therefore depend on the movement of vacancies down the channel. 


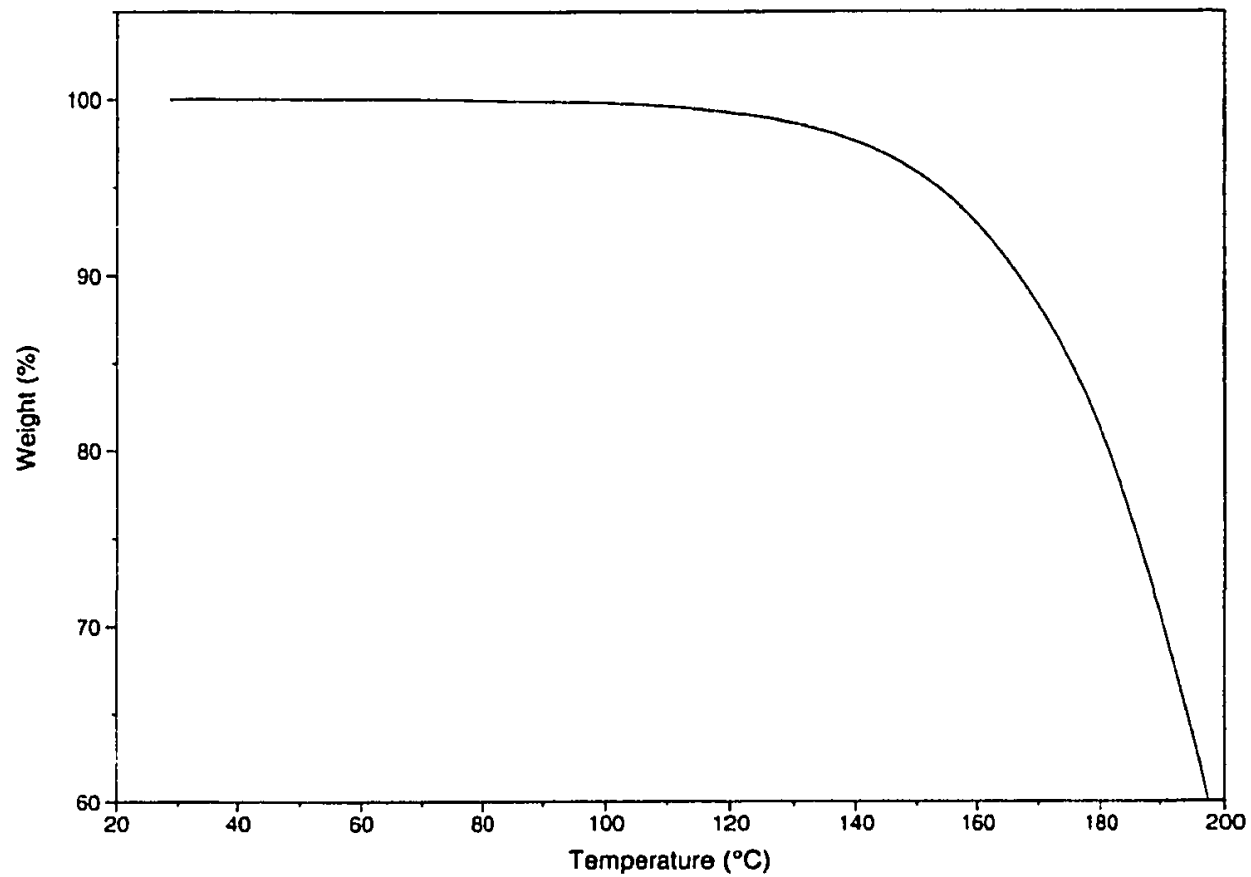

Figure XIV-4: Thermogravimetric (TGA) trace of water / TATM as a function of temperature.

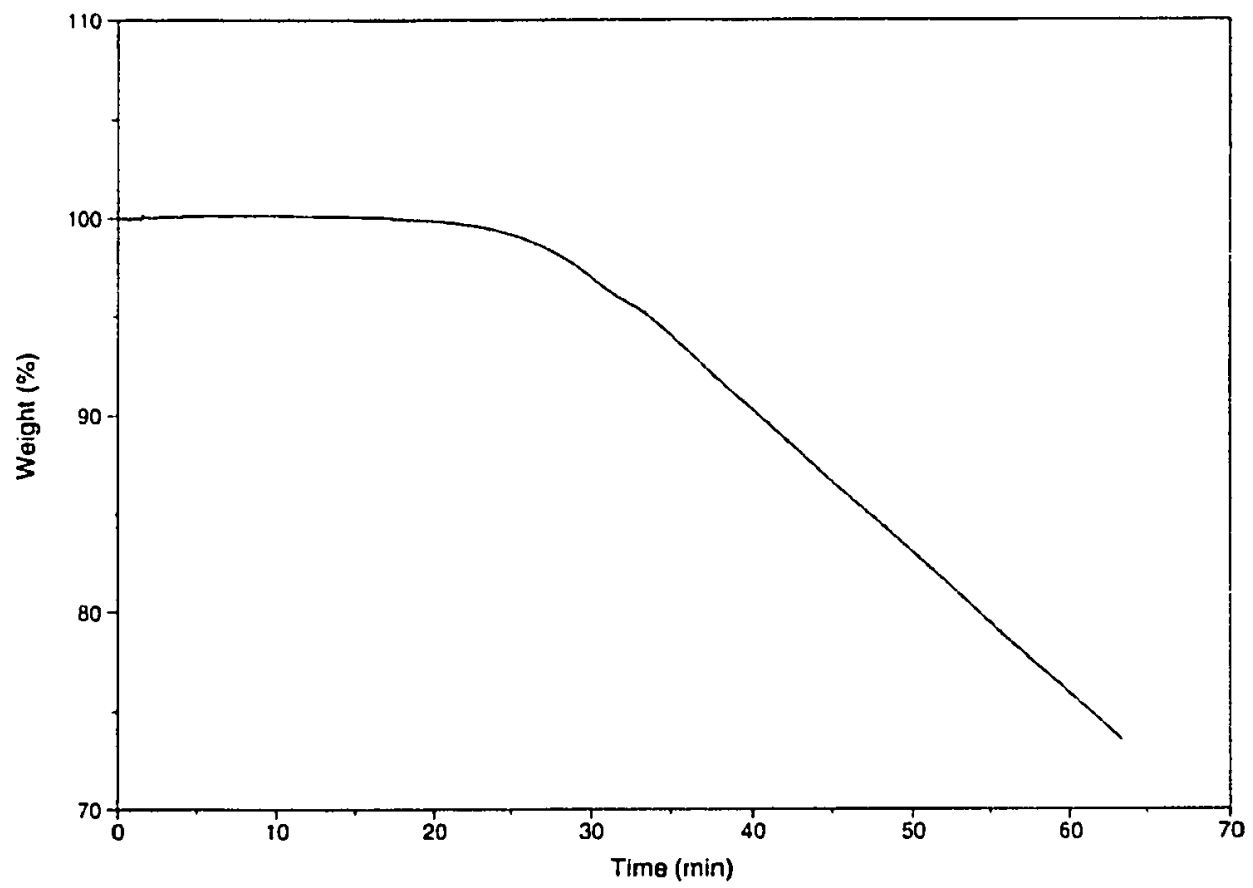

Figure XIV-5: Isothermal $\left(100^{\circ} \mathrm{C}\right)$ thermogravimetric (TGA) trace of water / TATM as a function of time. 
In order to assess the general mobility of water in the channel, a ${ }^{2} \mathrm{H}$ NMR experiment was carried out on a deuterated version of the compound. A sample of $2 \mathrm{D}_{2} \mathrm{O} \cdot \mathrm{TATM}$ was prepared by repeating the preparative procedure, with the exception of a few drops of $\mathrm{D}_{2} \mathrm{O}$ that were added to the cold $\mathrm{SO}_{2}$ solution, and that the $\mathrm{SO}_{2}$ was evaporated through a drying tube to prevent the condensation of atmospheric water. The ${ }^{2} \mathrm{H}$ solid - state NMR spectrum at room temperature is shown in Figure XIV-6, and it consists of a sharp line superimposed on a typical ${ }^{2} \mathrm{H}$ doublet. The sharp line is a relatively small proportion of the total, and we attribute it to $\mathrm{D}_{2} \mathrm{O}$ on the outside of the crystals. The doublet can be characterized by an single effective quadrupolar coupling constant $\left(\chi_{\text {err }}\right)$ of $53.1( \pm 0.1) \mathrm{kHz}$, with an asymmetry parameter $(\eta)$ of $0.27( \pm 0.01)$. The rigid-lattice quadrupolar coupling constant for a hydrogen-bonded water molecule is $\sim 200 \mathrm{kHz}$, so that the reduced value observed here indicates that there is extensive motional averaging. Also, the observation of a single doublet suggests that exchange between the ordered and disordered water molecules is fast on the timescale of the quadrupolar coupling constant. This suggests that a vacancy - mediated mechanism would indeed be effective for water transport.

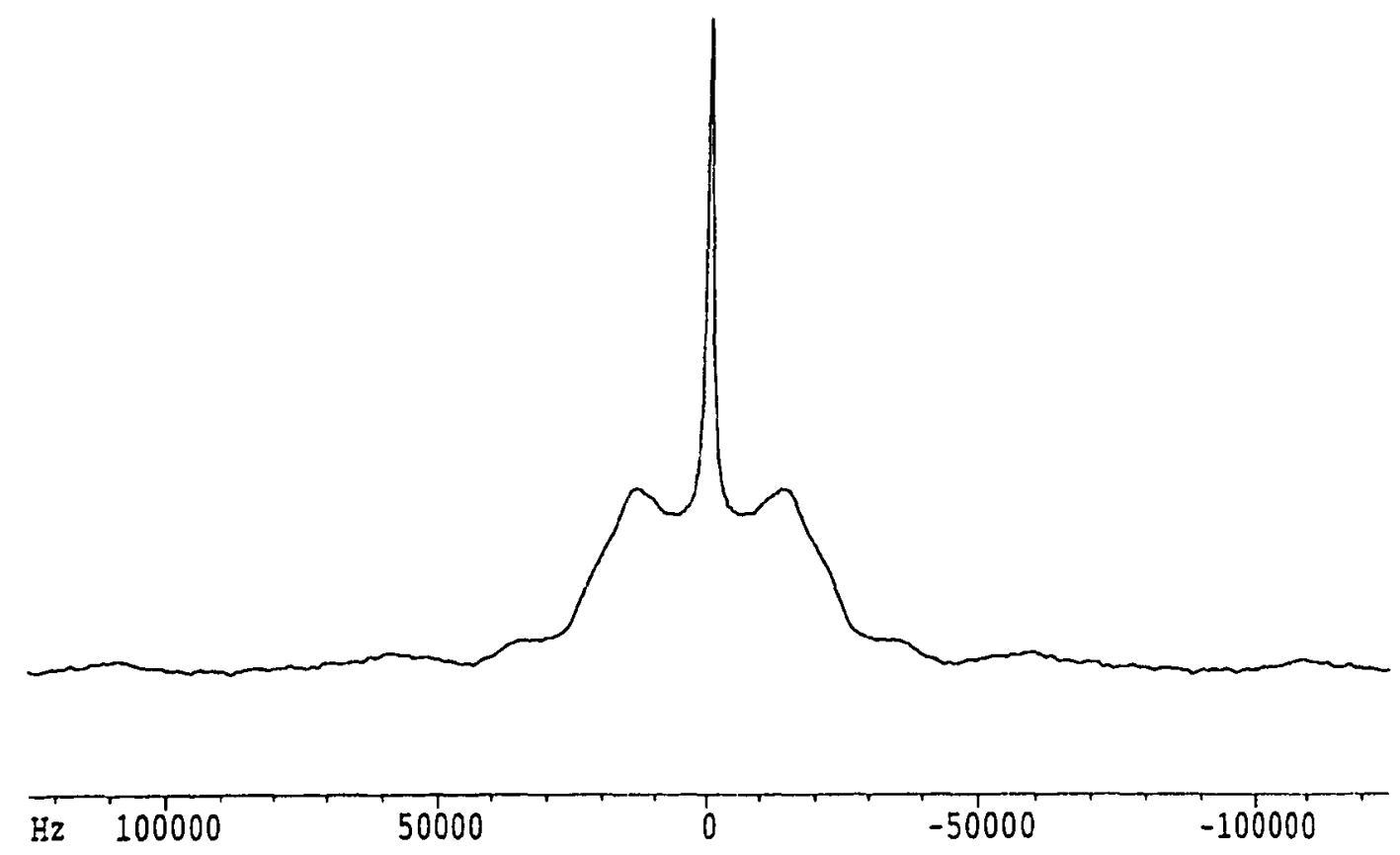

Figure XIV-6: Deuterium NMR spectrum of $2 \mathrm{D}_{2} \mathrm{O} \cdot \mathrm{TATM}$ at room temperature. 
Table XIV-1: Crystal data, data collection and final refinement parameters for the singlecrystal structure of $2 \mathrm{H}_{2} \mathrm{O} \cdot$ TATM

\begin{tabular}{ll} 
Crystal Data & \\
\hline Molecular formula & $1 \mathrm{C}_{19} \mathrm{H}_{16} \mathrm{O}_{3} \mathrm{~S}_{3} \cdot 2 \mathrm{H}_{2} \mathrm{O}$ \\
Molecular weight $(\mathrm{amu})$ & 424.53 \\
Temperature $(\mathrm{K})$ & $173(2)$ \\
Crystal system & triclinic \\
Space Group & $\mathrm{P} \overline{1}$ \\
$a(\AA)$ & $8.7607(13)$ \\
$b(\AA)$ & $10.7929(15)$ \\
$c(\AA)$ & $21.7940(30)$ \\
$\alpha\left({ }^{\circ}\right)$ & $88.980(3)$ \\
$\beta\left(^{\circ}\right)$ & $89.572(3)$ \\
$\gamma\left({ }^{\circ}\right)$ & $80.703(3)$ \\
$V\left(\AA^{3}\right)$ & $2033.3(5)$ \\
$Z$ & 4 \\
$d_{\text {calc }}\left(\mathrm{g} \mathrm{cm}^{-3}\right)$ & 1.387 \\
$d_{\text {meas }}\left(\mathrm{g} \mathrm{cm}^{-3}\right)$ & 1.341 \\
$F(000)$ & 888 \\
$\mu($ MoK $\alpha)\left(\mathrm{mm}^{-1}\right)$ & 0.391 \\
$\lambda(\AA)$ & 0.71073 \\
$2 \theta$ range $\left(^{\circ}\right)$ & $1.87^{\circ}<2 \theta<25.00^{\circ}$
\end{tabular}

$\begin{array}{ll}\text { Data Collection } & \\ \text { Crystal dimensions (mm) } & 0.30 \times 0.30 \times 0.20 \\ \text { Index ranges } & -10 \leq h \leq 10 \\ & -12 \leq k \leq 12 \\ & -25 \leq l \leq 25 \\ \text { Reflections measured } & 18418 \\ \text { Unique reflections } & 7151 \\ R(\text { int) } & 0.0673 \\ \text { Scan mode } & \text { omega } \\ \text { Absorption correction } & \text { multiscan } \\ & \\ \text { Final Refinement } & \\ \text { Refinement method } & \text { Full-matrix least squares } \\ \text { Data/restraints/parameters } & 7151 / 18 / 551 \\ \text { Goodness of fit on } F^{2} & 0.956 \\ \text { Final } R \text { indices }[I>2 \sigma(I)] & R_{1}=0.0739, w R_{2}=0.1827 \\ R \text { indices (all data) } & R_{1}=0.1206, w R_{2}=0.2057 \\ \text { Highest peak }\left(\mathrm{e} \AA^{-3}\right) & 0.85 \\ \text { Deepest hole }\left(\mathrm{e} \AA^{-3}\right) & -0.60 \\ & \end{array}$


(1) G.M. Preston, T.P. Carroll, W.B. Guggino and P. Agre. Science. 256, 385 (1992).

(2) B.L. de Groot, A. Engel and H. Grubmuller. J. Mol. Biol. 325, 485 (2003).

(3) C.H. Yu, S. Cukierman and R. Pomes. Biophys. J. 84, 816 (2003).

(4) H. Birkedal, D. Scwarzenbach and P. Pattison. Angew. Chem. Int. Edn. 41, 754 (2002).

(5) J. Heberle, G. Buldt, E. Koglin, J.P. Rosenbusch and E.M. Landau J. Mol. Biol. 281, 587 (1998).

(6) C.H. Goerbitz. Chem. Eur. J. 7, 5153 (2001).

(7) H. Birkedal, D. Scwarzenbach and P. Pattison. Angew. Chem. Int. Edn. 41, 754 (2002).

(8) A.P. Yakubov, Y.K. Sudarushkin, L.I. Belen'kii and L. Gol'dfarb. J. Org. Chem. (USSR). 9, 1549 (1973).

(9) L. Bin Din and O. Meth-Cohn. J. Chem. Soc. Chem. Commun. 741 (1977).

(10) R.E. Marsh. Acta Crystallogr. B50, 112 (1994).

(11) P.H. Van Rooyen and H.M. Roos. Acta Crystallogr. C47, 2468 (1991).

(12) J.L.M. Dillen and H.M. Roos. Acta Crystallogr. C48, 2229 (1992).

(13) H.M. Roos and J.L.M. Dillen. Acta Crystallogr. C48, 1882 (1992).

(14) P.H. Van Rooyen and H.M. Roos. Acta Crystallogr. C47, 2718 (1991).

(15) L. Pang, R.C. Hynes and M.A. Whitehead. Acta Crystallogr: C50, 615 (1994).

(16) L. Pang and F. Brisse. Acta Crystallogr. C50, 1947 (1994).

(17) L. Pang and F. Brisse. Can. J. Chem. 72, 2318 (1994).

(18) P.S. Sidhu and J.A. Ripmeester. J. Supramol. Chem. 1, 63 (2001).

(19) P.S. Sidhu and J.A. Ripmeester. Supramol. Chem. 15(6), 433 (2003).

(20) P.S. Sidhu, G.D. Enright, J.A. Ripmeester and G.H. Penner. J. Phys. Chem. 106B, 8569 (2002).

(21) P. Finocchiaro and S. Failla. Solid State Supramolecular Chemistry: Crystal Engineering. In Comprehensive Supramolecular Chemistry, Volume 6, Chapter 18, J.L. Atwood, J.E.D. Davies, D.D. MacNicol and F. Vogtle Eds. Pergamon / Elsevier: Oxford, 1996. 


\section{CHAPTER XV}

\section{The preparation and characterization of a novel guest-free crystalline form of the tris (5-acetyl-3- thienyl) methane (TATM) host compound. A single crystal and powder X-ray diffraction, ${ }^{13} \mathrm{C} \mathrm{CP} / \mathrm{MAS}$ NMR, TGA, DSC and polycrystalline density measurement study.}

$\begin{array}{ll}\text { Abstract } & 360 \\ \text { Introduction } & 361 \\ \text { Experimental } & 362 \\ \text { Results } & \\ \text { (A) Single Crystal X-Ray Diffraction } & 363 \\ \text { (B) Powder X-Ray Diffraction } & 373 \\ \text { (C) }{ }^{3} \text { C CP/MAS NMR } & 378 \\ \text { (D) Thermogravimetric Analysis } & 379 \\ \text { (E) Differential Scanning Calorimetry } & 381 \\ \text { (F) Polycrystalline Densities } & 388 \\ \text { (G) Vapor Diffusion } & 390 \\ \text { Discussion } & \\ \text { (H) Effect of guest size upon host lattice } & 393 \\ \text { (I) Gossypol } & 396 \\ \text { (J) Hydroquinone } & 398 \\ \text { (K) Perhydrotriphenylene } & 401 \\ \text { (L) Dianin's compound } & 403 \\ \text { Summary } & 405 \\ \text { Concluding remarks } & 406 \\ \text { References } & 407\end{array}$




\section{ABSTRACT}

In this contribution, we report the preparation and characterization of a novel crystalline guest-free form of the TATM host material. The compound was prepared by heating a TATM inclusion compound in warm water $\left(-70^{\circ} \mathrm{C}\right)$ for about a week. The water slowly removes the guest from its TATM lattice, with a rearrangement of the remaining guestfree structure. TGA and ' $\mathrm{H}$ solution NMR experiments both conclusively show that there is practically no guest left behind in the lattice. Single crystal structures were obtained for the three starting materials and the common guest-free sample. Powder $\mathrm{X}$-ray diffraction and ${ }^{13} \mathrm{C} C P /$ MAS solid state NMR were used for bulk phase identification, and indicated that the four bulk products (three starting materials and guest-free compound) were characteristic of their respective single crystal structures. Both bulk phase techniques suggested that the same guest-free lattice was obtained regardless of whether the starting material was the ethanol, methanol or acetone inclusion compound. The measured torsional angles of TATM in the guest-free lattice have never before been encountered, suggesting that the inter- and intramolecular interactions in guest-free TATM are significantly different than in its inclusion compounds. The DSC experiments show an interesting relationship between residual guest content and thermal behavior. When the guest-free sample has a greater residual guest content (ethanol and methanol starting materials), it has a significantly lower melting point (greater melting point depression) than the guest-free material with a lower residual guest content (acetone starting material). For all three starting materials, there is a considerable increase in polycrystalline density on going from the starting material to the guest-free product, suggesting more extensive attractive intermolecular interactions in the crystalline guestfree material. Finally, we attempted to incorporate guests into guest-free TATM from the vapor phase. Acetone vapor entered the guest-free TATM lattice, whereas methanol and ethanol vapor did not. We also incorporated two more very volatile guests, namely methylene chloride and bromoethane. For the three solvent vapors that entered the guestfree lattice, acetone and methylene chloride formed TATM inclusion compounds isostructural to that formed by recrystallization, while bromoethane formed a novel structure when synthesized from the vapor and guest-free TATM. 


\section{INTRODUCTION}

Tripodal host molecules form a versatile class of supramolecular materials including triphenylmethane $[1,2]$ and analogues, such as TATM [3-19]. TATM host-guest materials show a wide variety of stoichiometries and structural motifs [5-12,17-19]; they have also demonstrated some propensity for polymorphism [11,17]. Indeed, a recent study in this area culminated in the discovery of at least five different forms of the 2:1 host:guest compound of TATM with 1,3-dichloropropane [19]. What is puzzling about TATM is that a crystalline guest-free form of the pure host has yet to be reported. Upon heating or vacuum sublimation of TATM inclusion compounds, the remaining pure host residue is an amorphous solid, melting at $45^{\circ}-50^{\circ} \mathrm{C}$. It is likely that the difficulty in producing a crystalline guest-free material and the propensity for polymorphism have a similar basis: The TATM molecules lack the ability to interact with each other via strong directional interactions. The absence of strongly preferred orientations of the thienyl rings is likely to give a variety of conformational isomers in the liquid or vapour, thus making efficient packing difficult upon solidification. In almost all TATM structural studies, the host and guest generally interact very weakly through van der Waals forces, so the guest appears to play a key role in directing the solid inclusion compound to a structure that packs efficiently.

A number of phase transformations may occur upon the formation and decomposition of inclusion compounds [20]. Consider the general scheme given in Figure XV-1, which illustrates the host molecules in their unenclathrated $\alpha$-phase. When the $\alpha$-phase is exposed directly to the guest (in liquid or vapor form), a new $\beta$-phase inclusion compound is formed. The mechanism of this reaction is not yet fully understood, but in the case of recrystallization of the solid host from a solution in the liquid guest, the guest may act as a template for the new structure. When the inclusion compound is heated, and the guest is allowed to escape, it may melt incongruently in one of several ways. It may revert to its original $\alpha$-phase, with total loss of guest. Secondly, it may lose only part of the guest, and yield a new $\gamma$-phase with a different host-guest ratio. Finally, the host framework may hold, giving rise to an "empty" lattice, or $\beta_{0}$

phase. This last phenomenon is rare, but has been found to occur in, for example, the gossypol / dichloromethane inclusion compound $[\mathbf{2 1 , 2 2}$. On the other hand, congruent 
melting will convert both the host and guest into a liquid melt. As a final point, it is possible to combine the techniques of thermal analysis and X-ray crystallography to monitor the phase changes that occur during the progressive thermal decomposition of an inclusion compound, depicted in Figure XV-2.
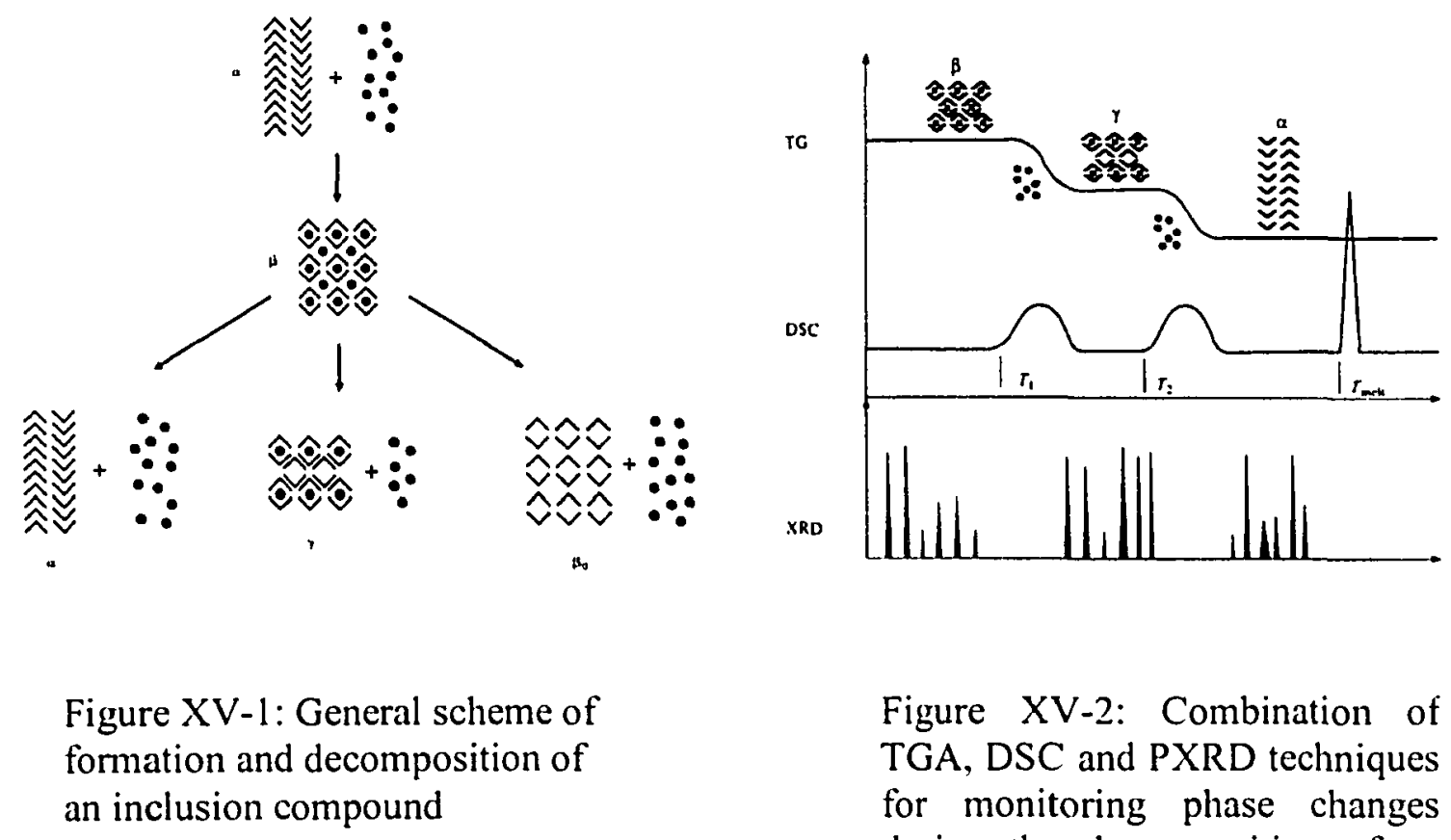

Figure XV-1: General scheme of Figure XV-2: Combination of TGA, DSC and PXRD techniques for monitoring phase changes during the decomposition of an inclusion compound.

\section{EXPERIMENTAL}

Commercially available reagents (Aldrich) were used as received. We used three starting materials to prepare the novel polycrystalline form of guest-free TATM, namely ethanol / TATM, methanol / TATM and acetone / TATM. Each of these starting materials ( 500 $\mathrm{mg}$ ) were separately heated in distilled water $(\sim 10 \mathrm{~mL})$ with vigorous stirring (in a small round bottom flask connected to a reflux condenser) for approximately one week at about $70^{\circ} \mathrm{C}$. This temperature was judged to be low enough to prevent melting or amorphization of the starting material. Higher temperatures resulted in melting, and thus collapse of the structure. Single crystals of guest-free TATM were prepared by layering distilled water over single crystals of the methanol / TATM inclusion compound in a 20 $\mathrm{mL}$ vial, and then placing the covered vial in a $70^{\circ} \mathrm{C}$ oven for $2-3$ weeks. 


\section{RESULTS}

\section{(A) Single crystal X-ray diffraction}

In this work, we employed three different starting materials to produce the crystalline guest-free TATM compound, namely acetone/TATM, ethanol/TATM and methanol/TATM. Single crystals of suitable size and quality of these three compounds were prepared, and subjected to single crystal X-ray diffraction. Crystal data, data collection and final refinement parameters are tabulated in Table XV-1. The room temperature structure of the ethanol/TATM inclusion compound has been previously reported [8]. The present structures, at $125 \mathrm{~K}$, resolve guest disorder in the methanol [63:37] and ethanol [55:45] inclusions. In all three starting materials, the guests are also disordered across an inversion center. A supposed guest-free single crystal that was screened prior to structural analysis was found to have almost the same unit cell parameters as its starting material (methanol/TATM). Subsequent structural analysis revealed that although the TATM framework was indeed isostructural to the starting material $\left(d_{\text {calc }}=1.378 \mathrm{~g} \mathrm{~cm}^{-3}\right)$, when the disordered guest was modeled as partially occupied methanol, only $\sim 90 \%$ of the host cages were guest-free $\left(d_{\text {calc }}=1.328 \mathrm{~g} \mathrm{~cm}^{-3}\right)$.

Table XV-I suggests that all three starting materials are isostructural, with one TATM molecule and $1 / 2$ guest molecule in each asymmetric unit. However the PXRD and ${ }^{13} \mathrm{C}$ CP/MAS NMR spectra (see below) suggest otherwise. However, one must realize that these two bulk characterization techniques are very sensitive to the significant differences in disposition of guest electron density between the three respective inclusion compounds, while the unit cell parameters as determined from SCXRD may be less sensitive. Nevertheless, the fact that they have identical lattice types (triclinic), space groups $(\mathrm{P} \overline{1})$ and near identical unit cell parameters $(a, b, c, \alpha, \beta, \gamma$ and $V)$, despite slight differences in bulk spectra, suggest that they are all isostructural. Indeed, they are also isostructural to the previously reported TATM inclusion compounds with 1,3difluoropropane (chapter X), ethyl acetate [5], 1,2-dichloroethane $\mathbf{A}$ (chapter VII) and cyclohexane A [11] guests. Another possibility for the disagreement is that the three starting materials may each crystallize in more than one form, so that the bulk samples from which the single crystal was drawn may not be exactly characteristic of the selected crystal. Polymorphism is very common among TATM inclusion compounds, with 1,2- 
dichloroethane (chapter VII) and cyclohexane [11] each crystallizing with TATM in at least two different forms, while 1,3-dichloropropane / TATM exists in five forms [19].

Table XV-1: Crystal data, data collection and final refinement parameters for guest-free TATM and the three starting materials.

Crystal Data

\begin{tabular}{|c|c|c|c|c|}
\hline Guest & acetone & ethanol & methanol & guest-free \\
\hline Molecular formula & $\begin{array}{l}2 \mathrm{C}_{19} \mathrm{H}_{16} \mathrm{O}_{3} \mathrm{~S}_{3} \\
1 \mathrm{C}_{3} \mathrm{H}_{6} \mathrm{O}\end{array}$ & $\begin{array}{l}2 \mathrm{C}_{19} \mathrm{H}_{16} \mathrm{O}_{3} \mathrm{~S}_{3} \\
1 \mathrm{C}_{2} \mathrm{H}_{6} \mathrm{O}\end{array}$ & $\begin{array}{l}2 \mathrm{C}_{19} \mathrm{H}_{16} \mathrm{O}_{3} \mathrm{~S}_{3} \\
1 \mathrm{CH}_{4} \mathrm{O}\end{array}$ & $2 \mathrm{C}_{19} \mathrm{H}_{16} \mathrm{O}_{3} \mathrm{~S}_{3}$ \\
\hline Molecular weight (amu) & 835.08 & 823.06 & 809.04 & 777.06 \\
\hline Temperature (K) & $125(2)$ & $125(2)$ & $125(2)$ & $173(2)$ \\
\hline Crystal system & triclinic & triclinic & triclinic & triclinic \\
\hline Space group & $\mathrm{Pl}$ & $\mathbf{P} \overline{\mathbf{l}}$ & P1 & $P \overline{1}$ \\
\hline$a(\AA)$ & $8.1161(4)$ & $8.2585(4)$ & $8.1244(4)$ & $10.5004(12)$ \\
\hline$b(\AA)$ & $10.5951(5)$ & $10.3109(4)$ & $10.4462(5)$ & $12.5300(15)$ \\
\hline$c(\AA)$ & $12.3577(6)$ & $12.4012(5)$ & $12.2124(6)$ & $14.8353(17)$ \\
\hline$\alpha\left(^{\circ}\right)$ & $94.9120(10)$ & $82.7280(10)$ & $94.5970(10)$ & $82.316(2)$ \\
\hline$\beta\left(^{\circ}\right)$ & $107.8570(10)$ & $70.5960(10)$ & $108.3720(10)$ & $72.311(2)$ \\
\hline$\gamma\left({ }^{\circ}\right)$ & $95.9140(10)$ & $85.7780(10)$ & $94.4360(10)$ & $88.529(2)$ \\
\hline$V\left(\AA^{3}\right)$ & $998.33(8)$ & $987.44(7)$ & $974.68(8)$ & $1842.7(4)$ \\
\hline$Z$ & 1 & 1 & 1 & 2 \\
\hline$d_{\text {calc }}\left(\mathrm{g} \mathrm{cm}^{-3}\right)$ & 1.389 & 1.384 & 1.378 & 1.400 \\
\hline$d_{\text {Ineas }}\left(\mathrm{g} \mathrm{cm}^{-3}\right)$ & 1.344 & 1.337 & 1.332 & 1.376 \\
\hline$F(000)$ & 436 & 430 & 422 & 808 \\
\hline$\mu(\mathrm{MoK} \alpha)\left(\mathrm{mm}^{-1}\right)$ & 0.392 & 0.395 & 0.399 & 0.417 \\
\hline Wavelength $(\AA)$ & 0.71073 & 0.71073 & 0.71073 & 0.71073 \\
\hline $2 \theta$ range $\left({ }^{\circ}\right)$ & $1.74<2 \theta<29.60$ & $1.75<2 \theta<29.59$ & $1.77<2 \theta<29.59$ & $1.45<2 \theta<25.00$ \\
\hline \multicolumn{5}{|l|}{ Data Collection } \\
\hline$\overline{\text { Crystal size (mm) }}$ & $0.40 \times 0.30 \times 0.25$ & $0.30 \times 0.25 \times 0.10$ & $0.35 \times 0.30 \times 0.10$ & $0.20 \times 0.20 \times 0.06$ \\
\hline \multirow[t]{3}{*}{ Index ranges } & $-11 \leq h \leq 11$ & $-11 \leq h \leq 11$ & $-11 \leq h \leq 11$ & $-12 \leq h \leq 12$ \\
\hline & $-14 \leq k \leq 14$ & $-14 \leq k \leq 14$ & $-14 \leq k \leq 14$ & $-14 \leq k \leq 14$ \\
\hline & $-17 \leq l \leq 17$ & $-17 \leq l \leq 17$ & $-16 \leq 1 \leq 16$ & $-17 \leq 1 \leq 17$ \\
\hline Reflections collected & 12597 & 12423 & 12189 & 16789 \\
\hline Independent reflections & 5516 & 5450 & 5351 & 6508 \\
\hline$R$ (int) & 0.0268 & 0.0205 & 0.0230 & 0.0761 \\
\hline Completeness to $2 \theta_{\max }$ & $98.0 \%$ & $98.3 \%$ & $97.8 \%$ & $100.0 \%$ \\
\hline Scan mode & omega & omega & omega & omega \\
\hline Absorption correction & multiscan & multiscan & multiscan & multiscan \\
\hline \multicolumn{5}{|l|}{ Final Refinement } \\
\hline \multirow[t]{2}{*}{ Refinement method } & full matrix & full matrix & full matrix & full matrix \\
\hline & least squares & least squares & least squares & least squares \\
\hline Data/restraints/params & $5516 / 0 / 328$ & $5450 / 6 / 322$ & $5351 / 2 / 314$ & $6508 / 0 / 507$ \\
\hline Goodness of fit on $F^{2}$ & 0.998 & 1.029 & 1.028 & 0.938 \\
\hline \multicolumn{5}{|l|}{ Final $R$ indices $[1>2 \sigma(I)]$} \\
\hline$R 1$ & 0.0415 & 0.0376 & 0.0388 & 0.0676 \\
\hline$w R 2$ & 0.1029 & 0.0975 & 0.1038 & 0.1626 \\
\hline \multicolumn{5}{|l|}{$R$ indices (all data) } \\
\hline$R 1$ & 0.0583 & 0.0464 & 0.0487 & 0.1087 \\
\hline$w R 2$ & 0.1107 & 0.1037 & 0.1107 & 0.1793 \\
\hline Highest peak $\left(\mathrm{e} \AA^{-3}\right)$ & 0.532 & 0.483 & 0.488 & 0.863 \\
\hline Deepest hole $\left(\mathrm{e} \AA^{-3}\right)$ & -0.257 & -0.242 & -0.288 & -0.604 \\
\hline
\end{tabular}


The unit cell parameters in a triclinic cell are determined by convention. The "reduced cell" is the term usually applied, and is characterized as the three shortest noncoplanar vectors that define the lattice. In addition, the lattice vectors must define a "right handed" coordinate system, and in order to be unique it requires that $a<b<c$ with all included angles greater than $90^{\circ}$ or all included angles less than $90^{\circ}$. Other conditions are used to ensure a unique choice in cases where two cell dimensions may be accidentally equal or where a cell angle approaches $90^{\circ}$.

For TATM inclusion compounds a number of reported structures are isostructural, but the triclinic cells as reported by convention are different:

Cell 1: 1,3-difluoropropane, (chap. VII) ethyl acetate, $|5|$ and 1,2-dichloroethane A [17] inclusions have been solved with unit cells $8.2 \AA, 10.5 \AA, 12.5 \AA$, $97^{\circ}, 107^{\circ}, 96^{\circ}$. This is similar to our methanol and acetone inclusion cells

Cell 2: On the other hand, ethanol [8] and cyclohexane $\mathbf{A}[11]$ inclusions have been solved with unit cells $8.2 \AA, 10.5 \AA, 12.5 \AA, 82^{\circ}, 71^{\circ}, 85^{\circ}$, similar to our ethanol result

Cell 1 will transform to a "non-conventional" setting similar to that of cell 2 with a

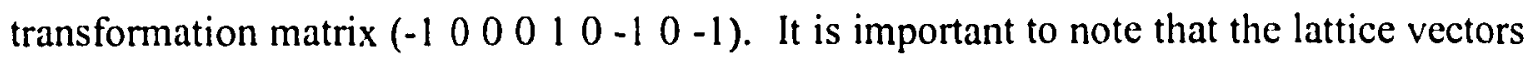
$a$ and $b$ are unchanged (except for a change of sign for $a$ ), but the new $c$ is a combination of the old $a$ and $c\left(c^{*}=-a-c\right)$. The direction is entirely different. As is happens, the lengths $c$ and $c^{\circ}$ are almost equal. This is the source of the setting ambiguity.

Since all three starting materials are isostructural, we chose to present two diagrams for each of the three inclusion compounds, all six views are different, and as they are all isostructural, any one diagram may be easily applied to the other two starting materials. In Figure XV-3 are presented two packing diagrams for acetone / TATM. The view in Figure $\mathrm{XV}-3 \mathrm{~A}$ is down the channel $(a)$ direction. In this figure, the structure is made up of ribbons running in the horizontal (011) direction. Two of the three thienyl rings of each TATM molecule participate in the formation of the channel, while the third thienyl ring links up, via $\pi-\pi$ stacking, with the non-participating thienyl ring in a neighbouring unit cell, to form the endless ribbons. The ribbons are laid side by side in the $b c$ plane to form sheets, and the sheets are stacked along the $a$ direction to form layers. Although the channel direction and the ribbon direction may vary, this ribbon and 
layer motif is almost universally encountered for TATM inclusion compounds whose structures have been reported in the literature. Similarly to all of the literature TATM inclusion compounds listed above that are isostructural to the three present starting materials, the channel is along the smallest unit cell dimension (a), varying from $8.033 \AA$ for 1,2-dichloroethane A / TATM [17] to $8.622 \AA$ for cyclohexane A / TATM [11]. The three starting materials have $a$ dimensions in between these two extremes. In Figure XV3B is presented a view of acetone / TATM in the $a b$ plane, showing four layers stacked in the $a$ direction. In this structure, as in the other two starting materials, neighbouring layers are related by a unit cell translation, and ribbons in adjacent layers run in parallel directions. In the view of Figure $X V-3 B$, the channels run slightly off the vertical direction, and illustrates that the $\pi$-stacked thienyl rings exist in isolated pairs, rather than the sometimes encountered infinitely $\pi$-stacked thienyl rings. Finally, notice that in Figure XV-3A, each guest molecule / host cavity is surrounded in all four directions (within any one sheet) by pairs of $\pi$-stacked thienyl rings, while each pair of $\pi$-stacked thienyl rings is surrounded in all four directions by a guest molecule / host cavity.
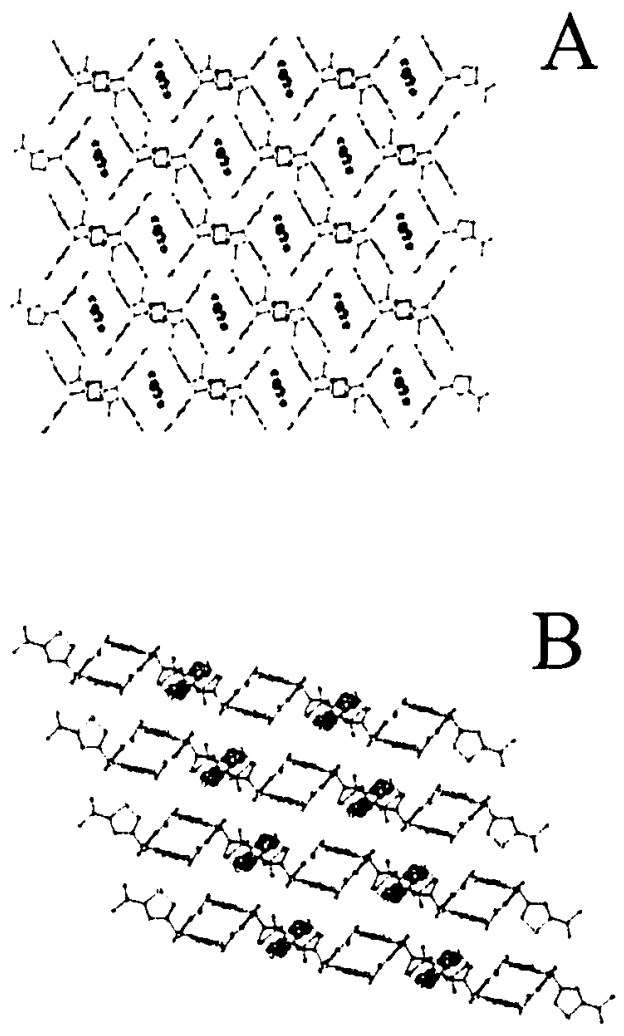

Figure XV-3: Structure of acetone/TATM.

(A) Down $a$ (channel) direction. Ribbons run along [011] (horizontal direction)

(B) View in $a b$ plane. Notice linear channels running along the $a$ (slightly off the vertical) direction, and isolated pairs of $\pi$-stacked thienyl rings. 
Two packing diagrams for ethanol / TATM appear in Figure XV-4. The first view, Figure XV-4A, is down $a$ ( $b c$ plane), the channel axis. As in acetone/TATM (Figure $\mathrm{XV}-3$ ), ribbons run parallel to [011] (NE - SW diagonal direction). The view may be compared to Figure XV-3A, but rotated by $45^{\circ}$ in the $b c$ plane. Also notice that two acetyl groups from two TATM molecules in adjacent unit cells also contribute to the formation of the channel. Figure XV-4B is a view down the $b$ axis ( $a c$ plane). The channel axis is in the plane of the figure, and slightly off vertical. Notice in this figure that there is a significant constriction of the channel, as one thienyl ring of each TATM molecule (of the two that participate in the formation of the channel) lie directly above, and another directly below the guest, partially blocking the channel between guest sites. In other words, there is, as is often encountered with other TATM structures, a bottleneck in the channel between guest sites. This may explain, in part, why TATM holds onto its guest very tenaciously, at least at room temperature, for TATM inclusion compounds generally still retain their original physicochemical properties for even years after first being prepared.
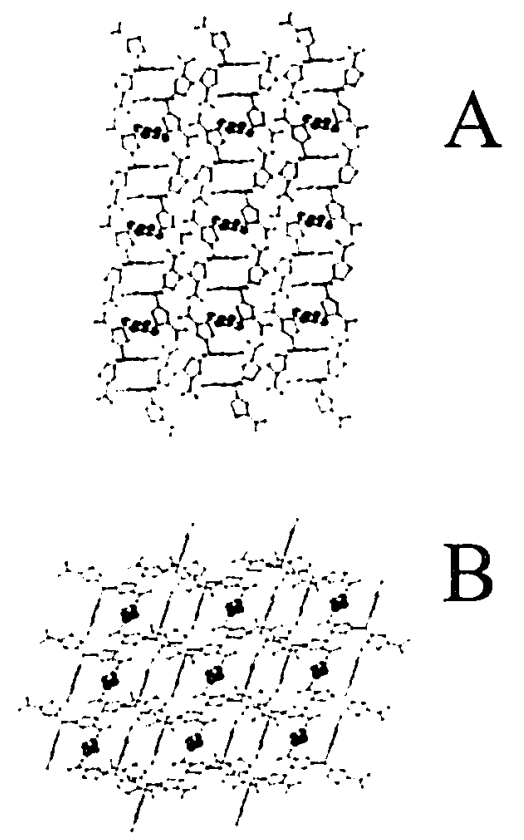

Figure XV-4: Structure of ethanol/TATM (A) Down $a$ (bc plane). The view is down the channel axis. As in acetone/TATM (Figure $\mathrm{XV}-3$ ), ribbons run parallel to [011] (diagonal direction), with channels along $a$.

(B) Down $b$ (ac plane). The channel axis is in the plane of the figure, and slightly off vertical. Note the bottleneck channels arising from thienyl rings in between adjacent guest sites in the channel.

In Figure XV-5 are presented two perspectives of methanol / TATM. Figure XV-5A depicts an ORTEP drawing of the unit cell of methanol / TATM. All three starting materials are isostructural, and have only $1 / 2$ TATM molecule in the asymmetric unit, and Figure XV-5A applies to all three starting materials. Figure XV-5B is a view slightly off 
the channel $(a)$ axis, shows the familiar ribbons running in the [011] horizontal direction in the figure. The view is similar to that of acetone / TATM in Figure XV-1A, in which the view is exactly down the $a$ axis.

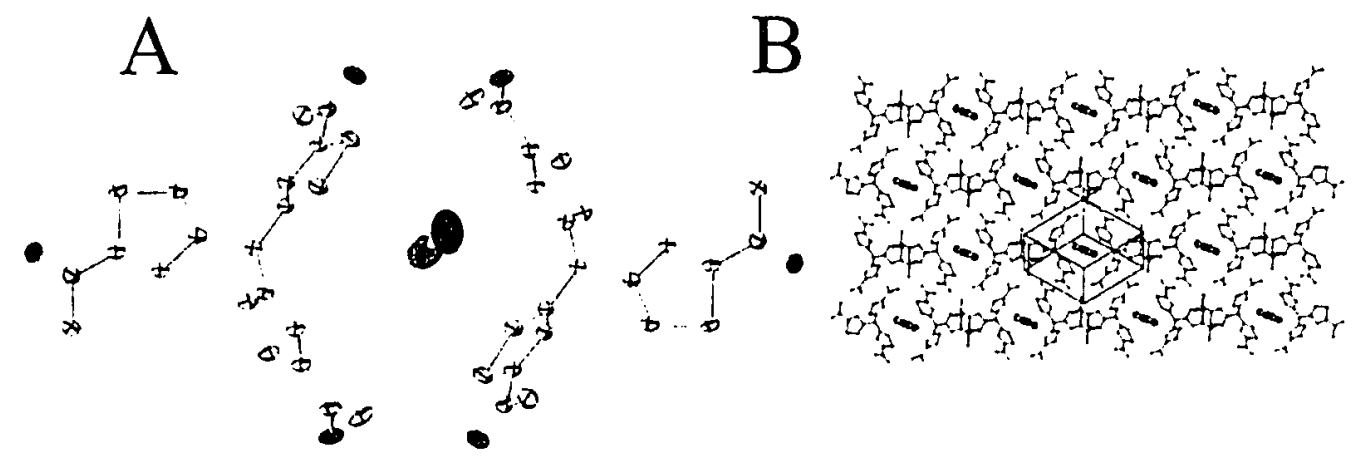

Figure XV-5: Structure of methanol/TATM (A) Schematic of the unit cell (B) Slightly off the channel $(a)$ axis. Ribbons run along [011] (horizontal direction). The view is a similar perspective to Figure XV-3A above (acetone / TATM).

The asymmetric unit of the guest-free compound is shown in Figure XV-6B. The structure is triclinic, space group P $\bar{l}$, with two crystallographically distinct TATM molecules. The TATM molecules appear to be arranged in bilayers that are stabilized by quartets of $\pi$-stacked thienyl rings. If we label the thienyl rings from the number of the carbon atom that is bonded to the central methine carbons $\left(\mathrm{Cl}\right.$ and $\left.\mathrm{Cl}^{\circ}\right)$, then rings 8 and 14' (ring separation $\delta \sim 3.7 \AA$ ) are $\pi$-stacked (Figure XV-6B). In addition, thienyl rings 8 ' and 14 of Figure XV-6B appear to weakly $\pi$-stack with rings 14 and 8 ' respectively ( $\delta$ $\sim 4.4 \AA$ ) from equivalent TATM molecules in adjacent unit cells, to produce ribbons of $\pi$-coupled TATM molecules extending along the $a$ direction. The ribbons are arranged in layers in the $a b$ plane. Adjacent layers interdigitate to form bilayers, as depicted in Figure XV-6A. It appears that additional $\pi$-stacking between rings 8 and $8(\delta \sim 3.8 \AA)$ and $8^{\prime}$ and $8^{\prime}(\delta \sim 4.0 \AA)$ stabilize this packing motif. As a result, two distinct quartets of $\pi$-stacked rings, $14-8^{\prime}-8^{\prime}-14$ and $14^{\prime}-8-8-14^{\prime}$ are observed within the bilayer. Adjacent bilayers interact through van der Waals forces and aromatic $\mathrm{C}-\mathrm{H} \cdots \mathrm{O}$ contacts. 


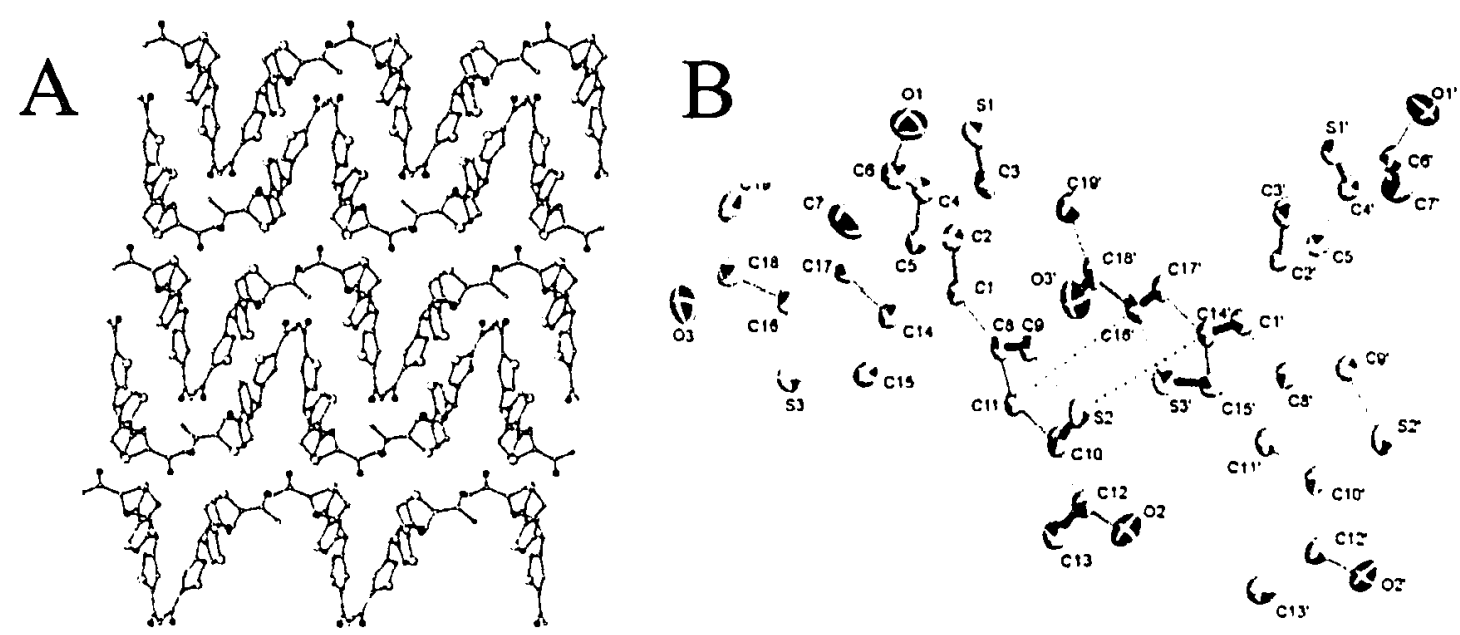

Figure XV-6: Structure of crystalline guest-free TATM

(A) View down the $a$ axis showing the bilayer packing motif. The $b$ axis is horizontal.

(B) ORTEP plot of the asymmetric unit of guest-free TATM. The dotted lines indicate $\pi-\pi$ stacking between thienyl rings 8 and $14^{\circ}$.

Table XV-2 summarizes the host TATM torsion angles for the three starting materials and crystalline guest-free TATM from the four crystal structures of the present work. The host torsion angles $\tau_{1}, \tau_{2}$ and $\tau_{3}$ are about the central methine $\mathrm{C}-\mathrm{H}$ carbon of the host, and the three carbons from the three thienyl rings (carbon 3 of TATM) that are directly bonded to the central methine $\mathrm{C}-\mathrm{H}$ carbon of the host. In addition, we also include in Table XV-2 the conformation of the carbonyl group of the host with respect to its respective ring sulfur, $S(s y n)$ designating a $\sim 0^{\circ}$ torsion angle, while $A$ (anti) denotes a $\sim 180^{\circ}$ torsional angle. The atomic numbering scheme and torsion angle ordering are the same as previously discussed by Herbstein [23].

Table XV-2: Torsional angles $\left( \pm 0.01^{\circ}\right)$ for the three starting materials and guest-free TATM.

\begin{tabular}{|c|c|c|c|c|c|}
\hline \multirow{2}{*}{\multicolumn{2}{|c|}{$\frac{\text { Compound }}{\text { ethanol / TATM }}$}} & $\tau_{1}\left({ }^{\circ}\right)$ & $\tau_{2}\left({ }^{\circ}\right)$ & $\tau_{3}\left({ }^{\circ}\right)$ & $\mathrm{C}=\mathrm{O}$ wrto $\mathrm{S}$ \\
\hline & & -3.14 & 110.59 & 160.71 & SSS \\
\hline methanol / TATM & & 0.78 & 111.98 & 161.21 & SSS \\
\hline acetone / TATM & & 1.24 & 110.55 & 164.07 & SSS \\
\hline guest-free TATM: & $\begin{array}{l}\text { A } \\
\text { B }\end{array}$ & $\begin{array}{l}-17.24 \\
-20.12\end{array}$ & $\begin{array}{l}117.06 \\
110.25\end{array}$ & $\begin{array}{l}125.64 \\
127.10\end{array}$ & $\begin{array}{l}S S S \\
\text { SSS }\end{array}$ \\
\hline
\end{tabular}


From the table, it appears that the three starting materials, which are all isostructural, possess very similar torsion angles, as perhaps would be expected. For these three compounds, the thienyl ring that is approximately coplanar to the $\mathrm{C}-\mathrm{H}$ bond has torsion angles ranging from $-3.14^{\circ}$ to $1.24^{\circ}$, the next largest torsion angle varies from $110.55^{\circ}$ to $111.98^{\circ}$, while the largest angle goes from $160.71^{\circ}$ to $164.07^{\circ}$. Furthermore, all three of these structures indicate that the universal orientation of the carbonyl bond of the acetyl groups is toward the direction of the sulfur atom of its associated thienyl ring (i.e. all 3 structures are SSS). Finally, perhaps as expected, the crystalline guest-free product contains TATM molecules that have a novel conformation, being never before observed in the TATM literature. The average angles are $-18.68^{\circ}\left( \pm 1.44^{\circ}\right), 113.65^{\circ}$ $\left( \pm 3.40^{\circ}\right)$ and $126.37^{\circ}\left( \pm 0.73^{\circ}\right)$, SSS. This suggests that the inter- and intramolecular interactions in guest-free TATM are significantly different when compared to its inclusion compounds.

Table XV-3: Literature host torsion angles $\left( \pm 0.1^{\circ}\right)$ for TATM materials [23].

\begin{tabular}{|c|c|c|c|c|c|}
\hline Guest & Host Molecule & $\tau_{1}\left({ }^{\circ}\right)$ & $\tau:\left(0^{\circ}\right)$ & $\left.\tau_{3} 0^{\circ}\right)$ & Orientation of $\mathrm{C}=\mathrm{O}$ wrto $\mathrm{S}$ \\
\hline ethyl acetate & $A$ & 7.0 & 102.9 & 157.0 & SSS \\
\hline ethanol & A & -3.2 & 110.7 & 160.0 & SSS \\
\hline cyclohexane $\mathbf{A}$ & $A$ & -12.6 & 109.4 & 162.8 & SSS \\
\hline benzene & $\begin{array}{l}\text { A } \\
B\end{array}$ & $\begin{array}{l}-16.2 \\
-10.1\end{array}$ & $\begin{array}{l}126.2 \\
124.2\end{array}$ & $\begin{array}{l}148.2 \\
148.7\end{array}$ & $\begin{array}{l}S S .4 \\
S S_{A}\end{array}$ \\
\hline carbon tetrachloride & $\begin{array}{l}\mathrm{A} \\
\mathrm{B}\end{array}$ & $\begin{array}{l}-20.0 \\
-10.3\end{array}$ & $\begin{array}{l}129.0 \\
131.6\end{array}$ & $\begin{array}{l}148.3 \\
156.7\end{array}$ & $\begin{array}{l}S S . A \\
S S . A\end{array}$ \\
\hline cyclohexane B & $\begin{array}{l}\text { A } \\
\text { B }\end{array}$ & $\begin{array}{l}-11.1 \\
-18.7\end{array}$ & $\begin{array}{l}129.5 \\
128.6\end{array}$ & $\begin{array}{l}151.0 \\
149.4\end{array}$ & $\begin{array}{l}S S .4 \\
S S .4\end{array}$ \\
\hline cycloheptane & $\begin{array}{l}\mathrm{A} \\
\mathrm{B}\end{array}$ & $\begin{array}{l}-12.2 \\
-17.1\end{array}$ & $\begin{array}{l}130.4 \\
129.6\end{array}$ & $\begin{array}{l}150.2 \\
148.0\end{array}$ & $\begin{array}{l}S S . A \\
S S . A\end{array}$ \\
\hline cyclooctane & $\begin{array}{l}\text { A } \\
\text { B }\end{array}$ & $\begin{array}{l}16.6 \\
-13.9\end{array}$ & $\begin{array}{l}130.1 \\
132.2\end{array}$ & $\begin{array}{l}146.5 \\
151.9\end{array}$ & $\begin{array}{l}S S A \\
S S A\end{array}$ \\
\hline$n$-hexane & $\begin{array}{l}\text { A } \\
B \\
C\end{array}$ & $\begin{array}{l}2.4 \\
0.6 \\
1.6\end{array}$ & $\begin{array}{l}108.2 \\
110.1 \\
110.5\end{array}$ & $\begin{array}{l}156.8 \\
157.6 \\
155.4\end{array}$ & $\begin{array}{l}\text { SSS } \\
\text { SSS } \\
\text { SSS }\end{array}$ \\
\hline cyclononanone & $A$ & -7.3 & 101.8 & -15.2 & SSS \\
\hline
\end{tabular}


Next, it is interesting to compare our torsion angles with those reported in 1997 by Herbstein [23]. Based on the crystal structures of TATM inclusion compounds that were available at the time, he classified the structures according to the torsional angles of the thienyl rings about the central methine $\mathrm{C}-\mathrm{H}$ bond of the host. He suggested that the data indicated that there were only three general types of host torsional conformation that pervaded his observations (see Table XV-3). They were as follows: (conformation \#1) $\tau_{1} \sim 0^{\circ}, \tau_{2} \sim 105^{\circ}, \tau_{3} \sim 160^{\circ}, S S S$; (conformation \#2) $\tau_{1} \sim 0^{\circ}, \tau_{2} \sim 130^{\circ}, \tau_{3} \sim 150^{\circ}, S S A$ and (conformation \#3) $\tau_{1} \sim 0^{\circ}, \tau_{2} \sim 100^{\circ}, \tau_{3} \sim-15^{\circ}$, SSS. In the present study, we see similar results, in that all three starting materials fall approximately into conformation \#1, as do the literature structures of ethyl acetate [5], ethanol [8], cyclohexane $\mathbf{A}[\mathbf{1 1}]$ and $n$-hexane [7] inclusions. The first three literature structures are isostructural to the three starting materials, while $n$-hexane/TATM is not. Herbstein noted that the range of torsion angles within each of the three conformational groups did not exceed $10^{\circ}$, while in our case the largest variation is $4.38^{\circ}$ (for the smallest torsion angle). The range for the other two torsion angles are both less than $3.5^{\circ}$. In Herbstein`s work, he found approximately equal occurrences of SSA (10) and SSS (7). In the present study, we encountered only SSS conformations, and in our study of 1,3-dichloropropane / TATM (Chapter XII), which crystallizes in at least five different forms, there were seven occurrences of SSS, but only two occurrences of $S S A$. So it seems that the relative abundance of $S S A$ conformations as found by Herbstein does not seem to be reproduced in our work

We may compare isostructural TATM inclusion compounds directly by measuring the diameter of the channel and comparing it to the nature of the included guest. For all three starting materials, the channel runs along the a direction, so a comparison of the product of $b \times c$ cell dimensions may give an indication of the channel cross section. We present this data in Table XV-4, and compare it with the molecular weight of the guest. Similar data for five TATM inclusion compounds as reported in the literature are also included. We also present the molecular weight, as a rough measure of guest size, to compare with the cross sections of the TATM channels. For the three starting materials, acetone / TATM has both the largest diameter channel (130.93 $\left.\AA^{2}\right)$ and the greatest guest molecular weight $\left(58.08 \mathrm{~g} \mathrm{~mol}^{-1}\right)$. Next comes ethanol / TATM (127.87 $\AA^{2}$ and $46.07 \mathrm{~g} \mathrm{~mol}^{-1}$ respectively), while methanol has both the smallest 
diameter channel $\left(127.57 \AA^{2}\right)$ and the smallest guest molecular weight $\left(32.04 \mathrm{~g} \mathrm{~mol}^{-1}\right)$. As far as the five literature structures in Table XV-4 are concerned, there is a complicating factor of differing acquisition temperatures, since unit cell parameters change somewhat with temperature. However, for the two structures at $298 \mathrm{~K}$ (ethyl acetate [5] and ethanol [8]), the molecular weight of ethyl acetate is almost twice that of ethanol, and accordingly, the $b c$ plane for ethyl acetate $\left(138.44 \AA^{2}\right)$ is significantly larger than that for ethanol $\left(129.52 \AA^{2}\right)$. Similarly, for the two structures at $173 \mathrm{~K}, 1,2-$ dichloroethane A (chapter VII) has a larger molecular weight than 1,3-difluoropropane (chapter $\mathrm{X}$ ) (98.96 $\mathrm{g} \mathrm{mol}^{-1}$ and $80.08 \mathrm{~g} \mathrm{~mol}^{-1}$ respectively) and as expected, a larger channel cross-section (134.21 $\AA^{2}$ and $132.98 \AA^{2}$ respectively). Finally, we acknowledge that it would be more accurate to correlate guest size (instead of mass), but the observed correlation with guest mass perhaps suggests that either measure is applicable.

Table XV-4: Dimensions (esd in brackets) of the channel for TATM inclusion compounds of the present study and for literature structures.

\begin{tabular}{llllll} 
& \multicolumn{2}{c}{ Channel along $a$} & & & \\
Guest & $b(\AA)$ & $c(\AA)$ & $b c\left(\AA^{2}\right)$ & $M W(\mathrm{amu})$ & $\mathrm{T}(\mathrm{K})$ \\
\hline ethanol & $10.3109(4)$ & $12.4012(5)$ & $127.867(9)$ & 46.07 & 125 \\
methanol & $10.4462(5)$ & $12.2124(6)$ & $127.573(12)$ & 32.04 & 125 \\
acetone & $10.5951(5)$ & $12.3577(6)$ & $130.931(12)$ & 58.08 & 125 \\
& & & & & \\
ethyl acetate & $11.229(5)$ & $12.329(5)$ & $138.44(12)$ & 88.11 & 298 \\
$\begin{array}{l}1,2 \text {-dichloroethane A } \\
\text { cyclohexane A }\end{array}$ & $10.9065(7)$ & $12.3063(8)$ & $134.219(17)$ & 98.96 & 173 \\
$\begin{array}{l}\text { 1,3-difluoropropane } \\
\text { ethanol }\end{array}$ & $10.194(2)$ & $12.795(2)$ & $130.43(5)$ & 84.16 & 220 \\
\hline
\end{tabular}

\begin{tabular}{llllll} 
& \multicolumn{2}{c}{ Channel along $c$} & & \\
Guest & $a(\AA)$ & $h(\AA)$ & $a b\left(\AA^{2}\right)$ & MW(amu) & $T(\mathrm{~K})$ \\
\hline benzene & $11.538(5)$ & $13.560(5)$ & $156.45(12)$ & 78.11 & 298 \\
carbon tetrachloride & $11.638(3)$ & $13.684(3)$ & $159.25(8)$ & 153.8 & 295 \\
cyclohexane B & $11.668(4)$ & $13.729(3)$ & $160.19(9)$ & 84.16 & 295 \\
cycloheptane & $11.721(5)$ & $13.734(6)$ & $160.98(14)$ & 98.19 & 220 \\
cyclooctane & $11.914(1)$ & $14.013(2)$ & $166.95(4)$ & 112.2 & 220 \\
\hline
\end{tabular}

In addition, we also present in Table XV-4 the channel dimensions for a second group of isostructural TATM inclusion compounds that have a channel direction along the crystallographic $c$ axis, as reported in the literature. For this group, a measure of the channel axis (down $c$ ) may be determined by the product of the $a \times b$ cell dimensions, as reported in Table XV-4, along with the molecular weight of the guests. From this table, 
an interesting relationship is demonstrated for the cyclic hydrocarbon guests cyclohexane B [11] (295K), cycloheptane [11] (220 K) and cyclooctane [11] (220 K), for which as the molecular weight of the guest increases $\left(84.16,98.19\right.$ and $112.12 \mathrm{~g} \mathrm{~mol}^{-1}$, respectively), the dimensions of the channels also increase $\left(160.19,160.98\right.$ and $166.95 \AA^{2}$, respectively). At $220 \mathrm{~K}$ (as opposed to $295 \mathrm{~K}$ ), the $a b$ plane dimension for cyclohexane B / TATM would be even smaller, which would make the correlation even better. For benzene [6] and $\mathrm{CCl}_{4}$ [9] guests (at $298 \mathrm{~K}$ and $295 \mathrm{~K}$ respectively), the heavier $\mathrm{CCl}_{4}$ guest has a larger channel cross-section $\left(159.25 \AA^{2}\right)$, while the half as heavy benzene guest has a significantly smaller channel cross-section $\left(156.45 \AA^{2}\right)$. So we see a pervasive relationship between the mass of guest and the resulting channel cross-section.

\section{(B) Powder X-ray diffraction}

Figure XV-7 depicts the indexed powder X-ray diffraction patterns of the three starting materials (ethanol, methanol and acetone inclusions). This PXRD patterns and especially the ${ }^{13} \mathrm{C}$ CP/MAS NMR spectra tentatively suggest that none of the three bulk starting materials are isostructural, neither to each other nor to any of the guest-free products, despite the near identical unit cell dimensions of the three starting material single crystals (see Table XV-1). As mentioned above, this disagreement may arise if the selected crystals are not characteristic of the bulk products, perhaps due to polymorphism.

By acquiring the single crystal unit cell parameters, it is possible to calculate a powder pattern for the compound, with an accompanying table of calculated peak positions $(2 \theta)$, and their respective calculated intensities (see supplemental material). Then we may correlate the experimental peak positions with the calculated ones, not only to index the reflections (see Figure XV-7), but also to allow us to perform a least-squares regression on the differences between each of the experimental and calculated peak positions by varying the unit cell parameters, using the single crystal unit cell parameters as a starting point. This then allows us to calculate unit cell parameters based on the powder X-ray diffraction pattern, and to compare them with the single crystal unit cell parameters. In Table XII-5, we tabulate the resulting single crystal and powder unit cell parameters for the three starting materials (ethanol, methanol and acetone inclusions). 


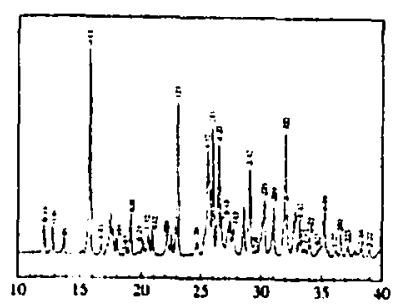

A
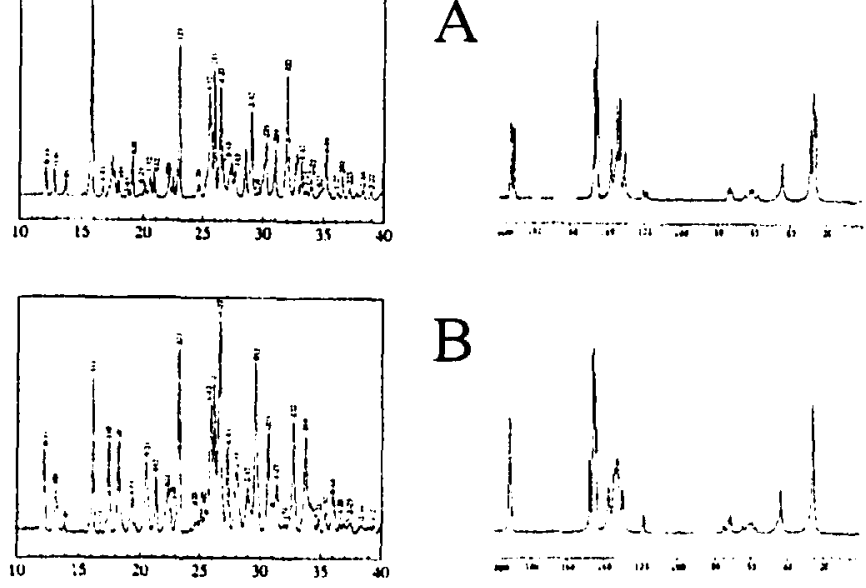

B
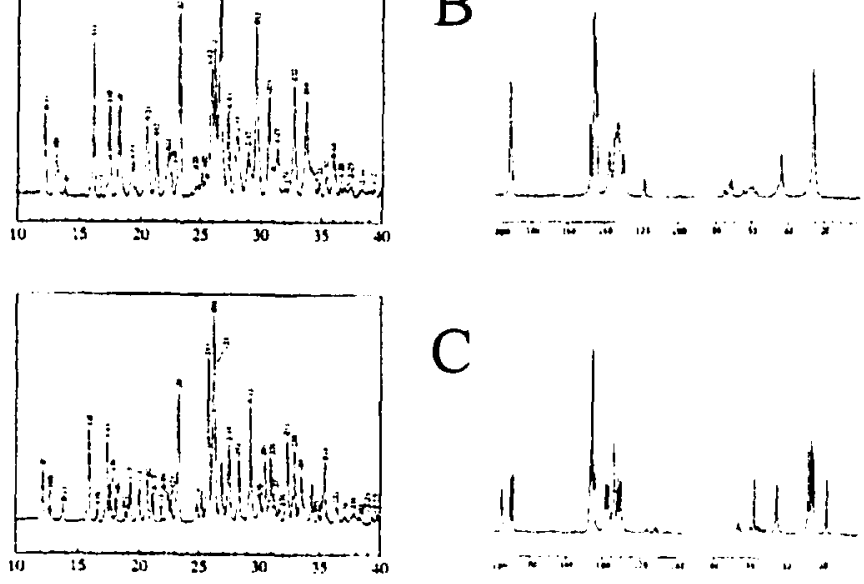

Figure XV-7: Indexed PXRD patterns (left column) and ${ }^{13} \mathrm{C} \mathrm{CP/MAS} \mathrm{solid-state} \mathrm{NMR}$ spectra (right column) for the three starting materials: (A) acetone / TATM (B) methanol / TATM and (C) ethanol / TATM

Table XV-5: Single crystal (esd in brackets) and PXRD (within $0.003 \%, 0.009 \%$ and $1.7 \%$ for unit cell dimensions, angles and volume, respectively) unit cell parameters for the three starting materials.

\begin{tabular}{|c|c|c|c|c|c|c|}
\hline & \multicolumn{2}{|c|}{ Ethanol/TATM } & \multicolumn{2}{|c|}{ Methanol/TATM } & \multicolumn{2}{|c|}{ Acetone/TATM } \\
\hline & $P X R D$ & $S C$ & $P X R D$ & $S C$ & PXRD & $S C$ \\
\hline & RT & $\mathrm{RT}$ & RT & $125 \mathrm{~K}$ & RT & $125 \mathrm{~K}$ \\
\hline$\overline{a(\AA)}$ & 8.203 & $8.2585(4)$ & 8.145 & $8.1244(4)$ & 8.181 & $8.1161(4)$ \\
\hline$b(\AA)$ & 10.290 & $10.3109(4)$ & 10.557 & $10.4462(5)$ & 10.684 & $10.5951(5)$ \\
\hline$c(\AA)$ & 12.361 & $12.4012(5)$ & 12.327 & $12.2124(6)$ & 12.394 & $12.3577(6)$ \\
\hline$\alpha\left({ }^{\circ}\right)$ & 82.30 & $82.728(1)$ & 94.86 & $94.597(1)$ & 94.57 & $94.912(1)$ \\
\hline$\beta\left(^{\circ}\right)$ & 70.68 & $70.596(1)$ & 108.79 & $108.372(1)$ & 108.02 & $107.857(1)$ \\
\hline$\gamma\left({ }^{\circ}\right)$ & 85.89 & $85.778(1)$ & 94.92 & $94.436(1)$ & 95.92 & $95.914(1)$ \\
\hline$V(A$ & 975.38 & $987.44(7)$ & 992.71 & $974.68(8)$ & 1017. & $998.33(8)$ \\
\hline
\end{tabular}

All powder X-ray diffraction experiments were performed close to room temperature. For the single crystal unit cell determinations, the methanol and acetone inclusions were measured at $125 \mathrm{~K}$, while the ethanol/TATM crystal was measured at room temperature. As far as methanol and acetone inclusions are concerned, one may see from Table XV-5 that the room temperature powder diffraction unit cell distances $(a, b, c)$ and volume $(V)$ 
are significantly and consistently larger than the single crystal unit cell parameters measured at $125 \mathrm{~K}$, due to thermal expansion of the lattice on the increase in temperature. On the other hand, for ethanol/TATM, the room temperature PXRD unit cell parameters are slightly smaller than the single crystal unit cell parameters (also at room temperature). This difference is likely because of circulating water that is used to cool the PXRD sample stage. The PXRD pattern was recorded in the winter, and at this time of the year, the stage is quite cold to the touch, due to the low temperature $\left(-2^{\circ} \mathrm{C}\right)$ of the circulating water. This is reflected in the respective unit cell parameters, where the calculated PXRD distances and volume for ethanol/TATM are slightly, but consistently, smaller than the experimental room temperature single crystal unit cell parameters.

The PXRD patterns of three polycrystalline guest-free samples, along with a calculated pattern based on the unit cell parameters for a guest-free single crystal, appear in Figure XV-8. From this figure, it is easy to conclude that all three starting materials produce practically identical guest-free phases when heated in water, and also that the bulk guest-free products were characteristic of the measured single crystal.

Table XV- 6 depicts the unit cell parameters for the guest-free TATM samples, both experimental single crystal and calculated PXRD. The single crystal unit cell parameters were obtained at $173 \mathrm{~K}$, while the powder unit cell parameters were calculated based on the PXRD patterns (Figure XV-8) at room temperature, as mentioned above. This again results in an increase in the unit cell dimensions and volume on going from $173 \mathrm{~K}$ to room temperature. Guest-free TATM prepared from ethanol/TATM seems to have the largest unit cell dimensions and volume, next comes guest-free TATM from acetone/TATM, and that from methanol/TATM is the smallest. These results may be correlated to the DSC results (see below). Empty TATM (from ethanol / TATM) has the largest unit cell volume and both the lowest melting point and enthalpy of decomposition. This is perhaps expected, as the guest-free form with the weakest intermolecular interactions should have both the largest volume and the lowest $\Delta_{\text {tus }} H$. Guest-free TATM from methanol and acetone have smaller, and practically identical unit cell dimensions and volume. As expected, they have both melting temperatures and enthalpies of decomposition that are significantly larger than the guest-free phase prepared from ethanol/TATM. 


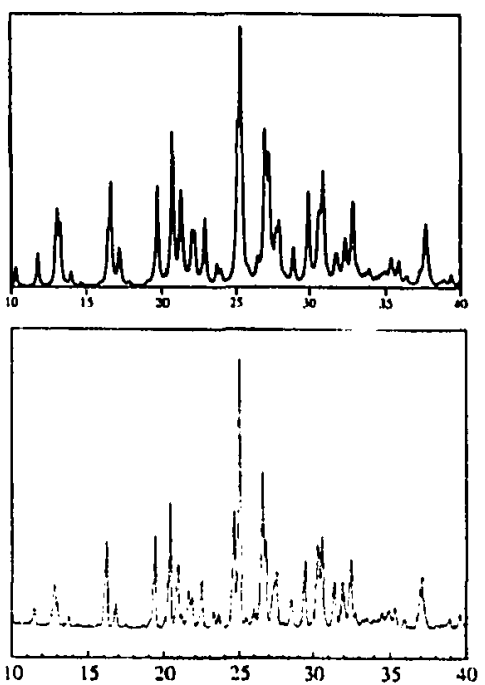

A

$\mathrm{B}$
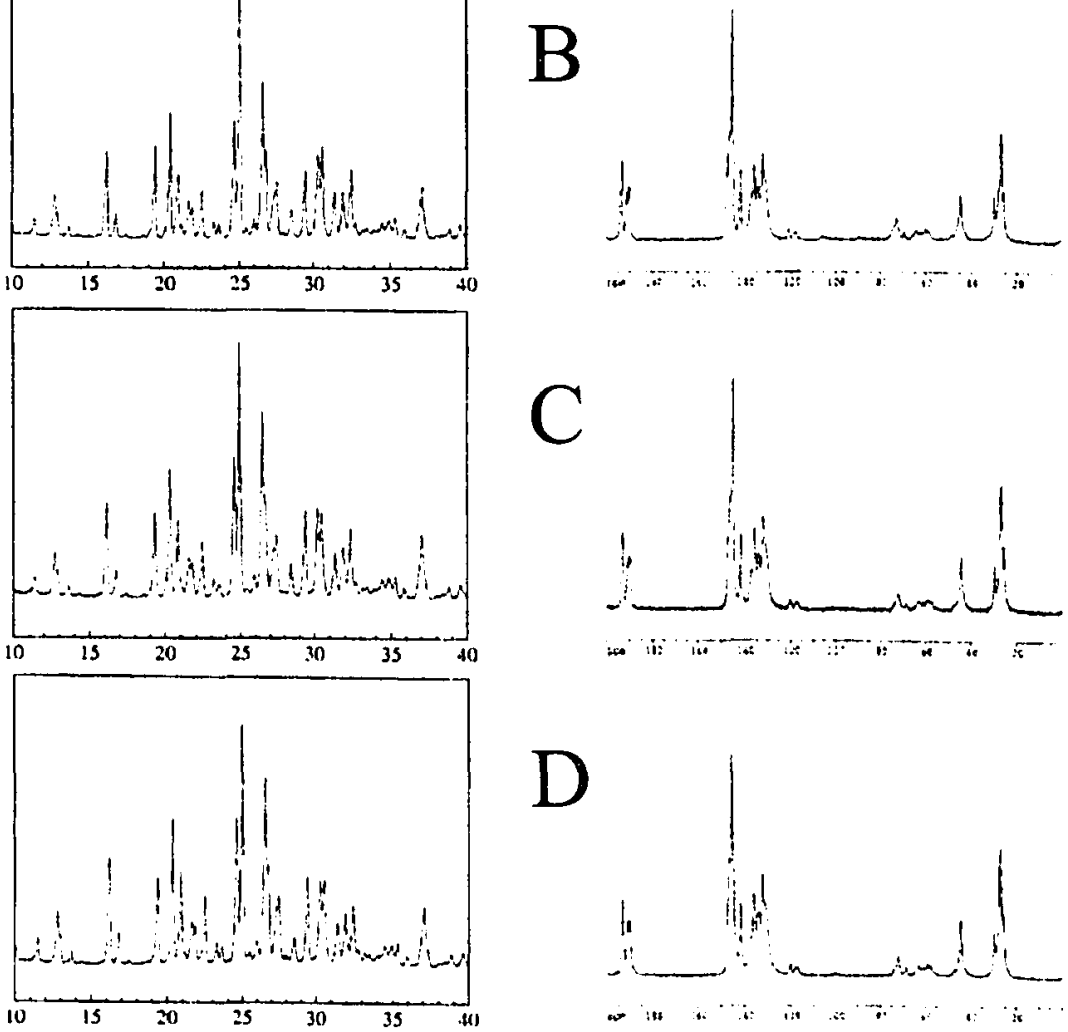

Figure XV-8: PXRD patterns (left column) and ${ }^{13} \mathrm{C} \mathrm{CP/MAS} \mathrm{solid-state} \mathrm{NMR} \mathrm{spectra}$ (right column) for the three TATM crystalline guest-free samples:

(A) calculated PXRD pattern based on single crystal unit cell parameters

(B) guest-free TATM (prepared from acetone / TATM)

(C) guest-free TATM (prepared from methanol / TATM)

(D) guest-free TATM (prepared from ethanol / TATM) 
Table XV-6: Single crystal (esd in brackets) and powder X-ray diffraction (within $0.003 \%, 0.009 \%$ and $1.7 \%$ for unit cell dimensions, angles and volume, respectively) unit cell parameters for the three polycrystalline TATM guest - free materials (at room temperature), and also for the single crystal of guest-free TATM, prepared from a sample of methanol / TATM single crystals (at $173 \mathrm{~K}$ ). Note expansion of unit cell dimensions on going from $173 \mathrm{~K}$ to room temperature $(a, b, c, V)$

$$
\begin{array}{llll}
\text { GF (methanol) } & \text { GF (ethanol) } & \text { GF (methanol) } & \text { GF (acetone) } \\
\text { SC (173 K) } & \text { POW (RT) } & \text { POW (RT) } & \text { POW (RT) }
\end{array}
$$

\begin{tabular}{lllll}
\hline$a(\AA)$ & $10.5004(12)$ & 10.5542 & 10.5352 & 10.5381 \\
$b(\AA)$ & $12.5300(15)$ & 12.6602 & 12.6552 & 12.6462 \\
$c(\AA)$ & $14.8353(17)$ & 14.9248 & 14.9007 & 14.9126 \\
$\alpha\left(^{\circ}\right)$ & $82.316(2)$ & 82.203 & 82.242 & 82.223 \\
$\beta\left(^{\circ}\right)$ & $72.311(2)$ & 72.384 & 72.321 & 72.340 \\
$\gamma\left({ }^{\circ}\right)$ & $88.529(2)$ & 88.405 & 88.406 & 88.430 \\
$V\left(\AA^{3}\right)$ & $1842.7(4)$ & 1882.9 & 1875.3 & 1876.1 \\
\hline
\end{tabular}

Finally, the PXRD pattern of vacuum sublimed (amorphous) guest-free TATM appears in Figure XV-9B. The pattern displays a broad, featureless hump from about $16^{\circ}<2 \theta<$ $40^{\circ}$, reflecting its amorphous character, which comes from the molecules in this phase experiencing a broad distribution of electronic environments.
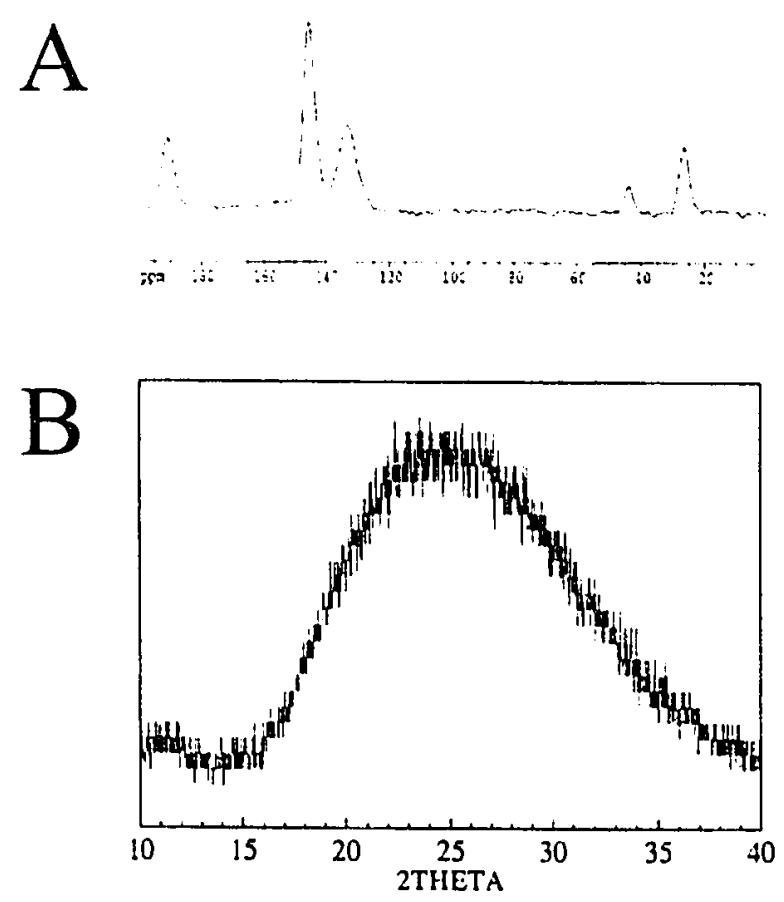

Figure XV-9: (A) ${ }^{13} \mathrm{C}$ CP/MAS solid-state NMR spectrum and (B) PXRD pattern of vacuum sublimed (amorphous) guest-free TATM. 


\section{${ }^{13} \mathrm{C}$ CP/MAS NMR}

In Figure XV-7 is depicted the ${ }^{13} \mathrm{C}$ CP/MAS NMR spectra of the three starting materials. As was already concluded above from PXRD observations, the ${ }^{13} \mathrm{C}$ CP/MAS NMR spectra also indicate that all three bulk starting materials have different structures, based on the uniqueness of the splitting patterns of the host resonances at 190-195 ppm (carbonyl), 130-150 ppm (thienyl) and 25-30 ppm (methyl). However, all three spectra indicate that there is one TATM molecule in each of the three respective asymmetric units. The multiplicity of the three carbonyl resonances and the three methyl resonances are all three, while the multiplicity of all three thienyl ring resonances are six for each of the two types of thienyl ring carbons (tertiary carbons at $\sim 134 \mathrm{ppm}$ and quaternary carbons at $\sim 146 \mathrm{ppm}$ )

It is also interesting to note that the carbonyl and methyl host ${ }^{13} \mathrm{C} \mathrm{CP} / \mathrm{MAS} N M R$ resonances for methanol/TATM appear as broad singlets. We have never encountered this before, namely all three carbons in the asymmetric unit (carbonyl and methyl) resonating at similar (non-resolved) frequencies. Despite this, we may still conclude that in this case there is only one host molecule in the asymmetric unit, since both the quaternary and tertiary thienyl ring signals each show a multiplicity of six. Since there are six quaternary thienyl carbons and six tertiary thienyl carbons in one TATM molecule, it is apparent that the asymmetric unit contains only one host molecule.

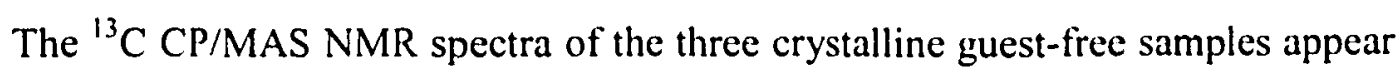
in Figure XV-8. Again, as was concluded above from PXRD, the ${ }^{13} \mathrm{C}$ CP/MAS NMR spectra indicate that the three guest-free samples are of the same phase. The splitting patterns from the carbonyl, thienyl and methyl carbons of the host are all practically identical. Furthermore, all three NMR spectra indicate that there are now two host molecules in the asymmetric unit. For all three carbonyl carbon signals at $190-195 \mathrm{ppm}$, the multiplicities from lowest to highest field are $3,1,1,1$. For all three methyl carbon signals at $25-30 \mathrm{ppm}$, the multiplicities from lowest to highest field are 1,2,2,1. These results suggest that there is a rearrangement of the host lattice as the guest is removed. If the guest was removed without rearrangement of the host lattice, one would likely see only one host molecule in the asymmetric unit for all three guest-free samples. This suggests that the original host lattice becomes unstable when the guest is removed, and a 
lower energy structure is attained in which intermolecular interactions change from a combination of host-host and host-guest to exclusively host-host.

The ${ }^{13} \mathrm{C}$ CP/MAS NMR spectrum of vacuum sublimed (guest-free) TATM appears in Figure XV-9A. As was shown above, the ${ }^{13} \mathrm{C}$ powder NMR spectrum also suggests that the material is amorphous, as is evidenced by the broad singlets for each of the five host NMR signals. This is in contrast to TATM inclusion compounds, where the detailed sharp splitting patterns give information on the crystal symmetry in each case.

(D) Thermogravimetric analysis (TGA)

The TGA traces for each pair of starting material and its associated guest-free product appear in Figure XV-10. For each pair, the solid line corresponds to the starting material, while the dashed line refers to the sample that was heated in water for 1 week at $70^{\circ} \mathrm{C}$. The fact that the TGA curve displays no significant guest loss for the latter samples clearly shows that the hot water removed practically all the guest from each of the three starting materials. For the three starting materials, significant guest loss starts around $75^{\circ} \mathrm{C}$, and continues up to about $225^{\circ} \mathrm{C}$. At higher temperatures, for both the starting materials and also the guest-free products, the TATM host residue starts to decompose. Another feature of the three starting material curves is the inflection point around $100^{\circ} \mathrm{C}$, which corresponds to the melting point of the compounds. Before the inflection point, guest loss occurs very rapidly, due to extensive guest desorption pathways arising from a large surface area. After the starting material melts, guest loss occurs much more slowly. This is because the sample now exists basically as a molten mass, with a much smaller surface area. The difference between the inflection point temperatures (from TGA) and the melting temperatures (from DSC), especially for acetone/TATM $\left(126.0^{\circ} \mathrm{C}\right.$ ) and ethanol/TATM $\left(115.1^{\circ} \mathrm{C}\right)$ may be due to the nature of different measuring techniques. TGA samples sit in an open pan with circulating nitrogen gas, so the sample may melt at a lower temperature. On the other hand, the samples for DSC were placed in hermetically sealed pans, specified as being capable of withstanding up to 5 atmospheres of pressure, which may make them melt at a higher temperature. Finally, the TGA curve for vacuum sublimed TATM (from ethanol/TATM) appears in Figure XV-11, and its 
negligible guest loss suggests that there is practically no ethanol solvent remaining inside the structure.
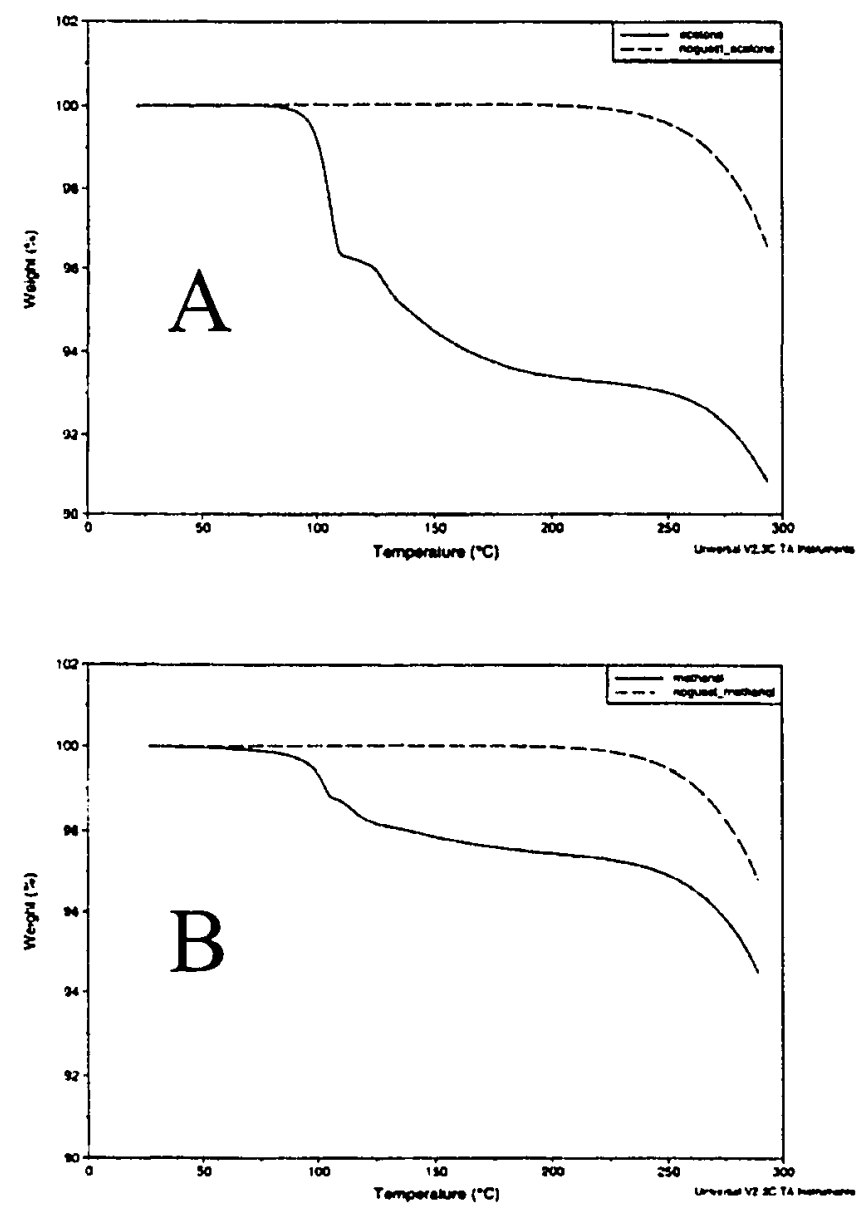

Figure XV-10: TGA traces of the three guest-free materials (dashed lines) along with their respective starting materials (solid lines):
(A) acetone / TATM
(B) methanol / TATM
(C) ethanol / TATM

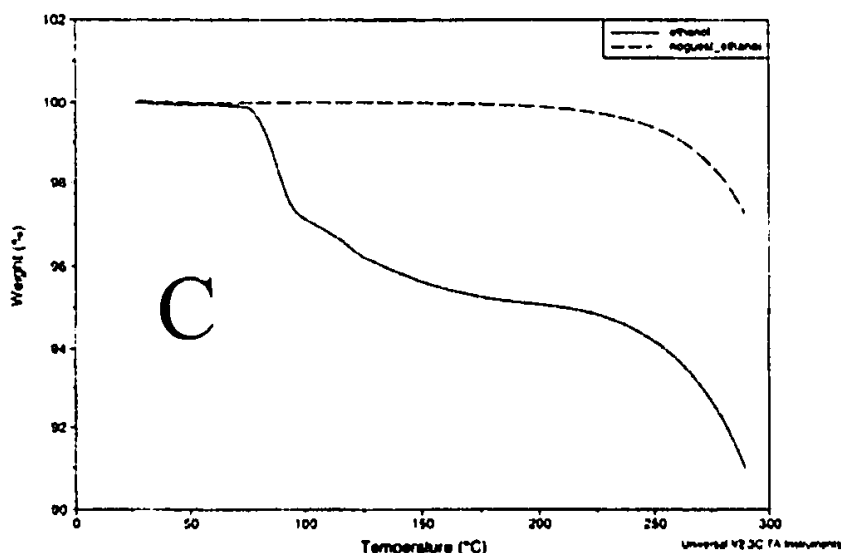




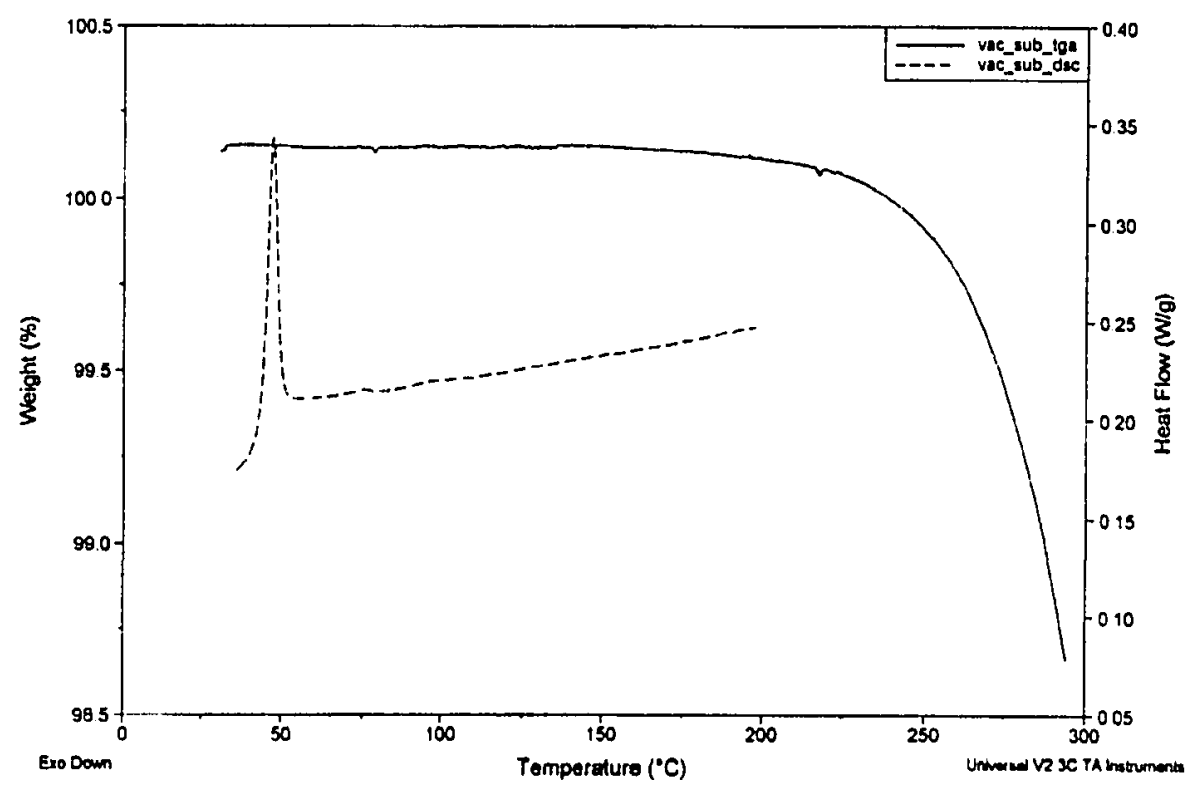

Figure XV-11: TGA (solid line-left axis) and DSC (dashed line-right axis) traces for vacuum sublimed (sample A) guest-free TATM.

(E) Differential scanning calorimetry (DSC)

The DSC traces for the three starting materials and the three guest-free samples are shown in Figure $X V-12$. The melting temperatures and the enthalpy of decomposition for these six samples are tabulated in Table XV-7. As depicted in the table, for all three starting materials, the melting temperature correlates with the heat of fusion. Acetone/TATM has the highest melting temperature of the three starting materials $T_{\text {m }}$ (onset) $=126.0^{\circ} \mathrm{C}$, and also the highest enthalpy of fusion, $\Delta_{\mathrm{fus}} H=46 \mathrm{~kJ} \mathrm{~mol}^{-1}$. Next comes ethanol/TATM $\left(115.1^{\circ} \mathrm{C}\right.$ and $\left.33 \mathrm{~kJ} \mathrm{~mol}^{-1}\right)$ and finally methanol/TATM $\left(101.0^{\circ} \mathrm{C}\right.$ and $27 \mathrm{~kJ} \mathrm{~mol}^{-1}$ ). Thus, in this case, the starting material with the highest enthalpy of fusion also has the highest melting temperature. In general, melting temperatures are not easily related to any particular property or properties of the sample, however in this case we see a definite correlation between the melting temperature and the enthalpy of fusion. Since $\Delta_{\text {fus }} S=\Delta_{\text {tus }} H / T_{\mathrm{m}}$, this implies that the entropy of fusion is relatively constant. However, the values vary significantly, namely $115 \mathrm{~J} \mathrm{~mol}^{-1} \mathrm{~K}^{-1}, 85 \mathrm{~J} \mathrm{~mol}^{-1} \mathrm{~K}^{-1}$ and $72 \mathrm{~J}$ $\mathrm{mol}^{-1} \mathrm{~K}^{-1}$, for the acetone, ethanol and methanol inclusions, respectively. 

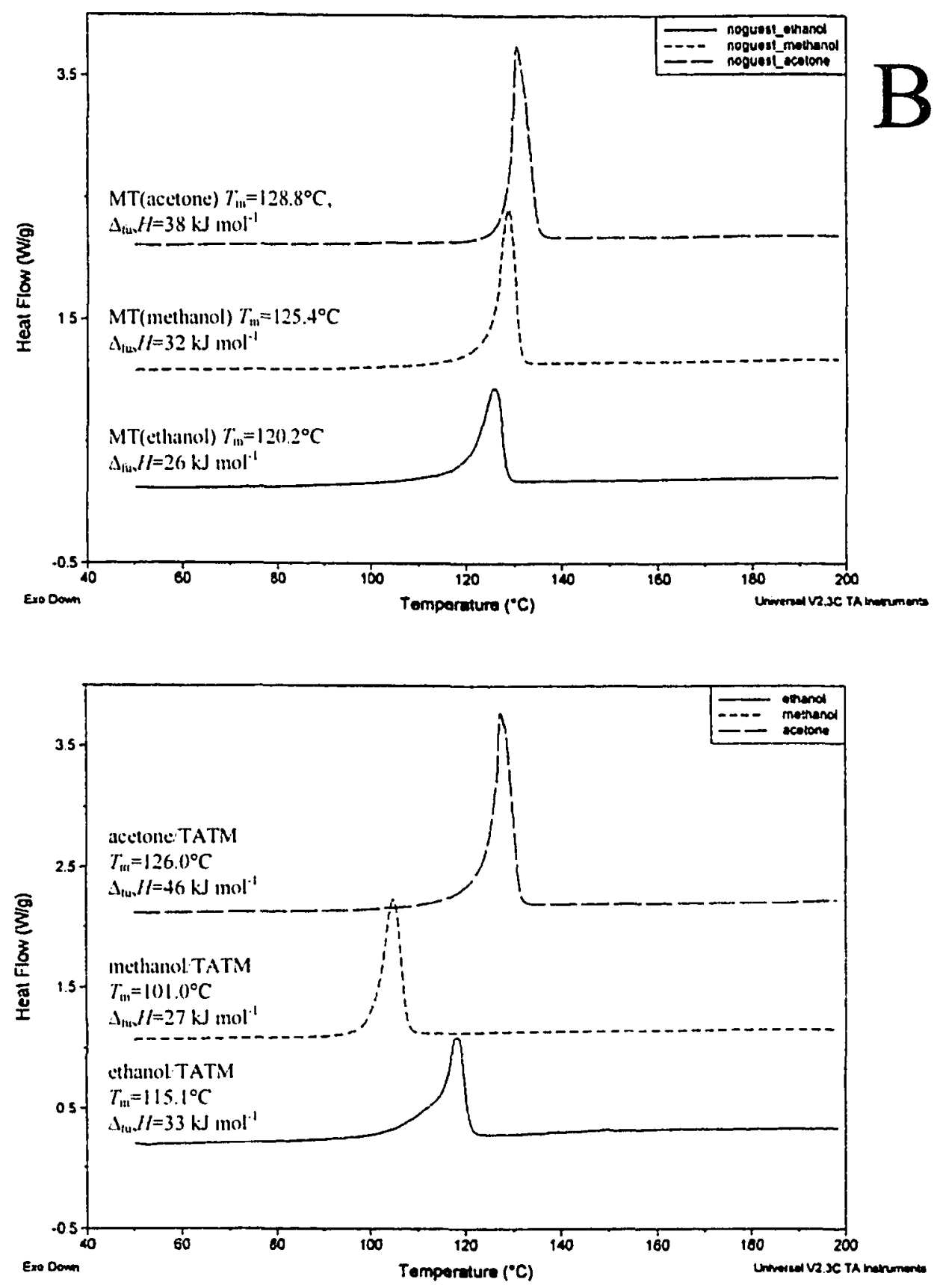

Figure XV-12: DSC traces for the three crystalline guest-free TATM samples, along with their respective starting materials. The traces were displaced along the vertical axis.
(A) Guest-free samples:
solid line - from ethanol / TATM
(B) Starting materials:
short dash - from methanol / TATM
solid line - ethanol / TATM
long dash - from acetone / TATM
short dash - methanol / TATM
long dash - acetone / TATM 
Table XV-7: Differential scanning calorimetric data for the three starting materials and the guest-free samples.

\begin{tabular}{|c|c|c|c|}
\hline Compound & $T_{\ln }\left( \pm 0.1^{\circ} \mathrm{C}\right)$ & $\Delta_{\text {fius }} H\left( \pm 1 \mathrm{~J} \mathrm{~g}^{-1}\right)$ & $\Delta_{\text {lus }} H\left( \pm 1 \mathrm{~kJ} \mathrm{~mol}^{-1} \mathrm{TATM}\right)$ \\
\hline acetone/TATM & 126.0 & 118 & 46 \\
\hline ethanol/TATM & 115.1 & 86 & 33 \\
\hline methanol/TATM & 101.0 & 69 & 27 \\
\hline guest-free (acetone) & 128.8 & 97 & 38 \\
\hline guest-free (methanol) & 125.4 & 82 & 32 \\
\hline guest-free (ethanol) & 120.2 & 67 & 26 \\
\hline
\end{tabular}

There are perhaps two ways in which one can interpret the DSC results of the three starting materials. The first would be to relate heat of fusion of the TATM inclusion compound with the molecular weight of the guest. Acetone/TATM has the highest molecular weight of the three guests $\left(58.1 \mathrm{~g} \mathrm{~mol}^{-1}\right)$, and the highest enthalpy of fusion. Next comes ethanol/TATM (46.1 $\left.\mathrm{g} \mathrm{mol}^{-1}\right)$, with an intermediate enthalpy of fusion. Finally, methanol/TATM, which has the smallest enthalpy of fusion, has the lowest guest molecular weight $\left(32.0 \mathrm{~g} \mathrm{~mol}^{-1}\right)$. So for these fairly polar guests, TATM seems to prefer larger, or perhaps more polarizable, guests. The other reasoning is to differentiate on the basis of hydrogen bonding capacity. For acetone/TATM, there is only a hydrogen bond acceptor on the guest (the carbonyl group). For ethanol, there is one hydrogen bond donor and one hydrogen bond acceptor. Finally, for the methanol/TATM inclusion compound, there is again one hydrogen bond donor and acceptor. However, in one gram of methanol, there are 46.1 / 32.0 more hydrogen bond donors and acceptors than in one gram of ethanol. These observations suggest that perhaps TATM prefers guest molecules with less hydrogen bond capacity.

As far as the guest-free samples are concerned, we see the same thermal behaviour, namely that each melting temperature may be related to its respective enthalpy of melting. The guest-free form from acetone/TATM has both the highest melting temperature $\left(128.8^{\circ} \mathrm{C}\right)$ and the highest enthalpy of melting $\left(38 \mathrm{~kJ} \mathrm{~mol}^{-1}\right)$. Next comes 
guest-free TATM from methanol/TATM $\left(125.4^{\circ} \mathrm{C}\right.$ and $\left.32 \mathrm{~kJ} \mathrm{~mol}^{-1}\right)$, while guest-free TATM from ethanol/TATM has both the lowest melting temperature $\left(120.2^{\circ} \mathrm{C}\right)$ and heat of melting $\left(26 \mathrm{~kJ} \mathrm{~mol}^{-1}\right)$. The entropy of fusion goes as $95 \mathrm{~J} \mathrm{~mol}^{-1} \mathrm{~K}^{-1}, 80 \mathrm{~J} \mathrm{~mol}^{-1} \mathrm{~K}^{-1}$ and $66 \mathrm{~J} \mathrm{~mol}^{-1} \mathrm{~K}^{-1}$ for the guest-free samples from the acetone, methanol and ethanol starting materials, respectively.

One may ask why the different guest-free materials have significantly different thermal behaviour, when, as judged by their powder X-ray diffraction patterns and ${ }^{13} \mathrm{C}$ CP/MAS NMR spectra, they are all practically isostructural. The answer may lie in their residual guest and water content. From ' $\mathrm{H}$ solution NMR, we found that each of the three guest-free forms have a small amount of guest that was not removed by the hot water, and also that some of the water was left behind. In Figure XV-13, the ' $\mathrm{H}$ solution NMR spectra of acetone/TATM (top), and the spectra of guest-free TATM from acetone/TATM (bottom) are shown. In the guest-free sample, the weak signals corresponding to the residual guest and water content is integrated. These integrals were compared to the signal from the central methine $\mathrm{C}-\mathrm{H}$ proton of the TATM host (at about $5.6 \mathrm{ppm}$ ), to obtain the residual guest and water content. The results are shown in Table XV-8. The guest-free sample from acetone/TATM has the lowest amount of residual guest $(0.042 \%$ by mass), and not surprisingly, has the highest melting temperature $\left(128.8^{\circ} \mathrm{C}\right)$ and highest heat of decomposition of the three guest-free samples. Next come the guest-free samples from ethanol/TATM and methanol/TATM, with higher residual guest content $(0.173 \%$ and $0.174 \%$ respectively). They have lower melting temperatures $\left(120.2^{\circ} \mathrm{C}\right.$ and $125.4^{\circ} \mathrm{C}$, respectively) and lower enthalpies of fusion. So we see that the residual guest acts as an "impurity" on the completely pure guest-free TATM, with larger residual contents giving rise to a larger and larger melting point depression. The DSC data suggest that the presence of more and more residual guest leads to a progressive decline in melting temperature and heat of fusion, when compared to the completely guest-free form, and that even miniscule amounts of impurities can have a profound effect on the thermal properties of a compound. 


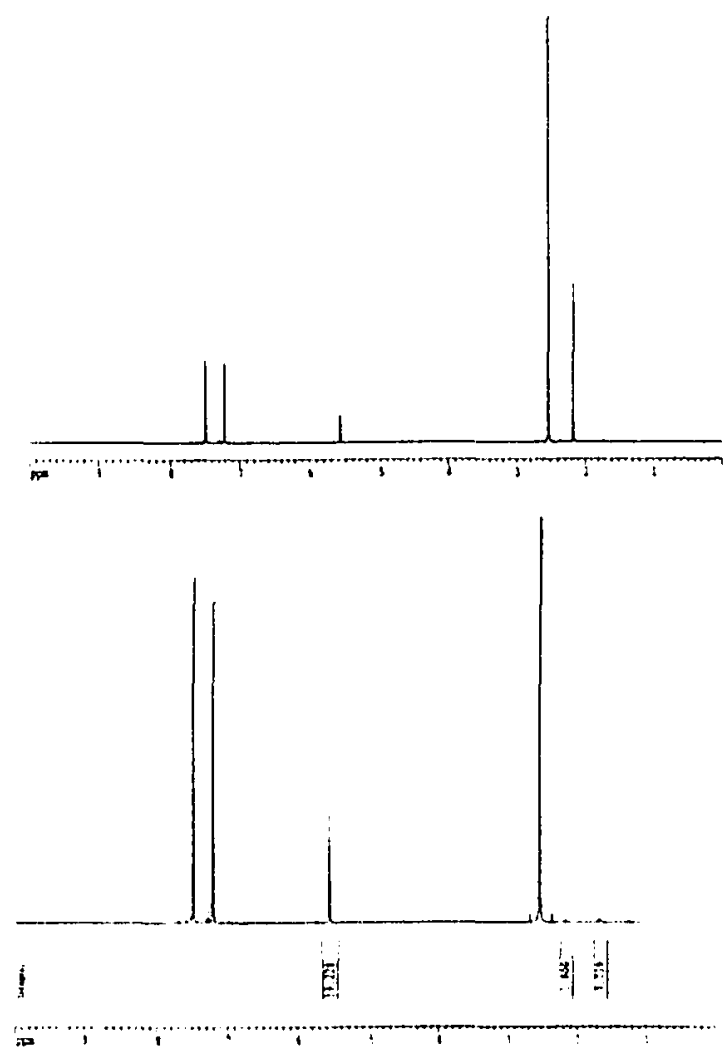

Figure XV-13: 'H solution NMR of acetone/TATM (above) and the guest-free material prepared from acetone/TATM (below). The integrals for the residual guest and left-over water are indicated. Please refer to Table XV-8 for details.

Table XV-8: Residual guest and residual water content of guest-free TATM samples (as measured by solution ' $\mathrm{H}$ NMR in $\mathrm{CDCl}_{3}$ )

\begin{tabular}{lllll} 
Fragment/molecule & $\begin{array}{l}\text { Signal integral } \\
( \pm 0.01)\end{array}$ & $\begin{array}{l}\mathrm{H} \text { integral } \\
( \pm 0.01)\end{array}$ & $\begin{array}{l}\text { Percent by mass } \\
( \pm 0.001 \%)\end{array}$ & $\begin{array}{l}\text { Mole fraction } \\
( \pm 0.001)\end{array}$ \\
\hline Acetone/TATM & 1.00 & 0.17 & 0.042 & \\
$\mathrm{CH}_{3}$ (guest) & 7.74 & 3.87 & 0.302 & 0.003 \\
$\mathrm{H}_{2} \mathrm{O}$ & 59.23 & 59.23 & & 0.069 \\
$\mathrm{CH}$ (host) & & & & \\
& 1.00 & 0.50 & 0.173 & 0.015 \\
Ethanol/TATM & 4.31 & 2.15 & 0.292 & 0.064 \\
$\mathrm{CH}_{2}$ (guest) & 34.01 & 34.01 & & \\
$\mathrm{H}_{2} \mathrm{O}$ & & & \\
$\mathrm{CH}$ (host) & & & & \\
Methanol/TATM & 1.00 & 0.33 & 0.174 & 0.021 \\
$\mathrm{CH}$ (guest) & 2.18 & 1.09 & 0.320 & 0.069 \\
$\mathrm{H}_{2} \mathrm{O}$ & 15.73 & 15.73 & & \\
$\mathrm{CH}$ (host) & & & &
\end{tabular}

On the other hand, the "left-over" water does not display as strong a correlation effect on the thermal properties as does the residual guest. The guest-free sample with greatest 
$\Delta_{\text {fus }} H$, from acetone/TATM, has a residual water content of $0.302 \%$ (see Table XV-8). The guest-free samples from ethanol/TATM and methanol/TATM have water contents of $0.292 \%$ and $0.320 \%$ respectively, one lower and one higher than that for the acetone/TATM guest-free product. This perhaps suggests that small amounts of water do not have as strong an influence on the thermal properties of guest-free TATM compounds as does the residual guest content. The fact that a TATM inclusion compound with water was reported [18] suggests that the water may actually form part of the lattice structure, rather than being simply adsorbed on the surface.

Finally, the DSC curve for amorphous vacuum sublimed (guest-free) TATM appears in Figure XV-11. The DSC melting endotherm for this sample is a relatively sharp peak with an onset melting temperature of $43.8^{\circ} \mathrm{C}$, much lower than most all TATM inclusion compounds. The anomalous thermal behaviour of this product is further demonstrated by the very low enthalpy of decomposition, only $3 \mathrm{~kJ} \mathrm{~mol}^{-1}$. This observation is unusual, as amorphous materials are generally not expected to show a melting endotherm, but rather usually exhibit a glass transition. This observation perhaps suggests that this sample (vacuum sublimed sample $\mathbf{A}$ ) is partially crystalline, whose melting may have given rise to the sharp (however not very intense) melting endotherm. It is possible that the long time lag in between vacuum sublimation and DSC characterization for this sample (vacuum sublimed sample $\mathbf{A}$ ), on the order of weeks, may have allowed some of the amorphous regions of the sample to crystallize (at room temperature). On the other hand, the sample in Figure XV-14 (vacuum-fused sample B, see below) was characterized almost immediately after vacuum fusion, which may have avoided the partial crystallization that characterized vacuum sublimed sample $\mathbf{A}$.

The transformation of an amorphous material (e.g. a polymer) from a glass to a rubber phase at the glass transition temperature, $T_{\mathrm{g}}$, is perhaps the most significant of the transitions observed in such a material. A simplistic view of $T_{\mathrm{g}}$ is the temperature below which the individual molecules have very little mobility. On a larger scale, polymers are rigid and brittle below $T_{\mathrm{g}}$ and can undergo plastic deformation above it. The glass transition temperature is usually applicable to amorphous phases such as glasses and plastics. 
The importance of the glass transition is reflected in the considerable body of literature on the subject. A number of review articles give a good overview of the main experimental observations and theoretical models associated with the glass transition [2434]. The technological significance of the glass transition is considerable, in that it marks the upper temperature limit of application for the glass phase, and the lower temperature limit for the rubber phase. The properties of a given material on either side of this dramatic boundary are so different that the two solid forms of glass and rubber would be hard to recognize as having the same chemical structure.

The main changes observed with increasing temperature through $T_{\mathrm{g}}$ are a dramatic reduction in the mechanical properties of both elastic modulus and viscosity in moving from a rigid glass to a relatively flexible rubber-like phase, and an increase in specific heat capacity and coefficient of thermal expansion. These changes in physical properties are generally affected by factors such as rate of observation, thermal history of the sample, pressure, chemical composition and molecular weight.

As was noted above, the observation of a melting endotherm is unusual for an amorphous material. In an attempt to ensure that the results were reproducible, a second sample (vacuum-fused sample B) was examined, however the DSC thermal program was started from low temperature $\left(-125^{\circ} \mathrm{C}\right)$, immediately after the sample was prepared by melting $\left(150^{\circ} \mathrm{C}\right)$ in a vacuum oven. In this case a transition was observed at $-16^{\circ} \mathrm{C}$, the nature of which (see Figure XV-14) is fairly typical of a glass transition, characterized by a change in baseline level, corresponding to a difference in heat capacity between the two phases. The most likely explanation for a melting endotherm of Figure XV-11 (vacuum sublimed sample $\mathbf{A}$ ) is that a significant amount of crystalline material formed on storage, which dissolved in the supercooled liquid when it softened around $50^{\circ} \mathrm{C}$. These observations suggest that sample $\mathbf{B}$ is the more definitive amorphous sample, with the sample A results suggesting that the sample may evolve on storage.

We wish to make note that the calibration of the two DSC traces (in Figures XV11 and $\mathrm{XV}-14)$ are different. In Figure XV-11, endothermic transitions point upward, while in Figure XV-14, endothermic transitions point downward; this also explains the differences in baseline level transition between the two figures. The same heating rate was used in both experiments $\left(5^{\circ} \mathrm{C}\right.$ per minute). 


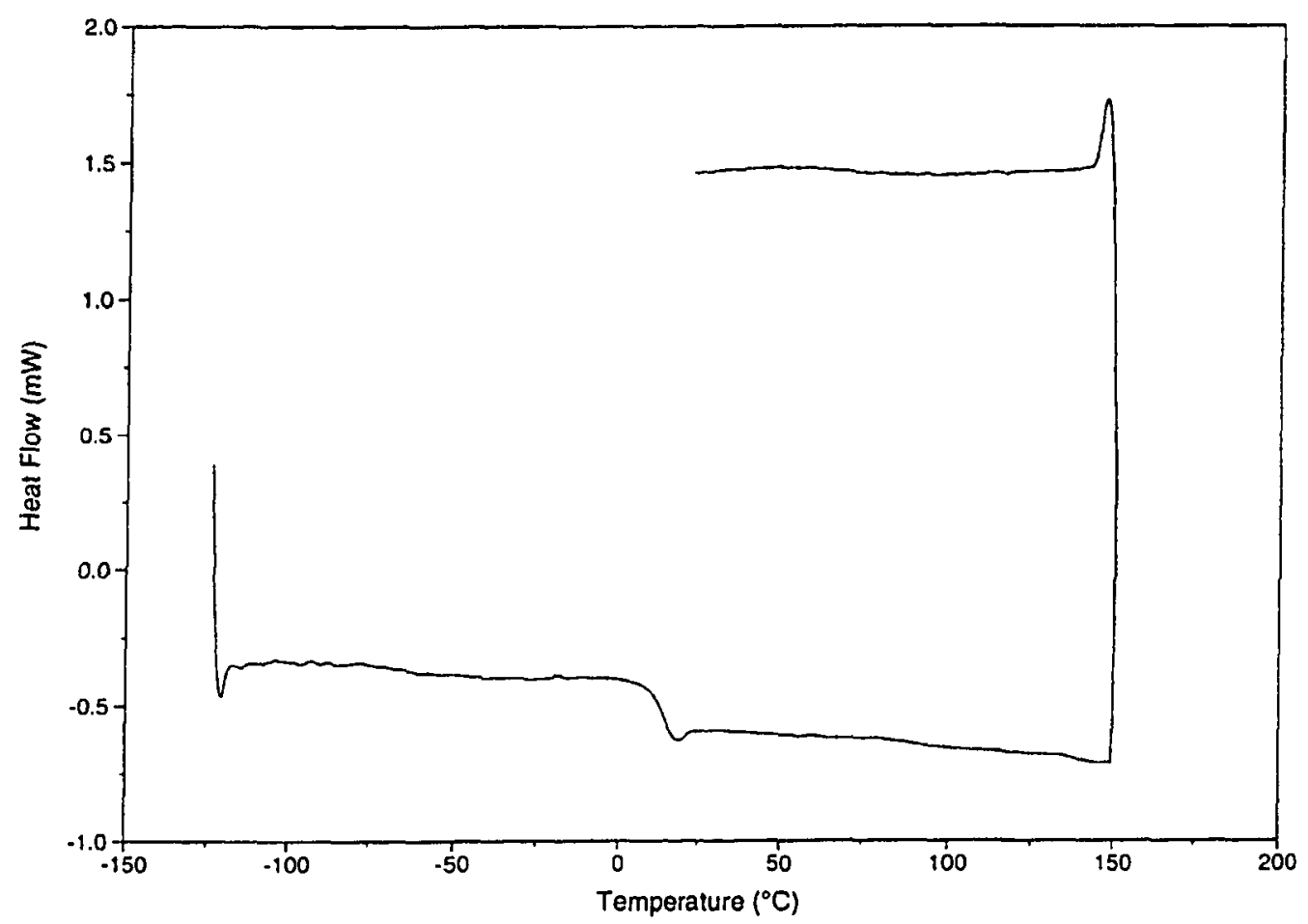

Figure XV-14: DSC trace for vacuum-fused (amorphous) guest-free TATM (sample B). The temperature program started at $-125^{\circ} \mathrm{C}$, heated at $5^{\circ} \mathrm{C}$ per minute to $150^{\circ} \mathrm{C}$, then cooled to room temperature at the same rate. Notice the change in baseline level at $18.6^{\circ} \mathrm{C}$, suggestive of a glass transition for this sample.

(F) Polycrystalline density measurements

The polycrystalline densities, as measured by a gas pycnometer, appear in Table XV-9. In this table, the densities of the three starting materials and the three guest-free samples are listed. For all three, the density of the guest-free form is significantly greater than its respective starting material. This suggests that there are more attractive intermolecular interactions between the TATM host molecules of the guest-free samples when compared to the combination of host-guest and host-host interactions of the three starting materials. This is also reflected in the DSC melting temperatures, where for all three samples, the melting point of the guest-free material is significantly greater than that of its parent compound (see Table XV-7). On the other hand, the heat of decomposition fails to show a similar correlation.

For the three starting materials, acetone/TATM has the greatest density $(1.344 \mathrm{~g}$ $\left.\mathrm{cm}^{-3}\right)$. This is reflected in its highest melting temperature $\left(126.0^{\circ} \mathrm{C}\right)$ and the highest enthalpy of decomposition $\left(46 \mathrm{~kJ} \mathrm{~mol}^{-1}\right)$. Next comes ethanol/TATM $\left(1.337 \mathrm{~g} \mathrm{~cm}^{-3}\right)$, 
with a lower melting temperature $\left(115.1^{\circ} \mathrm{C}\right.$ ) and a lower enthalpy of decomposition (33 $\left.\mathrm{kJ} \mathrm{mol}{ }^{-1}\right)$. Finally, methanol/TATM has the lowest density $\left(1.331 \mathrm{~g} \mathrm{~cm}^{-3}\right)$, and as expected, has the lowest melting temperature $\left(101.0^{\circ} \mathrm{C}\right)$ and the lowest enthalpy of decomposition $\left(27 \mathrm{~kJ} \mathrm{~mol}^{-1}\right)$. So we see a very interesting relationship between polycrystalline density and thermal behaviour.

Table XV-9: Polycrystalline densities measured in bulk using a pycnometer apparatus. Each experiment was repeated in duplicate.

\begin{tabular}{|c|c|c|c|c|}
\hline & Trial & $\left(\mathrm{g} \mathrm{cm}^{-3}\right)$ & Trial $2\left(\mathrm{~g} \mathrm{~cm}^{-3}\right)$ & Average $\left(\mathrm{g} \mathrm{cm}^{-3}\right)$ \\
\hline Ethanol / TATM & 1.329 & $( \pm 0.001)$ & $1.346( \pm 0.002)$ & $1.337( \pm 0.002)$ \\
\hline Guest-free (ethanol) & 1.375 & $( \pm 0.002)$ & $1.384( \pm 0.004)$ & $1.379( \pm 0.003)$ \\
\hline Methanol / TATM & 1.328 & $( \pm 0.005)$ & $1.335( \pm 0.003)$ & $1.331( \pm 0.004)$ \\
\hline Guest-free (methanol) & 1.375 & $( \pm 0.002)$ & $1.377( \pm 0.001)$ & $1.376( \pm 0.001)$ \\
\hline Acetone / TATM & 1.342 & $( \pm 0.003)$ & $1.347 \quad( \pm 0.002)$ & $1.344( \pm 0.002)$ \\
\hline Guest-free (acetone) & 1.380 & $( \pm 0.002)$ & $1.369( \pm 0.001)$ & $1.374( \pm 0.002)$ \\
\hline $\begin{array}{l}\text { Vacuum sublimed } \\
\text { (guest-free) TATM }\end{array}$ & 1.348 & $( \pm 0.002)$ & $1.348 \quad( \pm 0.001)$ & $1.348 \quad( \pm 0.001)$ \\
\hline
\end{tabular}

On the other hand, the guest-free samples show exactly the opposite behaviour. Guestfree TATM (from ethanol/TATM) has the highest density $\left(1.379 \mathrm{~g} \mathrm{~cm}^{-3}\right.$ ), but the lowest melting temperature $\left(120.2^{\circ} \mathrm{C}\right)$ and the lowest heat of decomposition $\left(26 \mathrm{~kJ} \mathrm{~mol}^{-1}\right)$. Guest-free TATM (from methanol/TATM) has an intermediate density $\left(1.376 \mathrm{~g} \mathrm{~cm}^{-3}\right)$, with an intermediate melting temperature $\left(125.4^{\circ} \mathrm{C}\right)$ and heat of decomposition $(32 \mathrm{~kJ}$ $\mathrm{mol}^{-1}$ ). Finally, guest-free TATM (from acetone/TATM) has the lowest density $(1.374 \mathrm{~g}$ $\left.\mathrm{cm}^{-3}\right)$, but perhaps unexpectedly, the highest melting temperature $\left(128.8^{\circ} \mathrm{C}\right)$ and the highest enthalpy of decomposition $\left(38 \mathrm{~kJ} \mathrm{~mol}^{-1}\right)$ of the three. Why we see these two completely opposite trends may be very difficult to reconcile simply on the basis of the nature of the materials involved.

One possibility for these observations may be simply that the residual guest content of the guest-free samples has a stronger correlation with both the melting temperature and enthalpy of decomposition than does the polycrystalline density. As was mentioned above, guest-free TATM (from acetone/TATM) had the lowest residual guest 
content, and perhaps as a result, the highest melting temperature and enthalpy of decomposition. Guest-free TATM from ethanol and methanol inclusions display higher residual guest content and subsequently $T_{\mathrm{m}}$ and $\Delta_{\text {fus }} H$ values that are both lower than those mentioned above.

The polycrystalline density of vacuum sublimed (amorphous) guest-free TATM, as listed in Table XV-9, is $1.348 \mathrm{~g} / \mathrm{mL}$. This density is significantly lower than those for the three crystalline guest-free samples, which are all greater than $1.37 \mathrm{~g} / \mathrm{mL}$, which suggests that the amorphous guest-free product exhibits weaker intermolecular interactions, when compared to the crystalline guest-free materials.

\section{(G) Vapor diffusion into guest-free TATM}

After preparing crystalline guest-free TATM, we wished to determine if it was possible to incorporate small organic molecules into the guest-free TATM lattice from the gas phase. As a first attempt, we used the three starting material guests, namely ethanol, methanol and acetone. After exposing guest-free TATM to each of these three vapors at room temperature, we determined, based on ' $\mathrm{H}$ solution NMR, that the only one of the three guests to enter the TATM lattice was acetone. This is perhaps because acetone has a higher ambient vapor pressure (bp $56^{\circ} \mathrm{C}$ ) than both methanol $\left(\mathrm{bp} 65^{\circ} \mathrm{C}\right)$ and ethanol (bp $78^{\circ} \mathrm{C}$ ), or alternatively that acetone has no hydrogen bond donors, in contrast to methanol or ethanol. Indeed, of the three starting materials, acetone/TATM has the highest melting temperature and the highest enthalpy of decomposition (see Table XV-7). So for acetone/TATM, the energy gained by nucleation and crystallization from the vapor is perhaps large enough to pass over the energy barrier required to rearrange the host lattice to incorporate the acetone guest molecules. For ethanol/TATM and methanol/TATM, this energy barrier is not overcome by the nucleation / crystallization steps, so the inclusion compound is not formed from the vapor. Furthermore, we attempted to incorporate methanol and ethanol at elevated temperatures $\left(-50^{\circ} \mathrm{C}\right)$, but this was also unsuccessful.

These results suggest that guest-free TATM will only incorporate small volatile molecules from the vapor. So we went further and attempted to incorporate methylene chloride $\left(\right.$ bp $40^{\circ} \mathrm{C}$ ) and bromoethane $\left(\right.$ bp $37^{\circ} \mathrm{C}$ ) at room temperature. As judged by ${ }^{\prime} \mathrm{H}$ 
solution NMR, we were successful on both counts. In Figures XV-15, XV-16 and XV-17 we present powder X-ray diffraction patterns and ${ }^{13} \mathrm{C} C P / M A S$ NMR spectra for the vapor diffused products from acetone, methylene chloride and bromoethane respectively. In the bottom row of each figure are depicted the spectra of the conventional products recrystallized from solution. The top row of each figure displays the spectra prepared by vapor deposition. From these figures, it is clearly evident that acetone/TATM (Figure XV-15) and methylene chloride/TATM (Figure XV-16) each have the same structure regardless of whether the product was prepared by recrystallization or vapor adsorption. On the other hand, the spectra for bromoethane/TATM (Figure XV-17) suggest that there is a dependence of structural type upon crystallization conditions, indicating that this guest may crystallize as a polymorph, as was previously observed for cyclohexane [11] 1,2-dichloroethane (chapter VII) and 1,3-dichloropropane (chapter XII).
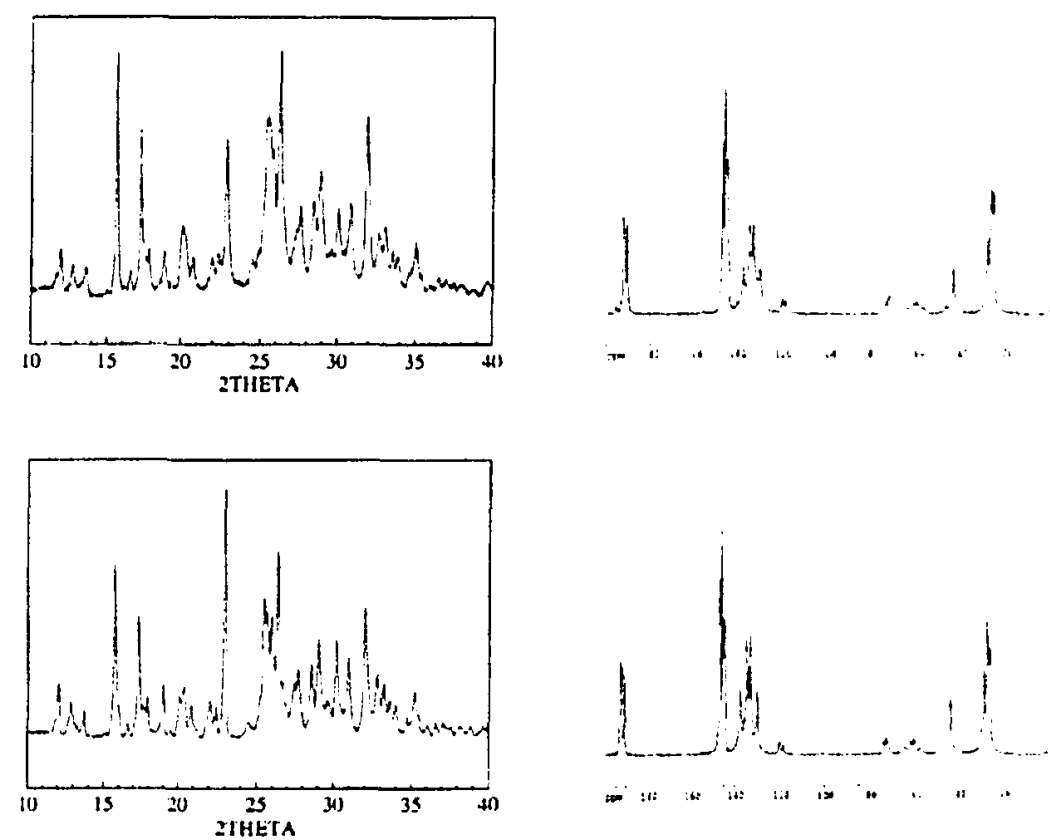

Figure XV-15: Powder X-ray diffraction patterns (left column) and ${ }^{13} \mathrm{C} C P / M A S ~ N M R$ spectra (right column) for acetone/TATM samples prepared by recrystallization from solution (bottom row) and by vapor deposition (top row). Note practically identical spectra in both columns. 

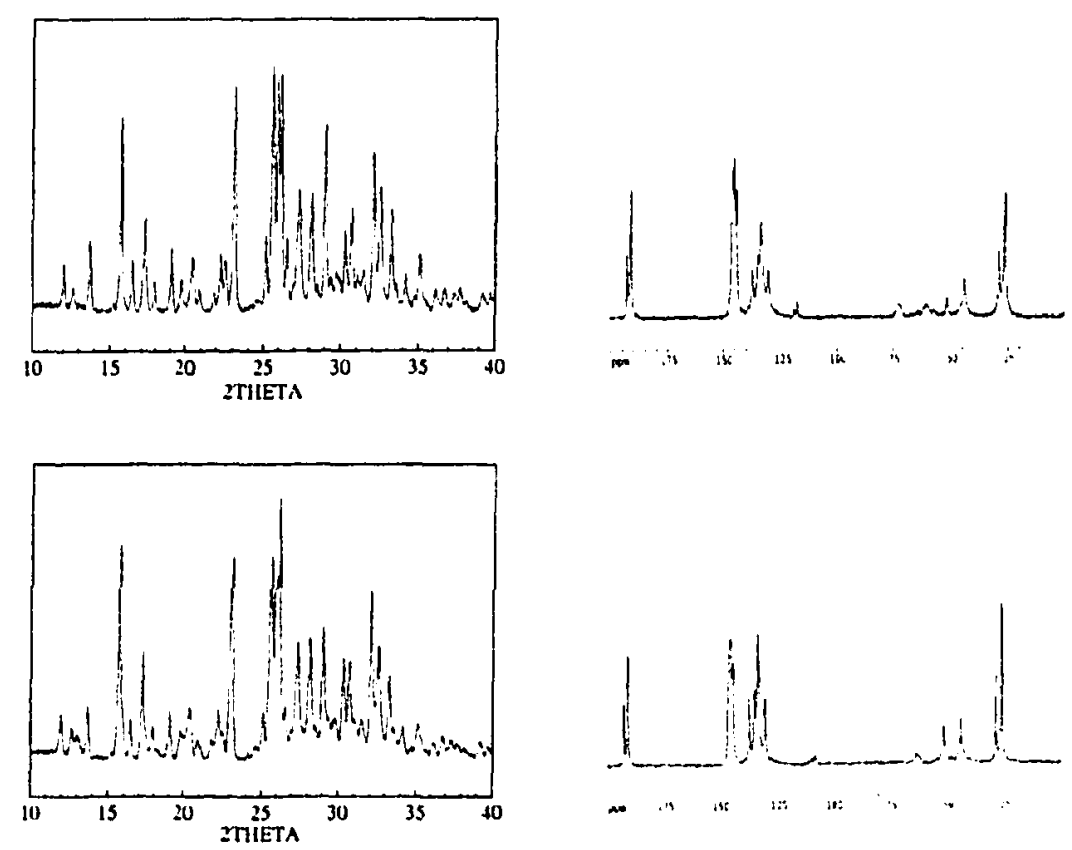

Figure XV-16: Powder X-ray diffraction patterns (left column) and ${ }^{13} \mathrm{C} \mathrm{CP/MAS} \mathrm{NMR}$ spectra (right column) for methylene chloride/TATM samples prepared by recrystallization from solution (bottom row) and by vapor deposition (top row). Note practically identical spectra in both columns.
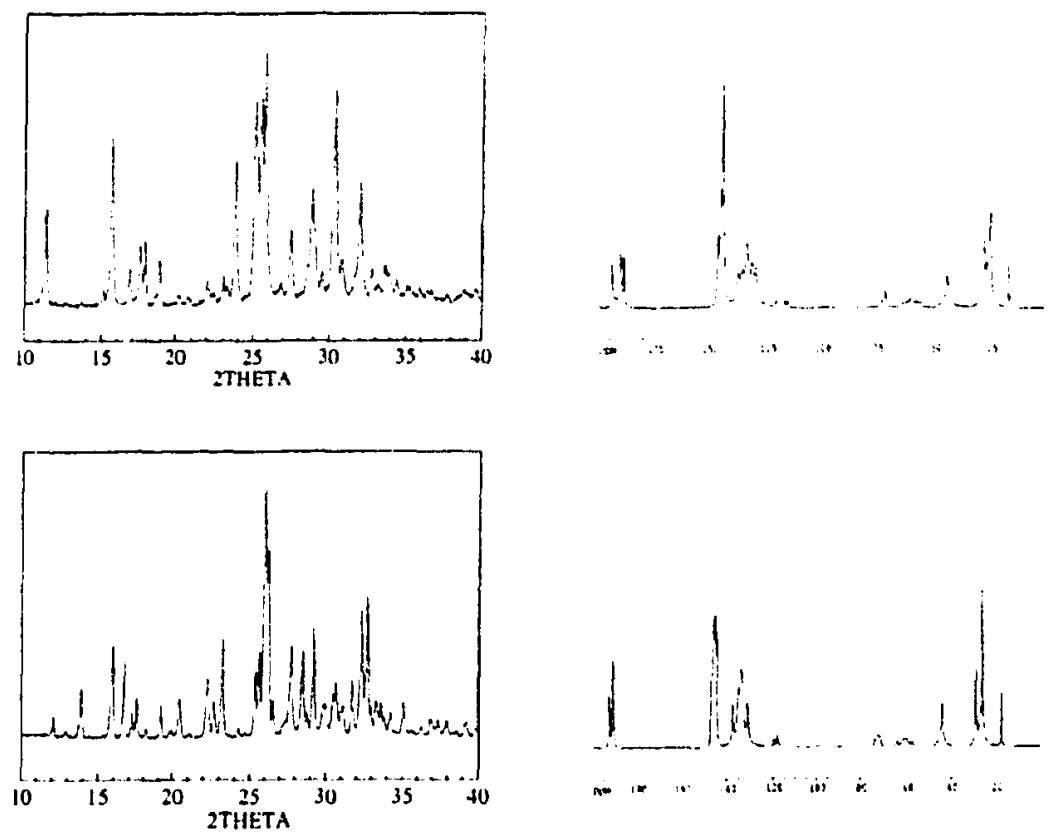

Figure XV-17: Powder X-ray diffraction patterns (left column) and ${ }^{13} \mathrm{C} \mathrm{CP/MAS} \mathrm{NMR}$ spectra (right column) for bromoethane/TATM samples prepared by recrystallization from solution (bottom row) and by vapor deposition (top row). Note significantly different spectra in both columns. 


\section{DISCUSSION}

(H) Effect of guest size upon host lattice

An interesting correlation, with regard to unit cell volume, as a function of guest size, concerning literature structures of tri-ortho-thymotide (TOT) inclusion compounds [3537], appears in Figure XV-18 [38]. For cage-type TOT structures of space group $P 3_{1}, 21$ [39], a direct relationship between the two variables is clearly evident. The guest volumes were calculated based on the sum of atom volume increments. In addition, similar statistically significant linear correlations have also been obtained for the cagetype TOT inclusion compounds within the (1) $P_{\mathrm{bca}}$ and (2) $P 3_{1}{ }^{*}$ space group structures [40]. The slopes of these three plots allow a quantitative determination of the "susceptibility" of the unit cell size to a change in volume of the guest. This calculation gives approximate cell expansion increments of about $1.5-2.0 \mathrm{~nm}^{3}$ per $1.0 \mathrm{~nm}^{3}$ increase in guest volume, suggesting a fairly small external response of the lattice dimensions, meaning that changes in the mutual arrangement of the host molecules are kept to a minimum under the action of internal influences, which suggests a relative invariance of the attractive forces between guest and host, which is a possible rationalization of TOT's very versatile host properties.

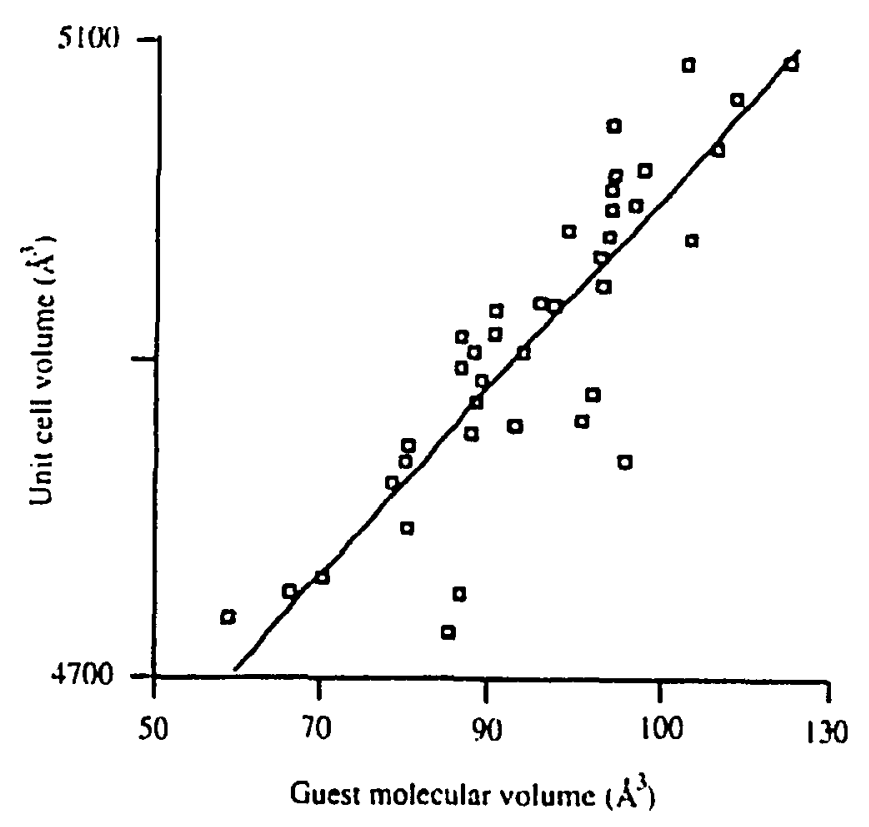

Figure XV-18: Correlation between unit cell volume and guest molecular volume for triortho-thymotide cage-type inclusion compounds [38] 
As far as channel-type TOT inclusions (with long, linear guests) are concerned ( $P 6_{1}, P 6_{2}$ or $P 3_{1}$ space groups) [41,42], one now observes ill-defined stoichiometric ratios between host and guest, which depends, to some extent, on the length of the guest molecule. The lack of congruence between host and guest periodicities is likely to blame for the scatter of a similar plot of unit cell volume vs. guest molecular volume (in Figure XV-19) [43], for these channel-type TOT complexes.

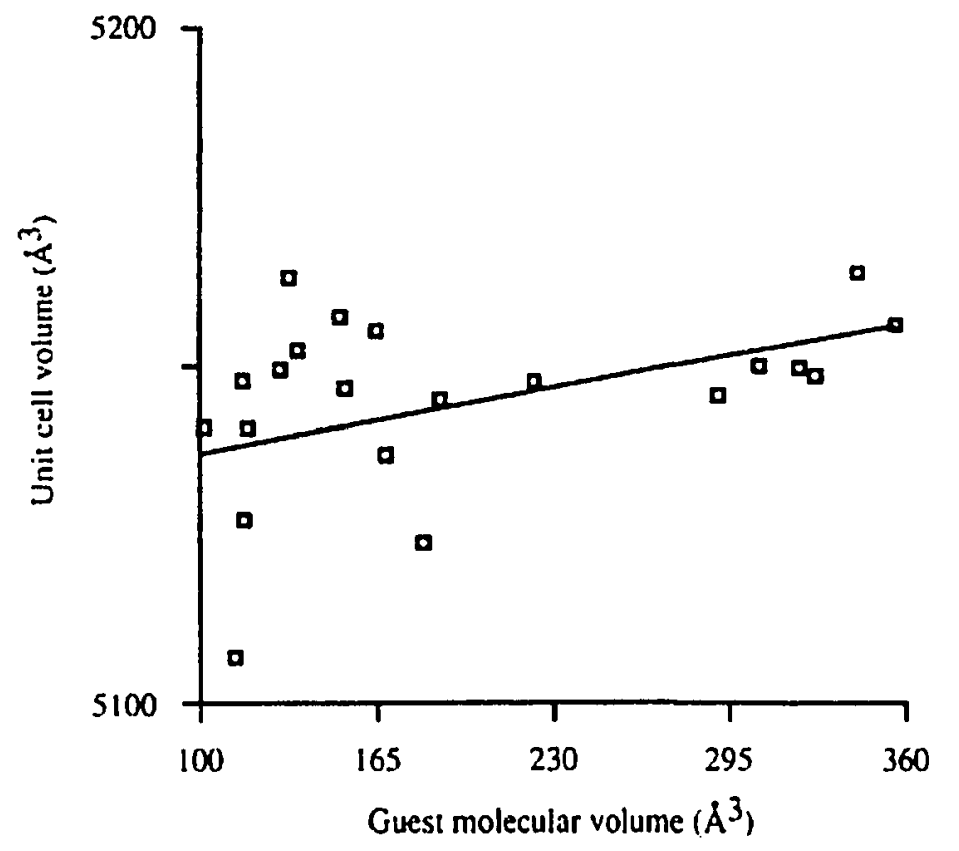

Figure XV-19: Correlation between unit cell volume and guest molecular volume for triortho-thymotide channel-type inclusion compounds [38]

The examination of an isomorphous series of perhydrotriphenylene (PHTP) inclusion compounds with $n$-paraffin guests $[\mathbf{4 4 , 4 5}]$, shows that an increase in the molecular weight of the guest corresponds to a slight reduction in the lateral dimensions of the channel, and that the repeat distance $P_{\mathrm{N}}$ along the channel axis may be expressed, to a first approximation by $(5 \leq n \leq 16)$ :

$$
P_{\mathrm{N}}(\AA)=\{1.215 \cdot(N-1)\}+3.44
$$

where $N$ is the number of carbon atoms of the guest hydrocarbon. This behaviour is similar to that found for urea/n-alkane inclusion compounds $[46,47]$. 
For isostructural urea inclusion compounds (space group $\mathrm{P} 2 / 1 \mathrm{n}$ ) with $1,6-$ dihalohexane guests, as one goes from $\mathrm{Cl}\left(\mathrm{CH}_{2}\right)_{6} \mathrm{Cl}$ to $\mathrm{Cl}\left(\mathrm{CH}_{2}\right)_{6} \mathrm{Br}$ to $\mathrm{Br}\left(\mathrm{CH}_{2}\right)_{6} \mathrm{Br}$, the crystal structures $[48]$ indicate a steady increase in $a b$ (the cross-sectional area of the channel), as perhaps is expected. On the other hand, the $c$ axis dimension (the host channel repeat distance) gradually decreases with increasing guest size. The structures were obtained at $99 \mathrm{~K}, 102 \mathrm{~K}$ and $108 \mathrm{~K}$ respectively (see Table XIII-7, p 337).

Furthermore, for $(\alpha+1),(\omega-1)$-alkanedione / urea complexes (2,7-octanedione, 2,9decanedione and 2,10-undecanedione guests - all at $292 \mathrm{~K}$ ) [49,50], again $a$ and $b$ (and therefore the cross-sectional area of the channel) increase with guest length, while the $c$ axis dimension decreases significantly with increasing size guest length (c.f. increasing guest size for the $\mathrm{X}\left(\mathrm{CH}_{2}\right)_{6} \mathrm{X}$ guests above). The crystal data for these three urea inclusion complexes appear in Table XV-10 [51].

Table XV-10: Crystal data for $(\alpha+1),(\omega-1)$-alkanedione/urea complexes [51] (see also Table XIII-7, p. 337)

\begin{tabular}{llllllll}
\hline Guest & $T(\mathrm{~K})$ & Space group & $a(\AA)$ & $b(\AA)$ & $c(\AA)$ & $\beta\left(^{\circ}\right)$ & Comments \\
\hline 2,7-octanedione & 292 & $P 6,22$ & 8.208 & 8.208 & 76.97 & 120 & $6 c_{\mathrm{g}}=7 c_{\mathrm{h}}$, weak H-G H-bonds \\
2,9-decanedione & 292 & $P 3,12$ & 8.229 & 8.229 & 44.16 & 120 & $3 c_{\mathrm{g}}=4 c_{\mathrm{h}}$, weak H-G H-bonds \\
2,10-undecanedione & 292 & $C 222$ & 8.345 & 13.94 & 32.98 & 90 & $2 c_{\mathrm{b}}=2 c_{\text {w w weak H-G H-bonds }}$ \\
\hline
\end{tabular}

As a further illustration, it is relevant to enquire whether the bulk of the guest component has any effect on the cavity shape of the Dianin's compound thia-analog host. The results are summarized in Table XV-11. On passing from the ethanol clathrate, which has two sterically undemanding guest molecules per cavity, to the increasingly bulky acetylene-derivative guests, only slight increases in the $a$ and $c$ axial dimensions are observed. On the other hand, significant increases in the $\mathrm{O} \cdots \mathrm{O}$ hexameric hydrogen bond distances are found with increasing guest size, and this corresponds to a weakening of the $[-\mathrm{OH}-]_{6}$ unit's hydrogen bonds. Thus, for these bulky guests, a modest expansion of at least the ends of the cage does occur. 
Table XV-11: Variation of lattice parameters and $\mathrm{O} \cdots \mathrm{O}$ distances with guest size for clathrates of the Dianin's compound thia-analogue [52]

\begin{tabular}{|c|c|c|c|c|}
\hline Guest & $\mathrm{H}: \mathrm{G} S \mathrm{SR}$ & $\mathrm{a}(\AA)$ & $c(\AA)$ & $0 \cdots O$ distance $(\AA)$ \\
\hline ethanol & $3: 1$ & 27.81 & 10.90 & $2.96(1)$ \\
\hline 2,5,5-trimethyl-3-yn-2-ol & $6: 1$ & 27.91 & 10.99 & $3.03(1)$ \\
\hline di-tert-butylacetylene & $6: 1$ & 28.00 & 11.08 & $3.07(1)$ \\
\hline
\end{tabular}

Finally, a study concerning guest selectivity upon complexation with the $\beta$-chitin host material was published in 2004 [53]. As part of their literature review, they noted that complexation with aliphatic alcohols [54] and aliphatic mono- and diamines [55] causes widening of the $\beta$-chitin sheet spacing proportional to the length of the alkyl chain of the guest, as measured by PXRD. All of these studies corroborate well with our data on TATM inclusion compounds, in that larger and larger TATM guests cause a progressive stretching of the cross-sectional area of the host channel.

\section{(I) Gossypol}

Gossypol, as mentioned in the introductory section of the thesis, forms inclusion compounds with a wide variety of small and medium sized organic molecules. In many cases, these compounds crystallize easily, and many of them were characterized by their crystal data [56]. Taking into account the unit cell parameters and crystal symmetry, all known inclusion compounds of gossypol have been classified into 12 groups, designated I - XII, with subtypes within several of the 12 main groups (22 groups in total) [57]. From this wide variety of structural motifs, it is evident that gossypol, with six potential proton donors and eight potential proton acceptors, can easily adjust its association modes to form voids with a shape, size and chemical nature that is complementary to those of the guest molecules to be accommodated within its crystal lattice [58].

In addition to the wide variety of host-guest inclusions, gossypol has also been obtained as a crystalline substance without included solvent molecules. Two forms, designated $P_{1}$ and $P_{2}$ (see Table XV-12), were crystallized directly from solution [59]. A third form $\left(\mathrm{P}_{3}\right)$ results from decomposition of type VIII clathrates at room temperature. When guest molecules leave the gossypol crystals, the host network does not collapse, and the crystals remain single. Slow removal (e.g. under vacuum) of solvent molecules 
from other gossypol inclusion compounds, especially the channel types, leads to several more polymorphic modifications of guest-free gossypol. Powder X-ray diffraction patterns of guest-free samples obtained by decomposition of the Ia, Ila, IVb and XI types of gossypol inclusions are characteristic of their respective starting materials, indicating that at least four other guest-free polymorphs $\left(\mathrm{P}_{4}-\mathrm{P}_{7}\right)$ are formed [59].

Table XV-12: Crystal data for gossypol guest-free forms $P_{1}, P_{2}$ and $P_{3}$ [59-61]

\begin{tabular}{llll}
\hline Form & $\mathrm{P}_{1}$ & $\mathrm{P}_{2}$ & $\mathrm{P}_{3}$ \\
\hline lattice type & monoclinic & monoclinic & monoclinic \\
space group & $\mathrm{P} 2 / \mathrm{c}$ & $\mathrm{C} 2 / \mathrm{c}$ & $\mathrm{C} 2 / \mathrm{c}$ \\
$a(\AA)$ & $13.468(2)$ & $42.032(12)$ & $21.208(8)$ \\
$b(\AA)$ & $21.376(3)$ & $32.785(5)$ & $19.079(4)$ \\
$c(\AA)$ & $8.794(1)$ & $15.890(4)$ & $15.267(2)$ \\
$\beta\left({ }^{\circ}\right)$ & $97.23(1)$ & $110.30(2)$ & $113.19(2)$ \\
$V\left(\AA^{3}\right)$ & 2512 & 20536 & 5678 \\
$\underline{d}$ calc $\left(\mathrm{g} \mathrm{cm}^{-3}\right)$ & 1.37 & 1.30 & 1.21 \\
\hline
\end{tabular}

Crystals of the $P_{1}$ polymorph [60] were obtained from a solution of gossypol in a mixture of diethyl ether $(60 \mathrm{mg} / \mathrm{mL})$ and $n$-hexane at $28^{\circ}-30^{\circ} \mathrm{C}$. The $P_{2}$ crystals were formed by adding an equal volume of $n$-hexane to a more dilute ether solution $(20 \mathrm{mg} / \mathrm{mL})$, at a temperature of $23^{\circ}-25^{\circ} \mathrm{C}$ [59]. In order to obtain the $\mathrm{P}_{3}$ guest-free form, crystals of the gossypol $/ \mathrm{CH}_{2} \mathrm{Cl}_{2}$ complex, at $23^{\circ}-25^{\circ} \mathrm{C}$, were removed from the mother solution, leading to immediate decomposition to the $\mathrm{P}_{3}$ desolvated polymorph [61]. The $\mathrm{P}_{4}$ polymorph was formed as a result of drying the gossypol complex with ethanol under vacuum (0.1 torr) for 3 hours at $23^{\circ}-25^{\circ} \mathrm{C}[62]$. The $P_{5}$ form was obtained by desolvating the gossypol / diethyl ether complex under vacuum $\left(0.1\right.$ torr) at $25^{\circ}-27^{\circ} \mathrm{C}$ for 3 hours, and then at 0.01 torr and $60^{\circ} \mathrm{C}$ for a further 20 hours $|63|$. Crystals of the $P_{6}$ structure were obtained from the gossypol/acetone complex under vacuum $\left(0.01\right.$ torr) for 20 hours at $60^{\circ} \mathrm{C}[64]$. Finally, the $\mathrm{P}_{7}$ guest-free form was prepared from gossypol/pyridine by drying in vacuum (0.01 torr) at $50^{\circ} \mathrm{C}$ for 20 hours $[65]$.

Of these seven guest-free gossypol crystal modifications, the structures of only $P_{1}$ [60], $P_{2}[59]$ and $P_{3}[61]$ have been determined crystallographically. It was impossible [59] to carry out a complete determination of the structure of the other 4 forms due to the inability to prepare suitable single crystals. 
Organic substances often form two or three (but seldom four or more) polymorphic modifications. Guest-free gossypol is therefore very unusual in forming such a large number of polymorphs. In general, decomposition of inclusion compounds with one host and different guests (and different structural types) usually gives rise to the same unsolvated host type. In this respect, gossypol behaves in a different manner. Upon decomposition of its complexes (belonging to different structural types), a guestfree polymorph specific for each group is formed, and on increasing the temperature, such different polymorphs are not transformed into each other. Such non-typical behaviour by gossypol shows that in its different crystal forms, packings with similar energy are achieved, among which there is no strongly preferred form.

\section{(J) Hydroquinone}

Hydroquinone \{para-dihydroxybenzene, $\left.p-\mathrm{C}_{6} \mathrm{H}_{4}(\mathrm{OH})_{2}\right\}$, also known as quinol, exists in three guest-free polymorphic forms. The $\alpha$-quinol form [66] is the most stable at room temperature, whereas the metastable $\gamma$-quinol form [67] can be prepared by sublimation or by rapid evaporation of a solution of hydroquinone in diethylether. Crystallization of hydroquinone from common solvents (such as methanol, acetonitrile etc.) generally yields a $\beta$-quinol clathrate, with guest solvent molecules trapped inside cavities of the $\beta$ quinol host lattice [68]. The existence of a solvent-free $\beta$-quinol polymorph (empty lattice), was obtained by crystallization from an n-octane solution [69] (see Figure XV20).
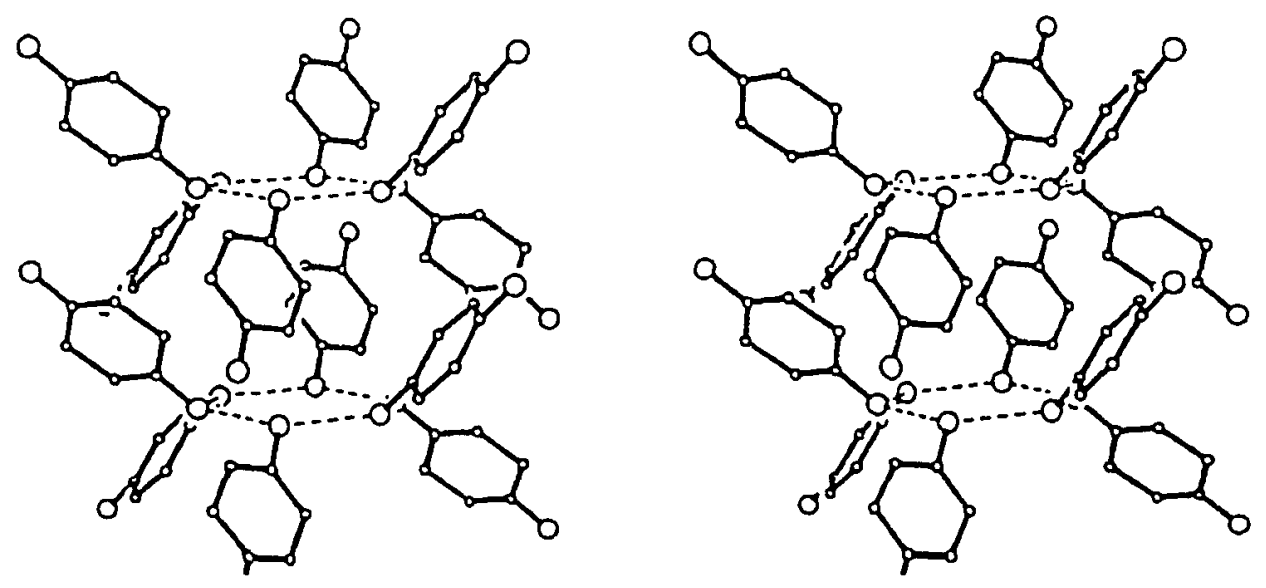

Figure XV-20: Stereoview illustrating the constructing of a clathration cage in unsolvated $\beta$-hydroquinone [69]. 
As far as forming inclusion compounds, however, the $\beta$-form is the most versatile, and it was established [70-75] that three crystallographically distinguishable forms of the $\beta$-quinol host lattice (denoted I, II and III) can exist, all having the same general formula $3 \mathrm{C}_{6} \mathrm{H}_{4}(\mathrm{OH})_{2} \cdot x \mathrm{G}$, where $\mathrm{G}$ represents the encaged guest molecule, and $x$ is a site occupancy factor such that $0 \leq x \leq 1$. Please refer to Table XV-13 for hydroquinone crystal data.

Table XV-13: Crystal data for $\beta$-quinol inclusion compounds $\left(3 \mathrm{C}_{6} \mathrm{H}_{4}(\mathrm{OH})_{2} \cdot x \mathrm{G}\right)[68]$

\begin{tabular}{llllll}
\hline Type & Lattice type & Space Group & $a(\AA)$ & $c(\AA)$ & guest \\
\hline I & hexagonal & $\mathrm{R} \overline{3}$ & $16.613(3)$ & $54.746(5)$ & no guest \\
I & hexagonal & $\mathrm{R} \frac{3}{3}$ & $16.610(3)$ & $55.24(1)$ & $\mathrm{Xe}$ \\
I & hexagonal & $\mathrm{R} \frac{3}{}$ & $16.616(3)$ & $54.89(1)$ & $\mathrm{H}_{2} \mathrm{~S}$ \\
& & & & & \\
II & hexagonal & $\mathrm{R} 3$ & $16.31(5)$ & $58.21(1)$ & $\mathrm{SO}_{2}$ \\
II & hexagonal & $\mathrm{R} 3$ & $16.621(2)$ & $55.62(1)$ & $\mathrm{MeOH}$ \\
II & hexagonal & $\mathrm{R} 3$ & $16.650(1)$ & $54.53(1)$ & $\mathrm{HCl}$ \\
II & hexagonal & $\mathrm{R} 3$ & $15.946(2)$ & $63.48(2)$ & $\mathrm{MeNC}$ \\
& & & & & \\
III & hexagonal & P3 & $16.003(2)$ & $62.45(2)$ & $\mathrm{MeCN}$ \\
\hline
\end{tabular}

The $\alpha$-quinol structure belongs to space group $\mathrm{R} \overline{3}$, with $54 \mathrm{C}_{6} \mathrm{H}_{4}(\mathrm{OH})_{2}$ molecules in a triply primitive hexagonal unit cell [66]. As shown in Figure XV-21 [76], of the three crystallographically independent $\alpha$-quinol molecules in the asymmetric unit, two are involved in forming two open interpenetrating hydrogen-bonded cageworks, similar to those in $\beta$-quinol, while the third molecule forms double helices consisting of hydrogenbonded chains of $\mathrm{C}_{6} \mathrm{H}_{4}(\mathrm{OH})_{2}$ molecules about a three-fold screw axis. The cages and helices are hydrogen bonded to each other in such a way that the two interpenetrating cageworks are directly linked to each other, as are the two strands of the double helix.

Crystals of the $\gamma$-form of hydroquinone are monoclinic, space group $P 2_{1} / \mathrm{c}[67]$ (see Table XV-14). The structure is built up of sheets of hydrogen bonded hydroquinone molecules (see Figure XV-22) [76]. As is perhaps expected, due both to the thermal instability of $\gamma$-quinol and the ability to easily cleave the single crystals, the sheets are apparently held together by only weak van der Waals forces. 

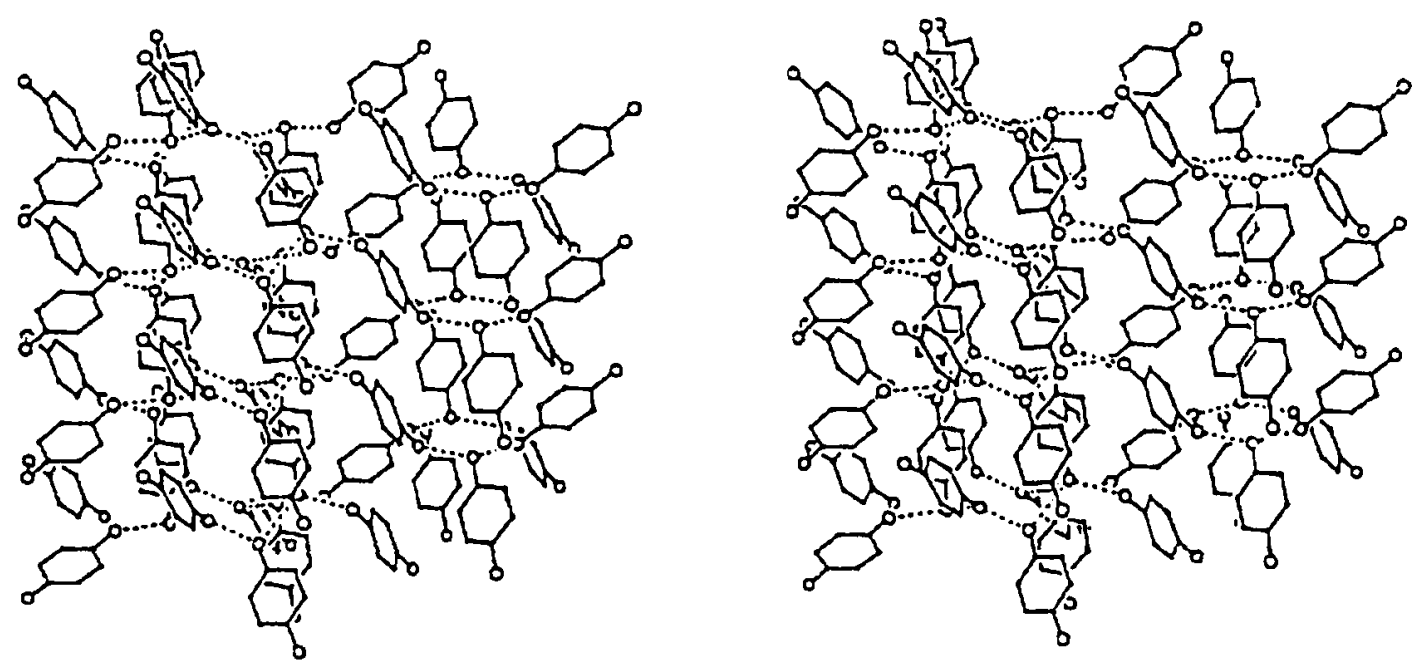

Figure XV-21: Stereoview of the structure of $\alpha$-hydroquinone, in which two crystallographically distinct $\mathrm{C}_{6} \mathrm{H}_{4}(\mathrm{OH})_{2}$ molecules are used to construct the unoccupied cages on the right, while a third independent molecule forms double helices around a threefold screw axis on the left [76].
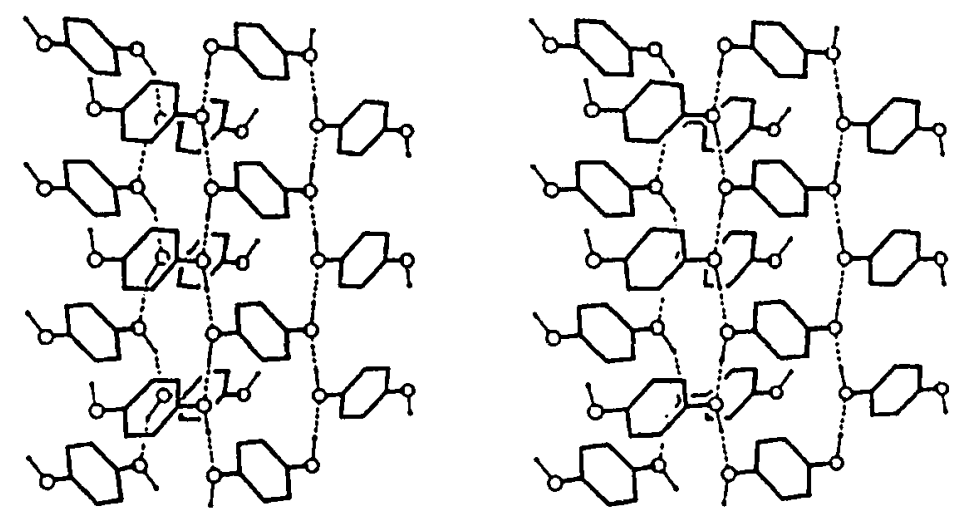

Figure XV-22: A stereoview of the crystal structure of $\gamma$-hydroquinone built from sheets of hydrogen-bonded molecules [76]

Table XV-14: Crystal data for $\alpha$-quinol [66] and $\gamma$-quinol [67]

\begin{tabular}{lll}
\hline Type & $\alpha$-quinol & $\gamma$-quinol \\
\hline lattice type & hexagonal & monoclinic \\
space group & $\mathrm{R} \overline{3}$ & $\mathrm{P} 2 \mathrm{~J} / \mathrm{c}$ \\
$\mathrm{a}(\AA)$ & $38.46(2)$ & 8.07 \\
$\mathrm{~b}(\AA)$ & - & 5.20 \\
$\mathrm{c}(\AA)$ & $5.65(3)$ & 13.20 \\
$\beta\left({ }^{\circ}\right)$ & - & 107 \\
$\mathrm{Z}$ & 54 & 4 \\
\hline
\end{tabular}




\section{(K) Perhydrotriphenylene}

Pure racemic perhydrotriphenylene (PHTP), exists in two different crystalline forms (see Table XV-15), both of which are monoclinic $[77,78]$. One is stable and melts at $125.2^{\circ} \mathrm{C}$, while the other is metastable throughout the temperature range explored by DSC, and melts at $117^{\circ} \mathrm{C}[79]$. The formation of the latter form is revealed in a DSC experiment by the amount of supercooling observed during crystallization [80]. At room temperature, the metastable solvent-free form converts to the stable form in a few hours, while at higher temperatures the conversion is more rapid $[\mathbf{8 1 , 8 2}]$. Neither form contains significant empty space, while all of the intermolecular distances are close to the normal van der Waals distances $[\mathbf{7 7 , 7 8}$. In the stable form, the PHTP molecules are tilted by an angle of $-63^{\circ}$ with respect to the $c$ axis, such that the average molecular planes of superimposed PHTP molecules are at an intermolecular distance of about $4.78 \AA$, nearly the same at that encountered for PHTP inclusion compounds [83].

Table XV-15: Crystal data for the two guest-free forms of perhydrotriphenylene [77-79]

\begin{tabular}{lll}
\hline Type & Modification I & Modification II \\
\hline lattice type & monoclinic & monoclinic \\
space group & P2 $1 /$ a & C2/c \\
$a(\AA)$ & $19.59(7)$ & $16.94(8)$ \\
$b(\AA)$ & $15.39(6)$ & $10.41(5)$ \\
$c(\AA)$ & $5.36(3)$ & $9.73(5)$ \\
$\beta\left({ }^{\circ}\right)$ & $109.7(3)$ & $113.5(5)$ \\
$V\left(\AA^{3}\right)$ & $1522(9)$ & $1574(12)$ \\
$Z$ & 4 & 4 \\
$d_{\text {calc }}\left(\mathrm{g} \mathrm{cm}^{-3}\right)$ & 1.07 & 1.04 \\
$\underline{d}_{\text {mbias }}\left(\mathrm{g} \mathrm{cm}^{-3}\right)$ & 1.05 & 1.03 \\
\hline
\end{tabular}

The most common crystal form of pure PHTP (modification I) occurs either by crystallization from a solvent that is unable to give an inclusion compound (e.g. methyl ethyl ketone or $\alpha$-pinene), or alternatively by sublimation. It has also been obtained by extraction of volatile guest molecules (e.g. $n$-heptane, chloroform or cyclohexane) from the corresponding inclusion compound, or by slow cooling of the melt. This modification is stable under normal conditions. The molecular packing is represented in Figure XV-23. 


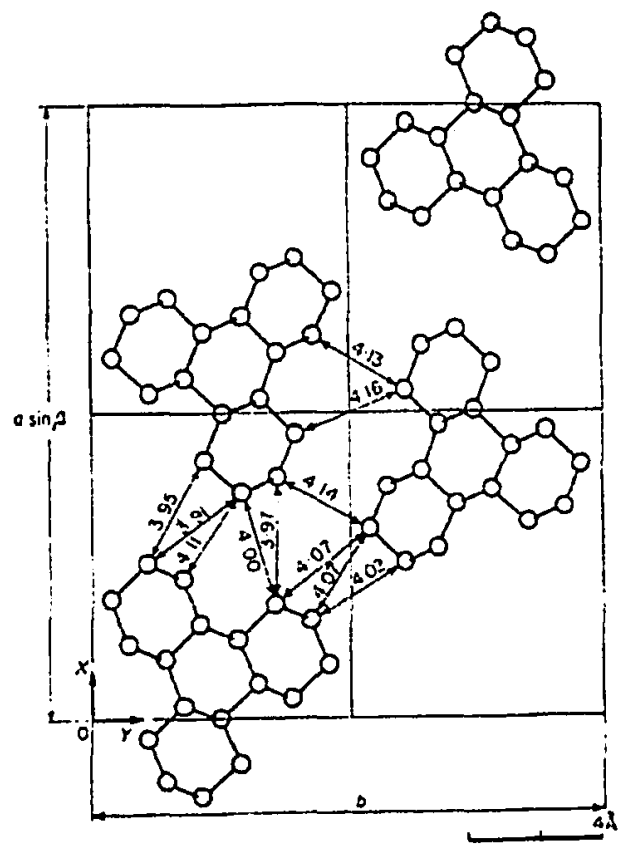

Figure XV-23: Molecular packing (down c) of modification I of crystalline PHTP. Short intermolecular C...C distances are illustrated. The hydrogen atoms have been removed [66]

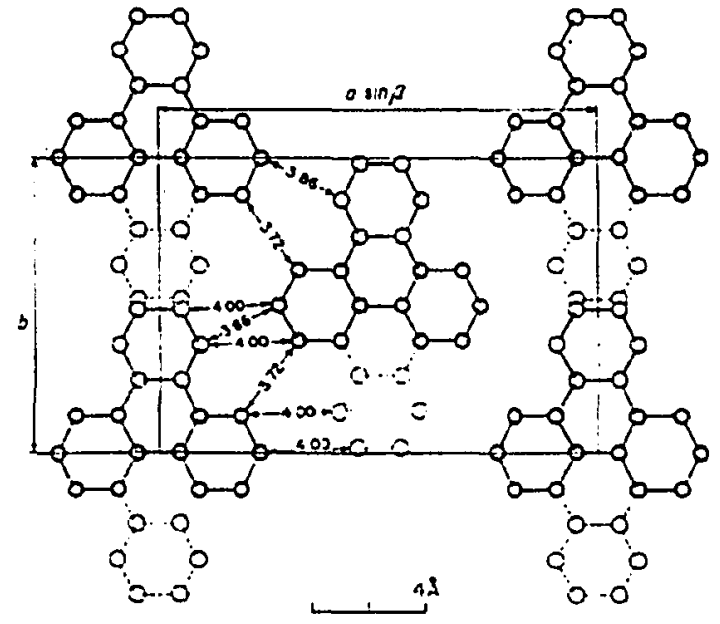

Figure XV-24: Molecular packing (down c) of modification II of crystalline PHTP. Short intermolecular $\mathrm{C} \cdots \mathrm{C}$ distances are illustrated. The hydrogen atoms have been removed [66]

A metastable form (modification II) was obtained by rapid cooling of the melt. It transforms completely, within a few hours, into the stable modification I. For this reason, no quantitative evaluation of the observed structure factors was possible. However, qualitative comparison of the $\mathrm{X}$-ray intensities with the calculated structure factors allowed the authors to determine the essential features of the molecular packing. On the basis of the systematic absences of the reflections with $(h k 0), h+k$ odd, and with $(h 0 l), l$ odd, both space groups $C_{\mathrm{c}}$ and $\mathrm{C} 2 / \mathrm{c}$ were considered. By trial and error, the authors showed that the latter space group is correct, with the PHTP molecules being located around crystallographic two-fold axes (see Figure XV-24), with their average molecular planes perpendicular to $c$. The most satisfactory molecular packing corresponds to the overlap (along $c$ ) of two outer rings between neighboring molecules in opposite orientations, which explains why the $c$ distance is nearly twice that in most PHTP inclusion compounds, where the PHTP molecules repeat exactly down their three fold $(c)$ axis. 
As far as vapor deposition is concerned, for volatile guests it is sufficient to expose the guest-free PHTP crystals to vapors of the guest in order to obtain the inclusion compound in a very short time (within 5-30 minutes). The difference in this regard with respect to urea is very clear: Urea requires (1) low temperatures (e.g. $-78^{\circ} \mathrm{C}$ ), (2) the presence of inclusion promoters such as methanol, and (3) reaction times lasting from many hours to days, or even weeks.

\section{(L) Dianin's Compound}

Dianin's compound is a host system known to intercalate a wide variety of compounds into an hourglass shaped cavity formed by six molecules of the host. This cavity has been considered to be invariant, regardless of whether guest molecules are or are not included [84]. In order to confirm the existence of empty cavities in guest-free (unsolvated) crystals of Dianin's compound, and the essential invariance of the cage structure independent of inclusion, the crystal and molecular structure of unsolvated Dianin`s compound was recently reported $[85]$.

In this publication, a comparison of the cage structures in clathrates of Dianin's compound with ethanol $[86]$, chloroform $[86]$ and carbon tetrachloride $[87,88]$ guests with that of guest-free Dianin's compound show no remarkable differences. The maximum differences in the positions of the respective non-hydrogen atoms of these three inclusion compounds, when compared to the guest-free structure, are $0.03 \AA, 0.08 \AA$ and $0.06 \AA$ for the ethanol, chloroform and carbon tetrachloride inclusions, respectively. Difference Fourier synthesis showed no electron cloud (i.e. no enclathration) in the cavities of the guest-free lattice. This confirms the existence of a significant volume of empty space in the guest-free crystal. The molecular structure of guest-free Dianin's compound itself is also very similar to that found for the host in the ethanol, chloroform and carbon tetrachloride inclusions. The maximum differences in the bond lengths, bond angles and torsion angles for these host molecules, when compared to the guest-free Dianin's compound molecules, are only $0.008 \AA, 0.4^{\circ}$ and $0.7^{\circ}$, respectively. Therefore, one can conclude that the nature of the cavities in the guest-free lattice is essentially invariant and independent of inclusion. Figure XV-25 displays a view of the empty, hourglass shaped cavity of guest-free Dianin`s compound. 


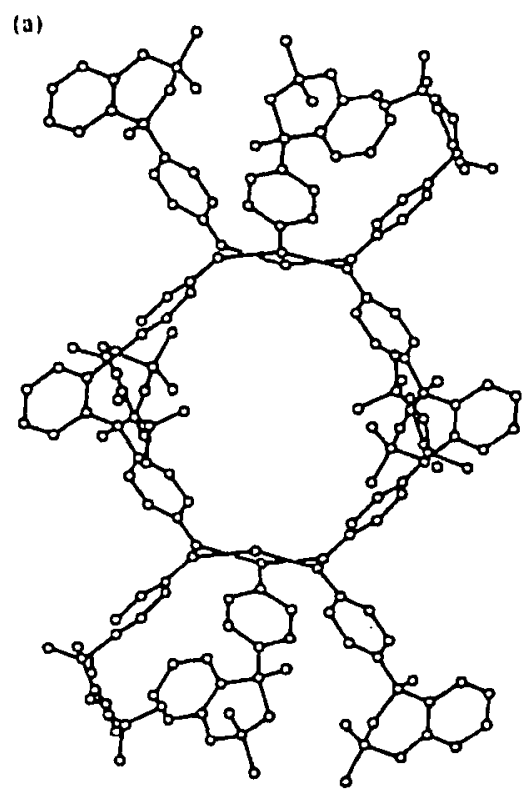

(b)

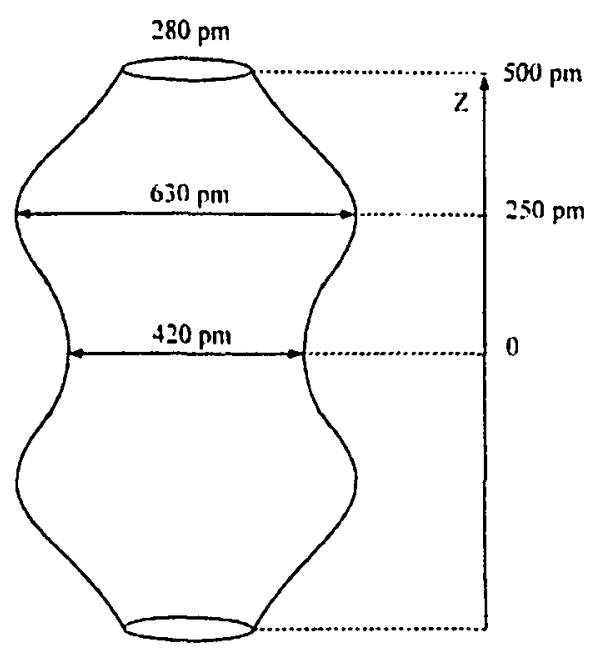

Figure XV-25: (a) The structure of unsolvated Dianin's compound, viewed normal to the ac plane. Two molecules, which lie in front of and behind the cavity, have been excluded to show the cage more clearly (b) The biggest figure of resolution that fits the cage, considering the excluded volume of all host atoms [85]

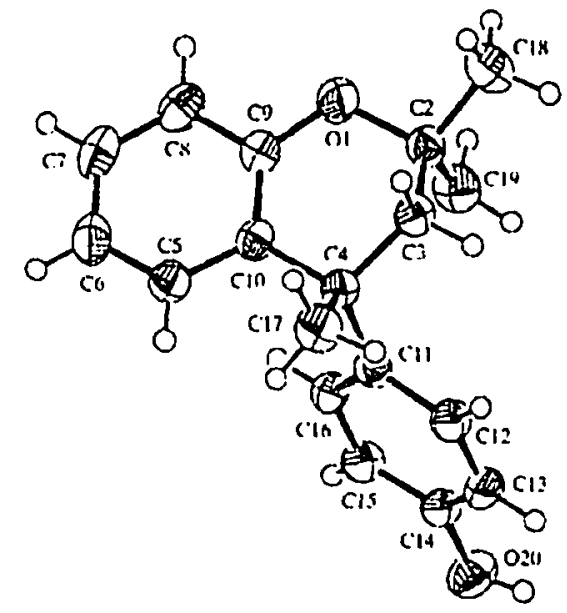

Scheme 1: ORTEP plot of Dianin's compound with atomic labelling scheme as used in the text [85]

In the hourglass shaped cavity of Dianin's compound, the $C_{18}$ methyl carbon atom (see Scheme 1) protrudes into the upper and lower halves of the cavity, while the $C_{19}$ methyl carbon atom is directed into the constricted neck of the cavity. On the other hand, the $\mathrm{C}_{17}$ methyl carbon atom does not explicitly participate in the formation of the cavity. The ${ }^{13} \mathrm{C}$ CP/MAS NMR chemical shift of $\mathrm{C}_{18}$ is, therefore, expected to be sensitive to the presence and nature of guest molecules, while that of $\mathrm{C}_{19}$ should be susceptible to interactions between guest molecules and the neck of the cage [89-91]. Accordingly, the 


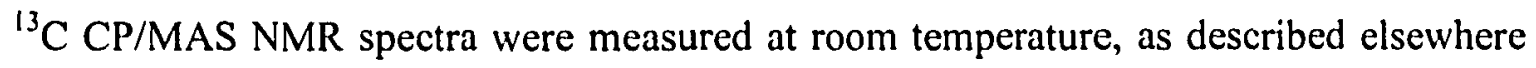
[92]. The ${ }^{13} \mathrm{C}$ NMR chemical shifts of these three methyl carbon atoms were measured both for guest-free Dianin's compound and for clathrates with $n$-pentane (1), $n$-hexane (2), $n$-heptane (3) and n-octane (4). The chemical shift of $\mathrm{C}_{18}$ for guest-free Dianin's compound $\{25.10 \mathrm{ppm}\}$ moves significantly downfield upon inclusion \{27.22, 27.40, 27.04 and $27.15 \mathrm{ppm}$ for (1) - (4) respectively\}, while that of $C_{17}\{34.62 \mathrm{ppm}\}$ for the guest-free sample is relatively unchanged in the complexes $\{34.26,34.61,34.45$ and $34.76 \mathrm{ppm}$ for (1) - (4) respectively\}. The chemical shift of $\mathrm{C}_{19}$ for the unsolvated host $\{30.74 \mathrm{ppm}\}$ is slightly downfield of (1) and (2) \{30.57 and $30.57 \mathrm{ppm}$, respectively\}, while it is further downfield than both (3) and (4) \{30.06 and $30.10 \mathrm{ppm}$, respectively\}. On the basis of MM2 molecular mechanics calculations [92], the conformations of $n$ pentane and $n$-hexane guest molecules are almost all trans, while those of the intercalated $n$-heptane and $n$-octane molecules are not. Steric interactions between the guest molecule and the neck of the cavity may become significant for $n$-heptane and n-octane guests, resulting in a significant decrease in the chemical shift of $\mathrm{C}_{19}$ for these two complexes. when compared to the $n$-pentane and $n$-hexane inclusions.

\section{SUMMARY}

The previously reported studies on other host systems mentioned above can be compared to the current TATM materials. It was shown in section $(\mathrm{H})$ that for the host systems TOT, perhydrotriphenylene, urea, thia-Dianin`s compound and $\beta$-chitin, there is a general dependence of the size of the cavities-channels-layers (void space) of the host upon the nature of the guest, such as volume, molecular weight, number of carbon atoms (or length), or bulkiness of the guest. This can be compared with the current TATM materials, where it was demonstrated that there was a direct correlation between the molecular weight of the guest and the resulting channel cross-section.

Gossypol is similar to TATM in its remarkable inclusion capabilities, and the existence of a number of different structural polytypes, suggestive of the ability of both TATM and gossypol to easily adjust their association modes to form voids with a size, shape and chemical nature that are complementary to those of the guest molecules to be included. On the other hand, TATM, at least so far, crystallizes in only one crystalline 
guest-free form, while gossypol forms many types of guest-free forms, dependent in part, on the nature of the gossypol inclusion starting material. In many cases the gossypol guest-free material is characteristic of the starting material, and when the guest molecules leave, the host lattice does not collapse, and the crystals remain single.

Hydroquinone, as was the case for gossypol, forms different guest-free structures, denoted as $\alpha$ - $\beta$ - and $\gamma$-quinol, of which $\beta$-quinol, as far as forming inclusion compounds, is the most versatile. On the other hand, $\beta$-quinol is very specific in forming inclusions with only small guest molecules that do not appreciably distort the host cage, suggesting a relatively limited ability of the host lattice to adjust to the size of the potential guest. Furthermore, $\beta$-quinol, as a host-guest material, forms only three structural polytypes, in contrast to TATM and gossypol, as mentioned above.

Perhydrotriphenylene, in a similar manner to TATM ( 1 form) and $\beta$-quinol (3 forms), forms only two guest-free materials, of which one is metastable. Furthermore, guest-free perhydrotriphenylene is easily able to form inclusion compounds on exposure to vapors of volatile guests, similarly to TATM, and in contrast to urea, which requires low temperatures, inclusion promoters such as methanol, and long reaction times.

Dianin`s compound is able to crystallize in a guest-free lattice with empty voids (in other words, a significant amount of empty space), in contrast to the dense guest-free phase of TATM. For Dianin's compound, the cage structure is independent of the presence or absence of guests, and the empty guest-free phase was stable enough for structural characterization.

\section{CONCLUDING REMARKS}

In summary, the knowledge of different crystal structures involving a given substance, both in a pure state and as a host component in a series of inclusion compounds, seems to be of great interest for a general understanding of the molecular aspects of crystallization.

In this study, we report an experimental technique to prepare a crystalline guestfree lattice of the TATM host material, namcly by heating a TATM inclusion compound (with e.g. ethanol, methanol or acetone guest) at $70^{\circ} \mathrm{C}$ with vigorous stirring over the course of about one week. For all intents and purposes, TATM is completely insoluble in water, so the removal of the guest by the hot water is a slow process, taking days or 
weeks. All three starting materials gave rise to the same guest-free TATM lattice, suggesting that there is a common rearrangement of the lattice once the guest is removed. Whether the same guest-free lattice is obtained from TATM inclusion compounds with other guests remains to be seen.

\section{CHAPTER XV}

\section{REFERENCES}

(1) P. Finocchiaro and S. Failla Solid-State Supramolecular Chemistry: Crystal Engineering, In Comprehensive Supramolecular Chemistry, J.L. Atwood, J.E.D. Davies, D.D. MacNicol and F. Vogtle, Eds. Volume 6, Chapter 18, Pergamon / Elsevier: Oxford, 1996.

(2) H. Hartley and N.G. Thomas, J. Chem. Soc. 89, 1013 (1906).

(3) A.P. Yakubov, Y.K. Sudarushkin, L.I. Belen`kii and L. Gol’dfarb, J. Org. Chem. (USSR) 9, 1549 (1973).

(4) L. Bin Din and O. Meth-Cohn, J. Chem. Soc., Chem. Commun. 741 (1977).

(5) P.H. van Rooyen and H.M. Roos, Acta Crystallogr. C47, 2468 (1991).

(6) P.H. van Rooyen and H.M. Roos, Acta Crystallogr. C47, 2718 (1991).

(7) H.M. Roos and J.L.M. Dillen, Acta Crystallogr. C48, 1882 (1992).

(8) J.L.M. Dillen and H.M. Roos, Acta Crystallogr. C48, 2229 (1992).

(9) L. Pang, R.C. Hynes and M.A. Whitehead, Acta Crystallogr. C50, 615 (1994).

(10) L. Pang and F. Brisse, Acta Crystallogr. C50, 1947 (1994).

(11) L. Pang and F. Brisse, Can. J. Chem. 72, 2318 (1994).

(12) R.E. Marsh, Acta Crystallogr. B50, 112 (1994).

(13) P.S. Sidhu and J.A. Ripmeester, J. Supramol. Chem. 1, 63 (2001).

(14) P.S. Sidhu and J.A. Ripmeester, Supramol. Chem. 15(6), 433 (2003).

(15) P.S. Sidhu, J. Bell, G.H. Penner and K.R. Jeffrey, Can. J. Chem. 73, 2196 (1995).

(16) P.S. Sidhu, J. Bell, G.H. Penner and K.R. Jeffrey, Can. J. Chem. 74, 1784 (1996).

(17) P.S. Sidhu, G.D. Enright, J.A. Ripmeester and G.H. Penner, J. Phys. Chem. B 106, 8569 (2002).

(18) P.S. Sidhu, K.A. Udachin and J.A. Ripmeester, Chem. Commum. 12, 1358 (2004).

(19) P.S. Sidhu, G.D. Enright, K.A. Udachin and J.A. Ripmeester, Crystal Growth and Design. 4(6), 1249 (2004).

(20) D.R. Bond, M.R. Caira, G.A. Harvey and L.R. Nassimbeni. Acta Ciyst. 46B, 771 (1990).

(21) S.A. Talipov, B.T. Ibragimov, G.B. Nazarov, T.F. Aripov and A.S. Sadikov. Khim. Prir. Soedin. 835 (1985).

(22) B.T. Ibragimov, Z.G. Tiljakov, K.M. Beketov and S.A. Talipov. J. Incl. Phenom. Mol. Recognit. Chem. 27, 99 (1997).

(23) F.H. Herbstein, Acta Crystallogr. B53, 168 (1997).

(24) A. Bondi, Physical Properties of Molecular Crystals, Liquids and Glasses, John Wiley and Sons Inc., New York (1968).

(25) D.W. van Krevelen, Properties of Polymers, $3^{\text {rd }}$ edition, Elsevier, Amsterdam (1993). 
(26) R. Zallen, The Physics of Amorphous Solids, John Wiley and Sons, New York (1983).

(27) D. Meier, Molecular Basis of Transitions and Relaxations, Gordon and Breach, New York (1978).

(28) G.B. McKenna, Glass Formation and Glassy Behaviour. In Comprehensive Polymer Science, Volume 2, G. Allen, Ed., Pergamon Press: Oxford (1989).

(29) F.W. Billmeyer, Textbook of Polymer Science, John Wiley and Sons, New York (1984).

(30) E.A. Turi, Thermal Characterization of Polymeric Materials, Academic Press, New York (1981).

(31) M.S.M. Alger, Polymer Science Dictionary, Elsevier, London (1989).

(32) J. Bicerano and D. Adler. Pure and Applied Chemistry 59, 101 (1987).

(33) J.A. Bryson, The Glass Transition, Melting Point, and Structure. In Polymer Science, A.D. Jenkins, Ed., North Holland Publishing Company: Amsterdam (1972).

(34) M.C. Shen and A. Eisenberg. Glass Transitions in Polymers, Progress in Solid State Chemistry 3, 407 (1966).

(35) R. Arad-Yellin, B.S. Green, M. Knossow and G. Tsoucaris Enantiomeric Selectivity of Host Lattices, In Inclusion Compounds. J.L. Atwood, J.E.D. Davies and D.D. MacNicol, Eds. Volume 3, Chapter 9, Academic Press: London, 1988.

(36) R. Gerdil. In Topics in Current Chemistry. E. Weber, Ed. Volume 140, Chapter 4. Springer-Verlag: Berlin, 1987.

(37) R. Arad-Yellin, B.S. Green, M. Knossow and G. Tsoucaris. J. Am. Chem. Soc. 105, 4561 (1983).

(38) R. Gerdil. Tri-o-Thymotide and Related Hosts. In Comprehensive Supramolecular Chemistry, J.L. Atwood, J.E.D. Davies, D.D. MacNicol and F. Vogtle, Eds. Volume 6, Chapter 8 (Figure 4, p 246). Pergamon / Elsevier: Oxford, 1996.

(39) Reference 38: Table 1, p 243.

(40) Reference 38: Tables 2 and 3, p 244.

(41) D. Lawton and H.M. Powell. J. Chem. Soc. 2339 (1958).

(42) Reference 38: Table 3, p 244.

(43) Reference 38: Figure 5, p 247.

(44) G. Allegra, M. Farina, A. Colombo, G. Casagrande-Tettamanti, U. Rossi and G. Natta. J. Chem. Soc. (B) 1028 (1967).

(45) A. Colombo and G. Allegra. Rend. Accad. Naz. Lincei. 43, 41 (1967)

(46) A.E. Smith. Acta Cryst. 5, 224 (1952).

(47) F. Laves, N. Nicolaides and K.C. Peng. Z. Kristallogr: 121, 258 (1965).

(48) M.D. Hollingsworth, U. Werner-Zwanziger, M.E. Brown, J.D. Chaney, J.C. Huffman, K.D.M. Harris and S.P. Smart J. Am. Chem. Soc. 121 (41), 9732 (1999)

(49) M.D. Hollingsworth, M.E. Brown, A.C. Hillier, B.D. Santarsiero and J.D. Chaney. Science - AAAS weekly paper edition 273(5280), 1333 (1996).

(50) M.E. Brown and M.D. Hollingsworth. Nature (London) 376, 323 (1995).

(51) M.D. Hollingsworth and K.D.M. Harris. Urea. Thiourea and Selenourea. In Comprehensive Supramolecular Chemistry, J.L. Atwood, J.E.D. Davies, D.D. MacNicol and F. Vogtle, Eds. Volume 6, Chapter 7 (Table 4, p 207). Pergamon / Elsevier: Oxford, 1996. 
(52) D.D. MacNicol. Hydroquinine, Phenol and Dianin's Compound In Inclusion Compounds. J.L. Atwood, J.E.D. Davies and D.D. MacNicol, Eds. Volume 2, Chapter 1. Academic Press: London, 1984.

(53) Y. Noishiki, S. Kuga, M. Wada, K. Hori and Y. Nishiyama. Macromolecules. 37, 6839 (2004).

(54) Y. Saito, T. Okano, J.L. Putaux, F. Gaill and H. Chanzy. In Advances in Chitin Science. A. Domard, G.A.F. Roberts, K.M. Varum, Eds. Volume 2, p 507. Jacques Andre Publishers: Lyon, France, 1997.

(55) Y. Noishiki, Y. Nishiyama, M. Wada, S. Okada and S. Kuga. Macromolecules. 4, 944 (2003).

(56) M. Gdaniec, B.T. Ibragimov and S.A. Talipov. Gossypol. In Comprehensive Supramolecular Chemistry, J.L. Atwood, J.E.D. Davies, D.D. MacNicol and F. Vogtle, Eds. Volume 6, Chapter 5 (Table 1, pp 122-123). Pergamon/Elsevier: Oxford, 1996.

(57) E. Weber and H.P. Josel. J. Inclusion Phenom. 1, 79 (1983).

(58) B.T. Ibragimov, S.A. Taipov, B.N. Dadabaev, G.B. Nazarov and T.F. Aripov. Khim. Prir. Soedin. 675 (1988).

(59) B.T. Ibragimov and S.A. Talipov. J. Inclusion Phenom. Mol. Recognit. Chem. 17, 325 (1994).

(60) S.A. Talipov, B.T. Ibragimov, B.N. Dadabaev, T.F. Aripov and A.S. Sadykov. Khimiya Prir. Soedin 112 (1986).

(61) S.A. Talipov, B.T. Ibragimov, G.B. Nazarov, T.F. Aripov and A.S. Sadykov. Khimiya Prir. Soedin 835 (1985).

(62) B.T. Ibragimov, S.A. Talipov, B.N. Dadabaev and A.A. Abduvakhabov. Khimiva Prir. Soedin 191 (1992).

(63) S.A. Talipov, B.T. Ibragimov, G.N. Tishenko, T.F. Aripov, G.N. Nazarov and B.V. Strokopytov. Krystallografiva 33, 384 (1988).

(64) B.T. Ibragimov, M. Gdaniec and B.N. Dadabaev. J. Incl. Phenom. 8, 333 (1990).

(65) B.T. Ibragimov, B.N. Dadabaev, S.A. Talipov and A.A. Abduvakhabov. Khimiya Prir. Soedin 186 (1992).

(66) S.C. Wallwork and H.M. Powell. J. Chem. Soc. Perkin Trans. 2. 641 (1980).

(67) K. Maartmann-Moe. Acta Cryst. 21, 979 (1966).

(68) T.C.W. Mak and B.R.F. Bracke. Hydroquinone Clathrates and Diamondoid Host Lattices. In Comprehensive Supramolecular Chemistry, J.L. Atwood, J.E.D. Davies, D.D. MacNicol and F. Vogtle, Eds. Volume 6, Chapter 2. Pergamon / Elsevier: Oxford, 1996.

(69) S.V. Lindeman, V.E. Shklover and Y.T. Struchkov. Civst. Struct. Commun. 10, $1173(1981)$.

(70) D.E. Palin and H.M. Powell. Nature (London). 156, 334 (1945).

(71) D.E. Palin and H.M. Powell. J. Chem. Soc. 208 (1947).

(72) D.E. Palin and H.M. Powell. J. Chem. Soc. 815 (1948).

(73) S.C. Wallwork and H.M. Powell. J. Chem. Soc. 4855 (1956).

(74) H.M. Powell. J. Chem. Soc. 298, 300, 468 (1950).

(75) H.M. Powell. J. Chem. Soc. 61 (1948).

(76) Reference 68: Figure 5, p. 29. 
(77) G. Allegra, M. Farina, A. Immirzi, A. Colombo, U. Rossi, R. Broggi and G. Natta. J. Chem. Soc B. 1020 (1967).

(78) A. Colombo, E. Torti and G. Allegra. Rend. Accad. Naz. Lincei. 43(8), 196 (1967).

(79) M. Farina. In Inclusion Compounds. J.L. Atwood, J.E.D. Davies and D.D. MacNicol, Eds. Volume 2, Chapter 3, Academic Press: London, 1984.

(80) M. Farina, G. DiSilvestro and P. Sozzani. Mol. Cryst. Liq. Cryst. 93, 169 (1983).

(81) M. Farina, G.D. Silvestro and M. Grassi. Makromol. Chem. 180, 1041 (1979).

(82) M. Farina and G.D. Silvestro. Gazz. Chim. Ital. 112, 91 (1982).

(83) R.L. Harlow and G.R. Desiraju. Acta Cryst. C46, 1050 (1990).

(84) D.D. MacNicol, J.J. McKendrick and D.R. Wilson. Chem. Soc. Rev. 7, 65 (1978).

(85) F. Imashiro, M. Yoshimura and T. Fujiwara. Acta Cryst. C54, 1357 (1998).

(86) J.L. Flippen, J. Karle and I.L. Karle. J. Am. Chem. Soc. 92, 3749 (1970).

(87) W. Abriel, A. DuBois, M. Zakrzewski and M.A. White. Can. J. Chem. 68, 1352 (1990).

(88) C. Ping, E.A.C. Lucken and G. Bernardinelli. J. Am. Chem. Soc. 112, 8754 (1990).

(89) J.A. Ripmeester. J. Inclus. Phenom. 1.87 (1983).

(90) P. Barker, N.E. Burlinson, B.A. Dunell and J.A. Ripmeester. J. Magn. Reson. 60, 486 (1984).

(91) F. Lee, E. Gabe, J.S. Tse and J.A. Ripmeester. J. Am. Chem. Soc. 110, 6014 (1988).

(92) F. Imashiro. J. Am. Chem. Soc. 115, 2231 (1993). 


\section{THESIS SUMMARY}

In this contribution, we have presented results from various physicochemical characterization techniques with regards to TATM inclusion compounds with an extremely wide and diverse variety of alkyl halide and aryl halide guests and guest families. In chapter VII, we employed long-range sensitive single crystal diffraction and short-range sensitive ${ }^{2} \mathrm{H}$ solid-state NMR together to arrive at a consistent model for the dynamics of the guest. In the next chapter (VIII), we reported structural characteristics and thermal stabilities of TATM inclusion compounds with alkyl halide guests using various bulk characterization techniques. The next two chapters (IX and X) dealt with competitive inclusion experiments and nature of inclusion mixtures for the same guest families as in Chapter VIII. We then reported results in a similar manner as in Chapter VIII for two different guest families, $\alpha, \omega$-dichloroalkanes and 1-bromoalkanes, in Chapters XI and XIII respectively. Data on five true polymorphic modifications of TATM / 1,3-dichloropropane were presented in Chapter XII. Finally, an inclusion compound between hydrophobic TATM and water was described in Chapter XIV, while a method to prepare crystalline guest-free TATM, along with its characterization, was reported in Chapter XV.

As is evident in this thesis, we have used an extremely wide variety of characterization techniques to supply information on these TATM inclusion compounds. This allows one to draw correlations (of a particular TATM inclusion or a TATM guest family) between the various techniques. This places our results on a firmer foothold. Indeed, the philosophy of our research group is to use as many physical techniques as are available to characterize our supramolecular materials. As an example, we were planning to submit for publication a manuscript about 1,2-dichloroethane/TATM (Chapter VII) that reported results only from deuterium NMR, which concluded that the guest was performing internal rotation about the $\mathrm{C}-\mathrm{C}$ bond to give a trans/gauche conformational equilibrium. Further characterization (particularly single crystal diffraction) debunked this theory, and provides a particularly striking example of the benefits of using as many techniques as are available to a particular researcher to characterize his/her materials. 


\section{SUGGESTIONS FOR FURTHER WORK}

Although we have characterized our materials using a wide range of techniques (however limited somewhat by time constraints), we wish to comment here on some suggestions for further experimentation. Concerning Chapter VII, which dealt with 1,2dichloroethane/TATM, we have obtained variable temperature ${ }^{2} \mathrm{H}$ NMR spectra of 1,2dibromoethane- $\mathrm{d}_{4} /$ TATM, which suggest that it is performing the same type of motion, but at a rate that is significantly less, at any particular temperature, than the 1,2dichloroethane- $d_{4}$ guest. This suggests that the guest molecules are engaged in motions that involve large scale halogen atom reorientation (the motion that was used in the simulations). Chapter VIII would benefit from single crystal structures within each guest family. It is not necessary to measure all three inclusion compounds within any one guest family, rather only one of the isomorphous ones, followed by indexing the PXRD patterns for the others. For example, one could obtain a structure of cyclohexyl chloride / TATM, and then fit the calculated pattern to those of cyclohexyl bromide / TATM and cyclohexyl iodide / TATM. It might also be worthwhile to measure kinetic parameters from variable heating rate TGA (namely the activation energy for guest release) for these inclusions. Indeed, the measured order of $E_{\mathrm{a}}$ for the monohalobenzene inclusions follows the order $\mathrm{C}_{6} \mathrm{H}_{5} \mathrm{~F}>\mathrm{C}_{6} \mathrm{H}_{5} \mathrm{Cl}>\mathrm{C}_{6} \mathrm{H}_{5} \mathrm{Br}>\mathrm{C}_{6} \mathrm{H}_{5} \mathrm{I}$. This provides complementary information to the DSC results. The next two chapters deal with competitive inclusion mixtures. The results from Chapters VIII and IX would be put on firmer ground by measurement of the pure-phase crystal structures, which may perhaps allow one to correlate structural features with thermal properties (Chapter VIII) and competitive inclusion (Chapter IX). Chapter $\mathrm{X}$ would benefit from single-crystal characterization of the inclusion mixtures, however one would have to be able to tell the two guests apart, so perhaps the chlorideiodide combination would be most appropriate for this purpose. For the $\alpha, \omega-$ dichloroalkane guests (Chapter XI), we have obtained crystal structures for the larger guests $(n=8,9,10)$, the motivation for which is because they crystallize in a novel $4 \mathrm{H}: 1 \mathrm{G}$ stoichiometric ratio. This inclusion family might also benefit from competitive inclusion experiments. The 1,3-dichloropropane results might be extended to other guests, for example by using the same preparatory techniques for other 1,3dihalopropanes (halo $=\mathrm{F}, \mathrm{Br}$ or I), to see if the extent of polymorphism is a characteristic 
of the 1,3-dihalopropane guest family in general, regardless of the nature of the halogen substituent. For the 1-bromoalkane guest family (Chapter XIII), we have obtained crystal structures of the inclusions of $\mathrm{Br} 2-\mathrm{Br}$, along with $\mathrm{Br} 2 \mathrm{Br}-\mathrm{Br} 6 \mathrm{Br}$ (ten structures in total). We plan on publishing them with the rest of the results. The water/TATM study (chapter XIV) would be complemented from a variable-temperature deuterium NMR study, rather than just at room temperature. We were limited in this regard by the very weak ${ }^{2} \mathrm{H}$ NMR signal in our measured sample, which did not allow acquisition at low temperatures. Further attempts at crystallization may yield a sample with a stronger signal. Finally, the chapter (XV) on guest-free TATM would be enhanced from a determination of whether the same guest-free TATM lattice is obtained from starting materials of different structures other than the acetone, ethanol or methanol inclusions. Preliminary results indicate that benzene/TATM, when heated in hot water, gives rise to the same guest-free lattice as the ones reported in Chapter XV. This suggests that the original lattice is destroyed, and the liberated guest has no influence on the nature of the subsequent guest-free material. It also suggests that the hot water can remove guests that are expressly hydrophobic and that do not dissolve to any measurable extent in the water. 


\section{FINAL REMARKS}

Organic compounds that form crystalline host-guest inclusions with suitable molecules are attracting increasing attention in view of their practical uses. These include chemical separation, stabilization and protection of labile species, topochemistry, and the development of novel solid materials. This has stimulated the development of new strategies in crystalline inclusion formation, and has motivated the design of novel host types. The most consistently reported results refer to inclusion compounds that are based on coordination-assisted clathrate formation between functionalized and/or polar host and guest molecules. The formation and stability of these crystalline complexes are affected by functional as well as by topological complementarity, and consequently are sensitive to small structural variations.

The field of supramolecular chemistry is concerned with selective molecular recognition between host and guest molecules. These interactions control specific physical functions of host molecules, which give rise to the process of molecular recognition, which is of vital importance to biological chemistry. The process of molecular recognition lies at the heart of host-guest chemistry, because it is the nonbonded intermolecular interactions that are responsible for phenomena such as selfassembly of molecules, cooperative phenomena in biological macromolecules and the equilibria established between proteins and ligands. Thus, supramolecular chemistry, which has drawn together physics and biology with chemistry, is witnessing rapid developments, and with it several changes in chemical concepts and theories. It is expected that improved synthetic methodology and a better understanding of weak intermolecular interactions may lead to the design of highly selective and sophisticated schemes of host-guest recognition. 